\title{
Quantum-state specific scattering of molecules from surfaces
}

\author{
Dissertation
}

zur Erlangung des mathematisch-naturwissenschaftlichen Doktorgrades

"Doctor rerum naturalium"

der Georg-August-Universität Göttingen

im Promotionsprogramm Chemie

der Georg-August University School of Science (GAUSS)

\author{
vorgelegt von \\ Kai Golibrzuch \\ aus Northeim \\ Göttingen, 2014
}




\section{Betreuungsausschuss}

Prof. Alec M. Wodtke, Abteilung Wodtke, Institut für Physikalische Chemie/ Max-Planck-Institut für Biophysikalische Chemie

Prof. Dirk Schwarzer, Abteilung Wodtke, Institut für Physikalische Chemie/ Max-Planck-Institut für Biophysikalische Chemie

\section{Mitglieder der Prüfungskommission}

Referent: $\quad$ Prof. Alec M. Wodtke, Abteilung Wodtke, Institut für Physikalische Chemie/ Max-PlanckInstitut für Biophysikalische Chemie

Korreferent: Prof. Dirk Schwarzer, Abteilung Wodtke, Institut für Physikalische Chemie/ Max-PlanckInstitut für Biophysikalische Chemie

Weitere Mitglieder der Prüfungskommission:

Prof. Jörg Schröder, Abteilung Troe, Institut für Physikalische Chemie

Prof. Martin Suhm, Abteilung Suhm, Institut für Physikalische Chemie

Prof. Jürgen Troe, Abteilung Troe, Institut für Physikalische Chemie

PD Dr. Thomas Zeuch, Abteilung Suhm, Institut für Physikalische Chemie

Tag der mündlichen Prüfung: 12.09.2014 


\section{Danksagung}

First, I would like to thank Prof. Alec M. Wodtke for giving me the opportunity to do my dissertation in his group and for supplying all the nice experimental tools. He was always available for helpful discussions of my results. I also thank him for all the suggestions he made how to improve the experiment and the ideas he provided for new measurements, although I ignored most of them.

I want to acknowledge Prof. Daniel J. Auerbach for his help in understanding surface scattering over the past years. He was involved in almost every experimental study I did, especially in the discussion and interpretation of results.

Next, I thank Dr. Christof Bartels for the help he provided throughout my whole Ph.D. time in the group. $\mathrm{He}$ was in charge with a main part of the data analysis, especially in the NO/Au(111) work, and managed to resist my data bombardment from the laboratory. Furthermore, he was always available for and involved in almost all discussions of experimental results.

I thank Dr. Pranav R. Shirhatti for the help in the laboratory and in data analysis. He joined me as a postdoctoral fellow at in the middle of my Ph.D. work and was involved in last stages of the NO/Au(111) scattering as well as in all the hard work on CO scattering from $\mathrm{Au}(111)$ and $\mathrm{Pt}(111)$.

My special thanks go to Prof. Igor Rahinov from the Open University of Tel Aviv in Israel. He joined our group for six weeks a year and was mostly involved in NO scattering experiments. Thank you for joining me in the laboratory. It was a pleasure to work with you in the lab.

More very special thanks to Dr. Russel J. Cooper, who worked as a Ph.D. student on the same experiment when I started. He was a brilliant teacher in my first year and I learned from him about how to do surface science and how the whole experiment works at all.

I also want to acknowledge the mechanical and electronics work shop at the Institute for Physical Chemistry for their support during my Ph.D. time. Without them, some experiments wouldn't have been possible.

Next, I want to thanks my colleagues in the lab, especially Nils Bartels and Dr. Tim Schäfer, who tried to underlie the scientific atmosphere of multiple $10 \mathrm{~Hz}$ discharges and the $1 \mathrm{~Hz}$ pumping of the cryostat with some ambient music. It was always fun to work with you in the lab. I will never forget the times when we shot laser across the complete room.

I also thank the whole Wodtke group for the nice atmosphere you provided. I enjoyed working in the group a lot, not only because of the tough table soccer games after lunch (and in between).

Ganz besonders möchte ich auch meiner Familie danken. Meinen Eltern, Schwiegereltern, Großeltern und meiner Schwester danke ich für die Unterstützung während meines gesamten Studiums, dass ich ja bereits 2006 begonnen habe.

Zum Schluss, möchte ich mich vor allem bei meiner Frau Sara Remiorz-Golibrzuch bedanken. Sie stand in all den Jahren hinter mir und hat mich aufgemuntert, wenn es nötig war. Danke auch für das ab und zu vorhandene Verständnis, wenn ich einmal nicht so viel Zeit für sie hatte.

Vielen Dank für Alles!

Great thanks to everybody! 


\section{Abstract}

In my work, I investigated the quantum-state resolved scattering of three different diatomic molecules (NO, CO, $\mathrm{N}_{2}$ ) from different surfaces, including $\mathrm{Au}(111)$ and $\mathrm{Pt}(111)$. I focused on measurements of the energy transfer between the various degrees of freedom available using both state-of-the-art and new methods developed in the course of this work. I strove to investigate a few simple model systems with the goal of discovering generally valid rules for the coupling between different degrees of freedom of these simple model systems.

As a first system, I investigated vibrationally inelastic scattering of nitric oxide (NO) from a single crystal $\mathrm{Au}(111)$ surface, a system that has been extensively studied in the past and is thought to be well understood. I measured absolute vibrational excitation probabilities for $v=0 \rightarrow 1,2,3$ scattering as a function of surface temperature and incidence translational energy and compared the results to firstprinciples independent electron surface-hopping (IESH) theory as well as to an empirical state-to-state kinetic rate model. The excitation probabilities of $\mathrm{NO}(v=1,2,3)$ increase with surface temperature $\left(T_{S}\right)$ in an Arrhenius-like fashion under all conditions of my work. For each final vibrational state, I find that the Arrhenius activation energy is equal to the vibrational energy required for excitation which shows that the NO vibrational energy is taken from a thermal bath. Narrow angular distributions and early, narrow arrival time profiles indicate a direct scattering mechanism leading to fast recoiling molecules. The experimental observations allow for the conclusion that excitation into all vibrational states occurs upon coupling of the NO vibration to electron-hole pairs (EHPs) of the metal surface and that adiabatic (mechanical) coupling to phonons or translation is negligible. The comparison to predictions of firstprinciples IESH theory reveals quantitative agreement for $v=0 \rightarrow 1$ excitation but the theoretical predictions slightly underestimate the probabilities for $v=0 \rightarrow 2$ excitation and clearly underestimate $v=0 \rightarrow 3$ excitation. A detailed comparison of the excitation mechanisms reveals that this disagreement for scattering into final vibrational states $v_{f}>1$ results from an underestimation of overtone excitations in the scattering process.

Further failures of the current implementation of the IESH model appear in a comparison to measurements of incidence energy $\left(E_{I}\right)$ dependent $\mathrm{NO}(v=3 \rightarrow 1,2,3)$ relaxation probabilities. The experiments show that the probabilities for vibrational relaxation increase with incidence energy while the IESH simulations predict the opposite trend. A detailed trajectory analysis reveals that the theoretical model predicts a large fraction of multi-bounce collisions that increases with decreasing $E_{I}$. A selection of only single-bounce collisions improves the $E_{I}$ dependence but still does not reproduce the experimental observations. The single bounce results predict relaxation probabilities that do not depend on $E_{I}$. My results indicate that the overestimation of multi-bounce collision in the IESH model is probably related to a corrugated potential energy surface (PES) because multi-bounce artifacts occur also for simple adiabatic calculations on the ground-state PES. The failure might be directly related to a failure of the density-functional theory (DFT) calculations from which the PES was obtained.

As a final study on the NO/Au(111) system, I performed state-to-state time-of-flight experiments on scattering of incident $\mathrm{NO}(v=2,3)$ from $\mathrm{Au}(111)$ into different final vibrational and rotational states at various incidence energies. For the first time, my data shows that vibrationally inelastic scattering of NO from a metal surface can influence the final translational energy $\left(E_{f}\right)$ of the scattered molecules. I find that vibrational excitation leads to a decrease of $E_{f}$ while vibrational relaxation increases $E_{f}$. The amount of vibrational energy that couples to the translational motion ( $T \leftrightarrow V$ coupling) depends on incidence energy as well as on surface temperature. I speculate that the $T \leftrightarrow V$ coupling results from an EHP mediated energy transfer mechanism in which vibrational energy is first released (taken) into (from) 
EHPs which then couple to the translation motion. Furthermore, I observe that the dependence of $E_{f}$ on the final rotational energy $\left(E_{\text {rot }}\right)$ depends on incidence energy as well as on the final vibrational state. At higher $E_{I}$, the decrease of $E_{f}$ with $E_{\text {rot }}$ is similar for all vibrational channels. With decreasing incidence energy, $E_{f}$ gradually becomes independent of $E_{\text {rot }}$. This effect is more pronounced and occurs already at higher $E_{I}$ for vibrationally inelastic scattering. The mechanism for this observation remains unclear but the observations are in agreement with the expectation for dynamical steering effects or multi-bounce collisions that might become important at low $E_{I}$. Nevertheless, the data can act as an ideal benchmark for future new or improved theoretical models, which have to treat nonadiabatic as well as adiabatic interactions of the NO molecules with the Au(111) surface correctly in order to obtain reasonable agreement.

As next model systems, I investigated the scattering of $\mathrm{CO}$ molecules from $\mathrm{Au}(111)$ and $\mathrm{Pt}(111)$. The experiments on $\mathrm{CO} / \mathrm{Au}(111)$ scattering involve measurements of $v=0 \rightarrow 1$ excitation probabilities as well as measurements of $v=2 \rightarrow 2,1$ branching ratios. In both cases, I find that the probabilities for vibrational (de-)excitation first decrease with increasing $E_{I}$ but then increase again for $E_{I}>0.4 \mathrm{eV}$. Overall, the absolute excitation probabilities are about a factor of three lower than observed for $\mathrm{NO} / \mathrm{Au}(111)$. The results on $v=0 \rightarrow 1$ excitation are partly in agreement with expectations for trapping followed by desorption at low $E_{I}$ if one assumes complete equilibration with the surface. However, the time-of-flight distributions for scattering of incident $\mathrm{CO}\left(v_{i}=2\right)$ show that the assumption of complete equilibration with the surface in trapping-desorption is probably not valid in this system. The experimental data shows that incident $\mathrm{CO}\left(v_{i}=2\right)$ molecules can be trapped at the surface but are desorbed in $v_{f}=1,2$ prior to complete equilibration. This observation is direct evidence for vibrationally hot molecules, often referred to as hot precursors, at the surface. The experiments raise the question about the vibrational lifetime of $\mathrm{CO}$ adsorbed on $\mathrm{Au}(111)$ and whether it is similar to observed picosecond lifetimes found for $\mathrm{CO} / \mathrm{Pt}(111)$ or $\mathrm{CO} / \mathrm{Cu}(100)$.

I further measured $\mathrm{CO}(v=0 \rightarrow 1)$ excitation probabilities in scattering from $\mathrm{Pt}(111)$. The $\mathrm{CO} / \mathrm{Pt}(111)$ system exhibits broad angular distributions and $T_{S}$ dependent arrival time distributions. The excitation probabilities agree with the thermal expectation and reflect complete equilibration with the surface. The data supports vibrational excitation occurring due to trapping followed by desorption after equilibration with the surface. This is further supported by measured speed distributions for desorbing/ recoiling $\mathrm{CO}\left(v_{f}=0,1\right)$, which only show significant direct scattering for $v=0 \rightarrow 0$ scattering.

Furthermore, I used a new velocity selected residence time technique to investigate the desorption kinetics of $\mathrm{CO}$ from $\mathrm{Pt}(111)$ in real-time with microsecond resolution. I measured the time dependent flux of molecules leaving the surface at well-defined final velocity, $s_{f}$, as a function of surface temperature. I compare the results of previous studies to the experimental data using a simple firstorder kinetic rate model. The comparison demonstrates the capability of the method to judge the reliability of previous results; it is very sensitive to the choice of the kinetic parameters. Furthermore, the experimental data shows clear deviations of the experimental data from the first-order desorption kinetics reported previously. By comparison to a kinetic model involving surface diffusion and adsorption at step sites, I am able to assign the two processes to direct desorption from terraces and to step-toterrace diffusion followed by desorption from the terrace sites. Finally, I derive a binding energy of $E_{0}=1.43-1.51 \mathrm{eV}$ for $\mathrm{CO}$ adsorption at $\mathrm{Pt}(111)$ terraces using transition-state theory; the value is in agreement with recent heat of adsorption measurements.

As a last system, I measured vibrational excitation probabilities for $\mathrm{N}_{2}$ scattering from $\mathrm{Pt}(111)$ at various incidence energies ranging from 0.1-1.1 eV. I find again an Arrhenius-like dependence of the $v=0 \rightarrow 1$ excitation probability on the temperature of the surface with an activation energy equal to the 
vibrational energy uptake. The Arrhenius prefactors increase with increasing incidence energy with zero threshold and are about one order of magnitude lower than for NO/Au(111). Narrow and $T_{S}$ independent angular and time-of-flight distributions clearly indicate a direct scattering mechanism. Consequently, the experimental results exhibit all possible fingerprints of nonadiabatic V-EHP coupling for a molecule-surface system in which the very low electron affinity of the gas phase molecules seems to make electron transfer processes very unlikely. 


\section{Index}

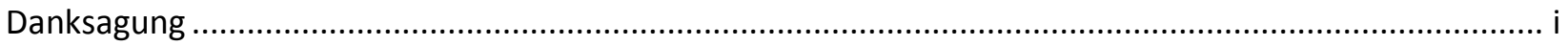

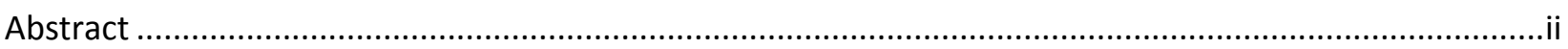

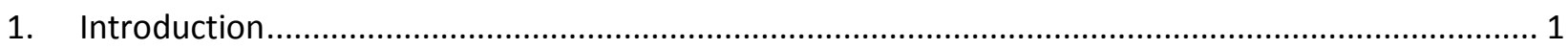

1.1. Born-Oppenheimer breakdown in gas-surface interactions ................................................ 3

1.1.1. Adiabatic and nonadiabatic vibrational energy transfer at surfaces .................................. 3

1.1.2. Nonadiabatic effects from first-principles theory ......................................................... 7

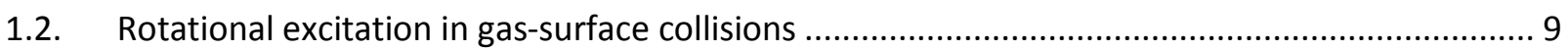

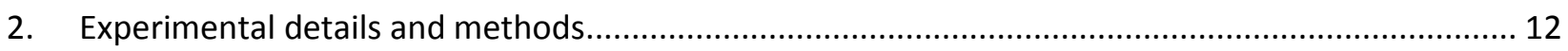

2.1. Molecular beam surface scattering in ultra-high vacuum ................................................... 12

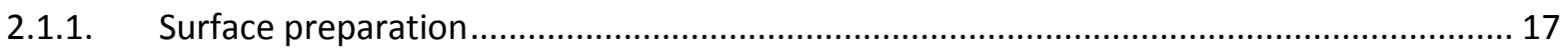

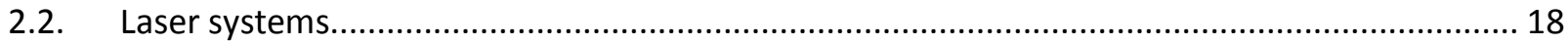

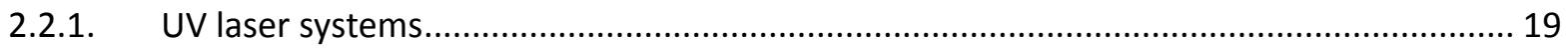

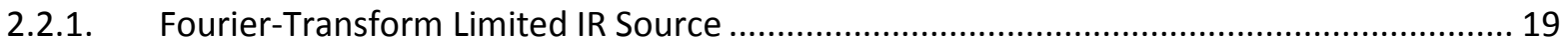

2.3. Resonance enhanced multi-photon ionization (REMPI) detection ......................................... 22

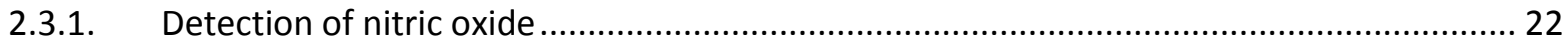

2.3.2. Electronic states and REMPI spectroscopy of carbon monoxide ...................................... 24

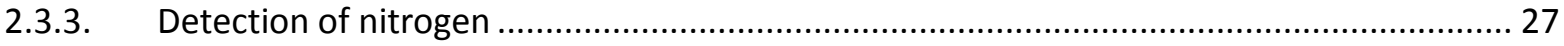

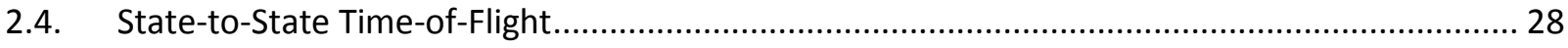

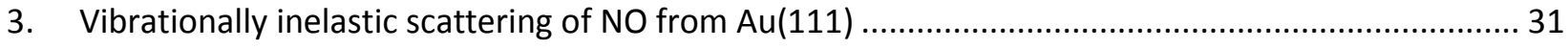

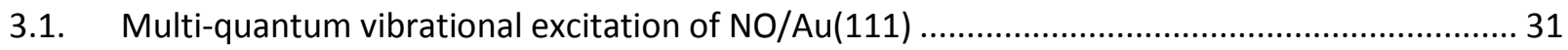

3.1.1. Determination of absolute excitation probabilities ....................................................... 32

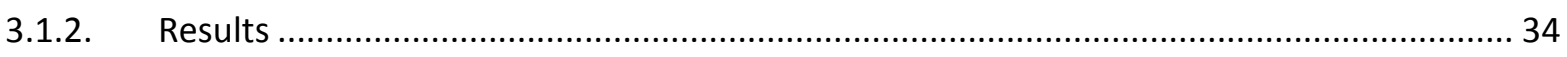

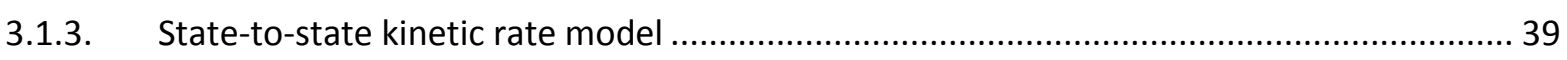

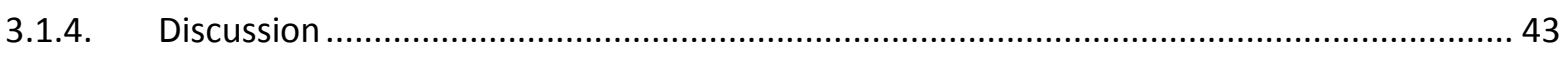

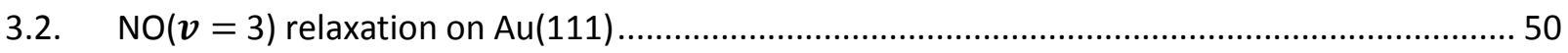

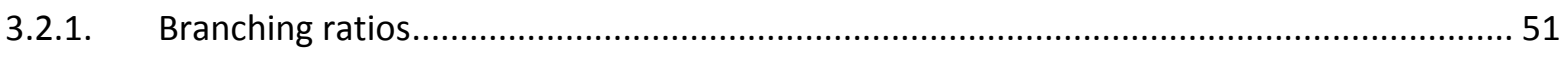

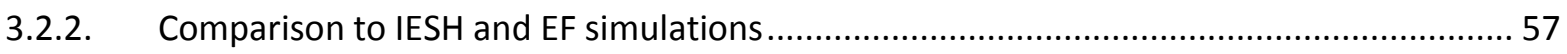

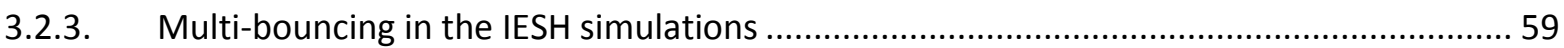

3.3. Translational energy transfer in rotationally and vibrationally inelastic $\mathrm{NO}(v=3) / \mathrm{Au}(111)$

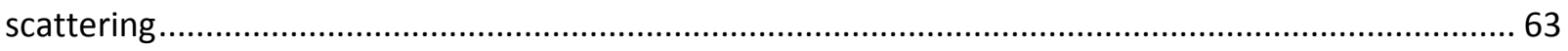

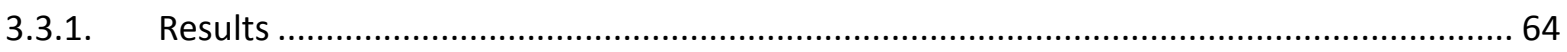

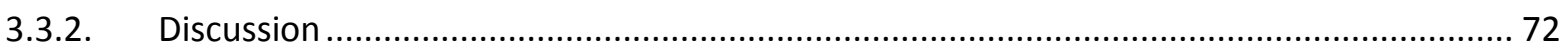

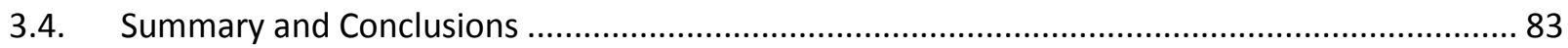

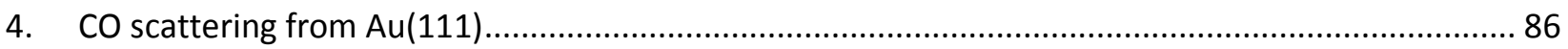

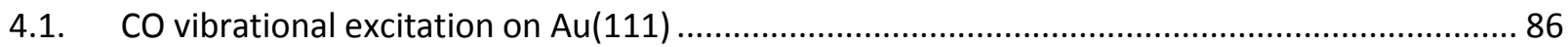




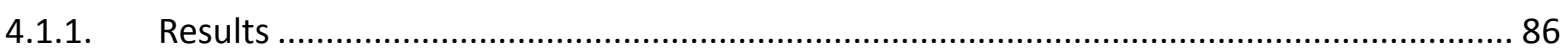

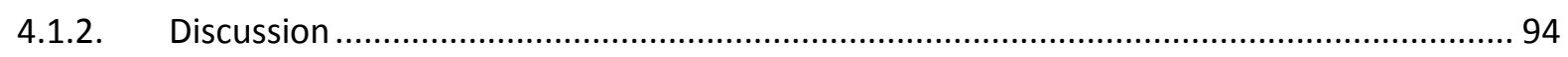

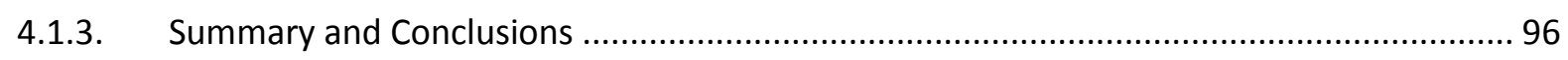

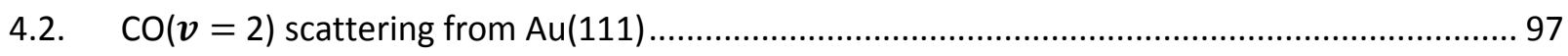

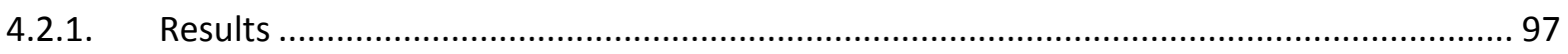

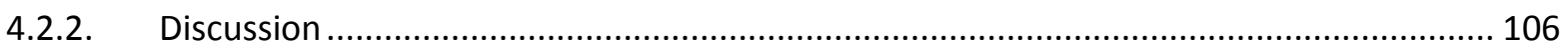

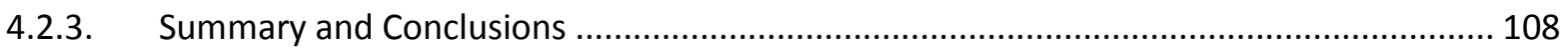

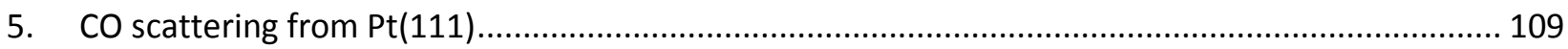

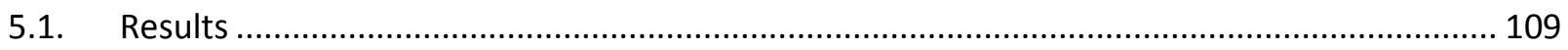

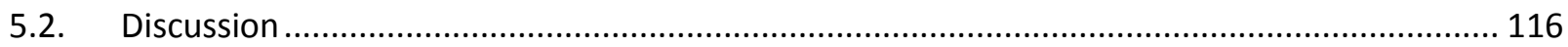

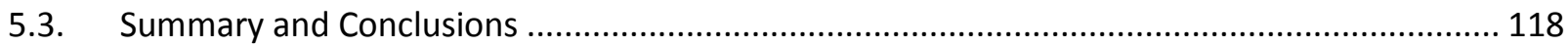

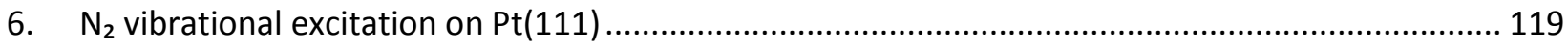

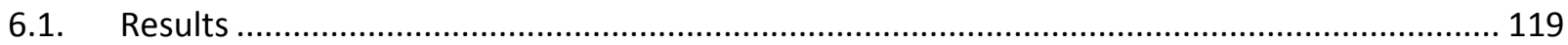

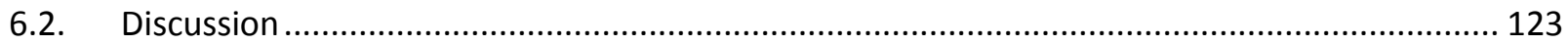

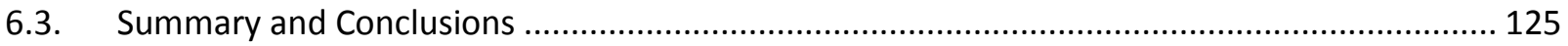

7. CO desorption from Pt(111) by velocity selected residence time measurements .......................... 126

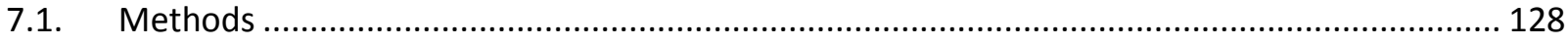

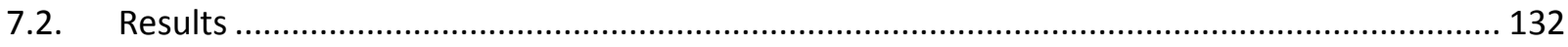

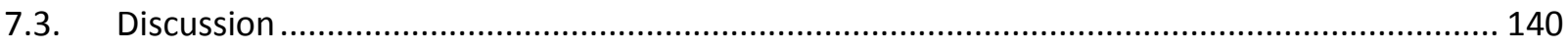

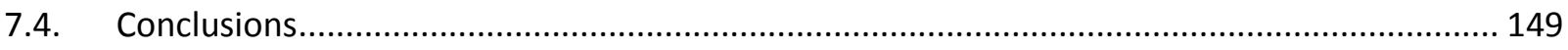

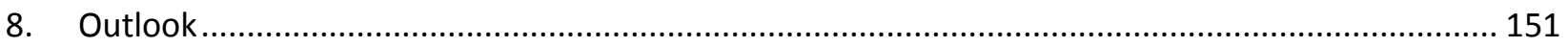

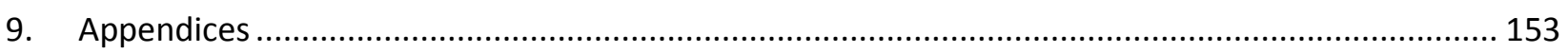

9.1. Appendix A: Gain curve of the micro-channel plate detector............................................... 153

9.2. Appendix B: Analysis of state-to-state time-of-flight spectra .............................................. 154

9.3. Appendix C: Forward convolution for fitting of NO time-of-flight distributions...................... 155

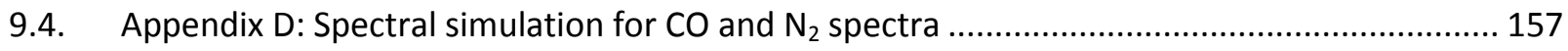

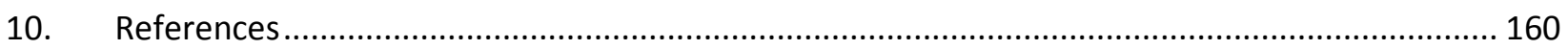

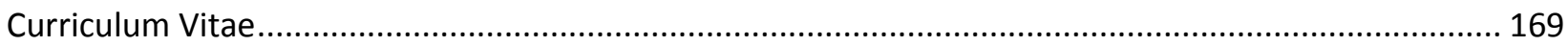




\section{Introduction}

Energy exchange at the gas-surface interface plays an important role in many phenomena that impact our daily lives. Examples include the production of commodity chemicals, fertilizer, and pharmaceuticals using heterogeneous catalysis, aerodynamic drag, heat transfer, and the processing of materials in microchip manufacturing. The processes occurring when a molecule collides with a surface are illustrated in Fig. 1.1. The impinging molecule can be scattered off the surface, accompanied with excitation of the internal degrees of freedom (DOFs) of the molecule (rotation, vibration) and the surface (phonons, electronic excitation), or it can be trapped in the physisorption or chemisorption well. The latter process can be followed by dissociation (dissociative chemisorption), a process that is involved in many chemical reactions at surfaces, e.g. the ammonia synthesis in the Haber-Bosch process (see Fig. 1.2) [6]. Over the last decades, molecular beam scattering from surfaces has been shown to be a powerful tool to study these elementary processes in detail [3, 9-35]. From a fundamental point of view, it is desirable to understand the different channels for energy transfer in detail for at least a few model systems that can act as benchmarks for comparison to predictions from first-principles theory. In order to achieve this goal, it is necessary to simplify the model system as much as possible to investigate the distribution of the final quantum states of the molecule and its dependence on the initial conditions. These requirements are met best for molecular beam scattering of diatomic molecules from clean low index single crystal surfaces [12] under ultra-high vacuum (UHV) conditions. Departures from these conditions introduce additional complexity. For example, even the simple change of considering already high index surfaces complicates matters because complexities associated with the high density of steps can lead to higher reactivity, which may dramatically affect the dynamics of the systems [36-38].

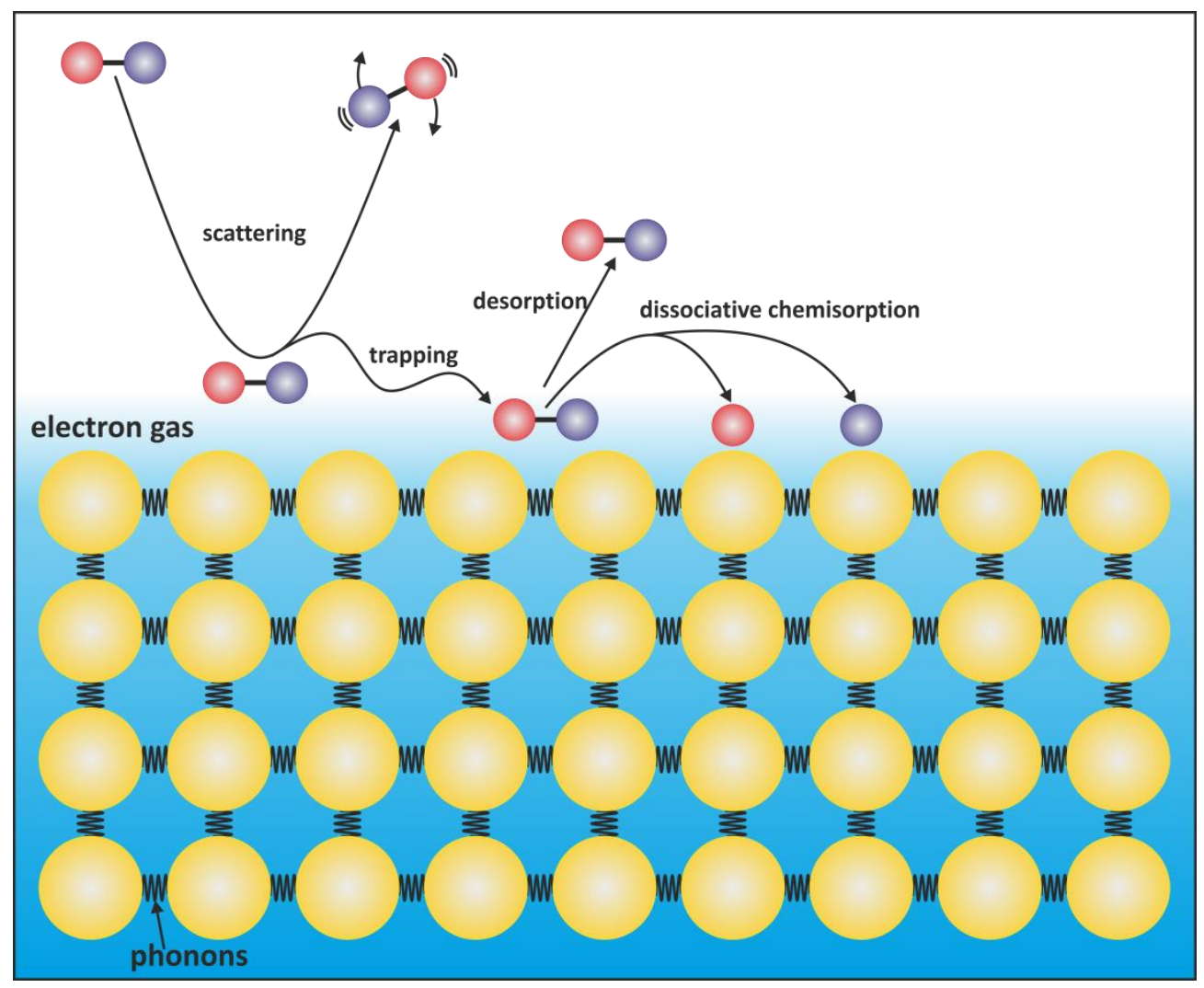

Fig. 1.1: Schematic overview of processes that can occur in gas surface collisions. 


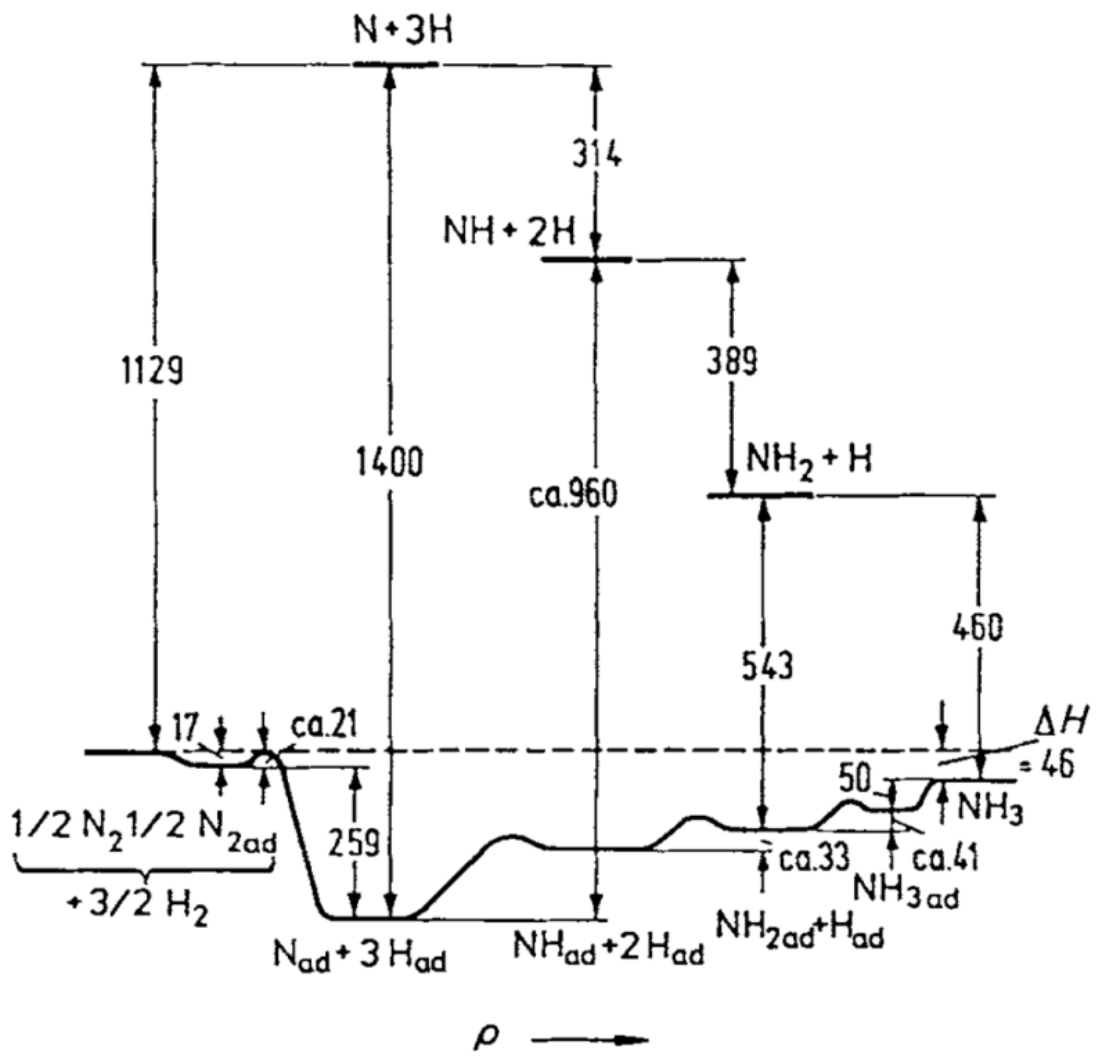

Fig. 1.2: Energy diagram of the ammonia synthesis and comparison of the energetics for heterogeneous catalysis and reaction in gas phase (energies in kJ/mole) [6]. Ertl, G., Elementary Steps in Heterogeneous Catalysis. Angew. Chem. Intern. Ed., 1990. 29(11), 1219-1227. Copyright Wiley-VCH Verlag GmbH \& Co. KGaA. Reproduced with permission.

The use of molecular beams as a source of gas phase molecules has the advantage that it provides very good control over the experimental conditions. The translational energy of the incident molecules is well-defined (narrow velocity distribution) and tunable (seeding in carrier gases) and the molecules appear predominantly in their rotational $\left(T_{\text {rot }} \approx 10 \mathrm{~K}\right)$ and vibrational ground states. Realistic conditions, like they occur in a reactor environment, would lead to an overly complex system that makes a detailed investigation of reaction mechanisms or coupling between different DOFs impossible. The control over the experimental conditions in quantum-state resolved scattering of diatomic molecules from clean, flat surface makes these systems ideal for comparisons to theoretical approaches from first principle that finally enables a detailed understanding of the dynamics of the system.

The different degrees of freedom - rotation, vibration and translation of the molecule as well as phonon and electronic excitation of the surface - of these simple systems can in principle couple to each other, which already illustrates the complexity of the observable interactions. In theoretical approaches the motion of the nuclei (translation, rotation, vibration and phonons) is often modeled as a classical motion on a single potential energy surface (PES) calculated from the energy of the system for different positions of the nuclei $[9,39]$. These kinds of calculations are based on the validity of the BornOppenheimer approximation (BOA) [40], the assumption that the nuclear motion can be decoupled from the electronic degrees of freedom of the system. This assumption holds quite often for gas phase molecules which is a result of the large gaps between different electronic states. However, there is an increasing number of studies showing that the BOA frequently fails to describe the interaction of gas phase molecules with a metal surface at which the electronic states of the metal form a continuum of states with infinitely small spacing. This phenomenon is often referred to as BOA breakdown [7, 41, 42]. 

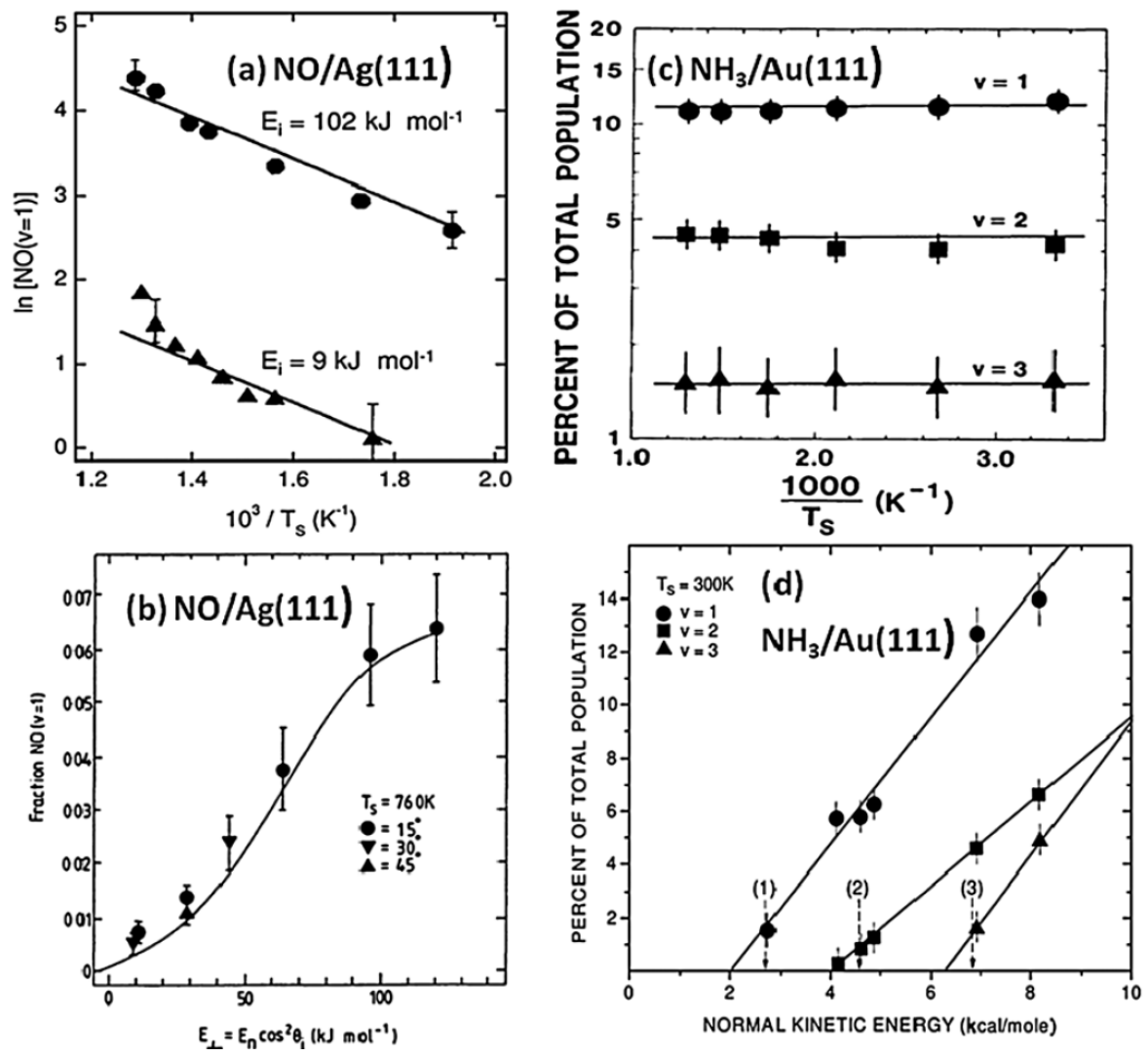

Fig. 1.3: Examples for adiabatic and nonadiabatic gas-surface interactions represented by vibrational umbrella-mode excitation of $\mathrm{NH}_{3} / \mathrm{Au}(111)$ [4] and vibrational excitation of $\mathrm{NO} / \mathrm{Ag}(111)$ [8]. Reprinted figure with permission from Kay, Raymond, and Coltrin, Physical Review Letters 59(24.), 2792-2794, 1987. Copyright 1987 by the American Physical Society. Reprinted figure with permission from Rettner et al., Physical Review Letters 55(18), 1904-1907, 1985. Copyright 1985 by the American Physical Society.

\subsection{Born-Oppenheimer breakdown in gas-surface interactions}

\subsubsection{Adiabatic and nonadiabatic vibrational energy transfer at surfaces}

The failure of the most frequently used approximation in theoretical chemistry at metal surfaces has attracted enormous attention over the last decades and is one of the topics treated in my work. Fig. 1.3 illustrates the basic difference between adiabatic and nonadiabatic gas-surface interactions for two of the most prominent examples in the field. Kay et al. studied the vibrational excitation of the $v_{4}$ umbrella vibrational mode of $\mathrm{NH}_{3}$ in scattering off $\mathrm{Au}(111)$ [4]. They found that the excitation probabilities increase linearly with incidence translational energy $\left(E_{I}\right)$ and exhibit clear thresholds when the kinetic energy reaches the energy required for vibrational excitation of one, two or three quanta of $v_{4}$ (Fig. $1.3 \mathrm{~d})$. Furthermore, the authors found that the $v_{4}$ excitation probability does not depend on surface temperature $\left(T_{S}\right)$ (Fig. 1.3c). Both features clearly indicate that the vibrational excitation of the umbrella mode in ammonia is due to energy transfer from incident translation and that it can be explained by a purely adiabatic (mechanical) mechanism.

For vibrational excitation of NO scattered off a $\mathrm{Ag}(111)$ surface, Rettner et al. observed a completely different behavior [8]. They found that the vibrational excitation probabilities increase exponentially with surface temperature. The $T_{S}$ dependence followed an Arrhenius-like behavior with an activation 

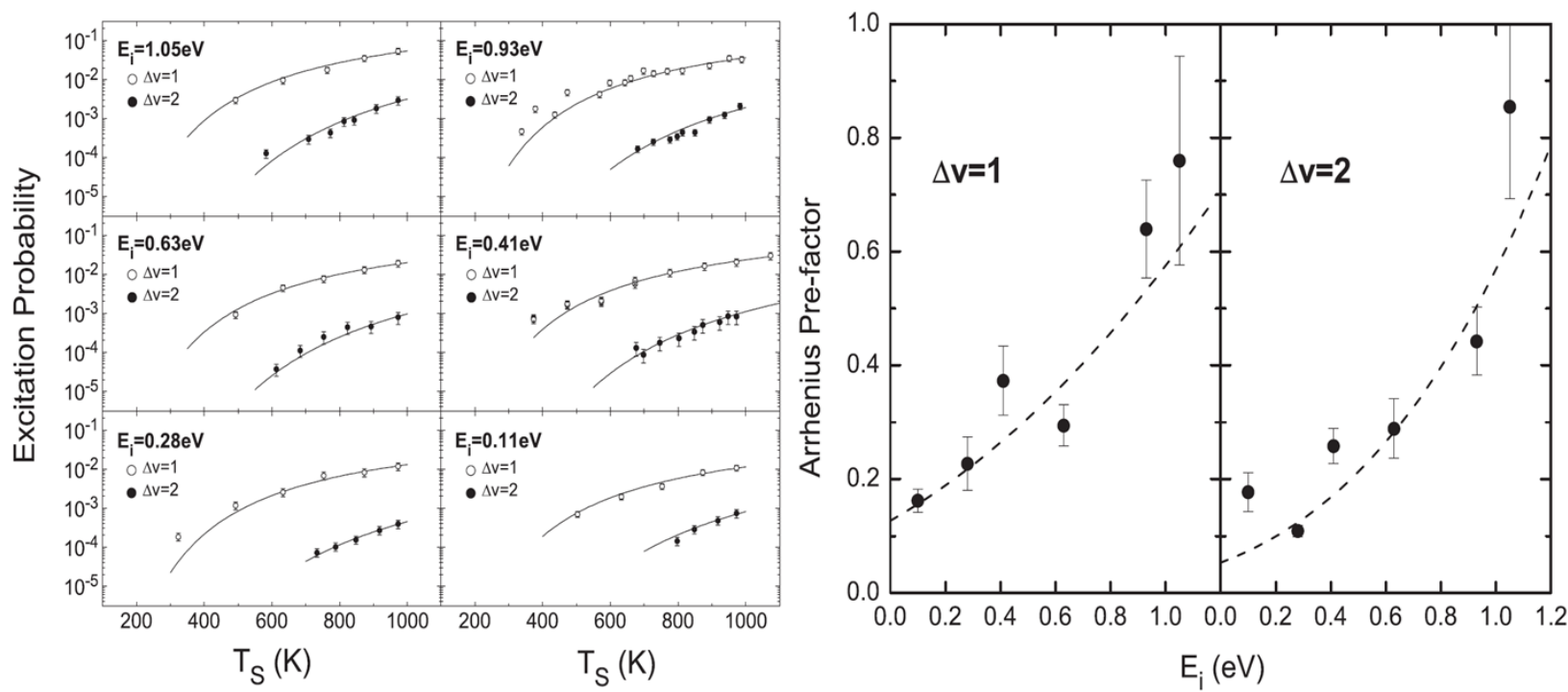

Fig. 1.4: Results of the absolute vibrational $\mathrm{NO}(v=0 \rightarrow 1,2) / \mathrm{Au}(111)$ excitation probability measurements by Cooper et al. [1]. Left panel: Absolute vibrational excitation probabilities for different incident energies ranging from $E_{I}=0.11-1.05 \mathrm{eV}$ with corresponding fits to Arrhenius functions of the form $P_{01}=A_{01} e^{\left(-\frac{0.236 e V}{k_{B} T S}\right)}$ and $P_{02}=A_{02} e^{\left(-\frac{0.476 e V}{k_{B} T S}\right)}$. Right panel: $E_{I}$ dependent Arrhenius prefactors for $\mathrm{NO}(v=0 \rightarrow 1)$ and $\mathrm{NO}(v=0 \rightarrow 2)$ excitation derived from the fits on the left. In both cases the prefactor increases with increasing $E_{I}$ with zero threshold. Reprinted with permission from Journal of Chemical Physics, 2012. 137(6), 064705-12. Copyright 2012, AIP Publishing LLC.

energy equal to the vibrational energy uptake of $0.236 \mathrm{eV}$ of the NO molecule (Fig. 1.3a). In addition, the authors studied the dependence of the $v=1$ excitation on the normal component of incident translational energy, $E_{\perp}=E_{I} \cos ^{2} \theta_{I}$. The excitation probability increases with increasing translational energy but, in contrast to the results for $\mathrm{NH}_{3}$ scattering, Rettner et al. did not observe any threshold for the excitation. The authors concluded that the experimental results were in agreement with a mechanism based on coupling of the NO vibration to thermally excited EHPs in the metal. Newns developed this idea further and related the mechanism for vibrational excitation to an electron transfer between the gas phase molecule and the Ag surface [43]. Consequently, vibrational excitation of NO scattering from $\mathrm{Ag}(111)$ is caused by nonadiabatic coupling of (nuclear) vibrational motion of the molecule to electron-hole pairs (EHPs) in the metal. The NO/Ag(111) system is an example for a system where the electronic DOFs influence the motion of the nuclei, thus for BOA breakdown.

The work of Rettner, Auerbach and co-workers [8, 10, 44] motivated several further studies on vibrational excitation of nitric oxide, especially on the NO/Au(111) system [1, 25, 27, 30]. These studies were focused on the understanding and modeling of the mechanism for vibrational excitation. Cooper et al. measured absolute vibrational $\mathrm{NO}(v=0 \rightarrow 1,2)$ excitation probabilities using various corrections to the experimentally observed temperature dependent REMPI spectra [1]. In agreement with the earlier results for $\mathrm{NO}(v=0 \rightarrow 1) / \mathrm{Ag}(111)$ scattering, they observed an Arrhenius-like dependence of the vibrational excitation probabilities on surface temperature for both final excited vibrational states $v_{f}=1,2$ with activation energies equal to one and two vibrational quanta. An overview of the results is shown in Fig. 1.4. Absolute excitation probabilities (left panel) were measured for different incidence energies ranging from $E_{I}=0.11-1.05 \mathrm{eV}$. All datasets are well represented by Arrhenius functions with activation energies equal to the vibrational energy uptake ( $0.236 \mathrm{eV}$ per quantum). In these Arrhenius fits, the exponential term corresponds to a thermal factor that reflects the temperature dependent availability of excited electron-hole-pairs that match the required energy, whereas the prefactor is related to the intrinsic coupling strength of the NO vibration to the EHPs of the metal $[1,8,27,30,43$, 44]. 

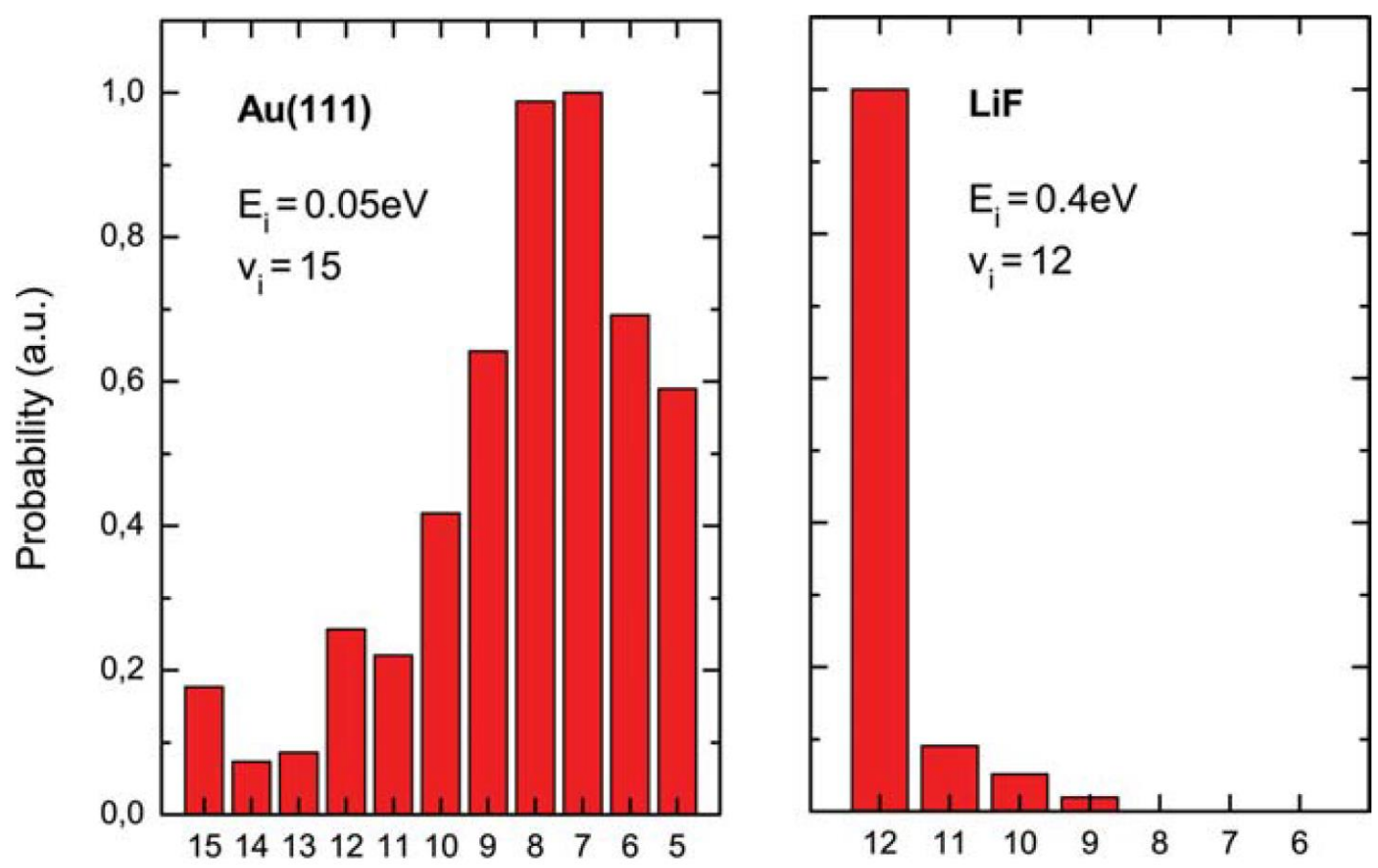

Final vibrational state

Fig. 1.5: Vibrational state distributions for scattering of highly vibrationally excited NO from Au(111) and LiF. The experiments showed multi-quantum relaxation on a $\mathrm{Au}(111)$ (metal) surface, whereas only little relaxation was found on LiF (insulator). From Science, 2000. 290(5489), 111-114. Reprinted with permission from AAAS.

The same authors also presented a more detailed analysis of the excitation mechanism. They introduced a kinetic rate model to describe the nonadiabatic transitions in the scattering event [27, 30]. This approach enabled a detailed analysis of the mechanism for excitation of $v=2$. Their results indicated that direct vibrational overtone excitation dominates $(\sim 70 \%)$ over a sequential $0 \rightarrow 1 \rightarrow 2$ mechanism. However, the model included several assumptions and therefore the result has to be treated with care.

In addition to experiments on vibrational excitation, several experiments were performed to study the opposite process of vibrational relaxation. Huang et al. scattered NO in high vibrational states, prepared by stimulated emission pumping [45], from $\mathrm{Au}(111)$ and LiF [3]. The results are shown in Fig. 1.5. For scattering of $\mathrm{NO}(v=12)$ off an insulating LiF surface, the authors found only very little relaxation into $v=11,10$ and 9 . A completely different behavior was observed for $\mathrm{NO}(v=15)$ scattering off $\mathrm{Au}(111)$ at $E_{I}=0.05 \mathrm{eV}$ : the experimental results showed multi-quantum vibrational relaxation that was observable down to $v=5$ while the distribution peaks at $v=7-8$. The difference between scattering off an insulator and a metal surface relies on the possibility of electron-hole pair excitations at the $A u(111)$ surface, which promote the vibrational energy transfer. A disadvantage of these experiments was the relatively low incidence energy of $E_{I}=0.05 \mathrm{eV}$ necessary to resolve the manifold of overlapping vibrational bands. However, this low $E_{I}$ certainly increases the chance that a substantial amount of the impinging NO molecules can be trapped at the surface.

While the experimental observation of vibrational excitation and relaxation represents a more or less indirect evidence for vibration-to-EHP (V-EHP) coupling, later work provided a more direct measure of the nonadiabatic interaction. White et al. used a Cs covered $\mathrm{Au}(111)$ surface in order to reduce the work function of the surface to about $1.3-1.6 \mathrm{eV}$ and to enable electron emission if sufficient vibrational energy is provided. Indeed the authors were able to show that electron emission from the surface occurs as soon as the vibrational energy of the incident NO molecules exceeded the work function of the 


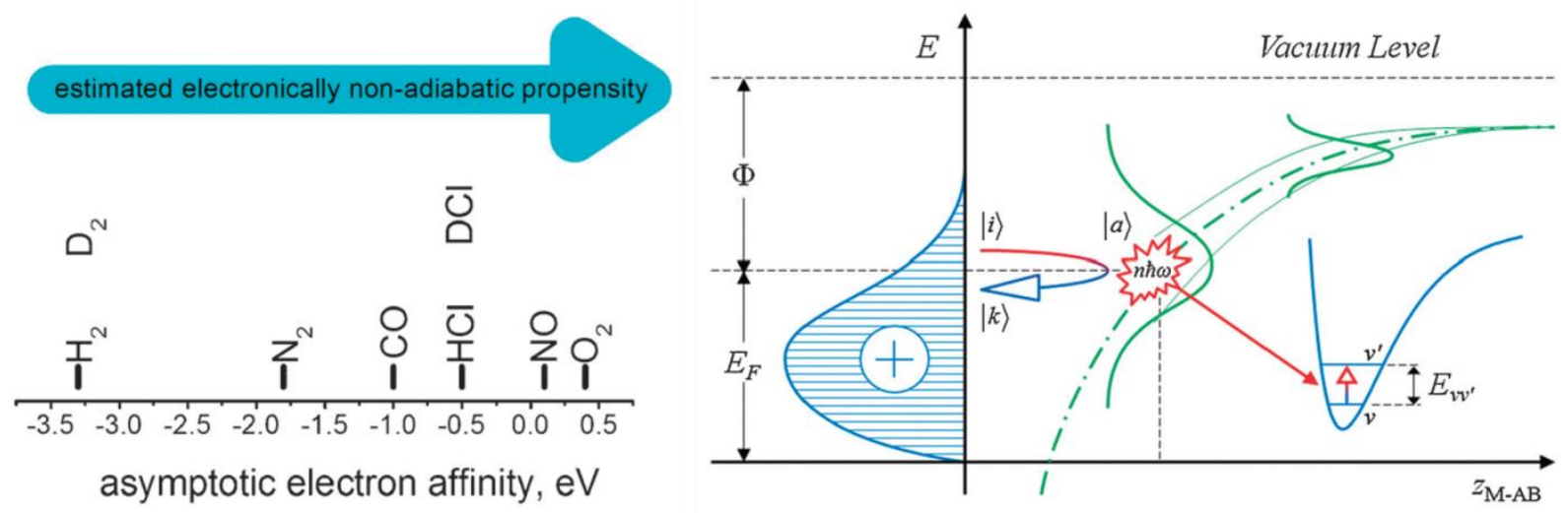

Fig. 1.6: Left: Estimated asymptotic electron affinity of different diatomic molecules [7]. It is assumed that the strength of the nonadiabatic coupling scales with the electron affinity of the molecule. Right: Lowering of the molecule electron affinity level upon approach to a metal surface [7]. The negative charge on the molecule is stabilized by Coulomb interaction with an image charge at the surface. Reproduced from ref. [7] with permission of the PCCP Owner Societies.

surface [34]. Moreover, the electron yield showed an inverse dependence on incident beam velocity [31] and finally measurements of the kinetic energy distributions of the emitted electrons demonstrated that nearly all the vibrational energy can be transferred to one single electron [29]. However, the modification of the surface raises the question how comparable a clean $\mathrm{Au}(111)$ and a $\mathrm{Cs}: \mathrm{Au}(111)$ surface really are. Au(111) itself is not reactive while the Cs layer on top will probably react with the colliding NO molecules. This stark difference in reactivity might change the dynamics of the system and lead to completely different results, for example regarding the dependence on $E_{I}$.

Another apparent issue is related to the open-shell character of NO: Do other molecules besides NO exhibit this nonadiabaticity? The number of other examples showing V-EHP coupling in molecular beam surface scattering is quite limited. Rettner tried to observe vibrational excitation in scattering of carbon monoxide from $\mathrm{Au}(111)$ [46]. He was able to observe rotational state distributions and trapping probabilities but could only derive the vibrational excitation probability for one set of parameters $\left(T_{S}, E_{I}\right)$ and estimated the upper limit for $\mathrm{CO}(v=0 \rightarrow 1)$ excitation to be less than $10^{-3}$. However, from his work it remained unclear whether the vibrational excitation occurs predominantly via direct scattering or trapping followed by desorption. A more complete picture of $\mathrm{CO}$ interacting with metal surfaces was derived from vibrational lifetime measurements of $\mathrm{CO}$ adsorbed on insulators and metals [47-53]. For $\mathrm{CO}$ adsorbed on a $\mathrm{NaCl}(100)$ surface, the vibrational lifetime was measured by infrared fluorescence to be $4.3 \mathrm{~ms}$, whereas it decreased dramatically to $2-5 \mathrm{ps}$ for $\mathrm{CO}$ adsorbed on $\mathrm{Cu}(100)$ and $\mathrm{Pt}(111)$. The difference is again the availability of EHPs for the metal surfaces. The vibrational energy dissipation into EHPs is supported by several further theoretical studies, which also showed that coupling of the high frequency $\mathrm{CO}$ stretching vibration to low frequency surface phonons is inefficient $[47,51,52,54-56]$.

Ran et al. observed vibrational excitation in $\mathrm{HCl}$ scattering from $\mathrm{Au}(111)$ [33]. Interestingly, the authors found a change in vibrational excitation mechanism with increasing surface temperature in this system. Below $600 \mathrm{~K}$ surface temperature the $\mathrm{HCl}(v=0 \rightarrow 1)$ excitation probability is independent of $T_{S}$ but increases with increasing incidence energy, whereas it increases exponentially with increasing $T_{S}$ in an Arrhenius-like fashion for higher surface temperatures $T_{S}>600 \mathrm{~K}$. The authors fitted the results to a simple model assuming an adiabatic and a nonadiabatic term. The adiabatic coefficient exhibited a linear increase with increasing $E_{I}$ with a threshold around $0.57 \mathrm{eV}$, which is $\sim 0.21 \mathrm{eV}$ higher than the vibrational energy uptake $\left(\Delta E_{v i b}=0.36 \mathrm{eV}\right)$ of the molecule. For the nonadiabatic Arrhenius term with $E_{A}=E_{v i b}$ the prefactors were found to also increase approximately linearly with $E_{I}$ but with zero threshold. Rahinov et al. studied the vibrational relaxation of $\mathrm{HCl}(v=2)$ scattering off $\mathrm{Au}(111)$ and 
observed about $10 \%$ relaxation into $v=1$. However, this study was focused on the effect of vibrational relaxation on the final translational energy of the scattered $\mathrm{HCl}(v=1,2)$ molecules [32].

$\mathrm{H}_{2}$ dissociation on and scattering off $\mathrm{Cu}(111)$ is another example that has been studied extensively experimentally as well as theoretically [57-59]. In this system, vibrational excitation of hydrogen was studied as function of incident kinetic energy. In case of the $\mathrm{H}_{2} / \mathrm{Cu}(111)$ system neither a nonadiabatic nor a mechanical excitation of the $\mathrm{H}_{2}$ vibration could explain the experimental observations. The coupling in this system was caused by the adiabatic potential energy surface (PES) used to describe the dissociative adsorption [60]. The curvature of the PES around the transition state, at which the $\mathrm{H}-\mathrm{H}$ bond is stretched, caused the observed coupling between incidence translational energy and vibrational excitation [61].

\subsubsection{Nonadiabatic effects from first-principles theory}

A first step toward understanding of gas-surface interactions is the comparison of experimental observations to first-principles theory. In general, both theory and experiment will benefit from such a comparison. Some experimental results are hard to understand in detail without comparison to theoretical models/ simulations whereas a theoretical model has to be tested by qualitative and quantitative comparison to high quality experimental data to prove its validity. The biggest problem of modern theoretical chemistry is still the fact that the accurate quantum mechanical description of a system is often far too complex to be treated with the available computational resources. This is especially the case for gas-surface interactions in which many atoms have to be considered. Therefore, modern theories still have to make certain approximations to enable a treatment of a system. First, many theoretical studies are based on the validity of the Born-Oppenheimer approximation [40], the decoupling of nuclear and electronic motion. The presented experimental studies, especially on NO scattering from metal surfaces, already illustrated that the BOA breaks down frequently at metal surfaces where the electronic states are infinitely closely spaced and form a continuum of states. Second, the size of the system requires the use of density functional theory (DFT) $[62,63]$ because more accurate methods are computationally too expensive. A problem with DFT calculations is that the results always have to be taken with care and often depend strongly on the choice of the exchange correlation (XC) functional. Furthermore, it is well-known that DFT frequently fails to describe charge transfer which is known to be important at metal surfaces. Third, a complete quantum mechanical treatment of all degrees of freedom is often not possible and theoretical studies use the classical approximation which, for example, neglects the zero point energy of the system. Finally, some theoretical studies use the BornOppenheimer static surface (BOSS) model and neglect the thermal motion of the surface atoms. All these approximations can influence the theoretical results if they are not applicable to the considered system. For example, theoretical calculations showed that the BOSS model fails in description of $\mathrm{H}_{2}$ dissociation on $\mathrm{Cu}(111)$ [58]. Another example is methane dissociation on $\mathrm{Ni}(111)$ where the movement of a $\mathrm{Ni}$ atom out of the surface can significantly influence the activation barrier of the reaction $[18,19]$. 

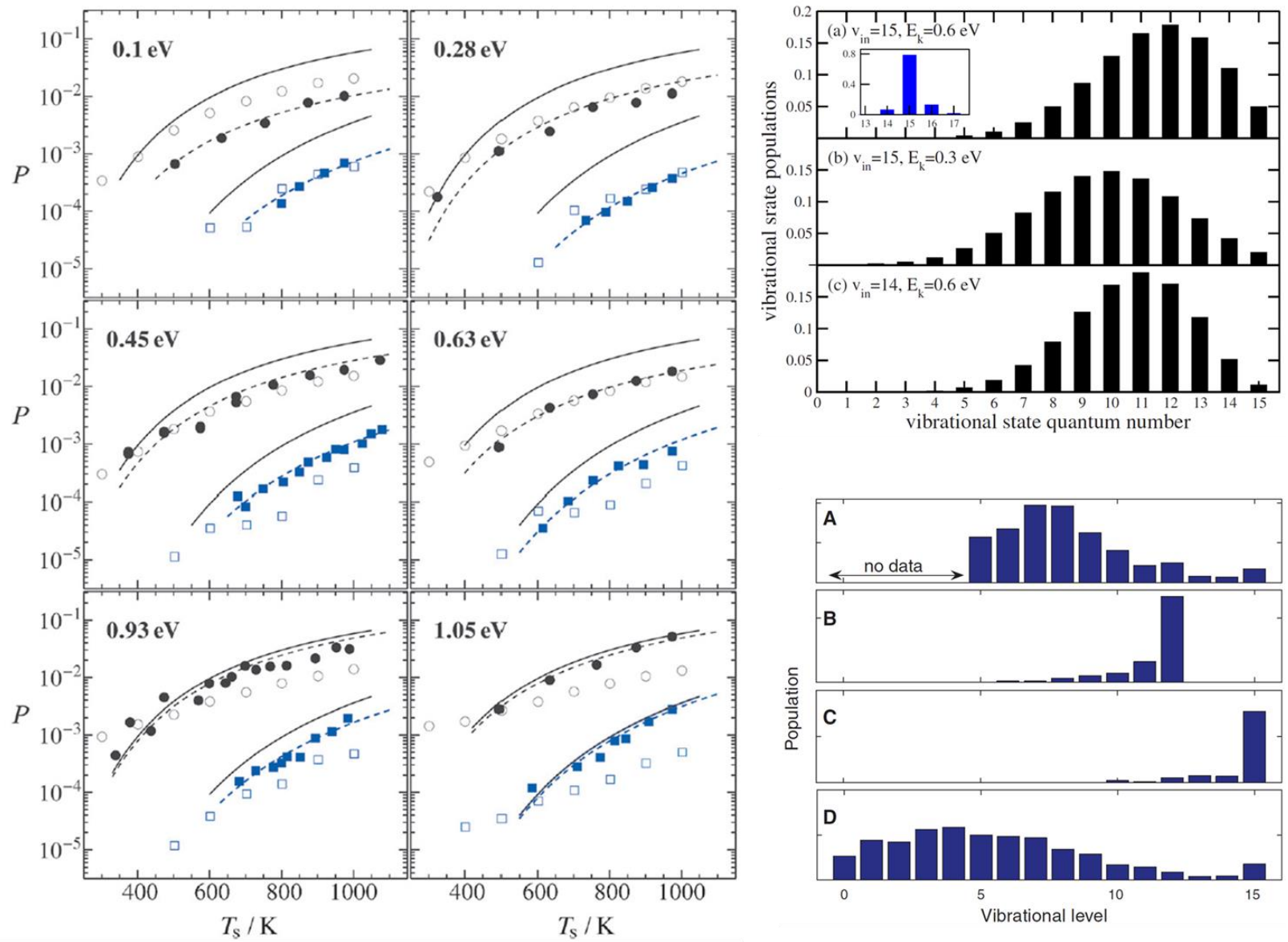

Fig. 1.7: Left panel: Comparison of experimental (solid symbols) and theoretical (open symbols) values for vibrational $\mathrm{NO}(v=0 \rightarrow 1,2)$ excitation probabilities on Au(111) [25] (Cooper et al., Multiquantum Vibrational Excitation of NO Scattered from Au(111): Quantitative Comparison of Benchmark Data to Ab Initio Theories of Nonadiabatic Molecule-Surface Interactions. Angewandte Chemie, 2012. 124(20), 5038-5042. Copyright Wiley-VCH Verlag GmbH \& Co. KGaA. Reproduced with permission.). For relaxation of highly vibrationally excited $\mathrm{NO}(v=12,15)$ from $\mathrm{Au}(111)$ experimental results (Fig. 1.5) could be qualitatively reproduced by electronic friction (upper right panel, Reprinted figure with permission from Monturet and Saalfrank, Physical Review B, 82(7), 075404, 2010. Copyright 2010 by the American Physical Society.) and IESH theory (lower right panel, from Science, 2009. 326(5954), 829-832. Reprinted with permission from AAAS). In both theories turning off the non-adiabatic coupling lead to almost no relaxation (inset in upper right panel, panel $\mathrm{C}$ in the lower right panel).

The failure of DFT, which is often used to calculate the potential energy surface for molecular dynamics (MD) simulations, is probably best demonstrated in the highly controversial studies on the (at first blush) simple dissociation of $\mathrm{O}_{2}$ on $\mathrm{Al}(111)$. The experimental results of Osterlund et al. [66] showed that the dissociative adsorption of oxygen on the surface is an activated process. The activation barrier is not present in DFT calculations, which predict a sticking probability of 1 independent of incidence energy [67]. The same authors also performed DFT calculations but restricting the system to stay on the triplet potential energy surface [67]; the resulting observation of a barrier raised the question about spin selection rules. However, more recent work of Libisch et al. showed that the absence of the barrier is probably related to a failure of DFT. The authors used embedded correlated wave function methods to calculate a 2D PES and observed a barrier in the reaction, in agreement with experiment [68]. The difference in this work to conventional DFT calculations is that the correlation wave functions method is able to treat charge transfer, likely from the Al surface to the $\mathrm{O}_{2}$ molecule, correctly. 
My work is mostly focused on nonadiabatic interactions in collisions of gas phase molecules with metal surfaces, thus on systems where the BOA breaks down. In surface chemistry there are mainly two different approaches to take nonadiabatic coupling of electron-hole pairs of the metal and the nuclear motion of the molecules into account. First, there is electronic friction theory, which is based on a weak coupling between the nuclear motion and the electronic system [47, 69-71], and second independent electron surface hopping (IESH), which, in principle, can be used for larger nonadiabatic couplings and is based on an approach where the system is allowed to switch between different adiabatic potential energy surfaces [72-74]. Electronic friction theories were successfully applied to calculate vibrational lifetimes of $\mathrm{CO}$ on metal surfaces $[47,72]$ as well as they could qualitatively reproduce the experimental observations for $\mathrm{NO}(v=15)$ relaxation in scattering from $\mathrm{Au}(111)$ (upper right panel in Fig. 1.7) [64]. However, electronic friction theory was not able to reproduce experimentally observed excitation probabilities [25]. The failure is likely related to the weak coupling approximation in the friction model that might not hold for the NO/Au(111) system.

Shenvi et al. showed that the IESH model gives qualitative agreement to the results for vibrational relaxation of $\mathrm{NO}(v=15)$ [65]. In contrast to friction approaches, the surface hopping model also produces vibrational excitation and the theoretical prediction shows semi-quantitative agreement with the experimental observations [25]. However, it appeared from comparison of Cooper et al. that the incidence energy dependence on the vibrational excitation is not treated correctly in the theoretical model. The simulation gave almost quantitative agreement at medium incidence energies $E_{I}=0.45$ $0.63 \mathrm{eV}$ but the model overestimated the excitation probabilities at low $E_{I}$ and underestimated excitation at high incidence energies. Before my work, a comparison for relaxation at low vibrational states has not been performed but offers a suitable additional test for the theoretical approaches that are available up to now. Moreover, relaxation of NO in low vibrational states can be measured experimentally with much higher precision than excitation probabilities or relaxation of high $v$ states.

\subsection{Rotational excitation in gas-surface collisions}

Studies on the vibrational energy exchange in gas-surface scattering demonstrated that this process mostly exhibits nonadiabatic interactions because mechanical coupling to high frequency stretching vibrations is inefficient. However, diatomic molecules provide two additional degrees of freedom, rotation and translational motion. The excitation of rotational degrees of freedom has been studied for various molecules and surfaces [10,11, 13, 46, 75-79] in the past. In systems for which direct scattering was observed, the rotational state distributions showed large excitation of high $J$ states. This phenomenon is frequently referred to as rotational rainbow scattering. Some examples of rotational state distributions for $\mathrm{NO} / \mathrm{Ag}(111)$ [10], $\mathrm{CO} / \mathrm{Au}(111)$ [46], $\mathrm{N}_{2} / \mathrm{Ag}(111)$ [11] and $\mathrm{HCl} / \mathrm{Au}(111)$ [75] scattering are shown in Fig. 1.8. In all these systems, the rotational distributions show thermal components (linear on a Boltzmann plot) at low $J$ with strong deviations from the thermal behavior at high $J$ states. In other words: the scattering produces a population in high rotational states that exceeds the expectation of the thermal Boltzmann distribution.

The reason for this behavior is an orientation dependent translation-to-rotation energy transfer that can be imagined in a simple classical picture. If the molecule collides with the surface exactly head-on or side-on, the collision cannot transfer any perpendicular momentum to angular momentum and the molecules will not be rotationally excited. If the impingent molecule approaches the surface in a tilted orientation, the molecule can become rotationally excited. The final rotational energy can be calculated from simple considerations of conservation of momentum and energy. The rotational distribution can be used to make conclusions about the interaction potential of the molecule with the surface [75]. In case 
of $\mathrm{NO}$ it is well known that the $\mathrm{N}-\mathrm{Ag}$ potential is attractive whereas the O-Ag potential has only repulsive interactions [9, 39]. Rotational state distributions for $\mathrm{NO}$ scattering from $\mathrm{Ag}(111)$ have been reproduced by stochastic trajectory calculation on a single PES constructed by Muhlhausen et al. [39]. Furthermore, a combined experimental and theoretical study demonstrated that the translational energy transfer to the surface (phonons) changes with the orientation of the molecule at impact. Kimman et al. measured the final translational energy for $\mathrm{NO}(v=0)$ scattering from $\mathrm{Ag}(111)$ as a function of the final rotational state and compared the results to trajectory simulations using the same interaction potential that could reproduce the rotational distributions (Fig. 1.9) [9]. Their observations indicated that the gain in rotational energy was not completely compensated by an equivalent loss of translational energy. The authors reported that both translation-to-rotation and translation-to-phonon energy transfer depend on the orientation of the molecule when it collides with the surface: a tilted orientation of the molecule leads to rotational excitation but only small energy transfer to the lattice phonons, whereas head-on collisions lead to no or little rotational but maximum phonon excitation. The same behavior was also observed for $\mathrm{N}_{2} / \mathrm{Ag}(111)$ scattering [13] and is expected to be a general phenomenon in direct scattering of molecules from surfaces.
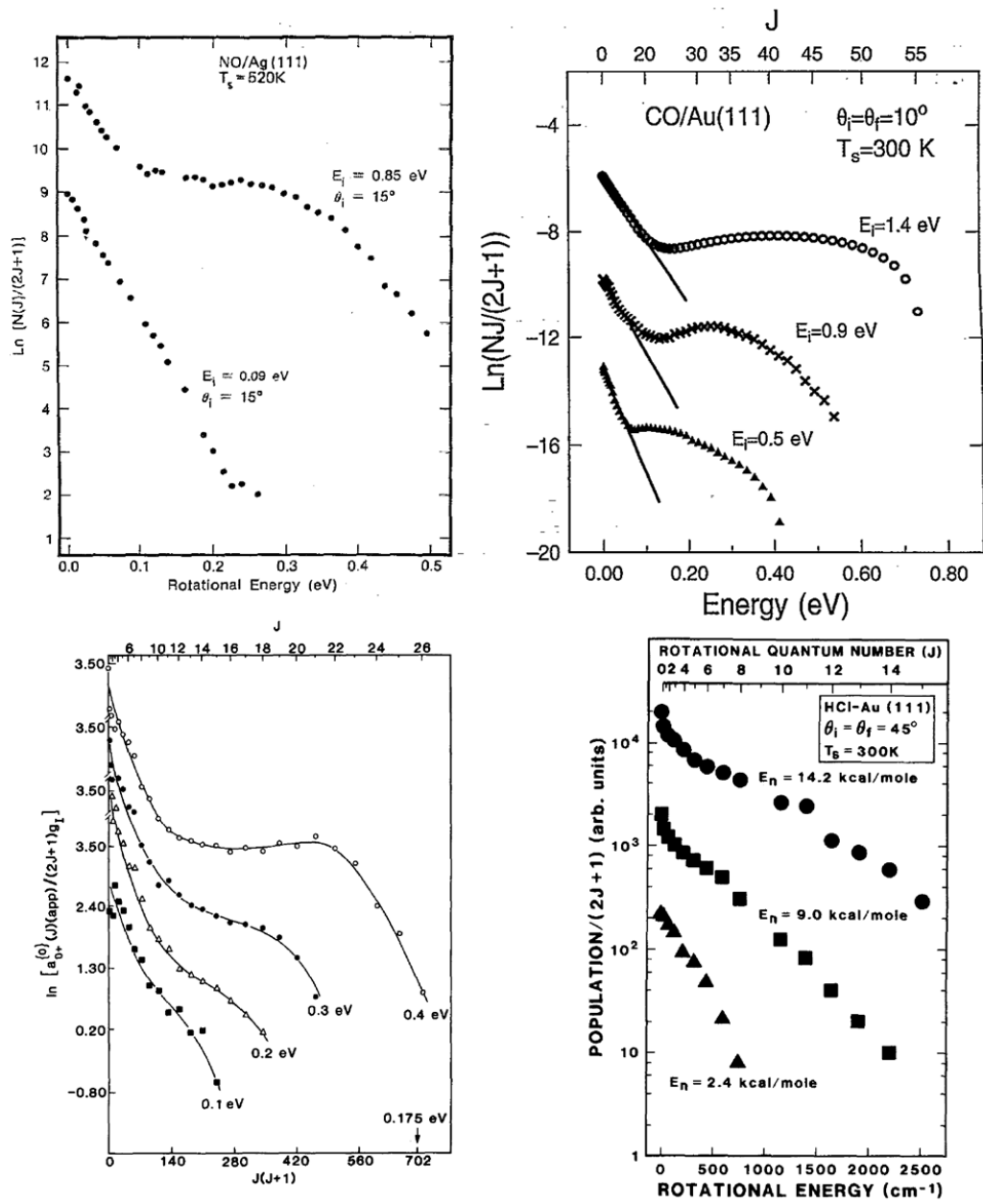

Fig. 1.8: Boltzmann plots of rotational state distributions for $\mathrm{NO} / \mathrm{Ag}(111)$ [10] (upper left panel, reprinted with permission from The Journal of Chemical Physics, 1991. 94(1), 734-750. Copyright 1991, AIP Publishing LLC.), CO/Au(111) [46] (upper right panel, reprinted with permission from The Journal of Chemical Physics, 1993. 99(7), 5481-5489. Copyright 1993, AIP Publishing LLC.), $\mathrm{N}_{2} / \mathrm{Ag}(111)$ [13] (lower left panel, reprinted with permission from The Journal of Chemical Physics, 1988. 89(4), $2558-2571$. Copyright 1988, AIP Publishing LLC.) and $\mathrm{HCl} / \mathrm{Au}(111)$ [75] (lower right panel, reprinted with permission from The Journal of Chemical Physics, 1990. 92(4), 2614-2623. Copyright 1990, AIP Publishing LLC.) scattering. All examples represent rotational distributions for direct scattering with pronounced rotational rainbows. 

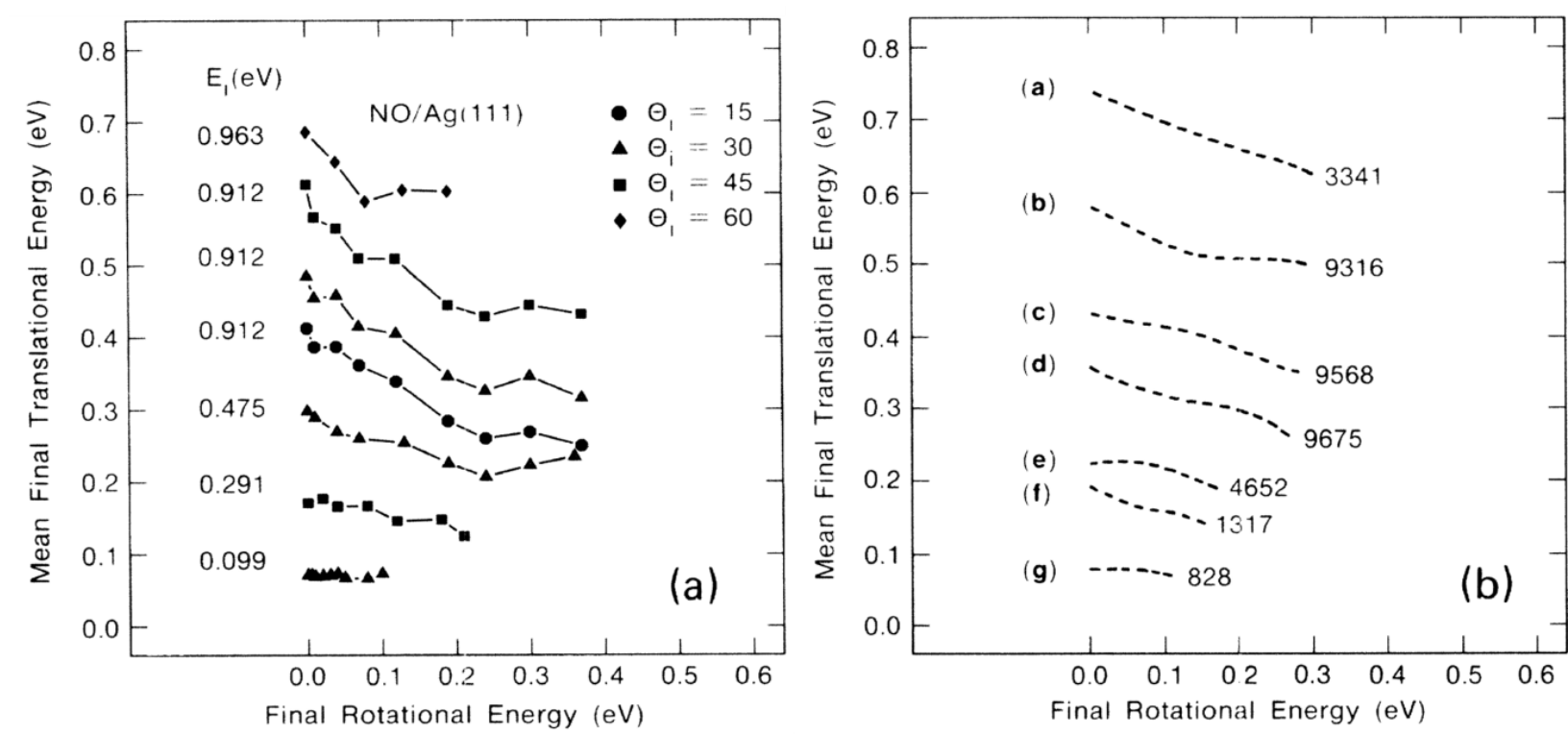

Fig. 1.9: Mean final translational energies as a function of final rotational energy for $\mathrm{NO}(v=0 \rightarrow 0)$ scattering from $\mathrm{Ag}(111)$ [9]. (a) Experimental results, (b) simulation using a semi-empirical model of Muhlhausen et al. [39]. Reprinted figure with permission from Kimman et al., Physical Review Letters 57(16), 2053-2056, 1986. Copyright 1986 by the American Physical Society.

In addition to this anti-correlation between translational energy transfer to phonons and to rotation (T-R anti-correlation), Kimman et al. found that the T-R curves approach a slope of zero with decreasing $E_{I}$. In other words, the final translational energy becomes independent of the final rotational energy of the molecule for low incidence energies. The authors were not able to make conclusions about the mechanism for this observation only from the experimental results. They used trajectory simulations, which showed quantitative agreement with the experiment, and concluded that this effect was related to an increasing fraction of multi-bounce collisions with decreasing $E_{I}$ that scrambled the T-R coupling. Similar behavior was observed and related to the same mechanism for NO/Pt(111) [80, 81] and $\mathrm{N}_{2} / \mathrm{Cu}(111)$ [11] scattering. However, all these studies were limited to scattering of molecules in their vibrational ground states. A work of $\mathrm{NO}(v=2)$ scattering from $\mathrm{Au}(111)$ showed that molecules that were scattered vibrationally inelastically were less rotationally excited than those molecules that were scattered into the same vibrational state [34]. This rotational cooling effect was associated with orientation effects promoting the V-EHP coupling. Therefore, orientations that favor nonadiabatic interaction lead to less rotational excitation. Recently it was shown that these effects are indeed present and that $\mathrm{N}$-first orientations lead to enhanced vibrational relaxation while $\mathrm{O}$-first orientations decrease the vibrational relaxation probability but increase the rotational excitation in the vibrationally elastic channel $[35,65]$. 


\section{Experimental details and methods}

\subsection{Molecular beam surface scattering in ultra-high vacuum}

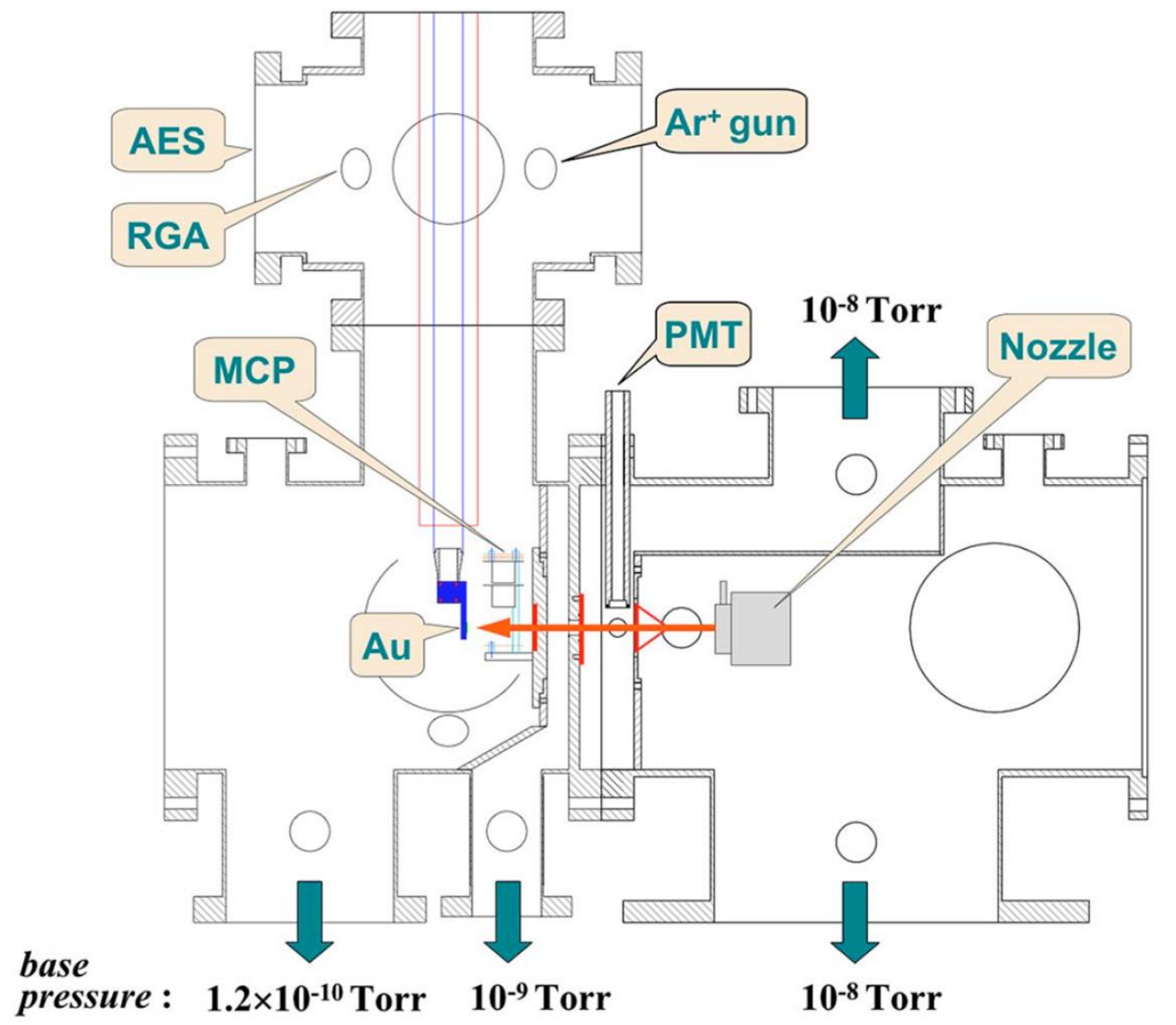

Fig. 2.1: Schematic drawing of the vacuum apparatus for molecular beam scattering [82]. A pulsed molecular beam is generated by supersonic expansion of gas mixtures in a piezo electrically driven nozzle (3atm stagnation pressure, $\mathrm{FWHM}=70 \mu \mathrm{s}$ ). It passes two stages of differential pumping before it enters the main chamber. The beam can be blocked by a slide valve in between the first and second differential pumping state. The ultra-high vacuum scattering chamber is equipped with the sample mounted on a 4-axes manipulator, an ion detector (ion lens, micro-channel plates), Auger electron spectrometer, Argon ion gun and residual gas analyzer. Reprinted with permission from Review of Scientific Instruments, 2007. 78(10), 104104. Copyright 2007, AIP Publishing LLC.)

The basic experimental apparatus has also been described elsewhere [82] and is shown schematically in Fig. 2.1. In order to ensure a clean sample surface throughout the duration of the experiment it is crucial to clean and store the sample under ultra-high vacuum conditions. The definitions of different ranges of vacuum conditions are given in Table 2.1 [83]. The most important criterion for scattering of molecules from clean single crystalline surfaces is the time for formation of one monolayer of adsorbed molecules on the surface. From this point on, the experiments would reflect scattering from an adsorbate rather than from a clean metal surface. Even under high vacuum conditions at pressures of $10^{-3} \ldots 10^{-6}$ Torr, the surface is covered within milliseconds to seconds. The calculations of Table 2.1 illustrate nicely that molecule-surface scattering experiments require ultra-high vacuum (UHV) conditions to ensure a clean surface throughout a complete day of experimental work. The UHV chamber used in this work typically reaches a base pressure of $2 \times 10^{-10}$ Torr, which corresponds to at least 7 hours until formation of a monolayer of adsorbed molecules at the surface. 
Table 2.1: Definition of different types of vacuum adapted from ref.[83].

\begin{tabular}{c|c|c|c|c|c} 
Naming & $\begin{array}{c}\text { Lower limit } \\
\text { for pressure } \\
/ \text { Torr }\end{array}$ & $\begin{array}{c}\text { Molecules per } \\
\mathbf{c m}^{\mathbf{3}}\end{array}$ & $\begin{array}{c}\text { Mean free } \\
\text { path } \\
/ \mathbf{~ c m}\end{array}$ & $\begin{array}{c}\text { Surface } \\
\text { collision } \\
\text { frequency } \\
/ \mathbf{c m}^{2} \mathbf{s}\end{array}$ & $\begin{array}{c}\text { Time for } \\
\text { formation of a } \\
\text { monolayer } \\
/ \mathbf{s}\end{array}$ \\
\hline Atmosphere & 760 & $2.7 \times 10^{19}$ & $7 \times 10^{-6}$ & $3 \times 10^{23}$ & $3.3 \times 10^{-9}$ \\
\hline Rough vacuum & $10^{-3}$ & $3.5 \times 10^{13}$ & 5 & $4 \times 10^{17}$ & $2.5 \times 10^{-3}$ \\
\hline High vacuum & $10^{-6}$ & $3.5 \times 10^{10}$ & $5 \times 10^{3}$ & $4 \times 10^{14}$ & 2.5 \\
\hline Very high vacuum & $10^{-9}$ & $3.5 \times 10^{7}$ & $5 \times 10^{6}$ & $4 \times 10^{11}$ & $2.5 \times 10^{3}$ \\
\hline Ultra-high vacuum & $10^{-12}$ & $3.5 \times 10^{4}$ & $5 \times 10^{9}$ & $4 \times 10^{8}$ & $2.5 \times 10^{6}$
\end{tabular}

In order to reach pressures below $10^{-9}$ Torr the vacuum chamber has to be heated to $\sim 140{ }^{\circ} \mathrm{C}$ for several days. This bake out procedure accelerates the degasing of the stainless steel chamber and reduces the amount of water in the gas phase significantly. Fig. 2.2 shows a mass spectrum of the residual gas in the UHV chamber for a base pressure of $3 \times 10^{-10}$ Torr. The main gases present in the UHV chamber after bake out are $\mathrm{H}_{2}(m / z=2), \mathrm{CO}(m / z=28)$ and $\mathrm{CO}_{2}(m / z=44)$ and their corresponding fragments. The relatively high amount of residual hydrogen in the chamber results from two factors: (1) the low pumping speed of the turbomolecular pumps for $\mathrm{H}_{2}$, which results from a lower compression ratio compared to other heavier gases, and (2) the high outgassing rate of $\mathrm{H}_{2}$ dissolved in the stainless steel vacuum chamber walls and components. $\mathrm{CO}$ and $\mathrm{CO}_{2}$ can results from degassing of the stainless steel walls of the vacuum chamber and are also produced from the hot filament used for electron impact ionization in the quadruple mass spectrometer (SRS RGA 200). The amount of water $(\mathrm{m} / z=18)$ is significantly reduced in the bake out procedure.

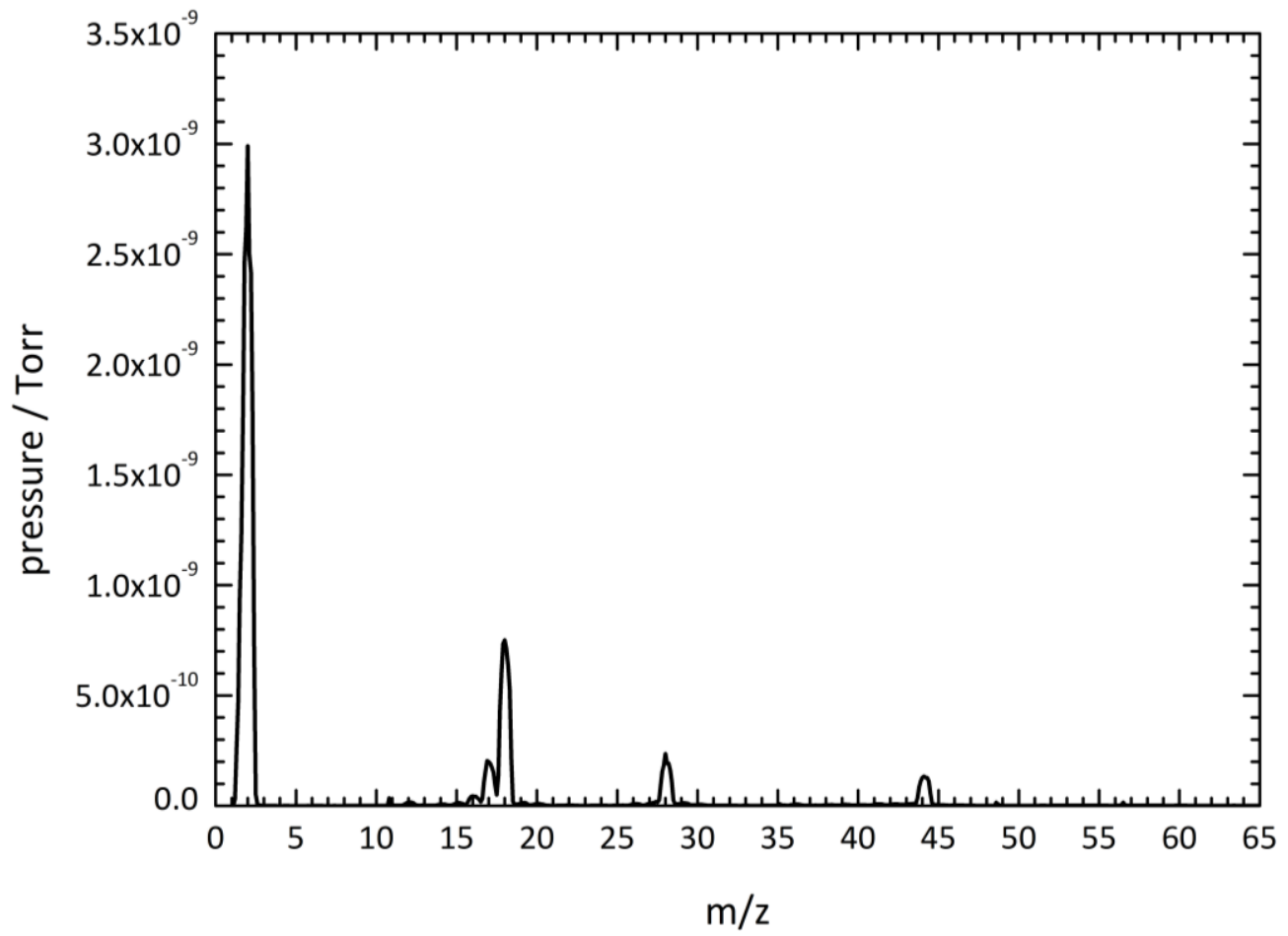

Fig. 2.2: Mass spectrum of the residual gas in the UHV chamber after bake out at a base pressure of $3 \times 10^{-10}$ Torr raising to $5.4 \times 10^{-10}$ Torr with the RGA operating. The main residual gases are $\mathrm{H}_{2}$, water, $\mathrm{CO}$ and $\mathrm{CO}_{2}$. Note that the displayed pressures do not necessarily reflect the real partial pressures because only one sensitivity factor is used for all different $\mathrm{m} / \mathrm{z}$. 
Another important reason for the usage of UHV conditions is related to the mean free path, that is, the average distance that the molecules travel before they collide with another molecule in the gas phase. The mean free path should be longer than dimensions of the experiment or at least longer than the distance that the molecules travel before they are detected.

The second important tool for gas-surface scattering experiments is the generation of a velocity controlled molecular beam [84]. Molecular beams are in general produced in an isentropic expansion of a gas or a gas mixture at pressure $P_{0}$ into a region of low pressure $P_{b}$ (vacuum) through a small aperture (nozzle). The gas flow causes a high collision rate at the opening which leads to narrow velocity distributions (translational cooling) and low rotational temperatures $T_{r o t}$ (rotational cooling). The final velocity $s_{\infty}$ of an ideal gas mixture can be calculated from the average molar heat capacity $\overline{C_{p}}$ and the average molar mass $\bar{W}$ using equation 2.1. By seeding of a sample gas in lighter or heavier carrier gases and varying the concentration, it is possible to tune the velocity and kinetic energy of the molecular beam. This is shown in Fig. 2.3 for $\mathrm{CO}$ molecular beams with different concentrations of $\mathrm{CO}$ in mixtures with $\mathrm{H}_{2}$ and $\mathrm{Ne}$. The molecular beam becomes faster with increasing concentration of $\mathrm{H}_{2}$ in the gas mixture. For pure hydrogen, equation 2.1 predicts a final velocity of $s_{\infty} \approx 3000 \mathrm{~m} \mathrm{~s}^{-1}$.

$$
s_{\infty}=\sqrt{\frac{2 \cdot \overline{C_{p}} \cdot T_{0}}{\bar{W}}}
$$

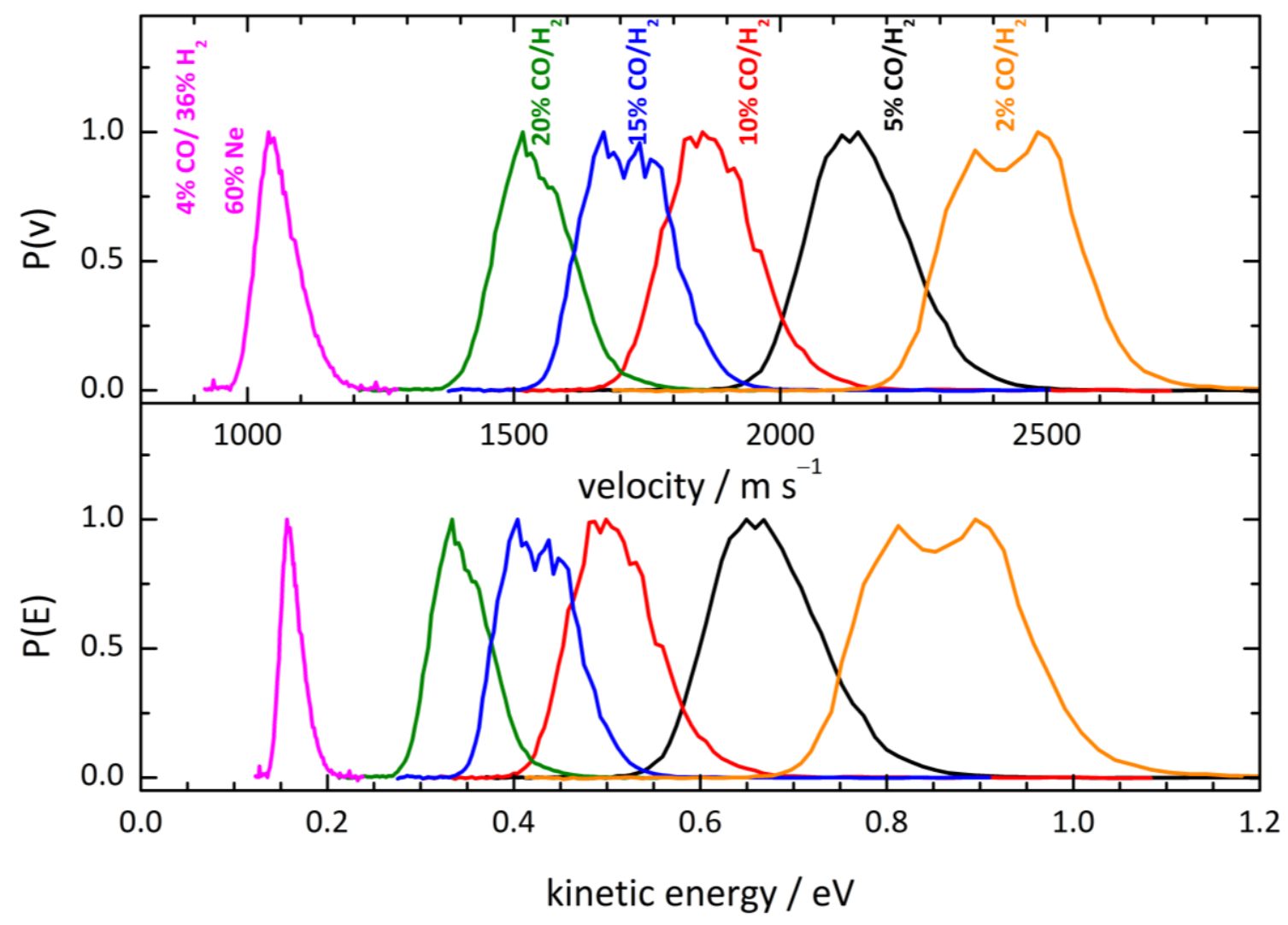

Fig. 2.3: Measured speed and kinetic energy distributions of six mixtures of $\mathrm{CO}$ in different carrier gases. All distributions were measured by $\mathrm{CO}(v=0 \rightarrow 2)$ excitation with an IR laser and (2+1) REMPI detection in 30-32 mm distance. 
In this machine, a molecular beam is generated by supersonic expansion of a gas mixture at 3 atm stagnation pressure into vacuum $\left(5 \times 10^{-6}\right.$ Torr) through a piezo-electrically driven nozzle (Fig. 2.4). A piezo crystal ( $\mathrm{PI}, \mathrm{P} 288.23$ ) is biased with a $70 \mu$ s pulse at $460 \mathrm{~V}$ which leads to deformation of the piezo away from the opening. A plunger, which is sealing the $1 \mathrm{~mm}$ opening is mounted on a hole in the middle of the piezo crystal, is pulled back by the deformation and opens the nozzle. The supersonic beam is skimmed (1.5 mm skimmer) and passes two stages of differential pumping which are separated by a $2 \mathrm{~mm}$ aperture. An additional $3 \mathrm{~mm}$ aperture separates the second differential pumping stage from the UHV chamber. The compact design of the machine results in a short nozzle-to-surface distance of only $\sim 180 \mathrm{~mm}$.

The source chamber is pumped by a cryogenic pump (ASC, Cryo Plex-10) operated by a helium compressor (ASC, M450). The advantage of this type of pumping is the very high pumping speed, especially for hydrogen $(3000 \mathrm{l} / \mathrm{s})$, which allows maintaining a pressure of $5 \times 10^{-6}$ Torr with the nozzle operating. A disadvantage of the cryogenic pump is that is does not allow the use of helium as a carrier gas. The first and second differential pumping stages are pumped by turbo molecular pumps (TMP) (Osaka, TG403 and TF160CA) with pumping speeds of $400 \mathrm{l} / \mathrm{s}$ and $120 \mathrm{l} / \mathrm{s}$. The second differential pumping stage can be separated from the source and first differential with a sliding valve which enables venting of the source/diff. I chambers without exposing the UHV part of the machine to air. The UHV scattering chamber is pumped by a turbo molecular pump (Leybold, NT360, $350 \mathrm{l} / \mathrm{s}$ ) which is backed by a second TMP (Pfeiffer-Balzers, TPU062, $70 \mathrm{l} / \mathrm{s}$ ) to reach the compression that is necessary to obtain UHV in the main chamber.

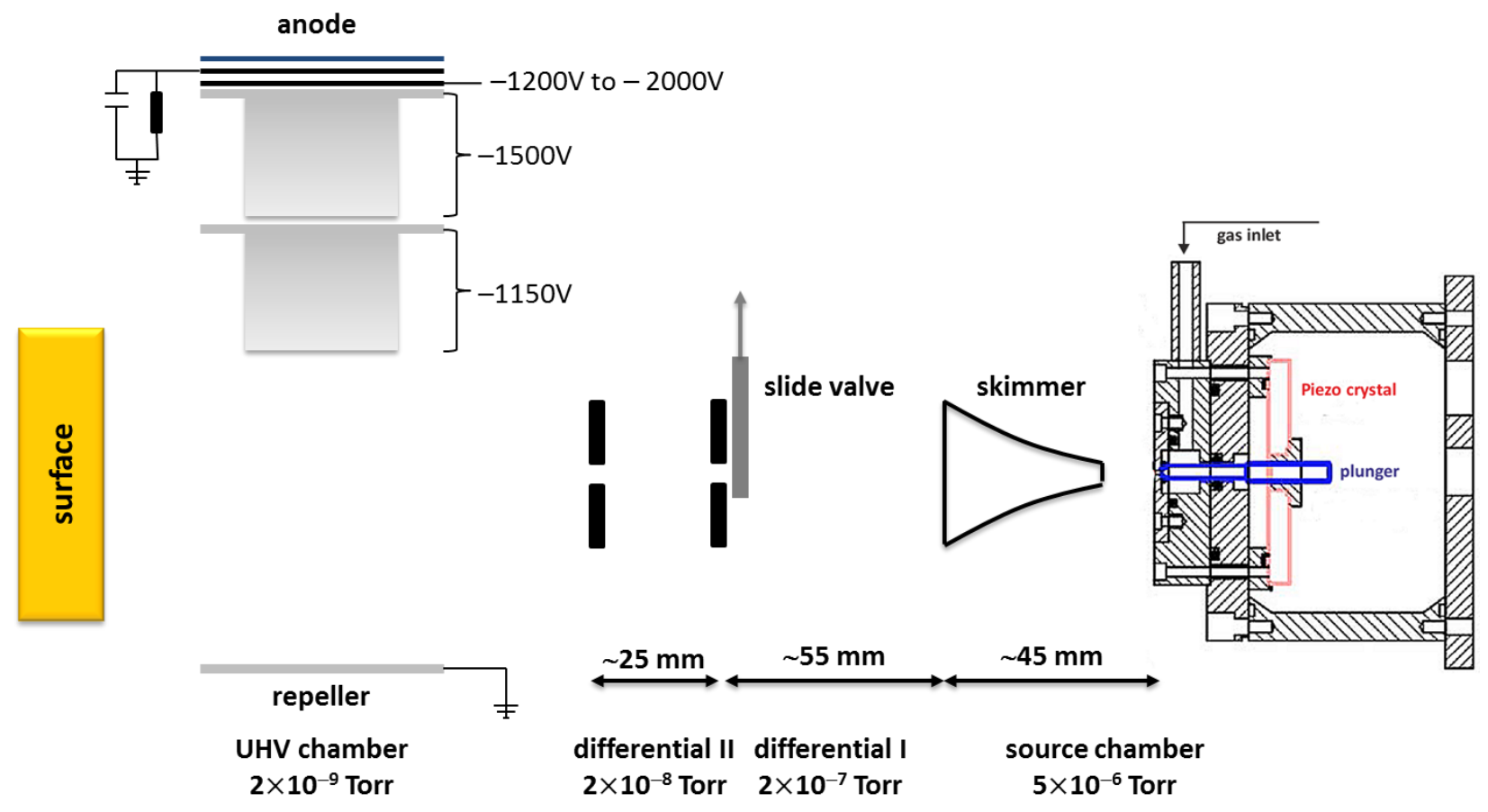

Fig. 2.4: Piezo nozzle (adapted from ref. [82] and path of the molecular beam into the UHV scattering chamber. The nozzle opening is about $45 \mathrm{~mm}$ away from the first wall on which a $1.5 \mathrm{~mm}$ skimmer is mounted. The beam passes two stages of differential pumping before it enters the UHV chamber after $\sim 125 \mathrm{~mm}$. The surface is about $180 \mathrm{~mm}$ from the nozzle opening. The ions are detected on a double Chevron MCP detector that is equipped with a repeller plate at the bottom and an ion lens consisting of two cylindrical elements. The pressures are given for operating nozzle. 
The UHV chamber itself is equipped with an ion detector (Fig. 2.4) consisting of a repeller plate, an ion lens with two cylindrical elements and a double micro channel plate detector in chevron configuration. The generated electrons are collected on an anode plate and the signal is displayed on an digital oscilloscope (LeCroy, WaveSurfer $104 \mathrm{WXs-B}$ ). The sample is mounted on a sample holder which is attached to a 4 axes $(x, y, z, \varphi)$ UHV manipulator (Vacuum Generator Fisons Instruments, Omniax 800). The assembly is shown in Fig. 2.5. The original sample holder consists of a pair of copper arms, which are electrically insulated from the rest of the sample holder by sapphire plates, and a cool finger. The sapphire plates ensure electrical insulation while allowing for good heat conduction. The copper arms are connected to $\sim 3 \mathrm{~mm}$ thick copper wires for electrical conductivity at low resistance. The sample is mounted using two tungsten wires (Alfa Aesar, $0.375 \mathrm{~mm}$, purity $99.95 \%$ ) that are mounted to the copper arms. Thermocouples (type K) are attached to the sample to monitor the temperature of the crystal. The sample can be resistively heated up to the melting point of the crystal and cooled down to liquid $\mathrm{N}_{2}$ temperature.

For comparison of different surfaces under identical conditions, I built a double sided sample holder which is shown in the middle and right panel of Fig. 2.5. The basic design is identical to that of the original sample holder described above. The new sample holder can be equipped with two different crystals mounted on opposite sides of the holder and that are electrically insulated from each other. A stainless steel heat shield is installed in between the samples in order to avoid evaporation/ sputtering of material from one onto the other surface. The thermal insulation of the two samples is satisfying. One sample can be heated to $1000 \mathrm{~K}$ while the other one warms up to only about $370 \mathrm{~K}$.
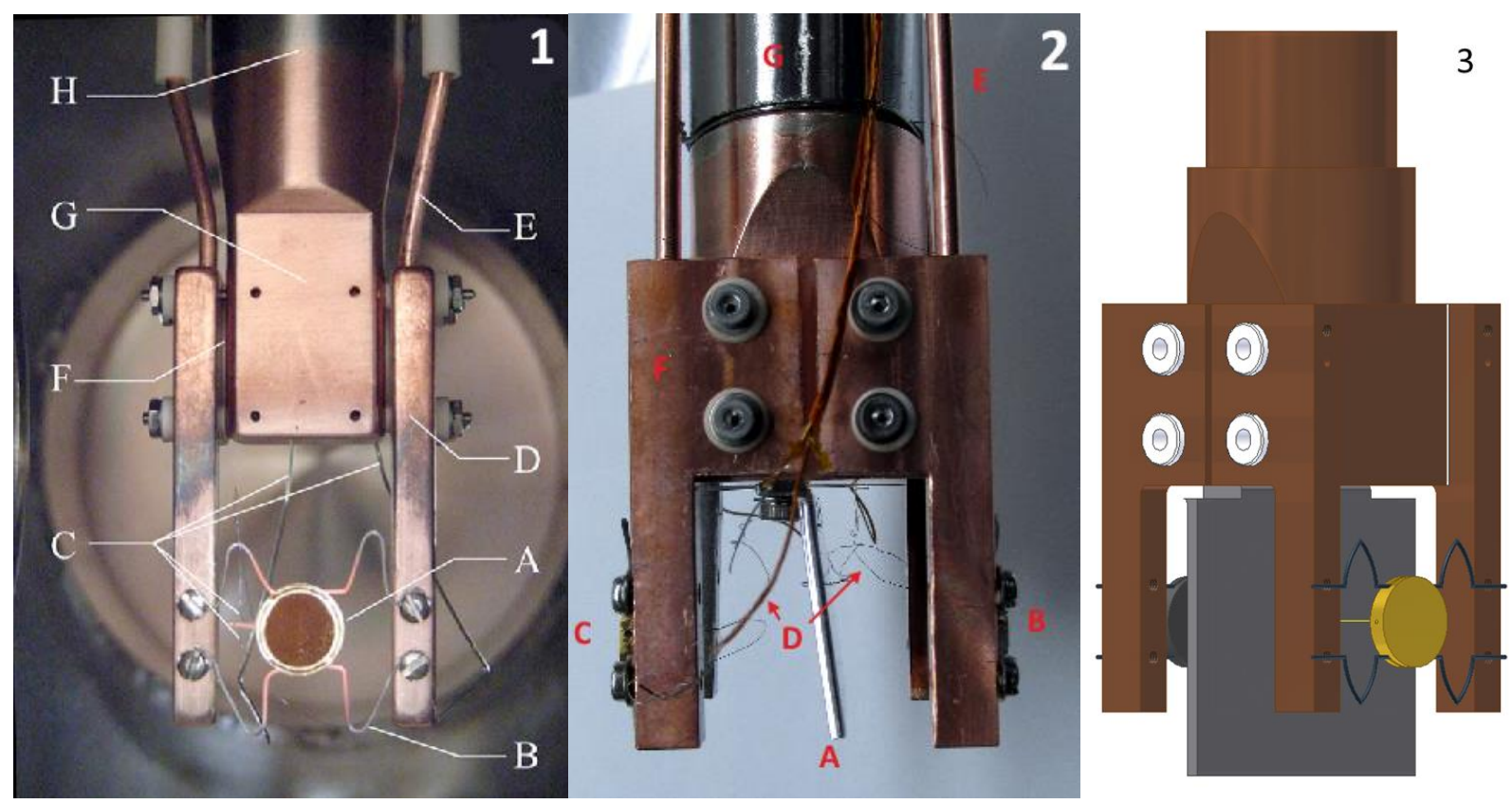

Fig. 2.5: Different sample holders used in my work: original single sample holder (1, left) and new double sided sample holder (middle and right). (1) Sample mount consisting of two copper arms (D) that mounted to a cold finger ( $H, G)$ [82]. The arms are electrically insulated by sapphire plates (F). In this picture the holder is equipped with a $A u(111)(A)$ crystal which is mounted to tungsten wires (B) and can be heated by resistive heating. Current is conducted at low resistance through copper rods (E) so only the tungsten wires heat up. The crystal temperature is monitored using type $\mathrm{K}$ thermocouples (C) which are pressed into the sample. Reprinted with permission from Review of Scientific Instruments, 2007. 78(10), 104104. Copyright 2007, AIP Publishing LLC.) $(2,3)$ : In the new double sided sample mount, two pairs of copper arms (F) are attached to the cold finger (G). They are electrically insulated again by sapphire plates. Here the holder is equipped with a Au(111) (C) and a Pt(111) (B) crystal. Both samples can be heated separately by resistive heating. A heat shield $(A)$ is installed in between the two samples to avoid evaporation/ sputtering of crystal material from one onto the other sample. The temperature is again monitored by type $\mathrm{K}$ thermocouples (D). 


\subsubsection{Surface preparation}

The upper part of the UHV chamber is equipped with various instruments for surface preparation. I use an Argon ion gun (LKTech NGI-3000) to sputter and clean the surface prior to every measurement. Afterwards the sample is inspected for impurities with an Auger electron spectrometer (Physical Electronics, Ф15-255G). Some examples for typically observed Auger spectra are shown in the left panel of Fig. 2.6 for different surfaces after sputtering. The main impurities prior to the cleaning procedure are in general carbon with a characteristic peak at $273 \mathrm{eV}$ and oxygen at $503 \mathrm{eV}$. After heating/ annealing I frequently detected a peak at $300 \mathrm{eV}$ resulting from calcium at the surface. This contamination possibly originates from impurities in the tungsten wires but is reduced after sufficient degasing. After the described procedure of Argon ion bombardment and Auger characterization, the Au and Ag surfaces are annealed to about $1000 \mathrm{~K}$ to reconstruct the (111) surface configuration that is destroyed in the cleaning process. In case of the Pt crystal, I found that $\mathrm{Ar}^{+}$sputtering alone is not sufficient for cleaning and I could still detect a significant amount of carbon at the surface. This residual contamination can be removed by heating the surface to $1000 \mathrm{~K}$ in about $5 \times 10^{-7}$ Torr oxygen $[77,78,85,86]$ for $30 \mathrm{~min}$. The $\mathrm{Pt}(111)$ surface is then finally recovered by heating in UHV to about $1200 \mathrm{~K}$ for $30 \mathrm{~min}$.
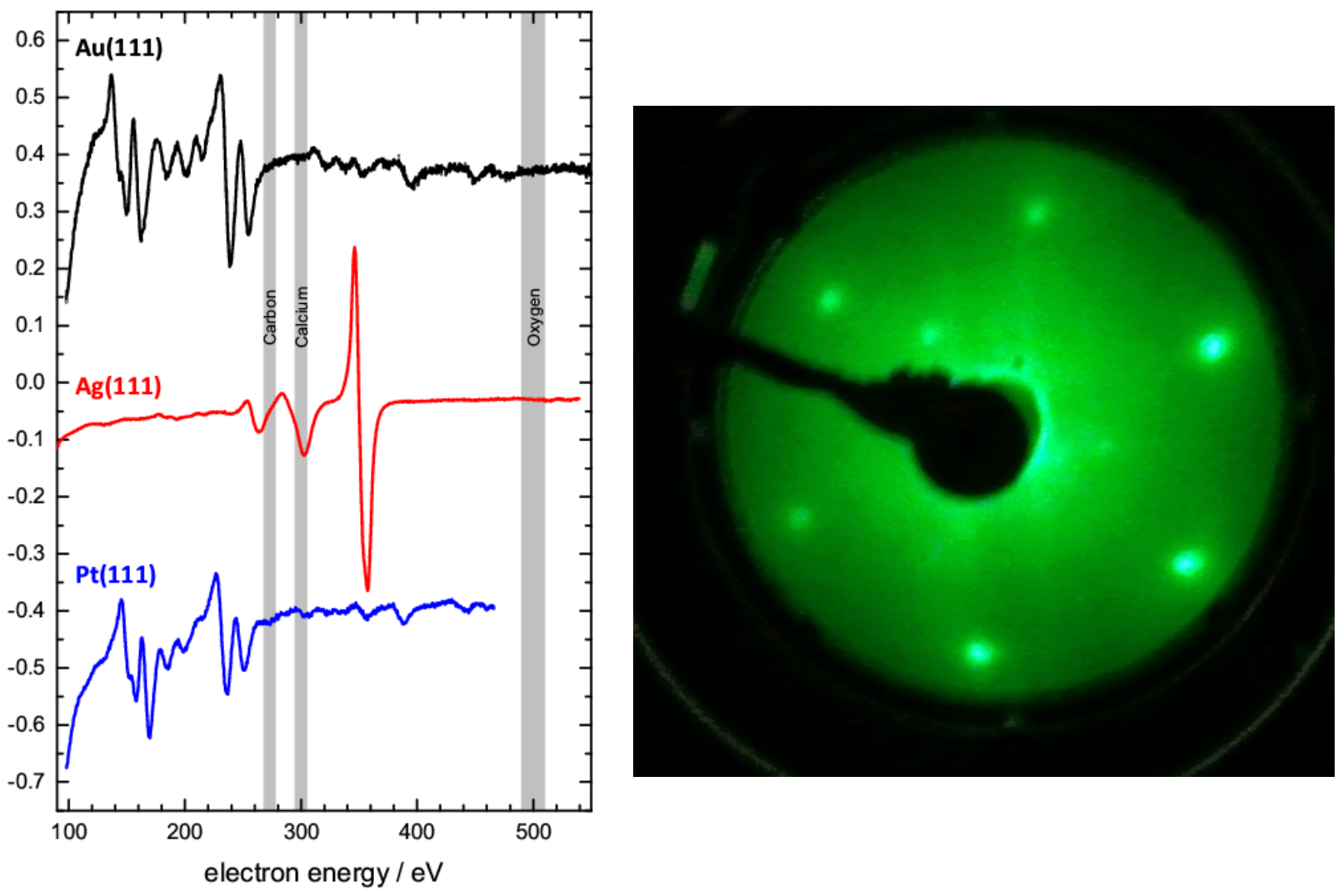

Fig. 2.6: Left panel: Typically observed Auger spectra for different single crystal surfaces: $\mathrm{Au}(111)$ (black), $\mathrm{Ag}(111)(\mathrm{red})$ and $\mathrm{Pt}(111)$ (blue). The main impurities under UHV condition are in general carbon (peak at $273 \mathrm{eV}$ ), calcium (300 eV) and oxygen (double peak at $500 \mathrm{eV}$ ). Carbon and oxygen are related to adsorption of background gas, i.e. CO, at the surface. Calcium impurities result from impurities of the tungsten wires that hold the sample. Right panel: LEED pattern of a Au(111) crystal measured with a SPECS ErLEED 150 at $50 \mathrm{eV}$ electron beam energy. The LEED pattern shows the characteristic 6-fold symmetry of the $\mathrm{Au}(111)$ surface. 


\subsection{Laser systems}

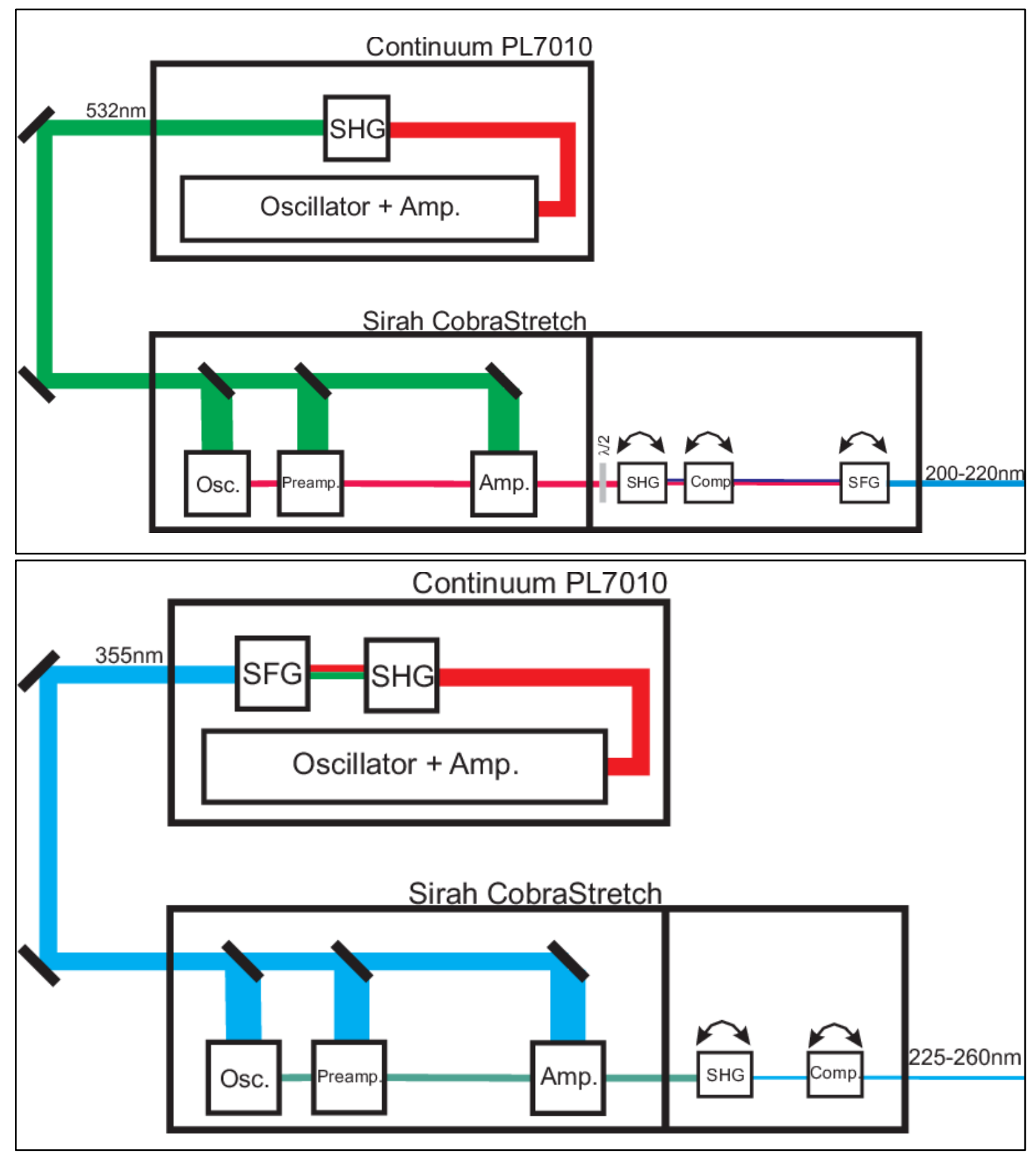

Fig. 2.7: Schematic set-up of the UV laser system used for state-specific detection of molecules. Lower panel: laser system used for generation of tunable UV radiation in the range from $220-260 \mathrm{~nm}$. A frequency doubled pulsed dye is pumped by the third harmonic of a pulsed Nd:YAG laser. The UV pulses (up to $5 \mathrm{~mJ}$ ) are separated from the fundamental by dichroic mirrors. Upper panel: Laser system for generation of UV light in the range from $200-220 \mathrm{~nm}$. A dye laser (600-660nm) is pumped by the second harmonic of a pulsed Nd:YAG laser. The dye laser output can be frequency doubled yielding UV radiation in the range from 300$330 \mathrm{~nm}$. The second harmonic can either be used for spectroscopy or for sum frequency mixing with the fundamental to obtain UV pulses in the range from 200-220nm (up to $9 \mathrm{~mJ}$ ). 


\subsubsection{UV laser systems}

I used resonance enhance multi-photon ionization (REMPI) spectroscopy (see section 2.3) for detection of scattered and incident molecules. This requires (1) the use of UV light to access appropriate electronic states, (2) sufficient photon flux/ intensity for absorption of two or more photons and (3) a narrow spectral bandwidth to achieve quantum state resolution. Nanosecond laser systems match all these requirements and represent a compromise of high intensity $\left(1 \mathrm{~mJ}\right.$ in a $10 \mathrm{~ns}$ pulse correspond to $10^{6} \mathrm{~W}$ peak power) and spectral resolution (bandwidth limit of $110 \mathrm{MHz}$ for a $8 \mathrm{~ns}$ pulse).

Tunable nanosecond pulsed dye lasers in combination with non-linear optical frequency conversion have shown to be an ideal tool for spectroscopic studies using laser induced fluorescence (LIF) or REMPI [12]. I used two different dye laser systems (Fig. 2.7) to provide the necessary tunable UV radiation for state selective detection. For detection of different molecules I used UV light in the range from 200-320 nm. The range of 220-260 $\mathrm{nm}$ is covered by the frequency doubled output of a dye laser (Sirah CobraStretch, $0.1 \mathrm{~cm}^{-1}$ bandwidth) operating with different coumarin dyes (C440, C450, C460, C480, C503) in the range from 435-530 nm (lower panel of Fig. 2.7). The dye laser is pumped by the third harmonic (355nm, up to $260 \mathrm{~mJ}$ ) of a pulsed Nd:YAG laser (Continuum PL7010, Continuum Surelite EX). First, the oscillator is pumped by a small portion of the pump light. It is equipped with a holographic grating $(2400$ lines/mm, $1^{\text {st }}$ order) at grazing incidence. The wavelength of the laser can be tuned by scanning the angle of a high reflective mirror with respect to the grating. The pulse of the resonator $(<1 \mathrm{~mJ})$ is pre- and main amplified in two additional stages yielding conversion efficiencies up to $16 \%$ with respect to the pump energy. The output of the laser is frequency doubled in a BBO ( $\beta$-barium borate, Sirah SHG-215) crystal. The displacement caused by the BBO crystal is compensated by a second plate (Sirah C-BBOL). The BBO crystal is mounted on a motor controlled rotational stage which is manually calibrated against the wavelength of the dye laser.

The second UV laser system is shown in the upper panel of Fig. 2.7. The dye laser is pumped by the second harmonic $(532 \mathrm{~nm}, 250 \mathrm{~mJ}$ ) of a pulsed Nd:YAG laser and is similar to the one described above. The basic difference between the two lasers is that the upper system is equipped with a different grating (1800 lines $/ \mathrm{mm}$ ) operating in the range from $540-920 \mathrm{~nm}$. The laser output can be frequency doubled in a BBO crystal (Sirah SHG-250-T). I can use the second harmonic for spectroscopic issues or it can be mixed with the fundamental (sum frequency generation, SFG) in a second BBO crystal (Sirah THG-197-T) for generation of UV light in the range from $200-220 \mathrm{~nm}$. Both BBO crystals are temperature stabilized to about $50^{\circ} \mathrm{C}$. I use dichroic mirrors to separate the different wavelengths $\left(1^{\text {st }}, 2^{\text {nd }}\right.$ and $3^{\text {rd }}$ harmonic) from each other.

\subsubsection{Fourier-Transform Limited IR Source}

For preparation of vibrationally excited molecules, I used a high power nanosecond infrared system with nearly Fourier transform limited bandwidth. The basic set-up is shown in Fig. 2.8. A continuous wave (cw) Nd:YLF laser (Coherent Verdi-10, up to $10 \mathrm{~W}$ ) is used to pump a cw ring dye laser (Sirah Matisse DR, $20 \mathrm{MHz}$ bandwidth). The ring dye laser is equipped with an optical diode and different frequency selective elements for single mode operation. The visible $\mathrm{cw}$ light $(400 \mathrm{~mW}, 660-690 \mathrm{~nm})$ is used for pulse amplification in a five stage pulsed amplifier (Sirah PulsAmp 5X), which is pumped by the second harmonic of an injection seeded pulsed Nd:YAG laser (Spectra Physics Quanta-Ray Pro-230), yielding transform limited visible $8 \mathrm{~ns}$ pulses with pulse energies of 30-60 mJ. The output of the pulsed amplifier is used for difference frequency mixing (DFM) in a $\mathrm{LiNbO}_{3}$ crystal (Sirah DFM-2400-T) with about $130 \mathrm{~mJ}$ of $1064 \mathrm{~nm}$ radiation of the injection seeded Nd:YAG laser. The DFM process typically generates IR light 
in the range from 1.5-2.4 $\mu \mathrm{m}$ with 3-5 mJ. The IR pulses are further amplified using optical parametric amplification (OPA) in a second $\mathrm{LiNbO}_{3}$ crystal (Sirah DFA-2400-T) pumped by additional $280 \mathrm{~mJ}$ of $1064 \mathrm{~nm}$ radiation. The OPA process generates a signal (up to $30 \mathrm{~mJ}$ ) and an idler (up to $20 \mathrm{~mJ}$ ) wave in the range of 1.8-2.4 $\mu \mathrm{m}$ and 2.2-4.2 $\mu \mathrm{m}$, respectively. Both beams can be used for spectroscopy. The resolution of the IR system is demonstrated on the upper panel of Fig. 2.8 which shows a spectral scan of the $\mathrm{R}(0.5)$ transition of the $3 \leftarrow 0$ vibrational band of ground state nitric oxide. The blue line indicates the FWHM of $0.006 \mathrm{~cm}^{-1}$ of the left peak, which is however slightly broadened due to unresolved hyperfine structures (see 2.3.1 for details).

The importance of narrow bandwidth lasers for pumping of vibrational transitions is illustrated in the lower panel of Fig. 2.9. The black solid curve demonstrates the natural linewidth of a transition with the resonance frequency $f_{0}$ and an excited state lifetime of $1 \mathrm{~ms}$, typical for an excited vibrational state, which limits the natural linewidth to $1 \mathrm{kHz}$. In the molecular beam, collisional broadening is basically absent while the beam divergence of about $1.2^{\circ}$ leads to a Doppler broadening of the transition to about $30 \mathrm{MHz}$ (black dashed curve), assuming a mean velocity of $2000 \mathrm{~m} \mathrm{~s}^{-1}$ and a transition frequency of $166 \mathrm{THz}(1.7 \mu \mathrm{m})$. The red and blue curves represent the spectral intensity distributions of laser beams with $3 \mathrm{GHz}\left(0.1 \mathrm{~cm}^{-1}\right)$ and $130 \mathrm{MHz}\left(0.004 \mathrm{~cm}^{-1}\right)$ bandwidth. The first case reflects the typical bandwidth of a pulsed dye laser that is equipped with a grating. The second case represents a nearly transform limited pulse as it is produced by the IR system presented here. A first estimation for the bandwidth limit of a laser pulse can be simply calculated from the uncertainty principle $\Delta E \Delta t \geq \hbar$ and gives $125 \mathrm{MHz}$ for a 8 ns pulse. A more accurate method uses Fourier transformation of, for example, a Gaussian laser pulse with a FWHM of $8 \mathrm{~ns}$. This calculation yields a bandwidth of $110 \mathrm{MHz}$. Fig. 2.9 shows that the transform limited pulse provides approximately 30 times more intensity at the resonance frequency $f_{0}$ than the one with $0.1 \mathrm{~cm}^{-1}$ bandwidth. Consequently, lasers with a broad bandwidth "waste" a lot of energy because they provide it at frequencies which are not in resonance with the molecular transition.

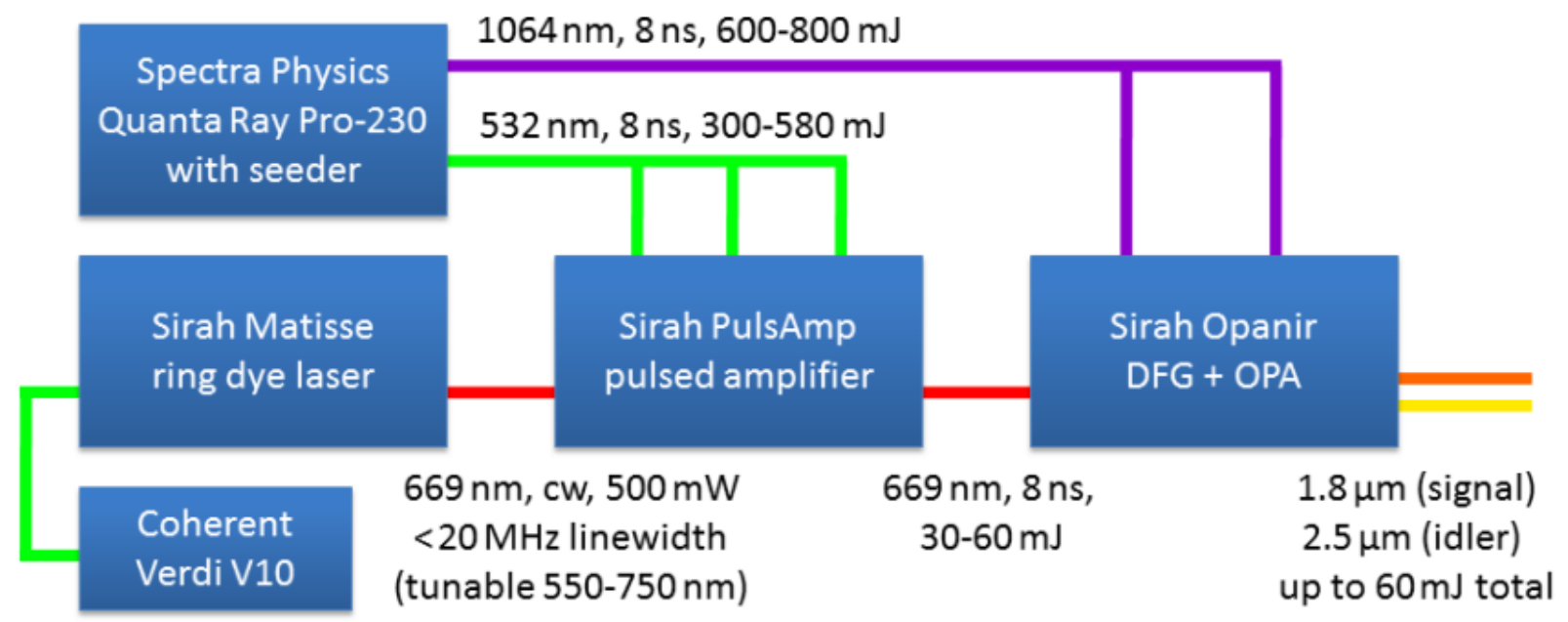

$532 \mathrm{~nm}, \mathrm{cW}, 5 \mathrm{~W}$

Fig. 2.8: Right: Schematic layout of the transform limited high power infrared laser system [87]. A cw single mode Nd:YLF laser (Coherent Verdi-10) is pumping a cw ring dye laser (Sirah Matisse DR, 400mW) that provides tunable single mode radiation with $20 \mathrm{MHz}$ bandwidth. The $\mathrm{cw}$ light is pulse amplified in a 5 stage pulsed amplifier (Sirah PulsAmp 5X) pumped by the second harmonic of an injection seeded Nd:YAG laser (Spectra Physics Quanta-Ray Pro-230). The single mode pulses ( 110 MHz

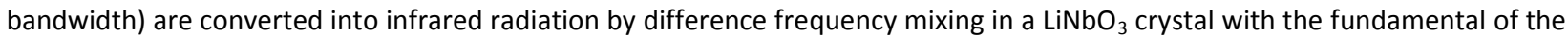
pulsed Nd:YAG laser. The IR pulses are further amplified in an OPA (optical parametric amplification) with additional Nd:YAG fundamental ( $280 \mathrm{~mJ}$ ) to obtain signal (up to $30 \mathrm{~mJ}$ ) and idler (up to $20 \mathrm{~mJ}$ ) wave. 

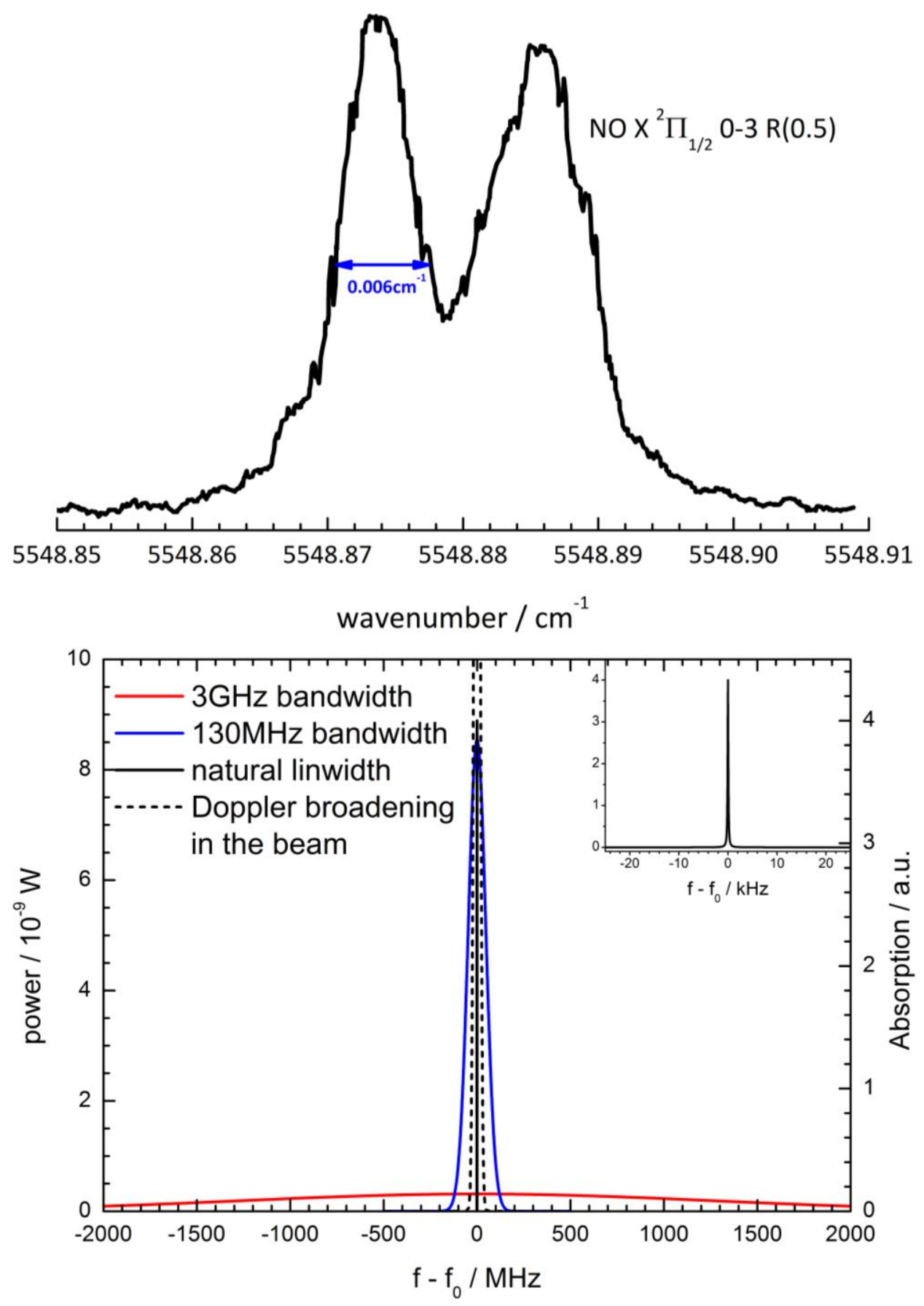

Fig. 2.9: Upper panel: Example for a scan of the IR laser over the $R(0.5)$ transition of the $3 \leftarrow 0$ band of ground state nitric oxide. Lower panel: Effect of the laser bandwidth on the excitation efficiency. The panel show a typical spectral absorption profile (black curve) and the spectral profiles of laser beams with $1 \mathrm{~W}$ power and $130 \mathrm{MHz} / 0.004 \mathrm{~cm}^{-1}$ (blue curve) and $3 \mathrm{GHz} / 0.1 \mathrm{~cm}{ }^{-1}$ (red curve) bandwidth. The dashed black line indicates Doppler broadening of the transition due to the divergence of the molecular beam The absorption profile reflects the natural linewidth of an excited state with 1 ms lifetime which is a typical value for excited vibrational states. The spectra profiles of the laser are simulated as Gaussians with areas of $1 \mathrm{~W}$ and $F W H M=130 \mathrm{MHz}$ and $3 \mathrm{GHz}$, respectively. The calculation shows that the nearly transform limited $130 \mathrm{MHz}$ profiles provides about 30 times more spectral intensity at $f_{0}$. 


\subsection{Resonance enhanced multi-photon ionization (REMPI) detection}

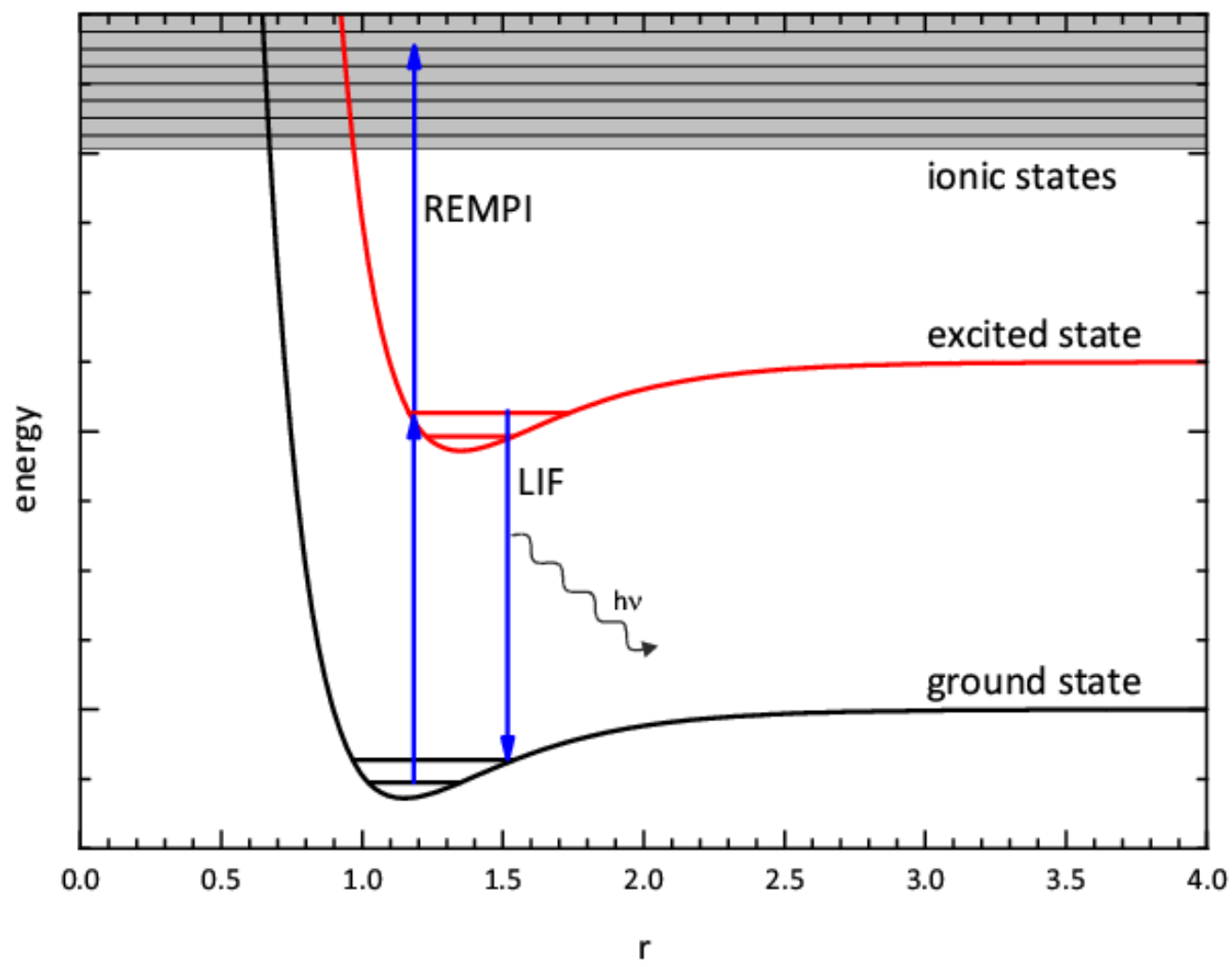

Fig. 2.10: Schematic principle of laser induced fluorescence and resonance enhance multi-photon ionization spectroscopy. The probe molecules are excited to an electronically excited state from which they can either decay back to the ground state upon emission of photons (fluorescence) or they can be ionized by adsorption of another photon.

In general, two different detection schemes have been used for quantum-state resolved detection of molecules: laser induced fluorescence (LIF) and resonance enhanced multi-photon ionization (REMPI) spectroscopy. The basic principles are shown in Fig. 2.10. Both methods require an intermediate electronic state that should not exhibit any dissociation. In case of LIF, the molecule is excited and the spontaneous emission is detected, either completely or spectrally resolved by a monochromator (dispersed fluorescence). REMPI spectroscopy, which is used in my work, involves multi-photon ionization (MPI) which is in principle also possible without any intermediate state. However the ionization probability increases strongly if the wavelength is in resonance with an electronic transition (resonant enhancement). I will briefly discuss the detection schemes used in my work for detection of $\mathrm{NO}, \mathrm{CO}$ and $\mathrm{N}_{2}$ molecules in the next sections 2.3.1-2.3.3.

\subsubsection{Detection of nitric oxide}

The potential energy diagram for the three lowest electronic states of nitric oxide is shown in Fig. 2.11. $(1+1)$ REMPI detection of NO offer two very well-known schemes using the $A^{2} \Sigma^{+}$or the $B{ }^{2} \Pi_{r}$ state as intermediates. For detection of low vibrational states, the strong $A \leftarrow X$ transition has been shown to provide very high sensitivity $[1,8,10,25,27,30,44,88]$. The equilibrium $\mathrm{N}-\mathrm{O}$ bond length in the $A$ state is slightly shorter than in the ground electronic state which leads to similar Franck-Condon factors for the $0-v^{\prime \prime}\left(v^{\prime \prime}=0,1,2,3\right)$ bands. For some of my experimental studies, it is necessary to use vibrational 
bands that are not overlapped with those originating from lower $v^{\prime \prime}$; this criteria is fulfilled for transitions into $A^{2} \Sigma^{+}\left(v^{\prime}=0\right)$, the so called $\gamma$ bands [89].

The $X^{2} \Pi_{r}$ ground state of nitric oxide is split into two spin-orbit states with $\Omega=\Lambda-S=1 / 2$ and $\Omega=\Lambda+S=3 / 2$. The ${ }^{2} \Pi_{1 / 2}$ is the electronic ground state but the ${ }^{2} \Pi_{3 / 2}$ state is located only about $123 \mathrm{~cm}^{-1}$ higher in energy. Because the electron spin $S$ and the orbital angular momentum $\Lambda$ of the ground state differ from zero they couple to nuclear rotation $N$ of the molecule, forming the total angular momentum $J$. The type of coupling is given by different Hund's cases. Hund's case (a) assumes that the interaction between nuclear rotation and the electronic motion is very weak but the electronic motion is coupled strongly to the internuclear axis. In this case $N$ and $\Omega$ form the total angular momentum $J=\Omega, \Omega+$ $1, \Omega+2 \ldots$ Hund's case (b) is dominant if the spin $S$ is not (always the case for $\Lambda=0$ ) or only very weakly coupled to the internuclear bond axis. In this case orbital angular momentum $\Lambda$ and nuclear rotation $N$ form a resultant quantum number $K=\Lambda, \Lambda+1$, $\Lambda+2 \ldots$ which represents the total angular momentum apart from spin. The spin couples to $K$ and forms the total angular momentum including spin $J=(\mathrm{K}+\mathrm{S}),(\mathrm{K}+$ $S-1) \ldots|K-S|$. For the ground state of nitric oxide, case (a) holds for low rotational states but switches to case (b) for high rotational excitation. In contrast, the $A^{2} \Sigma^{+}$ state always occurs in case (b) because the orbital angular momentum $\Lambda$ is zero and therefore not coupled to the internuclear axis [90]. All allowed transitions of the $A^{2} \Sigma^{+} \leftarrow X{ }^{2} \Pi_{r}$ band have to fulfill the

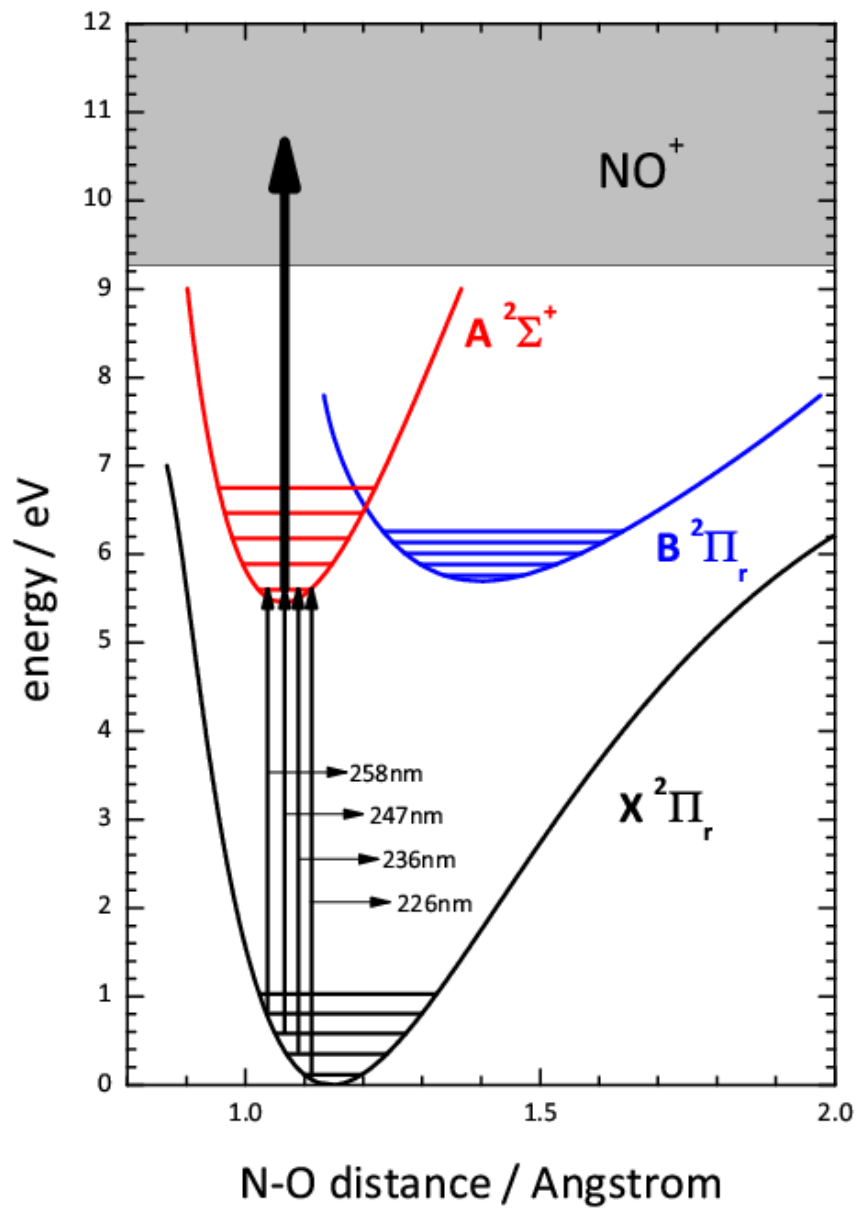

Fig. 2.11: Potential energy diagram of the three lowest electronic states of NO. The electronic ground state (black) and the $B$ state (blue) are split into two spin-orbit states with $\Omega=1 / 2$ and $3 / 2$. selection rule $\Delta J=0, \pm 1$.

The fact that $\Lambda=1$ for the electronic ground state of NO further leads to another kind of interaction that is neglected in Hund's case (a) and (b): the electronic orbital momentum $L$ can couple to the rotation of the nuclei which results in a splitting of the $J$ states into two states. This coupling is called $\Lambda$-doubling and the splitting generally increases with increasing rotational excitation [90]. Moreover, the non-zero nuclear spin of NO (nuclear spin of 1 for the nitrogen atom) leads to hyperfine splitting of both $\Lambda$ components. An example for resolved $\Lambda$-splitting is shown in the upper panel of Fig. 2.9 where the infrared overtone excitation excites $\mathrm{NO}\left(v^{\prime \prime}=0, J^{\prime \prime}=0.5\right)$ to $\mathrm{NO}\left(v^{\prime}=3, J^{\prime}=1.5\right)$. $J^{\prime \prime}=0.5$ has a $\Lambda$ splitting of about $0.012 \mathrm{~cm}^{-1}$ which increases to $0.023 \mathrm{~cm}^{-1}$ for $J=1.5$ [91]. Due to parity selection rules, IR excitation is only possible for $+\leftarrow-$ and $-\leftarrow+$ transitions (Fig. 2.12). Therefore, the two peaks in the spectrum of Fig. 2.9 are only spaced by the difference of the two splittings. 


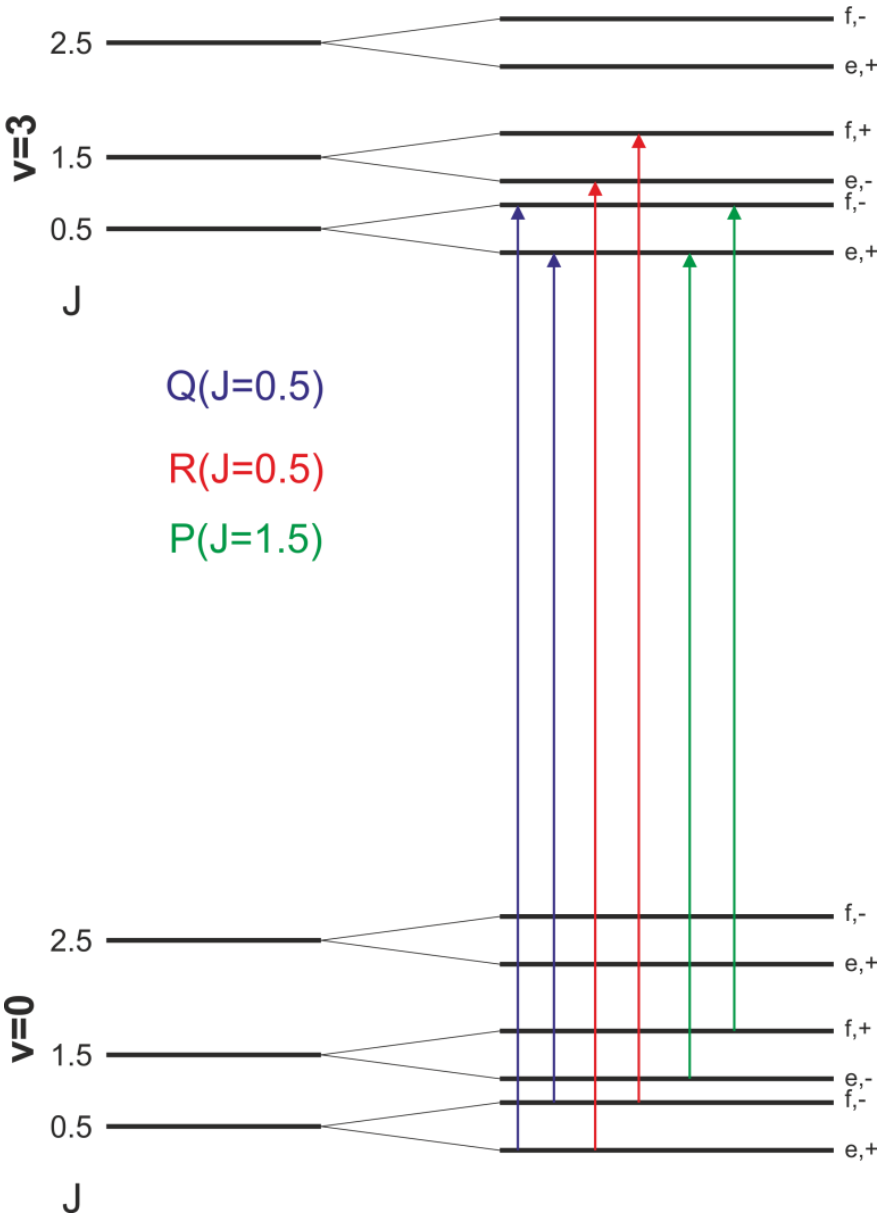

Fig. 2.12: Overview over possible IR transitions in the $X{ }^{2} \Pi_{1 / 2}$ ground state of nitric oxide. The arrows indicate transitions with $\Delta J=0$ (blue), $\Delta J=1$ (red) and $\Delta J=-1$ (green).

\subsubsection{Electronic states and REMPI spectroscopy of carbon monoxide}

An overview of the lowest electronic states of carbon monoxide is shown in Fig. 2.13. The CO ground state is the $X^{1} \Sigma^{+}$state and a distinction between Hund's cases a and $\mathrm{b}$ is therefore pointless [90]. In a ${ }^{1} \Sigma^{+}$state, even $J$ states have positive parity whereas odd $J$ states have negative parity. The first excited singlet state $\left(A{ }^{1} \Pi\right)$ is located about $8 \mathrm{eV}\left(65000 \mathrm{~cm}^{-1}\right)$ above the ground state. Consequently, a one photon transition requires vacuum ultraviolet radiation (VUV) at about $155 \mathrm{~nm}$. This wavelength is not accessible within the limitation of the experimental set-up and would require a four wave mixing scheme. Another possibility is (2+2) REMPI at about $309 \mathrm{~nm}$ (lower right panel in Fig. 2.14) which, however, involves absorption of overall four UV photons, a process that requires very high laser intensities (high pulse energies, tight focusing) to achieve the necessary sensitivity. These conditions are hard to generate. The $A$ state is very close to the $D{ }^{1} \Delta$ state; the transition from the ground state is one photon forbidden but two photon allowed [92]. Because the $D$ state is shifted toward longer bond lengths, good Franck-Condon factors are obtained for excitation into high intermediate vibrational levels [93]. In order to switch to an advantageous (2+1) REMPI scheme, excitation into $D{ }^{1} \Delta\left(v^{\prime}=10\right)$ would be required. Several transitions of the $D-X$ band have been observed and analyzed for perturbations [92, 93]. 
The $A$ and the $D$ state have the major drawback that they are only accessible by absorption of two UV photons but their vibrational ground states are so low in energy that they cannot be ionized in a $(2+1)$ REMPI process. The use of higher intermediate $v^{\prime}$ states leads to a strong overlap between different vibrational bands that is disadvantageous for detection on ground states molecules in different vibrational states with very different populations. However, the higher singlet states like the $B^{1} \Sigma^{+}, C^{1} \Sigma^{+}$and $E^{1} \Pi$ are high enough in energy to overcome this problem. Some examples for $(2+1)$ REMPI spectra for these transitions for rotationally excited ground-state molecules are shown in Fig. 2.14. In case of two photon ${ }^{1} \Sigma-{ }^{1} \Sigma$ transitions the selection rules are $\Delta J=0, \pm 2$. However, the use of linear polarized light enhances the Q-branch $(\Delta J=0)$ by a factor of about 200 compared to the O- $(\Delta J=-2)$ and Sbranch $(\Delta J=+2)$, thus only the Q-branch is visible in the upper right and lower left panel in Fig. 2.14 [94, 95]. For the use of circular polarized light, all three branches have comparable intensities, which is however due to a decrease in the Q-branch intensity and not due to an increase in the $\mathrm{O}$ - and S-branch intensities. From the point of sensitivity it is therefore favorable to use linear polarized light to obtain the maximum possible signal. For the $E-X$ transition the situation is again different

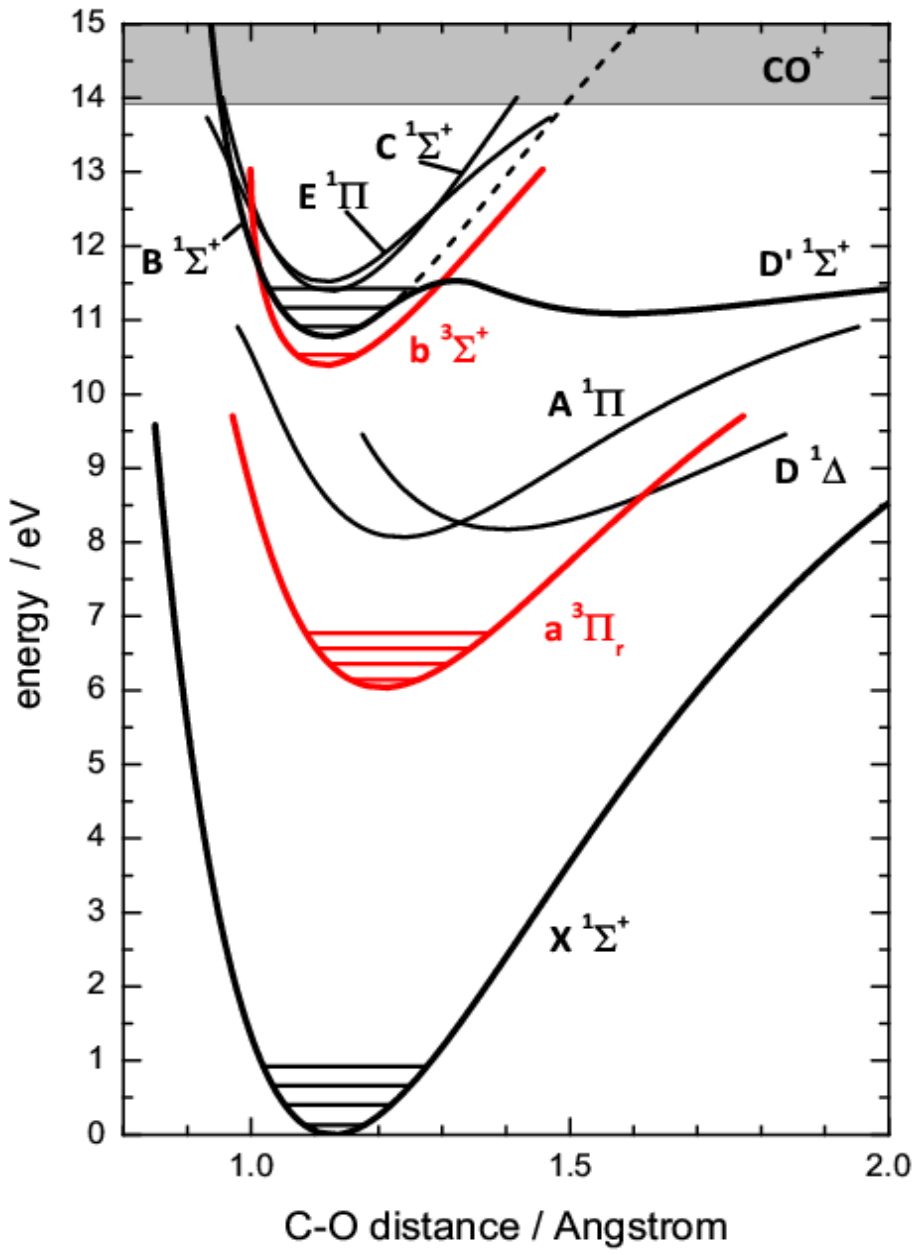

Fig. 2.13: Potential energy diagram for carbon monoxide. It shows the electronic states of up to $E{ }^{1} \Pi$ state. Singlet states are drawn black, triplet states are shown in red. Potential calculated from RKR parameters [5]. and the selection rules are $\Delta J= \pm 1, \pm 2$ [96], which leads to a congested rotational structure. Overall the most favorable scheme appeared to be $(2+1)$ REMPI via $B{ }^{1} \Sigma^{+}$state because the vibrational frequency is slightly lower $\left(2112.7 \mathrm{~cm}^{-1}\right)$ than the ground state frequency $\left(2169.81 \mathrm{~cm}^{-1}\right)$ which leads to a red shift of the (1-1) vibrational band with respect to the $(0-0)$ band. At the same time both bands are blue degrading $\left(B^{\prime \prime}=1.69124 \mathrm{~cm}^{-1}\right.$, $B^{\prime}=1.9612 \mathrm{~cm}^{-1}$ ). The Franck-Condon factors [97] for $\Delta v=0$ transitions are close to unity in the $B-X$ band but decrease rapidly for $\Delta v \neq 0$ transitions. A drawback of this scheme is the predissociation of the $B$ state for $v \geq 2$ which has the consequence that only ground state molecules in $v^{\prime \prime}=0$ and $v^{\prime \prime}=1$ can be detected with high sensitivity. 

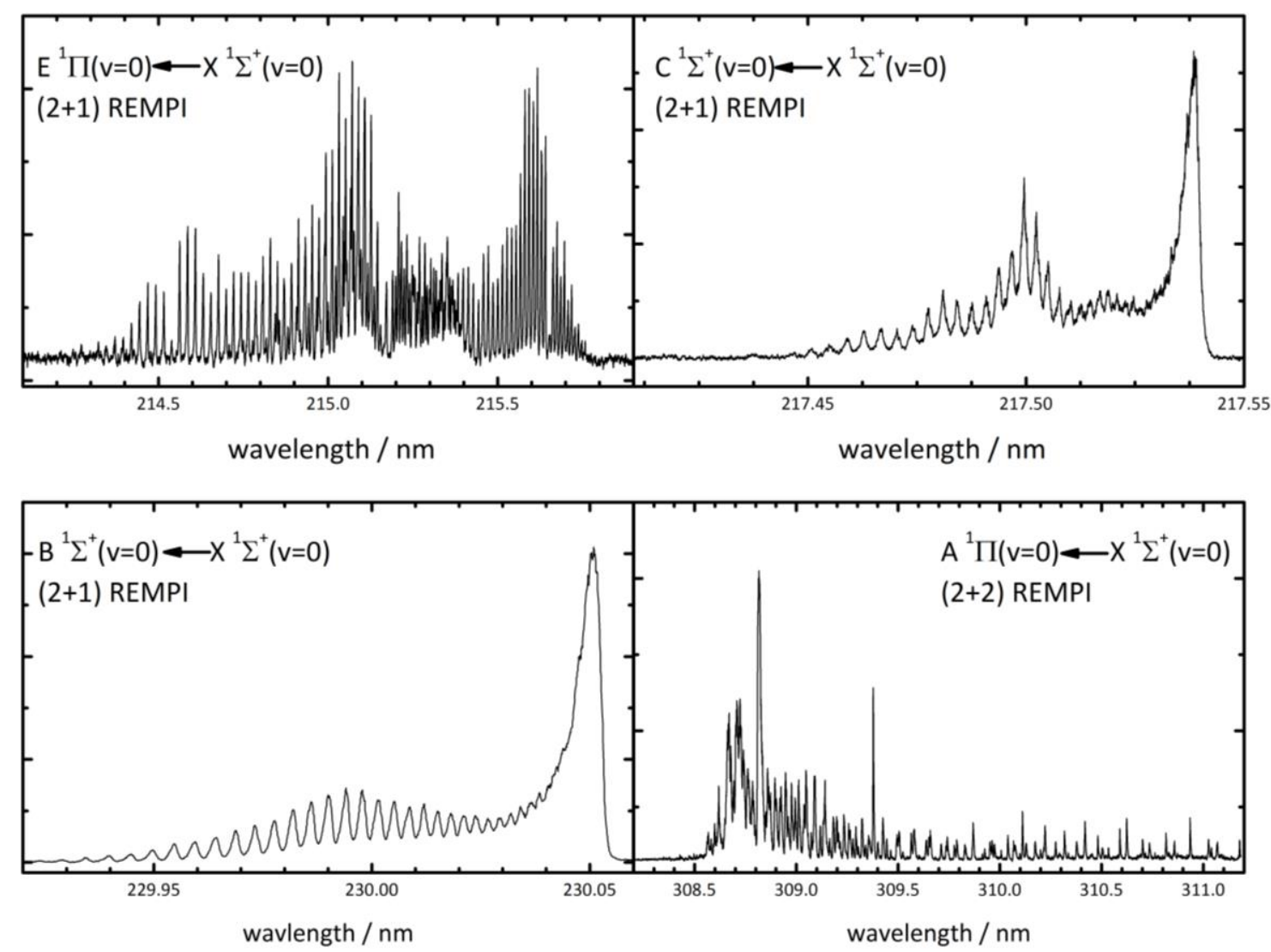

Fig. 2.14: Examples for possible REMPI schemes for ground state $\operatorname{CO}(v=0)$ via the $A{ }^{1} \Pi, B^{1} \Sigma^{+}, C^{1} \Sigma^{+}$and $E{ }^{1} \Pi$ state. All spectra were taken for a molecular beam $\left(E_{I}=0.6 \mathrm{eV}\right)$ scattered off $\mathrm{Au}(111)$.

In addition to its singlet states, CO has several accessible triplet states. Especially the $a^{3} \Pi_{\mathrm{r}}$ state has been investigated in great detail [98-103] and is located about $6 \mathrm{eV}\left(48400 \mathrm{~cm}^{-1}\right)$ above the ground electronic state. Although the optical excitation is spin-forbidden, the $\Omega=1\left(a^{3} \Pi_{1}\right)$ state has an unusually large transition probability caused by a perturbation from the $A{ }^{1} \Pi$ state. The relatively low energy of the $a$ state enables excitation with UV photons at about $206 \mathrm{~nm}$, which are however not sufficient in energy for $(1+1)$ ionization. Nevertheless, a (1+2) REMPI scheme is in principle possible and shown for a jet-cooled molecular beam in the left panel of Fig. 2.15. A more favorable detection of metastable CO in $a^{3} \Pi_{1}$ is a (1+1) REMPI detection scheme through the $b{ }^{3} \Sigma^{+}$state which is located at about $10.4 \mathrm{eV}\left(83800 \mathrm{~cm}^{-1}\right)$. Unfortunately the determination of rotational and vibrational state distributions is not straight forward in this $\left(1+1^{\prime}+1^{\prime}\right)$ UV-UV double resonance scheme but it represents a possible method for tagging (see 2.4) of $\mathrm{CO}$ molecules. Moreover, the scheme enables a rather sensitive detection of $\mathrm{CO}$ molecules in vibrational states $v \geq 2$. 


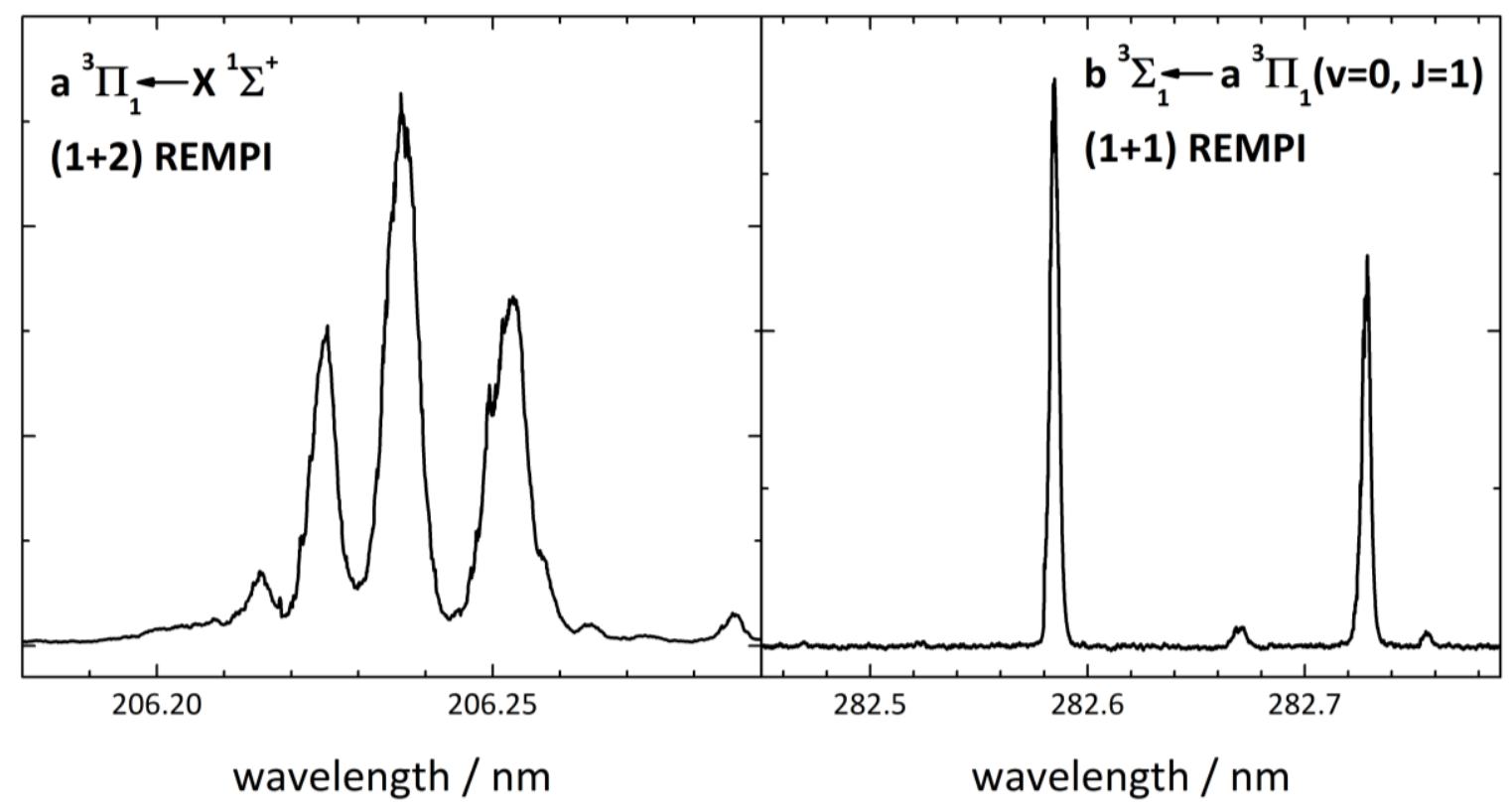

Fig. 2.15: REMPI detection of CO using intermediate triplet states. Left panel: (1+2) REMPI of jet-cooled $\mathrm{CO}(v=0)$ via $a{ }^{3} \Pi_{\mathrm{r}} \leftarrow$ $X^{1} \Sigma^{+}$. Right panel: (1+1) REMPI on metastable CO in the $a^{3} \Pi_{\mathrm{r}}(v=0, J=1)$ state via $b^{3} \Sigma^{3}(v=0)$.

\subsubsection{Detection of nitrogen}

Two different REMPI schemes have been used for detection of nitrogen scattered off metal surfaces in previous studies. Sitz et al. applied (2+2) REMPI via the $a^{1} \Pi_{g} \leftarrow X^{1} \Sigma_{g}^{1}$ transition in the range from 283$285 \mathrm{~nm}[13,104,105]$ whereas Lykke and Kay obtained rotational distributions from (2+1) REMPI using a" ${ }^{1} \Sigma_{g}^{+} \leftarrow X^{1} \Sigma_{g}^{+}$transition at 202-203 nm [79, 106]. The latter is favorable for several reasons: (1) the $(2+1)$ detection scheme is about 20 times more sensitive although much higher laser intensities can be provided for (2+2) REMPI, (2) (2+2) REMPI detection is alignment sensitive, which makes the analysis of rotational distributions not straight forward, whereas rotational distributions can be directly extracted from the line intensities of the (2+1) REMPI scheme. However, (2+1) REMPI via the $a^{\prime \prime}{ }^{1} \Sigma_{g}^{+}$state has the drawback that rotational resolution is rather poor because especially low rotational states are not or hardly resolved in the strongly enhanced $Q$ branch (for linear polarized light), similar to $(2+1) B \leftarrow X$ REMPI of CO. An example of the (2+1) REMPI spectrum of $\mathrm{N}_{2}$ used in my work is shown in Fig. 2.16. The left panel shows a room temperature spectrum taken with $6 \times 10^{-7}$ Torr background pressure of $\mathrm{N}_{2}$ in the UHV chamber. The intensity of the $S$ branch (red) was multiplied by 200 to make it comparable to the intensity of the $Q$ branch (black). The right panel shows a jet-cooled spectrum and illustrates the rotational cooling in the supersonic expansion. 


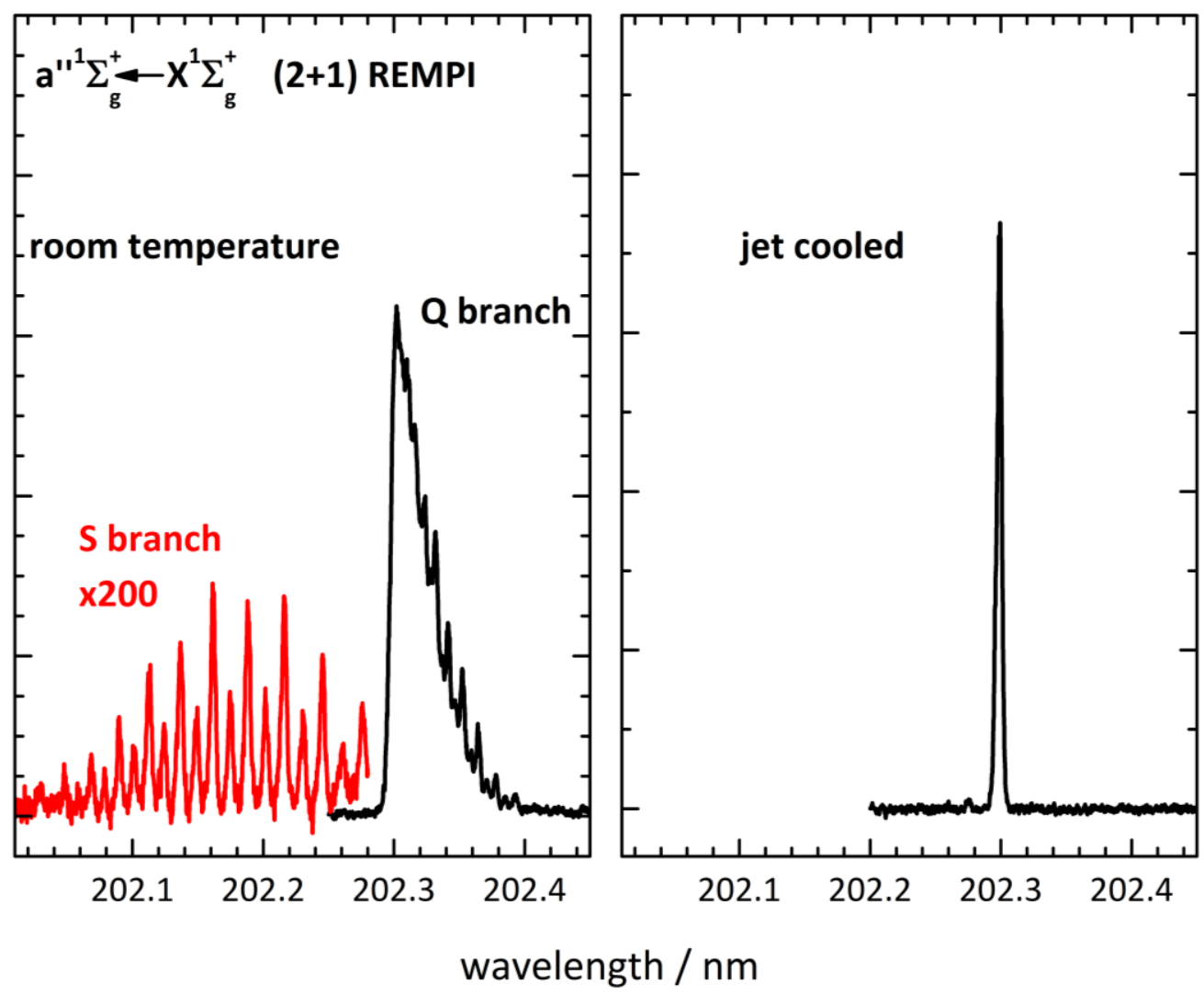

Fig. 2.16: Example for room temperature (left panel) and jet-cooled (right panel) (2+1) REMPI spectra of $\mathrm{N}_{2}$ using the $a^{\prime \prime}{ }^{1} \Sigma_{g}^{+} \leftarrow$ $X^{1} \Sigma_{g}^{+}$transition. The $\mathrm{Q}$ branch in the (2+1) REMPI spectrum is about 200 times stronger than the $\mathrm{O}$ - and S-branch for the use of linear polarized light.

\subsection{State-to-State Time-of-Flight}

The measurement of state-to-state time-of-flight distributions has been shown to be a powerful tool in order to reveal the coupling between different degrees of freedom in gas surface collisions [9-11, 32, 80, 107]. The basic principle of these experiments is rather simple: molecules with well-defined internal (rotational, vibrational) and translational energy are scattered off a flat single crystal surface at temperature $T_{S}$ and the final translational energy distribution after scattering is measured with quantum state resolution. In order to investigate as many details as possible it is desirable to improve the velocity resolution of the experiment as much as possible. The approaches to face this problem are the decrease in uncertainty in flight distance $\Delta L / L$ and to define the starting time $t_{0}$ for the time-of-flight as precisely as possible. The first issue is solved by maximizing $L$ within the limits of the experimental apparatus. The definition of $t_{0}$ has previously been implemented by introducing for example a chopper wheel in order to create short molecular beam pulses $[9,10]$. In my work the uncertainty in $t_{0}$ is minimized by an optical double resonance scheme similar to that used by Rahinov et al. [32]. In most cases, I used IR-UV double resonance in order to measure state-to-state time-of-flight distributions (see Fig. 2.17). In the easiest scheme, molecules are scattered in the vibrational ground-state $\left(T_{\text {rot }} \approx 10 \mathrm{~K}\right)$ and are 'tagged' by an IR laser (2.2.1) into a vibrationally excited state that is otherwise not or only weakly populated. The excited molecules are afterwards detected by REMPI while the REMPI laser beam is placed at a well-known distance from the IR beam. 


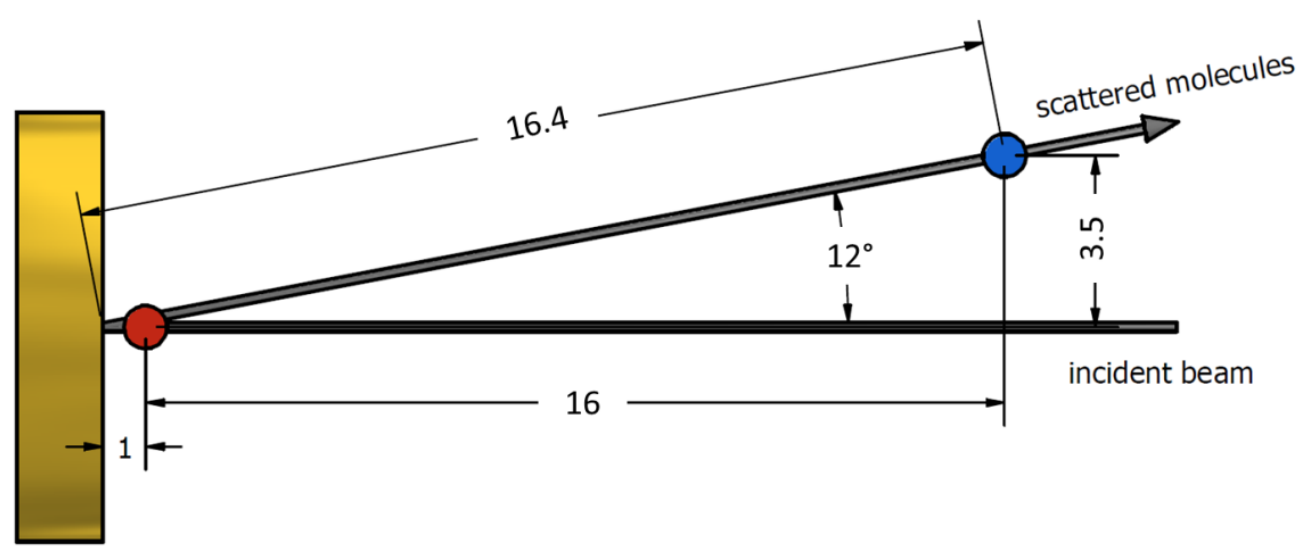

Fig. 2.17: Basic experimental set-up for state-to-state time-of-flight experiments using IR-UV double resonance.

In the same way the kinetic energy of the incident molecules can be measured (Fig. 2.18(a)). For this purpose the surface is moved up by about $10 \mathrm{~mm}$ to avoid any scattering and the IR laser is placed $30 \mathrm{~mm}$ upstream with respect to the REMPI laser. The IR laser produces vibrationally excited molecules which are selectively detected by REMPI. The velocity and energy distribution can then be calculated from the time-dependent density (REMPI signal) of molecules passing the REMPI beam (see Appendix B for details). In case of carbon monoxide, both schemes for time-of-flight experiments on incident and scattered molecules can also be realized by $b^{3} \Sigma^{+} \leftarrow a^{3} \Pi_{1} \leftarrow X{ }^{1} \Sigma^{+}$UV-UV double resonance.

In different experiments, I prepare incident molecules in a vibrationally excited state before they collide with the surface (Fig. 2.18). I carefully measure and optimize the distance between the IR laser and the surface plane by monitoring the transmitted IR light as a function of the surface position. A typical measurement of the IR distance from the surface is shown in the right panel of Fig. 2.18. The experimental approach of preparing incident molecules in low vibrational states $\left(v_{i}=2,3\right)$ enables detailed studies of vibrational energy dissipation in molecule-surface collisions. In addition to coupling between phonons, translational motion and rotation, these experiments enable me to investigate energy transfer between vibration and EHP and the motion of the nuclei. The interaction between all those possible channels for energy dissipation has so far only been investigated for $\mathrm{HCl}(v=2) / \mathrm{Au}(111)$ scattering but not for other, in case of NO/Au(111) better understood, systems.

A question that is always accompanied with the latter experiments is: do the measured time-of-flight spectra really only reflect the velocity/ energy distribution of the scattered molecules? Especially in systems where trapping-desorption is observed this question cannot be answered easily because the residence time that the trapped molecules spent at the surface can contribute to the observed arrival times in the experiment. In order to overcome this lack of understanding, I set up a two-dimensional time-of-flight scheme (2D-TOF) that involves excitation of incident molecules as well as tagging of molecules after the surface collision. In my work, I applied this scheme to two different systems: $\mathrm{CO}\left(v_{i}=2\right) / \mathrm{Au}(111)$ and $\mathrm{CO}\left(v_{i}=0\right) / \mathrm{Pt}(111)$. For scattering of $\mathrm{CO}\left(v_{i}=2\right)$ from $\mathrm{Au}(111)$ the IR laser excites incident $\mathrm{CO}\left(v_{i}=0\right)$ molecules about $1 \mathrm{~mm}$ in front of the surface. A first UV laser tags the final $\mathrm{CO}\left(v_{f}=2,1\right)$ molecules into the metastable $a^{3} \Pi_{1}$ state after scattering (UV-tag). The metastable CO molecules are finally detected after about $18 \mathrm{~mm}$ flight distance with $b^{3} \Sigma^{+} \leftarrow a^{3} \Pi_{1}(1+1)$ REMPI. Overall this scheme uses IR-UV-UV triple resonance. Because the UV-UV double resonance is established after the scattering process, the time-of-flight profile only reflects the velocity spread of the UV-tagged molecules. However, the temporal delay between IR excitation and UV tagging already causes a (coarse) 
pre-selection of velocities and I have to measure a complete 2D-TOF spectrum with several UV-UV TOF profiles for different IR-UV delays to construct the full speed distribution of the scattered molecules.

I used another type of 2D-TOF for the $\mathrm{CO}\left(v_{i}=0\right) / \mathrm{Pt}(111)$ system where I only applied the $b^{3} \Sigma^{+} \leftarrow a{ }^{3} \Pi_{1} \leftarrow X^{1} \Sigma^{+}$UV-UV double resonance for scattered $\operatorname{CO}\left(v_{f}=0,1\right)$ molecules. This enables on one hand the measurement of the speed distributions of the scattered molecules in different positions in the pulsed molecular beam and on the other I can use a constant delay between UV tagging and REMPI detection to filter out a narrow range of velocities. In the latter case, I finally measure the temporal profile of the molecular beam but suppressing any broadening related to velocity spread.

(a)
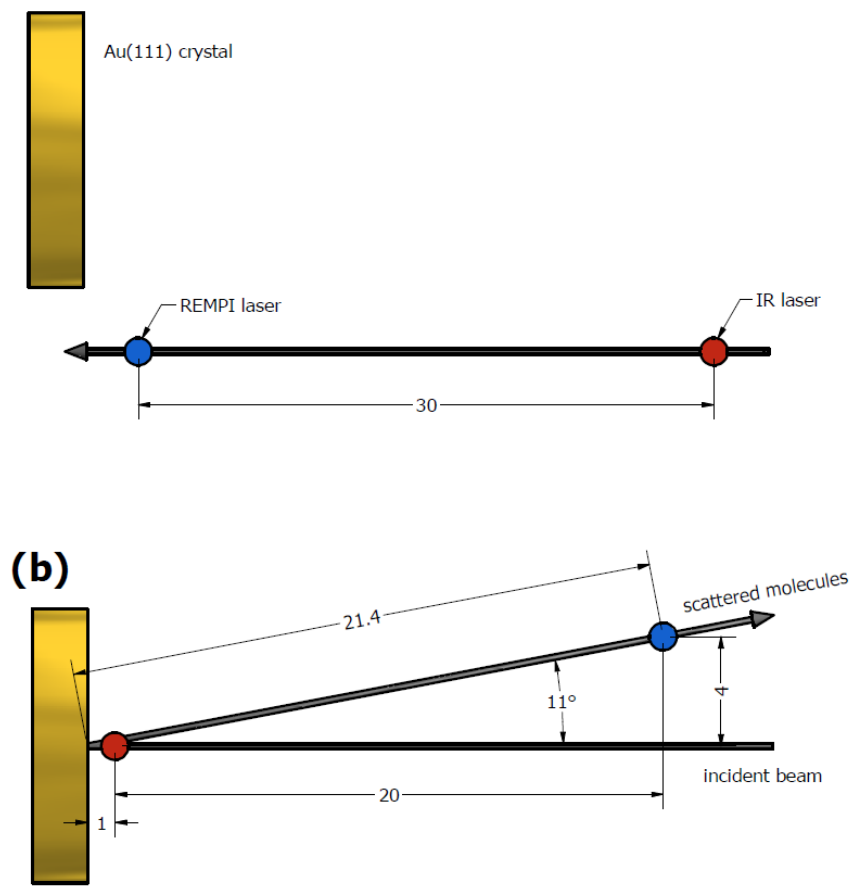

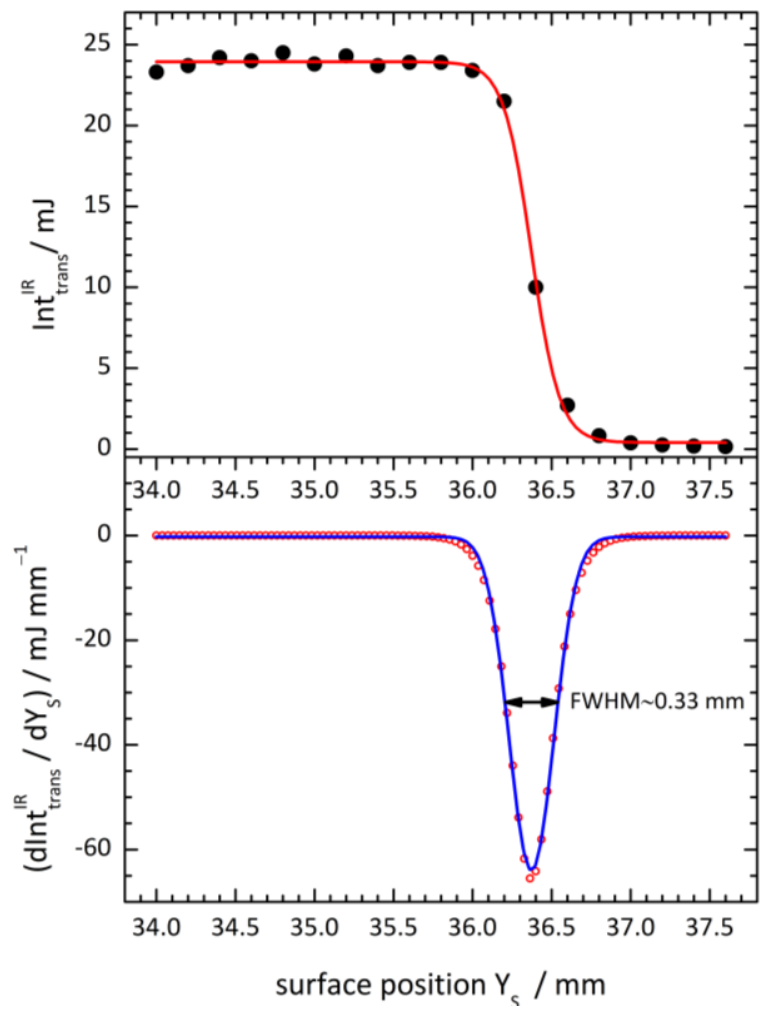

Fig. 2.18: Set-up for state-to-state time-of-flight experiments for determination of the incident beam velocity (left panel (a)) and for the scattered beam when incident molecules are prepared in vibrationally excited states (left panel (b)) [87]. The correct distance of the IR laser from the surface was in general determined by monitoring the transmittance through the vacuum chamber as function of the surface position $Y_{S}$ (right panel). The open symbols in the lower right panel reflect the first derivative of the fit to the experimental data. 


\section{Vibrationally inelastic scattering of NO from Au(111)}

\subsection{Multi-quantum vibrational excitation of NO/Au(111)}

Vibrational excitation of NO in direct scattering from metal surfaces has been studied in great detail over the last decades for scattering off $\operatorname{Ag}(111)[8,44]$ and $\operatorname{Au}(111)[1,25,27,30]$. Recently absolute vibrational excitation probabilities were measured [1] and semi-quantitative agreement with first principles IESH theory was obtained [25]. However, all these studies were so far limited to excitation of the lowest two excited vibrational states and no multi-quantum excitation similar to relaxation of $\mathrm{NO}(v=15) / \mathrm{Au}(111)$ was observed. The reason for this absence of experimental data is the extremely high sensitivity that is required for detection of $\mathrm{NO}(v=0 \rightarrow 3,4, \ldots)$ excitation. Previous work for $\mathrm{NO} / \mathrm{Au}(111)$ revealed that vibrational excitation and de-excitation result from nonadiabatic interaction of the molecular vibration with the electron-hole pairs of the metal surface $[3,8,25,26,30,31,34,43,44$, $65,88]$. The observed excitation probabilities follow an Arrhenius-like dependence on surface temperature with the activation energy equal to the vibrational energy uptake and a prefactor which depends on the incident kinetic energy $E_{I}$.

$$
P_{0 v}\left(T_{S}\right)=A_{0 v} e^{-\frac{\Delta E_{v i b}}{k_{B} T_{S}}}
$$

Newns [43] and later Matsiev et al. [30] have shown that this Arrhenius behavior originates from the $T_{S}$ dependent availability of excited EHPs that match the energy for vibrational excitation. For a simple estimation with identical prefactors for $v=0 \rightarrow 1,0 \rightarrow 2$ and $0 \rightarrow 3$ excitation, the thermal factor gives ratios of $v=1: 2: 3$ of about $1: 0.06: 0.004$ at $T_{S}=1000 \mathrm{~K}$. One expects then that the $\mathrm{NO}(v=3)$ population is only about $0.4 \%$ of $\mathrm{NO}(v=1)$ and $7 \%$ of $\mathrm{NO}(v=2)$. Conventional $(1+1) \mathrm{REMPI}$ does not provide the necessary sensitivity to study $0 \rightarrow 3$ excitation over a broad temperature range. However, one can use of the mismatch in $A \leftarrow X$ absorption cross-section $\left(\sim 3.5 \times 10^{-16} \mathrm{~cm}^{2}\right.$ [108]) and ionization crosssection $\left(\sim 7 \times 10^{-19} \mathrm{~cm}^{2}\right.$ [109]) out of the $A$ state. Therefore, the resonant $A \leftarrow X$ absorption step will be saturated at much lower laser intensities than the ionization step. Consequently, (1+1) REMPI will pump many more molecules into the $A$ state than are finally ionized and detected. Because the ionization is nonresonant, an increase in ion signal can be achieved by an intense second laser with sufficient photon energy $(\lambda \leq 328 \mathrm{~nm})$ to overcome the ionization threshold. Hippler and Pfab applied this two color $\left(1+1^{\prime}\right)$ REMPI scheme using an excimer laser $(308 \mathrm{~nm}, \sim 15 \mathrm{~mJ})$ for ionization and could enhance the $(1+1)$ REMPI signal by a factor of 20 [108].

Fig. 3.1 shows a comparison of conventional (1+1) REMPI (red) and two-color ( $\left.1+1^{\prime}\right)$ REMPI (black) for $\mathrm{NO}(v=0 \rightarrow 2)$ and $\mathrm{NO}(v=0 \rightarrow 3)$ excitation. All spectra were measured after scattering off $\mathrm{Au}(111)$ at $T_{S}=1000 \mathrm{~K}$ with identical laser intensities for the absorption step. In my work, the fourth harmonic (266 nm, 10-14 mJ) of a second Nd:YAG laser was used for ionization of the $A$ state for $\left(1+1^{\prime}\right)$ REMPI which leads to an enhancement in signal. The magnitude of the $\left(1+1^{\prime}\right)$ REMPI enhancement depends on the day-to-day temporal and spatial overlap of the two laser beams; I obtained enhancement factors of 5-10. The use of $266 \mathrm{~nm}$ radiation instead of $308 \mathrm{~nm}$ light for ionization has the major drawback that the photon energy of $266 \mathrm{~nm}$ radiation $(4.7 \mathrm{eV})$ can cause nonresonant two-photon ionization of $\mathrm{NO}(v=0)$ $(I P=9.26 \mathrm{eV})$, whereas $308 \mathrm{~nm}(4.0 \mathrm{eV})$ radiation requires absorption of three photons. Nonresonant ionization will mainly arise if the intense $266 \mathrm{~nm}$ beam hits the dense incident molecular beam; this situation is avoided in the experiments. 


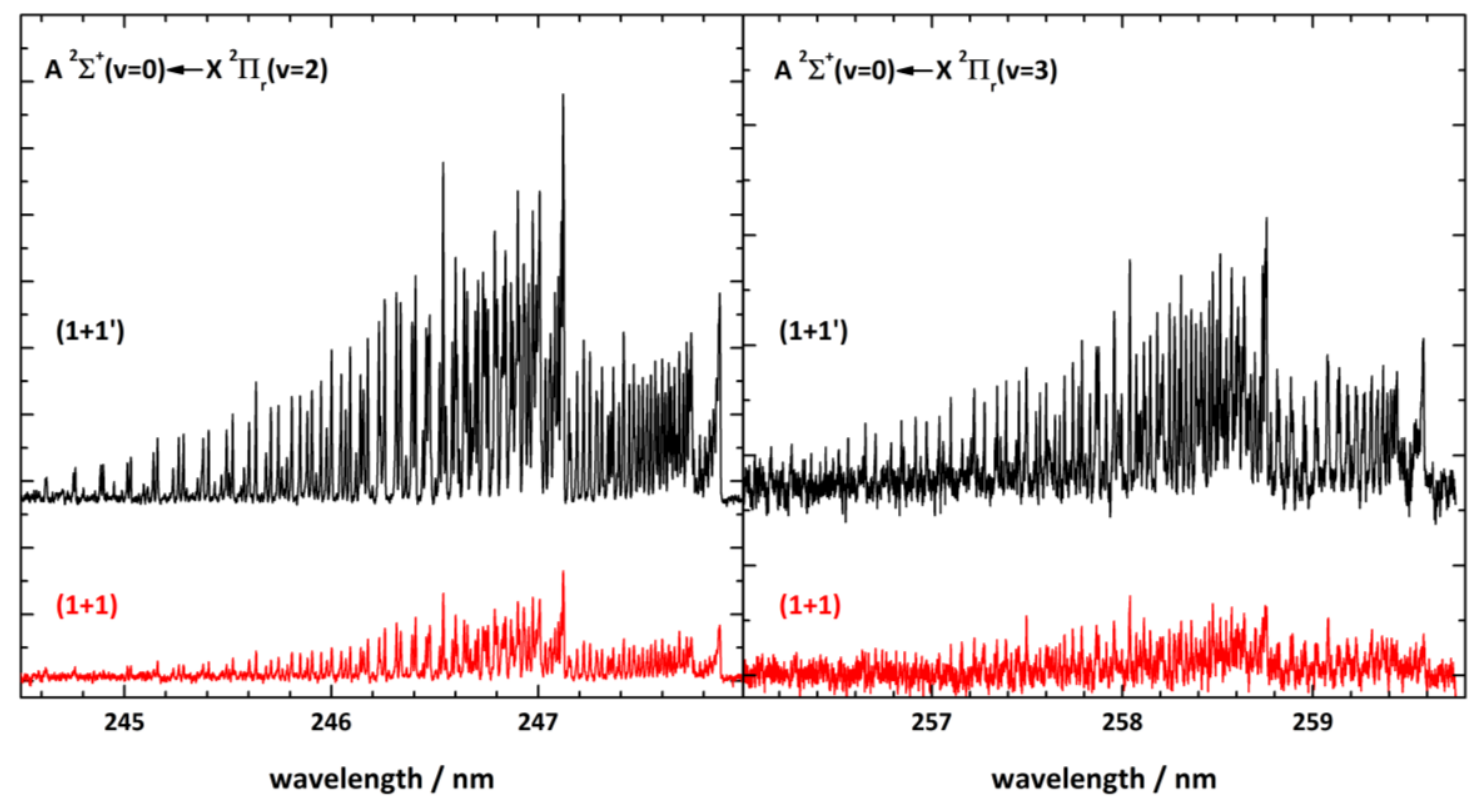

Fig. 3.1: Comparison of one-color (1+1) REMPI (red) and two-color (1+1') REMPI (black) for detection of $\mathrm{NO}(v=2)($ left) and $\mathrm{NO}(v=3)$ after scattering off $\mathrm{Au}(111)$ at $\mathrm{T}_{\mathrm{S}}=973 \mathrm{~K}$. For $\mathrm{NO}(v=2)$ the vibrational band can be detected with both REMPI schemes with sufficient $\mathrm{S} / \mathrm{N}$ ratio, whereas the $\mathrm{NO}(v=3)$ signal only exhibits a $\mathrm{S} / \mathrm{N}$ of less than 2 for $(1+1)$ REMPI. The comparison to two-color $\left(1+1^{\prime}\right)$ REMPI using additional $14 \mathrm{~mJ}$ of $266 \mathrm{~nm}$ shows that the signal is enhanced by a factor of about 5 while the noise-level is constant.

\subsubsection{Determination of absolute excitation probabilities}

I measured absolute vibrational excitation probabilities for $\mathrm{NO}\left(v_{i}=0\right)$ scattering into $v_{f}=1,2,3$ following the methodology of Cooper et al. [1]. In general, the probability for vibrational excitation is defined as:

$$
P_{0 v_{f}}=\frac{N_{v_{f}}}{\sum N_{v_{f}}} \approx \frac{N_{v_{f}}}{N_{v_{f}=0}}
$$

where $N_{v_{f}}$ is the number of molecules scattered into a specific final vibrational state $v_{f}$. Because excitation probabilities are small $\left(<10^{-2}\right.$ for $v=1$ [26] and even smaller for $\left.v>1\right)$ the denominator can be approximated by the number of molecules in the vibrational ground state $v_{f}=0 . N_{v_{f}}$ is proportional to the area $\int S(\lambda) d \lambda$ under the spectrum of the respective vibrational band, where $S(\lambda)$ is the wavelength dependent intensity of the REMPI signal. REMPI spectra for all detectable vibrational states are measured for a feasible range of surface temperatures using the 0-0, 0-1, 0-2 and 0-3 bands. Fig. 3.3 shows an example of typically observed REMPI spectra. The absolute spectral area depends on several factors like detector gain $\Gamma_{v}$, laser intensity $f\left(I_{v}\right)$, Franck-Condon factors $F C F_{0 v}$, and changes in the temporal $\Delta\left(\tau_{v}\right)$ and the angular distribution $\Theta\left(\theta_{v}\right)$ of the molecules. In case of $v_{f}=3$ an additional correction term TCRE for $\left(1+1^{\prime}\right)$ REMPI enhancement has to be included. All these correction factors are taken into account and used for correction of $\int S(\lambda) d \lambda$ :

$$
N_{v} \propto\left\langle s_{v}\right\rangle \cdot \Theta\left(\theta_{v}\right)^{-1} \cdot \Gamma_{v} \cdot f\left(I_{v}\right)^{-1} \cdot \Delta\left(\tau_{v}\right) \cdot F C F_{0 v}^{-1} \cdot\left\{T C R E^{-1}\right\} \cdot \int S(\lambda) d \lambda
$$


In addition, the density dependent REMPI signal must be converted into flux by multiplication with the scattered mean velocity $\left\langle s_{v}\right\rangle . \Gamma_{v}$ is measured experimentally by monitoring the REMPI signal as function of the voltage (1200-2000 V) across the micro-channel plate detector (see Appendix A for details). The laser power function is measured in a similar fashion by monitoring the REMPI signal for different laser pulse energies and can be described by a simple power function:

$$
f\left(I_{v}\right)=c \cdot I_{v}^{n}
$$

I obtain the exponent $n$ from double logarithmic plots which are shown in Fig. 3.2 for the different REMPI schemes. In case of (1+1) REMPI (red curve), I find $n=1.57$ which is consistent with saturation of one step in a two photon absorption process in the center of the beam profile [110]; the $A \leftarrow X$ absorption is most likely saturated due to the higher cross-section. For two-color $\left(1+1^{\prime}\right)$ REMPI $n=0.28$ (blue curve) and $n=0.44$ (black curve) are obtained for the resonant absorption (tunable dye laser) and the ionization step $(266 \mathrm{~nm})$, respectively. These numbers show that the $A \leftarrow X$ absorption is saturated and that the intense $266 \mathrm{~nm}$ laser beam even saturates the ionization step. The (at least partial) saturation of the absorption step in $(1+1)$ and $\left(1+1^{\prime}\right)$ REMPI suggests that the correction for FranckCondon factors can be neglected. Note that the Franck-Condon factors for the $0-v^{\prime \prime}$ bands, used here, are anyhow similar [89]. Consequently, their implementation in the analysis does not introduce a significant error to $N_{v_{f}}$ and $F C F_{0 v}$ is therefore included for the calculation of $N_{v_{f}}$.

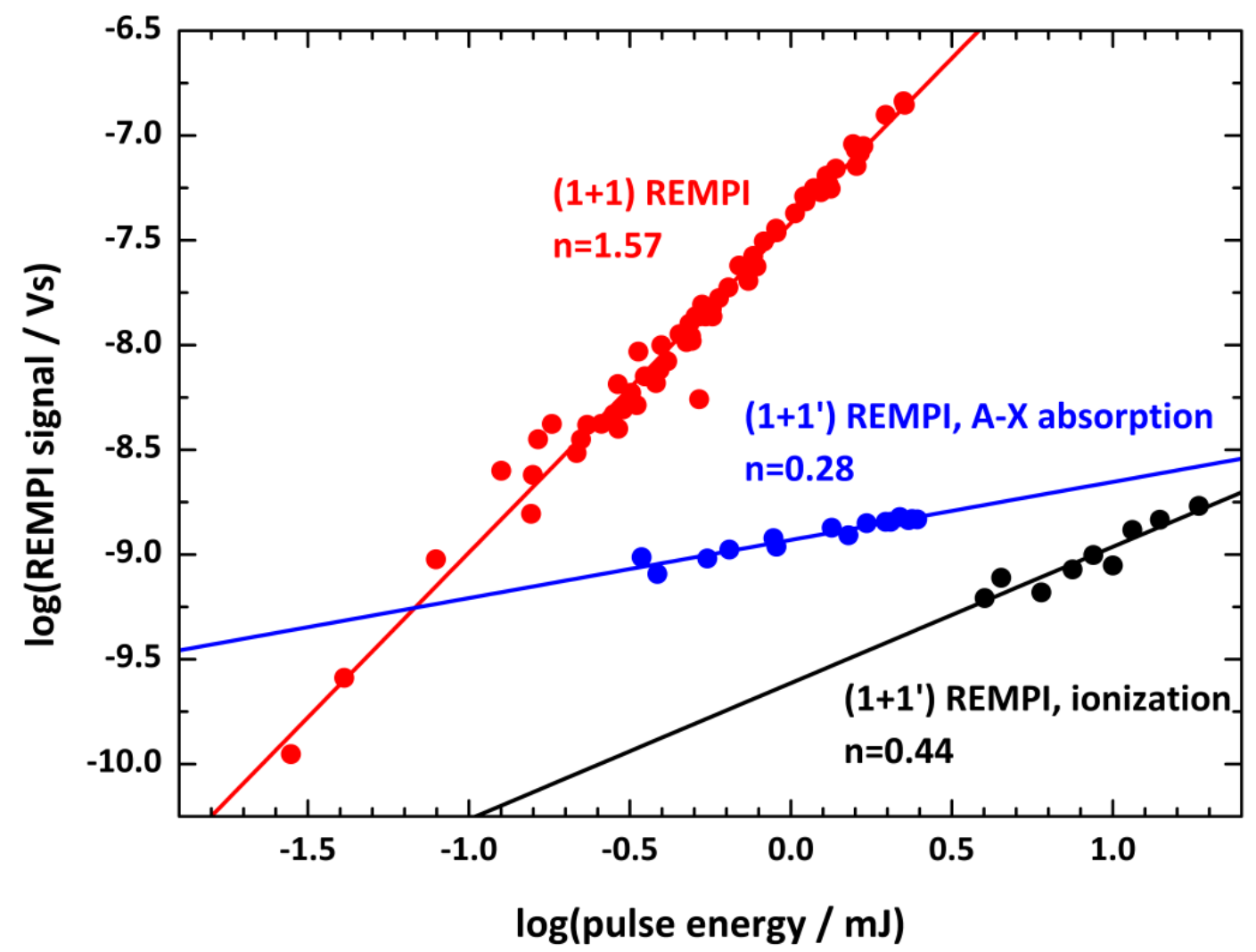

Fig. 3.2: Double logarithmic plot for the dependences of the ion signal on laser pulse energy for $(1+1)$ REMPI as well as for both lasers used for (1+1') REMPI. 
Angular distributions were measured by translating the REMPI beam parallel to the surface at a fixed distance, and monitoring the signal for different vibrational states and surface temperatures. The distributions are fitted to a Gaussian distribution of the type:

$$
\Theta\left(\theta_{v}\right)=\frac{e^{-\frac{1}{2}\left(\frac{\theta_{v}-\theta_{0}}{\sigma}\right)^{2}}}{\sqrt{2 \pi} \sigma}
$$

Here, $\theta_{0}$ is the center of the angular distribution and $\sigma$ is a width parameter that is related to the full width at half maximum, $F W H M=2 \sigma \sqrt{2 \ln 2}$. I measured three angular distributions for each $v_{f}$ and $E_{I}$ at different surface temperatures that covered the range of temperatures studied. I used linear interpolation in order to determine $\theta_{0}$ and $\sigma$ at the intermediate $T_{S}$ values.

$\Delta\left(\tau_{v}\right)$ accounts for the width of the temporal profile of the scattered molecular beam as well as for the intensity at the delay, $\tau_{v}$, at which the REMPI spectra were measured. Temporal profiles were measured at three different surface temperatures, and interpolation was used to find $\Delta\left(\tau_{v}\right)$ at intermediate $T_{S}$.

The correction TCRE is only applied for measurements of $\mathrm{NO}(v=0 \rightarrow 3)$ excitation where two-color $\left(1+1^{\prime}\right)$ REMPI must be used and the signal is enhanced with respect to conventional $(1+1)$ REMPI detection. The enhancement depends on the day-to-day temporal and spatial overlap of the tunable and the $266 \mathrm{~nm}$ laser beam. Therefore, it is determined prior to every $v_{f}=3$ excitation experiment by measuring the $\mathrm{NO}(v=0 \rightarrow 2)$ excitation probability at one single surface temperature with (1+1) and $\left(1+1^{\prime}\right)$ REMPI. This method is convenient for two reasons: (1) $v_{f}=2$ excitation probabilities provide sufficient S/N for (1+1) REMPI and (2) the 0-2 and the 0-3 band can be covered with the same laser dye allowing for quick switching and thus more reliable calibration.

\subsubsection{Results}

\section{REMPI spectra}

REMPI spectra of scattered $\mathrm{NO}\left(v_{f}=0,1,2,3\right)$ were measured at various surface temperatures ranging from 300-1100 K. An example of observed REMPI spectra is shown in Fig. 3.3 for scattering at $0.41 \mathrm{eV}$ incidence energy. The spectra are corrected for differences in detector gain, laser intensity and, in case of $\mathrm{NO}\left(v_{f}=3\right)$, for $\left(1+1^{\prime}\right) \mathrm{REMPI}$ enhancement. The left panel of Fig. 3.3 already allows predictions for the probabilities of excitation into different vibrational states. At $T_{S}=973 \mathrm{~K}$, the $\mathrm{NO}(v=0 \rightarrow 1)$ is $\sim 10^{-2}$, $\mathrm{NO}(v=0 \rightarrow 2)$ excitation is $\sim 4 \times 10^{-4}$ and $\mathrm{NO}(v=0 \rightarrow 3)$ excitation is $\sim 2 \times 10^{-5}$. The right panel of Fig. 3.3 illustrates the surface temperature dependence for excitation into $v_{f}=1,2,3$ by comparing spectra at $T_{S}=873 \mathrm{~K}$ and $1073 \mathrm{~K}$. The change in surface temperature results in an increase of about $50 \%$ for

$v_{f}=1$, of about $120 \%$ for $v_{f}=2$ and of about $160 \%$ in the $v_{f}=3$ signal. This strong surface temperature dependence of the vibrational excitation probability sets the lower limits for $T_{S}$ for observable signals with sufficient $\mathrm{S} / \mathrm{N}$ for $v_{f}=1, v_{f}=2$ and $v_{f}=3$ to $400 \mathrm{~K}, 650 \mathrm{~K}$ and $850 \mathrm{~K}$, respectively.

A qualitative comparison of the rotational structure of the different vibrational bands shows that the amount of rotational excitation seems to neither depend strongly on the final vibrational state nor on the temperature of the surface. None of the spectra exhibits pronounced features like rotational rainbow scattering. Note that the rotational structure of the $0-1,0-2$ and $0-3$ bands cannot be compared easily because the overlap of the rotational branches changes slightly for each vibrational band [111]. 

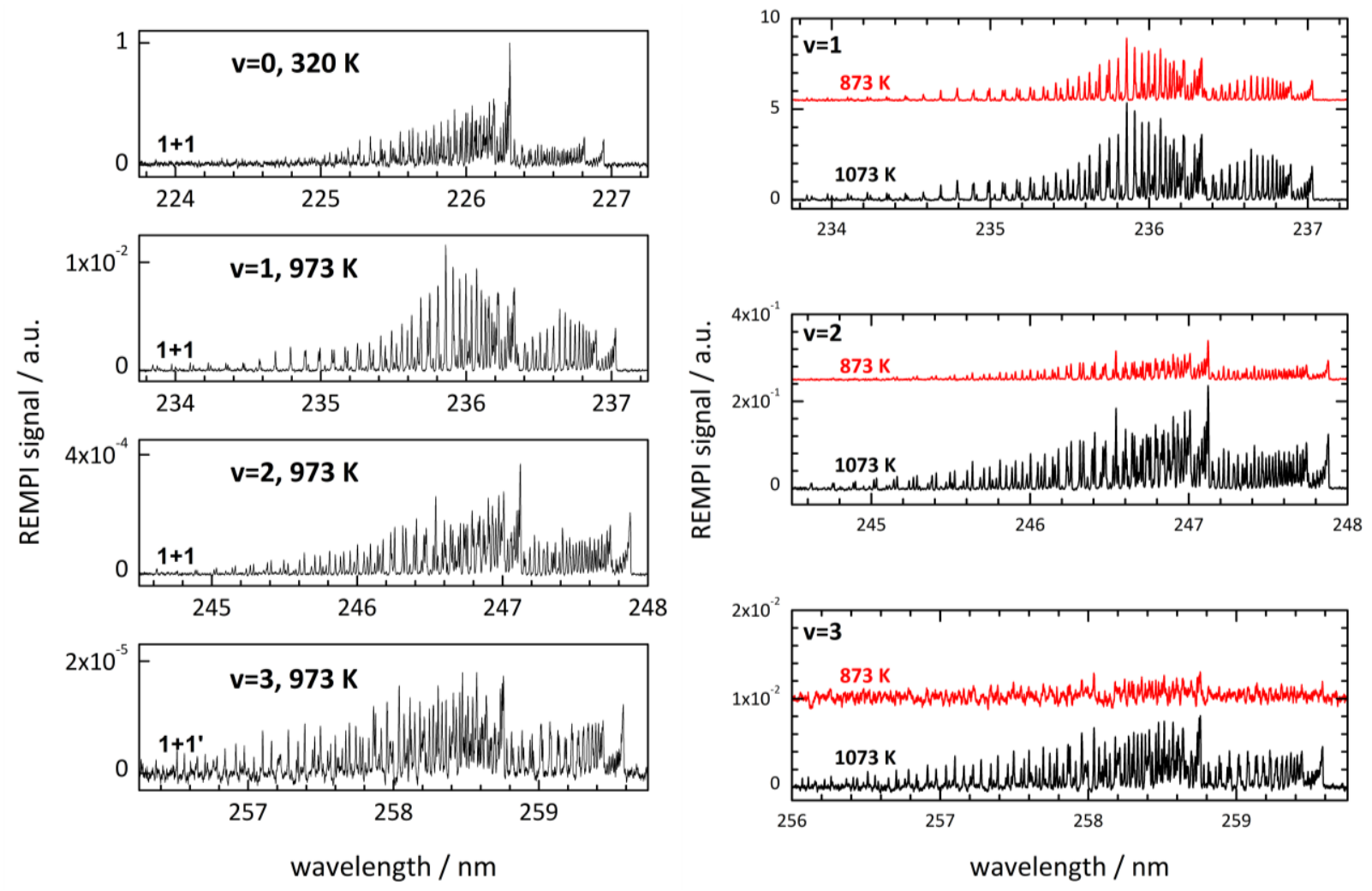

Fig. 3.3: REMPI spectra for the $0-0,0-1,0-2$ and $0-3 \gamma$-bands of NO scattered off $A u(111)$ at 0.41 eV incidence energy. Left: REMPI spectra for $\mathrm{NO}\left(v_{f}=0,1,2,3\right)$ at 320 for the vibrational ground state and $973 \mathrm{~K}$ for the vibrationally excited states.. The REMPI spectra were corrected for differences in detector gain, laser intensity and (in case of $\left.v_{f}=3\right)\left(1+1^{\prime}\right)$ REMPI enhancement. Reprinted with permission from Journal of Physical Chemistry A, 2013. 117(32), 7091-7101. Copyright 2013 American Chemical Society. Right: REMPI spectra for $\mathrm{NO}\left(v_{f}=1,2,3\right)$ at $873 \mathrm{~K}(\mathrm{red})$ and $1073 \mathrm{~K}$ (black) surface temperature. The spectra were corrected for changes in laser power, detector gain and $\left(1+1^{\prime}\right)$ REMPI enhancement. While the increase of REMPI signal with increasing $\mathrm{T}_{\mathrm{S}}$ is rather weak for $\mathrm{NO}\left(v_{f}=1\right)$, it is quite strong for $\mathrm{NO}(v=2)$ and even more dramatic for $\mathrm{NO}\left(v_{f}=3\right)$.

Furthermore, I carried out measurements for $\mathrm{NO}\left(v_{f}=3\right)$ excitation at different incidence translational energies ranging from 0.41 to $0.95 \mathrm{eV}$ in order to study the effect of $E_{I}$ on rotational and vibrational excitation. The influence on rotational excitation is illustrated in Fig. 3.4 which shows $\left(1+1^{\prime}\right)$ REMPI spectra of the NO $A\left(v^{\prime}=0\right) \leftarrow X\left(v^{\prime \prime}=3\right)$ band taken at different $E_{I}$. All spectra were measured after scattering off $\mathrm{Au}(111)$ at $T_{S}=973 \mathrm{~K}$. The area was normalized to 1 for comparison. The spectra clearly show that the rotational structure of the 0-3 band changes with increasing $E_{I}$. That is, higher incidence energy leads to more rotational excitation, which is most clear in the spectral area from $256-257.5 \mathrm{~nm}$. The effect of incidence energy on vibrational excitation is not easy to judge from the raw REMPI spectra themselves because the NO density in the molecular beam changes for different gas mixtures. I will therefore discuss the effect based on the final $E_{I}$ dependent excitation probabilities. 


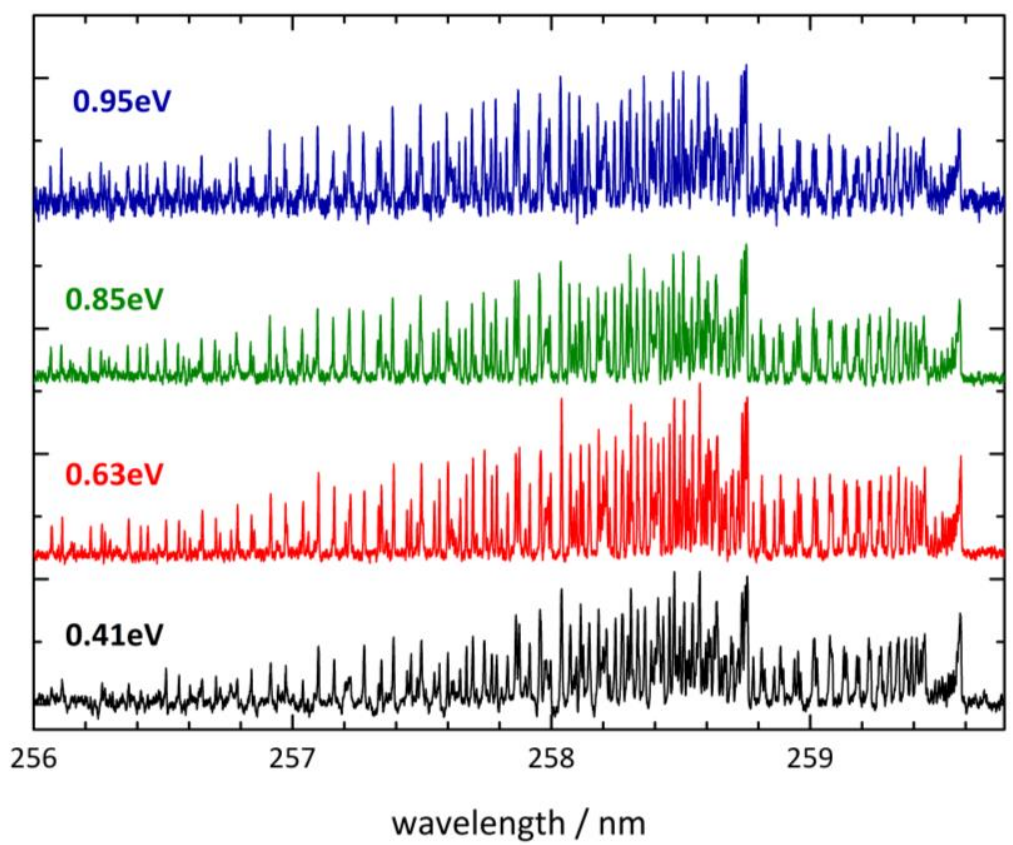

Fig. 3.4: $\left(1+1^{\prime}\right)$ REMPI spectra for $\mathrm{NO}(v=0 \rightarrow 3)$ excitation in scattering off $\mathrm{Au}(111)$ at $T_{S}=973 \mathrm{~K}$ for different incidence energies of $0.41 \mathrm{eV}$ (black), $0.63 \mathrm{eV}$ (red), $0.85 \mathrm{eV}$ (green) and $0.95 \mathrm{eV}$ (blue).

\section{Angular distributions}

Angular distributions of scattered molecules were measured by translating the REMPI beam parallel to the surface (perpendicular with respect to the molecular beam) at about $11 \mathrm{~mm}$ distance and monitoring the REMPI intensity as a function of beam position. Some representative results are shown in Fig. 3.5. The upper panel shows angular distributions for $\mathrm{NO} / \mathrm{Au}(111)$ scattering into $v_{f}=0,1,2,3$ at $E_{I}=0.41 \mathrm{eV}$. All angular distributions are narrow $\left(\cos ^{5} \theta-\cos ^{9} \theta\right)$ and peak near the specular angle at $0-5^{\circ}$ (angular resolution $\sim 10^{\circ}$ ). The angular distributions artificially appear broader than they are because they reflect a convolution of the size of the detection volume and the width of the real angular distribution. The detection volume is rather large because I use an unfocused REMPI beam $(\varnothing \approx 5 \mathrm{~mm})$ for the experiments. Furthermore, the REMPI laser will ionize all molecules along the laser beam direction with equal probability. The latter effect becomes significant for broad angular distributions and a $\cos \theta$ distribution will appear as $\cos ^{2} \theta$ in the experiment. The lower panel of Fig. 3.5 shows angular distributions for scattering into $v_{f}=3$ at different incidence energies. In the range from $E_{I}=0.4-$ $0.95 \mathrm{eV}$ all distributions have similar width and peak near the specular angle. The behavior of other vibrational states can be found in ref. [1] and is similar to that of $\mathrm{NO}(v=0 \rightarrow 3)$ excitation. In the previous work, angular distributions clearly broaden only at the lowest incidence energies of $E_{I}=0.1 \mathrm{eV}$ and $0.26 \mathrm{eV}$, a regime where trapping followed by desorption is known to be significant [112]. 

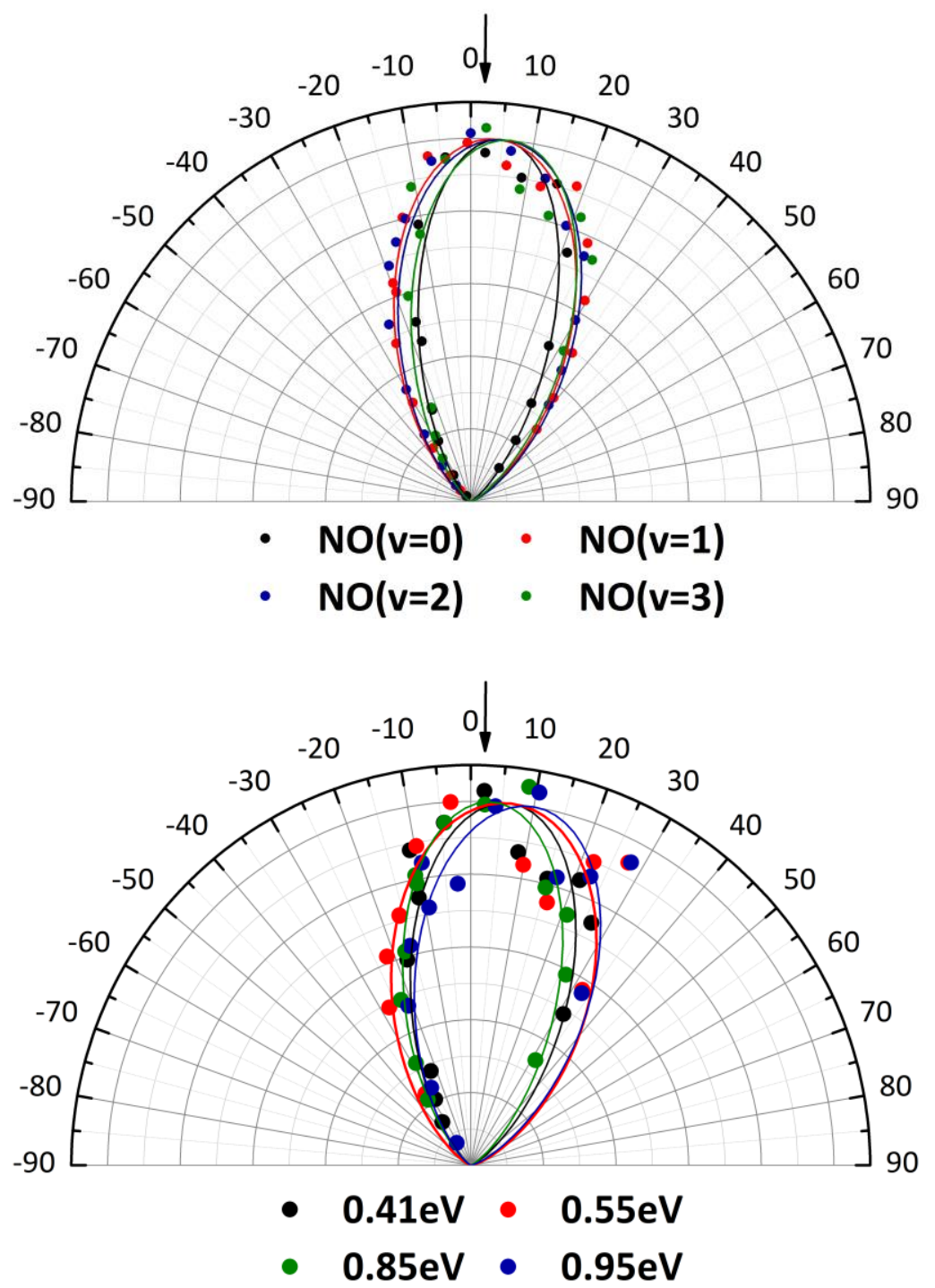

Fig. 3.5: Angular distributions for $\mathrm{NO}$ scattering from $\mathrm{Au}(111)$ for different vibrational states (top) and for $\mathrm{NO}(v=0 \rightarrow 3)$ scattering at different incidence energies (bottom). Angular distributions of $\mathrm{NO}\left(v_{f}=3\right)$ were corrected for non-resonant background caused by the intense ionization laser. The arrows indicate the incidence angle of about $2^{\circ}$.

\section{Temporal profiles}

An example of observed arrival time profiles for scattered $\mathrm{NO}\left(v_{f}=0,1,2,3\right)$ molecules at $E_{I}=0.41 \mathrm{eV}$ is shown in the left panel of Fig. 3.6. The right panel shows the dependence on surface temperature for $v=0 \rightarrow 3$ scattering. The arrival time distributions in the main panels are normalized to an area of 1 for comparison. The inset on the right shows the real (un-normalized) measured intensities for $\mathrm{NO}\left(v_{f}=3\right)$ at different $T_{S}$. The temporal profile of the scattered molecules is basically unaffected by the final vibrational state and only for $v_{f}=0$ a slightly more pronounced tail component is visible between 350 $600 \mu \mathrm{s}$. For all vibrational states, the width is slightly broader than that of the incident molecular beam $(\sim 70 \mu \mathrm{s})$ and exhibits a slight tail component. In the $v=0 \rightarrow 3$ channel the REMPI signal intensity increases significantly with increasing surface temperature (inset), an effect that results from higher vibrational excitation with increasing $T_{S}$ (see Fig. 3.3). The shape (width) of the arrival time distribution is however not affected by changes in surface temperature. Consequently, the term $\Delta\left(\tau_{v}\right)$ in equation 3.3 will only be a minor correction in the determination of absolute excitation probabilities. 


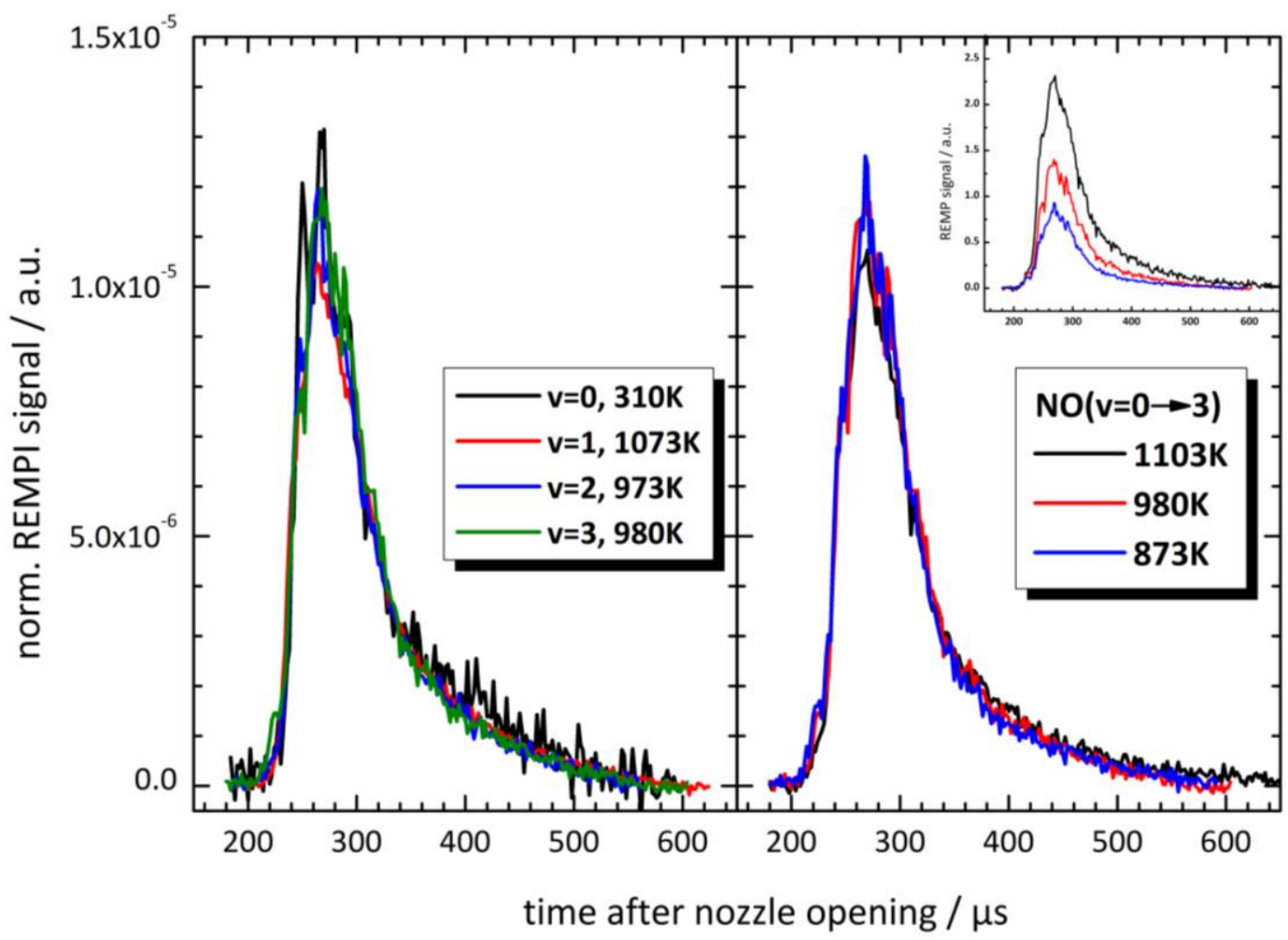

Fig. 3.6: Arrival time distributions for NO scattered off $A u(111)$. Left panel: Time-of-flight spectra for scattering into different vibrational states $v=0$ (black), $v=1$ (red), $v=2$ (blue) and $v=3$ (green). Right panel: Surface temperature dependence for the arrival time distribution of $\mathrm{NO}(v=3)$. The inset in the right shows the real measured intensities. The other time profiles were normalized to an area of 1 for comparison.

\section{Excitation probabilities}

Absolute vibrational excitation probabilities for $\mathrm{NO}\left(v_{f}=1,2,3\right)$ are derived from the data shown above and presented for incidence energies of $0.41,0.63$ and $0.95 \mathrm{eV}$ in Fig. 3.7. For all vibrational channels and incidence energies, the excitation probabilities show an Arrhenius-like dependence on surface temperature (solid lines). In every dataset the activation energy is fixed to the vibrational energy uptake of $0.236,0.472$ or $0.708 \mathrm{eV}$ for excitation into $v_{f}=1,2,3$, leaving the Arrhenius prefactors, $A_{0 v}$, as the only fit parameter. The derived values for $A_{0 v}$ are given in Fig. 3.7. For all vibrational channels they increase with increasing $E_{I}$ from $0.63 \mathrm{eV}$ to $0.95 \mathrm{eV}$. I find that the values for $A_{0 v}$ at $E_{I}=0.41 \mathrm{eV}$ are higher than those at $0.63 \mathrm{eV}$. This fluctuation in prefactors might however be within the uncertainty of the experiment and is also visible in the study of Cooper et al. [26].

Furthermore, the prefactors decrease systematically with increasing vibrational quantum number $v_{f}$. It is known that $A_{0 v}$ is related to the nonadiabatic coupling strength between the NO vibration and the EHPs in the metal $[27,30]$. Therefore, the $v_{f}$ dependence of the Arrhenius prefactors can be used to estimate the importance of different excitation pathways. The simplest case with only vibrational excitation steps involves transitions with $\Delta v=1,2,3$. Excitation into $v_{f}=2,3$ can then occur either in direct overtone excitation or in a sequential mechanism. A sequential mechanism with only $\Delta v=1$ excitation steps $(0 \rightarrow 1 \rightarrow 2$ or $0 \rightarrow 1 \rightarrow 2 \rightarrow 3)$ suggests prefactors of $A_{02}=A_{01}^{2}=0.149$ and $A_{03}=A_{01}^{3}=0.059$ for $v_{f}=2$ and $v_{f}=3$, assuming equal coupling for all single quantum transitions. These values do not agree with the experimental observation. 


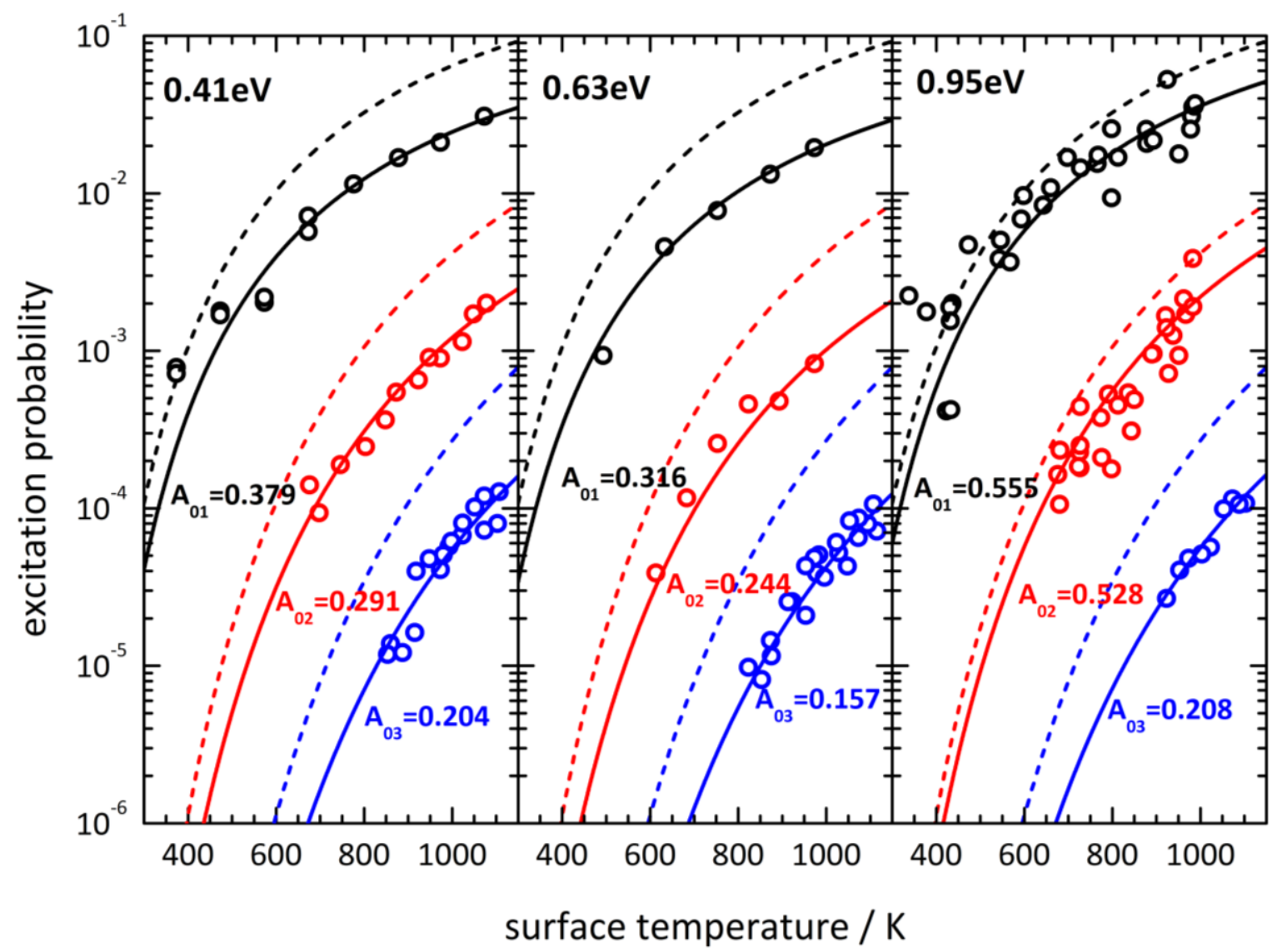

Fig. 3.7: Surface temperature dependent absolute excitation probabilities for $\mathrm{NO}\left(v_{f}=1,2,3\right)$ scattering from $\mathrm{Au}(111)$ for incidence energies of $0.41 \mathrm{eV}$ (left), $0.63 \mathrm{eV}$ (middle) and $0.95 \mathrm{eV}$ (right). The analysis includes corrections for detector gain, laser intensity, angular distribution and temporal profiles. The data for $v_{f}=1$ and $v_{f}=2$ excitation for $0.63 \mathrm{eV}$ and $0.95 \mathrm{eV}$ was taken from reference [1]. The solid line show Arrhenius plots for each vibrational state. The activation energy has been fixed to the vibrational energy uptake and only the pre-exponential factors are fitted. The dashed lines indicate the thermal limit for complete equillibration of the NO vibration with the surface temperature.

\subsubsection{State-to-state kinetic rate model}

Matsiev et al. previously introduced a state-to-state rate model in order to describe the kinetics of the vibrational excitation process [30]. The kinetic model involves basically all possible vibrational transitions. The rate for a transition $v \rightarrow v^{\prime}$ is described by:

$$
\frac{d n_{v^{\prime}}\left(t, T_{S}\right)}{d t}=\sum k_{v v^{\prime}} n_{v, v \prime}\left(t, T_{S}\right)
$$

The authors used the description of Persson and Persson [47] for the rate constant $k_{v v}$. This approach was originally developed for vibrational relaxation of $\mathrm{CO}$ adsorbed on $\mathrm{Cu}(100)$ and is based on first-order time-dependent perturbation theory and Fermi's Golden Rule: 


$$
k_{v v^{\prime}}=\frac{2 \pi}{\hbar} \sum_{i, k}\left|\left\langle i v\left|H^{\prime}\right| k v^{\prime}\right\rangle\right|^{2} \delta\left(\varepsilon_{i}-\varepsilon_{k}-\hbar \omega_{0}\right)
$$

where $v$ and $v^{\prime}$ are the initial and final vibrational states with the energies $\varepsilon_{i}$ and $\varepsilon_{k}, \omega_{0}$ is the vibrational frequency and $H^{\prime}$ is the perturbation corresponding to a Newns-Anderson type electronic Hamiltonian $[113,114]$. Equation 3.7 can be transferred into equation 3.8 following the procedure described in ref. [47]:

$$
k_{v v^{\prime}}=\alpha_{v v^{\prime}} \frac{E_{v v^{\prime}}}{e^{E_{v v^{\prime}} / k_{B} T}-1} \quad \text { with } \quad \alpha_{v v^{\prime}} \approx \frac{2 \pi}{\hbar} \lambda_{v v^{\prime}}^{2} \rho_{a}^{2}
$$

Here $E_{v v}$, is the vibrational energy change, $\lambda_{v v^{\prime}}=\left|\left\langle i v\left|H^{\prime}\right| k v^{\prime}\right\rangle\right|$ is the coupling constant describing the interaction of the electronic affinity level $|a\rangle$ and the molecular vibration, and $\rho_{a}$ is the projection of the density of states onto the molecular affinity level. The thermal factor $E_{v v^{\prime}} /\left(e^{E_{v v^{\prime}} / k_{B} T}-1\right)$ is derived from the Fermi-distribution and the density of states. Note that equation 3.8 can be approximated by $k_{v v^{\prime}} \approx \alpha_{v v^{\prime}} \times e^{-E_{v v^{\prime}} / k_{B} T}$ for $k_{B} T \ll E_{v v_{1},}$. This expression has the form of an Arrhenius equation as it has been observed empirically for NO excitation in scattering from $\operatorname{Ag}(111)[8,44]$ and $\operatorname{Au}(111)[1,25,27$, 30]. Using equations 3.6-3.8, one obtains the following system of coupled differential equations:

$$
\begin{aligned}
& \frac{d n_{0}}{d t}=-k_{01} n_{0}(t)-k_{02} n_{0}(t)-k_{03} n_{0}(t)+k_{10} n_{1}(t)+k_{20} n_{2}(t)+k_{30} n_{3}(t) \\
& \frac{d n_{1}}{d t}=k_{01} n_{0}(t)-k_{12} n_{1}(t)-k_{13} n_{1}(t)-k_{10} n_{1}(t)+k_{21} n_{2}(t)+k_{31} n_{3}(t) \\
& \frac{d n_{2}}{d t}=k_{02} n_{0}(t)+k_{12} n_{1}(t)-k_{23} n_{2}(t)-k_{20} n_{2}(t)-k_{21} n_{2}(t)+k_{32} n_{3}(t) \\
& \frac{d n_{3}}{d t}=k_{03} n_{0}(t)+k_{13} n_{1}(t)+k_{23} n_{2}(t)-k_{30} n_{3}(t)-k_{31} n_{3}(t)-k_{32} n_{3}(t)
\end{aligned}
$$

with the rate constants for the excitation processes:

$$
\begin{aligned}
& k_{01}=\alpha_{01} \frac{\hbar \omega_{0}}{e^{\hbar \omega_{0} / k_{B} T}-1}, \quad \quad k_{02}=\alpha_{02} \frac{2 \hbar \omega_{0}}{e^{2 \hbar \omega_{0} / k_{B} T}-1}, \quad k_{03}=\alpha_{03} \frac{3 \hbar \omega_{0}}{e^{3 \hbar \omega_{0} / k_{B} T}-1} \\
& k_{12}=\alpha_{12} \frac{\hbar \omega_{0}}{e^{\hbar \omega_{0} / k_{B} T}-1}, \quad k_{23}=\alpha_{23} \frac{\hbar \omega_{0}}{e^{\hbar \omega_{0} / k_{B} T}-1}, \quad k_{13}=\alpha_{13} \frac{2 \hbar \omega_{0}}{e^{2 \hbar \omega_{0} / k_{B} T}-1}
\end{aligned}
$$

and vibrational relaxation:

$$
\begin{aligned}
& k_{10}=\alpha_{21} \frac{\hbar \omega_{0}}{1-e^{-\hbar \omega_{0} / k_{B} T}}, \quad k_{20}=\alpha_{20} \frac{2 \hbar \omega_{0}}{1-e^{2 \hbar \omega_{0} / k_{B} T}}, \quad k_{30}=\alpha_{02} \frac{3 \hbar \omega_{0}}{1-e^{-3 \hbar \omega_{0} / k_{B} T}} \\
& k_{21}=\alpha_{21} \frac{\hbar \omega_{0}}{1-e^{-\hbar \omega_{0} / k_{B} T}}, \quad \quad k_{31}=\alpha_{31} \frac{2 \hbar \omega_{0}}{1-e^{-2 \hbar \omega_{0} / k_{B} T}}, \quad k_{32}=\alpha_{32} \frac{\hbar \omega_{0}}{1-e^{-\hbar \omega_{0} / k_{B} T}}
\end{aligned}
$$

Consequently, the model contains overall 12 coupling parameters $\alpha_{v v}$ and the interaction time $t$ as variable parameters. However, the experimental data provides only $T_{S}$ dependent excitation probabilities for three different vibrational states. Therefore, it is necessary to reduce the number of 40 
open fit parameters, which requires certain assumptions. Matsiev et al. assumed microscopic reversibility $\left(\alpha_{v v \prime}=\alpha_{v \prime v}\right)$ and that the probabilities for transitions with the same $\Delta v$ are equal. Furthermore, they related overtone transitions to single quantum transitions using a scaling parameter for fitting. Because my study involved one more vibrational state than observed in the previous experiments, I have to include two different overtone transitions $(0 \rightarrow 2,0 \rightarrow 3)$ resulting in two scaling parameters $\beta$ and $\gamma$.

$$
\begin{gathered}
\alpha_{01}=\alpha_{12}=\alpha_{23}=\alpha_{10}=\alpha_{21}=\alpha_{32}=\alpha_{\Delta v=1} \\
\alpha_{02}=\alpha_{20}=\alpha_{13}=\alpha_{31}=\beta \cdot \alpha_{\Delta v=1} \\
\alpha_{03}=\alpha_{30}=\gamma \cdot \alpha_{\Delta v=1}
\end{gathered}
$$

These assumptions reduce the number of adjustable parameters to four: $\alpha_{\Delta v=1}, \beta, \gamma$ and $t$. However, the interaction time $t$ cannot be fitted separately from the coupling parameter $\alpha_{\Delta v=1}$. Therefore, $\alpha$ is set to 1 and an 'effective interaction time $\tau$ ' is defined as:

$$
\tau=\alpha_{\Delta v=1} \cdot t
$$

The assumptions of Matsiev et al. have to be taken with some care because they have not been tested in detail so far. The work of Monturet and Saalfrank [64] raised doubts about the validity of the assumption that transition probabilities are equal for the same $\Delta v$. The authors used a ' $v$ scaling law' which gives higher transition probabilities for higher vibrational states and simply results from a calculation of the transition probabilities for a harmonic oscillator from first-order perturbation theory. This model changes the assumptions to:

$$
\begin{gathered}
\alpha_{01}=1 / 2 \cdot \alpha_{12}=1 / 3 \cdot \alpha_{23}=\alpha_{10}=1 / 2 \cdot \alpha_{21}=1 / 3 \cdot \alpha_{32} \\
\beta_{s}=(2 v+1) \beta
\end{gathered}
$$

Which of these two models is the better approximation is unclear and requires the investigation of excitation probabilities for $\mathrm{NO}\left(v_{i}>0\right)$, for example $v=2 \rightarrow 3$ excitation. In the following sections, I will refer to these models as case (a) for $v$ independent rate constants and as case (b) for $v$ scaling based on a harmonic oscillator.

The kinetic rate model can in principle be solved analytically but this method produces functions for $n_{v}(t)$ that are too complicated to treat them computationally efficiently. Therefore, the differential rate equations are solved numerically and $\tau, \beta, \gamma$ are fitted to reproduce the experimentally observed excitation probabilities. In contrast to a simple Arrhenius fit, the kinetic model provides the possibility to evaluate the pathway of excitation. In contrast to earlier work [30], this subpopulation analysis is performed by decomposition of the population of each vibrational level into a sum of populations arising from a specific pathway:

$$
n_{v}(t)=n_{v}^{0}(t)+\sum_{v_{1} \neq v} n_{v_{1}}, v(t)+\sum_{\substack{v_{1} \neq v \\ v_{2} \neq v_{1}}} n_{v_{1}, v_{2}, v}(t)+\cdots
$$


where $n_{v}^{0}(t)$ denotes the time-dependent subpopulation of the level $v$ at $t=0, n_{v_{1}, v}(t)$ represents the subpopulation of the state $v$ formed by a single-step transition from level $v_{1}, n_{v_{1}, v_{2}, v}(t)$ is the population of $v$ formed due to the two-step transition $v_{2} \rightarrow v_{1} \rightarrow v$, and so on.

The information for the pathways that lead to a specific vibrational level can be obtained by writing kinetic master equations for the subpopulations. The initial population $n_{v}^{0}(t)$ of a vibrational state can only decrease due to transitions into other vibrational states:

$$
\frac{d n_{v}^{0}}{d t}=-\kappa_{v} n_{v}^{(0)}, \quad \kappa_{v}=\sum_{v_{1} \neq v} k_{v v_{1}}
$$

where $\kappa_{v}$ denotes the total outgoing rate.

The master equation for a single-step subpopulation $n_{v_{1}, v}(t)$ is obtained by counting the gain in $v$ due to the initial subpopulation in $v_{1}$ and the loss of $v$ into other vibrational states.

$$
\frac{d n_{v_{1} v}}{d t}=-\kappa_{v} n_{v_{1} v}+k_{v_{1} v} n_{v_{1}}^{(0)}
$$

This structure holds for any $i$-step subpopulation that leads to population transfer into a vibrational level $v$ :

$$
\frac{d n_{v_{1} \ldots v_{i} v}}{d t}=-\kappa_{v} n_{v_{1} \ldots v_{i} v}+k_{v_{i} v} n_{v_{1} \ldots v_{i}}
$$

Here, $k_{v_{i} v}$ presents the $v_{i} \rightarrow v$ transition rate constant and $n_{v_{1} \ldots v_{i}}$ is the (i-1)-step subpopulation of the level $v_{i}$.

This system of differential equations completely describes the time evolution of all subpopulations and can be solved analytically yielding for example the following expressions for $n_{v}^{0}(t)$ and $n_{v_{1} v}(t)$ :

$$
\begin{gathered}
n_{v}^{0}(t)=n_{v}^{0}(t=0) e^{-\kappa_{v} t} \\
n_{v_{1} v}(t)=n_{v}^{0}(t) \frac{e^{-\left(\kappa_{v}-\kappa_{v_{1}}\right)}-1}{\kappa_{v}-\kappa_{v_{1}}}
\end{gathered}
$$

Equation 3.23 can be used to solve the further equations for 2-step subpopulation of $v$ and so on. Overall this approach represents a general framework for the analysis of population transfer pathways inside kinetic rate models. In contrast to earlier methods [30] which simply switched off overtone transitions and compared the remaining and original population, this formalism does not perturb the time evolution of the population of the single vibrational states. In the following paragraphs I will only discuss the simplest pathways that involve only excitation steps (see Table 3.1). More complicated pathways that involve also relaxation processes, e. g. $v=0 \rightarrow 1 \rightarrow 3 \rightarrow 2$, are not investigated in detail and summarized under 'other' pathways. A detailed comparison of kinetic model and experimental data reveals that these pathways do not contribute significantly to the observed excitation probabilities. 


\subsubsection{Discussion}

For the interpretation, the experimentally observed excitation probabilities from Fig. 3.7 are compared to the kinetic rate model as well as to full-dimensional first principles IESH theory. The latter uses molecular dynamics calculations applying an independent electron surface hopping model (IESH) [73, 74] that has been shown to faithfully describe vibrational excitation [25] and de-excitation [65] of NO scattered off a $A u(111)$ surface. The results of the IESH model (open symbols) as well as the kinetic rate model (lines) are shown in Fig. 3.8 for an incidence energy of $E_{I}=0.41 \mathrm{eV}$.

The full fit of the kinetic model to the experimental data is shown as solid lines in Fig. 3.8 and describes the experimental data equally well for both cases (a) and (b). However, the derived fit parameters (Table 3.1) depend strongly on the assumptions (a) and (b). If $v$ scaling for the transition rate constant is assumed, the overtone parameters $\beta$ and $\gamma$ decrease significantly whereas the interaction time $\tau$ is almost unaffected. Although it might influence the time evolution of the kinetic model, deactivation of overtone transitions can be used as a first test for the importance of overtone transitions with $\Delta v= \pm 2$, \pm 3 . The results are shown as dashed and dotted lines in Fig. 3.8. If the second overtone transition $(\Delta v=3)$ is turned off in the model ( $\gamma=0$, light blue dashed-dotted curve), the results for excitation into $v_{f}=1,2$ remain basically unchanged and only the result for $v_{f}=3$ excitation is influenced. However, this observation only holds for case (a); case (b) gives only very small changes to the predicted $v_{f}=3$ excitation probabilities. The latter is a result of the small $\gamma$ in case (b) which decreases from 0.103 to 0.014 , when $v$ scaling is taken into account.

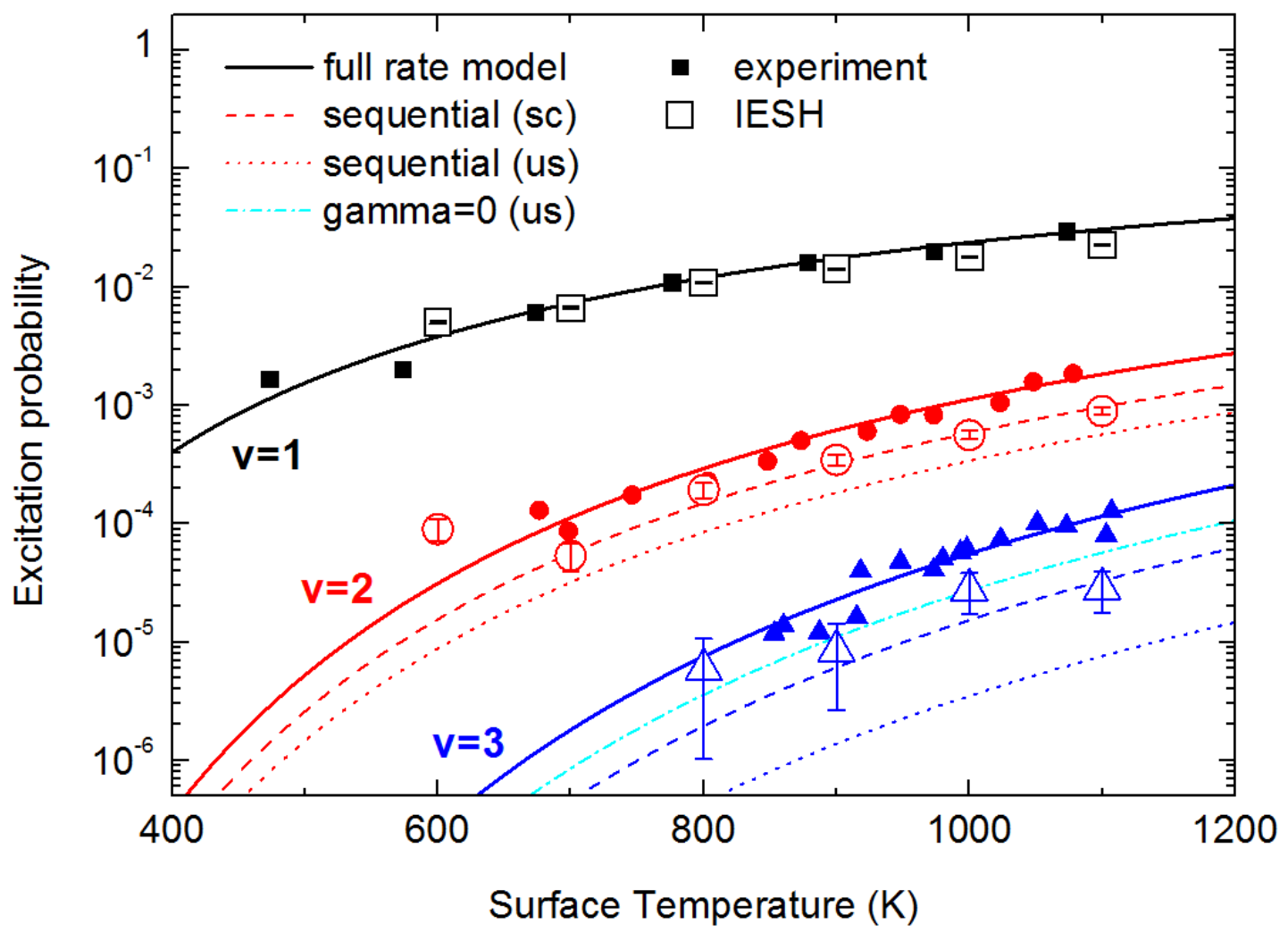

Fig. 3.8: Comparison of the experimentally observed excitation probabilities (solid symbols) to first-principle IESH theory (open symbols). The results of kinetic rate model are shown for the full fit (solid lines), with $\gamma=0$ (light blue) and purely sequential ( $\beta=0$ and $\gamma=0$ ) with $v$ scaling (dashed lines) and without $v$ scaling (dotted lines). Excitation probabilities from the IESH model were derived from one million trajectories using $M_{S}=80$ electronic states in for the metal. Reprinted with permission from Journal of Physical Chemistry A, 2013. 117(32), 7091-7101. Copyright 2013 American Chemical Society. 
Table 3.1: Fit results of the kinetic rate model and subpopulation analysis for kinetic model with (a) $v$ independent coupling and (b) linear $v$ scaling of the nonadiabatic coupling. The contributions of different pathways are given in \%. The right column presents an analysis of the excitation pathways resulting from analysis of the electronic hops occurring during trajectories that end up in a specific vibrational state. Note that only the simplest pure excitation channels are given here. More complicated pathways can be involved and will make the contribution of the given channel smaller than $100 \%$.

\begin{tabular}{c|c|c|c} 
channel & kinetic model (a) & kinetic model (b) & IESH \\
\hline & $\begin{array}{r}\tau=(1.797 \pm 0.004) \mathrm{eV}^{-1} \\
\beta=0.289 \pm 0.002 \\
\gamma=0.103 \pm 0.010\end{array}$ & $\begin{array}{r}\tau=(1.806 \pm 0.005) \mathrm{eV}^{-1} \\
\beta=0.243 \pm 0.003 \\
\gamma=0.014 \pm 0.010\end{array}$ & \\
\hline \multicolumn{3}{|c|}{$v=0 \rightarrow 2$} & 81 \\
\hline $\begin{array}{c}\text { sequential } \\
(0 \rightarrow 1 \rightarrow 2)\end{array}$ & 28 & 49 & 19 \\
\hline $\begin{array}{c}\text { direct-overtone } \\
(0 \rightarrow 2)\end{array}$ & 71 & 49 & $62 \pm 14$ \\
\hline $\begin{array}{c}\text { sequential } \\
(0 \rightarrow 1 \rightarrow 2 \rightarrow 3)\end{array}$ & $6 \pm 1$ & $23 \pm 1$ & $31 \pm 10$ \\
\hline $\begin{array}{c}\text { hybrid } \\
(0 \rightarrow 1 \rightarrow 3 \text { or } 0 \rightarrow 2 \rightarrow 3)\end{array}$ & $41 \pm 1$ & $71 \pm 2$ & $7 \pm 5$
\end{tabular}

Turning off the second $(\gamma=0)$ and the first overtone $(\beta=0)$ transition gives the dotted lines for case (a) and the dashed lines for case (b). In both models the procedure does not change the prediction for excitation of $\mathrm{NO}\left(v_{f}=1\right)$ which indicates the insignificance of mechanisms other than direct $v=0 \rightarrow 1$ excitation. In contrast, the kinetic model gives much lower excitation into $v_{f}=2$ and $v_{f}=3$ for both cases, while the effect is more pronounced for case (a). Most importantly, the kinetic model is not able to give good overall agreement anymore for excitation into all three vibrational states. This primary observation indicates the importance of overtone transitions for excitation into $v_{f}=2,3$.

However, this simple method has the risk of artificially disturbing the time evolution of the population of the different vibrational states. Application of the subpopulation analysis described in 3.1.3 provides a way to avoid this problem and I expect it to give more reliable results. In addition, the subpopulation analysis allows for a more detailed analysis and the importance of pathways that also involve relaxation can be estimated. The results of this detailed analysis are shown in Table 3.1; the time evolution of the different excitation pathways is shown in Fig. 3.9.

First, it is necessary to verify the assumption that only the simplest excitation pathways, which are noted in Table 3.1, contribute significantly to the population of a specific vibrational state. A comparison of the curves for $n_{v}$ (black solid lines) and $n_{v}^{(r e l)}$ (red dashed lines) in Fig. 3.9 verifies this assumption. For the interaction time $\alpha t$ at which the best fit to the dataset is observed (vertical dashed line), both lines basically fall on top of each other showing that contributions from other pathways than those noted above are negligible. For both cases (a) and (b), the kinetic model predicts production of $\mathrm{NO}\left(v_{f}=1\right)$ to be exclusively due to direct $v=0 \rightarrow 1$ excitation. Other excitation pathways (gray line) do not contribute significantly to the final $v_{f}=1$ population at $\alpha \tau_{a}$ or $\alpha \tau_{b}$. The slight increase for $\alpha t>2$ shows that these pathways only become important for long interaction times. 


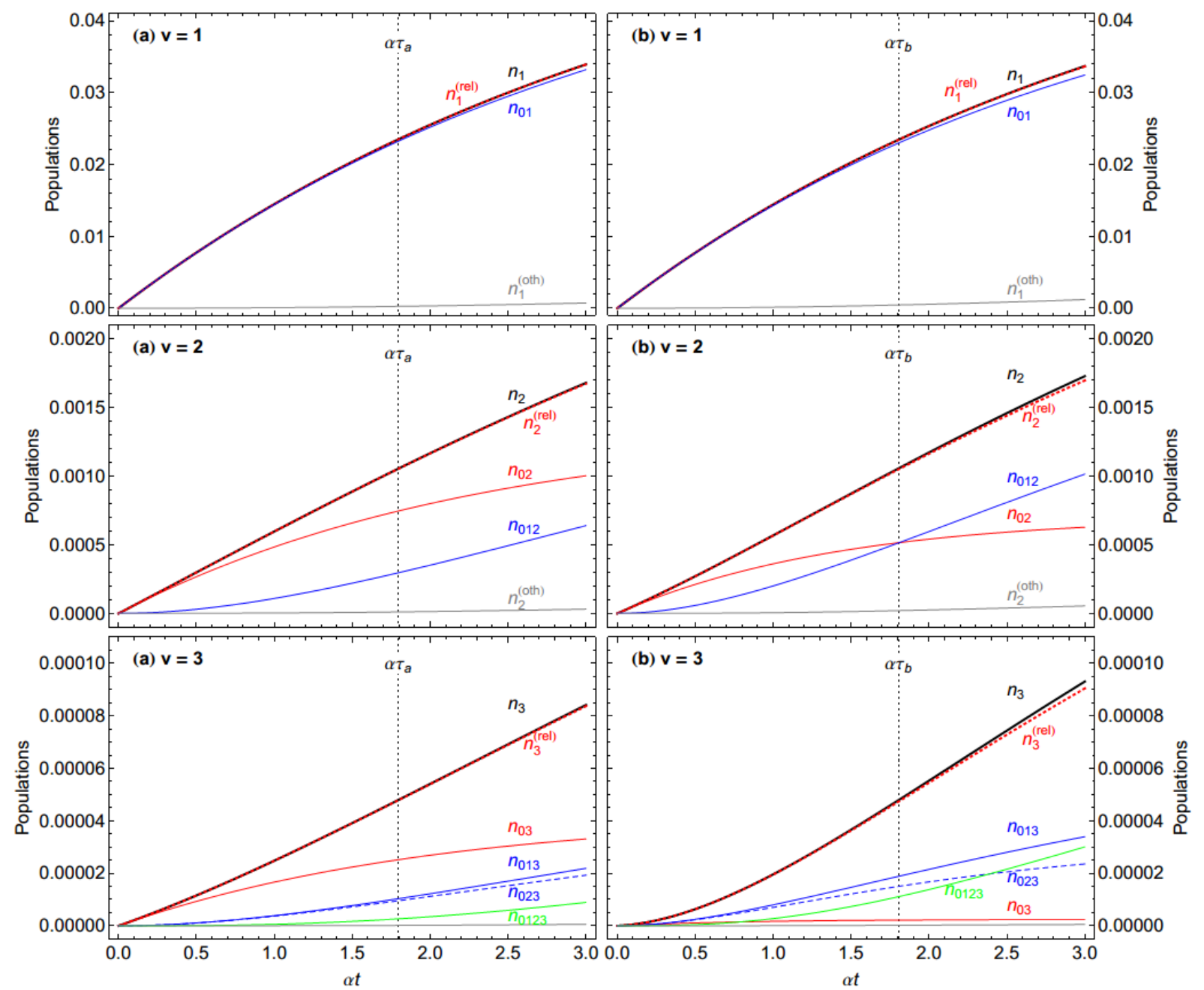

Fig. 3.9: Subpopulation analysis of $v_{f}=1,2,3$ as a function of dimensionless time, $\alpha t$, for $T_{S}=800 \mathrm{~K}$ and $E_{I}=0.41 \mathrm{eV}$. The kinetic model was fitted to the absolute excitation probabilities derived from the experiment. Case (a) assumes couplings independent of $v$ whereas case (b) takes $v$ scaling into account. The subpopulation analysis was performed as described above. The dotted lines indicate $\alpha t$ for which the model fits the experimental data best. The black solid lines correspond to full population of the vibrational states; blue solid lines indicate population via purely sequential mechanism, red solid lines show population due to direct overtone excitation, green and blue dashed lines reflect the hybrid mechanism of overtone and sequential excitation. The red dashed line shows the sum of sequential, overtone and hybrid mechanisms but ignoring more complicated pathways (gray lines). The good agreement between $n_{v}$ and $n_{v}^{r e l}$ indicates that mainly pure excitation pathways significantly contribute to the final state distribution at the interaction times observed here. The comparison of case (a) and case (b) shows that the $v$ scaling approximation clearly enhances the predicted importance of sequential excitation. Reprinted with permission from Journal of Physical Chemistry A, 2013. 117(32), 7091-7101. Copyright 2013 American Chemical Society.

As already indicated in the preliminary discussion above, cases (a) and (b) behave differently for $v_{f}=2$ and $v_{f}=3$ excitation. The $v$ scaling assumption strongly enhances the sequential excitation of both states for case (b). Case (a) predicts $71 \%$ direct overtone excitation for $v_{f}=2$ whereas case (b) gives equal contributions of $49 \%$ for sequential and direct-overtone excitation. The residual $2 \%$ of the $v_{f}=2$ population result from other pathways. The time evolution of the different subpopulations shows that direct overtone excitation dominates at early times $\alpha t$ independent of the initial assumption for case (a) or (b). The explanation for this observation is rather simple: at $\alpha t=0$ the system has only population in $v=0$ and none in $v=1$; the latter first has to build up as $\alpha t$ evolves. At later times, the sequential pathway becomes more and more important because $v=1$ is now populated due to $v=0 \rightarrow 1$ 
excitation. The transition probability from $v=1 \rightarrow 2$ exceeds that for $v=0 \rightarrow 2$ for two reasons: first, more thermally excited EHPs with $0.236 \mathrm{eV}$ energy are available than there are with $0.472 \mathrm{eV}$; second, the coupling parameter $\alpha_{02}$ is only $28 \%$ compared to $\alpha_{12}$ for case (a) and only $\sim 12 \%$ compared to $\alpha_{12}$ for case (b).

For production of $\mathrm{NO}\left(v_{f}=3\right)$, there are overall four different pathways that involve only vibrational excitation steps. In addition to the direct $0 \rightarrow 3$ overtone excitation and a purely sequential $0 \rightarrow 1 \rightarrow 2 \rightarrow 3$ mechanisms, two hybrid excitation pathways with $0 \rightarrow 1 \rightarrow 3$ and $0 \rightarrow 2 \rightarrow 3$ are possible. For the present set of data, the kinetic model gives a ratio of sequential : hybrid : direct-overtone excitation of 6:41:53 for case (a) and 23:71:5 for case (b). As in the case of $v_{f}=2$, the $v$ scaling assumption of case (b) enhances the importance of sequential pathways. For $v_{f}=3$, it further promotes the hybrid mechanism and almost completely suppresses the direct-overtone excitation. The fully sequential mechanism that involves three $\Delta v=1$ excitations, however, is now less favorable than a hybrid pathway consisting of only one $\Delta v=1$ and one $\Delta v=2$ transition. The kinetic rate model in case (b) clearly predicts that $v_{f}=3$ excitation is dominated by a hybrid mechanism. Interestingly, only case (a) produces a significant amount of $v_{f}=3$ molecules at early times $\alpha t$; the time evolution for $v_{f}=2$ showed this behavior for both assumptions (a) and (b). The reasons might be that the thermal factors, thus the availability of EHPs with $0.708 \mathrm{eV}$ energy is even worse than for $0.472 \mathrm{eV}$, and that $v$ scaling reduces the coupling constant by a factor of almost 10 .
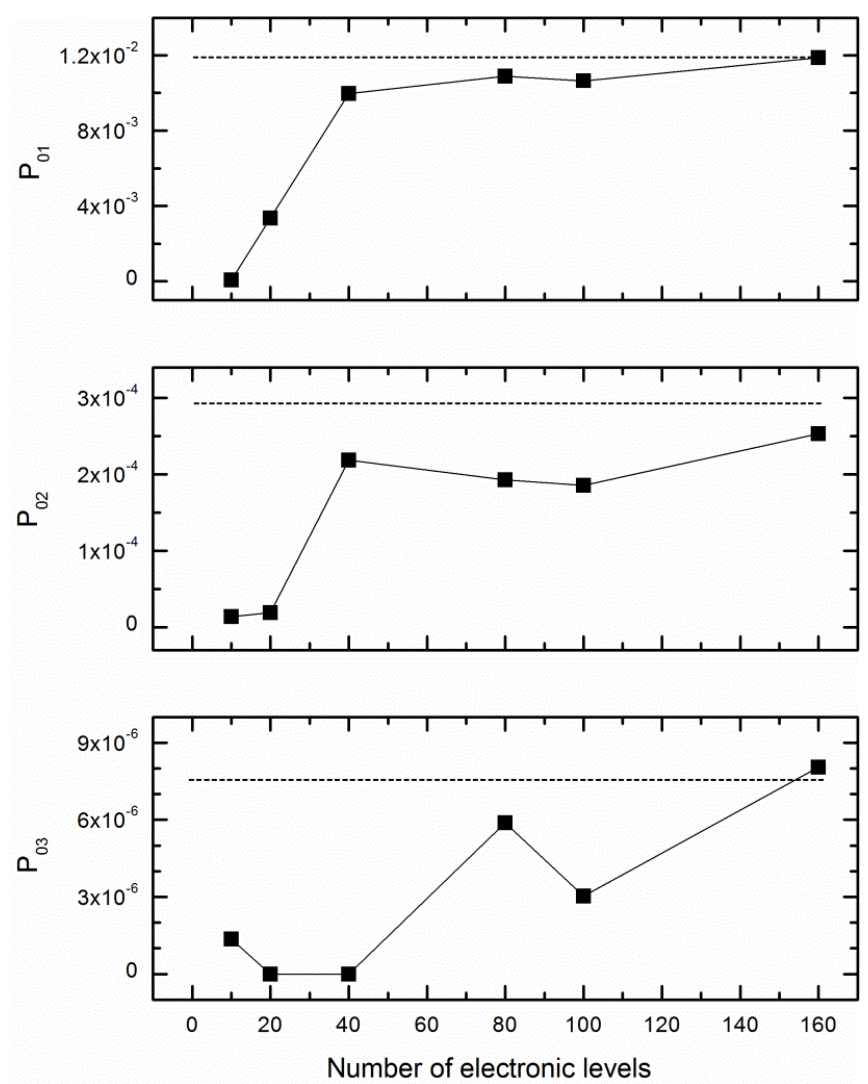

Fig. 3.10: Predictions of the IESH model for vibrational excitation probabilities into $v_{f}=1,2,3$ for $T_{S}=800 \mathrm{~K}$ if the electronic states of the metal is modelled by an increasing number of electronic states $M_{S}=10,20,40,80,100,160$. The excitation probability for $v_{f}=1$ converges already between 40-80 electronic state whereas $v_{f}=2$ seems to converge at a slightly higher number. For $v_{f}=3$ it is hard to judge if the excitation probabilities have converged already at $M_{S}=160$. The dashed lines correspond to the experimental values. Reprinted with permission from Journal of Physical Chemistry A, 2013. 117(32), 70917101. Copyright 2013 American Chemical Society. 
Overall, the kinetic rate model predicts the importance of direct-overtone excitations although the predictions for the contributions for $v_{f}=2,3$ vary whether $v$ scaling is assumed or not. Nevertheless, the kinetic model is empirical and fitted to the experimental data; it cannot replace an approach from first-principle theory in order to explain the nonadiabatic interactions.

The IESH model used to calculate the excitation probabilities of Fig. 3.8 is identical to that in ref. [25] and [65]. A detailed description of the model can be found in ref. [73, 74]. In contrast to previous simulations, the calculations of $\mathrm{NO}\left(v_{f}=3\right)$ excitation probabilities, which range from $10^{-6} \ldots 10^{-5}$, requires about one million trajectories for each surface temperature. An adjustable parameter in the calculation is the number of discrete electronic levels $M_{S}$ used to model the electronic continuum of the metal. The IESH results in Fig. 3.8 show the results for $M_{S}=80$ electronic states which are filled with 40 electrons. The effect of $M_{S}$ on the final excitation probabilities is shown in Fig. 3.10. Note that a higher number of electronic states causes an increase in computational time and $M_{S}=160$ represents the upper limit that can be performed in a reasonable time.

The comparison between the IESH results and the experimental data in Fig. 3.7 shows that the theory faithfully reproduces the experimental excitation probabilities for $\mathrm{NO}\left(v_{f}=1\right)$ (black open symbols). However, the model slightly underestimates $\mathrm{NO}\left(v_{f}=2\right)$ excitation and systematically underestimates the experimental values derived for $\mathrm{NO}\left(v_{f}=3\right)$. Thus, the disagreement between theory and experiment becomes worse with increasing $v_{f}$. Surprisingly, the IESH calculations agree very well with the predictions of the kinetic rate model if overtone transitions are turned off. Although a comparison of the empirical model to first-principles theory is not very reasonable and should be taken with care, it might still provide a first hint for the reason of IESH failure at $v_{f}=2,3$. The comparison would suggest that overtone transitions are underestimated in the IESH model. In order to prove this hypothesis, it is necessary to analyze the excitation pathways in the simulations. Table 3.1 gives the results of a pathway analysis based on the number of electronic hops that occur during those trajectories that end up in a specific vibrational state. Some examples for typical trajectories are shown in Fig. 3.11. In detail, the magnitude and sign of the electronic energy change is analyzed along the trajectory and the transitions corresponding to $\Delta v=1,2,3$ are counted. Transitions that lead to de-excitation are subtracted from those that caused vibrational excitation. The derived numbers are given in the right column of Table 3.1.

The results show that the IESH model in its current implementation strongly favors sequential excitation. It predicts $81 \%$ sequential excitation of $v_{f}=2$ and $62 \%$ sequential excitation for $v_{f}=3$. This behavior is in stark contrast with the predictions of the kinetic rate model that clearly revealed the importance of overtone transitions. The observation that the IESH model underestimates the excitation into $v_{f}=2,3$ indicates that this might be a possible weakness of the theoretical model. Comparison of the kinetic rate model and the IESH calculations supports this view, although the comparison might be artificial. Note that the agreement is best for case (b) of the rate model indicating that $v$ scaling is probably the correct assumption. The comparison does also show why the agreement is still better for $v_{f}=2$ for which the empirical model (case (b)) predicts $49 \%$ sequential excitation whereas it predicts only $23 \%$ full sequential excitation for $v_{f}=3$.

A possible explanation for underestimation of overtone transitions in the IESH simulation is the way the metal continuum is modeled. The simulations shown in Fig. 3.8 use $M_{S}=80$ discrete electronic levels to model the electronic continuum of the metal. Furthermore, the model used Gaussian quadrature to increase the density of states around the Fermi level $\left(E_{F}\right)$ which leads, however, to a decreasing density with increasing distance from $E_{F}$. The density increases again at the edges of the modeled conduction and valence bands but these regions do not play a role in the simulations. The first question to answer is then: how well do $M_{S}=80$ discrete electronic states describe the continuum of states in the metal? 

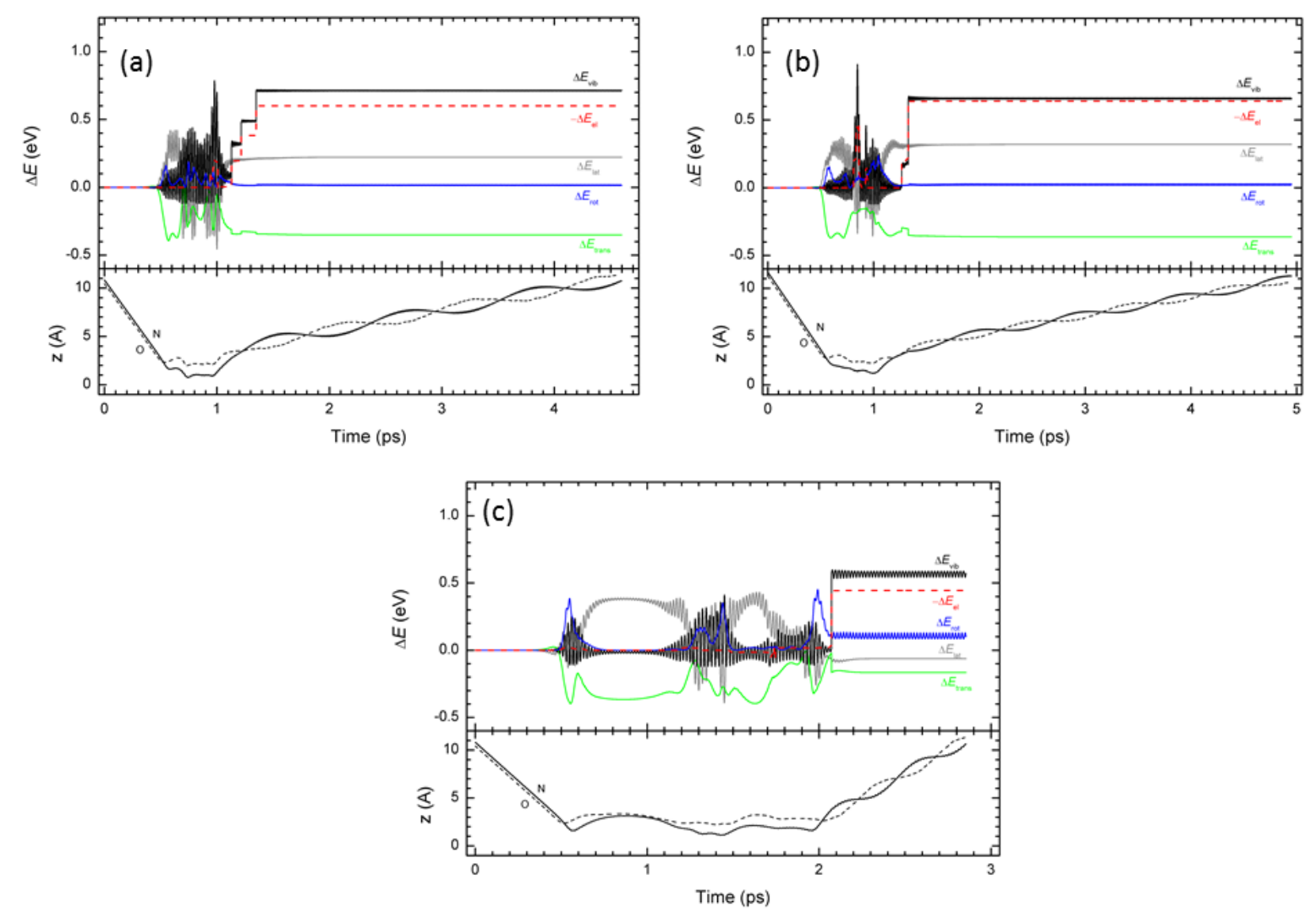

Fig. 3.11: Examples for IESH trajectories that end up in $v_{f}=3$ and show purely sequential (a), hybrid (b) and direct overtone excitation (c). The lower section of each panel shows the distances $z$ of the $\mathrm{N}$ - (solid line) and O-atom (dashed line) from the surface. The upper parts show the time evolution of vibrational energy $\Delta E_{\text {vib }}$ (black), rotational energy $\Delta E_{\text {rot }}$ (blue) and translational energy $\Delta E_{\text {trans }}$ (green), electronic energy $-\Delta E_{e l}$ (red) and lattice (phonon) energy $\Delta E_{\text {lat }}$ (gray). The upper right and the lower panel seem to represent impulsive scattering while the upper left trajectory involves a rather long interaction with the surface of about 1.5 ps with apparently three bounces. Reprinted with permission from Journal of Physical Chemistry A, 2013. 117(32), 7091-7101. Copyright 2013 American Chemical Society.

Unfortunately, the computational time for the simulations scales non-linearly with $M_{S}$. Fig. 3.10 shows calculated excitation probabilities for $\mathrm{NO}\left(v_{f}=1,2,3\right)$ for $M_{S}=10-160$. The results show that 10, 20 or 40 levels are not sufficient to describe any excitation probability correctly. Calculation of $\mathrm{NO}\left(v_{f}=1\right)$ excitation requires at least $M_{S}=60$ electronic states. For $v_{f}=2$ excitation the simulations approach the experimental value for 160 electronic levels but some convergence seems to be present already for $M_{S} \leq 80$. In the case of $v_{f}=3$ the uncertainty about convergence is even bigger although the excitation probabilities reach the experimental value at $M_{S}=160$. In addition, the distribution of electronic states across the conduction and valence bands can influence the results, e. g. using equal spacing instead of Gaussian quadrature. A comparison of calculations performed with equally spaced states reveal, however, even worse agreement with the experiment. The high density of states is necessary to increase the probability for nonadiabatic transitions (statistical effect) in a sense that it provides more states with the correct energy spacing to match the vibrational energy of the NO molecule.

Another issue of the current IESH model arises from Fig. 3.11 which shows different trajectories for $v_{f}=3$ excitation. The shown examples represent three different excitation pathways: fully sequential $0 \rightarrow 1 \rightarrow 2 \rightarrow 3$ (panel (a)), hybrid $0 \rightarrow 1 \rightarrow 3$ (panel (b)) and direct $0 \rightarrow 3$ overtone excitation (panel (c)). The top parts of each panel show the evolution of vibrational (black), electronic (red), rotational (blue), lattice (gray) and translational (green) energy along the trajectory. The lower parts show the distance of 
the $\mathrm{N}$-(solid line) and O-atom (dashed line) from the surface. The surface distances for trajectories (a) and (b) show a double bounce scattering event in which the $\mathrm{O}$-atom collides with the surface several $100 \mathrm{fs}$ prior to the $\mathrm{N}$-atom. In contrast, the trajectory $(\mathrm{c})$ shows a completely different scattering behavior: the NO molecule stays very close to the surface for about 1.5 ps and bounces three times before escaping. Note that the electronic transition that leads to $0 \rightarrow 3$ excitation occurs $\sim 100 \mathrm{fs}$ after the molecule started to leave the surface. This observation indicates that high vibrational excitation might not always be produced in a direct scattering event in the simulations but in a collision with multiple bounces and a rather long interaction time with the surface. The investigation of this issue requires a detailed analysis of the number of bounces during each trajectory, which is a rather complex task for the rare process of vibrational excitation that requires simulation of $10^{6}$ trajectories. Therefore, an according analysis is much easier applicable for processes with higher statistical probability that will need less trajectories to be captured. A suitable example is vibrational relaxation of NO in low initial vibrational states which is the topic of section 3.2. 


\section{2. $\mathrm{NO}(v=3)$ relaxation on $\mathrm{Au}(111)$}

Although there is an extensive literature on vibrational excitation of $\mathrm{NO}\left(v_{i}=0\right)[8,25-27,30,44]$, and vibrational de-excitation of highly vibrationally excited $\mathrm{NO}\left(v_{i}=15\right)[3,115]$, surprisingly little work has been done on vibrational relaxation of $\mathrm{NO}$ in low vibrational states. $\mathrm{NO}\left(v_{i}=2\right)$ scattering from $\mathrm{Au}(111)$ was previously studied experimentally $[34,88,112]$ but no direct detailed comparison to first-principles theory is available. The earlier studies revealed evidence for EHP mediated $\mathrm{NO}(v=2 \rightarrow 1)$ relaxation and $\mathrm{NO}(v=2 \rightarrow 3)$ excitation [88], showed insensitivity of the trapping probability to the incident vibrational state [112] and provided first evidence for an orientation dependence of the nonadiabatic interaction by means of rotational cooling in vibrationally inelastic scattering [34]. The experimental results for trapping probabilities and rotational cooling were compared to IESH simulations and qualitative agreement was obtained [65].

The results in section 3.1 already demonstrated that the IESH model, in its current implementation, has several problems regarding overtone transitions and direct scattering. Furthermore, a detailed comparison of $v=0 \rightarrow 1,2$ vibrational excitation probabilities at various incidence energies recently showed that the experimentally observed $E_{I}$ dependence is not reproduced by the theoretical model. However, the determination of absolute vibrational excitation probabilities is not trivial: measurements for one vibrational state require one full day of experiment. This causes a correction procedure (see section 3.1.1) in order to account, for example, for changes in laser power or laser beam position. Consequently, the measurements of $\mathrm{NO}(v=0 \rightarrow 1,2,3)$ vibrational excitation probabilities for one single incidence energy require at least four days of experiment. In addition, comparison to first-principles IESH theory requires the calculation of $10^{6}$ trajectories. In contrast, experiments on $\mathrm{NO}\left(v_{i}=3\right)$ relaxation are rather simple: first, vibrational relaxation probabilities are essentially independent of surface temperature and the determination at room temperature is sufficient, and, second, all vibrational states in $v=3 \rightarrow 1,2,3$ scattering can be measured under identical experimental conditions (scattering angle, time delay, etc.). This reduces the number of corrections and therefore possible errors significantly.

I performed experiments for $\mathrm{NO}(v=3 \rightarrow 3,2,1)$ scattering from $\mathrm{Au}(111)$ for different incidence energies ranging from $0.1-1.1 \mathrm{eV}$ and measured branching ratios between the observable final vibrational states $v_{f}$. The results are compared to first principles IESH theory. Because relaxation probabilities are in the order of $10^{-1}$, it is sufficient to simulate only 1000 trajectories for each $E_{I}$. The incident molecules are prepared in $v_{i}=3, J_{i}=1.5$ at about $14 \mathrm{~mm}$ distance from the surface by overtone excitation of the $3 \leftarrow 0 \mathrm{R}(0.5)$ transition using the transform limited IR laser system (see 2.2.1). The IR laser is focused into a vertical sheet by a $500 \mathrm{~mm}$ cylindrical lens. The scattered $\mathrm{NO}\left(v_{f}=1,2,3\right)$ molecules are detected with REMPI via the $A \leftarrow X$ transition using the 0-1, 0-2 and 1-3 vibrational bands. Unfortunately, I do not obtain absolute relaxation probabilities because the relaxation into $v_{f}=0$ is covered by a large background of NO molecules in the molecular beam that were not excited by the IR laser and that undergo $v=0 \rightarrow 0$ scattering. In principle, a comparison of incident molecules versus scattered molecules is possible but several partially unknown correction factors have to be taken into account. Determination of simple branching ratios between the observable vibrational states gives much more accurate and reliable results providing a better benchmark for comparison to first-principles theory. 


\subsubsection{Branching ratios}

Branching ratios $R_{v}$ for $\mathrm{NO}\left(v_{i}=3\right)$ scattering into $\mathrm{NO}\left(v_{f}=1,2,3\right)$ were obtained by a similar formalism used for the determination of excitation probabilities (see 3.1.1 or ref. [26]) and are given by the following expression:

$$
R_{v_{f}}=\frac{N_{v_{f}}}{N_{1}+N_{2}+N_{3}}
$$

where $N_{v_{f}}$ is the population of the vibrational state $v_{f}$. As for the determination of vibrational excitation probabilities, the calculation of $N_{v_{f}}$ starts with the measurement of REMPI spectra that are afterwards corrected for influences of detector gain, laser power, angular distributions and temporal profiles (see equation 3.3). An example of REMPI spectra is shown in Fig. 3.12. Because relaxation probabilities are much higher $\left(\sim 10^{-1}\right)$ than excitation probabilities, the scattering event produces comparable populations in $v_{f}=1,2,3$. In order to simplify the experiments and to minimize necessary correction factors, I used the $A-X(0-1)$ (red) and (0-2) (black) bands for detection of $\mathrm{NO}\left(v_{f}=1,2\right)$ but the A-X (1-3) band (blue) for detection of $\mathrm{NO}\left(v_{f}=3\right)$. This choice has the advantage that all three vibrational states can be covered within a spectral range of $15 \mathrm{~nm}$. The only disadvantage that results from these experiments is the small overlap between the (1-3) band with high rotational states of the (0-2) band.

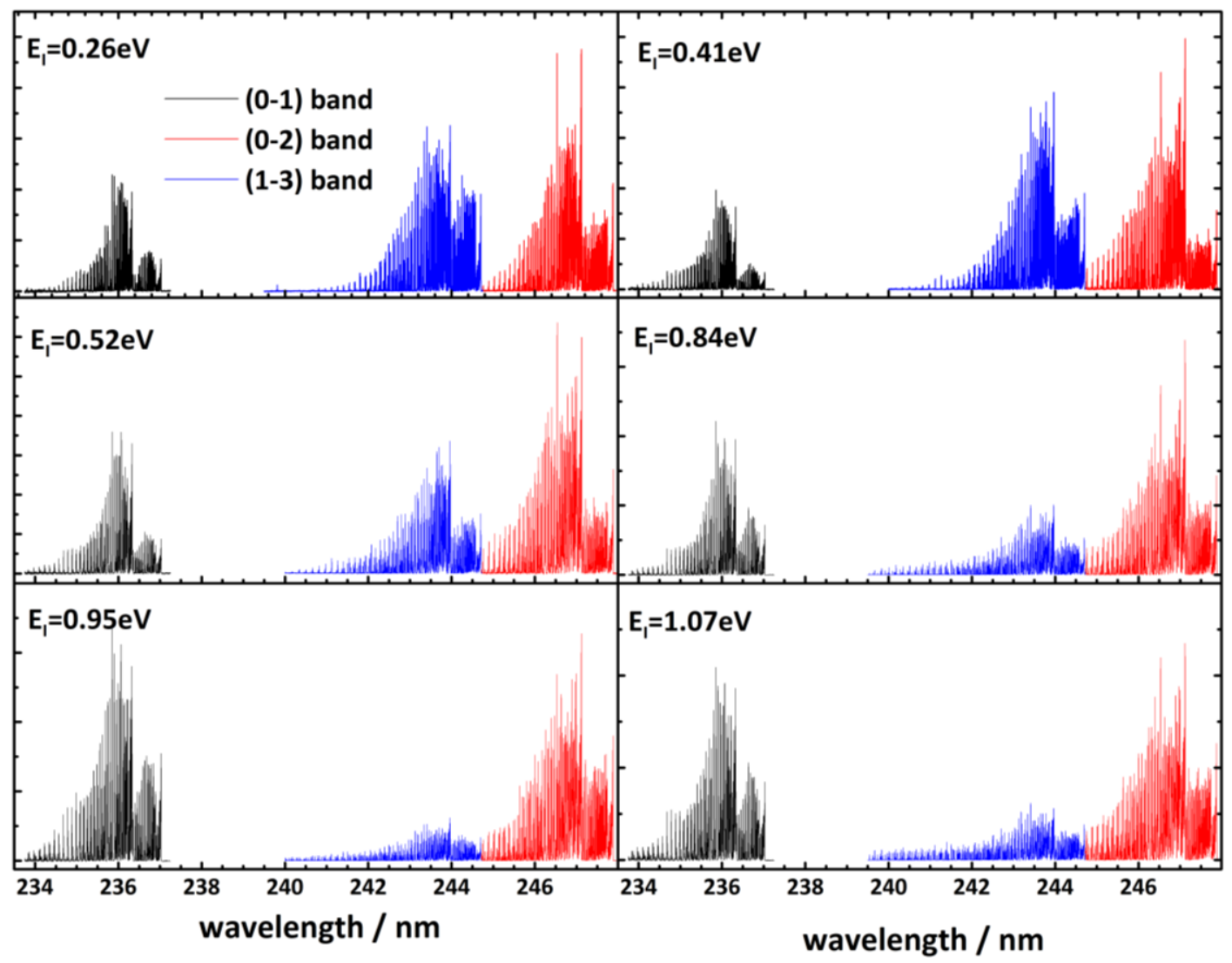

Fig. 3.12: REMPI spectra for $\mathrm{NO}\left(v_{i}=3, J_{i}=1.5\right)$ scattering from $\mathrm{Au}(111)$ at various incidence energies. I used the $A-X(1-3)$ (blue), (0-1) (black) and (0-2) (red) bands for the detection of scattering molecules. The spectra are corrected according to changes in laser power and were measured with the same detector gain. 


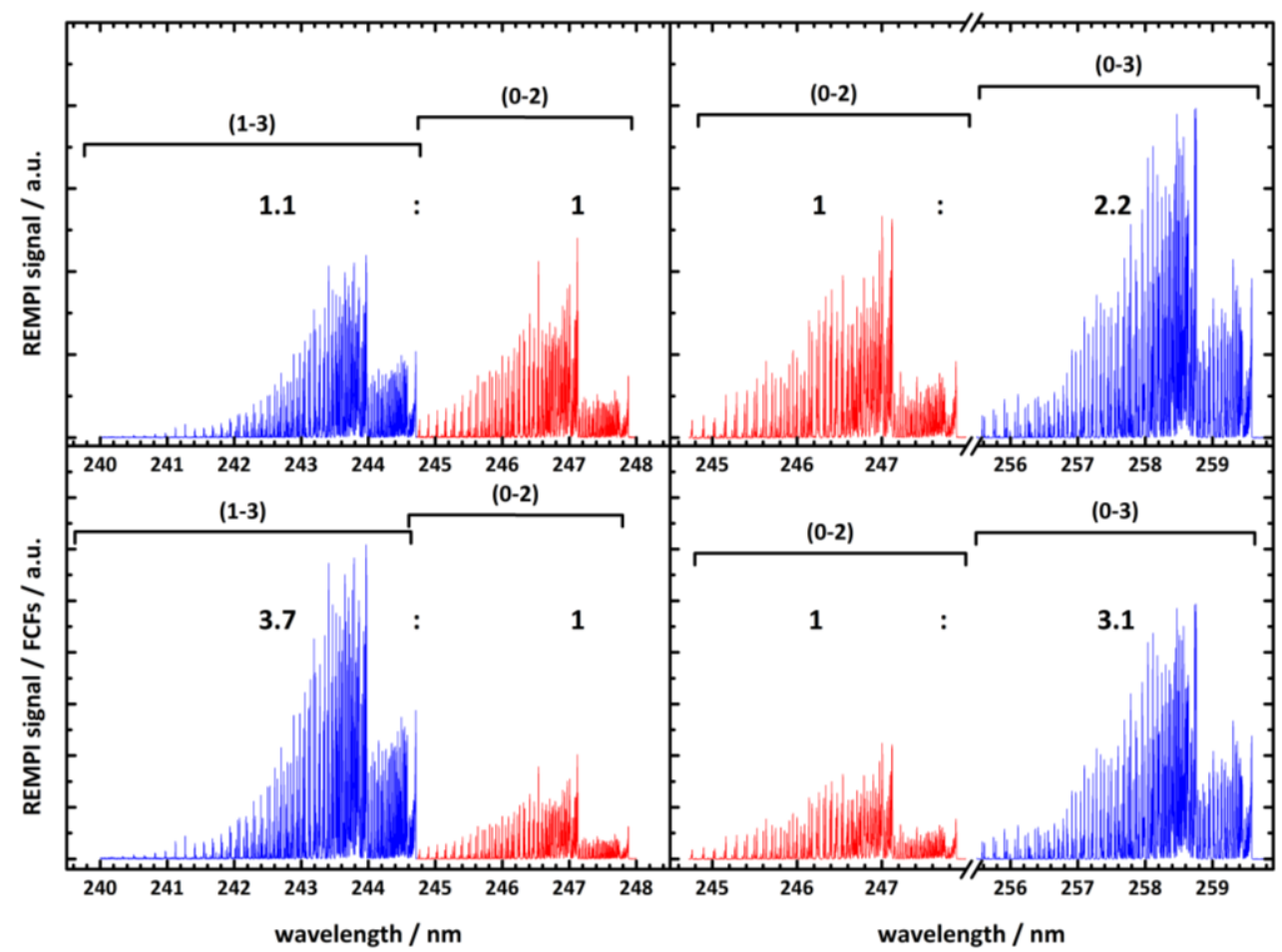

Fig. 3.13: REMPI spectra of $\mathrm{NO}(\mathrm{v}=3 \rightarrow 2,3)$ for the detection of $v_{f}=3$ (blue) via the (1-3) (left panels) or (0-3) (right panels) band. The red spectra show the (0-2) band for comparison. All spectra were measured for $E_{I}=0.41 \mathrm{eV}$ and corrected for differences in laser intensity. The numbers indicate the ratio in the spectral area referenced to the (0-2) band.

A drawback of the (1-3) band is the small Franck-Condon factor of only $\sim 0.07$, compared to $\sim 0.16$ and $\sim 0.23$ for the (0-3) and (0-2) band [89], respectively. Whether it is taken into account or not can change the results by a factor of 3 . The $A$ state ionization cross-section has been measured to be only weakly dependent on the intermediate vibrational state and wavelength [116]. The laser power dependences (equation 3.4) of the (1-3) and (0-2) transitions give exponents of $n_{(1-3)}=1.74$ and $n_{(0-2)}=1.14$ and suggest that the resonant transition is only partly saturated. Whether FCFs have to be applied for a partly saturated transition is not trivial but a comparison to an additional vibrational band can be used to answer the question experimentally. Thus, I measured REMPI spectra for $v=3 \rightarrow 2,3$ scattering using either the (1-3) or the (0-3) band for detection of $v_{f}=3$. The results are shown Fig. 3.13 for $E_{I}=0.41 \mathrm{eV}$. The upper panels show the measured REMPI signal which is only corrected for changes in laser intensity. The lower panels show the same spectra with the intensity divided by the respective Franck-Condon factor. The numbers indicate the integrals $\int S(\lambda) d \lambda$ normalized to the area under the spectrum of the (0-2) band. The values show that the (0-3) bands gives about two times more intensity than the (1-3) if no FCFs are included. The agreement is better if FCFs are taken into account. The remaining differences can be a result of the incomplete (0-3) band, which clearly extends to shorter wavelengths than observed here, or of partial saturation effects.

A first effect of $E_{I}$ on the relaxation probability is already visible from the spectra in Fig. 3.12. The relative amount of $\mathrm{NO}\left(v_{f}=3\right)$ (blue spectra) decreases with increasing incidence energy while the intensity of $v_{f}=1,2$ increases. Furthermore the spectra exhibit an increase of rotational excitation with increasing $E_{I}$ that is most prominent for vibrationally elastic $v=3 \rightarrow 3$ scattering. This effect is consistent with the previous observation of rotational cooling for vibrationally inelastic scattering of $\mathrm{NO}\left(v_{i}=2\right) / \mathrm{Au}(111)$ [117]. 
As mentioned above, the choice of vibrational bands makes the measurement of branching ratios essentially easier than that of vibrational excitation probabilities where the determination of the population of one single vibrational state basically requires already one complete day. For relaxation studies on $\mathrm{NO}\left(v_{f}=3\right)$, I obtain all data for one incidence energy within a few hours of experiment under identical conditions. This situation has the advantage that corrections for changes in angular distribution can be dropped as long as the angular distributions are similar for all three vibrational states. The upper panel of Fig. 3.14 shows experimental angular distributions for $v_{f}=1,2,3$ for an incidence energy of $E_{I}=0.51 \mathrm{eV}$. Note that the angular distributions were measured at about $12 \mathrm{~mm}$ distance from the surface with an unfocused REMPI beam $(\varnothing \sim 5 \mathrm{~mm})$. Within the uncertainty in angle the angular distributions are similar for all vibrational states and can therefore be neglected in the calculation of $N_{v_{f}}$.
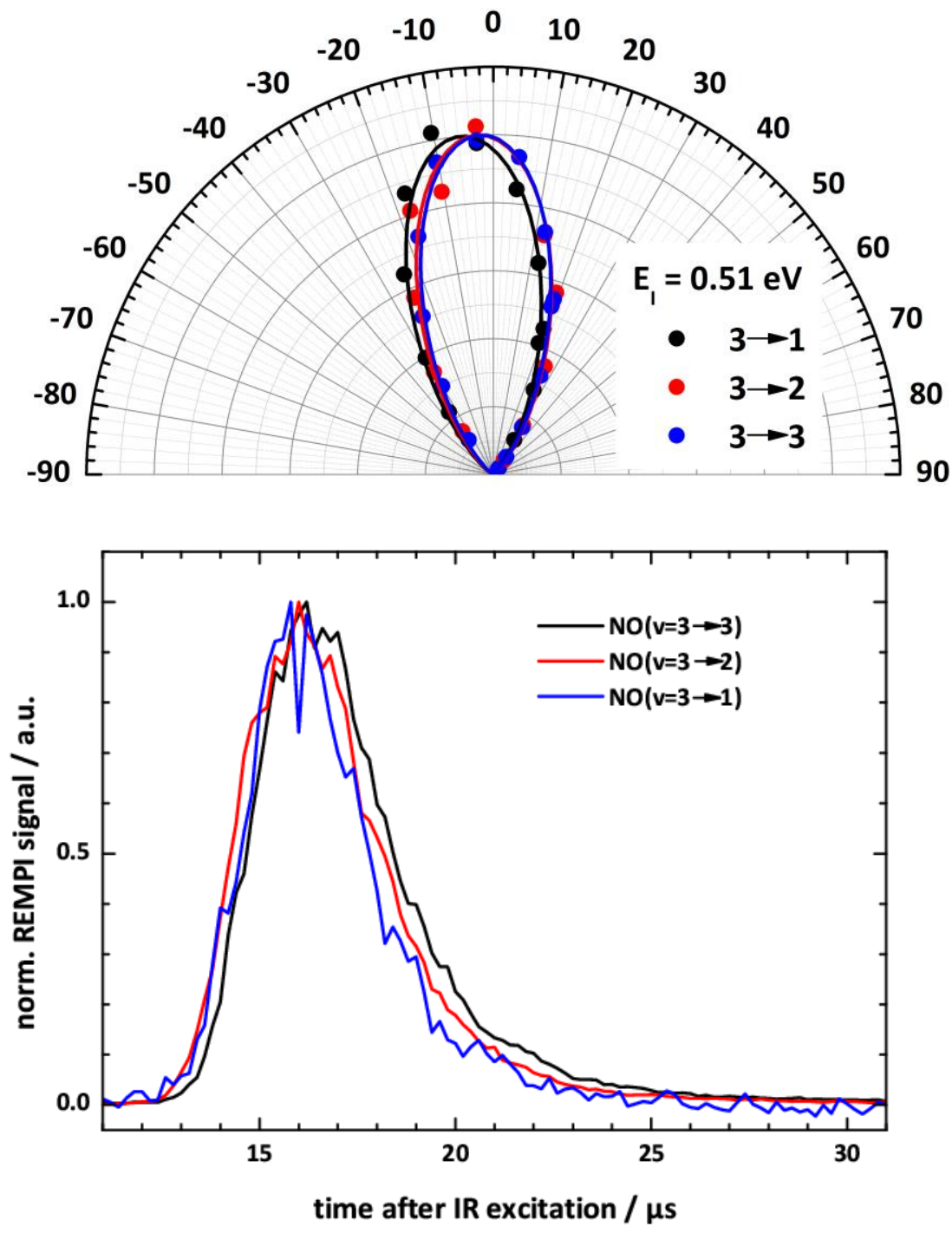

Fig. 3.14: Angular and arrival time distributions of $\mathrm{NO}(v=3 \rightarrow 3,2,1)$ scattering from $\mathrm{Au}(111)$ at 0.51 eV incidence energy. The narrow angular distributions for all vibrational states are identical within the angular resolution $\left(\sim 10^{\circ}\right)$ of the experiment. The arrival time distributions are also similar for all vibrational states but shift slightly towards earlier times for vibrationally inelastic scattering. 


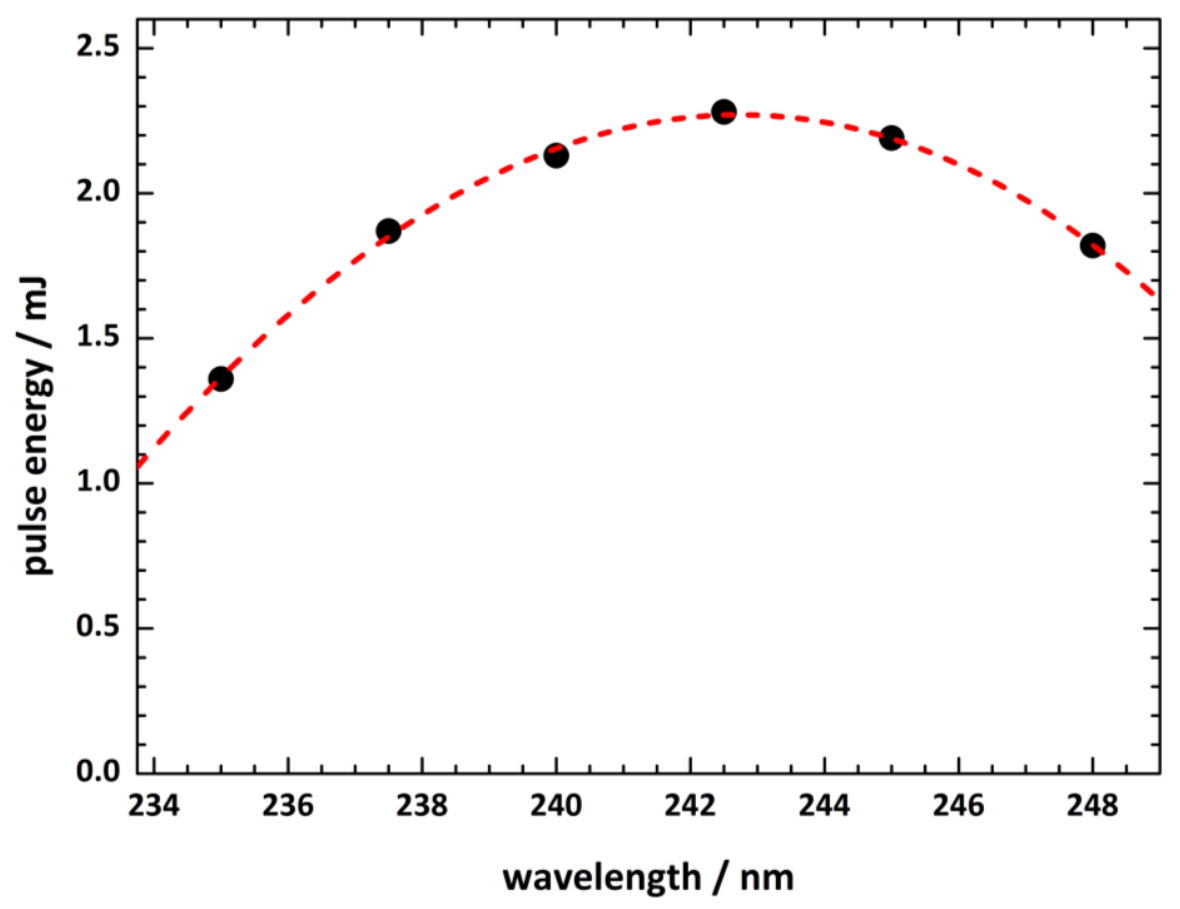

Fig. 3.15: Tuning curve of the REMPI detection laser in the range from $234-248 \mathrm{~nm}$. The data points were taken on a pyroelectric detector with averaging of $\sim 50$ laser pulses.

Fig. 3.14 further shows examples for arrival time distributions for $\mathrm{NO}\left(v_{f}=1,2,3\right)$ for $E_{I}=0.51 \mathrm{eV}$. The width of the temporal profiles is much narrower than those presented in Fig. 3.6 because the IR excitation creates a very well defined start time $t_{0}$ and excites only a small part of the molecular beam. All three temporal profiles are very similar but those molecules that underwent vibrational relaxation arrive slightly earlier at the detection laser. This effect is the topic of section 3.3 and will not be discussed here. The width and shift of the peak position is taken into account in the determination of $N_{v_{f}}$.

In addition, I have to take changes in the detector gain and fluctuations in laser power into account. The first effect can be avoided for relaxation measurements because relaxation probabilities are large $\left(\sim 10^{-1}\right)$ compared to excitation probabilities $\left(10^{-6} \ldots 10^{-2}\right)$ and all vibrational states can be detected using the same detector gain. Fluctuations of the laser intensity can however not be avoided in the experiment and have to be considered. I measured the laser intensity as a function of wavelength (see Fig. 3.15) prior to the measurements to obtain a tuning curve of the REMPI laser. The laser intensity is then measured at the beginning and at the end of each REMPI scan and used to scale the tuning curve of Fig. 3.15 accordingly. This procedure enables a wavelength dependent correction of the laser intensity that is already included in the spectra of Fig. 3.12 and Fig. 3.13. Changes in the IR laser intensity are considered by averaging the measured IR pulse energy at the beginning and at the end of each scan. The REMPI signal is then divided by the respective laser power and using the measured laser power dependences (equations 3.25-27).

$$
\begin{aligned}
f\left(I_{I R}\right) & \sim I_{I R}^{0.81} \\
f\left(I_{(1-3)}\right) & \sim I_{(1-3)}^{1.75} \\
f\left(I_{(0-1,2)}\right) & \sim I_{(0-1,2)}^{1.14}
\end{aligned}
$$


Finally, two more corrections have to be applied: (1) a correction for the $v_{f}=1$ signal originating from thermal $\mathrm{NO}\left(v_{i}=1\right)$ that is present in the incident molecular beam $\left(T_{\text {vib }} \approx T_{\text {nozzle }}\right)$, and (2) the overlap of the (0-2) band with the (1-3) band. I realize the first correction by measuring the spectrum of the (0-1) band twice, with and without the IR laser. The integral of the second (background) spectrum is then simply subtracted from that with the IR laser turned on. The second correction requires the suppression of the $v_{f}=3$ signal while the $v_{f}=2$ signal and rotational structure remains unchanged. I reach this situation by an additional REMPI scan for the (0-2) band without IR excitation but the surface heated to $T_{S} \approx 870 \mathrm{~K}$. In this experiment, the probability for $v=0 \rightarrow 3$ excitation is at least one order of magnitude lower than for $v=0 \rightarrow 2$ excitation (see section 3.1). Therefore, the intensity in the $A \leftarrow X(1-3)$ band is negligible. However, this method assumes that the rotational structure for $v_{f}=2$ resulting from $v=3 \rightarrow 2$ relaxation at $T_{S}=320 \mathrm{~K}$ is similar to that resulting from $v=0 \rightarrow 2$ excitation at $T_{S}=870 \mathrm{~K}$. Verification of this assumption has been given in the work of Cooper et al. [26] where the rotational temperature for $v=0 \rightarrow 1,2$ excitation was found to be only weakly dependent on surface temperature. Fig. 3.16 shows a comparison of REMPI spectra of the (0-2) band (red) in the region from 243-248 nm for $v=3 \rightarrow 2$ relaxation at $T_{S}=320 \mathrm{~K}$ and $v=0 \rightarrow 2$ excitation at $T_{S}=870 \mathrm{~K}$. The rotational structure is very similar for both processes and to a very good approximation independent of surface temperature. The similarity of $3 \rightarrow 2$ relaxation and $0 \rightarrow 2$ excitation results from the similar mechanisms on which both processes rely. Vibrational relaxation and excitation both occur upon nonadiabatic coupling to EHPs, that is, due to electron transfer. The probability for electron transfer is supposed to depend on the orientation of the NO molecule $[35,65]$. Furthermore, the amount of rotational excitation in a direct scattering event is known to depend on the orientation at impact $[9,10]$. Therefore, similar orientation effects will lead to similar mechanical excitation of rotation [9]. The observations of Fig. 3.16 support this argumentation. For correction of $R_{v}$, I integrated the spectrum taken for high $T_{S}$ until the band head of the (1-3) at $\sim 244.7 \mathrm{~nm}$ and compared the area to that of the complete spectrum. The difference is subtracted from the $N_{3}$ and added to $N_{2}$.

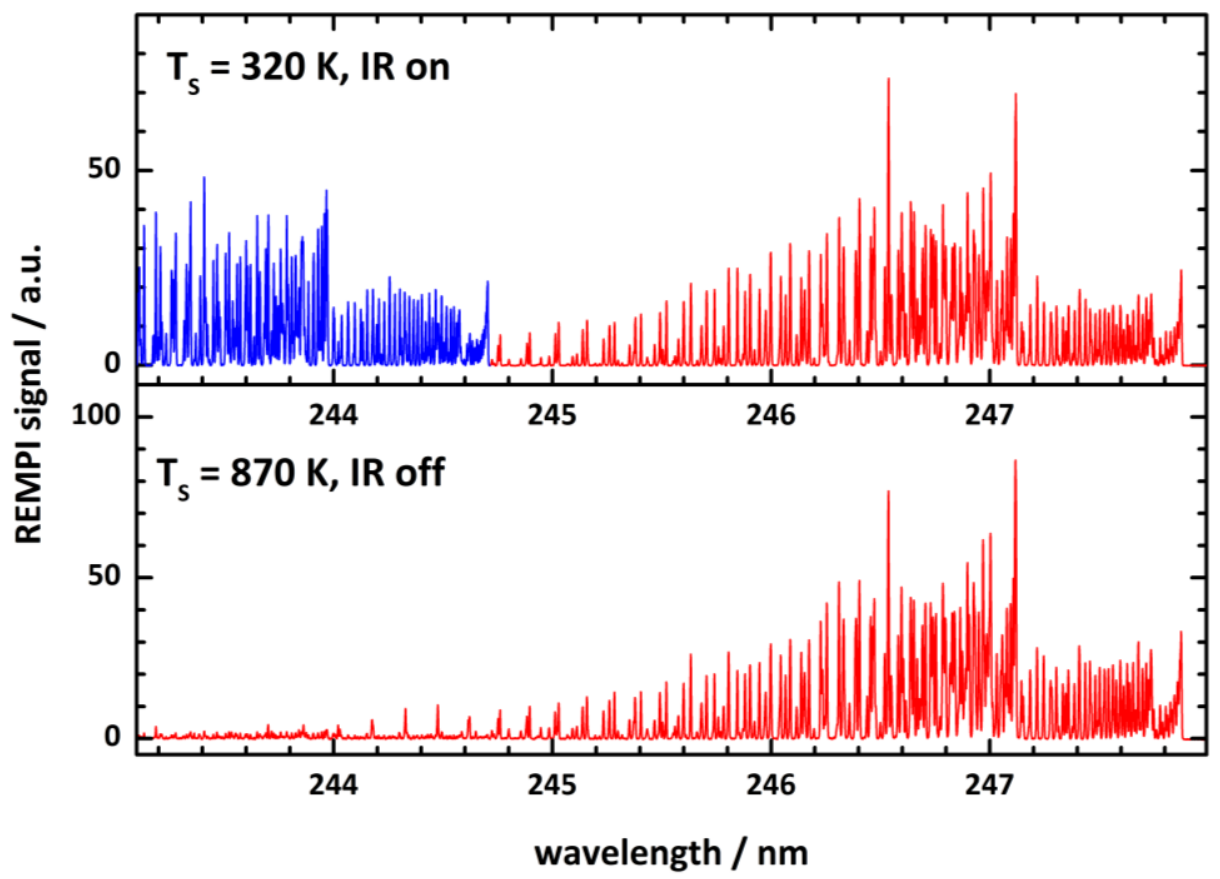

Fig. 3.16: Comparison of $\mathrm{NO}\left(v_{f}=2\right)$ REMPI spectra for $E_{I}=0.65 \mathrm{eV}$. The upper panels shows a spectrum of the (0-2) band (red) and a part of the (1-3) band (blue) with the IR laser turned on and surface at $T_{S}=320 \mathrm{~K}$. The lower panel shows the same spectral region but without the IR laser and the surface heated to $T_{S}=870 \mathrm{~K}$. 
Table 3.2: $E_{I}$ dependent branching ratios $R_{v_{f}}$ for $\mathrm{NO}\left(v_{i}=3, J_{i}=1.5\right)$ scattering from $\mathrm{Au}(111)$ at $T_{S}=320 \mathrm{~K}$. The errors were calculated from estimated $10 \%$ pulse-to-pulse fluctuations in laser intensity.

\begin{tabular}{c|c|c|c}
$E_{I} / \mathrm{eV}$ & $R_{1}$ & $R_{2}$ & $R_{3}$ \\
\hline 0.11 & $0.093 \pm 0.008$ & $0.144 \pm 0.017$ & $0.763 \pm 0.065$ \\
\hline 0.26 & $0.068 \pm 0.006$ & $0.246 \pm 0.025$ & $0.686 \pm 0.046$ \\
\hline 0.41 & $0.057 \pm 0.005$ & $0.246 \pm 0.060$ & $0.697 \pm 0.177$ \\
\hline 0.51 & $0.114 \pm 0.016$ & $0.345 \pm 0.051$ & $0.540 \pm 0.071$ \\
\hline 0.65 & $0.186 \pm 0.013$ & $0.386 \pm 0.066$ & $0.428 \pm 0.067$ \\
\hline 0.82 & $0.158 \pm 0.036$ & $0.427 \pm 0.081$ & $0.414 \pm 0.079$ \\
\hline 0.95 & $0.208 \pm 0.027$ & $0.474 \pm 0.048$ & $0.318 \pm 0.019$ \\
\hline 1.07 & $0.221 \pm 0.022$ & $0.424 \pm 0.114$ & $0.356 \pm 0.098$
\end{tabular}

The resulting branching ratios of $\mathrm{NO}\left(v_{f}=1,2,3\right)$ are shown in Fig. 3.17 and given specifically in Table 3.2. The data clearly exhibits enhanced vibrational relaxation at high incidence energies. The relative amount of molecules that survive the collision in $v_{f}=3$ decreases from $76 \%$ at $E_{I}=0.11 \mathrm{eV}$ to only $36 \%$ at the highest $E_{I}$. I find the opposite behavior for vibrationally inelastic scattering into $v_{f}=1$ and $v_{f}=2$. The relative population in both channels increases with increasing incidence energy, with $R_{v=2}$ ranging from $14 \%-47 \%$ and $R_{v=1}$ ranging from $6-22 \%$.

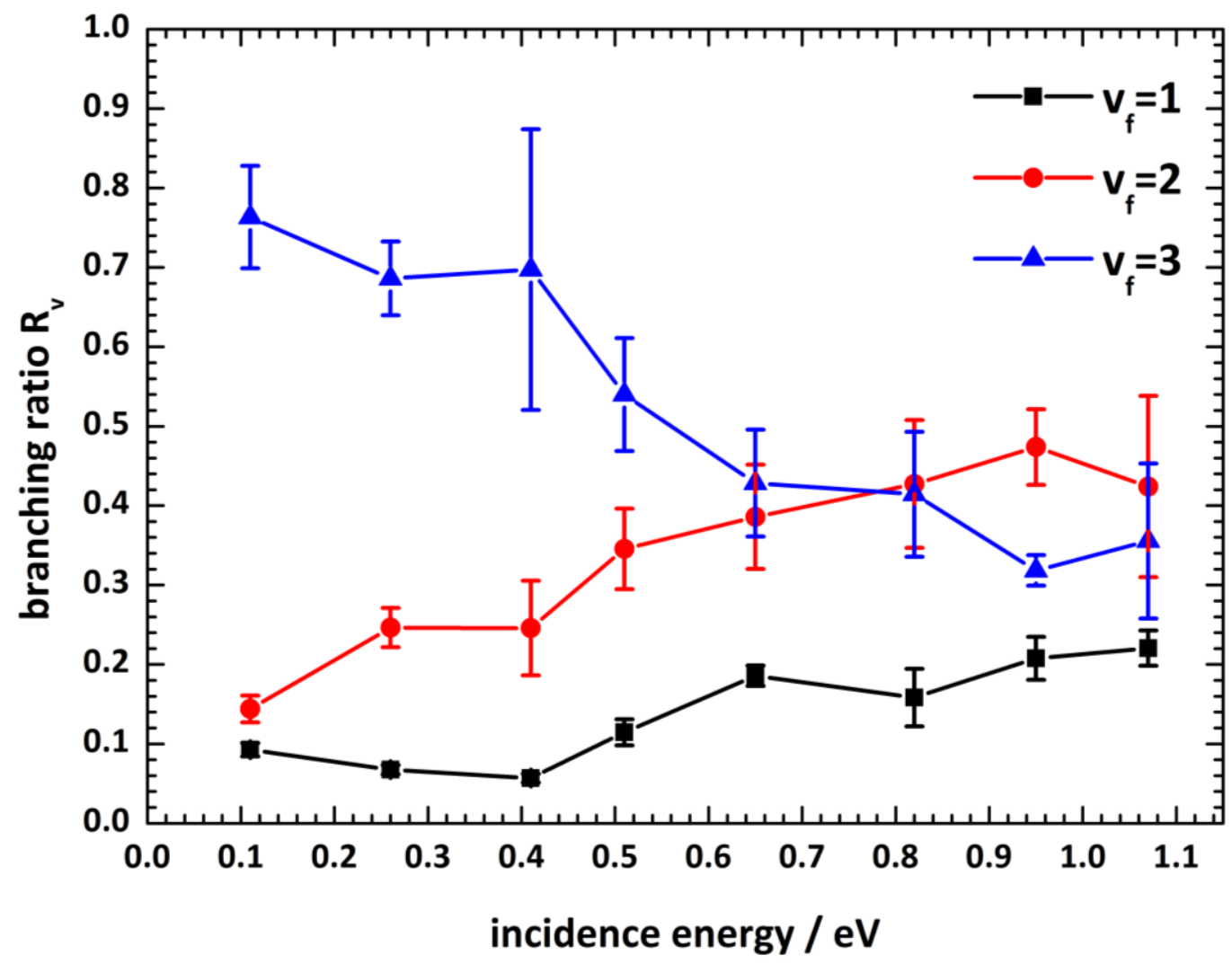

Fig. 3.17: Experimentally observed branching ratios for $\mathrm{NO}\left(v_{i}=3, J_{i}=1.5\right)$ scattering from $\mathrm{Au}(111)$ into $v_{f}=1$ (black), $v_{f}=2$ (red) and $v_{f}=3$ (blue) for incidence energies ranging from $E_{I}=0.1-1.1 \mathrm{eV}$. Error bars were calculated from estimated $10 \%$ pulse to pulse fluctuations in laser power and the change of the IR laser intensity during the scan. 


\subsubsection{Comparison to IESH and EF simulations}

The experimental branching ratios are suitable as benchmark data for comparison to first-principle IESH theory. I used the identical program code that was used to describe vibrational relaxation [74] and excitation [25] (section 3.1) for NO/Au(111). The initial conditions are the only differences to the calculations in section 3.1. The simulation starts with a surface temperature of $T_{S}=320 \mathrm{~K}$ and three quanta of vibrational energy in the NO molecule. Because vibrational relaxation probabilities lie in the range of several percent, it is sufficient to calculate only 1000 trajectories per incidence energy to obtain good statistics. The simulation program further provides the possibility to disable surface hopping and to enable electronic friction (EF) or to disable both nonadiabatic methods and to perform adiabatic calculations. Trajectories, for which the NO molecule did not reach a distance of $10 \AA$ from the surface after $20 \mathrm{ps}$, were excluded from the analysis (trapping). The comparison of the experimental to the results of the simulations is shown in Fig. 3.18. The left panel contains the theoretical prediction from the simulation when all trajectories are simply analyzed with respect to the final vibrational state $v_{f}$. The difference between the theoretically predicted and the experimentally observed branching ratios is quite striking: both IESH and EF simulations predict a dependence of the relaxation probabilities which is opposite to that observed in the experiment. In the simulations, the $\mathrm{NO}\left(v_{f}=3\right)$ survival probability increases with increasing $E_{I}$ while the $v=3 \rightarrow 1$ relaxation probability decreases.
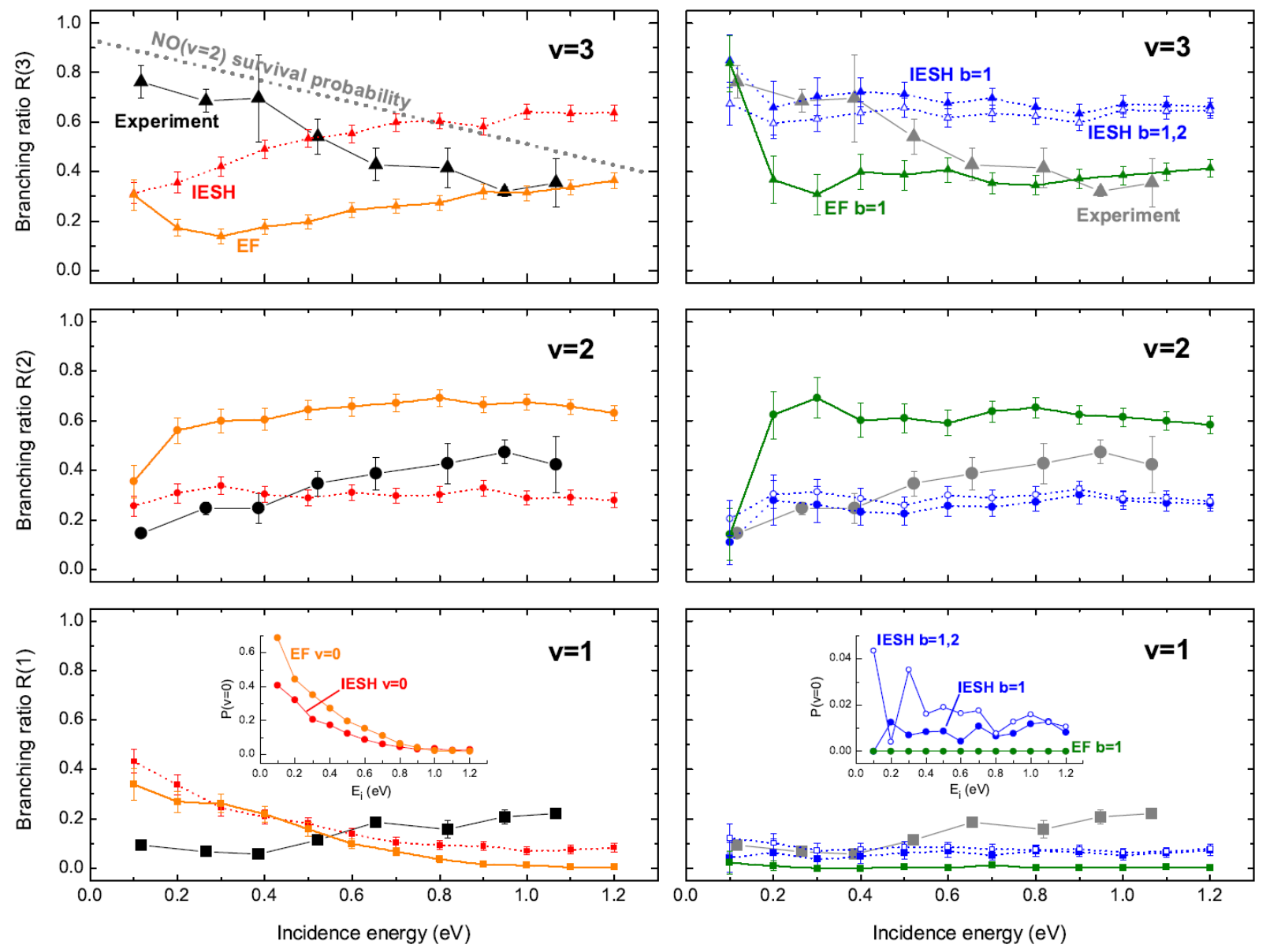

Fig. 3.18: Comparison of experimental $\mathrm{NO}\left(v_{f}=3,2,1\right)$ branching ratios (black) to IESH and EF simulations. Left panel: Comparison of experiment (black) to IESH (red) and EF (orange) results analyzing all trajectories. Right panel: Comparison of the experiment (gray) to EF (green) and IESH (blue) results but restricting the analysis to only single bounce and single and double bounce collisions. The insets show the $\mathrm{NO}(v=3 \rightarrow 0)$ relaxation probabilities [118]. Reproduced with permission from Golibrzuch et al., Journal of Chemical Physics, 2014. 140(4), 044701. Copyright 2014, AIP Publishing LLC. 
The search for the origin of this apparent failure of the theoretical model requires a detailed analysis of the trajectories produced in the simulations. Examples for different types of trajectories, observed in the IESH simulations at $E_{I}=0.6 \mathrm{eV}$, are shown in Fig. 3.19. The upper panel shows a single-bounce collision $(b=1)$ which is expected for direct scattering events. The middle panel shows a typical double-bounce collision $(b=2)$ where the O-atom first hits the surface but the $\mathrm{N}$-atom keeps on moving toward the surface and collides about $100 \mathrm{fs}$ later. This type of collision can, in principle, also be assigned as direct scattering. The lower panel shows a multi-bounce collision $(b>2)$ : the molecule collides with the surface at about $500 \mathrm{fs}$ and stays nearby at distances $z \leq 2.5 \AA$ for approximately 3 ps before it can escape. This kind of collision does clearly not represent a direct scattering event. I did not observe evidence for such multi-bounce collisions in the experimental angular and time-of-flight distributions at $E_{I}=0.51 \mathrm{eV}$ (Fig. 3.14). However, I cannot completely exclude multi-bounce event at lower $E_{I}$ because I did not measure angular distributions at $E_{I}<0.51 \mathrm{eV}$. Cooper et al. [26] measured angular distributions for $\mathrm{NO}(v=0 \rightarrow 0,1,2) / \mathrm{Au}(111)$ scattering and found a broadening of the angular distribution at low $E_{I}$. This broadening is possibly an indication for an increasing fraction of multi-bounce collisions with decreasing incidence energy but the IESH model can still significantly overestimate the importance of multi-bouncing. Therefore, my hypothesis is that the potential energy surface used in the IESH model leads to too many multi-bounce trajectories. As a test of this hypothesis, I drop multi-bounce trajectories which do not correspond to direct scattering.

The restriction of the trajectory analysis to only single, or alternatively single and double bounce, collisions requires an algorithm that counts the number of bounces at the surface. This raises the question how to define a bounce. It turns out that this question is rather difficult to answer. In principle, one can count the sign changes in $d z / d t=v_{z}$ or the second derivative, the acceleration $a_{z}$, along $z$ for the single atoms or the center of mass of the molecule. The most promising analysis counts how often $a_{z}$ of the center of mass exceeds a certain threshold, $1 / 8$ of its maximum along the trajectory. The choice of a variable threshold is necessary because the acceleration/ deceleration upon the molecule-surface collision is different for different $E_{I}$. Prior to the bounce analysis, the trajectory has to be smoothened to avoid artificial counts. The time dependent positions of the single atoms are first averaged over $1 \mathrm{fs}$ (trajectories calculated with $0.1 \mathrm{fs}$ time steps). Afterwards a moving average over $17.5 \mathrm{fs}$ (corresponding to one vibrational period) is applied to remove fast oscillations resulting from the classical NO vibration. Finally, the result is analyzed according to the acceleration $a_{z}$.

The result for restriction of the analysis to only single bounce trajectories is shown as blue solid lines in the right panels of Fig. 3.18. Especially the branching ratios at low incidence energy change dramatically; the survival probability of $\mathrm{NO}\left(v_{f}=3\right)$ increases whereas the relaxation probabilities drop accordingly. The incidence energy dependence does improve in a sense that the theoretical model does not predict the opposite dependence anymore. However, the simulations still do not show an increase in relaxation with increasing $E_{I}$. The simulations predict that the branching ratios stay more or less constant over the whole range of incidence energies. If the analysis is restricted to single and double bounce collisions, this theoretical prediction does not change significantly and only the probability for vibrational relaxation increases slightly. The same observations hold for the EF calculations.

Additionally, the molecular dynamics simulations give probabilities for relaxation into $\mathrm{NO}\left(v_{f}=0\right)$ which are not observable in the experiment. The results are shown as insets in the lower panels of Fig. 3.18. The values correlate basically with the calculations for $v=3 \rightarrow 1$ relaxation. The $\mathrm{NO}(v=3 \rightarrow 0)$ relaxation probability decreases with increasing $E_{I}$ if all trajectories are considered and stays almost constant if a restriction so single and double bounce collision is applied. Note that the IESH and the EF model give very similar results for analysis of all trajectories but EF yields basically no relaxation into $v_{f}=0,1$ if the analysis is restricted to only single and double bounce trajectories. 

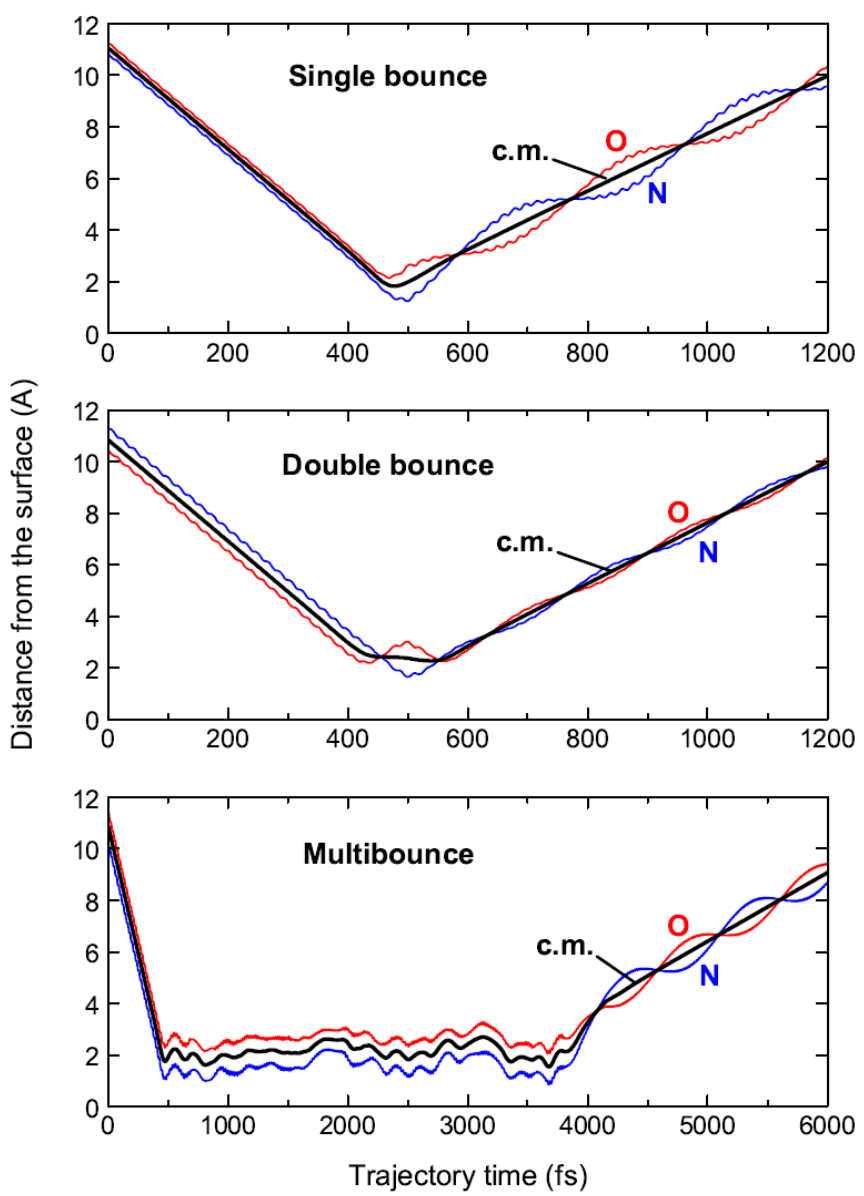

Fig. 3.19: Examples for single, double and multi-bounce collisions produced in the IESH simulations at $E_{I}=0.6 \mathrm{eV}$. Shown are the distances $z$ of the $\mathrm{N}$-atom (blue), O-atom (red) and the center of mass (black) of the molecule from the surface during the corresponding trajectory [118]. Reproduced with permission from Golibrzuch et al., Journal of Chemical Physics, 2014. 140(4), 044701. Copyright 2014, AIP Publishing LLC.

\subsubsection{Multi-bouncing in the IESH simulations}

In order to investigate the apparent failure of the theoretical model in detail, it is necessary to analyze all trajectories with respect to the number of bounces and the effects that result from these multi-bounce collisions. The left panel of Fig. 3.20 shows the fraction of single, double and multi-bounce collisions as a function of incidence translational energy for three different kinds of calculations: IESH, electronic friction and adiabatic. All three models use the same neutral potential energy surface for the NO-Au interaction. For electronic friction and adiabatic simulations, however, the NO molecule can only move on the ground-state PES. The comparison shows that all three approaches give basically the same results. The fraction of single-bounce collisions increases from about $5 \%$ at $E_{I}=0.1 \mathrm{eV}$ to $70 \%$ at $E_{I}=1.2 \mathrm{eV}$. Multi-bouncing shows the opposite trend and decreases from $85 \%\left(E_{I}=0.1 \mathrm{eV}\right)$ to $5 \%\left(E_{I}=1.2 \mathrm{eV}\right)$. The fraction of double bounce collisions first increases from up to a maximum of $30 \%$ at $E_{I}=0.6 \mathrm{eV}$ and then decreases again at higher $E_{I}$. The identical behavior of nonadiabatic (IESH, EF) and adiabatic calculations shows that the implementation of nonadiabaticity in the model is not responsible for the multi-bounce events. Note that the theoretical calculations predict about $40 \%$ multi-bounce collisions at $E_{I}=0.5 \mathrm{eV}$, an incidence energy at which my experimental angular and time-of-flight distributions clearly indicate direct scattering. This comparison does not exclude multi-bounce collisions in the experiment but it shows that the fraction of multi-bounce trajectories in the theoretical model is unreasonably high. 

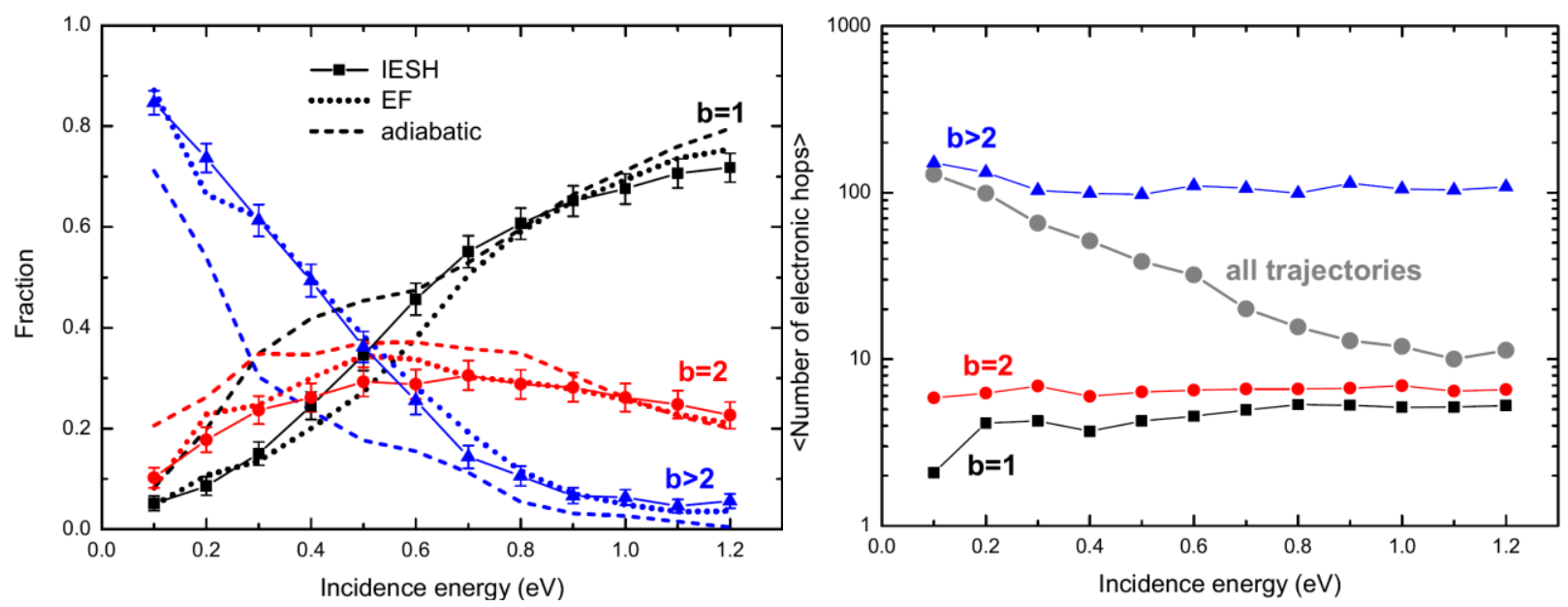

Fig. 3.20: Left panel: $E_{I}$ dependent fraction of single (black), double (red) and multi-bounce (blue) collisions when running the MD simulations with IESH enabled (solid lines), only electronic friction (dotted lines) or performing adiabatic (dashed lines) calculations. All three calculations show basically the same result. That is, the fraction of multi-bounce collisions increases dramatically with decreasing $E_{I}$. Right panel: Number of electronic hops for single (black), double (red) and multi-bounce (blue) collisions. The number of electronic transitions is at least one order of magnitude higher for multi-bouncing than for single and double bounce collisions [118]. Reproduced with permission from Golibrzuch et al., Journal of Chemical Physics, 2014. 140(4), 044701. Copyright 2014, AIP Publishing LLC.

The comparison in Fig. 3.18 already showed that removal of multi-bounce trajectories from the analysis improves the agreement between experiment and theory. Therefore, it is interesting to investigate how multiple collisions influence the nonadiabatic energy transfer. The data presented in Fig. 3.18 shows that multi-bouncing strongly enhances the relaxation probabilities at low $E_{I}$. In the IESH model, changes in vibrational energy are always related to electronic transitions (hops) during the trajectory. An analysis of the average number of electronic hops per trajectory is shown in the right panel of Fig. 3.20. If no restriction regarding the number of bounces is made (gray), the number of electronic hops decreases with increasing $E_{I}$ by about one order of magnitude, in accordance with the decrease in relaxation probability. A more careful analysis with restriction to single, double and multiple bounces shows that the average number of electronic hops does not change significantly with $E_{I}$ for all cases. Nevertheless, multi-bounce trajectories exhibit 10-15 times more hops than observed for single and double bouncing. The decrease in the number of electronic transitions in all trajectories with increasing $E_{I}$ results from a decreasing fraction of multi-bounce events. This observation supports the view that multi-bounce collisions lead to higher relaxation probabilities and disturb the corresponding dependence on $E_{I}$. Another feature appears from Fig. 3.20: even with restriction to single bounce collisions, the number of electronic transition does not change systematically with $E_{I}$. This is in agreement with the absent $E_{I}$ dependence for the branching ratios for $b=1$ and $b=1,2$.

In addition, the simulations enable the investigation of the effects of the number of bounces on the distance from the surface as well as on the orientation of molecules at the surface. The left panel of Fig. 3.21 shows this analysis for $E_{I}=0.1 \mathrm{eV}$. The results show that the smallest NO-surface distance $z_{\min }$ during the trajectory decreases with increasing number of bounces. For single-bounce collisions, I observe two maxima at about $2.6 \AA$ and $1.8 \AA$. For $b>2$, the distribution peaks at $z_{\text {min }} \approx 0.9 \AA$ while the orientation of the molecule improves towards the favorable $\mathrm{N}$-down orientation $\left(\theta=180^{\circ}\right)$. Note that $\theta_{\min }$ reflects the orientation of the molecule at $z_{\min }$ and not the orientation at impact (first collision). Molecules that are oriented with the $\mathrm{N}$-atom toward the surface can always approach the surface closer than if they are oriented with the O-atom first. This is a result of the attractive $\mathrm{N}$-Au potential whereas the O-Au potential is mainly repulsive $[65,73]$ in the model. The improved orientation and closer approach with increasing $b$ allow two possible interpretations: (1) multi-bouncing occurs predominantly 60 
for those molecules that collide approximately at the $\mathrm{N}$-first orientation, or (2) the molecules re-orient towards the $\mathrm{N}$-down orientation during the multiple bounces. In the latter case, one can imagine the multi-bounce event as some kind of pathway toward trapping which orients the NO molecule toward the binding orientation with the $\mathrm{N}$-atom pointing toward the surface.

The right panels of Fig. 3.21 show $z_{\min }$ and $\theta_{\min }$ values for all trajectories (no restriction to the number of bounces) as a function of incident kinetic energy. At low $E_{I}$, all $z_{\min }$ distributions show a maximum at $\sim 0.8 \AA$ which decreases with increasing $E_{I}$. Simultaneously, a peak at $1.6 \AA$ builds up that slightly shifts toward lower values with increasing $E_{I}$. The inset in the upper right panel demonstrates for $E_{I}=0.3 \mathrm{eV}$ that the peaks at $z_{\min }=0.8 \AA$ results exclusively from multi-bounce collisions. Trajectories with $b=1,2$ only reach a distance of $z_{\min } \geq 1.2 \AA$. Surprisingly, an increase of $E_{I}$ does almost not affect $z_{\min }$ for single and multi-bouncing. However, exactly this property has been identified to be responsible for the kinetic energy dependence of the probability for vibrational energy transfer [8, 43, 44]. The absence of this effect in the IESH model might be the reason for the absence of an $E_{I}$ dependence for NO excitation [25] and de-excitation.

The $\theta_{\min }$ distributions (lower right panel of Fig. 3.21) show that the orientation of the molecule improves toward perfect $\mathrm{N}$-down orientation for low $E_{I}$. The distributions are rather broad (flat from $115-170^{\circ}$ ) at high incidence energy and become narrower and peak at $180^{\circ}$ with for low incidence energy. This was previously observed by Shenvi et al. [65] using the identical program code. The authors addressed this effect to dynamical steering, a re-orientation of the molecule as it approaches the surface. The conclusions were made based on a comparison of $z_{\min }$ and $\theta_{\min }$ for $E_{I}=0.05 \mathrm{eV}$ and $E_{I}=0.8 \mathrm{eV}$ and smaller distances and better orientations were found for $E_{I}=0.05 \mathrm{eV}$. My detailed trajectory analysis, however, puts the previous observations into doubt: the analysis shows that the reorientation does not occur prior to the (first) collision with the surface but improves while the molecule bounces multiple times off the surface.
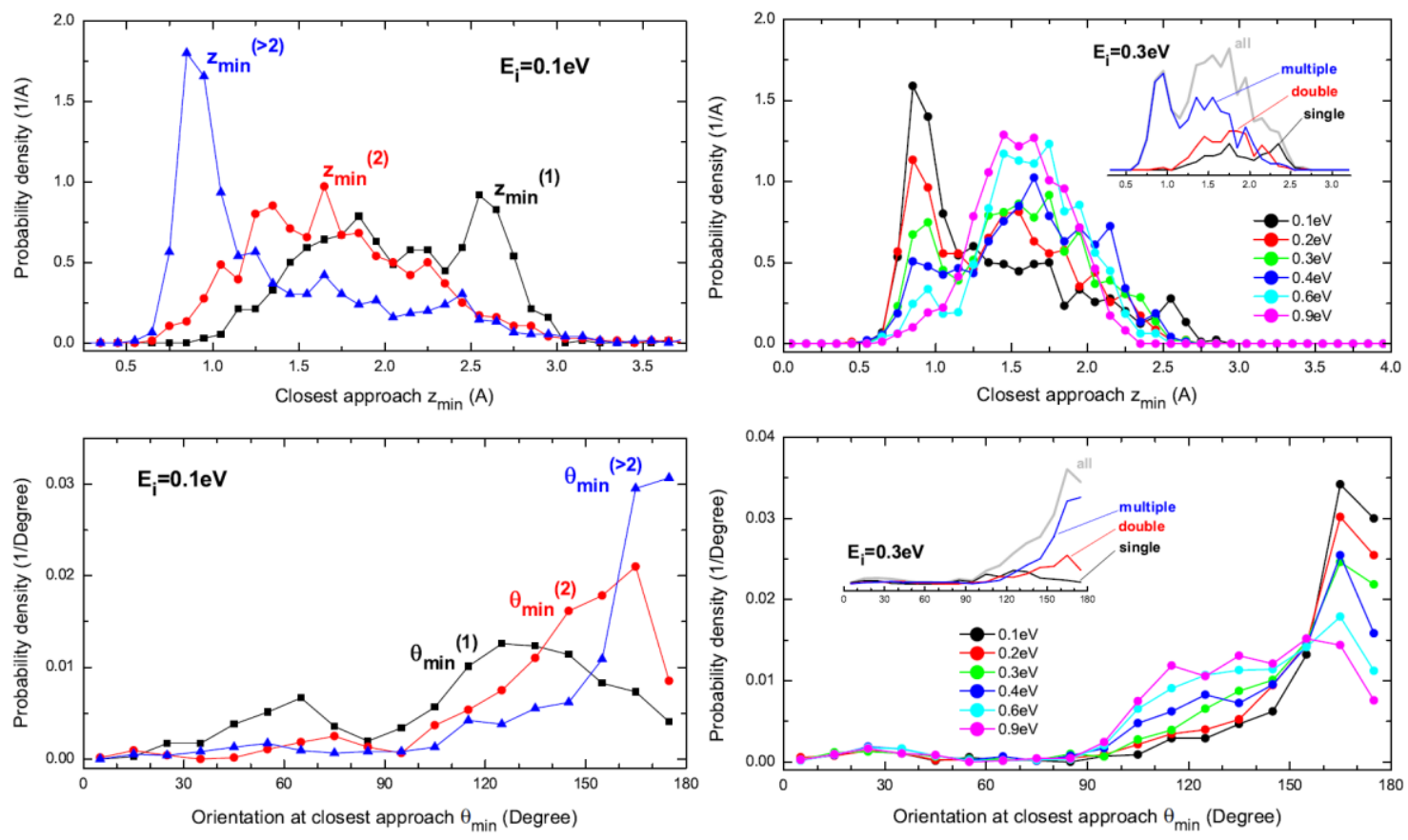

Fig. 3.21: Analysis of the distance of closest approach $z_{\min }$ (upper panels) and the orientation of the molecule $\theta_{\min }$ at $z_{\min }$ (lower panels) with restriction for the number of bounces (left) and as a function of incident kinetic energy (right) [118]. Reproduced with permission from Golibrzuch et al., Journal of Chemical Physics, 2014. 140(4), 044701. Copyright 2014, AIP Publishing LLC. 
The results show that the IESH model in its current implementation has serious problems to describe NO scattering from $\mathrm{Au}(111)$. The simulations produce a too large fraction of multi-bounce trajectories. Even if those trajectories are excluded from the analysis of vibrational relaxation probabilities, the theoretical model fails to reproduce the experimental results. Especially, I find no dependence of the relaxation probability on incidence translational energy, in contrast to the experimental results. The reason for this apparent failure of the model is not clear so far. Similar multi-bounce fractions for IESH, EF and adiabatic calculations show that the surface hopping, which is necessary to include the nonadiabaticity, is not to blame. Other sources can be the Au-Au or the NO-Au interaction potential. A wrong Au-Au interaction can produce a surface that is too soft and that can therefore absorb too much kinetic energy of the molecule. However, the Au-Au interaction potential was fitted to an experimental phonon spectrum [73] and it seems unlikely that it produces as dramatic effects as observed in the simulations. The NO-Au interaction potential was obtained by fitting physically reasonable pair potentials to data obtained from DFT calculations [73]. DFT is known to insufficiently describe charge transfer and is likely to give wrong energies for systems where charge transfer is important. The potential energy surface can be too corrugated which can result in perpendicular-to-parallel momentum transfer and therefore to a lower effective collision energy. Solution to this problem can be a recalculation of the PES with a higher density of DFT point to reduce the corrugation or using a different DFT functional. However, this does not solve the problems that might be introduced by the DFT method itself. More accurate correlated wavefunction methods [119] become available and provide a more accurate description of gas-surface interactions.

The most important result of the presented comparison for $\mathrm{NO}(v=3 \rightarrow 1,2,3) / \mathrm{Au}(111)$ scattering is that correct description of the adiabatic properties (like translational energy transfer) of a system is crucial to obtain correct results for the nonadiabatic features. 


\subsection{Translational energy transfer in rotationally and vibrationally inelastic $\mathrm{NO}(v=3) / \mathrm{Au}(111)$ scattering}

Vibrational energy exchange in NO collisions with metal surfaces has attracted much attention over the last decades $[1,3,8,25,27,29,30,34,44]$ and I presented new features in sections 3.1 and 3.2. However, very little work has been done in order to understand the influence of vibrationally inelastic scattering on translational energy of the scattered molecules. So far, translational motion is often believed to be a spectator in nonadiabatic NO-metal interactions that can influence the strength of the V-EHP coupling $[8,25,26,43]$, but cannot couple efficiently to the NO vibration as it was observed for $\mathrm{NH}_{3} / \mathrm{Au}(111)$ scattering [4]. To my knowledge, this picture has not been tested or verified so far, neither experimentally nor theoretically. Modern nonadiabatic theories have so far mainly been tested to be able to reproduce nonadiabatic effects by means of vibrational excitation and de-excitation of NO in scattering from $\mathrm{Au}(111)[25,64,73]$. However, the results from section 3.2 already showed that adiabatic effects like translational inelasticity, e. g. translational energy transfer to the lattice, are at least as important as nonadiabaticity for the correct description of the dynamics of a system.
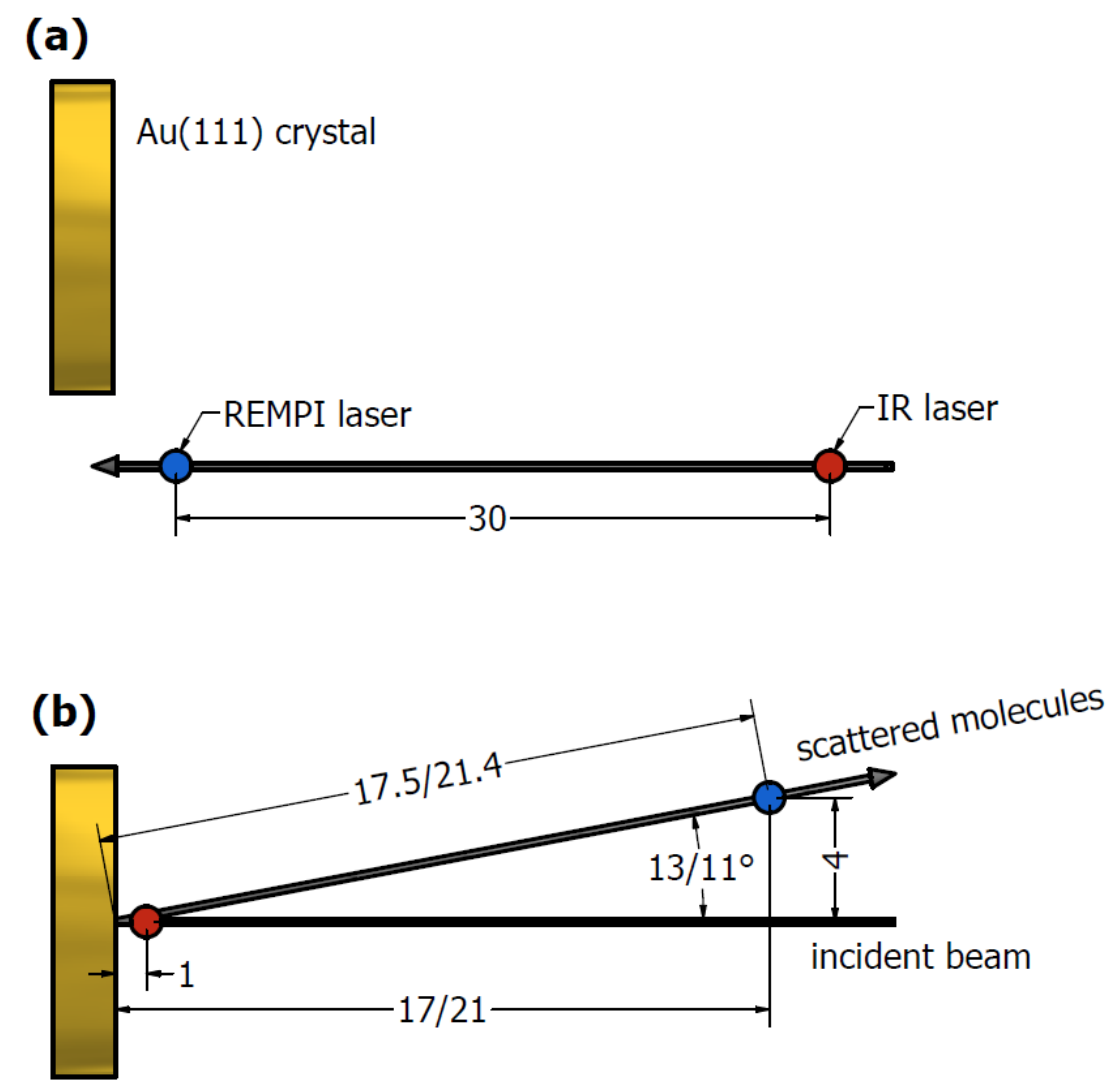

Fig. 3.22: Experimental set-up for measurement of high resolution IR-UV double resonance state-to-state time-of-flight experiments. Panel (a) shows the laser position for determination of the incidence energy. Panel (b) shows the conditions for experiments on scattered molecules. The two numbers in (b) indicate different geometries for excitation and relaxation experiments. Reprinted with permission from Golibrzuch et al., Journal of Physical Chemistry A, 2013. 117(36), 8750-8760. Copyright 2013 American Chemical Society.

Therefore the main questions that this section will try to answer are: How does the nonadiabatic vibrational energy transfer influence other degrees of freedom? Is translational motion really a spectator in vibrationally inelastic NO/Au(111) scattering? I measured state-to-state time-of-flight profiles using IRUV double resonance (see section 2.4 for a detailed description of this method) as an experimental tool 
to answer these questions. Briefly, an IR laser prepares incident $\mathrm{NO}\left(v_{i}=2,3, J_{i}=1.5\right)$ molecules by vibrational overtone pumping before they collide with the surface. Fig. 3.22 shows the experimental setup for these experiments. The translational energy of the incident molecular beam is measured by positioning the IR laser about $30 \mathrm{~mm}$ upstream with respect to the REMPI beam; the surface is moved up by $10 \mathrm{~mm}$ to avoid scattering (Fig. 3.22 (a)). For experiments on scattered molecules, the surface is moved in the path of the molecular beam and the IR laser excites incident molecules less than $1 \mathrm{~mm}$ away from the surface. The scattered molecules are detected at about $17 \mathrm{~mm}$ or $21 \mathrm{~mm}$ distance with quantum state resolution. The time-of-flight profiles were fitted following the procedure described in Appendix C. I investigated the final translational energy $E_{f}$ for different vibrational channels with loss and gain of one and two vibrational quanta $(-2 \leq \Delta v \leq+2)$ and compared the results to vibrationally elastic scattering. Furthermore, I studied the influence of surface temperature on the translational energy change as well as the effect of incidence energy and final rotational excitation.

\subsubsection{Results}

\section{Vibrationally inelastic and rotational quasi-elastic scattering at different surface temperatures}

The first part of this study is focused on the experimental results for vibrationally elastic and inelastic scattering with vibrational (de-)excitation of one and two vibrational quanta. In order to minimize effects resulting from rotational excitation, the measurements are limited to rotationally quasi-elastic scattering into $J_{f}=5.5$, which corresponds to a rotational energy of $E_{\text {rot }} \leq 2 \mathrm{meV}$. The measured arrival time distributions for scattering of $\mathrm{NO}\left(v_{i}=2,3\right)$ into $v_{f}=1,2,3,4$ are shown in Fig. 3.23 as open circles for various surface temperatures and $E_{I}=0.63 \mathrm{eV}$. The solid lines represent the corresponding fits to the experimental data. A detailed description of the model used to fit the time-of-flight spectra can be found in Appendix C. The good overall agreement shows that the model is suitable for description of the data. Already the raw data reveals that vibrational relaxation $(3 \rightarrow 2,3 \rightarrow 1,2 \rightarrow 1)$ leads to earlier arrival times at the detection laser and therefore to higher kinetic energies $E_{f}$. This effect is present for all examples in Fig. 3.23 comparing the black $(\Delta v=0)$ to the green $(\Delta v=-1)$ and blue $(\Delta v=-2)$ traces.

For vibrational excitation the observations are slightly different. At $T_{S}=570 \mathrm{~K}$, the $v=2 \rightarrow 3$ channel is shifted toward later arrival times by about $1 \mu \mathrm{s}$. This shift is similar, but opposite, to that observed in the $2 \rightarrow 1$ channel. The higher surface temperature (compared to the data in the left column) is necessary to observe sufficient excitation into $v_{f}=3$ (compare section 3.1). For excitation of two vibrational quanta $(2 \rightarrow 4)$ the surface temperature has to be raised even more to $T_{S}=970 \mathrm{~K}$ (right column) which still results in a much lower $\mathrm{S} / \mathrm{N}$ level than for the other scattering channels. At this surface temperature, I investigate only vibrational excitation because observation of $2 \rightarrow 1$ relaxation is now disturbed by a significant background resulting from $v=0 \rightarrow 1$ excitation. Compared to the time-of-flight profiles for $T_{S}=570 \mathrm{~K}$, the profiles at $T_{S}=970 \mathrm{~K}$ are clearly broadened. A decrease in $E_{f}$ for vibrationally inelastic $v=2 \rightarrow 3,4$ is not clearly visible anymore.

The fits to the time-of-flight data can be transferred into energy space and flux using the procedure described in Appendix B. The results are shown in Fig. 3.24 with the same color code used in Fig. 3.23. The gray dashed lines reflect the translational energy distribution of the incident molecular beam. A comparison to the final translational energy distributions shows that molecules that are scattered vibrationally elastically (black), lose about $45 \%$ of the incident kinetic energy in the surface collision. Furthermore, the $E_{f}$ distributions are much broader than the $E_{I}$ distribution. This broadening effect seems to increase with increasing surface temperature. Note that an increase of $T_{S}$ leads to a significant fraction of molecules with $E_{f}>E_{I}$. 


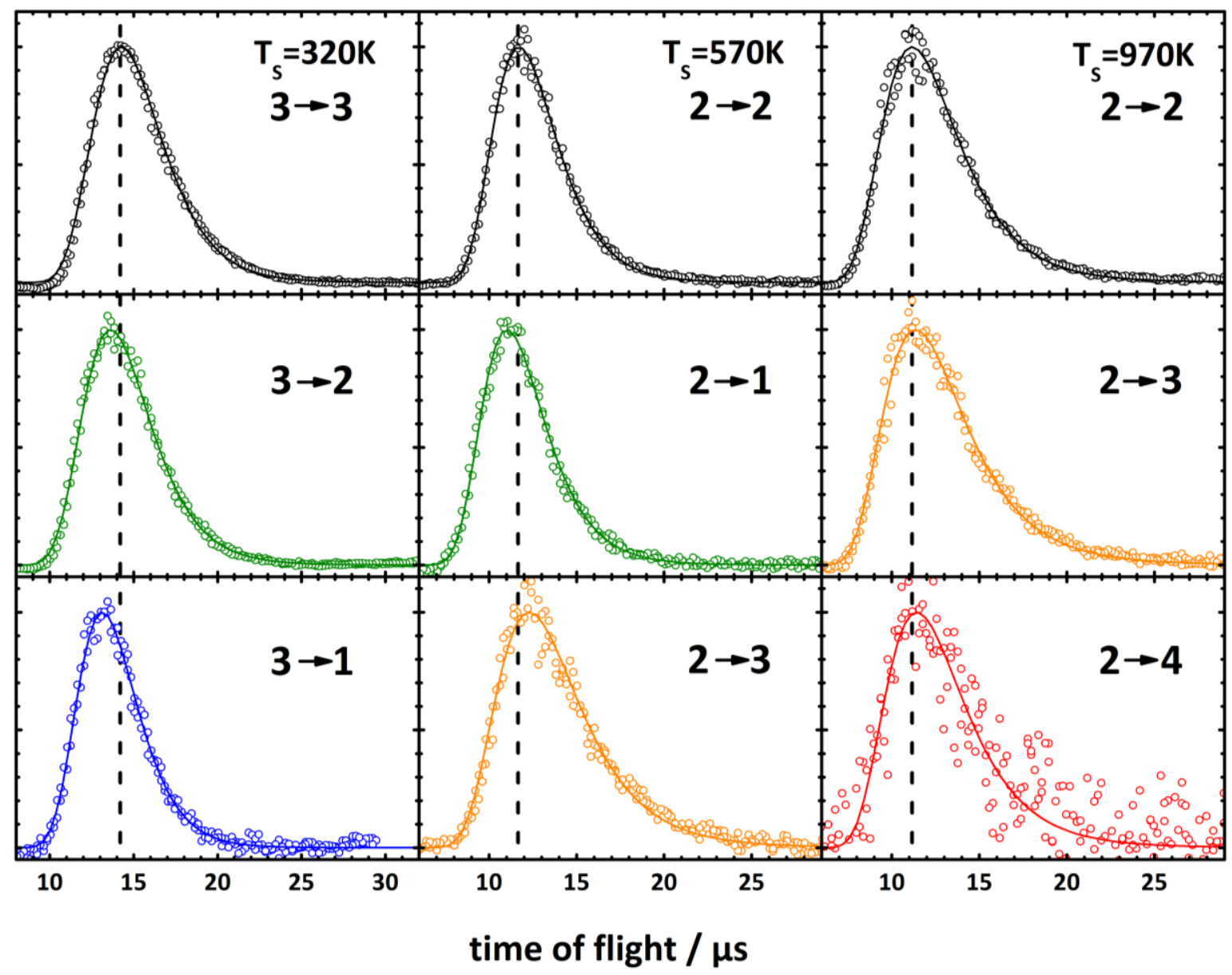

Fig. 3.23: Experimentally observed state-to-state time-of-flight profiles for rotationally quasi-elastic $\left(U_{f}=5.5\right)$ but vibrationally elastic and inelastic scattering of $\mathrm{NO}\left(v_{i}=2,3, J_{i}=1.5\right)$ scattering from $\mathrm{Au}(111)$ at $E_{I}=0.63 \mathrm{eV}$ and surface temperatures of $320 \mathrm{~K}$ (left), $570 \mathrm{~K}$ (middle) and $970 \mathrm{~K}$ (right). Colors indicate vibrationally elastic (black) and vibrationally inelastic scattering with loss of one (green) and two (blue) vibrational quanta and gain of one (yellow) and two (red) vibrational quanta. The dashed lines are drawn through the peaks of the vibrationally elastic channel to guide the eye. The fits to the experimental data were obtained according to the procedure described in Appendix C. Reprinted with permission from Golibrzuch et al., Journal of Physical Chemistry A, 2013. 117(36), 8750-8760. Copyright 2013 American Chemical Society.

The general trends that were already visible from the raw data are retained in these distributions. The peak of the translational energy distribution shifts by about $\sim 0.03 \mathrm{eV}$ towards higher $E_{f}$ for loss of one vibrational quantum and about twice as much for loss of two quanta of vibration. The opposite trend is observed for vibrational excitation at $T_{S}=570 \mathrm{~K}$ : loss of one vibrational quantum leads to an increase in $E_{f}$ by about $\sim 0.04 \mathrm{eV}$ while gain of one vibrational quantum leads to a decrease by the same amount. The effect for vibrational excitation decreases at the highest surface temperature of $T_{S}=970 \mathrm{~K}$, where all energy distributions become very similar. I observed basically no difference for uptake of one or two quanta of vibrational energy.

For better comparison it is reasonable to compress the experimental data. A translational energy distribution can for example be represented by the mean final translational energy $\left\langle E_{f}\right\rangle=\int E_{f} P\left(E_{f}\right) d E_{f}$. Fig. 3.25 shows the results for all vibrational scattering channels presented in Fig. 3.23 and Fig. 3.24 with the same color coding. In addition, it illustrates the effect of final rotational energy $E_{r o t}$ on $E_{f}$. Rotational excitation leads to a decrease in $\left\langle E_{f}\right\rangle$ for all nine vibrational channels. The dependence of final translation on rotational excitation is approximately linear but the slopes depend on 
$\Delta v$. An overview of the intercepts (extrapolation to $E_{\text {rot }}=0$ ) and slopes $\left(\Delta\left\langle E_{f}\right\rangle / \Delta E_{\text {rot }}\right)$ is given in Table 3.3. Vibrationally inelasticity systematically results in more positive slopes, especially for the case of vibrational excitation.

The intercepts, reflecting $E_{\text {rot }}=0$, can now be chosen to obtain the pure coupling between translation and vibration. At $T_{S}=320 \mathrm{~K}$ the loss of one and two vibrational quanta leads to an increase in $\left\langle E_{f}\right\rangle$ by about $0.03 \mathrm{eV}$ and $0.06 \mathrm{eV}$, respectively. I find a slightly larger shift of $0.045 \mathrm{eV}$ for loss of one vibrational quantum at $570 \mathrm{~K}$ surface temperature. The data shows the opposite behavior for $v=2 \rightarrow 3$ excitation, and in the excitation channel decreases $\left\langle E_{f}\right\rangle$ by $0.035 \mathrm{eV}$. The effect is significantly weaker at $T_{S}=970 \mathrm{~K}$ and vibrational excitation $(\Delta v=1,2)$ decreases $\left\langle E_{f}\right\rangle$ by only $0.016 \ldots 0.019 \mathrm{eV}$. Beside these effects caused by vibrational inelasticity, the data shows an additional effect on surface temperature. I find an increase in translational energy for vibrational elastic scattering $(3 \rightarrow 3,2 \rightarrow 2)$ with increasing surface temperature from $0.361 \mathrm{eV}$ at $T_{S}=320 \mathrm{~K}$ to $0.404 \mathrm{eV}$ at $T_{S}=970 \mathrm{~K}$.

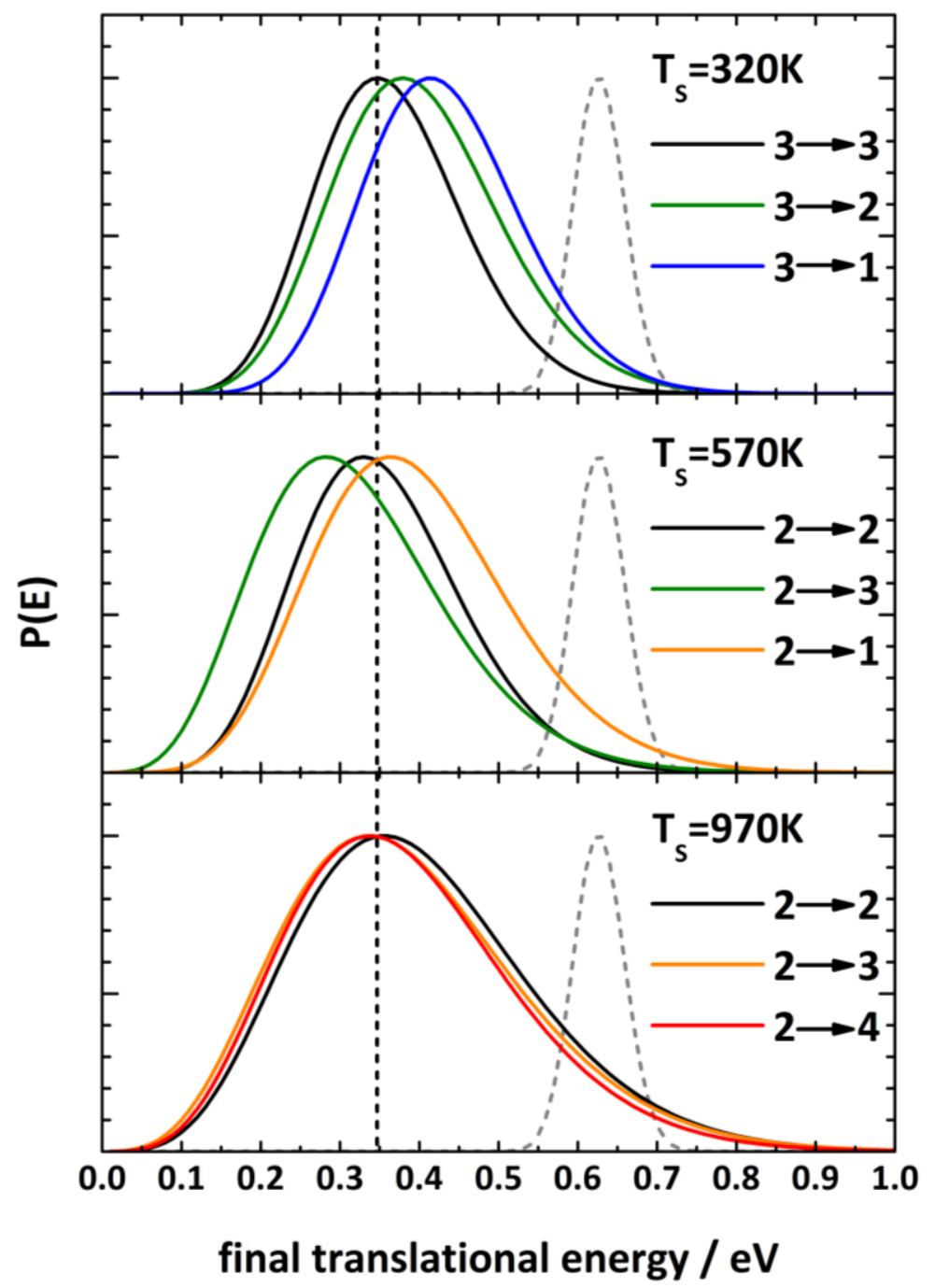

Fig. 3.24: Final translational energy distributions obtained from conversion of the fits shown in Fig. 3.23 into flux and energy space using the procedure described in Appendix C. The color code is identical with that used in Fig. 3.23. The gray dashed lines show the translational energy distribution of the incident molecular beam with $\left\langle E_{I}\right\rangle=0.63 \mathrm{eV}$. Reprinted with permission from Golibrzuch et al., Journal of Physical Chemistry A, 2013. 117(36), 8750-8760. Copyright 2013 American Chemical Society. 


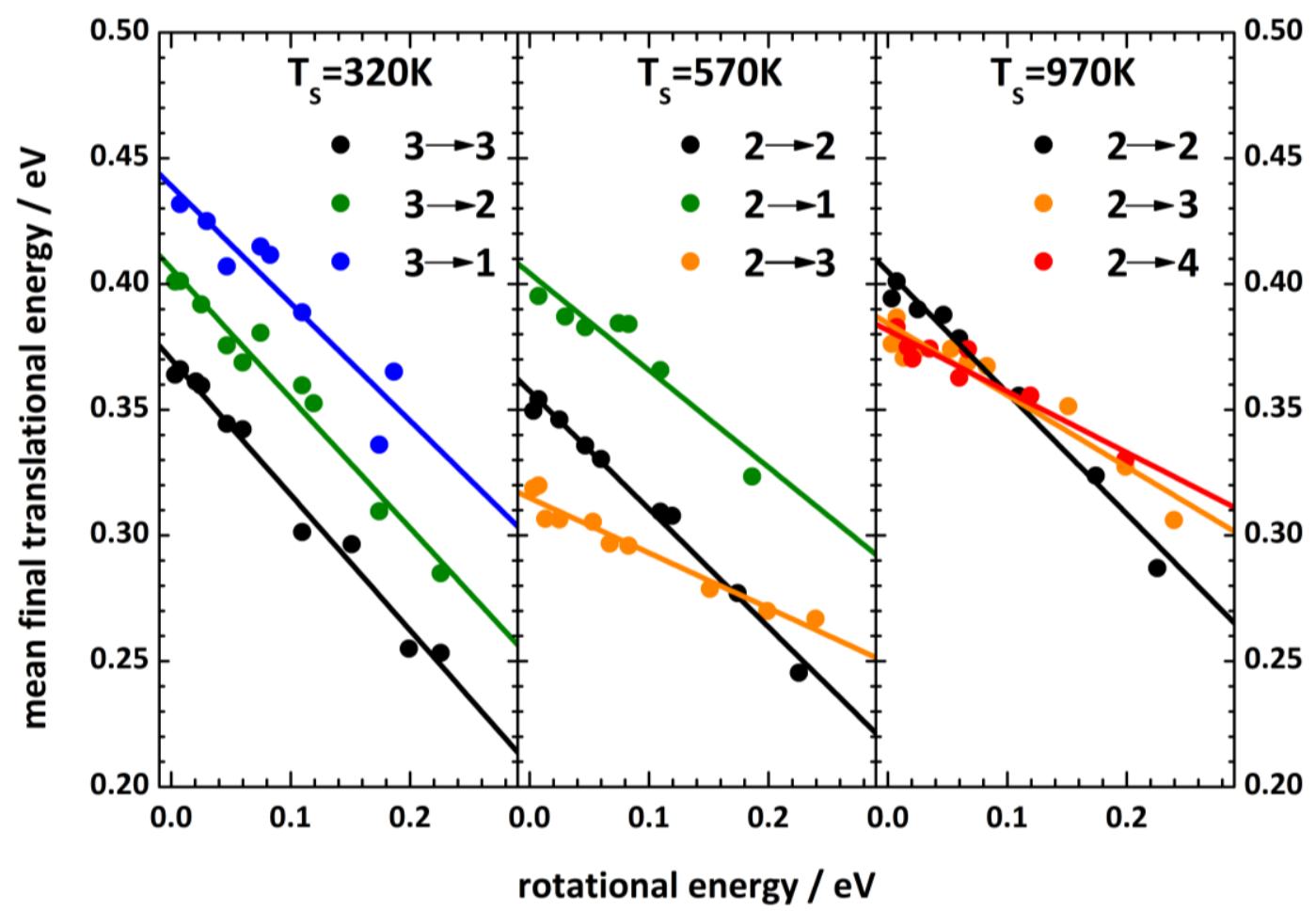

Fig. 3.25: Mean final translational energies as a function of final rotational excitation under the conditions given in Fig. 3.23 $\left(\left\langle E_{I}\right\rangle=0.63 \mathrm{eV}\right.$ ), applying the same color code for the vibrational scattering channels. At $T_{S}=320 \mathrm{~K}$ (left panel), vibrationally elastic and inelastic channels are almost parallel but the $3 \rightarrow 2$ and $3 \rightarrow 1$ channels are shifted towards higher $\left\langle E_{f}\right\rangle$ by about $35 \mathrm{meV}$. At $T_{S}=570 \mathrm{~K}$, vibrational relaxation $(2 \rightarrow 1)$ leads to an increase in $\left\langle E_{f}\right\rangle$ of $45 \mathrm{meV}$ and vibrational excitation to a decrease of $45 \mathrm{meV}$. For $T_{S}=970 \mathrm{~K}$, the effect is almost absent. The slopes of the approximately linear dependence of $\left\langle E_{f}\right\rangle$ on $E_{\text {rot }}$ are less negative for vibrational excitation than for relaxation and vibrationally elastic scattering. Reprinted with permission from Golibrzuch et al., Journal of Physical Chemistry A, 2013. 117(36), 8750-8760. Copyright 2013 American Chemical Society.

The slopes of the linear fits in Fig. 3.25 provide additional information because they are related to the energy transfer of initial translation to the surface and to final rotation. The data systematically shows values for $\Delta\left\langle E_{f}\right\rangle / \Delta E_{\text {rot }}$ that are bigger than -1 and that depend on $\Delta v$. For vibrationally elastic $2 \rightarrow 2 /$ $3 \rightarrow 3$ scattering, $\Delta\left\langle E_{f}\right\rangle / \Delta E_{\text {rot }}$ does not show any significant dependence on surface temperature or on initial vibrational state. However, vibrationally inelastic scattering systematically leads to an increase of $\Delta\left\langle E_{f}\right\rangle / \Delta E_{\text {rot }}$. The effect is much more pronounced for vibrational excitation than for de-excitation.

Table 3.3: Dependence $\Delta\left\langle E_{f}\right\rangle / \Delta E_{\text {rot }}$ of the final translational energy on rotational excitation and linear extrapolation of $\left\langle E_{f}\right\rangle$ to $E_{\text {rot }}=0$ for $\mathrm{NO}\left(v_{i}=2,3\right) / \mathrm{Au}(111)$ scattering into different final vibrational states at different surface temperatures and $E_{I}=0.65 \mathrm{eV}$. The corresponding linear fits are shown in Fig. 3.25.

\begin{tabular}{c|c|c|c} 
Vibrational channel & Surface temperature & $\boldsymbol{\Delta}\left\langle\boldsymbol{E}_{\boldsymbol{f}}\right\rangle / \boldsymbol{\Delta}_{\text {rot }}$ & $\left\langle\boldsymbol{E}_{\boldsymbol{f}}\right\rangle$ extrapolated to $\boldsymbol{E}_{\text {rot }}=\mathbf{0} / \mathbf{e V}$ \\
\hline $3 \rightarrow 3$ & $320 \mathrm{~K}$ & $-0.52 \pm 0.02$ & $0.361 \pm 0.003$ \\
\hline $3 \rightarrow 2$ & $320 \mathrm{~K}$ & $-0.50 \pm 0.04$ & $0.397 \pm 0.004$ \\
\hline $3 \rightarrow 1$ & $320 \mathrm{~K}$ & $-0.45 \pm 0.07$ & $0.429 \pm 0.008$ \\
\hline $2 \rightarrow 2$ & $570 \mathrm{~K}$ & $-0.47 \pm 0.02$ & $0.357 \pm 0.002$ \\
\hline $2 \rightarrow 1$ & $570 \mathrm{~K}$ & $-0.38 \pm 0.06$ & $0.403 \pm 0.005$ \\
\hline $2 \rightarrow 3$ & $570 \mathrm{~K}$ & $-0.22 \pm 0.02$ & $0.314 \pm 0.002$ \\
\hline $2 \rightarrow 2$ & $970 \mathrm{~K}$ & $-0.48 \pm 0.03$ & $0.404 \pm 0.003$ \\
\hline $2 \rightarrow 3$ & $970 \mathrm{~K}$ & $-0.28 \pm 0.03$ & $0.384 \pm 0.003$ \\
\hline $2 \rightarrow 4$ & $970 \mathrm{~K}$ & $-0.24 \pm 0.03$ & $0.381 \pm 0.003$
\end{tabular}




\section{Vibrationally and rotationally inelastic scattering of $\mathrm{NO}\left(v_{i}=3\right)$ at $\mathrm{T}_{S}=320 \mathrm{~K}$}

The previous section illustrated how vibrational inelasticity and surface temperature can influence the final translational energy of the scattered molecules. So far, I neglected two other parameters that can influence $E_{f}$ : incidence translational energy $\left(E_{I}\right)$ and rotational inelasticity. The latter effect was already briefly addressed in the context of Fig. 3.25 and will be investigated in more detail here. Fig. 3.26 shows normalized arrival time distributions for $\mathrm{NO}\left(v_{i}=3, J_{i}=1.5\right)$ scattering from $\mathrm{Au}(111)$ at $T_{S}=320 \mathrm{~K}$ surface temperature for three different incidence energies $E_{I}=0.26 \mathrm{eV}$ (left panels), $0.65 \mathrm{eV}$ (middle panels) and $0.98 \mathrm{eV}$ (right panels). Under the conditions of the experiment, I observe only vibrational relaxation into $v_{f}=1,2,3$ and essentially no vibrational excitation (see section 3.1). Because a thermal population of $\mathrm{NO}\left(v_{i}=1\right)$ is present in the incident molecular beam, the time of flight spectra for $v=3 \rightarrow 1$ scattering were recorded twice, with and without the IR laser. The scan without IR excitation ( $v=1 \rightarrow 1$ scattering) was used for baseline correction of the $v=3 \rightarrow 1$ data. The data was fitted using forward convolution taking estimated angular distributions [26] and the measured speed distribution of the incident molecular beam into account to determine the final translational energy distributions (see Appendix $C$ for details).
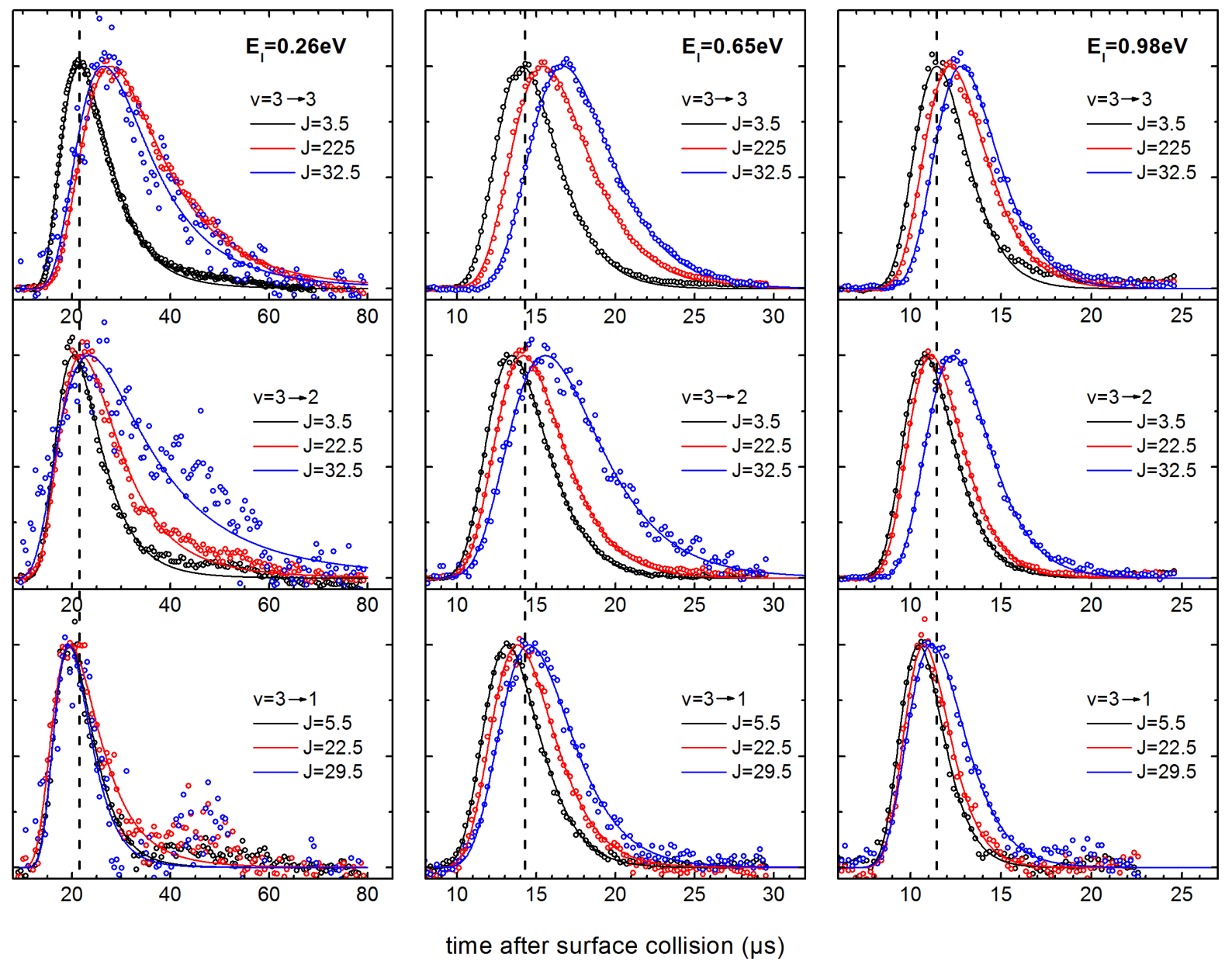

Fig. 3.26: Arrival time distributions for $\mathrm{NO}\left(v_{i}=3, J_{f}=1.5\right)$ scattering into $v_{f}=1,2,3$ and $J_{f}=3.5,5.5,22.5,29.5,32.5$ at $T_{S}=320 \mathrm{~K}$ and incidence energies of $0.26,0.65$ and $0.98 \mathrm{eV}$. The colors indicate low rotational excitation $\left(\mathrm{black}, J_{f}=3.5 / 5.5\right)$, final rotational excitation of $E_{\text {rot }}=0.109 \mathrm{eV}$ (red, $J_{f}=22.5$ ) and high rotational excitation of $E_{\text {rot }}=0.189 / 0.226 \mathrm{eV}$ (blue, $J_{f}=29.5 / 32.5$ ). The second peak around $50 \mu \mathrm{s}$ for $v=3 \rightarrow 1$ scattering at $E_{I}=0.26 \mathrm{eV}$ (lower left panel) is attributed to an artifact from the background correction due to low S/N under these conditions [121] - Published by the PCCP Owner Societies. 

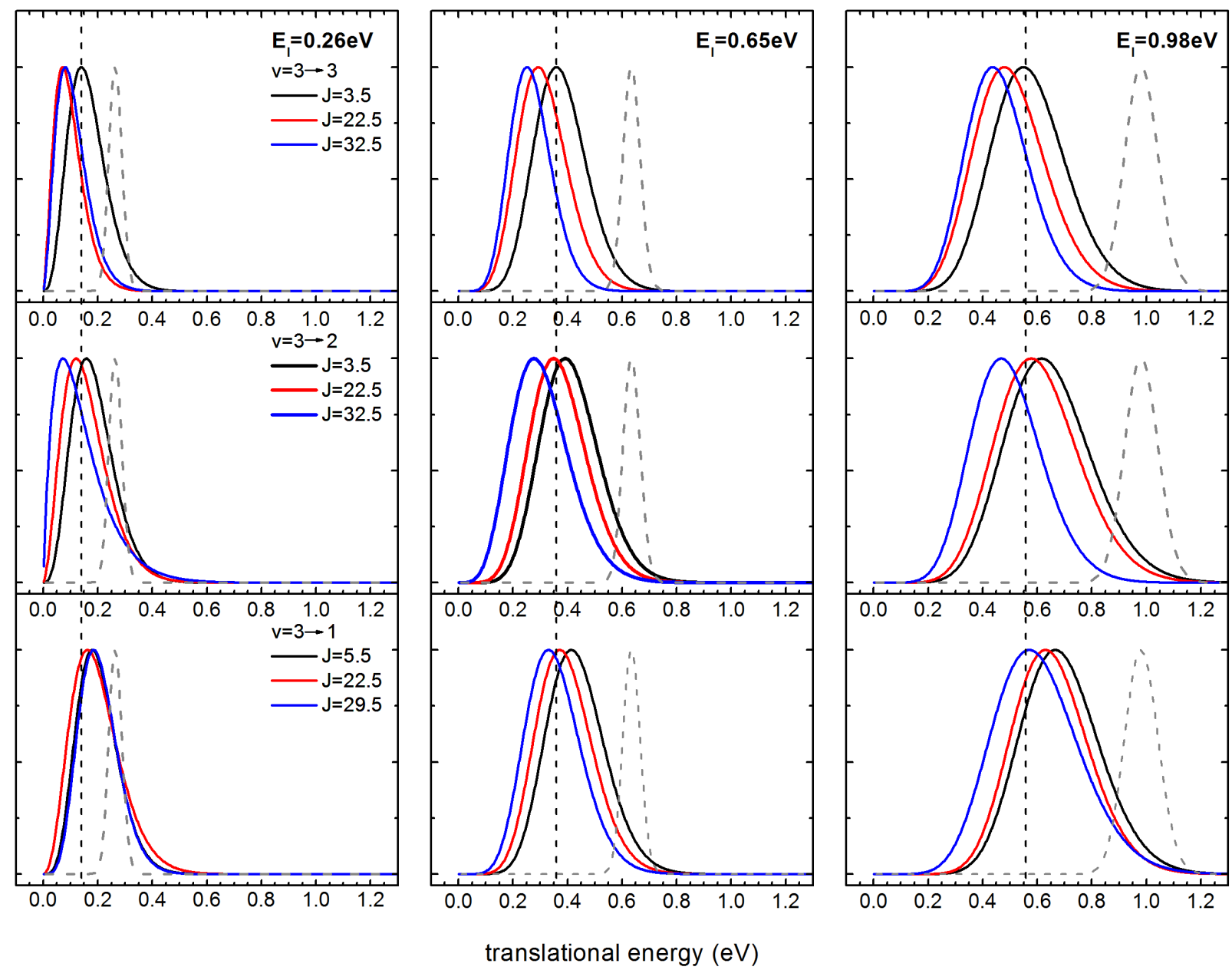

Fig. 3.27: Final translational energy distributions converted from the fits shown in fig. 3.26 using the identical color code. The gray dashed lines show the translational energy distribution of the incident molecular beam. The vertical black dashed lines are drawn through the peaks of the distributions for vibrationally elastic and rotationally quasi-elastic scattering $\left(v_{f}=3, J_{f}=3.5\right.$, 5.5) to guide the eye [121] - Published by the PCCP Owner Societies.

The black traces show rotationally quasi-elastic $\left(E_{r o t} \leq 1 \mathrm{meV}\right)$ scattering into $v_{f}=1,2,3$. Molecules that underwent vibrational relaxation arrive (on average) slightly earlier at the detection laser, which is in agreement with the observations of Fig. 3.23. The vertical dashed lines are drawn through the peaks of the traces for vibrationally elastic and rotationally quasi-elastic scattering into $\mathrm{NO}\left(v_{f}=3, J_{f}=3.5\right)$ to illustrate this observation. A new feature is the effect of rotational inelasticity on the arrival time distribution. The rotational energies cover a range from $E_{r o t} \leq 1 \mathrm{meV}\left(U_{f}=3.5,5.5\right)$ up to $E_{\text {rot }}=226 \mathrm{meV}\left(U_{f}=32.5\right)$. In general, the peak of the arrival time distributions shifts toward later times with increasing final rotational quantum number $J_{f}$.

For a better comparison, Fig. 3.27 shows the time-of-flight fits of Fig. 3.26 converted into energy space (see Appendix B for details). The tendencies that were visible in the raw experimental data are retained in the kinetic energy distributions. In agreement with the results of Fig. 3.24, vibrational relaxation leads to an increase in final translational energy which depends on $\Delta E_{v i b}$. Loss of two vibrational quanta increases $E_{f}$ approximately twice as much as loss of only one quantum of vibrational energy. The data of Fig. 3.27 further allows studying the effect of incidence translational energy on the vibration-to- 
translation $(\mathrm{V} \rightarrow \mathrm{T})$ energy transfer. The comparison of the three different incidence energies shown exhibits a decrease in $\mathrm{V} \rightarrow \mathrm{T}$ energy transfer with decreasing $E_{I}$.

Rotational excitation leads to a decrease in $E_{f}$ whereas the absolute loss of translational energy depends on $E_{I}$ as well as on $\Delta v$. In case of $v_{f}=3$, the peak of the distribution shifts by $0.11 \mathrm{eV}$ from $J_{f}=3.5$ to $J_{f}=32.5$ for $E_{I}=0.98 \mathrm{eV}$ but by only $0.06 \mathrm{eV}$ for $E_{I}=0.26 \mathrm{eV}$. The effect of $\Delta v$ on the (anti-)correlation between $E_{f}$ and $E_{\text {rot }}$ is most clearly seen for $v=3 \rightarrow 1$ scattering at $E_{I}=0.26 \mathrm{eV}$. Here, an increasing amount of rotational excitation does almost not affect the translational energy distributions of the scattered $\mathrm{NO}\left(v_{f}=1\right)$ molecules. At the higher incidence energies of $0.65 \mathrm{eV}$ and $0.98 \mathrm{eV}$, this effect is not clearly visible and all three vibrational scattering channels show similar dependences of $E_{f}$ on $E_{\text {rot }}$.

A further investigation of the effect of incidence energy of translation on $\Delta E_{f} / \Delta E_{v i b}$ and $\Delta E_{f} / \Delta E_{\text {rot }}$ requires again a reduction of the experimental $E_{f}$ distributions to only the mean final translational energy, $\left\langle E_{f}\right\rangle$. Fig. 3.28 shows the mean recoil energies $\left\langle E_{f}\right\rangle$ for six different incidence energies between $E_{I}=0.26 \mathrm{eV}$ and $E_{I}=0.98 \mathrm{eV}$. The colors indicate vibrationally elastic $v=3 \rightarrow 3$ (black) and vibrationally inelastic $3 \rightarrow 2$ (green) and $3 \rightarrow 1$ (blue) scattering. The final translational energy decreases approximately linearly with increasing rotational excitation for the rotational energies studied. Furthermore, molecules that were scattered into $v_{f}=1,2$ are shifted towards higher $\left\langle E_{f}\right\rangle$ while $v_{f}=1$ recoils at higher energy than $v_{f}=2$. The values for $\left\langle E_{f}\right\rangle$ at $E_{\text {rot }}=0$ and for $\Delta E_{f} / \Delta E_{\text {rot }}$ obtained from the linear fits are listed in Table 3.4.

For all three vibrational scattering channels, the linear dependence of $\left\langle E_{f}\right\rangle$ on $E_{\text {rot }}$ is similar at high incidence energies $E_{I} \geq 0.64 \mathrm{eV}$. For $E_{I}<0.64 \mathrm{eV}, \Delta\left\langle E_{f}\right\rangle / \Delta E_{\text {rot }}$ increases (approaches 0 ) much more rapidly for vibrationally inelastic scattering compared to vibrationally elastic $3 \rightarrow 3$ scattering. This dependence is more pronounced for the loss of two vibrational quanta $(3 \rightarrow 1)$ than for loss of only one quantum of vibrational energy $(3 \rightarrow 2)$. The $3 \rightarrow 1$ channels even reaches the limit at which the outgoing translational energy becomes independent of final rotational excitation $\left(\Delta\left\langle E_{f}\right\rangle / \Delta E_{r o t} \rightarrow 0\right)$.

Table 3.4: Dependence $\Delta\left\langle E_{f}\right\rangle / \Delta E_{\text {rot }}$ of mean final translational energy $\left\langle E_{f}\right\rangle$ on final rotational excitation $E_{\text {rot }}$ as a function of incidence energy $E_{I}$ for vibrationally elastic $v=3 \rightarrow 3$ and inelastic $v=3 \rightarrow 2$ and $v=3 \rightarrow 1$ scattering. The table further shows the final translational energy extrapolated to $E_{\text {rot }}=0$. All values were obtained from the linear fits shown in Fig. 3.28.

\begin{tabular}{|c|c|c|c|c|c|c|}
\hline$E_{I} / \mathrm{eV}$ & $\Delta\left\langle E_{f}\right\rangle / \Delta E_{\text {rot }}$ & $\begin{array}{c}\longrightarrow 3 \\
\left\langle E_{f}\right\rangle \\
\text { extrapolated } \\
\text { to } E_{r o t}=0 \\
/ \text { eV }\end{array}$ & $\Delta\left\langle E_{f}\right\rangle / \Delta E_{r o t}$ & $\begin{array}{c}\left\langle E_{f}\right\rangle \\
\text { extrapolated } \\
\text { to } E_{r o t}=0 \\
/ \text { eV }\end{array}$ & $\Delta\left\langle E_{f}\right\rangle / \Delta E_{r o t}$ & $\begin{array}{c}\mathbf{3} \rightarrow \mathbf{1} \\
\left\langle E_{f}\right\rangle \\
\text { extrapolated } \\
\text { to } E_{\text {rot }}=0 \\
\quad / \mathrm{eV}\end{array}$ \\
\hline 0.1 & $-0.21 \pm 0.14$ & $0.072 \pm 0.008$ & $-0.14 \pm 0.14$ & $0.082 \pm 0.006$ & $-0.07 \pm 0.57$ & $0.072 \pm 0.018$ \\
\hline 0.26 & $-0.34 \pm 0.12$ & $0.163 \pm 0.013$ & $-0.19 \pm 0.06$ & $0.184 \pm 0.006$ & $0.09 \pm 0.10$ & $0.190 \pm 0.011$ \\
\hline 0.41 & $-0.43 \pm 0.07$ & $0.228 \pm 0.0$ & $-0.19 \pm 0.15$ & $0.246 \pm 0.010$ & $0.05 \pm 0.26$ & $0.254 \pm 0.017$ \\
\hline 0.48 & $-0.57 \pm 0.05$ & $0.315 \pm 0.006$ & $-0.41 \pm 0.05$ & $0.342 \pm 0.006$ & $-0.32 \pm 0.13$ & $0.364 \pm 0.014$ \\
\hline 0.52 & $-0.51 \pm 0.08$ & $0.317 \pm 0.009$ & $-0.44 \pm 0.08$ & $0.348 \pm 0.009$ & $-0.39 \pm 0.11$ & $0.369 \pm 0.011$ \\
\hline 0.58 & $-0.55 \pm 0.05$ & $0.376 \pm 0.005$ & $-0.52 \pm 0.07$ & $0.413 \pm 0.007$ & $-0.47 \pm 0.15$ & $0.446 \pm 0.016$ \\
\hline 0.65 & $-0.53 \pm 0.05$ & $0.383 \pm 0.005$ & $-0.48 \pm 0.05$ & $0.416 \pm 0.005$ & $-0.52 \pm 0.13$ & $0.450 \pm 0.014$ \\
\hline 0.84 & $-0.56 \pm 0.08$ & $0.492 \pm 0.010$ & $-0.58 \pm 0.08$ & $0.540 \pm 0.009$ & $-0.57 \pm 0.16$ & $0.578 \pm 0.018$ \\
\hline 0.98 & $-0.55 \pm 0.11$ & $0.579 \pm 0.012$ & $-0.68 \pm 0.12$ & $0.662 \pm 0.013$ & $-0.59 \pm 0.20$ & $0.703 \pm 0.021$ \\
\hline
\end{tabular}




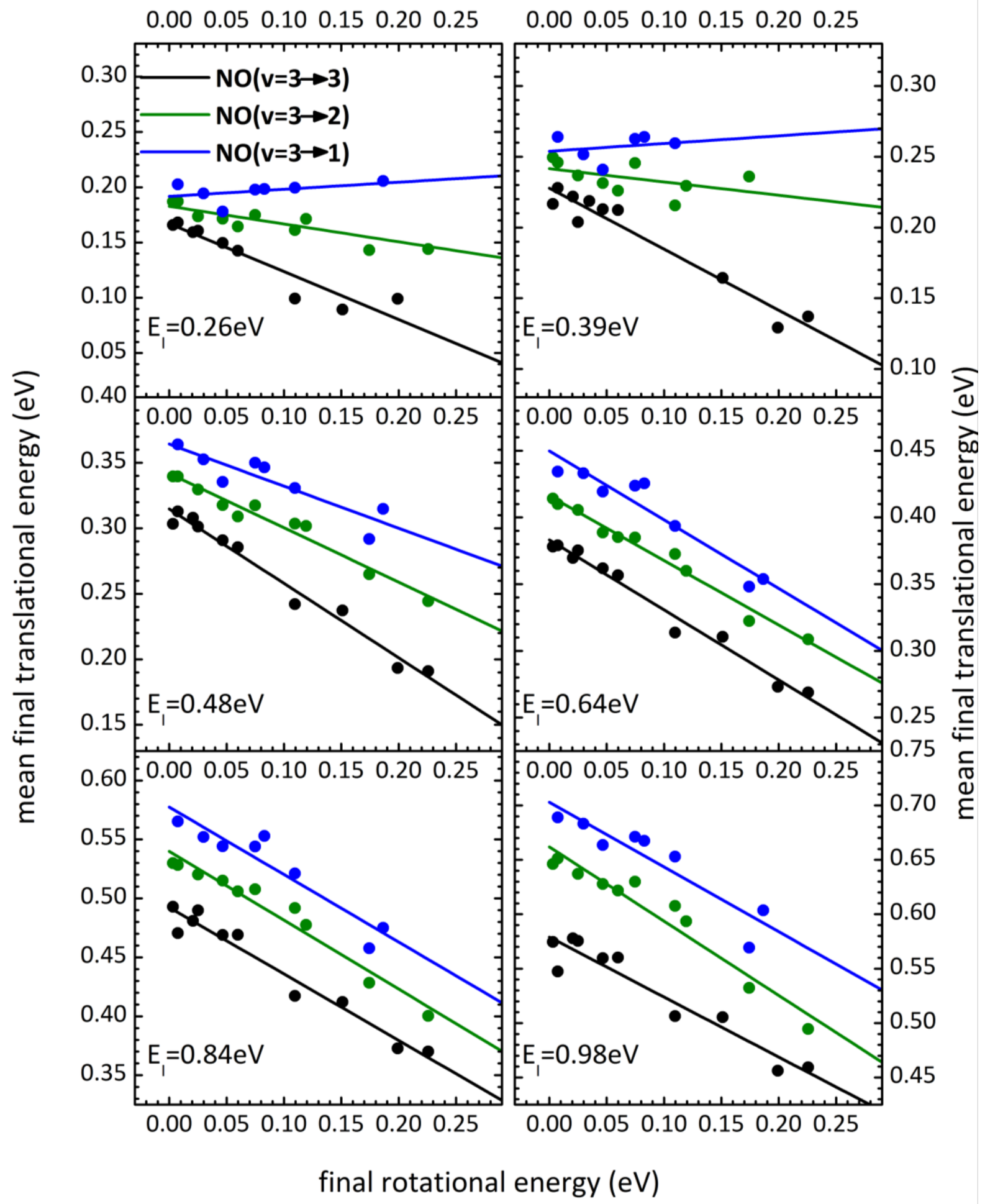

Fig. 3.28: Mean final translational energy as a function of final rotational energy for $E_{I}=0.2-0.98 \mathrm{eV}$ at $T_{S}=320 \mathrm{~K}$ for vibrationally elastic $3 \rightarrow 3$ (black) and vibrationally inelastic $3 \rightarrow 2$ (green), $3 \rightarrow 1$ (blue) scattering. The dependence of $\left\langle E_{f}\right\rangle$ on $E_{\text {rot }}$ is approximately linear at all incidence energies studied. At $E_{I} \geq 0.64 \mathrm{eV}$ the dependences for all vibrationally channels are similar within the scatter of the data points. At lower incidence energy $\Delta\left\langle E_{f}\right\rangle / \Delta E_{\text {rot }}$ approaches zero for all channels but the transition is more abrupt for vibrationally inelastic scattering [121] - Published by the PCCP Owner Societies. 
Furthermore, the amount of vibrational energy that is channeled into final translation decreases from about $73 \mathrm{meV}$ per vibrational quantum ( $30 \%$ of $\Delta E_{\text {vib }}$ ) at $E_{I}=0.98 \mathrm{eV}$ to $16 \mathrm{meV}$ per vibrational quantum ( $7 \%$ of $\Delta E_{v i b}$ ) at $E_{I}=0.26 \mathrm{eV}$. This $E_{I}$ dependence is in contrast to a previous study on $\mathrm{HCl}(v=2 \rightarrow 1,2) / \mathrm{Au}(111)$ scattering where a constant fraction of $26 \%$ of $\Delta E_{v i b}$ was found to be released into final translation for all incidence energies ranging from $0.28-1.27 \mathrm{eV}$.

\subsubsection{Discussion}

The previous section showed that the scattered translational energy $\left(E_{f}\right)$ depends strongly on the final quantum state of the molecule $\left(v_{f}, J_{f}\right)$ as well as on the experimental conditions $\left(E_{I}, T_{S}\right)$. It is not surprising that higher incidence energies in general lead to higher $E_{f}$. The fraction of $E_{I}$ that is lost to the surface bath $(S)$ depends in the temperature of the surface in a way that $E_{f}$ increases with increasing $T_{S}$. Rotational excitation causes a decrease in translational energy of the scattered molecules, an effect that was attributed to translational-to-rotation $(T \rightarrow R)$ energy transfer previously $[9,13,80,81]$. A completely new feature of the $\mathrm{NO}\left(v_{i}=3\right) / \mathrm{Au}(111)$ system is the observation of gain/ loss in translational energy upon vibrational relaxation/ excitation, thus vibration-translation $(T \leftrightarrow V)$ coupling. Although vibrational energy change has been investigated in great detail, T-V interactions have been neglected or thought to be negligible so far.

In the following sections I will discuss the different possible mechanisms for energy transfer between the different degrees of freedom. In addition, I will investigate the relations between all possible pathways.

\section{Energy transfer to the solid and thermal broadening}

The translational energy transfer to the solid can be estimated by extrapolation of the mean final translational energy shown in Fig. 3.25 and Fig. 3.28 to $E_{\text {rot }}=0$. This is shown for vibrationally elastic $\mathrm{NO}(v=3 \rightarrow 3)$ scattering for the peak of the translational energy distribution $E_{f}^{M P}$ (circles) and the mean final translational energy $\left\langle E_{f}\right\rangle$ (squares) in Fig. 3.29. The red dotted line shows the expected translational energy for complete equilibration with the surface temperature $\left(T_{S}=320 \mathrm{~K}\right)$. The red dashed line corresponds to the prediction of a simple hard cube model taking only conservation of energy and momentum in a collision of two spheres with masses of $\mathrm{NO}\left(m_{N O}=30 \mathrm{amu}\right)$ and $\mathrm{Au}\left(m_{A u}=197 \mathrm{amu}\right)$ into account. This model is often referred to as the Baule limit [122]. The final translational energy for collision of a moving NO molecule with a non-moving $A u$ atom is then given by $E_{f}=E_{I}\left(m_{A u}-m_{N O}\right)^{2} /\left(m_{A u}+m_{N O}\right)^{2}=0.54 \times E_{I}$. Because this model does not contain any internal excitation of the molecule only a comparison of the prediction to vibrationally elastic $\left(v_{f}=3\right)$ scattering is reasonable. The extrapolated values for $E_{\text {rot }}=0$ of the $v=3 \rightarrow 3$ data are shown as open symbols in Fig. 3.29. The error bars indicate $90 \%$ confidence intervals of the linear fit to the experimental data. The agreement between the data and the simple Baule limit is quite striking. It shows that the scattered molecules "remember" the incidence translational energy. The results for $\left\langle E_{f}\right\rangle$ are systematically above the predicted limit while the values of $E_{f}^{M P}$ are in better agreement. In any case, the observed final translational energies clearly exceed the thermal expectation, even at the lowest incidence energy, which indicates a direct scattering mechanism.

A remaining issue is the broadening of the scattered translational energy distributions with respect to the incident beam (see Fig. 3.27), which increases with increasing surface temperature. This dependence on surface temperature might indicate that the broadening is due to the thermal motion of the surface atoms that increases with increasing $T_{S}$. The simplest way to incorporate this movement in a model is to drop the assumption of a non-moving Au atom in the Baule model. In this thermal hard cube model, the 
interaction is still described by a hard cube potential, thus taking only conservation of energy and momentum into account at zero impact parameter. The thermal motion of the surface atom is now modeled by a 1D Maxwell-Boltzmann speed distribution:

$$
F_{\text {thermal }}\left(s_{A u}, T_{S}\right) d s_{A u}=\sqrt{\frac{m_{A u}}{2 \pi k_{B} T_{S}}} e^{-\frac{m_{A u} s_{A u}^{2}}{2 k_{B} T_{S}}} d s_{A u}
$$

where $m_{A u}$ is the mass and $s_{A u}$ is the speed of a single Au atom. Furthermore one has to take into account that collisions with surface atoms moving towards the impingent NO molecules have higher probability than those with both collision partners moving in the same direction. This can be taken into account by a collision probability $P_{\text {coll }}=\left(s_{N O, i}-s_{A u, i}\right) / 2 s_{N O, i}$ [123]. The final speed distribution of the scattered $\varphi_{f}(s)$ molecules is then given by a multiplication of $F_{\text {thermal }}\left(s_{A u}\right) d s_{A u}$ and the speed distribution $\varphi_{i}\left(s_{N O}\right)=A \times s^{3} \exp \left[-\left(s_{N O}-s_{0}\right)^{2} / \alpha_{0}{ }^{2}\right]$ of the incident NO molecules and integrating over incident velocity of the impingent molecules.

$$
\varphi_{f}\left(s_{N O}, T_{S}\right)=\int P_{\text {coll }} \times F_{\text {thermal }}\left(s_{A u, i}, T_{S}\right) \times \varphi_{i}\left(s_{N O, i}\right) d s_{N O, i}
$$

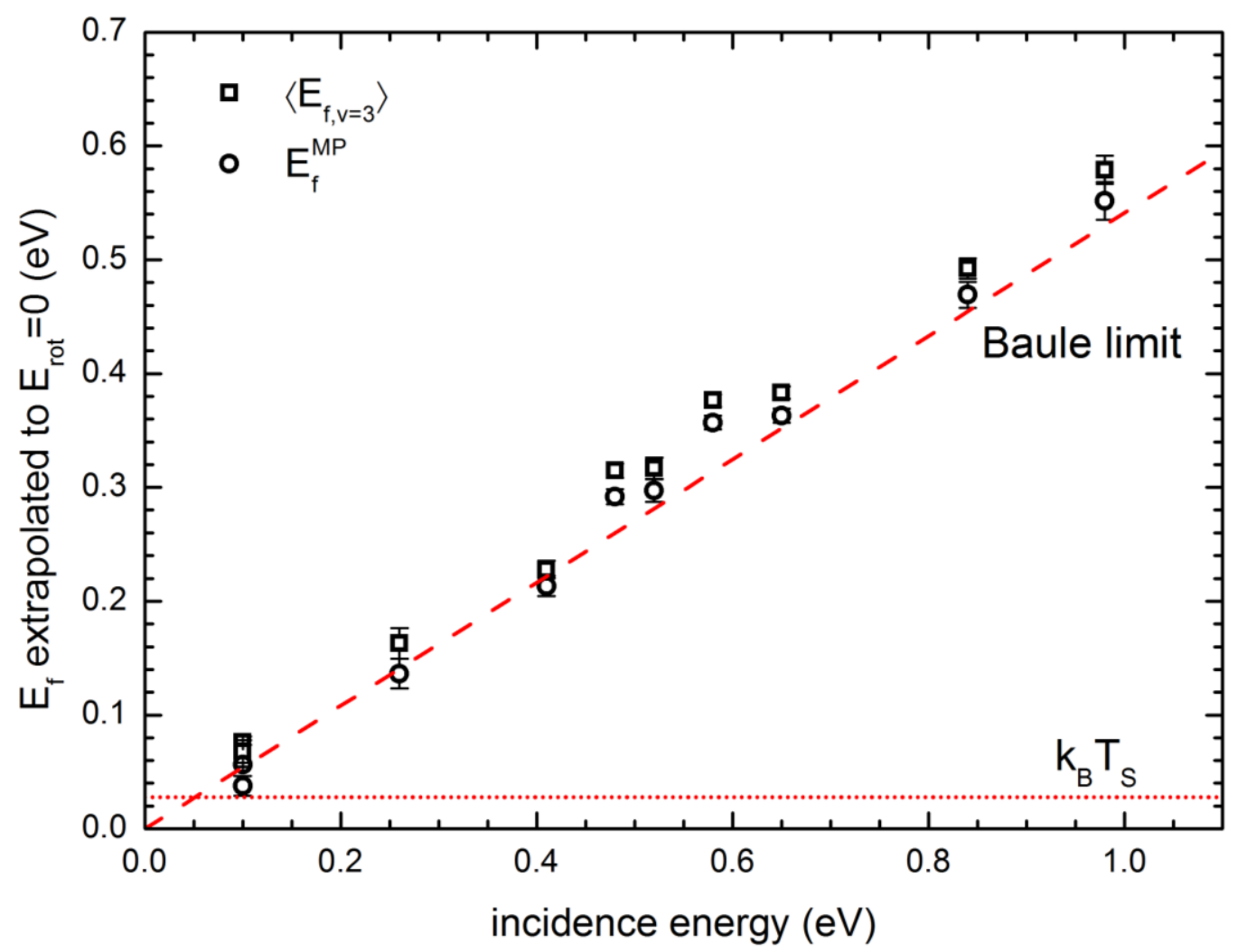

Fig. 3.29: Comparison of the $E_{f}^{M P}$ (circles) and $\left\langle E_{f}\right\rangle$ (squares) values for vibrationally elastic $v=3 \rightarrow 3$ and rotationally elastic scattering of $\mathrm{NO}\left(v_{i}=3\right)$ from $\mathrm{Au}(111)$. The data points were obtained from linear extrapolation of rotational energy dependences like those shown in Fig. 3.28 to $E_{\text {rot }}=0$. The red dashed line shows the expectation from a simple hard cube model while the red dotted line indicates the expectation for equilibration with the surface temperature [121] - Published by the PCCP Owner Societies. 
where $s_{A u, i}$ by can be replaced by applying the laws for conservation of energy and momentum:

$$
s_{A u, i}=\frac{m_{A u} \cdot s_{N O, i}-m_{N O} \cdot s_{N O, i}-m_{A u} \cdot s_{N O}-m_{N O} \cdot s_{N O}}{2 \cdot m_{A u}}
$$

where $s_{N O, i}$ and $s_{N O}$ are the initial and final speed of the NO molecule and $s_{A u, i}$ is the initial speed of the Au atom. $\varphi_{f}\left(s_{N O}, T_{S}\right)$ can be converted into energy space using the procedure described in Appendix $\mathrm{B}$. The results of the thermal Baule model are shown as red lines in the right panel of Fig. 3.30. The translational energy distributions observed in the experiment $(v=2 \rightarrow 2)$ are shown as black dots for comparison. The result shows that the simple mechanical model with thermal motion of the surface atom leads to a clear broadening of the predicted translational energy distribution; the broadening increases with increasing surface temperature. The agreement with the measured kinetic energy distribution is best on the low energy edge for $T_{S}=470 \mathrm{~K}$ as well as at the high energy edge for $T_{S}=973 \mathrm{~K}$. The deviations are possibly related to the assumptions of this still very simple model. First, the description of the surface by a single Au atom is artificial even if collisions at the top sites would dominate the scattering event. Second, the real system cannot be described by a hard cube potential but has an attractive well for the $\mathrm{N}$-atom pointing toward the surface $[65,73,74]$, which leads to an acceleration prior to the collision. With respect to the simplicity of the thermal Baule model, the observed overall agreement with the experimental data is quite satisfying.
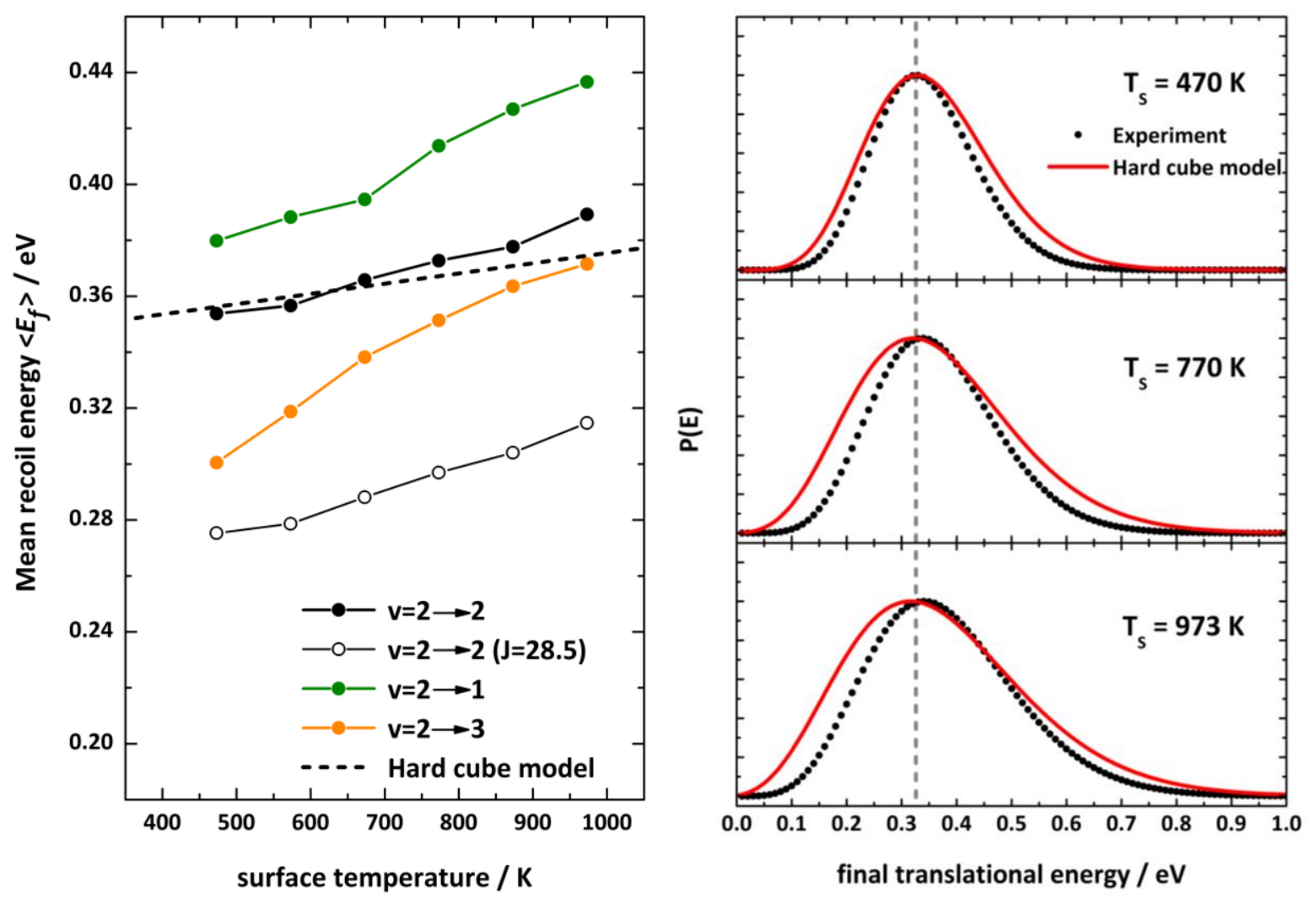

Fig. 3.30: Left panel: mean final translational energy as a function of surface temperature for vibrationally elastic $(2 \rightarrow 2)$ and inelastic $(2 \rightarrow 1,2 \rightarrow 3)$ scattering. The dashed line indicates the prediction of the thermal Baule limit. The black open symbols show vibrationally elastic but rotationally inelastic scattering. Right panel: Comparison of the experimentally observed $P\left(E_{f}\right)$ for vibrationally elastic $2 \rightarrow 2$ scattering (black dots) to hard cube model with thermal motion of the surface atom (red lines) at different surface temperatures. Reprinted with permission from Golibrzuch et al., Journal of Physical Chemistry A, 2013. 117(36), 8750-8760. Copyright 2013 American Chemical Society. 
The left panel of Fig. 3.30 shows the $T_{S}$ dependence of $\left\langle E_{f}\right\rangle$ for vibrationally elastic (black solid symbols) and inelastic scattering with loss (green) and gain (yellow) of one vibrational quantum. In addition, vibrationally elastic but rotationally inelastic scattering $\left(U_{f}=28.5\right)$ is shown as black open symbols. The thermal Baule model (black dashed line) predicts a linear increase of $\left\langle E_{f}\right\rangle$ with increasing surface temperature. The only reasonable channel to compare to this model is vibrationally elastic and rotationally quasi-elastic scattering (low $J_{f}$, black solid symbols). The experimental data also shows an approximately linear increase with increasing $T_{S}$ and agrees within $\pm 10 \mathrm{meV}$ with the thermal Baule model. However, the dependence $\Delta\left\langle E_{f}\right\rangle / \Delta T_{S}$ is stronger than predicted. This leads to an overestimation of $\left\langle E_{f}\right\rangle$ at $T_{S}<700 \mathrm{~K}$ and an underestimation of $\left\langle E_{f}\right\rangle$ at $T_{S}>700 \mathrm{~K}$ by the model and is further consistent with the agreement at the high and low energy edges of the $E_{f}$ distributions at different surface temperatures noted above.

Furthermore, Fig. 3.30 shows that rotational excitation does not significantly affect the $T_{S}$ dependence of $\left\langle E_{f}\right\rangle$. The curve for $J_{f}=28.5\left(E_{\text {rot }}=0.19 \mathrm{eV}\right)$ exhibits a similar linear dependence as in case of $J_{f}=3.5$ but is shifted toward lower energies by about $0.11 \mathrm{eV}$. The situation is different for vibrationally inelastic scattering: the increase of final translational energy with increasing surface temperature is about twice as strong as in case of vibrationally elastic scattering. This also affects the influence of vibrational inelasticity on $\left\langle E_{f}\right\rangle$. The kinetic energy gained upon vibrational relaxation increases from $0.03 \mathrm{eV}$ at $470 \mathrm{~K}$ to $0.05 \mathrm{eV}$ at $970 \mathrm{~K}$. At the same time, the loss in translational energy upon vibrational excitation decreases from $0.05 \mathrm{eV}$ at $T_{S}=470 \mathrm{~K}$ to $0.015 \mathrm{eV}$ at $970 \mathrm{~K}$. This observation of a $T_{S}$ dependent coupling between translation and vibration has to be kept in mind in the further discussion of the results. A possible explanation for different $\Delta\left\langle E_{f}\right\rangle / \Delta T_{S}$ might be that vibrationally inelastic scattering samples predominantly those collisions for which the Au atom moves towards the impingent NO molecule. Such collisions have higher effective collision energies than those for which the Au atom is moving in the same direction as the NO molecules. Sections 3.1 and 3.2 as well as reference [1] already showed that the nonadiabatic interaction and therefore the excitation/ relaxation probability increases with increasing incidence energy. Therefore, it is likely that vibrationally inelastic scattering favorably occurs in those collisions with the Au atom moving out of the surface.

According to the observed different $T_{S}$ dependences for $E_{f}$ in vibrationally elastic and inelastic scattering, the mean energies shown in Fig. 3.25 have to be corrected to the same surface temperature for a reasonable comparison. Fig. 3.31 shows the results if all vibrational channels of Fig. 3.25 are shifted towards $T_{S}=570 \mathrm{~K}$ and equal (linear) $\Delta\left\langle E_{f}\right\rangle / \Delta T_{S}$ is assumed for $\Delta v= \pm 2$ and $\Delta v= \pm 1$. 


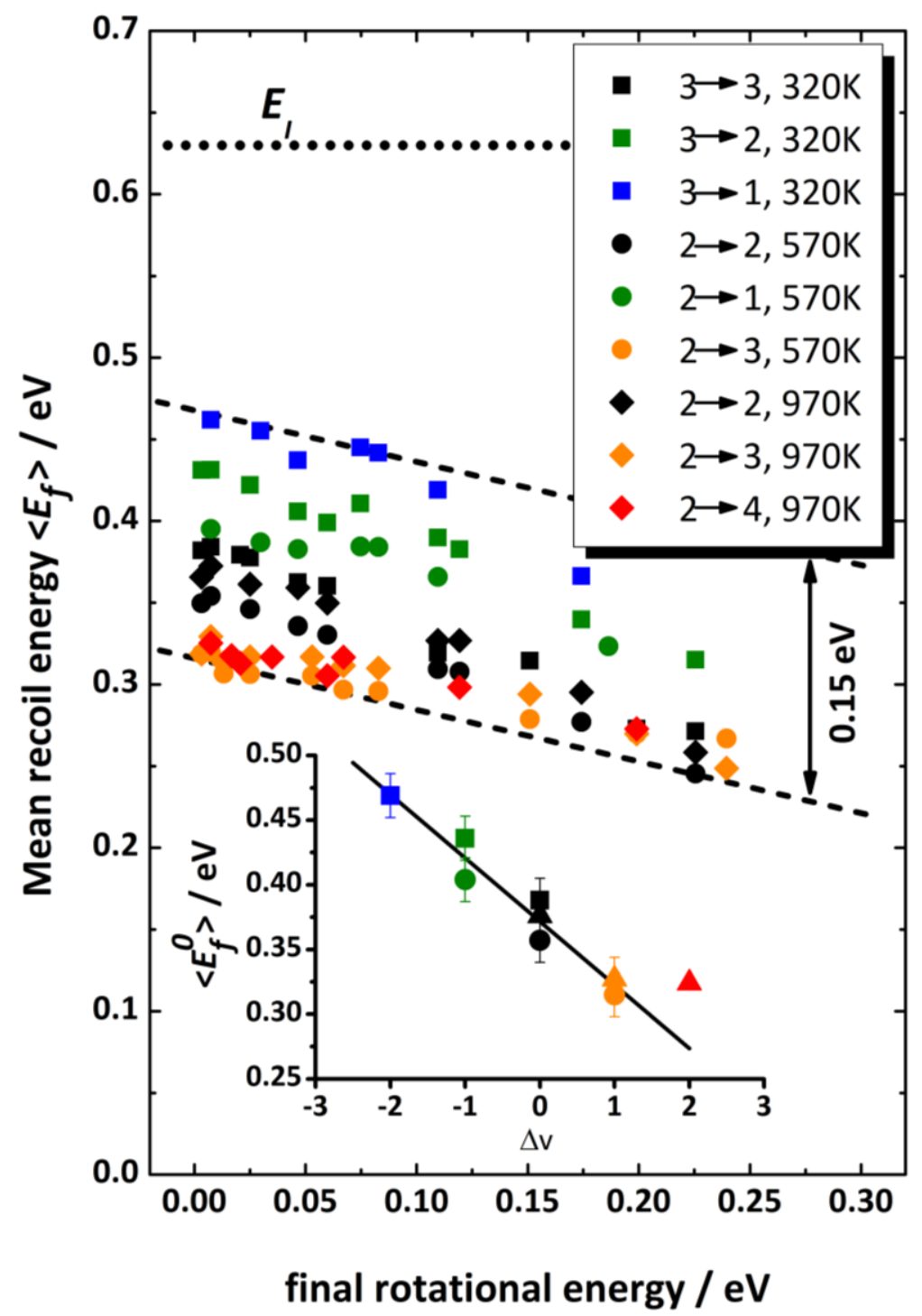

Fig. 3.31: Surface temperature corrected mean recoil energies for vibrationally elastic and inelastic scattering as function of final rotational energy. The data of Fig. 3.28 was corrected to $T_{S}=570 \mathrm{~K}$ using the dependences shown in Fig. 3.30. The dotted line indicates the mean incidence energy. Although the vibrational scattering channels of the data cover a range of $-0.47 \leq$ $\Delta E_{\text {vib }} \leq+0.47 \mathrm{eV}$ the observed translational energy covers only a range of $0.13 \mathrm{eV}$ (dashed lines). The inset shows the $T_{S}$ corrected recoil energies extrapolated to $E_{\text {rot }}=0$ as a function $\Delta v$. A linear fit yields a slope of $\Delta\left\langle E_{f}\right\rangle / \Delta v=-49 \mathrm{meV}$ per vibrational quantum ( $\Delta v=2$ was excluded from the fit). Reprinted with permission from Golibrzuch et al., Journal of Physical Chemistry A, 2013. 117(36), 8750-8760. Copyright 2013 American Chemical Society.

\section{Energy transfer between translation and vibration}

The new unknown feature of this work is the energy transfer between translation and vibration $(\mathrm{T} \leftrightarrow \mathrm{V})$. While vibrational energy transfer at metal surfaces has been extensively studied over the last decades in the sense of vibrational state distributions $[1,3,4,8,27,29,30,34,44,47,48,50,60,88,107,117,124]$, only little is known about how vibrational energy transfer influences other degrees of freedom, for example translational motion. Especially at metal surfaces, where nonadiabatic coupling of the molecular vibration to electron-hole-pairs of the metal (V-EHP) is known to be important, translational motion is often believed to be a spectator that can influence the V-EHP coupling strength but is not 
directly coupled to vibration. The results of Fig. 3.23-3.28 and Fig. 3.31 clearly show that this view can only be true to a first approximation. Vibrational de-excitation of incident $\mathrm{NO}\left(v_{i}=2,3\right)$ molecules leads to an increase in final translational energy while vibrational excitation results in a decrease of $E_{f}$. Moreover, the amount of $\mathrm{T} \leftrightarrow \mathrm{V}$ energy transfer seems to depend on the temperature of the surface as well as on incidence translational energy. At $E_{I}=0.65 \mathrm{eV}$, the surface temperature corrected data (Fig. 3.31) shows that the final translational energy only varies by $\sim 0.13 \mathrm{eV}$ (dashed lines) for a vibrational energy range of $-0.47 \mathrm{eV} \leq \Delta E_{v i b} \leq 0.47 \mathrm{eV}$. This is only $14 \%$ of the vibrational energy change and verifies the approximate spectatorship of molecular translation in nonadiabatic NO interactions with a metal surface, at least at this specific incidence energy.

The $E_{I}$ dependence of $\mathrm{V} \rightarrow \mathrm{T}$ energy transfer for $\mathrm{NO}(v=3 \rightarrow 1,2,3)$ scattering is shown in Fig. 3.32. The difference in translational energy between $v_{f}=3$ and $v_{f}=1,2$ increases linearly with increasing $E_{I}$. At low incidence energies, vibrational relaxation leads to increase of less than $10 \mathrm{meV}\left(<4 \%\right.$ of $\left.\Delta \mathrm{E}_{v i b}\right)$. At the highest $E_{I}=0.98 \mathrm{eV}$, the recoil energy increases by 80 meV for $v=3 \rightarrow 2$ scattering $\left(\sim 34 \%\right.$ of $\left.\Delta \mathrm{E}_{v i b}\right)$ and $120 \mathrm{meV}$ for $v=3 \rightarrow 1$ scattering $\left(\sim 25 \%\right.$ of $\left.\Delta \mathrm{E}_{v i b}\right)$. This result is in contrast to previously reported vibration-to-translation coupling in $\mathrm{HCl}(v=2 \rightarrow 1) / \mathrm{Au}(111)$ [32] scattering which is the only comparative study available. Independent of $E_{I}$, the authors observed a constant fraction of $\sim 26 \%$ of $\Delta E_{v i b}$ that was transferred into final translational energy upon vibrational relaxation. However, the $\mathrm{HCl} / \mathrm{Au}(111)$ system is much less understood than $\mathrm{NO} / \mathrm{Au}(111)$ scattering and no theoretical predictions are available so far. The authors suggested several possible mechanisms but were not able to make final conclusions which one is present for $\mathrm{HCl} / \mathrm{Au}(111)$.

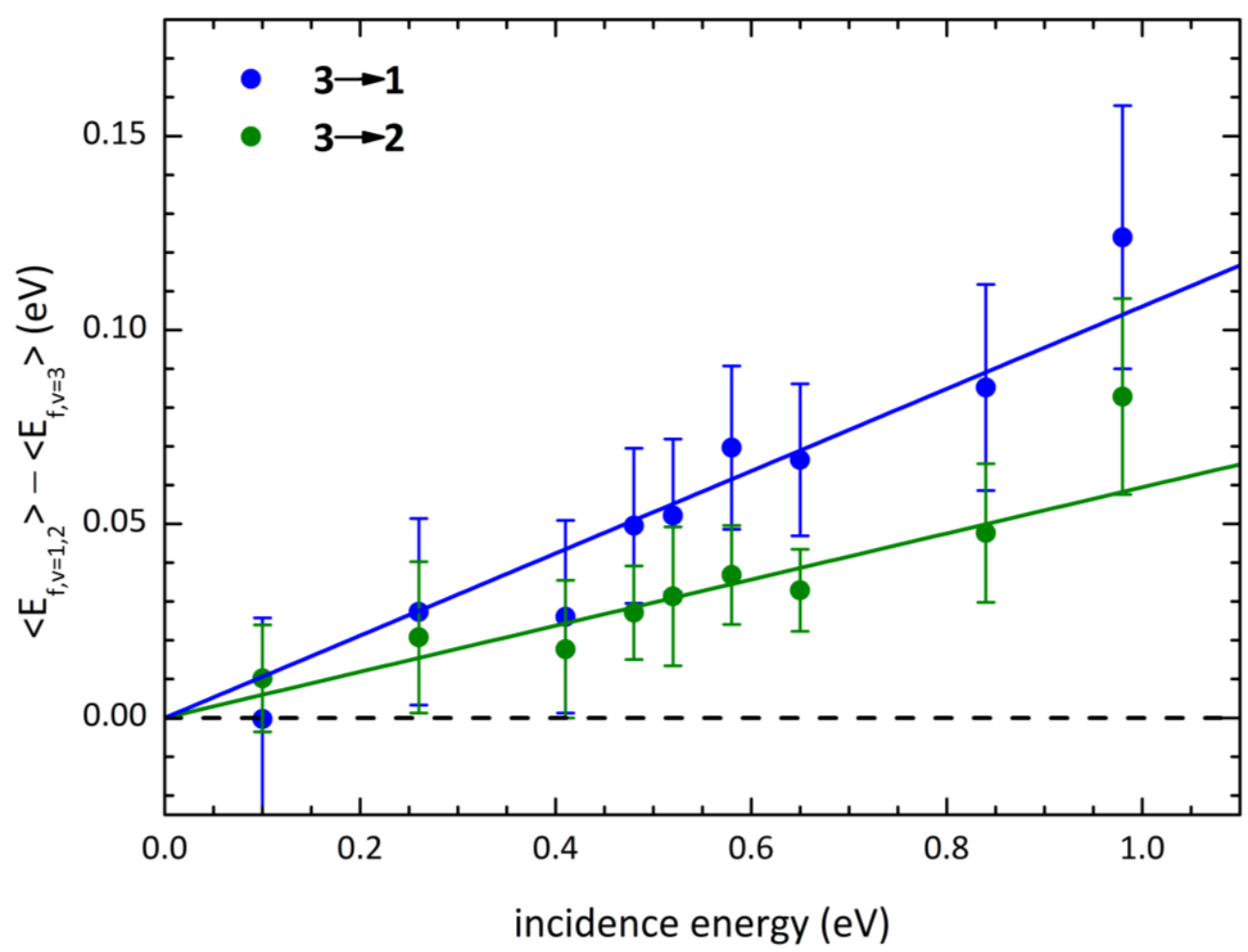

Fig. 3.32: Vibrational energy transfer to final translation for vibrationally inelastic $3 \rightarrow 2$ (red) and $3 \rightarrow 1$ (blue) scattering as function of incident kinetic energy. The values were obtained from linear extrapolation of the data shown in Fig. 3.28 to $E_{\text {rot }}=0$ and taking the difference to the vibrationally elastic $3 \rightarrow 3$ channel. Both channels show an approximately linear dependence on $E_{I}$ but loss of two vibrational quanta in general leads to higher recoil energies [121] - Published by the PCCP Owner Societies. 
In this context, I would like to note the advantages of the state-to-state time-of-flight technique. It maps out the translational energy distributions of for scattering into specific vibrational and rotational quantum states. Therefore, it enables deep insights into the dynamics of a system. However, the detail of information has the drawback that the results are often hard to explain only with the help of experimental data. A careful interpretation requires advanced theoretical models that cover all features of the system. In case of vibrationally inelastic NO/Au(111) collisions adiabatic models, like they were used for NO/Ag(111) [9], will fail to describe the dynamics of the system and nonadiabaticity has to be taken into account. The results of section 3.2 showed that the most promising IESH approach dramatically fails to describe the translational inelasticity of the NO/Au(111) system correctly. This failure makes it inapplicable for comparison to experimental translational energy distributions discussed here. For this reason, the following view on the $T \leftrightarrow V$ interaction is limited to the discussion of different possible mechanisms. I am not aware of a theoretical model that can describe all the features seen in the experiment.

First, vibrational energy exchange with translation could occur via a mechanical (adiabatic) coupling. The most prominent example for this interaction is $\mathrm{NH}_{3} / \mathrm{Au}(111)$ scattering [4] with $E_{I}$ dependent but $T_{S}$ independent excitation of the $\mathrm{NH}_{3}$ umbrella vibrational mode (see Fig. 1.3). However, for vibrational excitation and de-excitation in NO collisions with metal surfaces, it is well established that vibrational energy change happens upon coupling to EHPs. Furthermore, the observed $\mathrm{V} \leftrightarrow T$ energy transfer is much lower than the vibrational energy transferred.

Second, a mixed adiabatic and nonadiabatic mechanism is, in principle, possible and cannot be ruled out from the (de-)excitation results at different surface temperature. In this picture the vibrational energy is partially taken (released) from (into) translation and the rest couples to EHPs. The mechanism would be in agreement with $\mathrm{T} \leftrightarrow \mathrm{V}$ coupling at $E_{I}=0.65 \mathrm{eV}$ which is presented in Table 3.3. However, the $E_{I}$ dependence of the $\mathrm{V} \rightarrow \mathrm{T}$ energy transfer (Fig. 3.32) puts this model into doubt. For $\mathrm{NO}(v=3 \rightarrow 1,2$ ) relaxation, there is only the possibility that vibrational energy is released into $E_{f}$. If such a coupling would take place because of partially adiabatic interactions, it should not depend on incidence energy.

A third mechanism is based on the picture that the NO molecule forms a transient $\mathrm{NO}^{-}$ion due to charge transfer from the surface. The stabilizing positive image charge at the surface could accelerate the NO molecule toward the surface and lead to higher effective incidence energy. Because vibrational excitation and de-excitation involve such charge transfer, this model predicts the same effect for both processes which is not in agreement with the experiment; I observed opposite trends for vibrational relaxation and excitation (middle panel of Fig. 3.24). The only plausible way how the image charge based model could lead to such an effect is that relaxation occurs upon electron transfer in the incident molecule whereas excitation predominantly happens upon electron transfer after the collision (molecules leaving the surface). However, the charge transfer event is a rather statistical process where the probability depends mainly on the distance from the surface and the availability of EHPs [43]. Therefore, it seems unlikely that such an effect is important here. Nevertheless, it is well-known that charge transfer is important in the NO/Au(111) system $[1,25,27,30,65,88]$ and therefore the image charge acceleration should occur and possibly affect the $E_{f}$ distributions. The important question is then: Why does the translational energy distributions do not show evidence for this mechanism? A possible reason might simply be that the postulated transient anion is too short lived to have an effect on the translational motion of the nuclei and to cause significant Coulomb interaction between anion and image charge.

A fourth mechanism can be site specific scattering. For example, the strength of the nonadiabatic coupling can be stronger at hollow sites than it is if the NO molecule collides on top of a surface atom. 
However, this mechanism should again lead to similar effects for excitation and relaxation and can therefore be ruled out because the opposite trend is observed in the experiment.

Finally, another plausible mechanism relies on a fully nonadiabatic picture where EHPs of the metal would mediate the energy transfer between translation and vibration. In this mechanism, the vibrational energy is, in a first step, transferred to or taken from EHPs. This energy transfer process is supported by previous studies $[1,3,8,25,27,29-31,34,44,88]$ as well as by the results of section 3.1 and 3.2 . The process of vibrational relaxation creates excited EHPs while vibrational excitation de-excites thermally excited EHPs. Consequently, both processes disturb the thermal EHP distribution $\left(T_{e} \neq T_{S}\right)$. Although the thermalization is fast, it might be that a part of the excess (missing) electronic energy is channeled into (taken from) the kinetic energy of the molecule. Therefore, not only vibration but also the translational motion of the NO molecules would show nonadiabatic coupling to EHPs. Recent calculations by Janke et al. [125] indicated that such a T-EHP coupling is important in H-atom scattering from Au(111). However, a comparison of $\mathrm{H}$-atom and $\mathrm{NO}$ scattering from $\mathrm{Au}(111)$ is questionable and should be taken with great care. Another study on scattering of hyperthermal $\mathrm{N}$ atoms from $\mathrm{Ag}(111)$ revealed a negligible contribution of EHPs to the translational energy loss in the surface collision [126]. Nevertheless, the EHP mediated $T \leftrightarrow V$ coupling is in agreement with the experimental data. It can decrease the translational energy of molecules that were excited in the surface collision and accelerate molecules that lost vibrational energy to the surface. The $E_{I}$ dependence of this nonadiabatic T↔EHP coupling might be similar to the $E_{I}$ dependences for $\mathrm{NO}(v=0 \rightarrow 1,2)$ excitation [1] and $\mathrm{NO}(v=3 \rightarrow 1,2)$ de-excitation (section 3.2). More incidence translational energy might increase the coupling between nuclear motion (translation, vibration) and EHPs due to deeper penetration into regions of high electron density. Therefore, also the T↔EHP coupling will increase with increasing $E_{I}$ which is consistent with the experimental observations (Fig. 3.32).

\section{$E_{I}$ and $\Delta E_{v i b}$ dependence of translational energy transfer to rotation}

After the discussion of the influences of surface temperature and vibrational inelasticity, this section will focus on the influences of rotational inelasticity on $E_{f}$. The results of Fig. 3.25 and Fig. 3.28 already showed that the translational energy of the scattered molecules decreases approximately linearly with increasing final rotational energy $E_{\text {rot }}$. I obtain the relation $\Delta\left\langle E_{f}\right\rangle / \Delta E_{\text {rot }}$ from the slopes of linear fits to the experimental data. The $E_{I}$ dependent values are shown in Fig. 3.33 for vibrationally elastic ( $3 \rightarrow 3$, $2 \rightarrow 2)$ and vibrationally inelastic $(3 \rightarrow 2,3 \rightarrow 1,2 \rightarrow 1,2 \rightarrow 3)$ scattering. I only measured a systematic dependence on incidence energy for $\mathrm{NO}(v=3 \rightarrow 1,2,3)$ scattering. Experiments with $v_{i}=2$ are only compared at one single incidence energy $E_{I}=0.65 \mathrm{eV}$. The values for $\Delta\left\langle E_{f}\right\rangle / \Delta E_{\text {rot }}$ are similar (approximately -0.6) for all vibrational channels for $E_{I} \geq 0.6 \mathrm{eV}$ but become significantly different at lower incidence energies. In case of vibrationally elastic $3 \rightarrow 3$ scattering, $\Delta\left\langle E_{f}\right\rangle / \Delta E_{\text {rot }}$ increases smoothly up to -0.2 at $E_{I}=0.1 \mathrm{eV}$. For the $3 \rightarrow 2$ channel, the transition occurs more abruptly at about $E_{I}=0.5 \mathrm{eV}$ and seems to level off at $\Delta\left\langle E_{f}\right\rangle / \Delta E_{\text {rot }} \approx-0.15$ below $E_{I}=0.4 \mathrm{eV}$. This sharp transition is even more pronounced for the loss of two vibrational quanta in $v=3 \rightarrow 1$ scattering. This channel even reaches the limit of $\Delta\left\langle E_{f}\right\rangle / \Delta E_{\text {rot }}=0$ for $E_{I} \leq 0.4 \mathrm{eV}$, within the uncertainty of the experiment.

In addition, I can compare $\Delta\left\langle E_{f}\right\rangle / \Delta E_{\text {rot }}$ for loss and gain of one quantum of vibration for incident $\mathrm{NO}\left(v_{i}=2\right)$ at $E_{I}=0.64 \mathrm{eV}$ and $T_{S}=570 \mathrm{~K}$ (triangles). The $2 \rightarrow 2$ channel agrees reasonably well with the observed value for the $3 \rightarrow 3$ scattering at $T_{S}=320 \mathrm{~K}$. The vibrationally inelastic $(2 \rightarrow 1,2 \rightarrow 3)$ channels are shifted towards less negative values of $\Delta\left\langle E_{f}\right\rangle / \Delta E_{\text {rot }}$. These observations show that vibrational inelasticity influences the coupling between translation and rotation. 


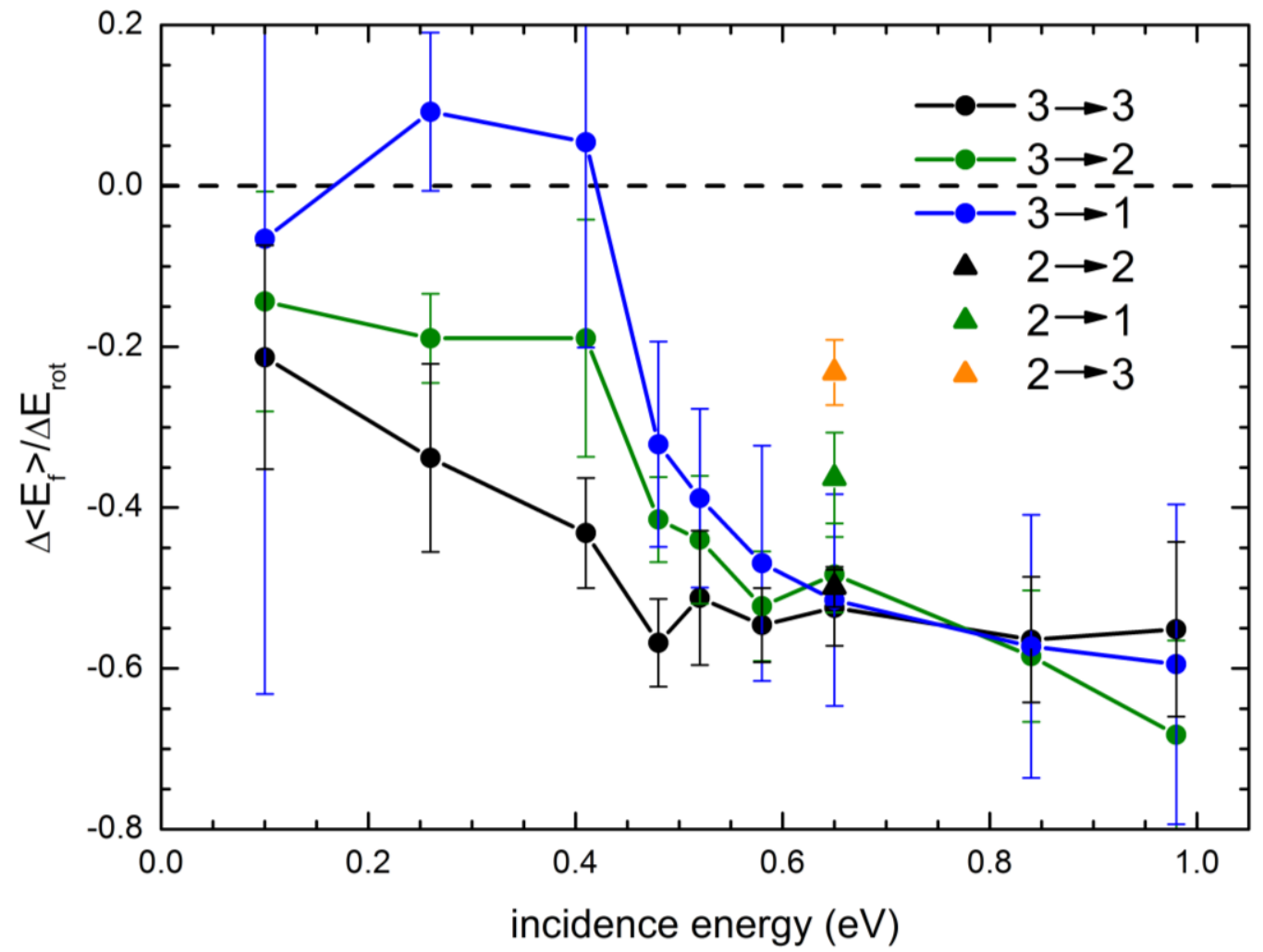

Fig. 3.33: Incidence energy dependence of $\Delta\left\langle E_{f}\right\rangle / \Delta E_{\text {rot }}$ for vibrationally elastic (black) and vibrational inelastic (red, blue, green) scattering from $\mathrm{Au}(111)$ at $T_{S}=320 \mathrm{~K}$. The connected dots are obtained from linear fits to the experimental data of Fig. 3.28, the triangles are obtained from linear fits to the experimental data in the middle panel of Fig. 3.25. $\Delta\left\langle E_{f}\right\rangle / \Delta E_{\text {rot }}$ is similar and constant at about -0.55 for all vibrational channels for $E_{I}>0.6 \mathrm{eV}$ and approaches zero for decreasing $E_{I}$. The transition is smooth for the $3 \rightarrow 3$ channel and more abrupt for vibrationally inelastic $3 \rightarrow 2$ and $3 \rightarrow 1$ scattering. The values for $2 \rightarrow 1$ and $2 \rightarrow 3$ scattering at $E_{I}=0.64 \mathrm{eV}$ and $T_{S}=570 \mathrm{~K}$ are even higher [121] - Published by the PCCP Owner Societies.

The further discussion of the observations requires an understanding of what the different values of $\Delta\left\langle E_{f}\right\rangle / \Delta E_{\text {rot }}$ mean. $\Delta\left\langle E_{f}\right\rangle / \Delta E_{\text {rot }}=-1$ indicates complete anti-correlation between translational and rotational energy meaning that rotational excitation is completely caused by energy transfer from translation ( $T \rightarrow R$ coupling). In a simple mechanical picture, the amount of final rotational energy depends on the orientation of the molecule at impact and the translational energy transfer to the surface (phonons) is independent of the final rotational energy. An example of such behavior is NO scattering from a very stiff surface like diamond [127]. If $\Delta\left\langle E_{f}\right\rangle / \Delta E_{r o t}=0$, it means that recoil and rotational energy are completely uncorrelated. The rotational energy might then be taken from the phonon bath rather than from $E_{I}$ ( $\mathrm{S} \rightarrow \mathrm{R}$ coupling). In general, $\Delta\left\langle E_{f}\right\rangle / \Delta E_{\text {rot }}$ values fall in between these two limiting cases which has been related to an anti-correlation of translational energy transfer to the surface (phonons, $T \rightarrow S$ ) and to rotational motion $(T \rightarrow R)[9,13,80,81]$ that depends on the orientation of the molecule when it collides with the surface. Perfect head-on or side-on collisions will not cause any rotational excitation but will transfer a maximum energy (e.g. Baule limit) to the lattice because the center of mass falls on the collision axis. For a tilted orientation, the collision will lead to rotational excitation of the molecule but will transfer less energy to the lattice.

There are a few earlier studies on other systems that were focused on the dependence of $E_{f}$ on $E_{\text {rot }}[9$, $13,80,81$ ] for vibrationally elastic $v=0 \rightarrow 0$ scattering. The work on the $\mathrm{NO} / \mathrm{Ag}(111)$ system [9] (Fig. 1.9) is probably the one that suits best for a comparison to NO/Au(111) scattering because both systems exhibit similar features and are non-reactive. An important difference between my work and that of 80 
reference [9] is the use of incident vibrationally excited molecules. This difference can lead to several difficulties in the interpretation of the experimental results. $\mathrm{NO}\left(v_{i}=0\right)$ molecules scattering off a room temperature surface end up (almost) exclusively in the same vibrational state, independent of the scattering dynamics. Only the final rotational state $J_{f}$ will influence the translational energy distribution. For scattering of vibrationally excited molecules, there is a possibility that vibrational inelasticity can act as a filter for certain scattering properties (e.g. orientations). Another important point is the lower mass of Ag (108 amu) compared to Au (197 amu). In the framework of the Baule model, this would mean that the translation energy transfer to the lattice would be higher for $\mathrm{Ag}(\sim 68 \%)$ than for $\mathrm{Au}(\sim 46 \%)$.

Note that already in the previous work on translational inelasticity in the $\mathrm{NO} / \mathrm{Ag}(111)$ system, the authors were only able to interpret the observed dependence of $E_{f}$ on $E_{\text {rot }}$ by comparison of the experimental data to molecular dynamics simulations using an empirical adiabatic potential energy surface. Such a rather simple model is not applicable for the experimental data of my work because nonadiabatic V-EHP interactions are necessary in order to obtain any vibrational relaxation/ excitation. The IESH model is probably the best current approach for taking these interactions into account. Unfortunately, the results of section 3.2 have shown that the model especially fails to reproduce the translational inelasticity in the NO/Au(111) system. The failure of the IESH model is again illustrated in Fig. 3.34, which shows the final translational energy distributions obtained in the experiment (blue curve) and predicted from the IESH model for vibrationally elastic scattering. The comparison clearly shows that the theoretical recoil energy of the molecules is much slower than obtained experimentally. Furthermore, restriction of the analysis to single and double bounce collision does not improve the agreement. Therefore the interpretation of the experimental results is again limited to the discussion of different mechanisms.

A first mechanism is related to conclusions made by Kimman et al. for $\mathrm{NO} / \mathrm{Ag}(111)$ scattering [9]. The authors observed a behavior of $\Delta\left\langle E_{f}\right\rangle / \Delta E_{\text {rot }} \rightarrow 0$ at low $E_{I}$ similar to that observed in this work. The trajectory calculations, which showed reasonable agreement with the experimental data, indicated that this behavior is due to an increasing fraction of multi-bounce collisions with decreasing $E_{I}$. The good agreement with the experiment made this interpretation reasonable. The change in $\Delta\left\langle E_{f}\right\rangle / \Delta E_{r o t}$ is then related to a thermalization of the NO rotational motion which could be interpreted as phonon to rotation $(\mathrm{S} \rightarrow \mathrm{R}$ ) coupling. For the $\mathrm{NO} / \mathrm{Au}(111)$ system the threshold at which such multi-bounce collisions might occur should in general be shifted toward lower incidence energy due to the lower translational inelasticity ( $\mathrm{T} \rightarrow \mathrm{S}$ energy transfer). My experiments have the additional complexity in that vibrational energy transfer can happen for the use of $\mathrm{NO}\left(v_{i}=2,3\right)$ instead of $\mathrm{NO}\left(v_{i}=0\right)$. The main question is then how multi-bounce collisions affect the different vibrational channels. The first consequence of a multibounce collision is that it increases the interaction time of the NO molecule with the surface. In other words, the molecule spends more time close to the surface than if it were scattered directly in a single bounce collision. The kinetic model presented in section 3.1.3 already showed that the longer interaction times lead to higher probabilities for vibrational excitation. The same behavior is expected in case of vibrational relaxation because the system will reach thermal equilibrium for $t \rightarrow \infty$ which corresponds to trapping/ adsorption. Furthermore, multiple collisions can steer the molecule into the favorable N-down orientation like observed in the IESH simulations for $\mathrm{NO}(v=3 \rightarrow 1,2,3)$ relaxation (Fig. 3.21). Both effects increase the probability for vibrational energy transfer between the NO molecule and the EHPs of the $\mathrm{Au}(111)$ surface. If such a mechanism is present here, multiple bounces will mainly be reflected in vibrationally inelastic scattering. Therefore, $\mathrm{S} \rightarrow \mathrm{R}$ coupling would be more important for scattering with $\Delta E_{v i b} \neq 0$ and $\Delta\left\langle E_{f}\right\rangle / \Delta E_{\text {rot }}$ will approach 0 already at higher incidence energies than for the vibrationally elastic channel. This model is consistent with the experimental observations of Fig. 3.33. However, there is no direct experimental evidence for multi-bounce events in the time-of-flight data at low $E_{I}$. The translational energy distributions do not appear broader or have multiple peaks. In addition, 
the Baule limit seems to hold for the whole range of incidence energies from 0.1-0.98 eV, which is not in agreement with thermalization with the surface. Nevertheless, the expectation for the final $E_{f}$ distribution at the lowest incidence energies is already close to a thermal Maxwell-Boltzmann distribution and might not be easy to distinguish. The contribution of multi-bouncing can neither be unequivocally verified nor completely excluded from the experimental data.

Another possible mechanism results from the idea of dynamical steering which was first suggested by Shenvi et al. for $\mathrm{NO}(v=15) / \mathrm{Au}(111)$ scattering [65]. In this picture, the NO molecules are steered into the $\mathrm{N}$-first orientation on approach toward the surface due to the attractive well for the $\mathrm{N}$-Au interaction potential; the O-Au potential is purely repulsive. The forces that the molecule experiences from the potential always try to rotate the molecules into the position with the $\mathrm{N}$-atom pointing towards the surface. If the molecule is fast (high $E_{I}$ ), it will not have enough time for re-orientation. However, if the incidence energy is lowered, there are more and more initial orientations that can turn around prior to the surface collision. In addition, the nonadiabatic coupling is stronger for the $\mathrm{N}$-first than for the O-first orientation, an effect that has been verified in recent experimental studies [128, 129]. Consequently, the steered molecules have a higher probably for V-EHP coupling, and, therefore, for vibrational excitation/ relaxation. Bartels et al. showed that the orientation effect for $\mathrm{NO}\left(v_{i}=3\right)$ molecules colliding with $\mathrm{Au}(111)$ decreases or disappears at low incidence and explained their observation by a dynamical steering effect [128]. However, their observations would also be consistent with the multi-bounce mechanism mentioned above. The main question is how steering can influence $\Delta\left\langle E_{f}\right\rangle / \Delta E_{\text {rot }}$. A possible explanation is that the potential causes a torque that leads to rotational excitation. The rotational energy will then be taken from the surface, which can also be seen as energy transfer from the surface bath.

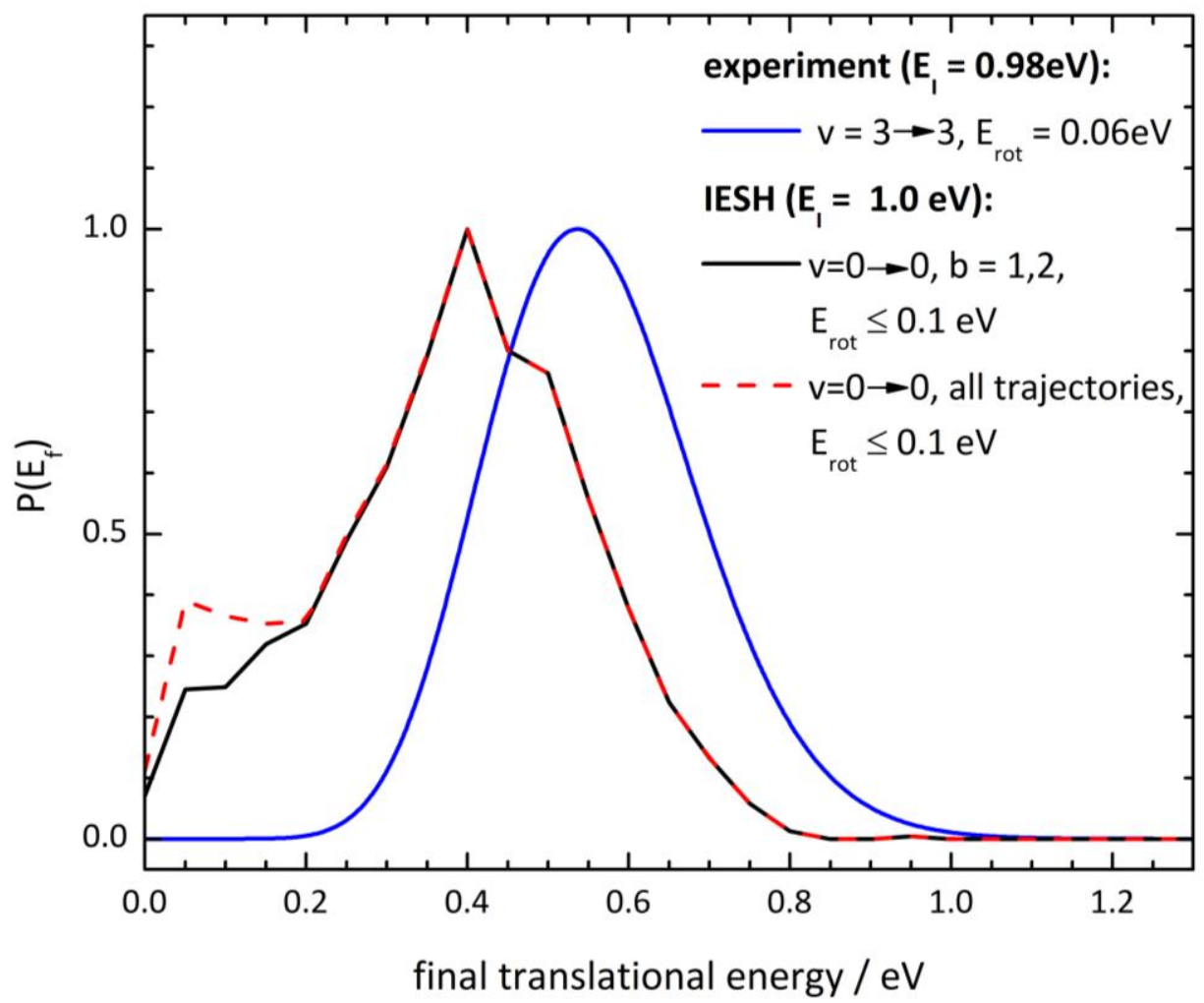

Fig. 3.34: Comparison of experimental $E_{f}$ distributions to the prediction of the IESH model. The experimental curve (blue) shows the results for $\mathrm{NO}(v=3 \rightarrow 3)$ scattering from $\mathrm{Au}(111)$ at $T_{S}=320 \mathrm{~K}$ and $E_{I}=0.98 \mathrm{eV}$. The theoretical curves show the results for $v=0 \rightarrow 0$ scattering at $T_{S}=300 \mathrm{~K}$ and $E_{I}=1.0 \mathrm{eV}$ for molecules scattering with $E_{\text {rot }} \leq 0.1 \mathrm{eV}$. The black and dashed red curves compare the $E_{f}$ distributions for selection of single and double bounce collisions and for all trajectories (no restriction). 
A detailed explanation is only possible by comparison to first-principles theories like IESH. I emphasize that any theoretical model, which is applied to this set of data, has to incorporate nonadiabatic coupling as well as it has to be based on a correct adiabatic interaction potential.

\subsection{Summary and Conclusions}

In summary, I have performed detailed experiments for vibrationally inelastic NO scattering from Au(111). I measured $v=0 \rightarrow 1,2,3$ vibrational excitation probabilities over a wide range of surface temperatures from $T_{S}=400-1100 \mathrm{~K}$. I compared the results to first-principles IESH theory that is known to give semi-quantitative agreement for vibrational relaxation [65] and excitation [25] of NO scattering from $\mathrm{Au}(111)$. All three vibrational channels exhibit excitation probabilities that depend on $T_{S}$ in an Arrhenius-like fashion with the activation energies equal to the vibrational energy uptake. The preexponential factors, which are related to the intrinsic nonadiabatic coupling strength, increase with increasing $E_{I}$. Angular distributions for scattering into $v_{f}=3$ are as narrow as $\cos ^{6-7} \theta$ indicating that direct scattering dominates the scattering mechanism. This is in agreement with earlier results where excitation into $v_{f}=1[8,44]$ and $v_{f}=1,2[1,27]$ was observed.

I compared the results for $E_{I}=0.41 \mathrm{eV}$ to an empirical kinetic rate model as well as to first-principles IESH theory. Independent of the assumption for coupling strength between states with the same $\Delta v$, the kinetic model suggested the importance of overtone transitions for the excitation pathways yielding $v_{f}=2$ and $v_{f}=3$. In contrast, the IESH model strongly favored pure sequential excitation. However, the approach from first-principles clearly underestimated the experimentally observed excitation probabilities for $v_{f}=2$ and even more for $v_{f}=3$. The reason for this disagreement is not completely clear but I speculate that the representation of the continuum of electronic states in the metal by $M_{S}$ discrete electronic states is not sufficient. Moreover, the electronic states in the model are not equally spaced but have a higher density around the Fermi-level decaying quickly with increasing energetic distance from $E_{F}$. This higher density is on the one hand necessary to provide enough electronic states for the electronic transitions in a region that is important for the non-adiabatic interaction of NO with a metal, but on the other hand it involves the risk that single quantum transitions are artificially enhanced with respect to overtone transitions. I observed a surprisingly good agreement for the IESH model with the kinetic rate model when overtone transitions are switched off in the empirical model. Although this agreement might be a co-incidence, it is further support for the suggested underestimation of overtone excitation in the IESH model.

A more detailed test of the approach from first-principles required a detailed trajectory analysis of the simulations. For this purpose, I measured branching ratios for $\mathrm{NO}(v=3 \rightarrow 3,2,1) / \mathrm{Au}(111)$ vibrational relaxation as a function of incidence translational energy. The experimental results showed that the relaxation probability increases with increasing incidence energy. Although the branching ratios calculated from first-principles are in the same order of magnitude as observed experimentally, the theoretical model predicted the opposite dependence on $E_{I}$. A detailed analysis of the trajectories showed that the apparent failure is due to an increasing, and at least overestimated, fraction of multibounce trajectories in the simulation at low $E_{I}$. These multi-bounce collisions lead (1) to an increasing number of electronic transitions (V-EHP coupling), (2) to a closer approach towards the surface and (3) to re-orientation towards favorable $\mathrm{N}$-down orientation. Especially the latter observation puts the previously postulated dynamical steering effect [65] into doubt. I used the identical program code in this work as the authors of reference [65] for their calculations at $E_{I}=0.05 \mathrm{eV}$, an incidence energy at which the IESH model will almost exclusively (>90\%) produce multi-bounce trajectories. An important point to 
note is that the surface hopping algorithm is not causing the problem in the simulations because I obtained very similar results for electronic friction or adiabatic calculations. Therefore, the implementation of nonadiabaticity by the surface hopping model is not necessarily to blame for the multi-bounce artifacts but rather the adiabatic potential energy surface, which has been calculated from density-functional theory (DFT) [73]. It is possible that the PES is too corrugated (perpendicular to parallel momentum transfer), that the interaction itself is treated wrong by the functional or that the translation to phonon energy transfer is too big. The latter argument is weakened by the fact that the $\mathrm{Au}-\mathrm{Au}$ interaction potential was optimized to fit the corresponding phonon spectrum [73]. The first two possibilities are more related to the failure of DFT, which is known to fail in the treatment of electron transfer events. Alternatives are just becoming available by means of embedded correlated wave function theory [130] where a small cluster is calculated by accurate correlated wave function theory and is afterwards embedded into the larger system which is treated with DFT.

The results on vibrational relaxation of $\mathrm{NO}\left(v_{i}=3\right) / \mathrm{Au}(111)$ scattering and the comparison to firstprinciples IESH theory clearly shows the importance of accurate adiabatic potential energy surfaces for the correct treatment of nonadiabatic interactions. In other words: it is crucial to obtain the adiabatic effects, like translational inelasticity, correct in order to be able to make correct predictions for nonadiabatic effects.

In order to provide additional benchmark experimental data to test future improved theoretical models, I measured translational energy distributions of vibrationally excited $\mathrm{NO}\left(v_{i}=2,3\right)$ molecules after scattering from a single crystal $\mathrm{Au}(111)$ surface into various vibrational and rotational states. My experiments show that $E_{f}$ and $P\left(E_{f}\right)$ depend on the temperature of the surface as well as on the final quantum state $\left(v_{f}, J_{f}\right)$ of the molecule. The translational energy distributions of the scattered molecules are much broader than those of the incident molecular beam. The mean recoil energy increases with increasing surface temperature. $\left\langle E_{f}\right\rangle$ is in reasonably good agreement with a simple hard cube (Baule) model that considers only conservation of energy and momentum. A modification of the simple Baule model into a thermal hard cube model with a moving Au atom is partly able to explain the broadening of the $E_{f}$ distributions with increasing $T_{S}$.

I found that vibrationally inelastic scattering influences the final translational energy in a way that vibrational relaxation increases $E_{f}$ and vibrational excitation decreases $E_{f}$. The amount of this $\mathrm{T} \leftrightarrow \mathrm{V}$ energy transfer depends on the temperature of the surface as well as on incidence energy. For vibrationally inelastic scattering $(\Delta v \neq 0), E_{f}$ increases about twice as fast with increasing $T_{S}$ than for vibrationally elastic scattering $(\Delta v=0)$. A possible explanation for this observation is that collisions with $\Delta E_{v i b} \neq 0$ discriminate against collisions where the surface atom is moving toward the incident molecule (out of the surface). The resulting higher effective collision energy could increase the nonadiabatic V-EHP coupling in a similar way to the $E_{I}$ dependence of vibrational relaxation/ excitation probabilities. Furthermore, the results show that the $T \leftrightarrow V$ coupling increases with increasing incidence energy. $A$ mechanism that is in agreement with the experimental observations is based on an EHP mediated T↔V energy transfer where the vibrational energy is first transferred to EHPs which then couple to the translational motion of the molecule. The T-EHP interaction strength might then increase with increasing $E_{I}$ similar to the (de-)excitation probabilities.

Finally, the dependence of recoil energy on the final rotational excitation of the molecule depends on the final vibrational state as well as on incidence energy. This effect might be related to multi-bounce scattering or to dynamical steering. However, a final interpretation of the experimental data is not straight forward and requires more information from first-principles theory. At this point, I can only speculate about the details of the dynamics that lead to the observed effects or rule out certain 
mechanisms. The most promising theoretical approach using the IESH model revealed significant weaknesses, especially regarding translational inelasticity. Therefore, a comparison of the IESH-based model in its current implementation is pointless and the model first has to be improved, for example by calculation of a more accurate PES. The data presented in this section represent an ideal benchmark for testing future improved versions of existing or new models. A first-principles approach capable to describe the experimental observations quantitatively has to include nonadiabatic V-EHP (and maybe T$E H P)$ coupling as well as it has to cover the adiabatic features $(T \rightarrow S, T \rightarrow R)$ of the system correctly.

Nevertheless, the IESH model for NO/Au(111) scattering is so far the only available approach from firstprinciples that succeeded to reproduce vibrational relaxation and excitation in the NO/Au(111) system. Electronic friction theories are known to fail for systems that exceed the weak coupling limit, like in case of NO scattered from metal surfaces [25]. Although the nonadiabatic interaction is reasonably well described in the IESH model, it fails in describing other observations: for example, angular distributions are predicted much broader than observed in the experiment and the incidence energy dependence for vibrational excitation into $v_{f}=1,2$ is not reproduced correctly [25]. 


\section{CO scattering from $\mathrm{Au}(111)$}

While nonadiabatic interactions in NO scattering from $A u(111)$ and $A g(111)$ have been studied extensively in the past only little work has been done to investigate nonadiabatic molecule-surface interactions for other molecules. This often raises the question: Do molecules other than the NO radical show nonadiabatic effects? Judging from the picture that the nonadiabaticity correlates with the electron affinity of the molecule (Fig. 1.6), as it makes electron transfer energetically more probable, only molecular oxygen should show even stronger effects. However, state specific detection of $\mathrm{O}_{2}$ is not straightforward and available REMPI schemes offer only poor sensitivity [131]. Studies on $\mathrm{HCl}$ scattering from $\mathrm{Au}(111)[32,33,107]$ showed that nonadiabatic effects probably play a role in the system but so far no theoretical work has been performed that can be compared to the experimental results.

Carbon monoxide is the next in the line of electron affinities. CO spectroscopy is well-known and sensitive $(2+1)$ REMPI detection is possible. Prior to this work, there was only one study by Rettner on the search of vibrational excitation in CO scattering off $\mathrm{Au}(111)$ [46]. The author observed trapping probabilities, rotational state distributions and $\operatorname{CO}(v=1)$ excitation. However, Rettner was not able to determine whether the vibrational excitation was due to direct scattering (as in case of $\mathrm{NO} / \mathrm{Ag}(111)$, $\mathrm{NO} / \mathrm{Au}(111))$ or due to trapping followed by desorption.

\subsection{CO vibrational excitation on $\mathrm{Au(111)}$}

I performed experiments to measure absolute vibrational excitation probabilities for $\mathrm{CO}(v=0 \rightarrow 1)$ scattering from $\mathrm{Au}(111)$ as a function of incident translational energy. The CO/Au(111) system is a reasonable choice to study vibrational excitation probabilities because of the detailed knowledge of the $\mathrm{NO} / \mathrm{Au}(111)$ system $[3,25-27,88,112,117]$. The goal of this study was to answer the questions raised by the work of Rettner [46]. Does CO excitation show the typical fingerprints of nonadiabatic V-EHP coupling like Arrhenius-like surface temperature dependence and an $E_{I}$ dependence with zero threshold? Is the vibrational excitation due to direct scattering or dominated by trapping/ desorption?

The absolute excitation probabilities observed in the experiment reveal the typical Arrhenius dependence on surface temperature with an activation energy equal to the vibrational energy uptake $\Delta E_{v i b}=0.265 \mathrm{eV}$. The prefactors are about a factor of three lower than for NO/Au(111) (see section 3.1). However, the $E_{I}$ dependence shows a clearly different behavior. The prefactor first decreases with increasing incidence energy, has a minimum at about $E_{I}=0.35 \mathrm{eV}$ and starts to increase again. This behavior is in agreement with vibrational excitation that is partly due to direct scattering, which leads to an increase of the prefactor with increasing $E_{I}$, and is partly due to trapping followed by desorption, which increases the prefactor with decreasing $E_{I}$.

\subsubsection{Results}

As for the determination of vibrational excitation probabilities for NO/Au(111), the determination of absolute vibrational excitation probabilities for $\mathrm{CO}$ also requires the measurement of surface temperature dependent REMPI spectra as well as $T_{S}$ and $v_{f}$ dependent angular and arrival time distributions. However, the determination of absolute excitation probabilities for $\mathrm{CO}(v=0 \rightarrow 1) / \mathrm{Au}(111)$ is significantly easier than in case of NO. The only applicable REMPI transitions that offer sufficient sensitivity to obtain vibrational excitation probabilities are the $B\left(v^{\prime}=v^{\prime \prime}\right) \leftarrow X\left(v^{\prime \prime}\right)$, thus the (0-0) and (1-1) bands which show an intense $Q$ branch in the (2+1) REMPI scheme. 


\section{REMPI spectra}
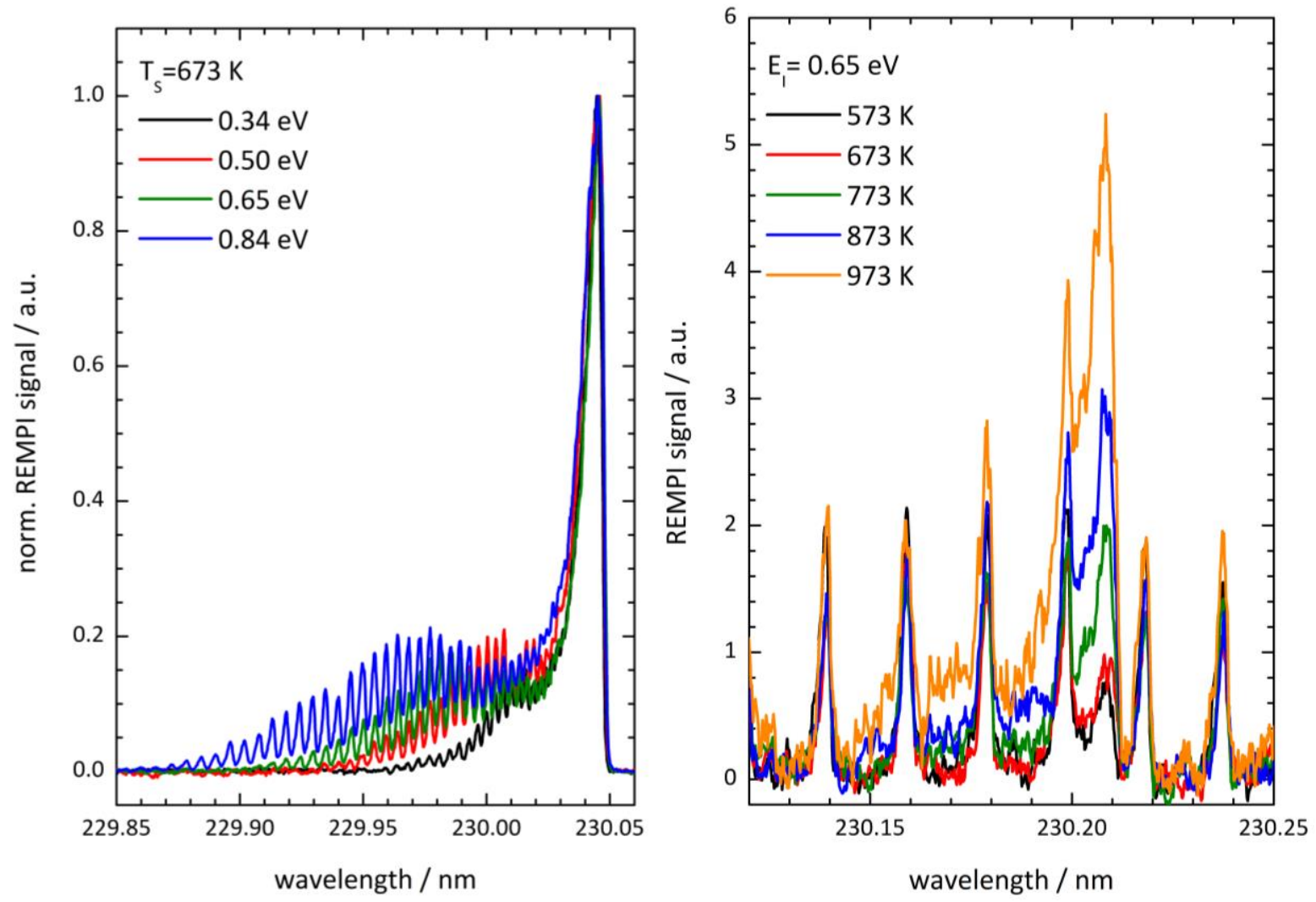

Fig. 4.1: $(2+1)$ REMPI spectra for $\operatorname{CO}\left(v_{\mathrm{f}}=0,1\right)$ scattered off $A u(111)$. Left panel: $B\left(v^{\prime}=0\right) \leftarrow X\left(v^{\prime \prime}=0\right)$ Q branch for different incident translational energies at $673 \mathrm{~K}$ surface temperature. At all four $E_{I}$, the spectrum shows a band head at about $230.04 \mathrm{~nm}$ and a rotational rainbow feature that shifts towards higher rotational states with increasing $E_{I}$. Right panel: Surface temperature dependence for the $B\left(v^{\prime}=1\right) \leftarrow X\left(v^{\prime \prime}=1\right) \mathrm{Q}$ branch which is overlapped with peaks of the $(0-0) 0$ branch. The band head of the (1-1) Q branch is located at about $230.208 \mathrm{~nm}$. The intensity increases strongly with increasing $T_{S}$.

Some examples for $B \leftarrow X(2+1)$ REMPI spectra for $\mathrm{CO}\left(v_{f}=0,1\right)$ after scattering from $\mathrm{Au}(111)$ are shown in Fig. 4.1. The left panel illustrates the dependence of the rotational structure of the (0-0) $Q$ branch on incidence energy. The shown spectra are measured at a surface temperature of $T_{S}=673 \mathrm{~K}$ but are basically independent of $T_{S}$. Under all conditions, the $\mathrm{Q}$ branch reveals a thermal-like component at low rotational states in the range from $230.05-230.02 \mathrm{~nm}$. The further decrease in intensity that would be expected from a thermal rotational distribution is interrupted by a second maximum at higher rotational states. This feature is often referred to as rotational rainbow scattering [10, 11, 13, 46, 75-79]. The rotational rainbow shifts toward higher $J_{f}$ with increasing $E_{I}$ while the thermal part remains basically unchanged. I model this rotational distribution according to the procedure described in Appendix $D$ by the sum of a thermal distribution and an additional Gaussian function centered at $J_{c}$ :

$$
N_{J}=\frac{(2 J+1) \cdot e^{-\frac{E_{r o t}(J)}{k_{B} T_{r o t}}}}{\sum_{J}(2 J+1) \cdot e^{-\frac{E_{r o t}(J)}{k_{B} T_{r o t}}}}+a \cdot e^{-\frac{\left(J-J_{C}\right)^{2}}{b^{2}}}
$$




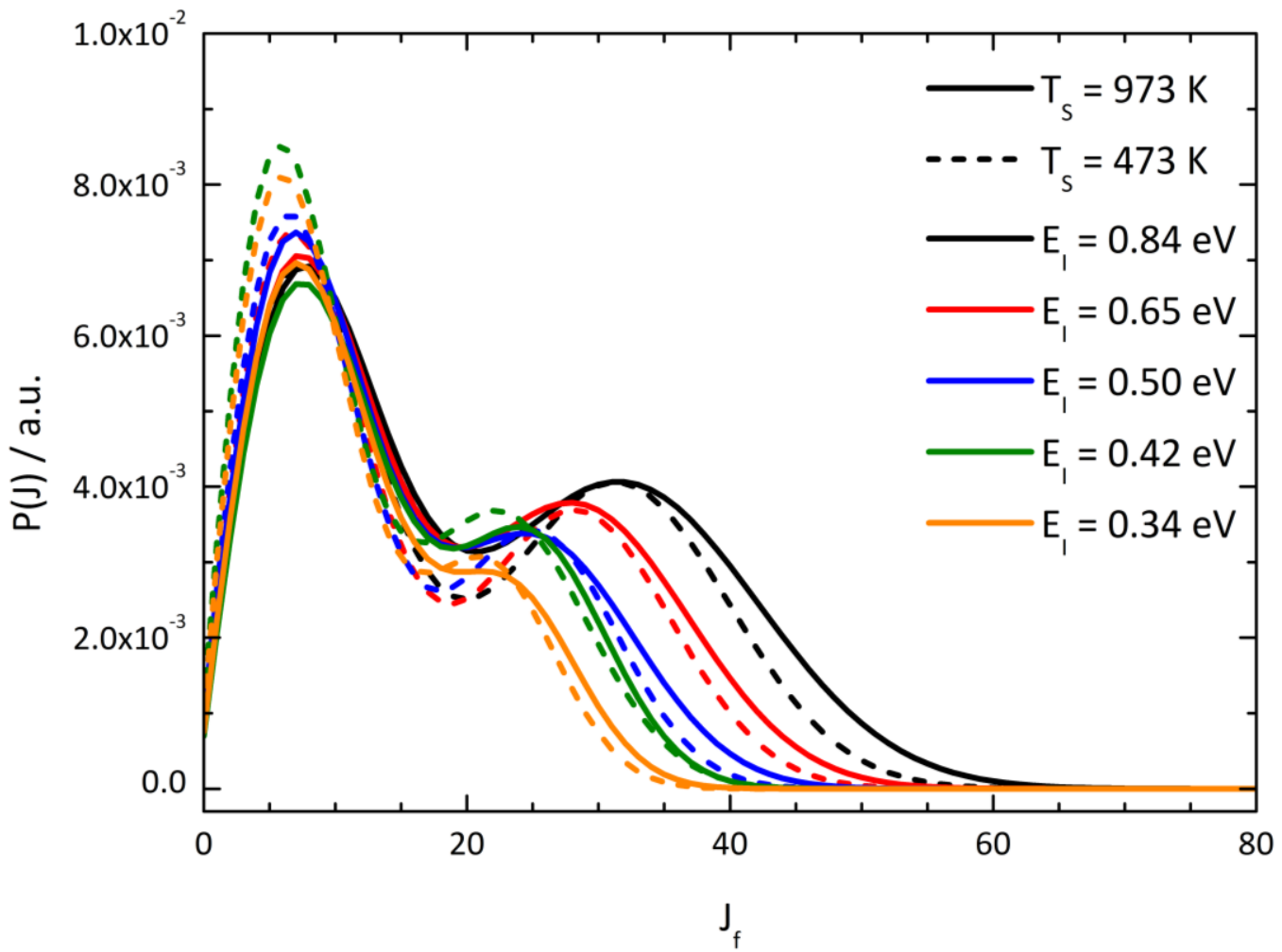

Fig. 4.2: Rotational distributions for $\mathrm{CO}\left(v_{f}=0\right)$ scattered off $\mathrm{Au}(111)$ at $T_{S}=473 \mathrm{~K}$ (dashed lines) and $973 \mathrm{~K}$ (solid lines) and different incidence energies ranging from $E_{I}=0.34-0.84 \mathrm{eV}$. The rotational distribution was derived from a fit (see Appendix D for details) to the experimentally obtained REMPI spectra. An increase of surface temperature only slightly increases the rotational excitation. All data show a pronounced rotational rainbow that shifts towards higher $J_{f}$ with increasing incidence energy.

where $a$ is a parameter that describes the amplitude, $J_{c}$ is the center rotational state and $b$ is a parameter that reflects the width of the rotational rainbow feature. Fig. 4.2 shows rotational state distributions obtained from fitting experimental rotational distributions like those in the left panel of Fig. 4.1. The solid and dashed lines indicate surface temperatures of $T_{S}=973 \mathrm{~K}$ and $473 \mathrm{~K}$; the colors indicate different incident translational energies. Some examples of obtained fit parameters are shown in Table 4.1 for different surface temperature and incidence energies. The results show that the temperature of the thermal distributions increases slightly with increasing incidence energy. Within the scatter of the data, I do not observe a clear dependence on surface temperature. The amplitude $a$ of the rotational rainbow exhibits a similar behavior. I observe a more systematic dependence on $E_{I}$ and $T_{S}$ for the width $b$ and the center $J_{c}$ of the rotational rainbow contribution. The parameter $b$ increases with increasing $T_{S}$ as well as with increasing $E_{I}$. $J_{C}$ is independent of surface temperature but shifts systematically towards higher values with increasing $E_{I}$.

An advantage of the use of the (0-0) and (1-1) bands lies in the fact that both vibrational bands are only separated by about $0.15 \mathrm{~nm}$ and can therefore be covered under identical scattering conditions. In the experiment, I measured REMPI spectra for the (0-0) and (1-1) Q branch at each surface temperature using similar laser power and just changing the detector gain. This procedure will allow neglecting the effect of angular dilution as long as the angular distributions are similar for both vibrational channels. 
Table 4.1: Examples for parameters resulting from fitting the rotational distributions of the (0-0) Q branch for different surface temperatures and incidence energies. All parameters show only a weak or no dependence on $T_{S}$ but increasing with increasing $E_{I}$.

\begin{tabular}{c|c|c|c|c|c}
$\boldsymbol{E}_{\boldsymbol{I}} / \mathbf{e V}$ & $\boldsymbol{T}_{\boldsymbol{S}} / \mathbf{K}$ & $\boldsymbol{a} / \mathbf{1 0}^{-\mathbf{3}}$ & $\boldsymbol{b}$ & $\boldsymbol{T}_{\text {rot }} / \mathbf{K}$ & $\boldsymbol{J}_{\boldsymbol{c}}$ \\
\hline \multirow{3}{*}{0.34} & 473 & 2.92 & 7.14 & 232 & 22 \\
& 723 & 2.63 & 6.67 & 259 & 22 \\
& 973 & 2.53 & 7.31 & 316 & 23 \\
\hline \multirow{3}{*}{0.42} & 473 & 3.62 & 9.22 & 216 & 23 \\
& 723 & 3.08 & 8.68 & 279 & 24 \\
& 973 & 3.24 & 8.12 & 343 & 25 \\
\hline \multirow{3}{*}{0.50} & 473 & 3.38 & 8.68 & 265 & 25 \\
& 723 & 3.25 & 9.05 & 288 & 26 \\
& 973 & 3.29 & 10.5 & 293 & 25 \\
\hline \multirow{3}{*}{0.65} & 473 & 3.68 & 10.3 & 283 & 28 \\
& 723 & 3.72 & 10.8 & 308 & 28 \\
& 973 & 3.75 & 12.3 & 322 & 28 \\
\hline \multirow{3}{*}{0.84} & 473 & 4.05 & 12.1 & 323 & 31 \\
& 723 & 3.98 & 13.4 & 322 & 31 \\
& 973 & 4.06 & 14.8 & 345 & 32
\end{tabular}

\section{Angular distributions}

In order to verify this assumption I measured angular distributions of $\operatorname{CO}\left(v_{f}=0,1\right)$ for various incidence energies and surface temperatures. Some results are shown in Fig. 4.3. The upper panel illustrates the effect of incidence energy on the width of the angular distribution. The angular distributions of scattered $\mathrm{CO}\left(v_{f}=0\right)$ molecules do not show a significant change with incidence energy in the range from 0.34$0.65 \mathrm{eV}$. The angular distributions are very narrow $\left(\cos ^{8-9} \theta\right)$ and peak near the specular angle of $\sim 5^{\circ}$. The middle and right panel of Fig. 4.3 show the effects of surface temperature and vibrational state $v_{f}$ for $E_{I}=0.65 \mathrm{eV}$. For $v_{f}=0\left(v_{f}=1\right)$, the width of the angular distributions increases slightly from $\cos ^{7.3(7.6)} \theta$ at $T_{S}=573 \mathrm{~K}$ to $\cos ^{6.4(6.0)} \theta$ at $T_{S}=973 \mathrm{~K}$, indicating that the final vibrational state has only little influence on the angular distribution. This behavior has been observed previously also for $\mathrm{NO} / \mathrm{Au}(111)$ scattering [26]. In any case, the measured angular distributions are much narrower than a $\cos \theta$ distribution that is expected for trapping followed by desorption. Note that I could not observed angular distributions for $v_{f}=1$ at $E_{I}=0.16 \mathrm{eV}$ and $0.84 \mathrm{eV}$ because of a too low $\mathrm{S} / \mathrm{N}$ level in the experiment. As I will discuss below, I have to make assumption for the corresponding angular distributions. 

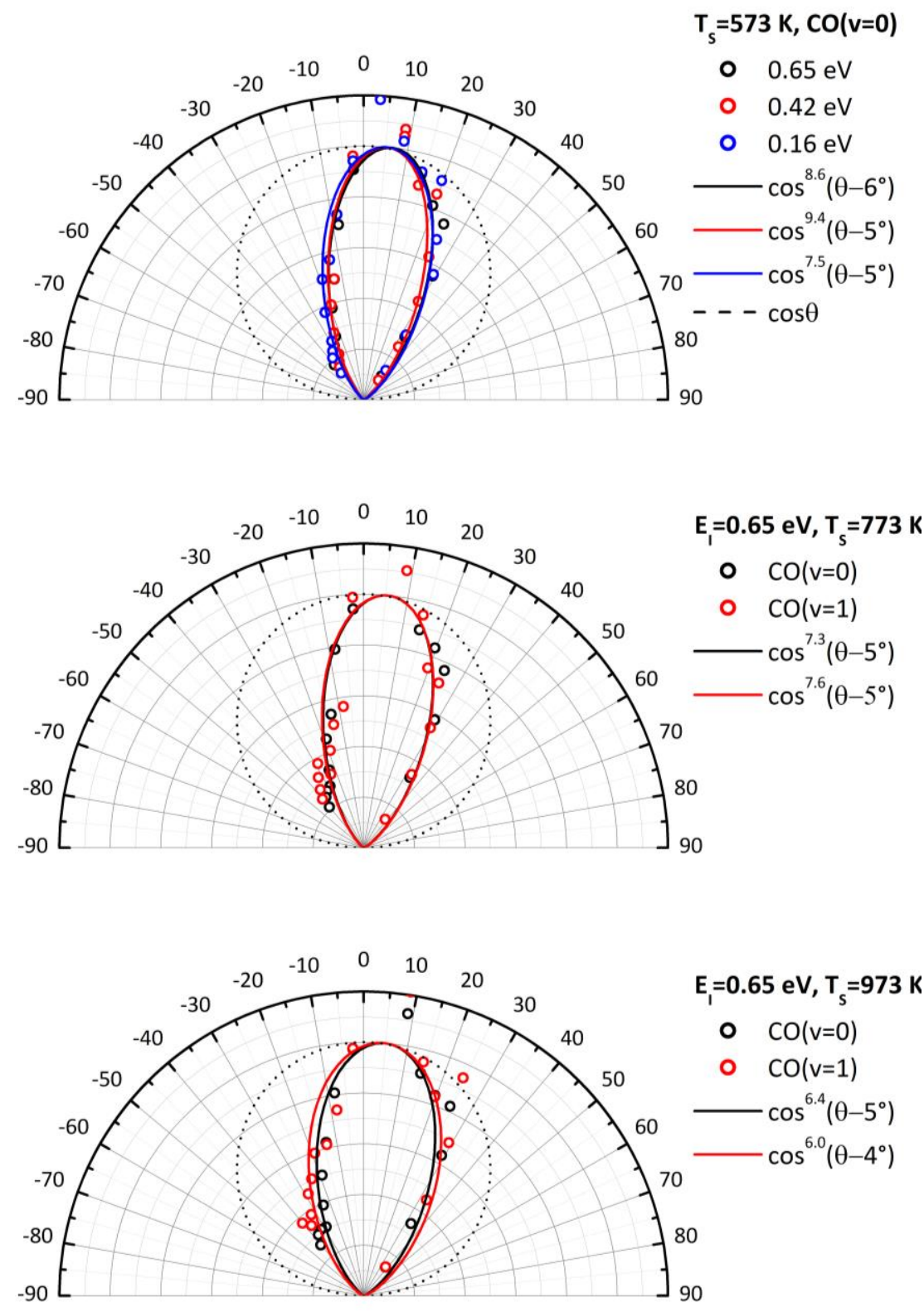

Fig. 4.3: Angular distributions for $\operatorname{CO}\left(v_{f}=0,1\right)$ scattered off $\operatorname{Au}(111)$ under various conditions fitted with a $\cos ^{n}\left(\theta-\theta_{0}\right)$ distribution. The dashed line indicates a $\cos \theta$ distribution that is expected for a trapping/ desorption mechanism. Top panel: $0 \rightarrow 0$ scattering for three different incidence energies $E_{I}=0.34,0.42$ and $0.65 \mathrm{eV}$ at $T_{S}=573 \mathrm{~K}$. All three incidence energies show a similar behavior and reveal narrow angular distributions. Middle and bottom panel: Angular distributions for $0 \rightarrow 0$ (black) and $0 \rightarrow 1$ (red) scattering at $773 \mathrm{~K}$ and $973 \mathrm{~K}$ surface temperature. Both dataset are shown for $E_{I}=0.65 \mathrm{eV}$. The angular distributions are almost identical for $v_{f}=0$ and $v_{f}=1$ and broaden slightly with increasing $T_{S}$. The points at the center had to be excluded from the fit due to an increase in intensity resulting from passing the incident molecular beam.

\section{Arrival time distributions}

Fig. 4.4 shows typical arrival time distributions for $\mathrm{CO}(v=0 \rightarrow 0,1)$ scattering from $\mathrm{Au}(111)$ at different surface temperatures for $E_{I}=0.65 \mathrm{eV}$. The large panels show the normalized intensity while the insets show the uncorrected measured intensities. Note that the scales of the two insets are not comparable because of the use of different detector gains for detection of $\operatorname{CO}\left(v_{f}=0\right)$ and $\operatorname{CO}\left(v_{f}=1\right)$. The shape of 
the temporal profiles does not change significantly with $v_{f}$ or surface temperature. They exhibit a broad maximum centered at $250 \mu \mathrm{s}$ and a slight tail at longer times. In addition, the intensity is not affected for $v=0 \rightarrow 0$ scattering. In contrast, the signal in the $v=0 \rightarrow 1$ channel increases strongly with increasing $T_{S}$ which is in agreement with the observed increase in REMPI signal with increasing $T_{S}$ (see Fig. 4.1). The observations for higher and lower incidence energy are similar but the arrival distributions shift in time. Note that the temporal profile of the molecular beam does not reflect the speed distribution of the scattered molecules. I measured all arrival time distributions at $\sim 12 \mathrm{~mm}$ distance from the surface, which corresponds to a flight-time of about $8 \mu$ from the surface to the detection laser for a final speed of $1500 \mathrm{~m} / \mathrm{s}$. This is much shorter than the temporal width of the molecular beam pulse (FWHM 70$100 \mu \mathrm{s})$.
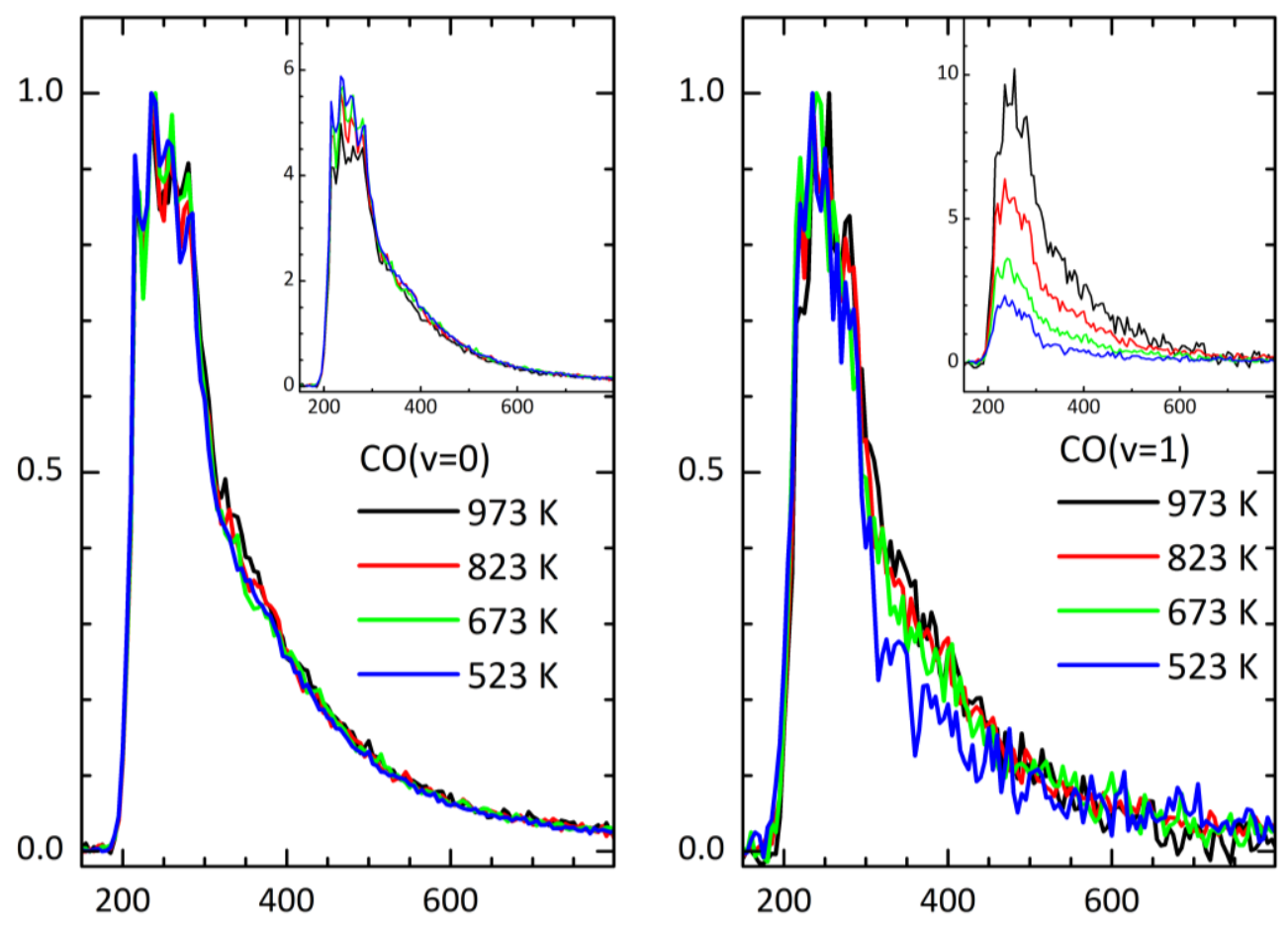

time after nozzle opening / $\mu \mathrm{s}$

Fig. 4.4: Arrival time distributions for $\operatorname{CO}\left(v_{f}=0,1\right)$ after scattering from $\mathrm{Au}(111)$ at different surface temperatures and $0.65 \mathrm{eV}$ incidence energy. The large panels show the profiles normalized to the peak while the insets show the measured intensity in arbitrary units. The shape of the temporal profiles does not depend on the temperature of the surface as well as the intensity of the $0 \rightarrow 0$ channel (left panels). The measured intensity of the $0 \rightarrow 1$ channel increases with increasing surface temperature (inset in the right panel) but the profile does not change its shape.

\section{Excitation probabilities}

The presented data is sufficient for the determination of absolute vibrational excitation probabilities. I correct the integral of the (0-0) and (1-1) Q branch for changes in laser power and detector gain as well as for differences in angular and arrival time distribution. The latter is only a minor correction because the shape of the arrival time distribution does not change significantly with surface temperature or final vibrational state $v_{f}$. The dependence of the REMPI signal on laser intensity follows equation 4.2. The exponent of $n=2.17$ indicates saturation of the ionization step. The gain curve of the MCP detector is shown in Appendix A. 


$$
f\left(I_{v}\right) \propto I_{v}^{2.17}
$$

Because I could not observe the $v_{f}=1$ angular distributions at the lowest and highest $E_{I}$, I have to make assumptions for these values. For $E_{I}=0.84 \mathrm{eV}$ it is reasonable to assume that the angular distributions does not change significantly compared those at $E_{I}=0.65 \mathrm{eV}$. The situation is different at $E_{I}=0.16 \mathrm{eV}$ at which trapping desorption might become important, although I do not observe clear evidence that trapping-desorption is important. However, I point out that this can well be related to the experimental conditions. Scattering at near normal incidence angles makes the observation of small trappingdesorption contributions difficult. For example, if I assume a trapping probability of $30 \%$, like observed by Rettner [46] at $T_{S}=500 \mathrm{~K}$, with a $\cos \theta$ distribution, and $70 \%$ direct scattering, with a $\cos ^{9} \theta$ distribution, the resulting angular distribution broadens to about $\cos ^{6.6} \theta$ and still shows specular scattering (Fig. 4.5). In addition, the angular distribution exhibits a small increase in intensity at large scattering angles $\theta_{f}>40^{\circ}$ which are not detectable within the limits of the experiment. This estimation of the appearance of trapping-desorption in the angular distributions of CO scattering from Au(111) illustrates that trapping can be significant although the experimental angular distributions are narrow. However, this hypothesis only holds when direct scattering and trapping-desorption both contribute to the final population in the detected quantum state. This is likely to be the case for vibrationally elastic $v=0 \rightarrow 0$ scattering.

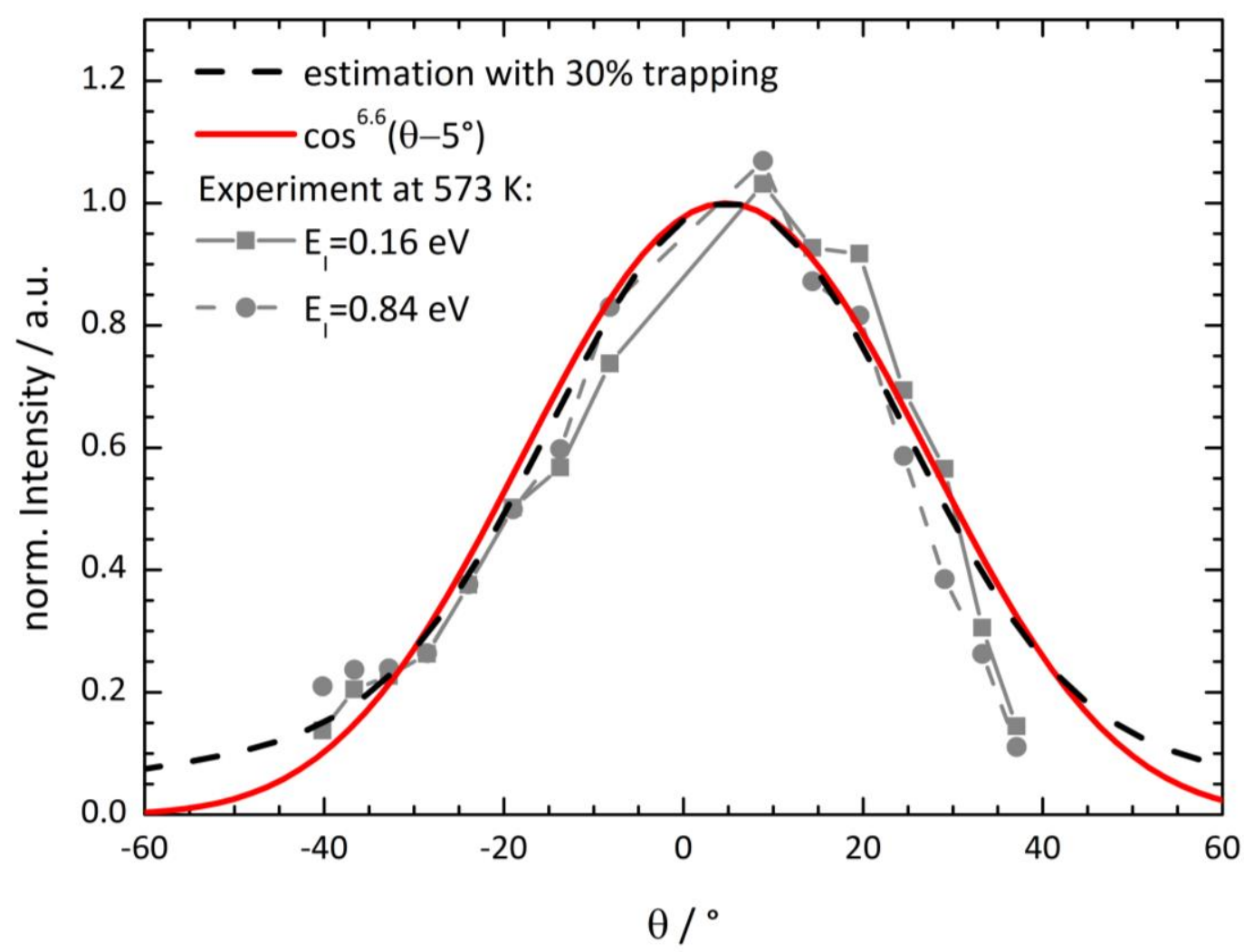

Fig. 4.5: Influence of trapping-desorption onto the angular distribution of CO scattering from Au(111). The dashed line shows the prediction for $30 \%$ trapping-desorption appearing in $\cos \theta$ and $70 \%$ direct scattering decribed by a $\cos ^{9}\left(\theta-5^{\circ}\right)$ distribution. The red line shows a fit to the predicted angular distribution. The gray points show the experimental data for $E_{I}=0.16 \mathrm{eV}$ and $0.84 \mathrm{eV}$ to illustrate the scatter in the data. 

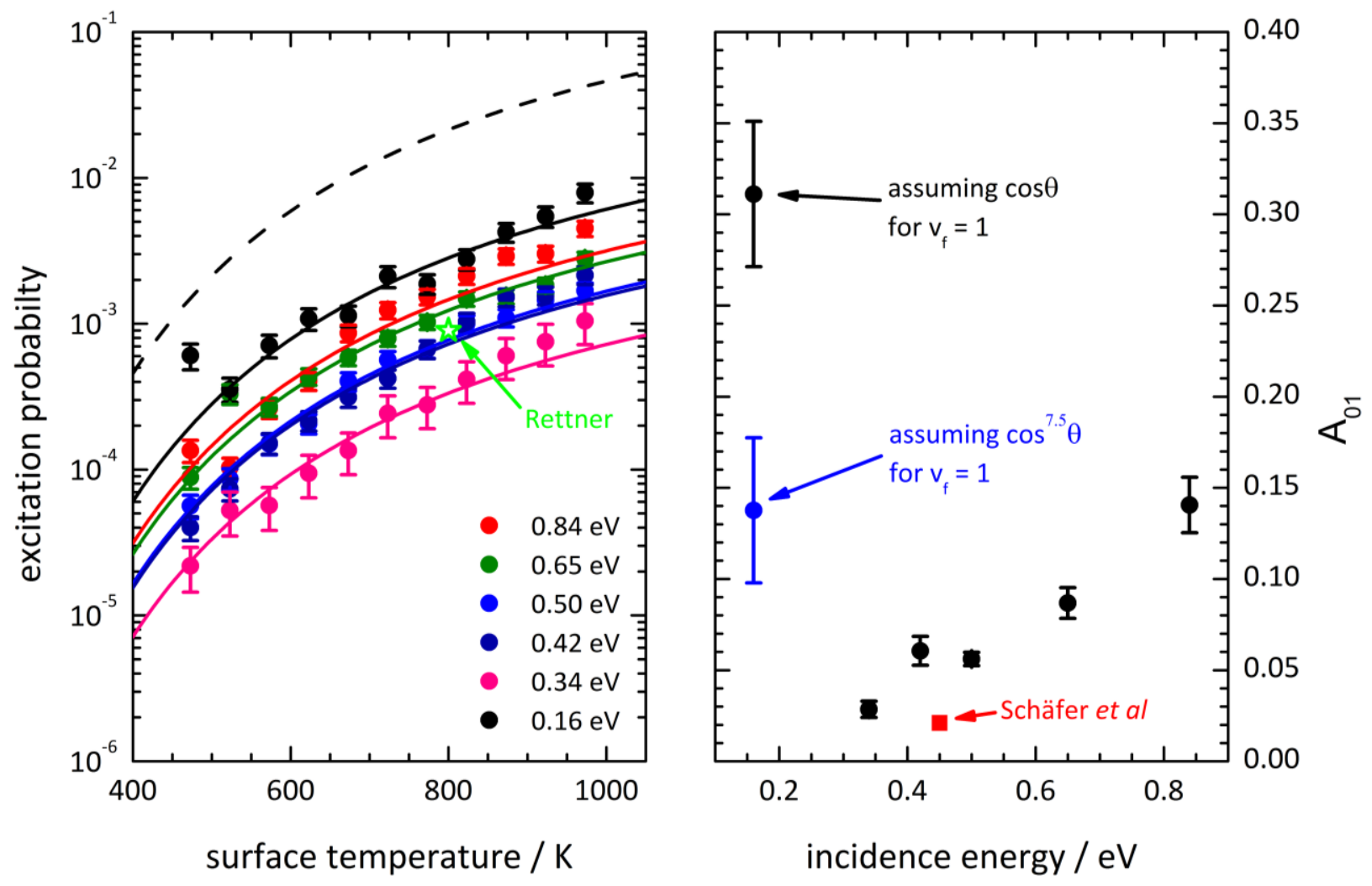

Fig. 4.6: Left panel: $\mathrm{CO}(v=0 \rightarrow 1)$ excitation probabilities as function of surface temperature for incidence energies ranging from 0.16-0.84 eV. The solid lines represent Arrhenius fits where the activation energy is fixed to $\Delta E_{v i b}=0.265 \mathrm{eV}$. The dashed curve corresponds to the thermal limit which is expected for complete equilibration at the surface temperature. The green star shows the only excitation probability observed by Rettner [46], which I like to refer to as Checkpoint Charlie. Right panel: Arrhenius prefactors resulting from the fits as function of incident kinetic energy. The error bars indicate $90 \%$ confidence interval. The red square show the prefactor observed by Schäfer et al. [132]. For $E_{I}=0.16 \mathrm{eV}$, I show two different prefactor resulting from different assumptions for the not observable angular distribution of $v_{f}=1$.

The angular distribution for $\mathrm{CO}\left(v_{f}=1\right)$ can be significantly different. It can either be similar to that for $v=0 \rightarrow 0$ or it can follow a $\cos \theta$ distribution. The first case assumes that vibrational excitation probabilities in direct scattering and trapping-desorption processes are similar whereas the latter assumption intends that $v=0 \rightarrow 1$ excitation is exclusively caused by trapping-desorption while excitation upon direct scattering is negligible. The difference in the correction for angular dilution to the excitation probability between these two assumptions is a factor of $\sim 2.3$, resulting from the ratio $\int_{-\pi / 2}^{\pi / 2} \cos ^{7.5} \theta d \theta / \int_{-\pi / 2}^{\pi / 2} \cos \theta d \theta$.

The resulting vibrational excitation probabilities (solid symbols) are shown as function of surface temperature in the left panel of Fig. 4.6 for different incidence energies ranging from $0.16-0.84 \mathrm{eV}$. The solid lines are Arrhenius fits (equation 4.3) for which the activation energy is fixed to the vibrational energy uptake $\Delta E_{v i b}=0.265 \mathrm{eV}$ and only the preexponential factor $A_{01}$ is fitted. The dashed line indicates complete equilibration with surface (thermal limit).

$$
P_{01}\left(T_{S}\right)=A_{01} \times e^{-0.265 e V / k_{B} T_{S}}
$$




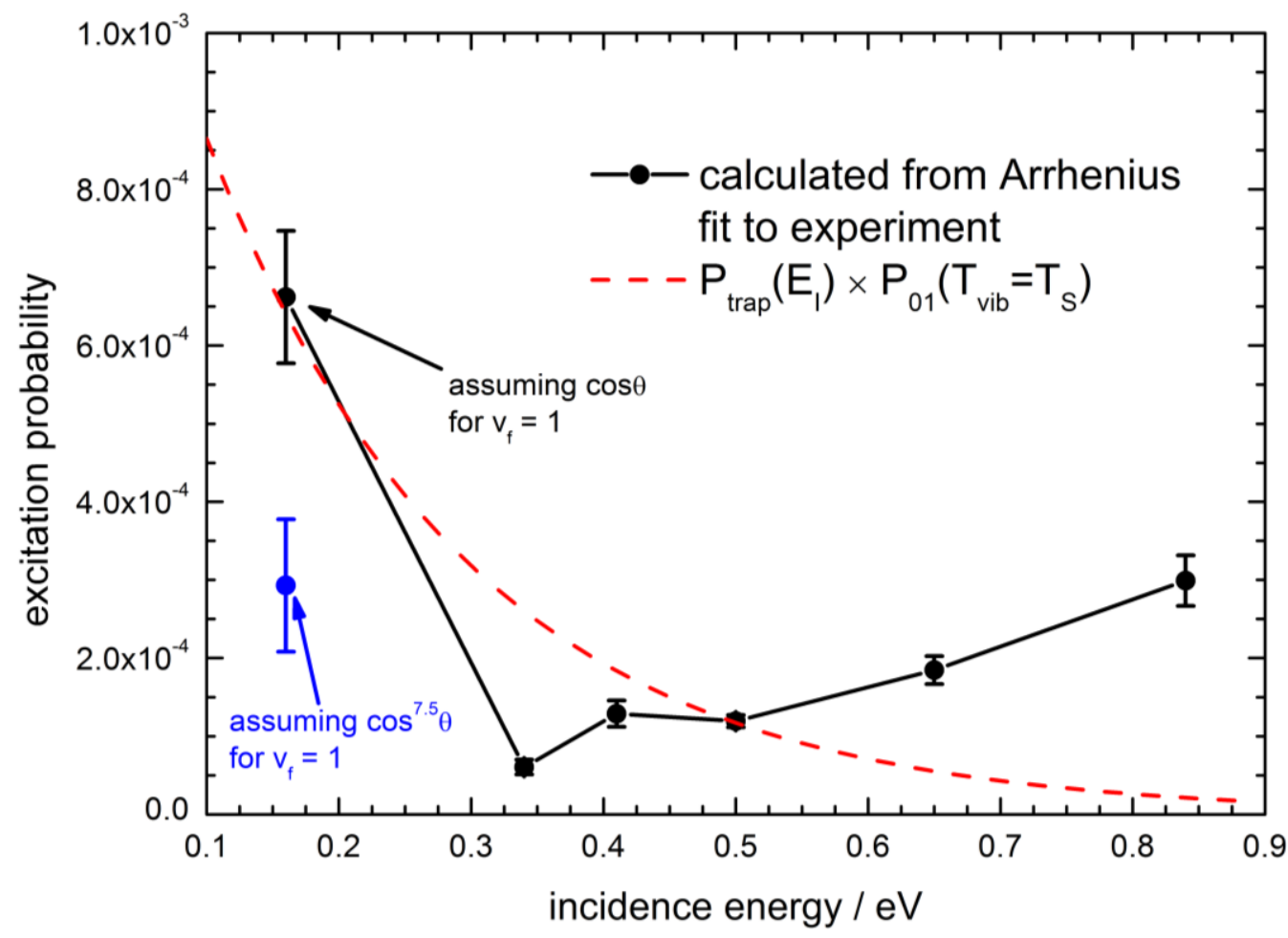

Fig. 4.7: Comparison of the observed $E_{I}$ dependent $\mathrm{CO}(v=0 \rightarrow 1)$ vibrational excitation probabilities (black) at $T_{S}=500 \mathrm{~K}$ to the expectation (red dashed curve) for trapping followed by desorption after complete equilibration with the surface. The excitation probabilities are calculated from the Arrhenius fits to the experimental data (Fig. 4.7). The trapping prediction was calculated using the trapping probability of Rettner [46], $P_{\text {trap }}\left(E_{I}\right)=0.67 \times e^{-E_{I} / 0.2 \mathrm{eV}}$, multiplied with the thermal population of $\mathrm{CO}(v=1)$ at the temperature of the surface.

The surface temperature dependence of the vibrational excitation probabilities follows the Arrhenius prediction over almost the complete range of temperatures. The only deviations occur for $T_{S}<500 \mathrm{~K}$. The $E_{I}$ dependence of the excitation probabilities is reflected in a change of the Arrhenius prefactor, $A_{01}$. This dependence is shown in the right panel of Fig. 4.6. Starting at high incidence energies, the prefactor first drops from about $A_{01}=0.14$ at $E_{I}=0.84 \mathrm{eV}$ to less than 0.03 at $E_{I}=0.34 \mathrm{eV}$. For further decrease of $E_{I}$ to $0.16 \mathrm{eV}$, the prefactor $A_{01}$ increases again to 0.15 or 0.3 , depending on the assumption for the $v_{f}=1$ angular distribution. This is in contrast to the observation for $N O / A u(111)[25,26]$ or $\mathrm{NO} / \mathrm{Ag}(111)[8,44]$ scattering which represent comparable and well understood benchmark systems.

\subsubsection{Discussion}

The primary experimental observation is that vibrational excitation of $\mathrm{CO}$ scattered off $\mathrm{Au}(111)$ is possible and takes place. One of the main questions is whether this excitation is related to direct scattering, like for $\mathrm{NO} / \mathrm{Au}(111)[25-27,30]$ and $\mathrm{NO} / \mathrm{Ag}(111)[8,44]$, or results from trapping followed by desorption, as suggested by Rettner [46]. Angular and arrival time distributions are in general the main indicators to distinguish between the two scattering mechanisms. Direct scattering yields narrow angular distributions and specular scattering while trapping-desorption causes broad angular distributions peaking at the surface normal. The temporal profile of the scattered molecular beam (Fig. 4.4) does not provide the required time resolution to distinguish the two components because the distributions reflect the temporal profiles of the incident molecular beam pulse and are not related to velocity spread. 
The experimental angular distributions in Fig. 4.3 are narrow and peak near the specular angle under all conditions of this work. Even at the lowest incidence energy of $E_{I}=0.16 \mathrm{eV}$ the angular distribution is as narrow as $\cos ^{7.5} \theta$ and exhibits specular scattering for $\operatorname{CO}\left(v_{f}=0\right)$. However, this does not exclude a significant fraction of molecules undergoing trapping followed by desorption (see Fig. 4.5).

Furthermore, the angular distributions broaden slightly with increasing surface temperature. This effect, which has been previously observed for $\mathrm{NO} / \mathrm{Au}(111)$ [26] scattering, is likely related to more thermal motion of the surface atoms with increasing surface temperature. Furthermore, the angular distributions are narrow for both vibrational channels indicating that direct scattering should dominate the excitation mechanism under the conditions of the experiment. As mentioned above, the signal-to-noise ratio for $v=0 \rightarrow 1$ excitation at $E_{I}=0.16 \mathrm{eV}$ was not sufficient in the experiment to obtain angular distributions for $v_{f}=1$. Therefore, it is not clear whether the observation also holds for vibrational excitation at the lowest incidence energy. For both assumptions of the angular distributions, whether it is similar to that of $v_{f}=0$ (blue point in Fig. 4.6) or follows a $\cos \theta$ distribution (black point in Fig. 4.6), the observed excitation probabilities are higher than those observed for higher $E_{I}$ with a prefactor between $A_{01} \sim 0.14$ and $\sim 0.32$, respectively.

In general, the measured excitation probabilities are about a factor of 3-5 lower than observed for $\mathrm{NO}(v=0 \rightarrow 1)$ excitation upon scattering from $\mathrm{Au}(111)$. The excitation probabilities are in good agreement with the previous observation by Rettner [46] who used a $0.7 \mathrm{eV}$ beam and measured an excitation probability of $9 \times 10^{-4}$ at $800 \mathrm{~K}$ surface temperature (green star in Fig. 4.6). However, Rettner could not exclude that vibrational excitation happens upon a trapping-desorption mechanism whereas my results indicate excitation in a direct scattering process at similar incidence energies. The experimental data can be well described by an Arrhenius function with an activation energy equal to the vibrational spacing of $0.265 \mathrm{eV}$. This indicates that the vibrational energy is taken from a thermal bath and not from translational energy, like in the case of $\mathrm{NH}_{3} / \mathrm{Au}(111)$ scattering [4]. From the previous observations on vibrational lifetimes of $\mathrm{CO}$ at metal surfaces [48, 50] and insulators [53], it seems reasonable that the vibrational energy is taken from excited EHPs of the metal, especially for short interaction times as they are expected for direct scattering.

Another indicator for nonadiabatic V-EHP coupling is the dependence of the excitation probabilities on incidence energy. The $E_{I}$ dependence is included in the Arrhenius prefactor which is related to the intrinsic coupling strength between the molecular vibration and the EHPs of the metal [27, 30]. In case of $\mathrm{NO} / \mathrm{Au}(111)$ the prefactors for $0 \rightarrow 1$ and $0 \rightarrow 2$ excitation increased with increasing incidence energy with zero threshold $[25,26] . \mathrm{CO}(v=0 \rightarrow 1)$ excitation on $\mathrm{Au}(111)$ exhibits a slightly different behavior. The prefactor decreases first with decreasing incidence energy between $E_{I}=0.84 \mathrm{eV}$ and $0.34 \mathrm{eV}$ but increases again at lower $E_{I}$. At $E_{I}=0.16 \mathrm{eV}$ the Arrhenius prefactor is similar to that at the highest incidence energy $\left(E_{I}=0.84 \mathrm{eV}\right)$ if I assume similar angular distributions for $v_{f}=0$ and $v_{f}=1$, and even exceeds the high $E_{I}$ value by a factor of $\sim 2$ for assumption of a $\cos \theta$ angular distribution. The rise at low $E_{I}$ is probably related to an increasing fraction of $v_{f}=1$ produced by trapping-desorption. The prediction for trapping and desorption after complete equilibration of the CO vibration with the surface temperature can be calculated from the trapping probability and the vibrational population at $T_{S}=T_{v i b}$ $\left(A_{01}=1, P_{v=1} \ll P_{v=0}\right)$. The trapping probability for $\mathrm{CO}$ on $\mathrm{Au}(111)$ was measured by Rettner [46] and can be described by a simple exponential decay $P_{\text {trap }}\left(E_{I}\right)=A \times e^{-E_{I} / \gamma}$ with the $T_{S}$ dependent parameters $A$ and $\gamma$. For a surface temperature of $500 \mathrm{~K}$, Rettner obtained parameters of $A=0.67$ and $\gamma=0.20 \mathrm{eV}$. The predicted excitation probability from trapping-desorption is then the product of trapping probability and thermal population, $P_{\text {exc }}\left(E_{I}\right)=A \times e^{-E_{I} / \gamma} \times e^{-0.265 \mathrm{eV} / k_{B} T_{S}}$. A comparison of the trapping-desorption prediction (red dashed curve) to the vibrational excitation probability at $T_{S}=500 \mathrm{~K}$ calculated from the derived Arrhenius parameters (black dots) is shown in Fig. 4.7. For 
$E_{I}=0.16 \mathrm{eV}$, this calculation predicts an excitation probability of $6.4 \times 10^{-4}$ which is in good agreement with the measured excitation probability of $(6.6 \pm 0.8) \times 10^{-4}$ under the assumption of a $\cos \theta$ angular distribution for $v=0 \rightarrow 1$ excitation. However, the prediction clearly overestimates the excitation probability at $E_{I}=0.16 \mathrm{eV}$ if I assume a $\cos ^{7.5} \theta$ angular distribution as observed for $v=0 \rightarrow 0$ scattering. The trapping probability decreases with increasing incidence energy and the predicted excitation into $v_{f}=1$ will follow the same trend. The trapping prediction for vibrational excitation further clearly overestimates the experimental values for $E_{I}=0.34 \mathrm{eV}$ and $0.42 \mathrm{eV}$ and agrees again with the measured value at $E_{I}=0.50 \mathrm{eV}$. However, the latter agreement is probably a coincidence because of the missing agreement at $E_{I}=0.34 \mathrm{eV}$ and $0.42 \mathrm{eV}$. In addition, I clearly observe angular distributions that are as narrow as $\cos ^{4-5} \theta$ which favor vibrational excitation upon direct scattering but do not exclude significant trapping-desorption. This comparison shows that the simple prediction for vibrational excitation based on the $E_{I}$ dependent trapping of $\mathrm{CO}$ on $\mathrm{Au}(111)$ fails to reproduce the experimental excitation probability at medium $E_{I} \geq 0.34 \mathrm{eV}$. However, the increase in excitation probability at the lowest $E_{I}$ is an indication that trapping followed by desorption becomes important. The high uncertainty due to the not observable angular distribution for $v_{f}=1$ at this incidence energy makes a judgment about the agreement with the trapping-desorption prediction difficult but the assumption of a $\cos \theta$ angular distribution gives an upper limit for the excitation probability.

Nevertheless, the general trend in the $E_{I}$ dependent vibrational excitation probability supports the view that the $\mathrm{CO}\left(v_{f}=0 \rightarrow 1\right)$ excitation is caused by two different mechanisms. The excitation upon direct scattering decreases with decreasing incidence energy similar to NO/Au(111) $[25,26]$ and $\mathrm{NO} / \mathrm{Ag}(111)[8$, 44]. Vibrational excitation resulting from trapping-desorption increases with decreasing incidence energy and starts being important for $E_{I}<0.4 \mathrm{eV}$. However, this increase in $P_{01}$ is only partly in agreement with the thermal expectation for trapping followed by desorption after equilibration with the surface. The deviations, especially at $E_{I}=0.34-0.42 \mathrm{eV}$, might be an indication that the trapped CO molecules are desorbed before complete equilibration. The residence time at the surface can be estimated by a simple Arrhenius equation, which is frequently referred to as Wigner-Polanyi equation in surface science. If I assume a reasonable prefactor of $10^{13} \mathrm{~s}^{-1}$ and a binding energy of $0.02 \mathrm{eV}$ [133], this calculated residence time $\left(\tau_{\text {des }}=1 / k_{\text {des }}\right)$ is only $\sim 0.2 \mathrm{ps}$ at room temperature and decreases further at higher surface temperatures. For weakly bound molecules it might therefore very well happen that the residence time at the surface reaches a regime at which it is comparable to the vibrational lifetime.

\subsubsection{Summary and Conclusions}

In summary, I measured vibrational $\mathrm{CO}(v=0 \rightarrow 1)$ excitation probabilities in scattering off $\mathrm{Au}(111)$ for different incident translational energies ranging from $0.16-0.84 \mathrm{eV}$. Under all conditions, the excitation probabilities increase with surface temperature in an Arrhenius-like fashion with an activation energy equal to the vibrational energy uptake. The preexponential factors of the Arrhenius fits are about a factor 3-5 lower than observed for the NO/Au(111) system. Furthermore, the prefactor first decreases from $E_{I}=0.16 \mathrm{eV}$ to $0.34 \mathrm{eV}$ before it starts increasing again with increasing incidence energy. To first approximation, this behavior is consistent with an increasing fraction of $\operatorname{CO}\left(v_{f}=1\right)$ produced by a trapping-desorption mechanism. 


\section{2. $\operatorname{CO}(v=2)$ scattering from $\operatorname{Au}(111)$}

Besides the study of vibrational excitation in gas-surface collisions, vibrational relaxation is an appropriate process to learn about the nonadiabatic effects in gas-surface interactions. While NO relaxation in scattering from $\mathrm{Au}(111)$ has been studied in detail $[88,112,117]$, I am not aware of any study on vibrational relaxation of $\mathrm{CO}$ at a metal surface. Bartels et al. showed recently that preparation of CO molecules in high vibrational states is possible [134] but rather complicated due to the lack of electronic states suitable for stimulated emission pumping. An easier approach is the preparation of $\mathrm{CO}$ molecules in low vibrational states using direct IR overtone pumping. However, detection of CO molecules in low $v$ states is challenging. (1+1) REMPI using the $A^{1} \Pi \leftarrow X{ }^{1} \Sigma^{+}$transition can be used to detect high vibrational states [134] but turns into an undesirable (2+2) REMPI scheme for low $v<11$ states [135]. (2+1) REMPI using the $B^{1} \Sigma^{+} \leftarrow X^{1} \Sigma^{+}$transition only works with high sensitivity for $\mathrm{CO}(v=0,1)$ because the $B$ state predissociates for $v^{\prime}>1$ and Franck-Condon factors decrease dramatically for $v^{\prime} \neq v^{\prime \prime}$ [97]. Therefore only detection of $\mathrm{CO}(v \leq 2)$ is possible via the $B \leftarrow X$ transition using the (0-0), (1-1) and (1-2) band. This scheme is already less sensitive for $\operatorname{CO}(v=2)$ than it is for $\mathrm{CO}(v=0,1)$ by a factor of about 50 because of the decrease in Franck-Condon factor.

In this section, I present results for scattering of incident $\mathrm{CO}\left(v_{i}=2\right)$ molecules from $\mathrm{Au}(111)$ which are prepared at a well-defined distance from the surface using IR overtone pumping. I investigated the scattering into the final vibrational states $v_{f}=1,2 ; 2 \rightarrow 0$ scattering cannot be observed due to the background of $v_{i}=0$ molecules in the molecular beam that were not excited by the IR laser. I measured branching ratios between the two vibrational states for different incidence energies ranging from $E_{I}=0.26-0.9 \mathrm{eV}$. The relative population of $\mathrm{CO}\left(v_{f}=1\right)$ is only $2-5 \%$ of the final $v_{f}=2$ population, which is about one order of magnitude lower than in case of $\mathrm{NO}(v=3 \rightarrow 1,2)$ scattering (section 3.2). The low $2 \rightarrow 1$ relaxation rate indicates that $2 \rightarrow 0$ relaxation is probably negligible and that the branching ratios are very close to absolute probabilities.

I observed an additional effect in the high resolution time-of-flight profiles at low incidence energies $\left(E_{I}<0.6 \mathrm{eV}\right)$. The arrival time profiles of the $2 \rightarrow 1$ channel show a bimodal distribution with a fast component that is present at all $E_{I}$ and a second slow component that is only present at $E_{I}<0.6 \mathrm{eV}$. The two components are likely related to contributions from direct scattering and a trapping-desorption mechanism. The latter is rather surprising because vibrational lifetimes of molecules at metal surfaces are believed to be only a few picoseconds $[48,50]$. Therefore, vibrational energy is expected to be almost immediately dissipated to EHPs if the molecule is trapped. For the $2 \rightarrow 2$ channel, I could not observe a trapping component in a simple IR-UV double resonance experiment. However, I applied a new two-dimensional time-of-flight experiment using IR-UV-UV triple resonance to show that the trapping component is also present in the $2 \rightarrow 2$ channel but simply made more difficult to observe by a dominant direct scattering component.

\subsubsection{Results}

For the determination of relaxation probabilities the IR laser is placed about $15 \mathrm{~mm}$ away from the surface and focused with a $750 \mathrm{~mm} \mathrm{CaF}_{2}$ lens. The REMPI laser detects the scattered molecules at $11 \mathrm{~mm}$ distance from the surface at $\sim 24^{\circ}$ scattering angle and is focused with a $500 \mathrm{~mm} \mathrm{CaF}$ lens. Like in the measurements on $\mathrm{NO}\left(v_{i}=3\right.$ ) relaxation (section 3.2), I can only determine branching ratios $R_{v_{f}}$ between the final vibrational states $v_{f}=1,2$. The branching ratio is defined as the number of molecules in one vibrational states divided by the number of molecules in all observable vibrational states: 


$$
R_{v_{f}}=\frac{N_{v_{f}}}{\sum_{v_{f}} N_{v_{f}}}=\frac{N_{v_{f}}}{N_{1}+N_{2}}
$$

The population is again given by equation 3.3 and is proportional to the observed REMPI signal corrected for differences in angular and arrival time distribution, laser power, detector gain and Franck-Condon factors. The dependence of the REMPI signal on laser intensity follows equation 4.2 for the (1-1) and the (1-2) band. The gain curve of the MCP detector is shown in Appendix A.

\section{REMPI spectra}

Fig. 4.8 shows examples for typically observed REMPI spectra for $\operatorname{CO}\left(v_{f}=2,1\right)$ at different incidence energies ranging from $0.25-0.92 \mathrm{eV}$. The spectra reflect the raw data and were recorded with similar laser intensity and identical detector gain. As pointed out above, $\operatorname{CO}\left(v_{f}=2\right)$ molecules can only be detected in the (1-2) band because $v^{\prime}=2$ in the $B$ state predissociates. Because the Franck-Condon factor of this vibrational band is low $\left(1.41 \times 10^{-2}\right.$ [97]) compared to the (1-1) band (0.972 [97]), the REMPI signal for $\mathrm{CO}\left(v_{f}=2\right)$ molecules is comparable or even smaller to that of molecules that lost one vibrational quantum in the surface collision.
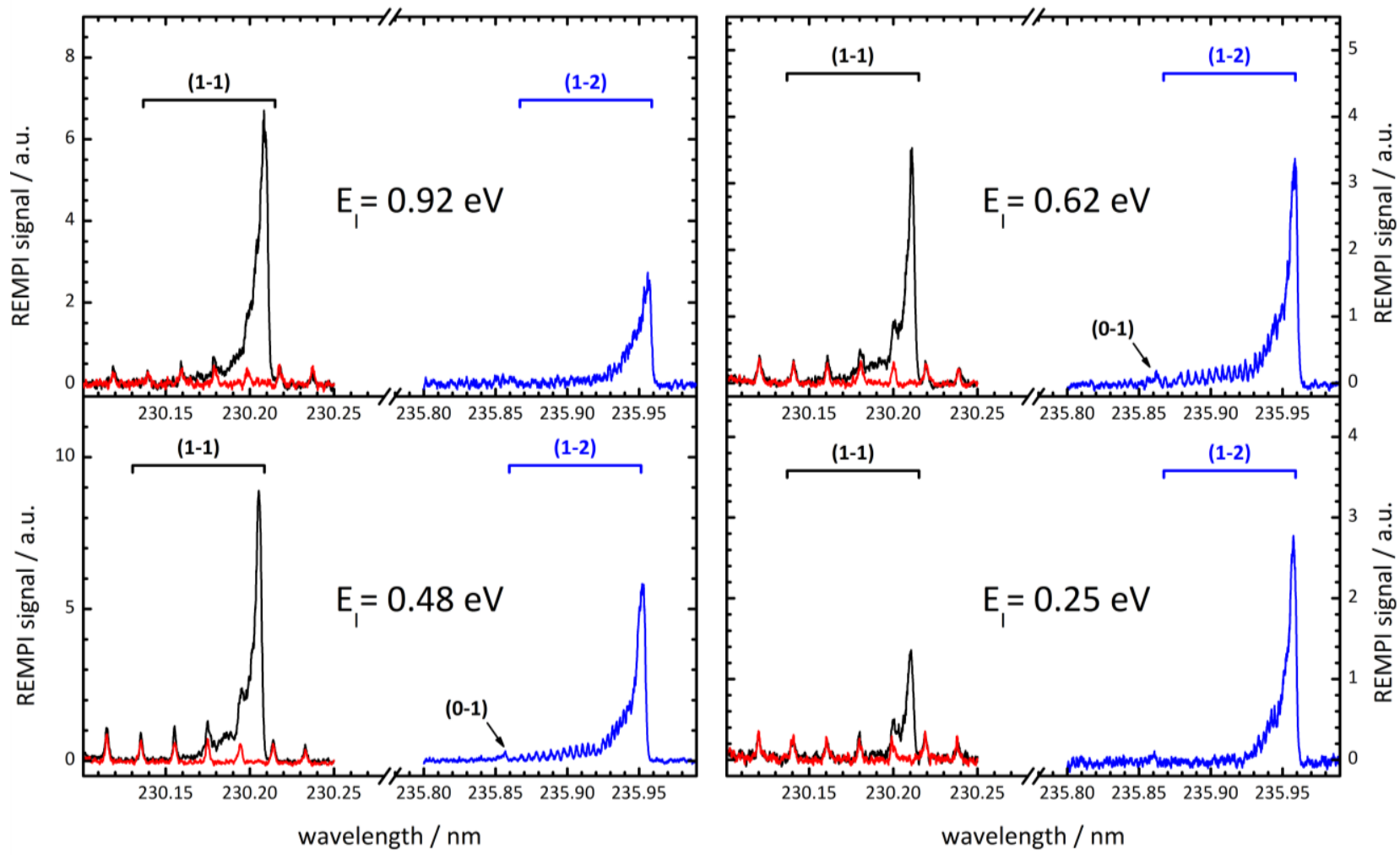

Fig. 4.8: REMPI spectra for $\mathrm{CO}\left(v_{i}=2\right)$ scattering into $v_{f}=1,2$ at different incidence energies and $320 \mathrm{~K}$ surface temperature. All spectra were measured at the peak of the arrival time distribution (see Fig. 4.9). The blue spectra show the $Q$ branch of the $B^{1} \Sigma^{+}\left(v^{\prime}=1\right) \leftarrow X^{1} \Sigma^{+}\left(v^{\prime \prime}=2\right)$ band. The black spectra show the (1-1) Q branch of the same electronic transition which is overlapped with the (0-0) O branch. The red spectra show the same spectral range with the IR laser blocked. The spectra for one single incidence energy were recorded with similar laser intensities and identical detector gain and laser beam positions. Note that the Franck-Condon factor of the (1-2) band is about 50 times lower than for the (1-1) band which results in comparable intensities for the (1-2) and (1-1) band. The ratio can also be estimated from the small peaks at $235.86 \mathrm{~nm}$ which correspond to the (0-1) band which has a Franck-Condon factors comparable to that of the (1-2) band. The relative intensity of $v_{f}=1$ decreases with respect to $v_{f}=2$ with decreasing incidence energy. 
Consequently, the population of $v_{f}=2$ molecules after scattering is significantly higher than for $v_{f}=1$. This effect is also illustrated by the rather small signal arising from the $B \leftarrow X$ (0-1) Q branch at about $235.86 \mathrm{~nm}$ for which the Franck-Condon factor is comparable to the (1-2) transition. Because of the lower $\mathrm{S} / \mathrm{N}$ at the highest and lowest incidence energy, this peak is only clearly visible at $E_{I}=0.48 \mathrm{eV}$ and $0.62 \mathrm{eV}$. The black spectra show the (1-1) Q branch which is overlapped with the (0-0) O branch. The red spectra were taken with the IR laser turned off. A comparison of the red and black spectra illustrates that $v_{f}=1$ is almost exclusively produced upon relaxation from $v_{i}=2$. Furthermore, I use the spectra with the IR turned off to subtract the intensity resulting from the (0-0) O branch from the (1-1) $Q$ branch. The (1-1) and (1-2) Q branches are then integrated to obtain the population of the respective vibrational states summed over all rotational states.

Already from the relative intensities of the (1-1) and (1-2) Q branches one can conclude on the $E_{I}$ dependence of the vibrational relaxation probability. That is, the intensity of the $\mathrm{CO}\left(v_{f}=1\right)$ signal with respect to the (1-2) intensity increases with increasing incidence energy. In addition, the rotational excitation in the $2 \rightarrow 2$ channel depends slightly on incidence energy while the rotational contour of the (1-1) band is independent of $E_{I}$.

\section{Arrival time distributions}

The arrival time distributions for different $E_{I}=0.92 \mathrm{eV}, 0.62 \mathrm{eV}$ and $0.25 \mathrm{eV}$ are shown in Fig. 4.9. All profiles are normalized to the peak for comparison. The displayed flight time corresponds to the time that the molecules travel from the IR excitation until they are detected by REMPI. This includes traveling about $\sim 15 \mathrm{~mm}$ at the incident beam velocity, $s_{i}$, from the IR laser to the surface collision and $11 \mathrm{~mm}$ from the surface to the REMPI laser at the (possibly different) scattered velocity, $s_{f}$. The arrival time estimated from a simple hard cube (Baule) model is shown as vertical gray dashed lines in Fig. 4.9. Because the IR excitation produces a short pulse of $\mathrm{CO}\left(v_{i}=2\right)$ molecules the temporal profiles of the scattered molecules are much narrower compared to those presented in Fig. 4.4. Consequently, effects like differences in the speed distribution of scattered molecules (velocity spread) become visible in these measurements.

At $E_{I}=0.92 \mathrm{eV}$, the time-of-flight distributions for $2 \rightarrow 2$ (black) and $2 \rightarrow 1$ (red) scattering are almost indistinguishable from each other and both peak at around $11 \mu \mathrm{s}$. This is in contrast to vibrational relaxation of $\mathrm{NO}\left(v_{i}=2,3\right)$ scattering from $\mathrm{Au}(111)$ where I observed a shift towards earlier arrival time for loss of one or two quanta of vibrational energy (see section 3.3). At $E_{I}=0.62 \mathrm{eV}$, both vibrational channels still show the same behavior but are shifted towards later arrival times by $\sim 3 \mu \mathrm{s}$. Furthermore, the $2 \rightarrow 1$ signal does not go exactly back down to zero at $t \geq 20 \mu$ s but stays at about $5 \%$ of the peak intensity. This effect becomes clearly visible at $E_{I}=0.25 \mathrm{eV}$. The black trace is now shifted to about $22 \mu \mathrm{s}$. The red profile also shows a first maximum at the same timing but exhibits an additional pronounced and broad tail at long flight times. Consequently, there is a significant fraction of $\mathrm{CO}\left(v_{f}=1\right)$ molecules that are slower than expected from the profile of the $2 \rightarrow 2$ channel. This effect is absent or only very slightly visible for the higher incidence energies. The first peak agrees very well with the expectation of the hard cube model and reflects direct scattering which is present at all incidence energies. The slow tail component in the time-of-flight profiles probably results from trapping followed by desorption for which a speed distribution is expected that is close to a thermal Maxwell-Boltzmann distribution at $T_{S}$. Because this effect is visible in a vibrationally excited state, my experimental results indicate that $\mathrm{CO}\left(v_{i}=2\right)$ molecules are trapped at the surface and desorbed before complete vibrational equilibration $\left(P_{v=0} \gg P_{v=1} \gg P_{v=2}\right.$ at $\left.T_{v i b}=T_{S}\right)$. 


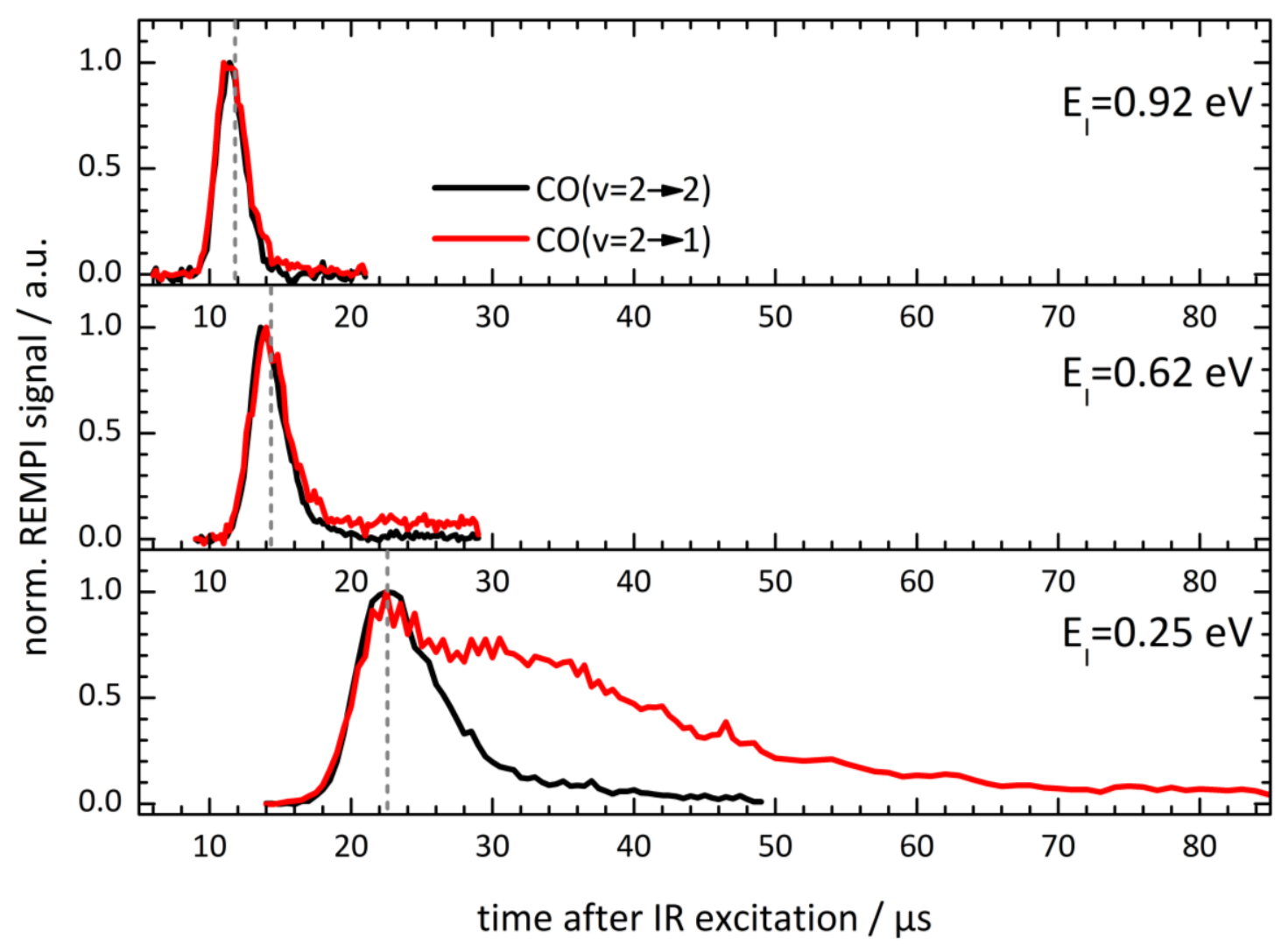

Fig. 4.9: Normalized time-of-flight profiles for $\mathrm{CO}(v=2 \rightarrow 2,1)$ scattering from $\mathrm{Au}(111)$ for three different incidence energies $E_{I}=0.25,0.62,0.92 \mathrm{eV}$. The black traces show the arrival time profiles for vibrationally elastic $2 \rightarrow 2$ scattering while the red traces show the $2 \rightarrow 1$ channel. The flight time corresponds to the time that the molecules travel from the IR excitation until they are detected with REMPI. It therefore represents the sum of the time from IR excitation until surface collision and the time from the surface collision to the REMPI detection. The gray dashed line shows the estimated arrival time from a simple hard cube model. At $E_{I}=0.92 \mathrm{eV}$, both vibrational channels exhibit basically identical time-of-flight distribution. The $2 \rightarrow 2$ channels show similar narrow profiles at all three incidence energies while those for $2 \rightarrow 1$ scattering change to a bimodal distribution with an additional late component that increases with decreasing $E_{I}$.

\section{Relaxation probabilities}

I used the presented data to calculate $\mathrm{CO}\left(v_{f}=1,2\right)$ branching ratios. The REMPI spectra of the (1-2) and (1-1) Q branches are fitted with a corresponding simulation (see Appendix D) and the fit is integrated. The integrals are corrected for differences in detector gain, laser intensity and width of the arrival time profiles. The resulting branching ratios $R_{1}$ and $R_{2}$ are shown in the left panel of Fig. 4.10 as a function of incidence energy. The right panel shows the $E_{I}$ dependent Arrhenius prefactors for $\mathrm{CO}(v=0 \rightarrow 1)$ excitation which were already presented in Fig. 4.6. The overall probabilities for $2 \rightarrow 1$ relaxation are about one order of magnitude lower than observed for NO/Au(111) [88]. The branching ratios show that about $97-99 \%$ of $\mathrm{CO}\left(v_{i}=2\right)$ is scattered vibrationally elastically and only $1-3 \%$ lose one quantum of vibrational energy in the surface collision. The $E_{I}$ dependence of $R_{1}$ is similar to that of the Arrhenius prefactor for $v=0 \rightarrow 1$ excitation. The probability for $2 \rightarrow 1$ relaxation decreases with increasing $E_{I}$ for $0.25 \mathrm{eV} \leq E_{I} \leq 0.45 \mathrm{eV}$. A further increase of incidence energy from $E_{I}=0.45 \mathrm{eV}$ up to $0.92 \mathrm{eV}$ leads to a linear increase of $R_{1}$. I found the same dependence on $E_{I}$ for vibrational excitation which indicates that excitation and relaxation result from the same mechanism. 


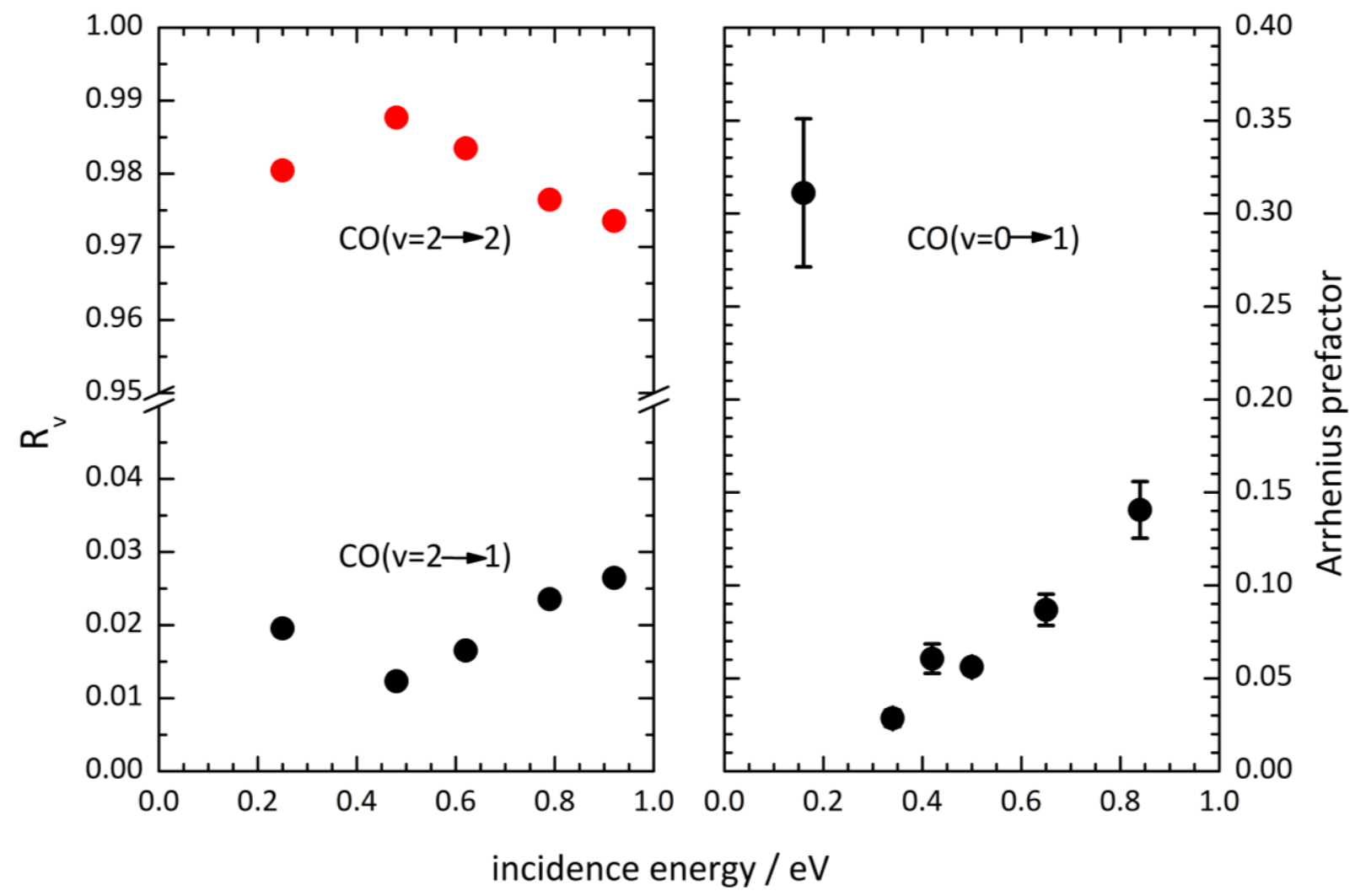

Fig. 4.10: Left panel: branching ratios for $\mathrm{CO}\left(v_{f}=2\right)(\mathrm{red})$ and $\mathrm{CO}\left(v_{f}=1\right)$ (black) as a function of incident translational energy. The fraction of $\mathrm{CO}\left(v_{f}=2\right)$ increases with decreasing incidence energy between $E_{I}=0.48-0.92 \mathrm{eV}$. The relaxation probability into $v_{f}=1$ increases accordingly. If the incidence energy is lowered further the amount of $\mathrm{CO}(v=2 \rightarrow 1)$ scattering increases again. Right panel: Arrhenius prefactors for $\mathrm{CO}(v=0 \rightarrow 1)$ excitation as already shown in Fig. 4.6. The prefactor for $E_{I}=0.16 \mathrm{eV}$ is only shown for the $\cos \theta$ assumption of the $v_{f}=1$ angular distribution. The prefactors for vibrational excitation and the relaxation probabilities show a very similar dependence on incidence energy.

\section{Two-dimensional time-of-flight experiments}

The observed differences in the arrival time distributions for $\operatorname{CO}\left(v_{f}=1\right)$ and $\operatorname{CO}\left(v_{f}=2\right)$ at low incidence energies (lower panel of Fig. 4.9) are very interesting results that require further experimental investigation. A problem of the conventional state-to-state time-of-flight experiments using IR excitation prior to the surface collision is that one cannot distinguish between residence time effects and velocity spread. In contrast, tagging of scattered molecules is only sensitive to effects caused by velocity spread. In order to distinguish residence time from velocity effects, I set-up a two-dimensional state-to-state time-of-flight (2D-TOF) experiment which is sketched in Fig. 4.11. The incident molecular beam of $\mathrm{CO}\left(v_{i}=0\right)$ molecules (black pulse) approaches the surface. Less than $1 \mathrm{~mm}$ in front of the surface the focused IR laser produces a short pulse (narrow red pulse) of incident $\operatorname{CO}\left(v_{i}=2, J_{i}=1\right)$ molecules. The excited molecules are scattered and slightly spread in time due to different velocities. Again less than $1 \mathrm{~mm}$ away from the surface, a first UV pump laser excites the scattered $\operatorname{CO}\left(v_{f}=1,2\right)$ molecules into $v^{\prime}=0$ of the metastable $a^{3} \Pi_{1}$ state (UV tagging). Because of the velocity spread that occurred between surface collision and UV tagging, the laser only excites molecules within a certain range of velocities $s_{f} \pm \Delta s_{f}$ (blue pulse). I finally detect the metastable $\mathrm{CO}^{*}(v=0)$ molecules in $\sim 18 \mathrm{~mm}$ distance via $b{ }^{3} \Sigma^{+} \leftarrow a^{3} \Pi_{1}(1+1)$ REMPI. 


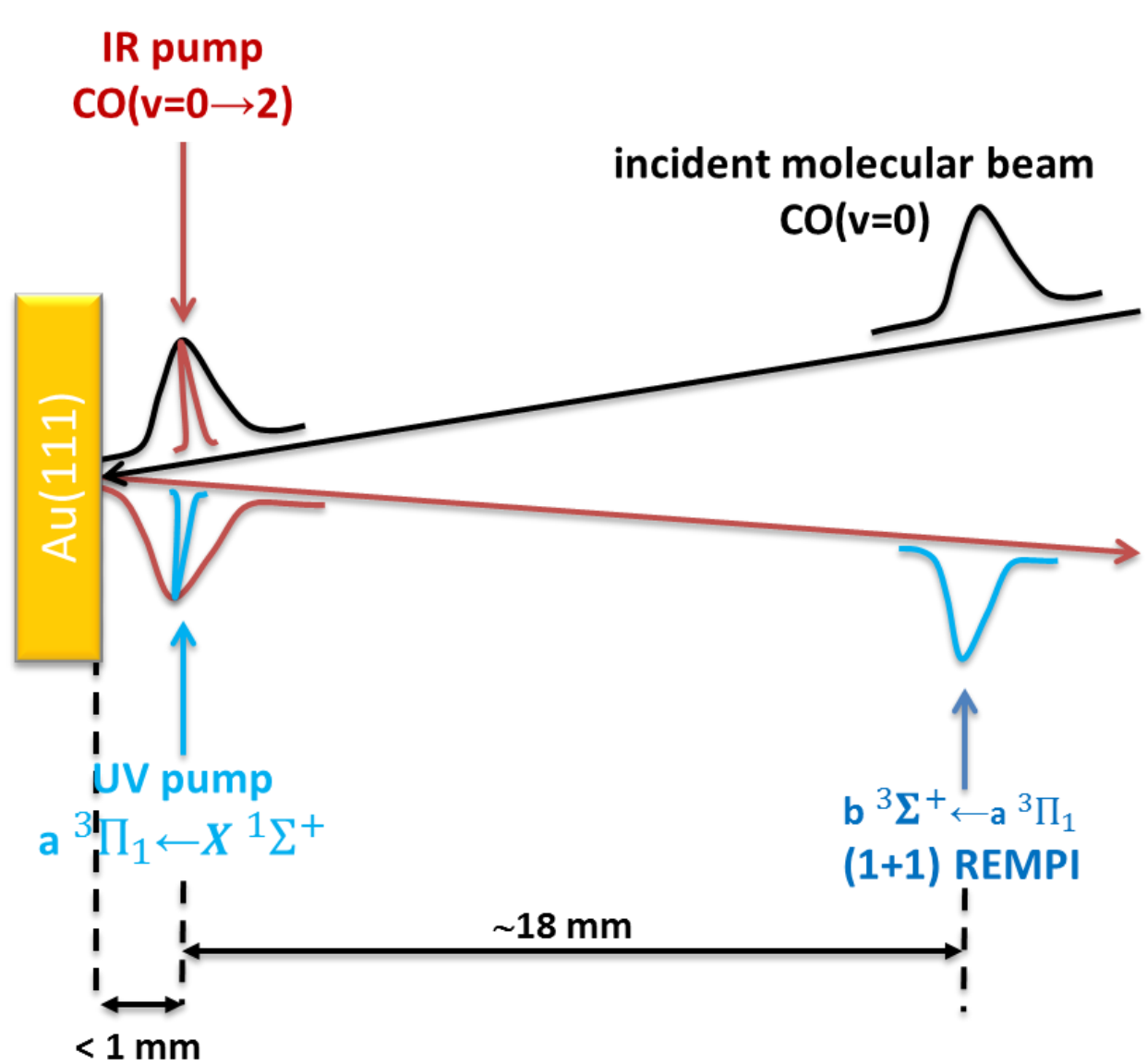

Fig. 4.11: Schematic set-up for the two-dimensional time-of-flight experiment on $\mathrm{CO}(v=2 \rightarrow 2,1)$ scattering from $\mathrm{Au}(111)$ using IR-UV-UV triple resonance. Incident $\mathrm{CO}\left(v_{i}=0\right)$ molecules (black pulse) approach the surface and a small part is pumped by the focused IR laser into $v_{i}=2$ less than $1 \mathrm{~mm}$ in front of the surface (red narrow pulse). The excited molecules are scattered and spread in time due to different velocities. A part of them is excited by the first UV laser into the metastable $a{ }^{3} \Pi_{1}$ state using the (0-2) and (0-1) bands around $226 \mathrm{~nm}$ and $215 \mathrm{~nm}$ (blue pulse). The metastable CO* molecules are finally detected at about $18 \mathrm{~mm}$ distance via $b^{3} \Sigma^{+} \leftarrow a^{3} \Pi_{1}(1+1)$ REMPI around $287 \mathrm{~nm}$.

Besides the selection of different rotational and vibrational states, this experimental technique has two variable parameters: the temporal delay $t_{1}$ between IR excitation and UV tagging and the delay $t_{2}$ between UV tagging and REMPI detection. First, the delay $t_{2}$ can be varied to detect the speed distribution of the scattered molecules. However, in contrast to conventional one-dimensional state-tostate time-of-flight measurements, the detected speed distribution does not necessarily reflect the complete speed distribution of the scattered molecules. The reason is that the first delay $t_{1}$ acts as a (coarse) velocity filter. Therefore, I have to measure time-of-flight spectra for different delays between IR excitation and UV tagging in order to obtain the full velocity distribution. In a second scheme, the delay between UV pumping and REMPI detection remains constant and the delay between IR excitation and UV tagging is varied. Now the constant UV-UV delay acts as a velocity filter with a resolution $\Delta s$ / $s \sim 5.6 \%$, assuming that both UV lasers are focused to a beam diameter of $\sim 0.5 \mathrm{~mm}$. The resulting scans then show the time dependent flux of molecules that leave the surface with a certain speed. The width of the temporal profiles reflects basically the quality of the velocity filter as well as the focal diameter of the IR and the UV pump laser. The latter does influence the experimental data quite strongly because the average flight distance (IR laser-to-surface + surface-to-UV tagging) is less than $2 \mathrm{~mm}$. Therefore a focal diameter of $\sim 0.5 \mathrm{~mm}$ reduces the time resolution dramatically as compared to the second flight distance of $18 \mathrm{~mm}$. 

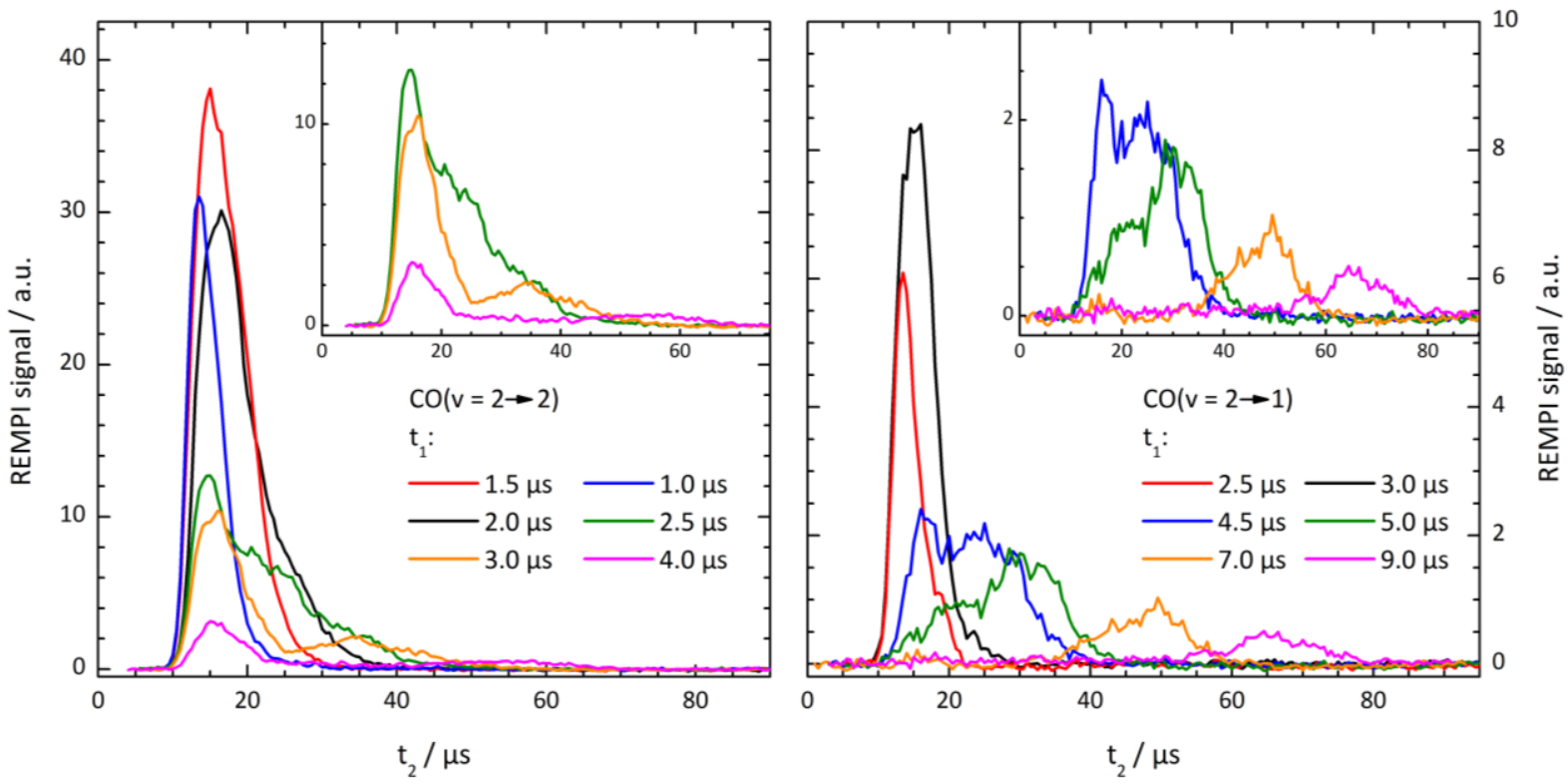

Fig. 4.12: Resulting time-of-flight distributions if the delay between UV tagging and REMPI detection is scanned for different delays between IR excitation and UV tagging. The right and left panels show the results for $2 \rightarrow 1$ and $2 \rightarrow 2$ scattering for $300 \mathrm{~K}$ surface temperature and $E_{I}=0.3 \mathrm{eV}$. The shown IR-UV delays are slightly different according to the differences in the IR and UV alignment with respect to the surface. The insets show a zoom into the data at large $t_{1}$. The different flight times corresponds to different velocities of the tagged molecules.

The results for the first method are shown for $v=2 \rightarrow 2$ and $v=2 \rightarrow 1$ scattering and $300 \mathrm{~K}$ surface temperature in Fig. 4.12. The insets show again the profiles for longer times $t_{1}$ in order to illustrate the changes at the time-of-flight distributions at later UV tagging. The data illustrates how the flight time between UV tagging and REMPI detection shifts towards later arrival times $t_{2}$ when I increase the temporal delay between IR excitation and UV tagging $\left(t_{1}\right)$. At low $t_{1}$, the temporal profiles show a sharp peak at $t_{2} \approx 10-20 \mu \mathrm{s}$ that decays rapidly with increasing $t_{1}$. This feature corresponds to the early component seen in Fig. 4.9. At larger values of $t_{1}$, the time-of-flight distributions shift further towards later arrival times $t_{2}$. The $2 \rightarrow 1$ channel shows a bimodal distribution for $t_{1}=4.5 \mu \mathrm{s}$ and $5.0 \mu \mathrm{s}$ (blue and green traces) with a significant second (slow) component. I observed this behavior in the onedimensional time-of-flight spectra of Fig. 4.9. However, the 2D-TOF technique now also reveals a bimodal distribution in the $2 \rightarrow 2$ channel. The effect is visible in the green $\left(t_{1}=2.5 \mu \mathrm{s}\right)$ and even more in the orange $\left(t_{1}=3.0 \mu \mathrm{s}\right)$ trace in the left panel of Fig. 4.12. I was not able to observe this behavior in the time-of-flight spectra of Fig. 4.9. A comparison of the bimodal distributions for $v_{f}=2$ and $v_{f}=1$ shows that the intensity in the direct scattering channel is much higher for $2 \rightarrow 2$ than for $2 \rightarrow 1$ scattering while the intensity of the trapping component is comparable.

The results of $t_{1}$ delay scans at constant $t_{2}$ are shown in Fig. 4.13 for final velocities ranging from $s_{f}=1500-300 \mathrm{~m} \mathrm{~s}^{-1}$. The differences in $t_{1}$ for $2 \rightarrow 1$ (left panel) and $2 \rightarrow 2$ scattering (right panel) are due to slightly larger distance of the IR excitation and UV tagging laser to the surface. The time-of-flight distributions systematically shift toward larger $t_{1}$ with decreasing velocity. I observe the highest intensities for $s_{f}=1500-900 \mathrm{~m} \mathrm{~s}^{-1}$ for both vibrational states. For lower final velocities, the intensity in the $2 \rightarrow 2$ channel decreases much faster than for $2 \rightarrow 1$ scattering, in agreement with the absence of a slow component for $v_{f}=2$ in the simple arrival time distributions of Fig. 4.9. The insets in Fig. 4.13 show the same time-of-flight profiles, but normalized to the peak for better comparison of the temporal width. As I pointed out above, this method is sensitive to residence time effects and broadening caused by velocity spread is minimized by the second constant UV-UV delay. The time resolution of the experiment 

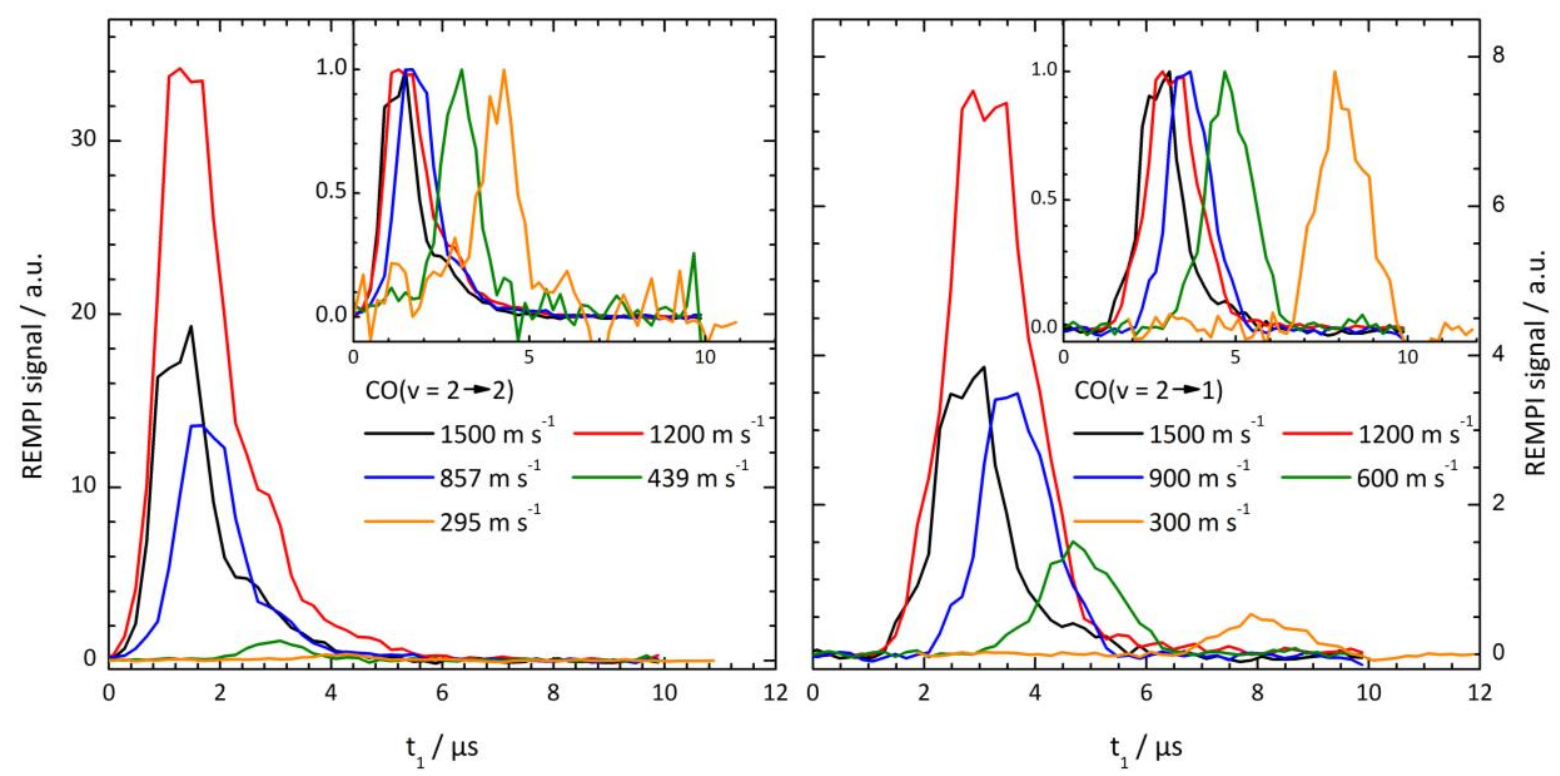

Fig. 4.13: Temporal distribution if the delay between IR excitation and UV tagging is varied while the UV-UV delay is constant and selects a certain velocity. The left and right panels show the results for $2 \rightarrow 1$ and $2 \rightarrow 2$ scattering at $T_{S}=300 \mathrm{~K}$ and $E_{I}=0.3 \mathrm{eV}$. The insets show normalized profiles for comparison of the widths of molecules leaving the surface at different speeds. For all final velocities the profiles exhibit a FWHM 1-2 $\mu \mathrm{s}$.

can be estimated from the width of the peaks for $s_{f}>1000 \mathrm{~m} \mathrm{~s}^{-1}$ because these profiles predominantly sample direct scattering events with zero residence time. In contrast, low $s_{f}$ discriminate against trapping-desorption events for which a certain residence time is expected.

For $s_{f} \geq 1000 \mathrm{~m} \mathrm{~s}^{-1}$ the arrival time distributions have a FWHM of $\sim 1.2 \mu$ s which represents the upper limit for detectable residence times in the experiment. I find the same FWHM for lower $s_{f}$, indicating that the residence time is much shorter than the time resolution of the experiment. The rate of desorption $\dot{r}_{\text {des }}$ follows an Arrhenius law and depends on the temperature of the surface $T_{S}$ and the surface coverage $\Theta$.

$$
\dot{r}_{d e s}=k_{d e s}\left(T_{S}\right) \times \Theta
$$

with

$$
k_{\text {des }}\left(T_{S}\right)=A_{d} \times e^{-E_{d} / k_{B} T_{S}}=1 / \tau_{\text {des }}
$$

where $k_{\text {des }}$ is the rate constant for desorption and $\tau_{\text {des }}$ represents the residence time at the surface. Therefore, the residence time will increase upon lowering of the surface temperature. In the experiments on vibrationally excited $\mathrm{CO}$ molecules, this effect will interfere with the limited lifetime of vibrationally excited states at the surface. In other words, if $\tau_{d e s}$ increases above a certain value the trapped $\operatorname{CO}(v=1,2)$ molecules relax down to $v_{f}=0$ prior to desorption and they will therefore not be detected in the experiment.

Fig. 4.14 shows the results of $t_{2}$ time-of-flight spectra for $2 \rightarrow 1$ (right panel) and $2 \rightarrow 2$ (left panel) scattering at three different surface temperatures $T_{S}=90,190,300 \mathrm{~K}$ and different delays $t_{1}$ between IR excitation and UV tagging. The figure shows only those $t_{1}$ delays for which I observed a bimodal arrival time distribution in the data presented in Fig. 4.12. Scattering into $v_{f}=2$ at $T_{S}=300 \mathrm{~K}$ results in a clear bimodal distribution for $t_{1}=3.0 \mu \mathrm{s}$ (red trace) and a narrow peak with a pronounced tail for $t_{1}=2.5 \mu \mathrm{s}$ 

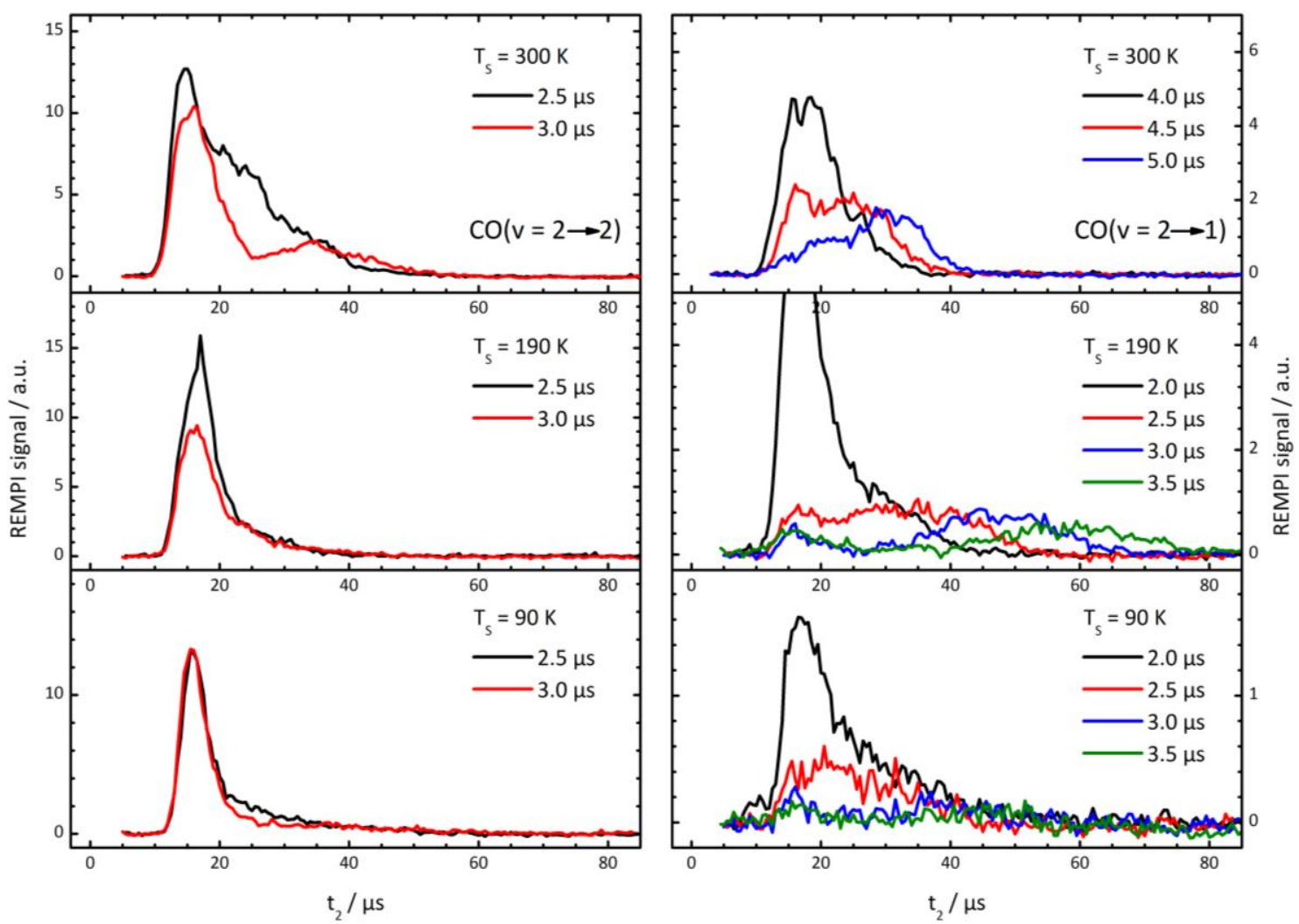

Fig. 4.14: Surface temperature dependence of the $t_{2}$ arrival time distributions for $2 \rightarrow 1$ (right panel) and $2 \rightarrow 2$ (left panel) scattering at different delays $t_{1}$ between IR excitation and UV tagging. The $t_{1}$ time scale for the room temperature data for $\mathrm{CO}\left(v_{f}=1\right)$ is shifted towards later times $t_{1}$ by about $2 \mu$ s due to slightly different laser beam alignment and surface position. The complete $2 \rightarrow 2$ data as well as the $2 \rightarrow 1$ data for $T_{S}=90 \mathrm{~K}$ and $190 \mathrm{~K}$ were taken at the same laser beam and surface position.

(black trace). Both effects are less or not visible at lower surface temperature. At $T_{S}=90 \mathrm{~K}$, both traces show only one narrow peak at $t_{2} \approx 18 \mu \mathrm{s}$. At $T_{S}=190 \mathrm{~K}$, the late component is only slightly visible in the red trace but appears only as a slight tail. The trapping component disappears in the time-of-flight spectra below $190 \mathrm{~K}$ for $v=2 \rightarrow 2$ scattering.

I observed a similar behavior for vibrationally inelastic $\mathrm{CO}(v=2 \rightarrow 1)$ scattering. Note that the $t_{1}$ values for $2 \rightarrow 1$ scattering at $300 \mathrm{~K}$ surface temperature are shifted by about $2 \mu$ s toward longer times due to a larger distance of the IR and UV pump laser from the surface. At room temperature, there is a pronounced trapping component which is especially visible in the red and blue trace. The bimodal distribution is also visible at $T_{S}=190 \mathrm{~K}$ for $t_{1}=2.5-3.5 \mu \mathrm{s}$. At the lowest surface temperature of $90 \mathrm{~K}$, the slow component disappears and only the first peak at $t_{2} \approx 20 \mu \mathrm{s}$ is observable.

It is the property of the experiment that residence time effects will only be visible for $t_{1}$ scans where the fixed $t_{2}$ delay acts as a velocity filter. The temperature dependent $t_{1}$ arrival time distributions are shown in Fig. 4.15 for $2 \rightarrow 1$ (right panel) and $2 \rightarrow 2$ scattering (left panel) at $T_{S}=90,190,300 \mathrm{~K}$. As before the room temperature data for $\mathrm{CO}\left(v_{f}=1\right)$ is shifted by $\sim 2 \mu$ s towards higher $t_{1}$. For scattering into $v_{f}=2$ the time-of-flight spectra do not change significantly with surface temperature. They only broaden slightly with decreasing $T_{S}$. The small shoulder in the black spectra at $t_{1} \approx 3 \mu$ s probably results from the laser beam profiles or back reflections of the IR or UV pump laser from the windows of the vacuum chamber. For vibrationally inelastic $v=2 \rightarrow 1$ scattering the profiles for $s_{f}=1200 \mathrm{~m} \mathrm{~s}^{-1}$ are unaffected by the surface temperature. However, the time-of-flight spectra at $s_{f}=600 \mathrm{~m} \mathrm{~s}^{-1}$ and $300 \mathrm{~m} \mathrm{~s}^{-1}$ 

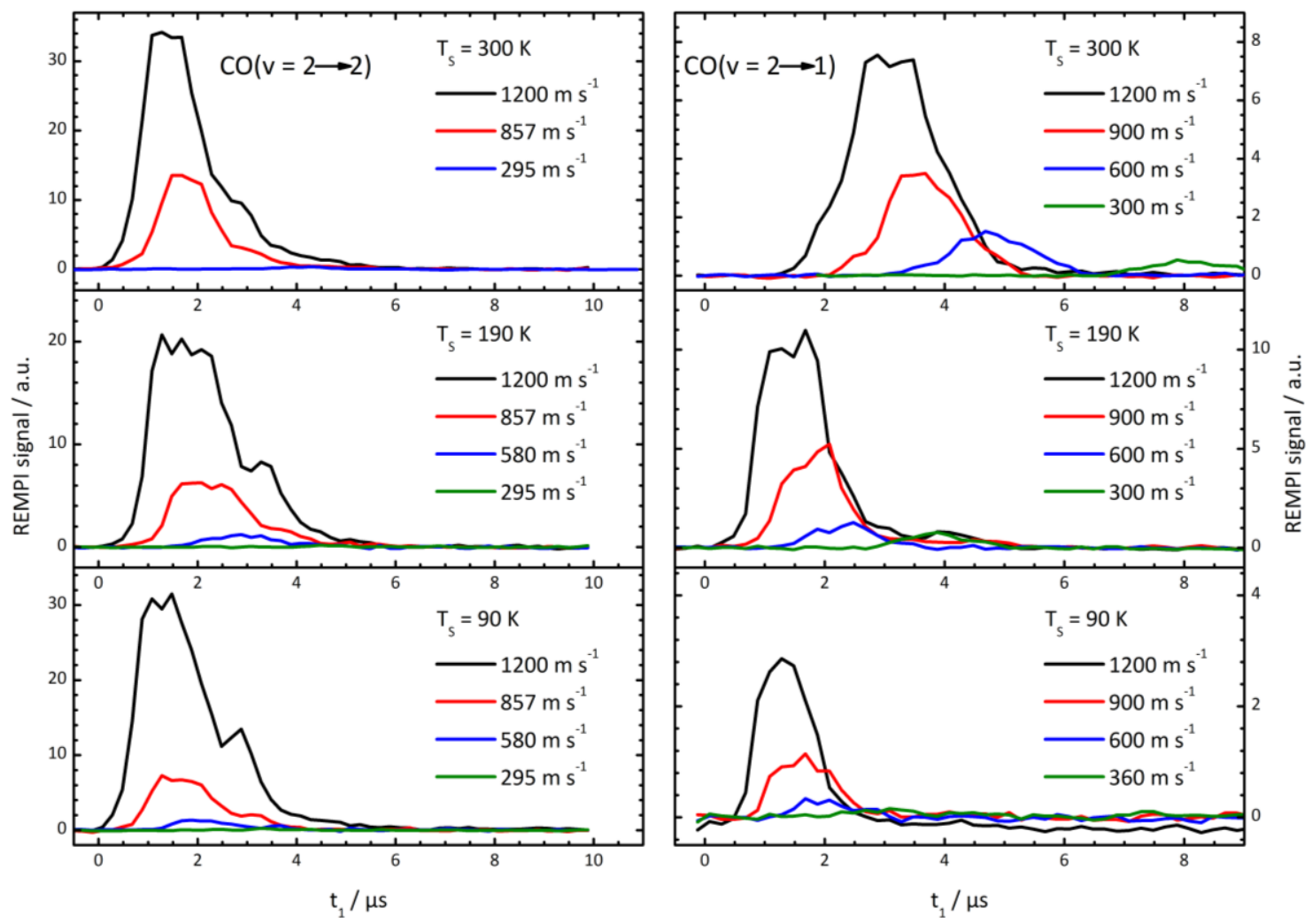

Fig. 4.15: Surface temperature dependence of the $t_{1}$ arrival time distributions at different selected final speeds for $2 \rightarrow 1$ and $2 \rightarrow 2$ scattering. For $2 \rightarrow 1$ scattering at $T_{S}=300 \mathrm{~K}$, the time scale is shifted towards longer times $t_{1}$ due to slightly different laser beam and surface positions. The black traces at $1200 \mathrm{~m} \mathrm{~s}^{-1}$ reflect mainly direct scattering with a width of $1.5-2.0 \mu \mathrm{s}$. The intensity of the REMPI signal decreases rapidly with decreasing velocity for both vibrational channels and all surface temperatures. Only for $2 \rightarrow 1$ scattering at $T_{S}=300 \mathrm{~K}$ and weakly at $190 \mathrm{~K}$ a significant signal is obtained for molecules that travel at $s_{f} \approx 300 \mathrm{~m} \mathrm{~s}^{-1}$.

decrease significantly in intensity with decreasing surface temperature. The width of the arrival time distributions does not change within the temporal resolution of the experiment. Consequently, the surface temperature dependence of the $t_{1}$ arrival time distributions does not exhibit a signature of residence times within the $\sim 1-2 \mu$ s time resolution of the experiment.

\subsubsection{Discussion}

The experimental observations of section 4.2.1 allow for a detailed interpretation of the interaction of vibrationally excited CO molecules with a Au(111) surface. I measured branching ratios $R_{v_{f}}$ for $\mathrm{CO}\left(v_{i}=2\right)$ scattering into $v_{f}=1$ and $v_{f}=2$ as a function of incidence kinetic energy in the range from $E_{I}=0.25-0.92 \mathrm{eV}$ (left panel Fig. 4.10). The $E_{I}$ dependence of $R_{1}$, thus the probability for $v=2 \rightarrow 1$ relaxation, is very similar to that for $v=0 \rightarrow 1$ excitation (right panel of Fig. 4.6/ Fig. 4.10). The probabilities for vibrational relaxation, as well as for vibrational excitation, decrease with increasing incidence energy between $E_{I}=0.25 \mathrm{eV}$ and $0.40 \mathrm{eV}$ and increase again for higher $E_{I}>0.41 \mathrm{eV}$. This behavior is remarkably different from the observations for $\mathrm{NO}(v=0 \rightarrow 1,2)[25,26]$ and $\mathrm{NO}(v=3 \rightarrow 1,2$, 3) (section 3.2) scattering from $\mathrm{Au}(111)$ where the (de-)excitation probability increases with increasing $E_{I}$ over the whole range of incidence energies from $E_{I}=0.11-1.2 \mathrm{eV}$. The time-of-flight profiles for $\mathrm{CO}(v=2 \rightarrow 1,2)$ of Fig. 4.9 provide a first explanation for the unexpected increase in relaxation/ excitation probability at $E_{I}<0.40 \mathrm{eV}$. The temporal profiles for the $v=2 \rightarrow 1$ scattering channel exhibit 
clear differences for $E_{I}=0.62,0.92 \mathrm{eV}$ and $E_{I}=0.25 \mathrm{eV}$. At high incidence energies, the time-of-flight distributions for $v_{f}=1$ and $v_{f}=2$ are well described by a single and narrow temporal distribution function. Vibrationally elastic $v=2 \rightarrow 2$ scattering also shows this behavior at $E_{I}=0.25 \mathrm{eV}$ while vibrationally inelastic $v=2 \rightarrow 1$ scattering shows a bimodal distribution with an early (fast) component, that is identical with the $2 \rightarrow 2$ channel, and a second slow and broad component.

The two features can be attributed to different scattering mechanisms. The fast feature results mainly from direct scattering; it is in reasonably good agreement with the prediction of a hard cube (Baule) model and is present at all incidence energies. The slow component only occurs at low incidence energies and is related to trapping followed by desorption. This observation itself is quite striking because the lifetime of excited vibrational states of $\mathrm{CO}$ adsorbed on a metal surface is expected to be only a few picoseconds based on previous measurements of vibrational lifetimes of CO/Pt(111) [48, 124] and $\mathrm{Cu}(100)$ [50]. Therefore, one would expect that $\mathrm{CO}\left(v_{i}=2\right)$ molecules that are trapped at the $\mathrm{Au}(111)$ surface will relax before they desorb and leave the surface. However, the results of Fig. 4.9 show that $\mathrm{CO}\left(v_{f}=1\right)$ molecules are desorbed with $T_{v i b} \gg T_{S}$. But why is the effect absent in the arrival time distribution for $v=2 \rightarrow 2$ scattering? The 2D-TOF experiments presented in Fig. 4.12 and Fig. 4.14 provide the answer to this question. The $t_{2}$ temporal profiles for $\operatorname{CO}\left(v_{f}=2\right)$ show that the trappingdesorption channel is also present for vibrationally elastic $2 \rightarrow 2$ scattering. However, the trapping component is now only a few percent of the direct scattering signal and is therefore covered by the intense direct component. Because the $v=2 \rightarrow 1$ relaxation probability resulting from direct scattering is only $2-3 \%$ (Fig. 4.10), the contributions of trapping-desorption and direct scattering become comparable for $v_{f}=1$. I can use the 2D-TOF approach to suppress most of the faster direct component in the $t_{2}$ time-of-flight spectra with the result that a bimodal distribution becomes visible for certain values of $t_{1}$. Consequently, $\operatorname{CO}\left(v_{i}=2\right)$ molecules are trapped at the surface and desorbed in $v_{f}=1$ and $v_{f}=2$. I note that this observation does not mean that no relaxation into $v_{f}=0$ occurs while the molecules are adsorbed at the surface.

A possible explanation for the experimental observation is that the probability for desorption in $v_{f}=1$, 2 is related to the ratio of vibrational lifetime for $\mathrm{CO}(v>0) / \mathrm{Au}(111)$ and the residence time $\tau_{\text {des }}$ at the surface. The vibrational lifetime depends on the strength of the nonadiabatic interaction between the $\mathrm{CO}$ molecule and the Au surface. The residence time can be calculated from an Arrhenius law given by equation 4.4 and increases with decreasing surface temperature. The results of Fig. 4.14 and Fig. 4.15 showed that the residence time is much less than $\sim 1-2 \mu \mathrm{s}$. Furthermore, the intensity of the trappingdesorption component decreases with decreasing surface temperature. It is still observable at $T_{S}=190 \mathrm{~K}$ but not present anymore at $T_{S}=90 \mathrm{~K}$. A calculation of the residence time requires the knowledge of the preexponential factor $A_{d}$ and the desorption (binding) energy for CO/Au(111). Peterson et al. [133] calculated binding energies for different surface sites and found a maximum of $0.02 \mathrm{eV}$ for adsorption at top-sites. For a prefactor of $A_{d} \approx 10^{13} \mathrm{~s}^{-1}$ the calculation yields residence times of $\tau_{\text {des }}=1.33,0.34$ and $0.21 \mathrm{ps}$ for $T_{S}=90,190$ and $300 \mathrm{~K}$. These values for $\tau_{\text {des }}$ are shorter or comparable to the vibrational lifetime of $\mathrm{CO}$ on $\mathrm{Pt}(111) / \mathrm{Cu}(100)$. However, $\tau_{\text {des }}$ depends very strongly on the assumed prefactor and activation energy and the calculated values can easily be wrong by one or two orders of magnitude. Furthermore, the vibrational lifetime for $\mathrm{CO}$ molecules adsorbed on a $\mathrm{Au}(111)$ surface has not been measured so far. The CO-Au attraction is very weak compared to Pt or Cu surfaces, where a chemical bond is formed, and it is well-known that the vibrational relaxation occurs upon electron transfer into the anti-bonding $2 \pi^{*}$ orbital of the CO molecule [47]. For CO/Au(111), the formation of a strong chemical bond is unlikely; the resulting weak interaction might also weaken the nonadiabatic V-EHP coupling. Consequently, the vibrational lifetime may be significantly longer for $\mathrm{CO} / \mathrm{Au}(111)$ than for $\mathrm{CO} / \mathrm{Pt}(111)$ or $\mathrm{CO} / \mathrm{Cu}(100)$. 
Another possible explanation is that the CO molecule is trapped at the surface in a precursor state that exhibits only weak coupling to the surface. This "hot precursor", which basically reflects an adsorbed molecules that has not yet thermalized, has been discussed earlier in the context of chemical reactions at surfaces [136]. My results clearly demonstrate the desorption of vibrationally excited $\mathrm{CO}\left(v_{f}=1,2\right)$ molecules that have not completely thermalized. Therefore, the experimental observations represent a direct evidence for hot precursor $\mathrm{CO}$ molecules adsorbed on $\mathrm{Au}(111)$. The corresponding hot precursor state is able to decay as the surface temperature is lowered and the residence time at the surface is increased.

\subsubsection{Summary and Conclusions}

In summary, I have performed detailed experiments to investigate the relaxation of $\mathrm{CO}\left(v_{i}=2\right)$ molecules scattering from $\mathrm{Au}(111)$ as a function of incidence kinetic energy. The experimental observations show that trapping-desorption influences the relaxation probabilities at low incidence energies $E_{I}<0.4 \mathrm{eV}$, which leads to an increase of $R_{1}$ with decreasing $E_{I}$. In contrast to the results for vibrational excitation, the arrival time distributions for $2 \rightarrow 2$ and $2 \rightarrow 1$ scattering provide sufficient temporal resolution to distinguish between direct scattering and trapping followed by desorption. A new 2D-TOF technique using IR-UV-UV triple resonance revealed that trapping-desorption is present in $v=2 \rightarrow 2$ as well as in $2 \rightarrow 1$ scattering at $300 \mathrm{~K}$ surface temperature. Experiments at lower surface temperatures showed that the trapping-desorption channel is still present but weaker in intensity at $190 \mathrm{~K}$. At $T_{S}=90 \mathrm{~K}$, I only observed direct scattering into $\operatorname{CO}\left(v_{f}=1,2\right)$.

My results clearly show that vibrationally excited $\mathrm{CO}$ molecules can be adsorbed on a Au(111) surface and can be desorbed prior to complete equilibration with the surface temperature. This unexpected behavior can be due to weak CO-Au attraction and the resulting short residence time. Furthermore, it is possible that the vibrational lifetime of $\mathrm{CO}$ molecules on a $\mathrm{Au}(111)$ surface is much longer than on Pt or $\mathrm{Cu}$ surfaces. Therefore, the difference between chemisorption ( $\mathrm{CO}$ on $\mathrm{Pt}, \mathrm{Cu}$ ) and physisorption (CO on $\mathrm{Au}$ ) might have a strong impact onto the nonadiabatic V-EHP coupling. In any case, the observation of non-equilibrated $\mathrm{CO}$ after trapping is a direct evidence for a hot precursor state, which was suggested to play a role in chemical reactions at metal surfaces. The final answer to the question which of these two mechanisms is responsible for the observed phenomena remains unclear and requires further investigation, for example from theory. Further key experiments could be the measurement of the vibrational lifetime for $\mathrm{CO} / \mathrm{Au}(111)$ by measuring the linewidth of the $\mathrm{CO}$ stretching vibration or direct lifetime measurements using sum frequency generation or transient IR absorption. These experiments would separate residence time and vibrational lifetime. 


\section{Co scattering from $\operatorname{Pt}(111)$}

The CO/Pt(111) system has been investigated intensively over the last decades, both experimentally and theoretically [48, 124, 137-148]. Most of these studies were focused on the adsorption of CO molecules by means of adsorption sites and binding energies. Furthermore, Beckerle et al. [48, 124] measured the vibrational lifetime for $\mathrm{CO}$ molecules adsorbed on $\mathrm{Pt}(111)$ at surface temperatures between $150 \mathrm{~K}$ and $300 \mathrm{~K}$ and found lifetimes of only a few picoseconds. This is in contrast to studies on $\mathrm{CO} / \mathrm{NaCl}(100)$, where a vibrational lifetime of $4.3 \mathrm{~ms}$ was observed [53], but similar to observations for $\mathrm{CO} / \mathrm{Cu}(110)$ with a vibrational lifetime of $\sim 2.0 \mathrm{ps}$ [50]. This strong decrease in vibrational lifetime by nine orders of magnitude on a metal surface shows that nonadiabatic effects play an important role in the $\mathrm{CO} / \mathrm{Pt}(111)$ system.

In contrast to the experiments on $\mathrm{NO} / \mathrm{Au}(111)$ and $\mathrm{CO} / \mathrm{Au}(111), \mathrm{CO}$ sticks to $\mathrm{Pt}(111)$ at room temperature and is only desorbed at higher surface temperatures $\left(T_{S}>500 \mathrm{~K}\right)$. The binding energy was measured to be in the range of 1.1-1.6 eV, obtained by different methods and for different surface coverages [138, 139, 144, 146-152]. Therefore, I had to carry out all measurements at elevated temperatures to ensure a clean surface throughout the whole experiment. I determined absolute vibrational excitation probabilities for $\mathrm{CO}(v=0 \rightarrow 1) / \mathrm{Pt}(111)$ for $673 \mathrm{~K} \leq T_{S} \leq 1123 \mathrm{~K}$ and $E_{I}=0.65 \mathrm{eV}$ in a similar way as described in section 3.1 .1 by measuring surface temperature dependent REMPI spectra, angular distributions and time-of-flight profiles for scattered $\operatorname{CO}\left(v_{f}=0,1\right)$ molecules. In order to investigate the dominant scattering mechanism for the CO/Pt(111) system, I used a UV-UV double resonance scheme to perform state-to-state time-of-flight experiments to measure the speed distributions of scattered $\mathrm{CO}\left(v_{f}=0,1\right)$ molecules at different surface temperatures.

The results show that trapping-desorption dominates the vibrational excitation in CO scattering off Pt(111); the vibrational excitation probabilities reach the thermal limit. They follow an Arrhenius dependence with a prefactor $A_{01} \approx 1.0$ and an activation energy equal to the vibrational spacing of $\mathrm{CO}$ $\left(E_{v i b}=0.265 \mathrm{eV}\right)$. The temporal profile of the scattered molecular beam as well as the angular distributions change significantly with surface temperature. The state-to-state time-of-flight results show that scattering into $v_{f}=0$ can happen upon two different mechanisms. A fast and narrow component peaks at $s_{f} \approx 1500 \mathrm{~m} \mathrm{~s}^{-1}$, is not affected by changes in $T_{S}$ and is assigned to direct scattering, while a second (slow) component changes strongly with surface temperature and is assigned to a trappingdesorption mechanism. For scattering into $v_{f}=1$, I only observe the slow component, an indication indicates that $v=0 \rightarrow 1$ excitation in CO scattering off $\mathrm{Pt}(111)$ is dominated by trapping-desorption. In addition, I find that the intensity of the trapping-desorption component decreases with decreasing surface temperature when measured at a specific delay in the molecular beam. This behavior is related to residence time of adsorbed $\mathrm{CO}$ molecules that reaches the time scale of the experiment for $T_{S}<700 \mathrm{~K}$.

\subsection{Results}

\section{REMPI spectra}

The general procedure for determination of absolute excitation probabilities has already been described in section 3.1.1 as well as in reference [1]. The first step is the measurement of surface temperature dependent REMPI spectra. In case of CO/Pt(111), the covered surface temperatures range from 673$1123 \mathrm{~K}$. I chose $T_{S}=673 \mathrm{~K}$ as a lower limit because it is already well above the desorption temperature 
of $\mathrm{CO}$ from $\mathrm{Pt}(111)(\sim 500 \mathrm{~K})$ at which the adsorbed molecules have enough thermal energy to escape from the surface. An example of REMPI spectra for scattered $\mathrm{CO}\left(v_{f}=0\right)$ and $\mathrm{CO}\left(v_{f}=1\right)$ at $T_{S}=673 \mathrm{~K}$, $773 \mathrm{~K}$ and $973 \mathrm{~K}$ at $E_{I}=0.65 \mathrm{eV}$ is shown in Fig. 5.1. All REMPI spectra were recorded at similar laser powers and identical focusing conditions, scattering angle and temporal delay ( $250 \mu \mathrm{s})$. The intensities of the $0 \rightarrow 0$ channel (left panel) reflect the measured intensities. The intensities of the $0 \rightarrow 1$ channel (right panel) are corrected for the higher detector gain and can directly be compared to those for $v=0 \rightarrow 0$ scattering. The intensity of the $\mathrm{CO}\left(v_{f}=1\right)$ REMPI spectra increases by a factor of about 6.5 if I raise the surface temperature from $673 \mathrm{~K}$ to $973 \mathrm{~K}$. It reaches about $3.5 \%$ of the $v=0$ intensity at $T_{S}=973 \mathrm{~K}$. In contrast to the results for CO/Au(111) scattering (Fig. 4.1), the $O$ branch of the $B \leftarrow X(0-0)$ band is almost invisible because of the high intensity in the (1-1) Q branch. I do not observe a prominent rotational rainbow feature in the (1-1) band.

The intensity of the (0-0) Q branch also changes with surface temperature; I will demonstrate below that this decrease in intensity is related to changes in the temporal profile of the molecules leaving the surface. The intensity at the band head from $230.05-230.00 \mathrm{~nm}$ approximately doubles upon increase of $T_{S}$ from $673 \mathrm{~K}$ to $773 \mathrm{~K}$ or $973 \mathrm{~K}$. This effect is absent at wavelengths below $230.00 \mathrm{~nm}$ where higher $J_{f}$ states are detected. This is also the regime where I observe a rotational rainbow that is especially visible at $T_{S}=673 \mathrm{~K}$ (blue). The rotational rainbow feature is much less pronounced at higher surface temperature because the intensity of the low $J_{f}$ band head $(230.00-230.05 \mathrm{~nm})$ increases significantly.
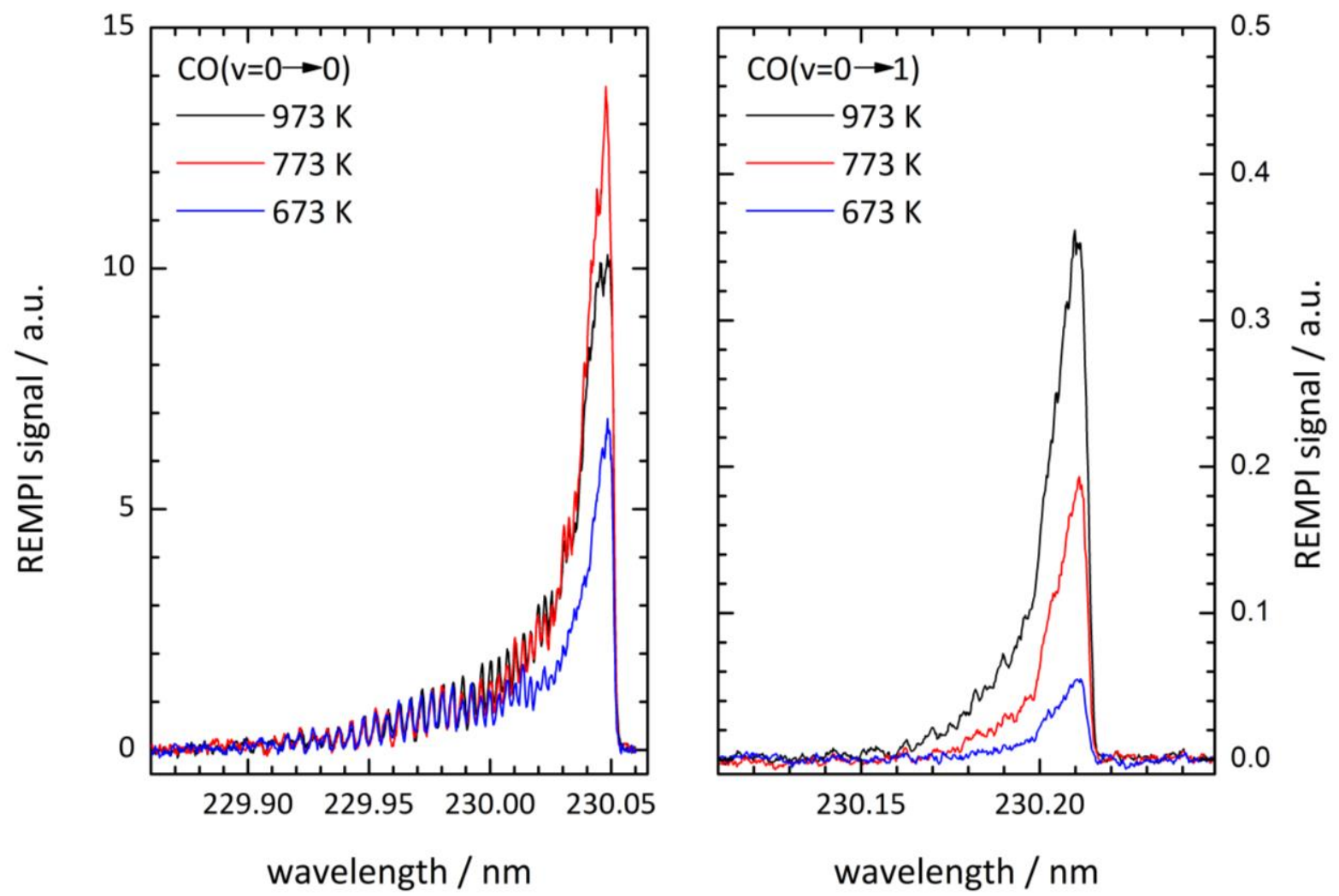

Fig. 5.1: Typically observed REMPI spectra for $\operatorname{CO}\left(v_{f}=0,1\right)$ scattered off $\mathrm{Pt}(111)$ at different surface temperatures and $E_{I}=0.65 \mathrm{eV}$. All spectra were recorded at similar laser intensities and identical focusing conditions, scattering angle and temporal delay. The REMPI spectra for $\mathrm{CO}\left(v_{f}=1\right.$ ) (right panel) are corrected for the higher detector gain. Therefore, the intensities can be compared to the $v=0$ spectra (left panel). The $v_{f}=1$ intensity increases by about a factor of 6.5 when the surface temperature is raised from $673 \mathrm{~K}$ to $973 \mathrm{~K}$ and reaches approximately $3.5 \%$ of the $v_{f}=0$ signal at $T_{S}=973 \mathrm{~K}$. The intensity of the 0-0 band also changes with $T_{S}$ at the band head (low $J_{f}$ ) but remains almost unchanged in the region below $230.00 \mathrm{~nm}\left(\operatorname{high} J_{f}\right)$. 


\section{Angular distributions}

I obtained angular distributions for scattered $\mathrm{CO}\left(v_{f}=0,1\right)$ molecules by translating the REMPI laser beam and the focusing lens parallel to the surface (perpendicular to the molecular beam) and monitoring the corresponding REMPI signal. The results for both vibrational states are shown in Fig. 5.2 for $T_{S}=673 \mathrm{~K}, 823 \mathrm{~K}$ and $973 \mathrm{~K}$ at $E_{I}=0.65 \mathrm{eV}$. The dashed line indicates a $\cos \theta$ distribution that is expected for trapping-desorption. The angular distributions are broader than those observed for $\mathrm{CO} / \mathrm{Au}(111)$ (Fig. 4.3). At all three surface temperatures, $\mathrm{CO}(v=0 \rightarrow 1)$ (red) scattering exhibits slightly broader distributions than scattering into $v_{f}=0$ (black). Angular distributions of $\mathrm{CO}\left(v_{f}=0\right)$ broaden with increasing surface temperature from $\cos ^{3.4} \theta$ at $T_{S}=673 \mathrm{~K}$ to $\cos ^{2.2} \theta$ at $T_{S}=973 \mathrm{~K}$. For scattering into $v_{f}=1$, I obtain angular distributions with $\cos ^{2.5} \theta$ at $T_{S}=673 \mathrm{~K}$ and $\cos ^{1.4} \theta$ at $T_{S}=973 \mathrm{~K}$. Especially the angular distribution for $\operatorname{CO}\left(v_{f}=1\right)$ and $T_{S}=973 \mathrm{~K}$ is very close to the expected $\cos \theta$ distribution for trapping followed by desorption.
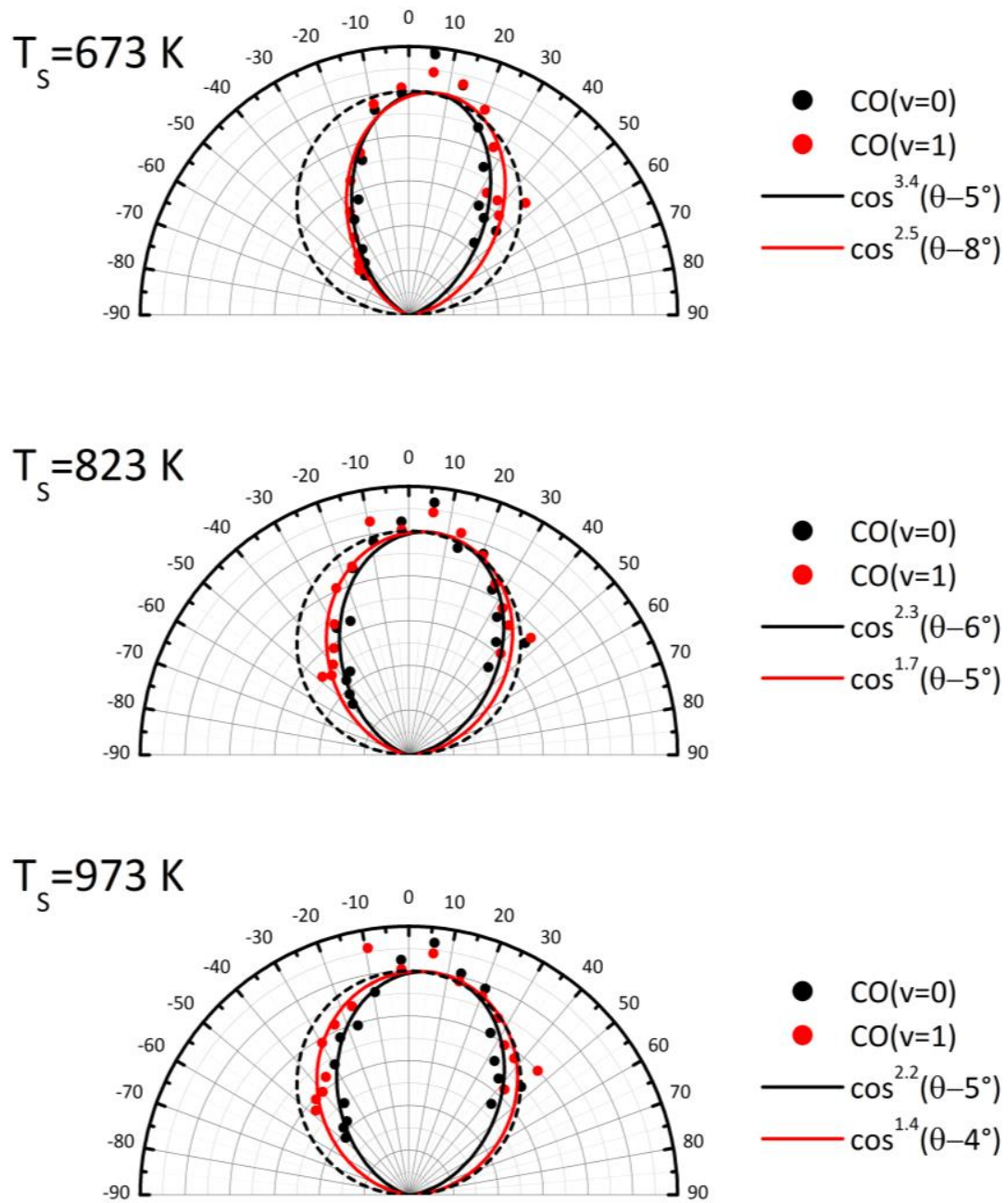

Fig. 5.2: Angular distributions for $\mathrm{CO}\left(v_{f}=0 \rightarrow 0,1\right)$ scattering off $\mathrm{Pt}(111)$ at three different surface temperatures $T_{S}=673 \mathrm{~K}$, $823 \mathrm{~K}$ and $973 \mathrm{~K}$. The black dashed line indicates a $\cos \theta$ distribution that is expected for trapping-desorption behavior. All angles are given with respect to the peak of the incident molecular beam. The angular distributions of $\operatorname{CO}\left(v_{f}=0\right)(\mathrm{black})$ and $\mathrm{CO}\left(v_{f}=1\right)(\mathrm{red})$ are relatively broad compared to those obtained for CO/Au(111) (see Fig. 4.3). In both cases the angular distributions broaden with surface temperature. In general the distributions for $0 \rightarrow 1$ scattering appear slightly broader than the angular distributions of the $0 \rightarrow 0$ channel and reach almost $\cos \theta$ at $T_{S}=973 \mathrm{~K}$. 


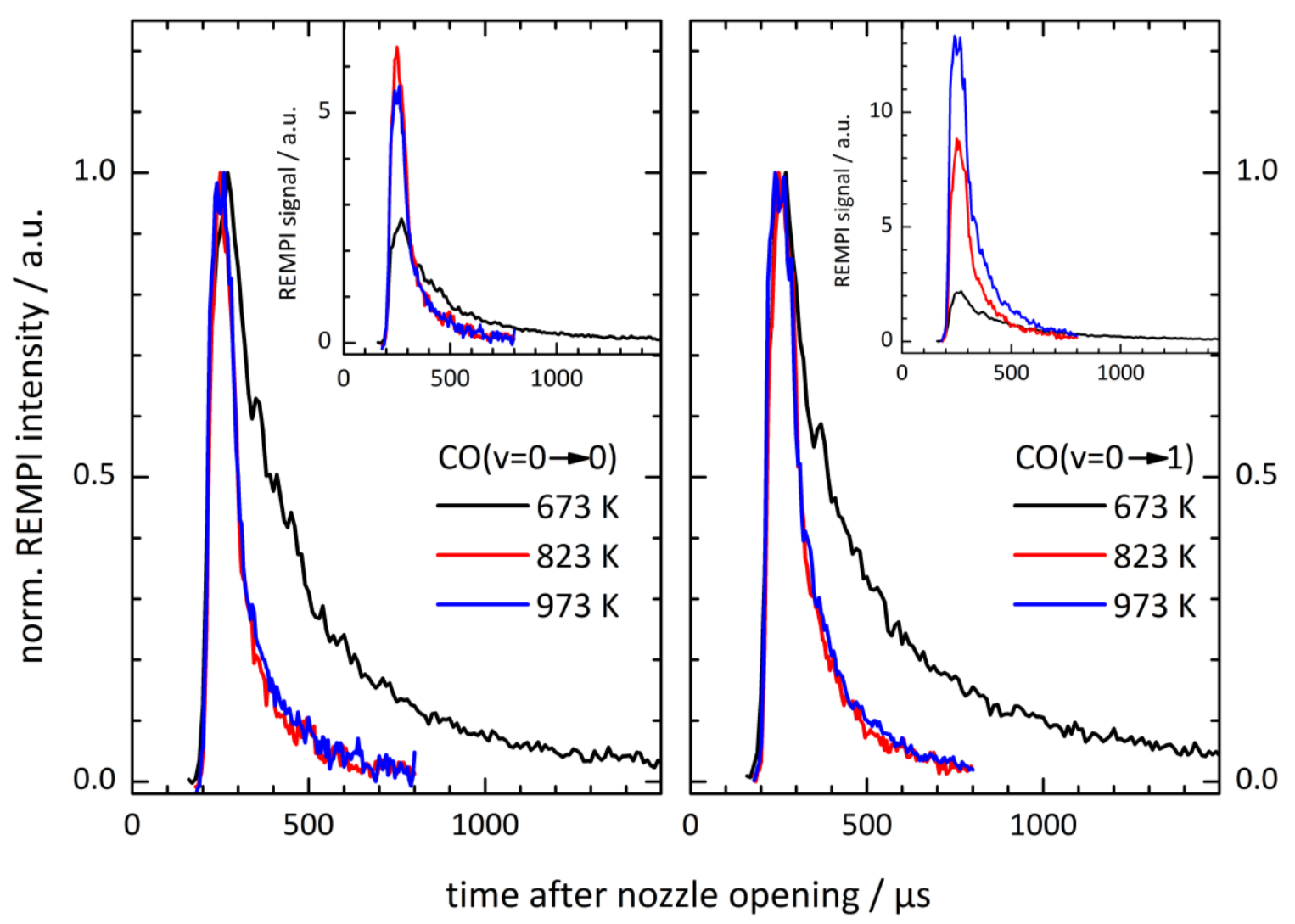

Fig. 5.3: Temporal profiles for $\mathrm{CO}$ scattering from $\mathrm{Pt}(111)$ at different surface temperatures. The left panel shows vibrationally elastic $v=0 \rightarrow 0$ scattering with normalized intensities. The inset shows the uncorrected measured intensities. The right panel shows temporal profiles for vibrationally inelastic $v=0 \rightarrow 1$ scattering with normalized intensities as well as uncorrected intensities as an inset.

\section{Temporal distributions}

The temporal profiles for $\mathrm{CO}(v=0 \rightarrow 0)$ (left panel) and $\mathrm{CO}(v=0 \rightarrow 1)$ (right panel) scattering at different surface temperatures are shown in Fig. 5.3. The intensities of the major panels are normalized to the peak for comparison. The insets show the (un-normalized) measured intensities that change strongly with $T_{S}$ in case of $0 \rightarrow 1$ scattering but also depend on surface temperature for the vibrationally elastic $0 \rightarrow 0$ channel. At $T_{S}=673 \mathrm{~K}$, the intensity of the $v_{f}=0$ signal drops significantly. The comparison of the normalized intensities clearly shows that the decrease in peak intensity for $v_{f}=0$ is accompanied by a broadening of the temporal profiles of the molecular beam. At $T_{S}=823 \mathrm{~K}$ and $973 \mathrm{~K}$, the shape remains basically unchanged for both vibrational states and only the intensity for $v_{f}=1$ decreases by $\sim 1 / 3$. At the lowest surface temperature (black traces), the temporal profiles broaden in time showing a pronounced tail at long flight times. The peak of the distribution only changes in intensity (insets) but does not shift in time. The strong change of the temporal profiles of the molecular beam with surface temperature is a new feature of the CO/Pt(111) system and was neither observed for NO/Au(111) nor for $\mathrm{CO} / \mathrm{Au}(111)$ scattering. 

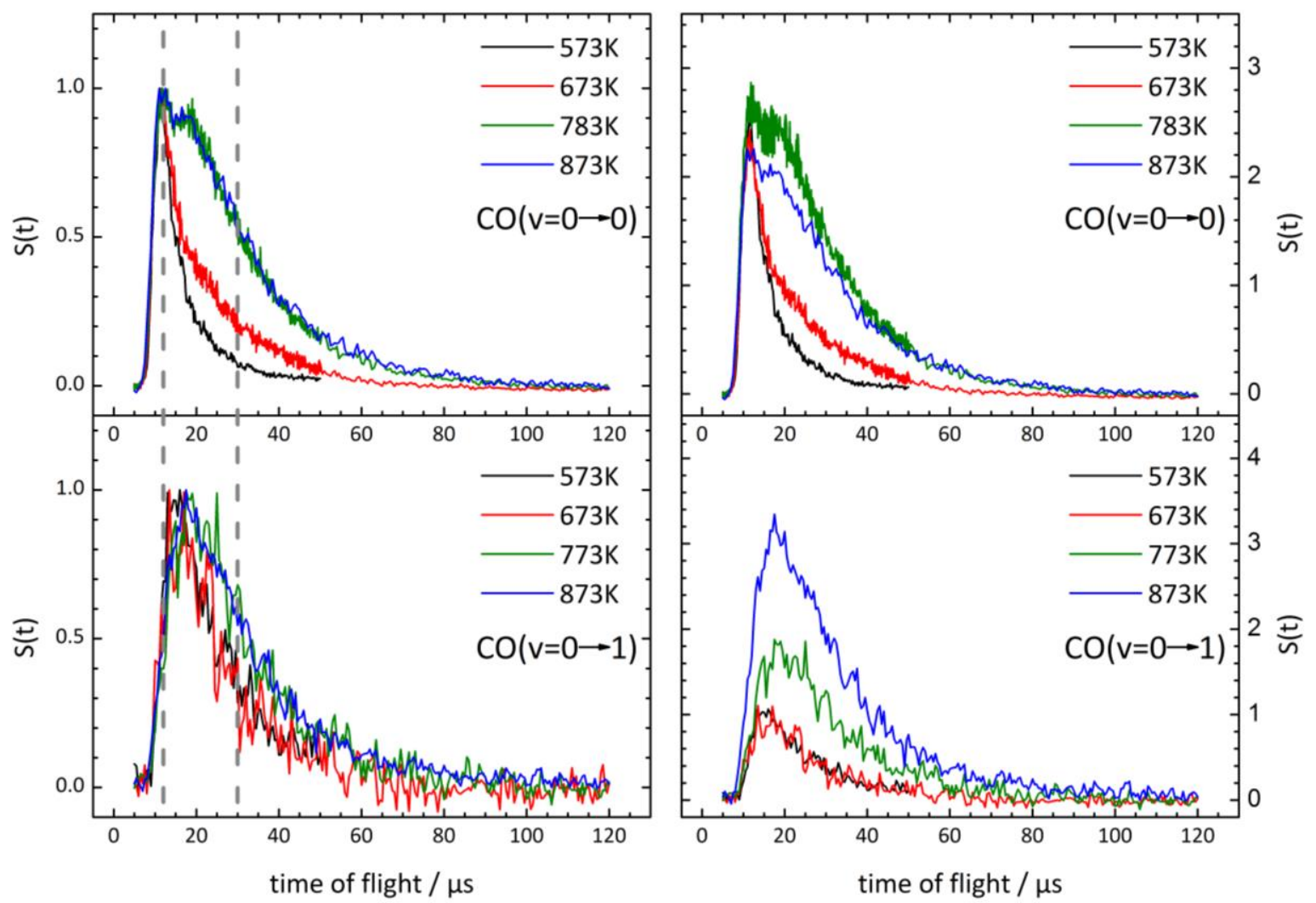

Fig. 5.4: Surface temperature dependent time-of-flight distributions using UV-UV double resonance. Scattering molecules are tagged into the metastable a ${ }^{3} \Pi_{1}$ state and detected with (1+1) $b \longleftarrow a$ REMPI. The timing of the tagging laser remains unchanged for the experiments. The left panels show normalized intensities for scattering into $v_{f}=0$ (upper panel) and $v_{f}=1$ (lower panel) while the right panels show the uncorrected real intensities. Especially for $0 \rightarrow 0$ scattering, a clear double peak structure is observed. If $T_{S}$ is lowered to below $673 \mathrm{~K}$, the second peak seems to disappear and only the first narrow feature peaking at about $12 \mu \mathrm{s}$ is observed. For $0 \rightarrow 1$ scattering, the effect is much less pronounced. The first peak is hardly visible in the time-offlight profile and only appears as a shoulder at around $12 \mu \mathrm{s}$. The temperature effect is probably hidden by the $T_{S}$ dependence of the $v_{f}=1$ excitation probability and the resulting low $\mathrm{S} / \mathrm{N}$ at the lowest surface temperatures.

\section{Speed distributions}

A possible reason for this $T_{S}$ dependence of temporal profile of the scattered molecular beam can be a strong change in the final speed distributions of the molecules leaving the surface. The measurement of speed distributions for $\mathrm{CO}\left(v_{f}=0,1\right)$ requires tagging and detection of both final vibrational states. An IR-UV double resonance scheme is difficult to apply for CO because $(2+1)$ REMPI detection of ground state molecules in $v^{\prime \prime}>1$ is not possible with high sensitivity. Therefore, I set up a UV-UV double resonance scheme that excites scattered $\operatorname{CO}\left(v_{f}=0,1\right)$ molecules into the metastable $a^{3} \Pi_{1}$ state via the $(0-0)$ and (0-1) bands. I then detect the prepared metastable CO* molecules at $18 \mathrm{~mm}$ distance with $b^{3} \Sigma^{+} \leftarrow a{ }^{3} \Pi_{1}(1+1)$ REMPI. This enables state-to-state time-of-flight experiments to measure the speed distributions of the scattered $\mathrm{CO}\left(v_{f}=0,1\right)$ molecules by varying the delay between the UV tagging and the REMPI detection laser. As I will show below, it is important to note that the timing of the tagging laser is fixed with respect to the molecular beam. Fig. 5.4 shows arrival time profiles for tagging of scattered $\mathrm{CO}\left(v_{f}=0\right)$ (upper panels) and $\mathrm{CO}\left(v_{f}=1\right)$ (lower panels) molecules after scattering from $\mathrm{Pt}$ (111) at different surface temperatures. The distributions on the left are normalized to the peak for comparison of the shape while the distributions on the right show the uncorrected measured intensity. 

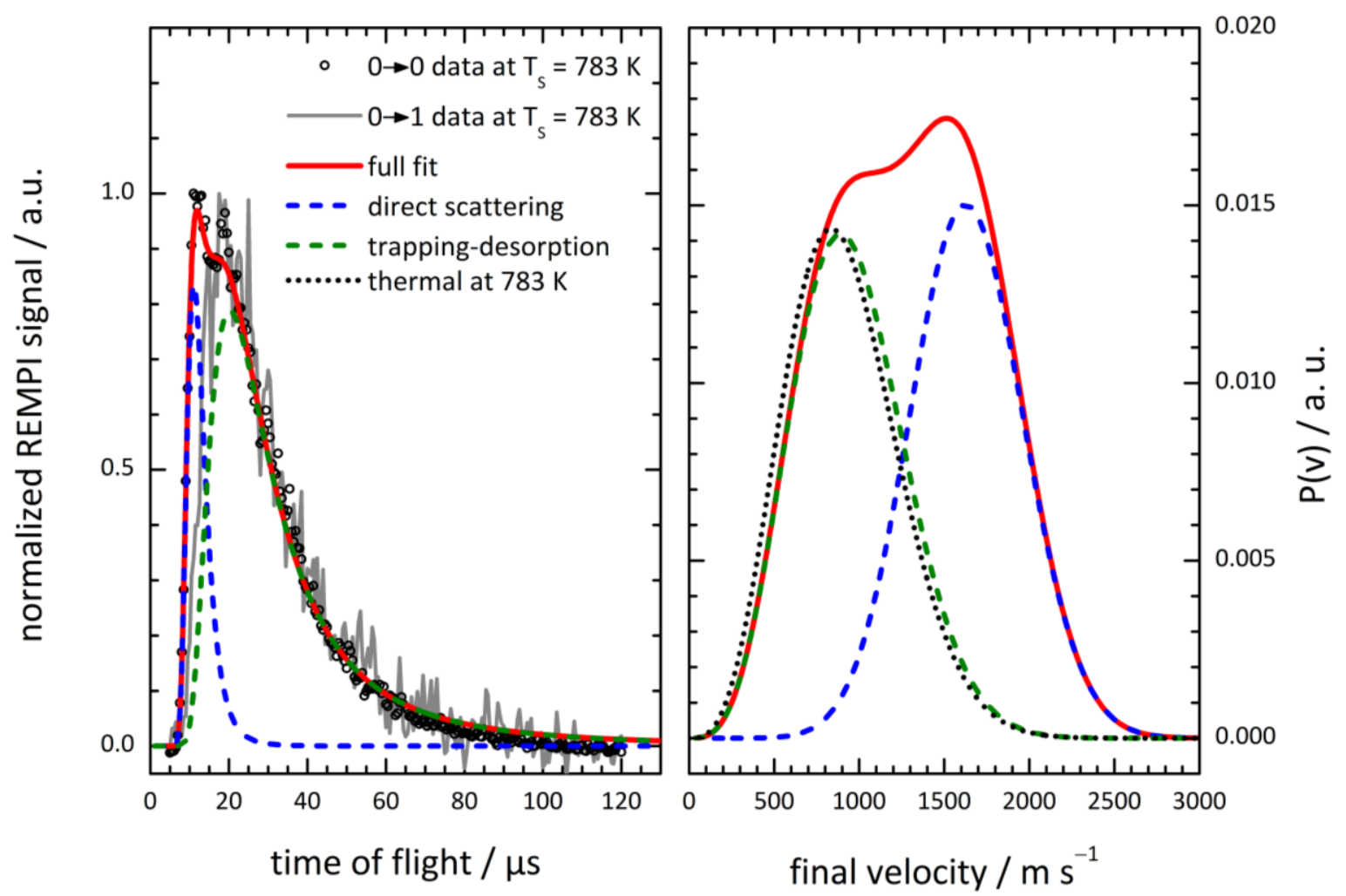

Fig. 5.5: Left panel: Fit of a bimodal time-of-flight distribution to the $v=0 \rightarrow 0$ experimental data (black open circles) at $T_{S}=783 \mathrm{~K}$. The red curve shows the full fit while the blue and green dashed curves show the contributions of direct scattering and trapping-desorption. The gray dashed line shows the arrival time distributions for $v=0 \rightarrow 1$ scattering at $T_{S}=783 \mathrm{~K}$. Right panel: The fit from the left panel converted into flux and velocity space. The black dotted line shows the expectation of a Maxwell-Boltzmann speed distribution at $783 \mathrm{~K}$.

At $T_{S} \geq 783 \mathrm{~K}$, the temporal profile of the $0 \rightarrow 0$ channel reveals a clear double peak structure with a narrow early, fast component peaking at $\sim 12 \mu \mathrm{s}$ and a late, slow and broad component peaking at $\sim 22 \mu \mathrm{s}$. The fast component is almost independent of surface temperature and is present for all surface temperatures. The slow component decreases with decreasing surface temperature and appears only as a small tail at $T_{S}=573 \mathrm{~K}$. I used a bimodal speed distribution to fit the $0 \rightarrow 0$ scattering experimental data at $T_{S}=783 \mathrm{~K}$. The right panel of Fig. 5.5 shows the speed distributions for $v_{f}=0$ compared to a thermal Maxwell-Boltzmann speed distribution at the surface temperature (black dotted line). The thermal expectation is in good agreement with the speed distribution of the slow component (green dashed line). Consequently, I assign the slow scattering component to trapping followed by desorption whereas the fast component reflects direct scattering.

$\mathrm{CO}(v=0 \rightarrow 1)$ does not show a clear bimodal distribution and can be very well described by a single broad speed distribution. The amplitude depends strongly on surface temperature, which is a result of the $T_{S}$ dependent $v=0 \rightarrow 1$ excitation probability (Fig. 5.6). In contrast to the arrival time distributions of the $0 \rightarrow 0$ channel, the temporal profile does not change its shape significantly at $T_{S}=673 \mathrm{~K}$ or $573 \mathrm{~K}$. A direct comparison of the time-of-flight distributions for $0 \rightarrow 0$ and $0 \rightarrow 1$ scattering at $T_{S}=783 \mathrm{~K}$ shows that the $v=0 \rightarrow 1$ time-of-flight profile (gray line in Fig. 5.5) agrees very well with the slow component of the $0 \rightarrow 0$ channel. This observation indicates that vibrational excitation of $\mathrm{CO}\left(v_{f}=1\right)$ scattering from $\mathrm{Pt}(111)$ is dominated by a trapping-desorption mechanism. 


\section{Absolute excitation probabilities}

The primary goal of this chapter is to use the experimental observations of section 5.1 to determine absolute vibrational excitation probabilities. For this purpose, I first corrected REMPI spectra like those shown in Fig. 5.1 for differences in laser power and detector gain. Because all spectra were recorded at the same scattering angle of $\sim 18^{\circ}$ and angular distributions broaden with increasing $T_{S}$ in a similar way for $\mathrm{CO}\left(v_{f}=0,1\right)$, I neglect the effect of angular dilution in the further analysis. However, the temporal profile of the molecular beam changes strongly with surface temperature and can influence the observed intensity in the REMPI spectrum. Therefore, I integrated the normalized time-of-flight distributions to apply a correction for the broadening effect at low $T_{S}$. Furthermore, I use the FranckCondon factors for the $B \leftarrow X(0-0)$ and (1-1) band to correct the REMPI intensities. This is a minor correction because they are near unity for both vibrational bands [97]. Fig. 5.6 shows the resulting absolute excitation probabilities as a function of surface temperature (black dots). The dashed line shows the expectation for complete equilibration with the surface temperature (thermal limit) and the red line corresponds to an Arrhenius fit with the activation energy equal to the vibrational energy uptake of $\Delta E_{v i b}=0.265 \mathrm{eV}$. The excitation probabilities observed in the experiment can be well described by the Arrhenius law. The prefactor, which is related to the intrinsic coupling strength, is near unity and the Arrhenius fit overlays almost perfectly with the thermal limit. Consequently, the measured excitation probabilities reflect the maximum possible vibrational excitation. The $\mathrm{CO}$ vibration is equilibrated with the surface $\left(T_{v i b}=T_{S}\right)$.
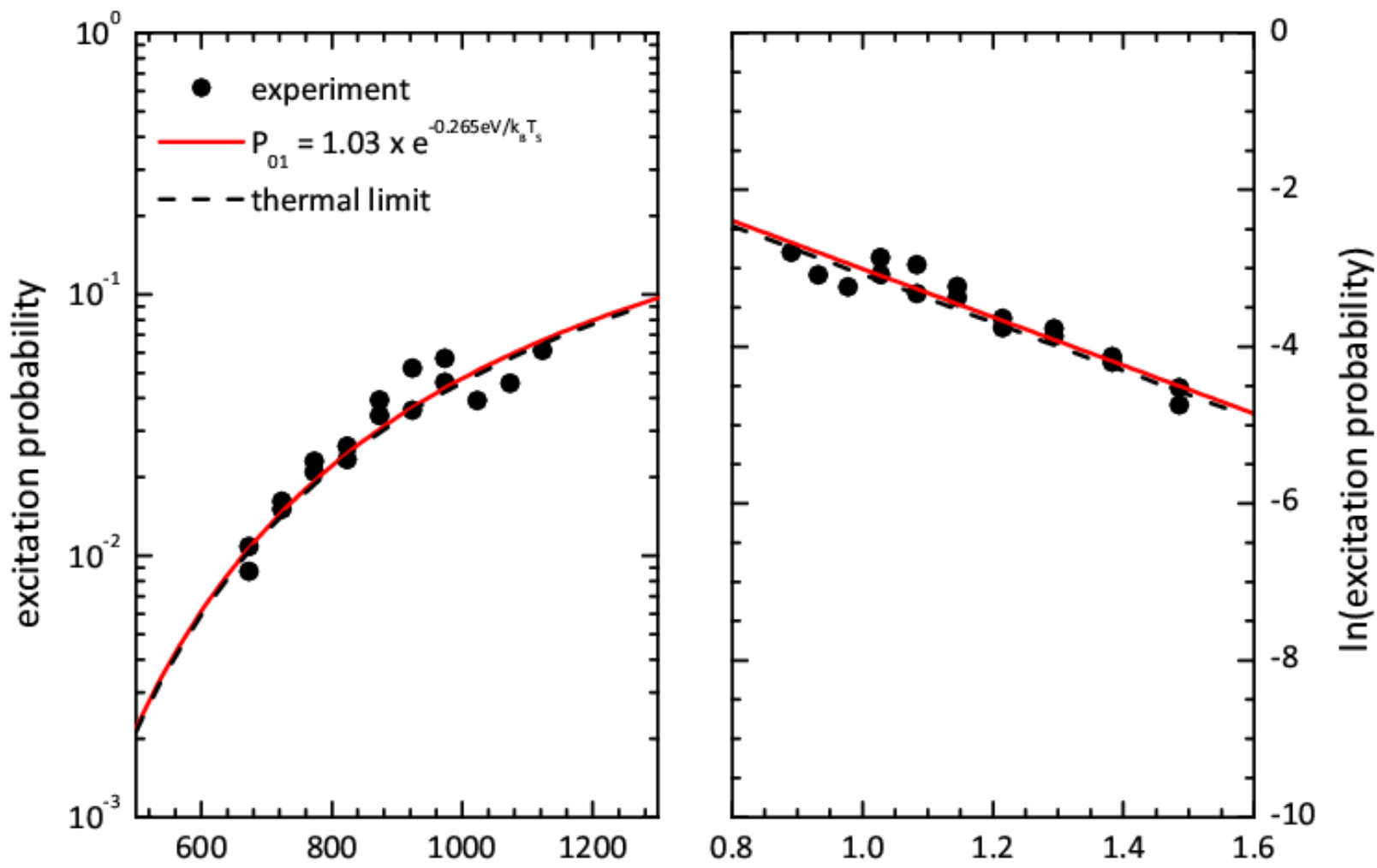

\section{surface temperature / $\mathrm{K} \quad$ inverse surface temperature / $1000 \mathrm{~K}^{-1}$}

Fig. 5.6: CO vibrational excitation probabilities for scattering from Pt(111). The two panels show the experimental data (black dots) together with an Arrhenius fit (red line) and the thermal limit (dashed black line) on a logarithmic scale (left panel) and on an Arrhenius plot (right panel). The Arrhenius activation energy was fixed to the vibrational energy uptake $\Delta E_{v i b}=0.265 \mathrm{eV}$. The experimental data agree well with the thermal expectation. 


\subsection{Discussion}

The presented results show that $\mathrm{CO}(v=0 \rightarrow 1)$ excitation probabilities after scattering from $\mathrm{Pt}(111)$ are in agreement with the thermal limit. I found a prexponential factor of $A_{01}=1.03 \pm 0.05$ while the expectation for complete equilibration is $A_{01}=1$. The first question to answer is whether the vibrational excitation happens upon a direct scattering or a trapping-desorption mechanism. In contrast to $\mathrm{NO} / \mathrm{Au}(111)$ scattering, the experimental results show that $\mathrm{CO}$ vibrational excitation in scattering off $\mathrm{Pt}(111)$ is dominated by a trapping-desorption mechanism. The angular distributions are broad for $\mathrm{CO} / \mathrm{Pt}(111)$, almost reaching $\cos \theta$, and the temporal profile of the scattered molecular beam changes significantly with surface temperature. Most importantly, the speed distribution of $\operatorname{CO}\left(v_{f}=1\right)$ is slow and broad whereas I find an additional fast component, attributed to direct scattering, in the speed distribution for $v=0 \rightarrow 0$ scattering. The slow component agrees reasonably well with a thermal Maxwell-Boltzmann distribution at $T_{S}$ and can therefore be assigned to trapping followed by desorption. All these results suggest that vibrational excitation in CO/Pt(111) scattering is caused by trapping followed by desorption after equilibration with the surface.

Previous results on the vibrational lifetime of $\operatorname{CO}(v=1)$ adsorbed on $\operatorname{Pt}(111)[48,124]$ already revealed a strong nonadiabatic interaction between the $\mathrm{CO}$ vibration and the EHPs of the metal. Therefore, it is very likely that $\mathrm{CO}(v=0 \rightarrow 1)$ excitation occurs upon V-EHP coupling even if the scattering is dominated by a trapping-desorption mechanism. This hypothesis is further supported by experiments on the $\mathrm{CO} / \mathrm{NaCl}(100)$ system that revealed a vibrational lifetime of $4.3 \mathrm{~ms}$ [53]. The basic difference between the two systems is the absence of EHPs for the insulating $\mathrm{NaCl}$ crystal. Therefore, the vibrational energy can only decay either through IR fluorescence or by coupling to the lattice phonons. The inefficiency of the latter effect is indicated by a vibrational lifetime that is similar to that in the gas phase.

Besides the nature of $\mathrm{CO}(v=0 \rightarrow 1) / \mathrm{Pt}(111)$ excitation, the $T_{S}$ dependence of the $\mathrm{CO}\left(v_{f}=0\right) \mathrm{REMPI}$ spectra (Fig. 5.1) and the temporal profiles (Fig. 5.3) requires detailed explanation and analysis. In contrast to $\mathrm{CO} / \mathrm{Au}(111)$ scattering (see section 4.1), the REMPI spectra of $\mathrm{CO}(v=0 \rightarrow 0) / \mathrm{Pt}(111)$ exhibit a significant dependence on surface temperature scattering. The spectra in the left panel of Fig. 5.1 only exhibit a weak rotational rainbow feature for $T_{S}=673 \mathrm{~K}$, which is in agreement with the fraction of $\mathrm{CO}\left(v_{f}=0\right)$ molecules that undergo direct scattering as identified in the bimodal speed distribution (Fig. 5.5). At higher surface temperatures the rainbow is covered by an increase of band head intensity. But why does the intensity in the (0-0) band head increase at all? The fraction of incident $v=0$ molecules that scatter back into $v_{f}=1$ is small and can only reduce the intensity with increasing $T_{S}$, thus it can only cause the opposite effect. The answer to this question results from the arrival time distributions of Fig. 5.3 , keeping in mind that all REMPI spectra were measured at the same temporal delay of $250 \mu \mathrm{s}$. The peak intensity of the (0-0) Q branch decreases because the temporal profile changes its shape strongly at $T_{S}=673 \mathrm{~K}$. This change in the temporal profile, which was measured for a REMPI wavelength of $230.04 \mathrm{~nm}$ (close to the (0-0) band head, probing $J_{f} \approx 7-10$ ), results from the fact that the surface temperature approaches the desorption temperature of 500-550 K for CO from $\mathrm{Pt}(111)[138,141]$. The residence time $\tau_{a d s}$ increases exponentially for decreasing $T_{S}$. Because the time scale of the experiment is limited by the length of the molecular beam pulse $\left(F W H M_{\text {in }} \approx 70 \mu \mathrm{s}\right)$ an increase in residence time will only become visible for $\tau_{a d s} \geq 10-100 \mu \mathrm{s}$. This effect appears more clearly in velocity resolved molecular beam profiles (Fig. 5.7). I applied the same double resonance scheme used to measure the speed distributions shown in Fig. 5.4 and Fig. 5.5. However, I now fix the temporal delay between UV tagging and REMPI detection to select a well-defined velocity and vary the delay between the opening of the pulse valve and the UV tagging laser. Consequently, I obtain the time-dependent flux of molecules that leave the surface with a certain final velocity, $s_{f}$. Therefore, this method suppresses all effects of velocity spread on the temporal profile of the scattered molecular beam. 


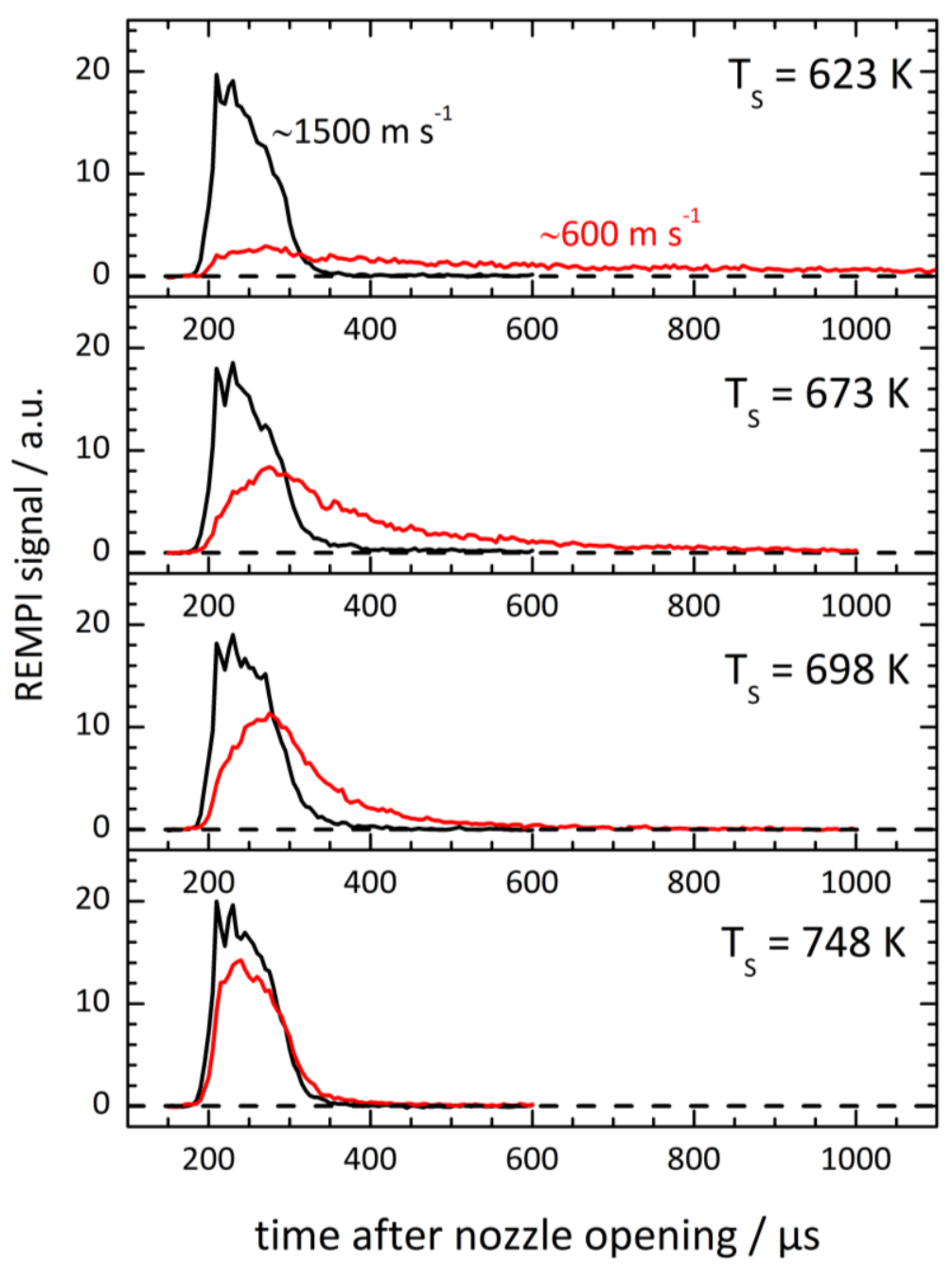

Fig. 5.7: Velocity resolved time-of-flight spectra for $\mathrm{CO}(v=0 \rightarrow 0) / \mathrm{Pt}(111)$ scattering as a function of surface temperature. The UV-UV double resonance is used to filter out a narrow range of final velocity. The data shows the time-dependent density at the position of the tagging laser which was placed less than $1 \mathrm{~mm}$ in front of the surface. The black traces show the results for a UVUV delay of $12 \mu \mathrm{s}\left(\sim 1500 \mathrm{~m} \mathrm{~s}^{-1}\right)$, the red lines show the results for a UV-UV delay of $30 \mu \mathrm{s}\left(\sim 600 \mathrm{~m} \mathrm{~s}^{-1}\right)$.

Fig. 5.7 shows the results for $\mathrm{CO}(v=0 \rightarrow 0)$ scattering if the UV-UV delay is set to $12 \mu \mathrm{s}\left(\sim 1500 \mathrm{~m} \mathrm{~s}^{-1}\right.$, black traces) or $30 \mu \mathrm{s}$ ( $600 \mathrm{~m} \mathrm{~s}^{-1}$, red traces) for different surface temperatures of the Pt(111) surface. The two chosen delays correspond to the two vertical dashed lines in Fig. 5.4. A final speed of $s_{f}=1500 \mathrm{~m} \mathrm{~s}^{-1}$ mainly samples the fast $T_{S}$ independent component in the speed distribution while $s_{f}=600 \mathrm{~m} \mathrm{~s}^{-1}$ is mainly sensitive to the $T_{S}$ dependent slow component.

Fig. 5.7 illustrates clearly the absence of a surface temperature effect in the direct scattering channel (black curves), which supports again the view of the origin of this component. In contrast, the trappingdesorption component (red traces) exhibits a strong $T_{S}$ dependence. At $T_{S}=748 \mathrm{~K}$, the temporal profile is very similar to the fast component and appears only slightly broader. With decreasing surface temperature the profile of the slow component spreads out in time and decreases in intensity. Because any velocity spread is suppressed in the measurement, this time spread can only be related to an increasing residence time, $\tau_{a d s}$, with decreasing surface temperature. An Arrhenius law using a activation energy for desorption $E_{d}=1.297 \mathrm{eV}$ and a prefactor of $A_{d}=2.9 \times 10^{13} \mathrm{~s}^{-1}$ [145] predicts a 
residence time of $\tau_{a d s}=179 \mu \mathrm{s}$ at $T_{S}=673 \mathrm{~K}$, which is on the time scale of the experiment. I further develop this method and the corresponding analysis in section 7.

In summary, the velocity resolved molecular beams profiles enable me to relate the broadening of the temporal profiles in Fig. 5.3 and the decrease in $\mathrm{CO}\left(v_{f}=0\right)$ REMPI signal (Fig. 5.1) to an increase in residence time. I note that this conclusion can only be made based on the results of Fig. 5.7. If I used only one laser to scan the temporal profile of the molecular beam, I cannot distinguish residence time and velocity spread.

Finally, I point out that the intensity of (0-0) Q branch stays almost constant below $230.00 \mathrm{~nm}\left(U_{f} \geq 25\right)$ for the whole range of surface temperatures. This observation results from the mechanism for scattering into these high $J_{f}$ states. The rotational population in this region is mainly caused by rotational rainbow scattering and therefore results from direct scattering, which is (almost) independent of surface temperature.

\subsection{Summary and Conclusions}

In summary, I have performed state-resolved experiments for $\mathrm{CO}(v=0 \rightarrow 0,1)$ scattering from $\mathrm{Pt}(111)$ at surface temperatures $623 \mathrm{~K} \leq T_{S} \leq 1123 \mathrm{~K}$ and $0.65 \mathrm{eV}$ incidence energy. The $\mathrm{CO}(v=0 \rightarrow 1)$ excitation probabilities agree well with the expectation for complete equilibration with the surface, exceeding the value for $\mathrm{CO}(v=0 \rightarrow 1)$ excitation in scattering from $\mathrm{Au}(111)$ by a factor of 10-15. I found broad angular and surface temperature dependent arrival time distributions that indicate the importance of trapping followed by desorption in this system. The high resolution state-to-state time-of-flight spectra for $\mathrm{CO}\left(v_{f}=0\right)$ molecules using a UV-UV double resonance scheme revealed a bimodal distribution with a narrow peak at early arrival times and a broad $T_{S}$ dependent feature at long flight times. The slow component is in agreement with a thermal Maxwell-Boltzmann distributions at $T_{S}$. In contrast, I observed only one single slow component in the state-to-state time-of-flight profiles for scattering into $v_{f}=1$.

Scans of the temporal profile of the scattered molecular beam using a constant UV-UV delay as a velocity filter show that the $T_{S}$ dependence in the arrival time distributions results from an increase in residence time of the trapped molecules with decreasing surface temperature. The residence time reaches the microsecond time scale of the experiment for $T_{S}<700 \mathrm{~K}$.

My results show that a mechanism that involves trapping followed by desorption dominates vibrational excitation of $\mathrm{CO}$ in scattering from $\mathrm{Pt}(111)$. Within the temperature regime of the experiment, the $\mathrm{CO}$ vibration shows complete equilibration with the surface. Even at $T_{S}=1100 \mathrm{~K}$, I estimate the residence time to $\tau_{a d s} \approx 30 \mathrm{~ns}$, which is much longer than the vibrational lifetime $\tau_{v i b} \approx 5 \mathrm{ps}$ of CO adsorbed on $\operatorname{Pt}(111)$ [48]. 


\section{6. $\mathrm{N}_{2}$ vibrational excitation on $\operatorname{Pt}(111)$}

$\mathrm{N}_{2}$ activation at metal surfaces is the key step in one of the most important reactions in industry: the ammonia synthesis from $\mathrm{N}_{2}$ and $\mathrm{H}_{2}$ in the Haber-Bosch process (Fig. 1.2). One of the main problems of the reaction is the high energy required for dissociation of $\mathrm{N}_{2}$ and $\mathrm{H}_{2}(\Delta H=1129 \mathrm{~kJ} / \mathrm{mole})$ which makes the use of a catalyst essential. Vibrational excitation due to nonadiabatic interactions with a hot surface could reduce the barrier for dissociation even more in the presence of a late transition state for dissociative adsorption [153]. However, nonadiabatic coupling of the $\mathrm{N}_{2}$ vibration to EHPs of a metal surface is expected to be rather weak from the point of electron affinity (see Fig. 1.6); the lowest anionic $\mathrm{N}_{2}^{-} X{ }^{2} \Pi_{g}$ state is located $\sim 2 \mathrm{eV}$ higher in energy than the neutral [154], about $0.5 \mathrm{eV}$ more than in case of $\mathrm{CO}$ [155]. In addition, even adiabatic excitation upon coupling to translation is unlikely due to the high frequency $\left(\omega_{e}=2358 \mathrm{~cm}^{-1}=0.292 \mathrm{eV}\right)$ of the $\mathrm{N}_{2}$ vibration.

Scattering of molecular nitrogen from metal surfaces has attracted a certain attention over the last decades $[11,13,104,156,157]$. The studies were mostly focused on rotationally inelastic scattering, desorption and alignment effects. To my knowledge, vibrational excitation in nonreactive scattering of $\mathrm{N}_{2}$ from a metal surface has not been observed so far.

Here, I present the first evidence for nonadiabatic vibrational $\mathrm{N}_{2}(v=0 \rightarrow 1)$ excitation in scattering from $\mathrm{Pt}(111)$. Narrow and $T_{S}$ independent angular distributions and time-of-flight profiles indicate a direct scattering event. The amount of $\mathrm{N}_{2}(v=1)$ observed in the experiments increases with increasing surface temperature in an Arrhenius-like fashion with an activation energy equal to the vibrational energy uptake of the molecule and prefactors that increase with incidence energy. In contrast to the experiments on $\mathrm{NO}$ and $\mathrm{CO}$ scattering, I simply calculated incidence energies for $\mathrm{N}_{2}$ molecular beams from the mixing ratio (equation 2.1) because IR-UV double resonance is not applicable for a homonuclear molecule. I expect the error introduced by this weakness of the experiment to be rather small. For seeded molecular beams of $\mathrm{NO}$ and $\mathrm{CO}, \mathrm{I}$ generally found a good agreement of measured and calculated beam energies. Furthermore, I could only apply surface temperatures above $473 \mathrm{~K}$ because all molecular beams contain $\mathrm{H}_{2}$ as a carrier gas which is known to dissociate on $\mathrm{Pt}(111)$ and is desorbed at about $420 \mathrm{~K}$ [137].

\subsection{Results}

\section{REMPI spectra}

I used $a^{\prime \prime}{ }^{1} \Sigma_{g}^{+} \leftarrow X^{1} \Sigma_{g}^{+}(2+1)$ REMPI for detection of $\mathrm{N}_{2}(v=0,1)$ due to good sensitivity and because rotational populations are directly extractable from the intensities of the spectrum (see section 2.3.3 for details). Examples for REMPI spectra of $\mathrm{N}_{2}\left(v_{f}=0,1\right)$ after scattering from $\mathrm{Pt}(111)$ are shown in Fig. 6.1. The left panel shows the effect of incident translational energy on the final rotational state distribution. The three spectra were normalized to the peak for comparison. It appears that the final rotational excitation produced in the scattering event strongly increases when $E_{I}$ is increased from $0.09 \mathrm{eV}$ to $1.08 \mathrm{eV}$. The spectra were fitted according to the procedure described in Appendix D. The fit results for $T_{\text {rot }}$ are shown in the left panel of Fig. 6.1 for different incidence energies and surface temperatures. The error of the fit can be estimated from the scatter in the magenta and light blue data points which show the results for the same incidence energy, $E_{I}=0.66 \mathrm{eV}$. I find that the rotational temperature of the vibrationally elastic $v=0 \rightarrow 0$ channel does not depend on surface temperature but systematically increases with $E_{I}$. 


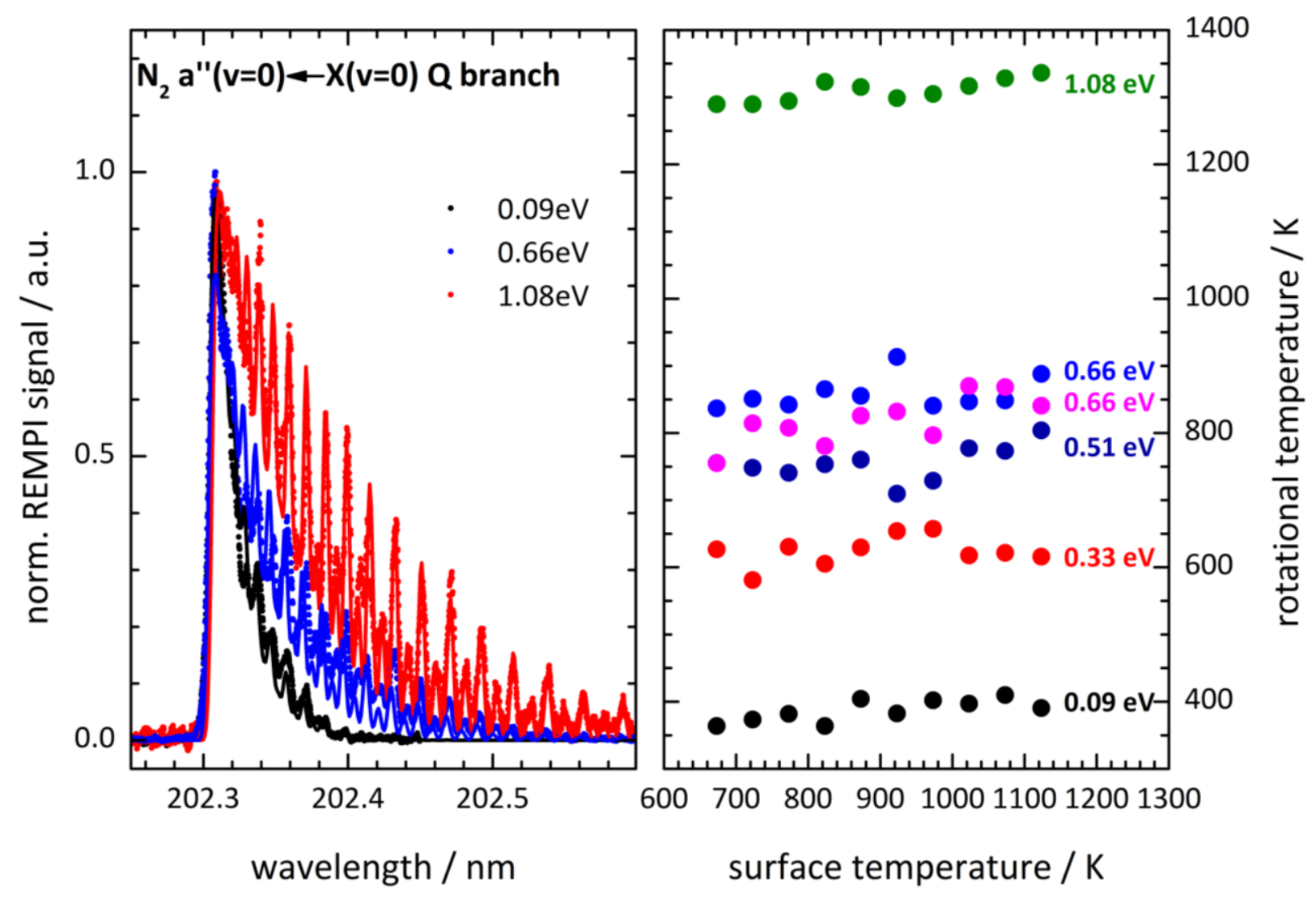

Fig. 6.1: REMPI spectra for $\mathrm{N}_{2}\left(v_{f}=0,1\right)$ after scattering from $\mathrm{Pt}(111)$. Left panel: Normalized REMPI spectra for the $\mathrm{N}_{2}(0-0) \mathrm{Q}$ branch for scattering at $E_{I}=0.09,0.66$ and $1.08 \mathrm{eV}$ with the dots reflecting the experimental data and the lines showing the corresponding fits. Although the spectra do not show prominent rotational rainbow features the rotational excitation clearly depends on $E_{I}$. Right panel: Rotational temperature for $\mathrm{N}_{2}\left(v_{f}=0\right)$ scattered at different surface temperatures and incidence energies.

$(2+1)$ REMPI spectra for the $a^{\prime \prime}\left(v^{\prime}=1\right) \leftarrow X\left(v^{\prime \prime}=1\right) \mathrm{Q}$ branch are shown in Fig. 6.2 for $E_{I}=0.66 \mathrm{eV}$ and $T_{S}=673-1073 \mathrm{~K}$. The band head of the (1-1) Q branch is located at about $202.69 \mathrm{~nm}$ and its intensity increases rapidly with increasing surface temperature. Note that some lines in the REMPI spectrum do not depend significantly on $T_{S}$. They originate from the (0-0) 0 branch which is about 200 times weaker than the (0-0) Q branch at $202.3 \mathrm{~nm}$ (Fig. 6.1). For very high rotational excitation in case of $E_{I}=1.08 \mathrm{eV}$, the red-degrading (0-0) $\mathrm{Q}$ branch also has significant intensity in this wavelength region.

In order to obtain a proper fit of the simulation to spectra like those shown in Fig. 6.2, I have to take all lines into account that result from the (0-0) bands. Because fitting of the rotational temperature for low vibrational excitation (e.g. black curve in Fig. 6.2) easily produces artificial rotational temperatures, I assumed that the rotational temperature for scattered $\mathrm{N}_{2}\left(v_{f}=1\right)$ is independent of $T_{S}$, which is in agreement with the results for $v=0 \rightarrow 0$ scattering (Fig. 6.1), and that it is produced in direct scattering (see below). I perform the complete fit only for the highest surface temperature with the most intense $\mathrm{N}_{2}\left(v_{f}=1\right)$ signal. A comparison of the fit to the experimental data is shown in the right panel of Fig. 6.2 for $T_{S}=973 \mathrm{~K}$. I finally use the obtained values for rotational temperature, $T_{\text {rot }}$, and wavelength offset, $\lambda_{o f f}$, to fit the complete dataset leaving only the amplitudes $A_{v_{f}=0}$ and $A_{v_{f}=1}$ as open fit parameters. 


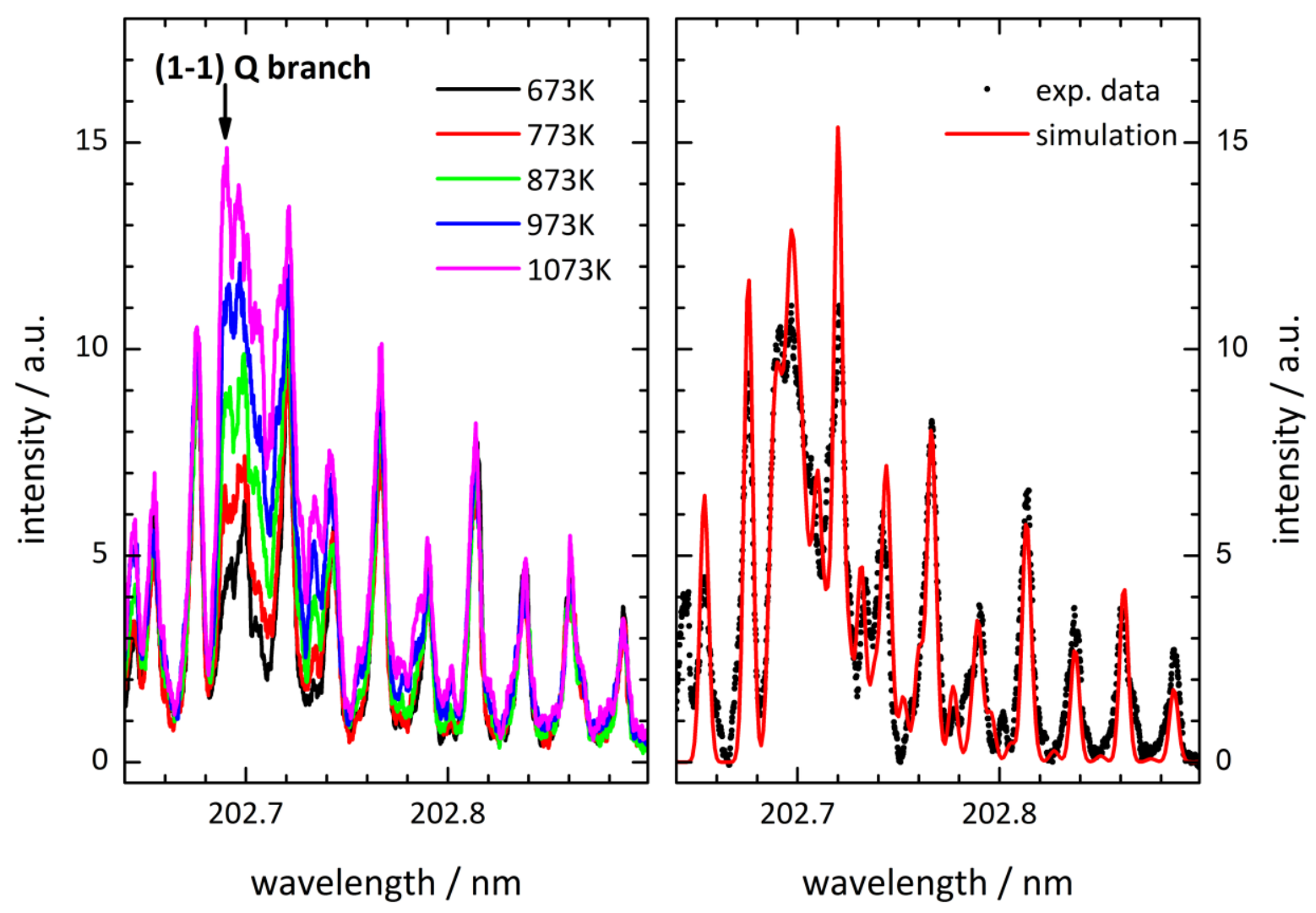

Fig. 6.2: (2+1) REMPI spectra for the (1-1) Q branch of the $\mathrm{N}_{2} a^{\prime \prime} \leftarrow \tilde{X}$ transition as a function of surface temperature for $E_{I}=0.66 \mathrm{eV}$. The intensity of the band head of the (1-1) Q branch at about $202.69 \mathrm{~nm}$ clearly increases with increasing $T_{S}$. Note that the $Q$ branch is overlapped with the (0-0) $O$ branch. Those lines do not depend significantly on $T_{S}$. The right panel shows the comparison for the experimental REMPI spectrum at $T_{S}=973 \mathrm{~K}$ (black dots) to a simulation (red line) using the method described in section 2.3.3.

I measured a spectrum of the (0-0) Q branch at every surface temperature, but with a lower detector gain, for comparison to the $v_{f}=1$ intensity. The fits for the (0-0) and (1-1) Q branches are finally integrated and corrected for detector gain and laser power fluctuations.

\section{Temporal profiles}

For every incidence energy, I measured arrival time distributions for scattered $\mathrm{N}_{2}$ molecules at various surface temperatures. The normalized time-of-flight profiles are shown in Fig. 6.3. Neither the peak of the profile nor the shape (width) exhibit a significant dependence on surface temperature or final vibrational state (left panel). Consequently, corrections originating from changes in the temporal profile of the molecular beam are small and almost negligible. The effect of incidence energy on the arrival time distribution is illustrated in the right panel of Fig. 6.3. I clearly observe a shift towards earlier times with increasing incidence energy. Note that the time axis shows the time of the REMPI detection with respect to the nozzle opening (trigger). Therefore, it contains the response time of the nozzle as well as the flight time from the nozzle to the surface and from the surface to the detection laser. Consequently, the $E_{I}$ dependence of the temporal profile can only be taken for qualitative comparison but I cannot determine the incidence energy from this data. 


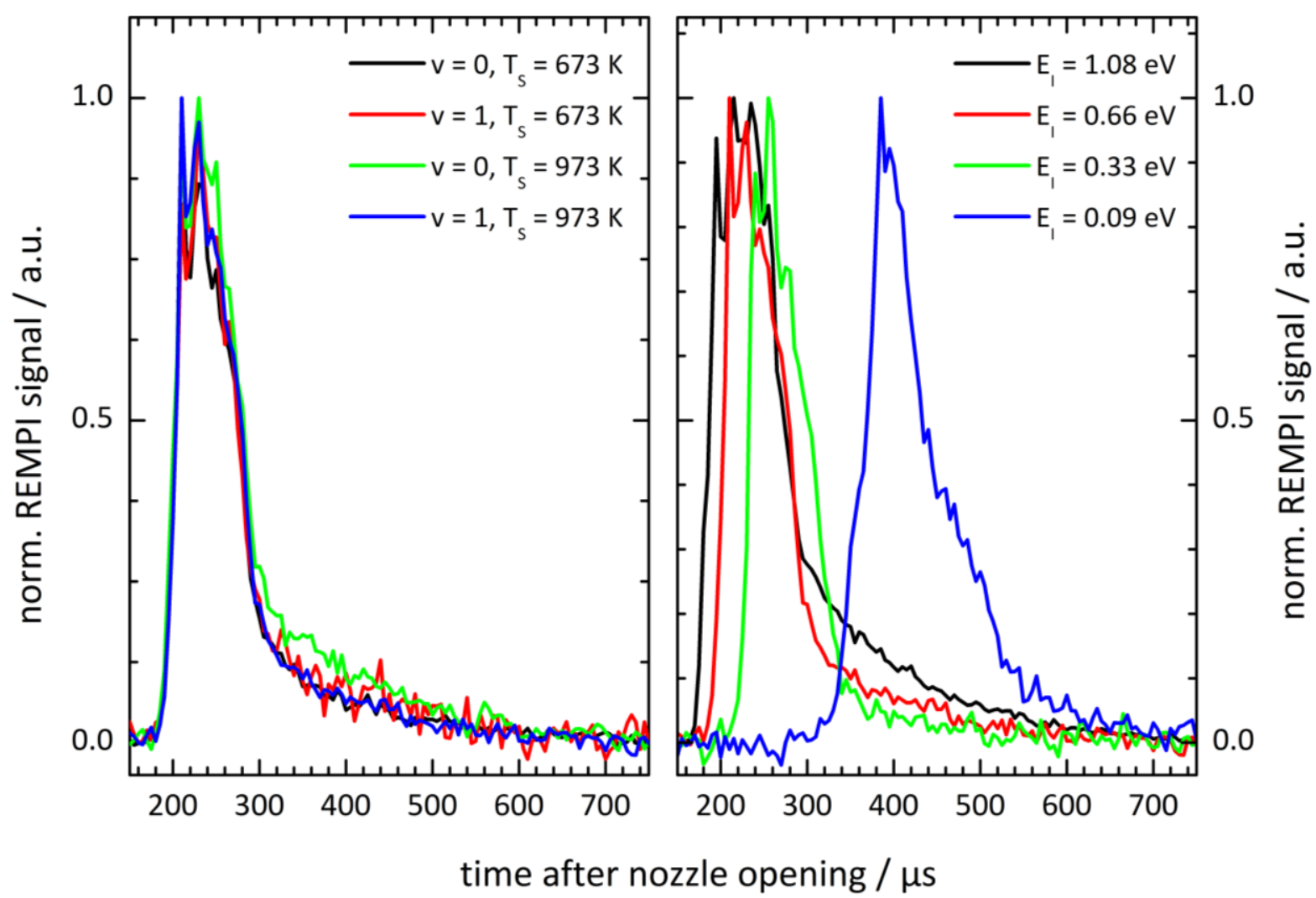

Fig. 6.3: Overview of observed arrival time distributions for $\mathrm{N}_{2} / \mathrm{Pt}(111)$ scattering. All distributions were normalized to the peak for comparison. Left panel: Temporal profiles for $\mathrm{N}_{2}\left(v_{f}=0,1\right)$ scattered off $\mathrm{Pt}(111)$ at $T_{S}=673$, $973 \mathrm{~K}$. Right panel: Time-offlight spectra for $\mathrm{N}_{2}\left(v_{f}=0\right)$ scattering off $\mathrm{Pt}(111)$ at $T_{S}=973 \mathrm{~K}$ but different incidence energies.

\section{Angular distributions}

I measured angular distributions for $\mathrm{N}_{2}\left(v_{f}=0,1\right)$ scattering off $\mathrm{Pt}(111)$ for different incidence energies and surface temperatures by translating the laser beam parallel to the surface, corresponding to perpendicular translation with respect to the incident molecular beam. Some examples for angular distributions of the scattered molecules are shown in Fig. 6.4. Under all conditions of this work, I observe very narrow angular distributions than can be characterized by a $\cos ^{25-30} \theta$ distribution. The angular distributions for scattered $\mathrm{N}_{2}\left(v_{f}=0\right)$ and $\mathrm{N}_{2}\left(v_{f}=1\right)$ are very similar, peak near the specular angle of about $4-7^{\circ}$ with respect to the incoming beam and broaden with increasing surface temperature. The effect of incidence energy on the scattered angular distributions is shown in the lower right panel of Fig. 6.4 for $E_{I}=0.09,0.51,0.66$ and $1.10 \mathrm{eV}$. For the whole range of incidence energies, the narrow width of the angular distribution is maintained and I observe only a slight broadening for the slowest molecular beam with $E_{I}=0.09 \mathrm{eV}$.

Because of the identical angular distributions for $v=0 \rightarrow 0$ and $v=0 \rightarrow 1$ scattering, I neglected the effects of angular dilution in the calculation of excitation probabilities. I compensated for the broadening effect with increasing $T_{S}$ by measuring the REMPI spectra for both vibrational states at each surface temperature. 


\section{$E_{1}=0.51 e V$}
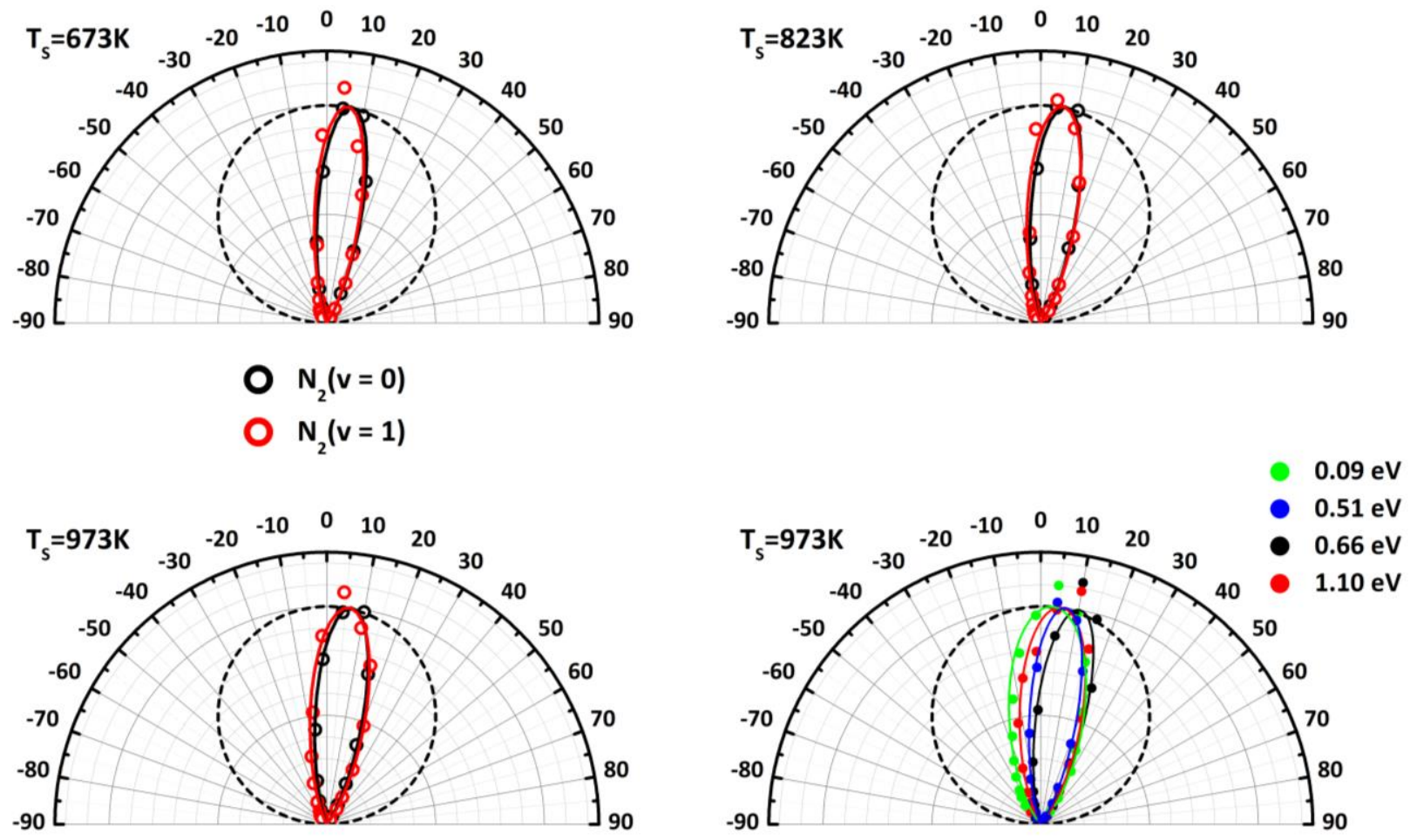

Fig. 6.4: Angular distributions for $\mathrm{N}_{2}\left(v_{f}=0,1\right)$ scattered from $\mathrm{Au}(111)$. Upper panels and bottom left panel: Angular distributions for $\mathrm{N}_{2}\left(v_{f}=0,1\right)$ scattered off $\mathrm{Pt}(111)$ at different surface temperatures and $E_{I}=0.51 \mathrm{eV}$. Lower right panel: Angular distributions for $\mathrm{N}_{2}\left(v_{f}=0\right)$ scattered from $\mathrm{Pt}(111)$ at $T_{S}=973 \mathrm{~K}$ and $E_{I}=0.09,0.51,0.66$ and $1.10 \mathrm{eV}$.

\subsection{Discussion}

Finally, I calculate vibrational excitation probabilities for $\mathrm{N}_{2}\left(v_{f}=1\right)$ in scattering from $\mathrm{Pt}(111)$ according to the procedure described in 3.1.1 and reference [26]. However, I do not take into account effects of angular dilution because angular distributions for $v_{f}=0,1$ are similar under all conditions of this work. I obtaine the spectral area $\int S(\lambda) d \lambda$ from integration of the simulations fitted to the experimental REMPI spectra. These integrals were corrected for laser power and detector gain. I include changes in the temporal profile of the scattered molecular beam but the corrections are small. Franck-Condon factors for the (0-0) and (1-1) band are similar and near unity [157] and can therefore be neglected. Some examples for the derived excitation probabilities are shown for three different incidence energies in the left panel of Fig. 6.5. The $v=0 \rightarrow 1$ excitation probabilities increase exponentially with increasing surface temperature. In all cases, the $T_{S}$ dependence is well described by an Arrhenius function with an activation energy $E_{a}=\Delta E_{v i b}=0.292 \mathrm{eV}$. Note that the higher noise level for $E_{I}=0.09 \mathrm{eV}$ is related to the very low $\mathrm{N}_{2}\left(v_{f}=1\right)$ signal in the experiment. Under all conditions of my work, the excitation probabilities are about one order of magnitude lower than the thermal limit (dashed line) which reflects the expectation for complete equilibration with the surface. 


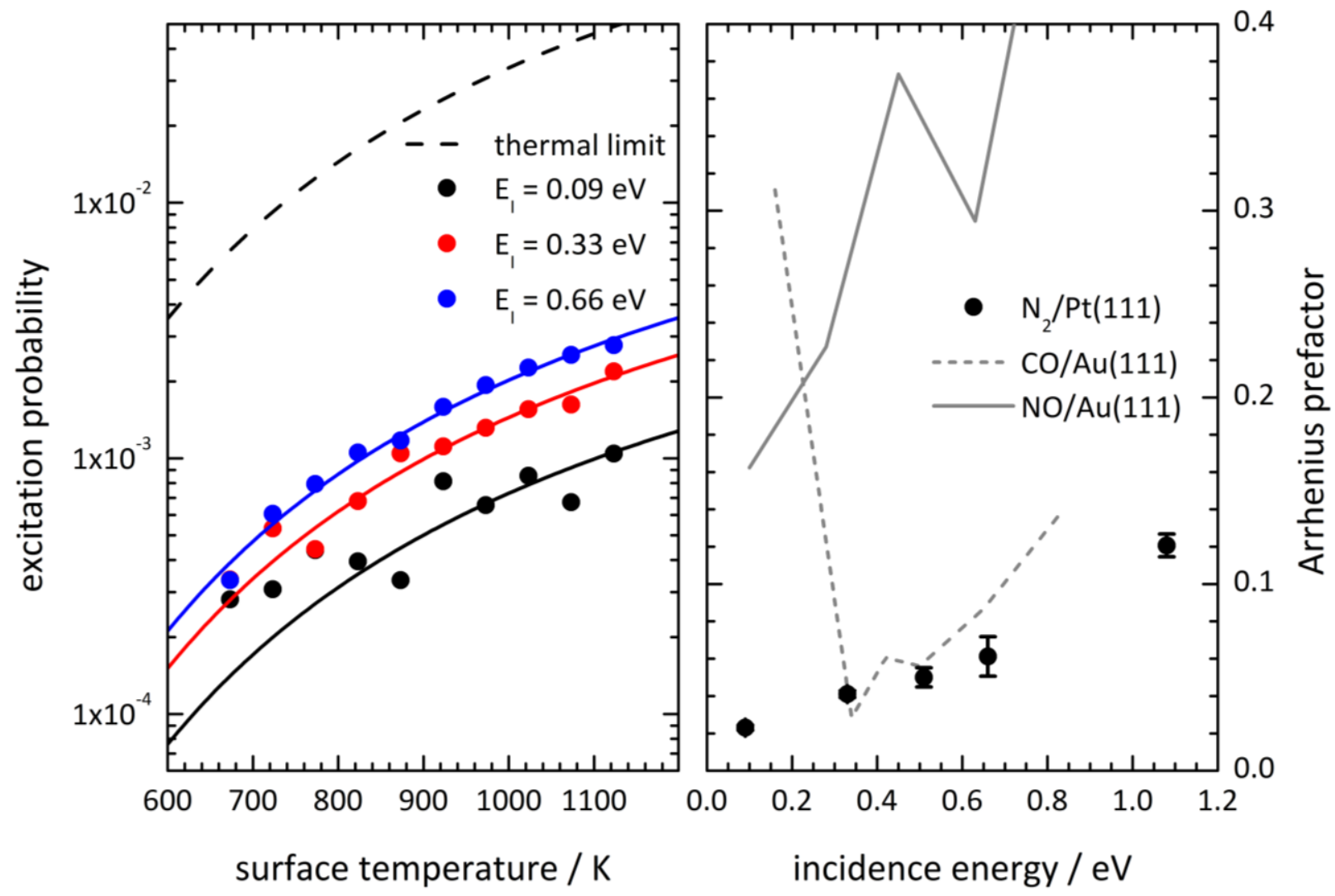

Fig. 6.5: Left panel: Absolute vibrational excitation probabilities for $\mathrm{N}_{2}(v=0 \rightarrow 1) / \mathrm{Pt}(111)$ for $E_{I}=0.09,0.33$ and $0.66 \mathrm{eV}$. The dashed line indicates the expectation for complete equilibration with the surface (thermal limit). Right panel: $E_{I}$ dependent preexponential factors from Arrhenius fits to the derived excitation probabilities (black symbol). Error bars were calculated from errors given for the Arrhenius fits and from the scatter of derived prefactors from different measurements. For comparison I show the preexponential factor $\mathrm{NO}(v=0 \rightarrow 1)$ (gray solid line) [26] and $\mathrm{CO}(v=0 \rightarrow 1)$ (section 4.1) excitation (gray dashed line) in scattering from $\mathrm{Au}(111)$.

For a comparison of different incident translational energies it is reasonable to eliminate the surface temperature dependence of the Arrhenius law and to only compare the preexponential factors. This comparison is shown in the right panel of Fig. 6.5. The Arrhenius prefactors increase with increasing incidence energy with zero threshold. All the observations for the $T_{S}$ and $E_{I}$ dependence of the $v=0 \rightarrow 1$ excitation probabilities are very similar to the observations for NO vibrational excitation in scattering from $\mathrm{Au}(111)$ and $\mathrm{Ag}(111)[8,10,25-27,44]$. The preexponential factors for $v=0 \rightarrow 1$ excitation in $\mathrm{NO} / \mathrm{Au}(111)$ [26] (gray solid line) and $\mathrm{CO} / \mathrm{Au}(111)$ (section 4.1) (gray dashed line) scattering are shown for comparison in the right panel of Fig. 6.5. The Arrhenius prefactors for NO/Au(111) are about 5-8 times higher than for $\mathrm{N}_{2} / \mathrm{Pt}(111)$ while $\mathrm{CO} / \mathrm{Au}(111)$ shows similar prefactors for $E_{I} \geq 0.34 \mathrm{eV}$ where trapping-desorption is negligible (see section 4.1).

Prior to a further discussion of the excitation probabilities, I first want to answer the question if vibrational excitation of $\mathrm{N}_{2}$ scattering from $\mathrm{Pt}(111)$ really results from a direct scattering mechanism. First, I observed narrow and $T_{S}$ independent arrival time distributions which are in agreement with a direct scattering mechanism but also with trapping-desorption if the residence time is much lower than the time resolution of the experiment $(\sim 10-100 \mu \mathrm{s})$. More direct evidence arises from measured angular distributions that are as narrow as $\cos ^{25} \theta$ and that exhibit specular scattering independent of $E_{I}$ and $T_{S}$. These observations are only in agreement with a direct impulsive scattering mechanism and rule out the importance of trapping followed by desorption. Furthermore, the rotational excitation of directly 
$\mathrm{N}_{2}$ vibrational excitation on $\mathrm{Pt}(111)$

scattered molecules is in general caused by energy transfer from incident translational energy [9, 13, $104,156]$. This will result in $E_{I}$ dependent but $T_{S}$ independent rotational excitation as well as in final velocities, $s_{f}$, that depend on the final rotational state $J_{f}$. The first two effects are reflected in the rotational temperatures of scattered $\mathrm{N}_{2}\left(v_{f}=0\right)$ molecules (Fig. 6.1). Consequently, my experimental observations suggest that $\mathrm{N}_{2}(v=0 \rightarrow 1)$ excitation occurs upon direct scattering from $\mathrm{Pt}(111)$. I note that I do not observe rotational rainbow scattering for this system like it was observed in earlier work for direct scattering from different surfaces $[11,13,104]$. The absence of rotational rainbows is possibly due to the interaction potential but molecular dynamics simulations with reasonable DFT or empirical potential are required to investigate this issue further.

$\mathrm{N}_{2}(v=0 \rightarrow 1)$ excitation in scattering from Pt(111) shows the typical fingerprints of nonadiabatic V-EHP coupling $[1,8,27,30,43,44]$. The excitation probabilities depend on surface temperature in an Arrhenius-like fashion with an activation energy equal to the vibrational energy uptake, and the Arrhenius prefactors increase with incident translational energy with zero threshold. A comparison to the well-studied $\mathrm{NO} / \mathrm{Au}(111)[1,25,27,30]$ and $\mathrm{NO} / \operatorname{Ag}(111)[8,44]$ systems shows that the Arrhenius prefactors for $\mathrm{N}_{2} / \mathrm{Pt}(111)$ are almost one order of magnitude lower than observed for NO scattering. This indicates that the V-EHP coupling itself is much weaker in this system. A possible explanation for the reduced nonadiabatic coupling strength is the almost $2 \mathrm{eV}$ lower electron affinity of $\mathrm{N}_{2}$ compared to NO (Fig. 1.6). This trend of lower vibrational excitation with decreasing electron affinity also holds for comparison of $\mathrm{CO} / \mathrm{Au}(111)$ and $\mathrm{NO} / \mathrm{Au}(111)$. Because the electron affinity of $\mathrm{N}_{2}$ is even lower than for $\mathrm{CO}$, it is actually very surprising that $\mathrm{N}_{2}$ shows any nonadiabatic coupling at all. Another question is whether the surface has an effect on the strength of the nonadiabatic V-EHP coupling? In order to answer this question, I also performed experiments to measure the excitation probabilities for $\mathrm{N}_{2}$ scattering from $\mathrm{Au}(111)$ but I was not able to observe any vibrational excitation. This observation allows the conclusion that $\mathrm{Pt}(111)$ - known to be reactive - might show stronger nonadiabatic interaction than (unreactive) $\mathrm{Au}(111)$. Another possible explanation is the higher density of states at the Fermi level for $\mathrm{Pt}(111)$ compared to $\mathrm{Au}(111)$ [133]; this can cause a purely statistical effect because the $\mathrm{N}_{2}$ molecule is able to couple to more electronic states in the metal. In any case, the hypothesis of stronger nonadiabaticity in scattering from $\mathrm{Pt}(111)$ is rather hard to test for other molecules like NO or CO because they might react or be trapped at the Pt(111) surface. The determination of vibrational lifetimes for different molecules at different surfaces, e.g. Pt(111) vs. Au(111), is a possible way to understand the influence of the surface on nonadiabatic gas-surface interactions.

\subsection{Summary and Conclusions}

I have derived absolute vibrational excitation probabilities for $\mathrm{N}_{2}(v=0 \rightarrow 1)$ scattering from $\mathrm{Pt}(111)$ at various incidence kinetic energies. The narrow angular and arrival time distributions and $E_{I}$ dependent but $T_{S}$ independent rotational distributions indicate a direct scattering mechanism. The $v=0 \rightarrow 1$ excitation probabilities show an Arrhenius surface temperature dependence with an activation energy equal to the vibrational spacing of $\mathrm{N}_{2}$ and prefactors that increase with increasing incidence energy without a threshold energy. All these observations are the typical fingerprints of nonadiabatic V-EHP coupling in molecule surface collisions and are similar to the first findings for NO vibrational excitation in collision with a $\operatorname{Ag}(111)$ surface $[8,44]$, a system for which nonadiabatic effects and charge transfer are known to be important. The observation of vibrational excitation for $\mathrm{N}_{2}$ is rather surprising because the electron affinity of $\mathrm{N}_{2}$ is low; the lowest anion state is $\sim 2 \mathrm{eV}$ higher in energy than the neutral molecule. For a charge transfer process, this energy plus the work function of the surface has to be compensated by image charge stabilization in order to stabilize a possible transient $\mathrm{N}_{2}^{-}$ion. 


\section{CO desorption from $\operatorname{Pt(111)~by~velocity~selected~residence~time~}$ measurements}

The accurate determination of binding energies between atoms and molecules in a system is crucial for the correct description of its properties. In gas-surface interactions the binding energy of the molecule to the surface is of particular interest but is not directly measureable. A frequent approach to face this problem is the determination the activation energy for desorption, $E_{d}$, in temperature programmed desorption (TPD) experiments [158]. In this method, the surface is dosed with molecules at a temperature well below the desorption temperature at which the adsorbate has sufficient thermal energy to leave the surface. The surface is afterwards heated with a constant heating rate $\beta$ and either the desorbing flux of molecules or the number of adsorbed molecules is monitored as a function of $T_{S}$. The experiments are in general repeated for different $\beta$ to determine the activation energy for desorption, $E_{d}$, and preexponential factor, $A_{d}$, of the corresponding Arrhenius equation (7.1), in surface science often referred to as Wigner-Polanyi equation:

$$
k_{d}\left(T_{S}\right)=A_{d} \times e^{-E_{d} / k_{B} T_{S}}
$$

The Redhead formula (7.2) gives the relation between the temperature $T_{\text {peak }}$ of the desorption peak, heating rate $\beta$, desorption energy $E_{d}$ and prefactor $A_{d}$ :

$$
\frac{A_{d}}{\beta}=\frac{E_{d}}{k_{B} T_{\text {peak }}^{2}} e^{-E_{d} / k_{B} T_{\text {peak }}}
$$

Equation 7.2 is only valid for first-order desorption kinetics and has different forms for higher order processes. I note that the reaction order can for example be extracted from the shape of the TPD peak. A detailed overview of the analysis of TPD spectra is given in ref. [159]. In general, TPD measurements rely on small shifts of $T_{\text {peak }}$ for different heating rates $\beta$ (typically several $\mathrm{K} / \mathrm{s}$ ). The experiments take place on a second time scale and can therefore not resolve fast kinetics. An additional problem is that TPD experiments are frequently performed only for one specific $\beta$ and simply assuming a 'reasonable' value for $A_{d}$ in order to derive $E_{d}$.

I used a new velocity selected time-of-flight approach to perform experiments on the desorption of CO from a single crystal Pt(111) surface on a microsecond to millisecond timescale. The CO/Pt(111) system was studied extensively in the past but different experimental approaches yielded surprisingly different values for $E_{d}$ and $A_{d}[138,139,144,146-152]$. The different studies reported activation energies for desorption ranging from $1.17-1.69 \mathrm{eV}$ and prefactors ranging from $10^{13}-10^{15} \mathrm{~s}^{-1}$. I demonstrate that the measurement of real-time desorption kinetics on a micro- and millisecond timescale is very sensitive to both parameters and that method has the potential to resolve fast kinetic processes from one another. Furthermore, I demonstrate the influence of steps on the adsorption-desorption dynamics.

Beside the power of the method, the accurate determination of $E_{d}$ from kinetic measurements is of fundamental interest for comparison to results from first-principles theory, mostly based on densityfunctional theory (DFT). There is a rising number of studies that consistently demonstrated the failure of DFT for description of CO binding to transition metals [160-166], an issue that is often referred to as the $\mathrm{CO} / \mathrm{Pt}(111)$ puzzle [142]. The calculations consistently prefer a high coordination number (adsorption at hollow/ bridge sites) [160,162, 163, 166], whereas experiments clearly demonstrated the preference for 
atop binding at low coverages [152, 167-169]. Moreover, the range of binding energies predicted by DFT depends strongly on the choice of the exchange correlation (XC) functional and varies by more than $1 \mathrm{eV}$ for different methods. For example, strongly gradient corrected XC functionals systematically decrease the binding energy. An accurate experimental determination of the binding energy can therefore be important in order to check the reliability of the theoretical calculations.

In order to compare to binding energies from first-principle, one needs to derive the binding energy from the experimental kinetic data. I show that transition-state theory (TST) is applicable for this problem. In general, transition-state theory can be used to calculate thermal rate constant from statistical mechanics. In absence of an activation barrier, the barrier height in TST is equal to the binding energy of the molecule. I demonstrate the application of TST to CO desorption from Pt(111) and one can derive the binding energy from the measured rate constants for desorption.

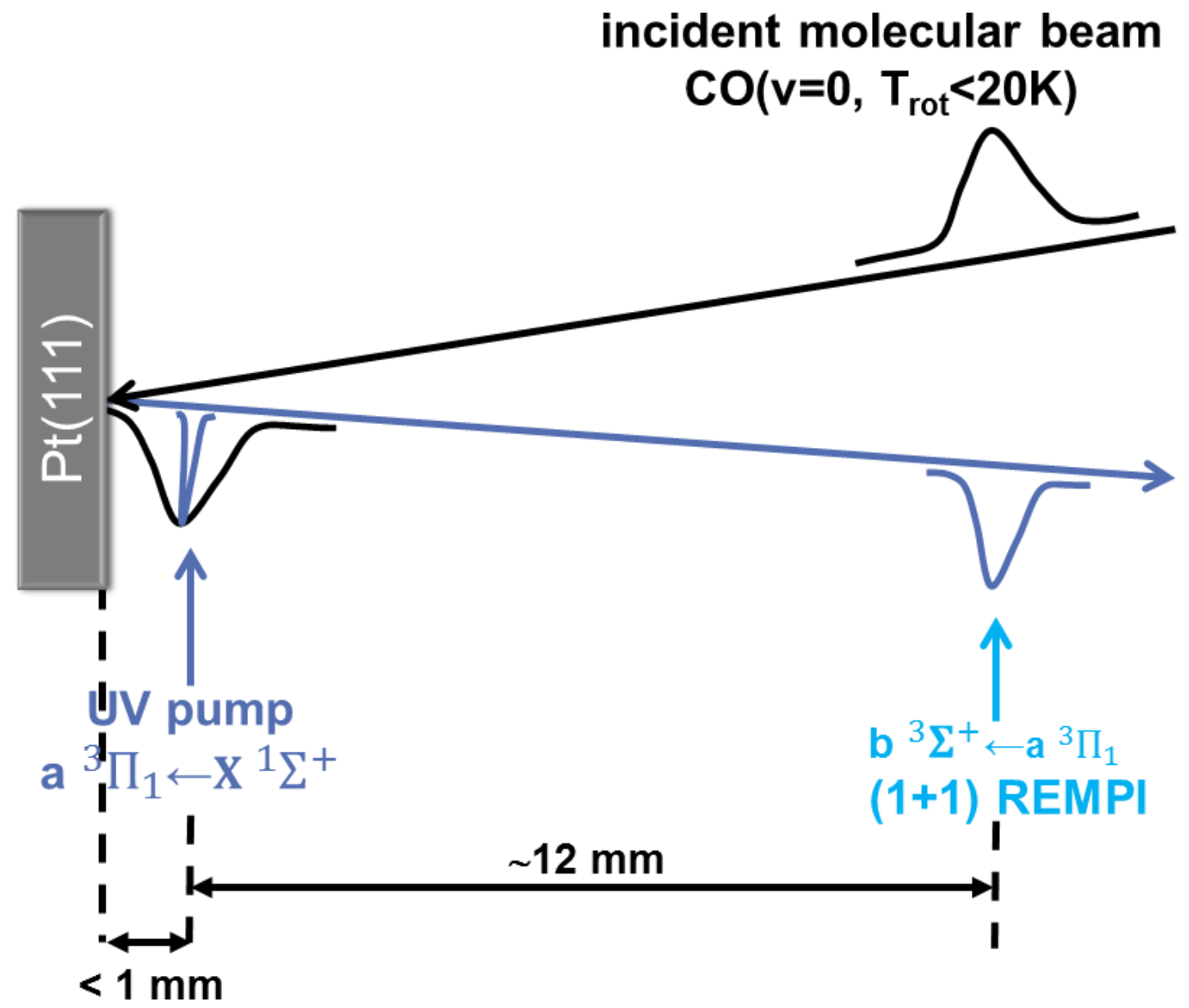

Fig. 7.1: Sketch of the experimental set-up used for experiments of CO desorption from Pt(111). A molecular beam of CO molecules (FWHM $\sim 170 \mu \mathrm{s}$ ) strikes a single crystal Pt(111) surface at $\sim 10-15^{\circ}$ incidence angle. The molecules leaving the surface are excited to the metastable $a^{3} \Pi_{1}(v=0)$ state and detected at $12 \mathrm{~mm}$ distance with $b^{3} \Sigma^{+} \leftarrow a^{3} \Pi_{1}(1+1) \operatorname{REMPI}\left(\theta_{f} \approx 9^{\circ}\right)$. 


\subsection{Methods}

\section{Velocity selected time-of-flight technique}

The method used to perform the desorption kinetics experiments is basically a simplified version of the two-dimensional time-of-flight approach presented in sections 2.4 and 4.2. Fig. 7.1 shows the basic experimental set-up for measurements of desorption kinetics. Compared to the set-up shown in Fig. 4.11 the only difference is that no IR excitation occurs prior to the surface collision. The incident molecular beam of pure $\mathrm{CO}\left(E_{I}=0.09 \mathrm{eV}\right)$ collides with a single crystal $\mathrm{Pt}(111)$ surface at $\sim 10-15^{\circ}$ incidence angle. The molecules are excited to the metastable $a^{3} \Pi_{1}(v=0)$ state less than $1 \mathrm{~mm}$ away from the surface. The metastable CO* molecules are finally detected at $\sim 12 \mathrm{~mm}$ distance by $b^{3} \Sigma^{+} \leftarrow a^{3} \Pi_{1}(1+1)$ REMPI. This set-up involves two delays $t_{1}$ and $t_{2}$ that can be varied. First, I can measure the speed distributions of the scattered/ desorbed molecules at different delays with respect to the nozzle opening by scanning the delay $t_{2}$ between the UV pump and the REMPI laser. Second, I can select a narrow range of velocities by the choice of a constant UV-UV delay, $t_{2}$, and then measure the temporal profile of the molecular beam. The second method yields the time dependent density of CO molecules that pass the UV pump laser. Because of the pre-selected final speed of the molecules and the small distance of the UV pump laser from the surface, the measured time behavior is only sensitive to residence time effects. Broadening due to velocity spread is suppressed by the constant UV-UV delay. This property of the method is the major advantage over the simple time-of-flight scans shown in Fig. 5.3. The temporal resolution of the velocity selected residence time measurements is mainly limited by the width of the molecular beam pulse of $\sim 170 \mu \mathrm{s}$.

\section{Surface coverage and step density}

An important parameter in the experiments is the dose of CO molecules to the surface by a molecular beam pulse. The number of molecules per pulse can be calculated from the average pressure, $\langle p\rangle \approx 2 \times 10^{-9}$ Torr, in the UHV chamber with the molecular beam operating. The pulsed molecular beam causes an initial pressure rise, $p_{\text {in }}$, that decays exponentially according to the pumping speed, $S=350$ $\mathrm{L} / \mathrm{s}$, and the volume, $V \approx 38 \mathrm{~L}$, of the UHV chamber.

$$
p(t)=p_{\text {in }} \times e^{-\frac{S}{V} t}
$$

Equation 7.3 results from the pumpdown time, $t=\frac{V}{S} \ln \left(\frac{p_{0}}{p}\right)$ [83], required to pump volume, $V$, from pressure $p_{0}$ to $p$. The average pressure in the chamber results from integration of equation 7.3 divided by the period time, $\tau=100 \mathrm{~ms}$, between two molecular beam pulses.

$$
\langle p\rangle=\int_{0}^{\tau} p_{\text {in }} \times e^{-\frac{S}{V} t} d t
$$

The initial pressure, $p_{\text {in }}$, caused by the molecular beam can be replaced by the ideal gas law:

$$
p_{\text {in }}=\frac{N_{i} k_{B} T}{V}
$$




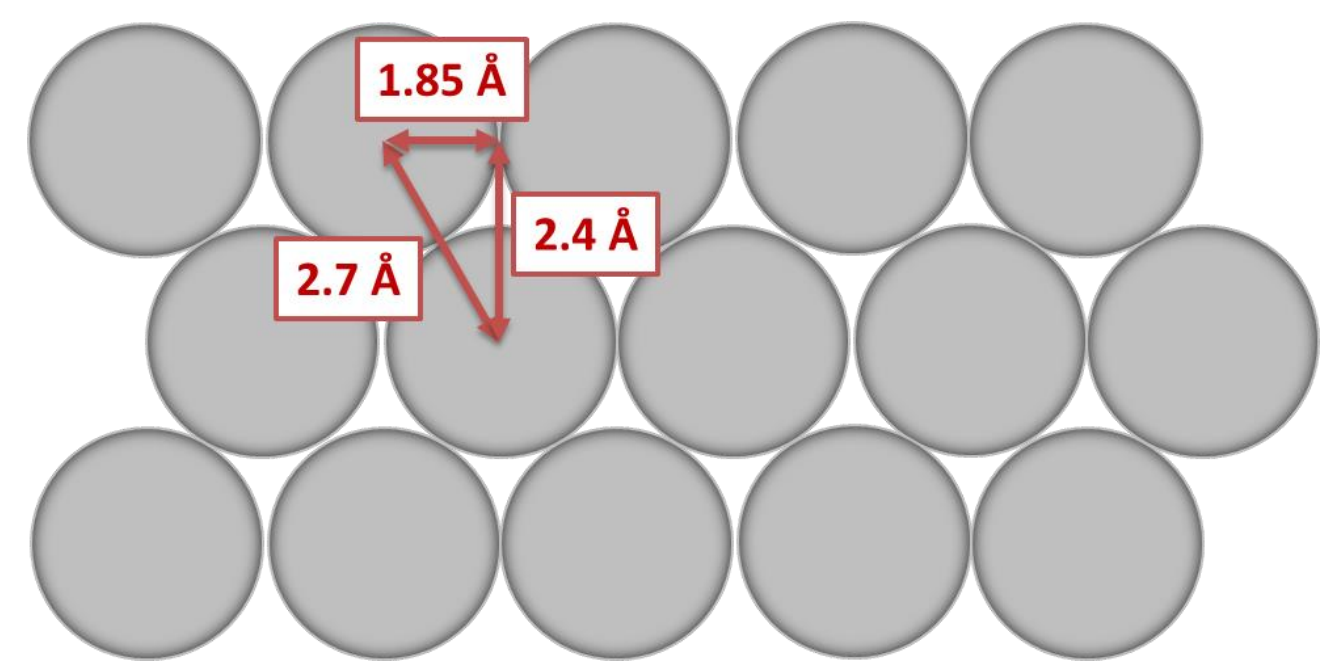

Fig. 7.2: Schematic structure of the closed packed fcc Pt(111) structure. The lattice constant of Pt is $a=3.91 \AA$ r resulting in a PtPt distance of $a / \sqrt{2}=2.7 \AA$.

From equation 7.4 and 7.5, the number of molecules, $N_{i n}$, that enter the UHV chamber in the molecular beam can be estimated. This calculation gives a dose of $5.2 \times 10^{13}$ molecules pulse $\mathrm{cm}^{-2}$, assuming a molecular beam diameter of $3 \mathrm{~mm}$ at the surface. For a Pt(111) surface (Fig. 7.2) with a lattice constant of $3.91 \AA$ [170], this dose corresponds to $\sim 3 \%$ of a monolayer.

The basic structure of a (111) surface of a face centered cubic (fcc) crystal is shown in Fig. 7.2. The distance between two Pt atoms is $\sim 2.7 \AA$, the distance between two rows of surface atoms is $2.4 \AA$. An important parameter is the step density of the Pt(111) crystal. Fig. 7.3 shows images obtained by atomic force microscopy (AFM) for a $1 \mu \mathrm{m} \times 1 \mu \mathrm{m}$ (top panels) and a $5 \mu \mathrm{m} \times 5 \mu \mathrm{m}$ (bottom panels) area. I find a high density of particles at the surface which probably result from exposure of the sample to air. Note that no cleaning procedure was applied prior to the AFM measurements. Nevertheless, the AFM images enable an estimation of the step density of the surface. The inset in the upper right panel shows the height profile along the short white arrow on the left; it demonstrates the presence of atomic steps with a height of $\sim 3 \AA$, in good agreement with the Pt-Pt distance of $2.7 \AA$. In addition, the profile illustrates that the (111) terraces can be as broad as $100-150 \mathrm{~nm}$. The large upper right panel also shows the effect of step bunching, that is, the accumulation of a high density of steps. In the shown profile, this effect is most clear in range from $400-700 \mathrm{~nm}$ with a height change of about $6 \mathrm{~nm}$.

For estimation of the step density, I use the height profile in the lower right panel of Fig. 7.3. The surface height increases by about $31.4 \mathrm{~nm}$ over a distance of $4.5 \mu \mathrm{m}$. The increase in height corresponds to 100 monoatomic steps ( $\sim 3 \AA$ per step), while the distance of $4.5 \mu \mathrm{m}$ reflects $\sim 1.9 \times 10^{4}$ rows of surface atoms with a distance of $2.4 \AA$. The comparison of the number of surface atoms and the number of steps yields a step density of $\sim 0.5 \%$. Although there might be areas with higher densities of steps, the surface dose of the molecular beam should on average be higher than the number of available step sites. Consequently, the intense molecular beam is able to saturate the steps at the Pt(111) surface as long as the sticking probability is higher than 0.3 . 

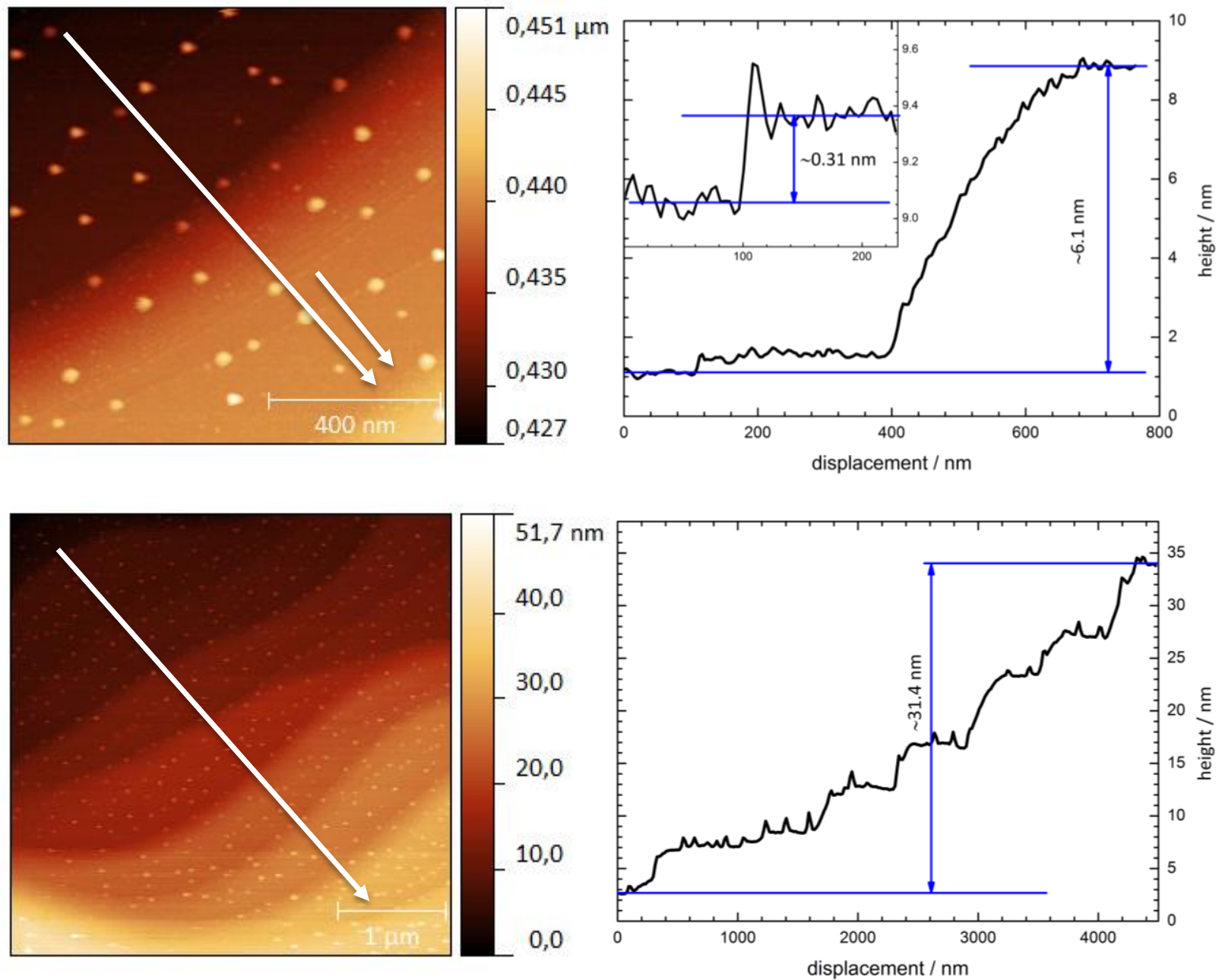

Fig. 7.3: Left panels: AFM images of the Pt(111) crystal used in this work for a $1 \mu \mathrm{m} \times 1 \mu \mathrm{m}$ (top) and $5 \mu \mathrm{m} \times 5 \mu \mathrm{m}$ (bottom) square area. The high density of small particles probably originates from the exposure to air prior to the AFM measurement for several weeks. Right panels: Height profiles along the white arrows in the images on the left. The inset in the top right panel shows the height profile along the small arrow on the left to illustrate the typical step height of a monoatomic step of about $0.3 \mathrm{~nm}$. Higher steps, as in the $400-650 \mathrm{~nm}$ in the main panel, correspond to step bunching. The lower right panel shows the height profile over a distance of $4500 \mu \mathrm{m}$. The height changes by about $31.4 \mathrm{~nm}$ which corresponds to 100 steps.

\section{Kinetic model for desorption and analysis}

In order to model the temporal behavior of the molecular beam profile after the surface collision as a function of surface temperature, I first applied a simple first-order kinetic model to simulate the time dependent flux of molecules that leave the surface. Previous studies on the adsorption and desorption of CO from Pt(111) suggested a simple first-order desorption kinetic [143, 145]. For this case, the timedependent number of adsorbed molecules increases according to the pulsed incident flux of $\mathrm{CO}$ molecules, $\Phi_{i n}(t)$, and decreases according to the first-order desorption rate constant $k_{d}$ :

$$
\frac{d N_{a d}(t)}{d t}=\Phi(t)_{i n}-k_{d}\left(T_{S}\right) N_{a d}(t)
$$


I determine the temporal behavior of the incident flux of molecules by measuring the temporal profile of the scattered molecular beam at $T_{S}=973 \mathrm{~K}$; here the residence time is short enough to be negligible. I model the high $T_{S}$ profile by a sum two Gaussian functions (equation 7.7) to obtain an analytical function for $\Phi_{\text {in }}(t)$. Alternatively, I can measure the temporal profile of the incident molecular beam at the position of the UV pump laser.

$$
\Phi_{\text {in }}(t)=A_{1} \times e^{-\left(t-t_{01}\right)^{2} / w_{1}^{2}}+A_{2} \times e^{-\left(t-t_{02}\right)^{2} / w_{2}^{2}}
$$

Fig. 7.4 shows a comparison between the temporal profile of the incident molecular beam (blue dashed line) and the temporal profile of desorbed/ scattered CO molecules at $T_{S}=973 \mathrm{~K}$ (open symbols). At this surface temperature, the residence time of the molecules is much smaller than the molecular beam pulse duration. Both profiles are very similar and the small differences might result from the different experimental methods. The temporal profile of the incident beam is measured with one single laser; therefore different velocities contribute to the shape of the temporal profile. Because the scattered profile is measured at conditions that are more similar to those in $T_{S}$ dependent measurements of $\Phi_{\text {out }}(t)$, I fit equation 7.7 to the open black symbols in Fig. 7.4 to model $\Phi_{\text {in }}(t)$.

The differential equation 7.6 can be solved analytically for $N_{a d}(0)=0$. The desorbing flux of molecules, $\Phi_{\text {out }}(t)$, that is measured in the experiment, is given by equation 7.8 .

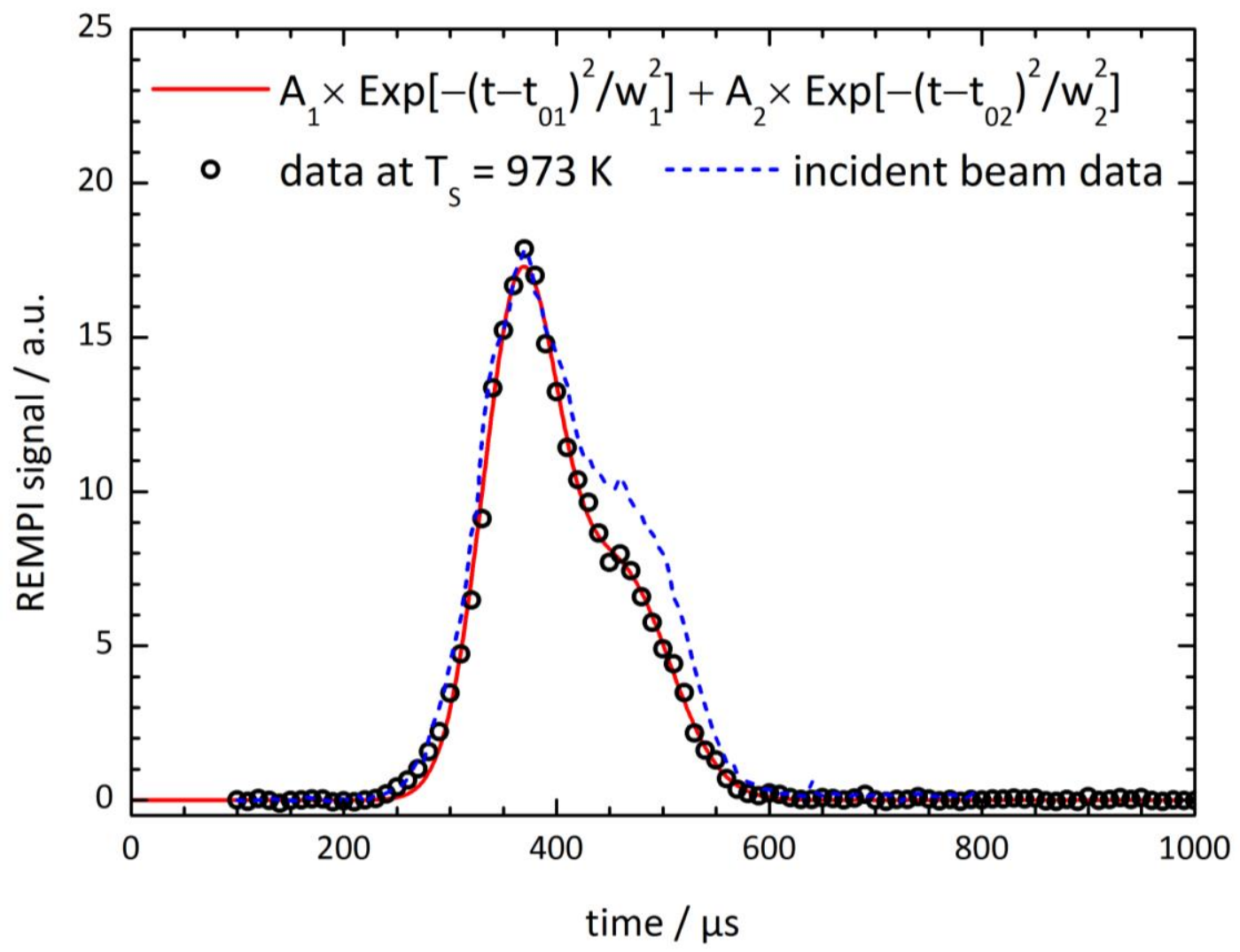

Fig. 7.4: Example for modeling of $\Phi_{\text {in }}(t)$ by a sum of two Gaussian functions. The analytical function is fitted to the temporal profile for $\mathrm{CO} / \mathrm{Pt}(111)$ scattering at $T_{S}=973 \mathrm{~K}$. At this temperature the trapped molecules should have a residence time at the surface of less than $1 \mu \mathrm{s}$ and therefore beyond the time scale of the experiment. The blue dashed line shows the temporal profile of the incident molecular beam for comparison. 


$$
\Phi_{\text {out }}\left(t, T_{S}\right)=\mathrm{B}_{\mathrm{TD}}\left(T_{S}\right) k_{d}\left(T_{S}\right) N_{a d}(t)
$$

$\Phi_{\text {out }}\left(t, T_{S}\right)$ is fitted to the experimental data at each surface temperature separately leaving the desorption rate constant $k_{d}\left(T_{S}\right)$ and an amplitude $B_{T D}\left(T_{S}\right)$ as the only adjustable fit parameters.

As the trapping probability is not unity at $E_{I}=0.09 \mathrm{eV}, \mathrm{I}$ have to add a second term to equation 7.8 to take direct scattering contributions into account. Since directly scattered molecules have a negligible residence time, their contribution to $\Phi_{\text {out }}(t)$ can be simply modeled by $\Phi_{\text {in }}(t)$ multiplied with an scaling factor $B_{D S}$. In principle, $B_{D S}$ is related to the sticking probability but its exact value can vary with $T_{S}$ according to changes in the final speed and rotational distribution of the CO molecules leaving the surface.

$$
\Phi_{\text {out }}\left(t, T_{S}\right)=\underbrace{\mathrm{B}_{\mathrm{DS}}\left(T_{S}\right) \Phi_{\mathrm{in}}(t)}_{\text {direct scattering }}+\underbrace{B_{T D}\left(T_{S}\right) k_{d}\left(T_{S}\right) N_{a d}(t)}_{\text {trapping-desorption }}
$$

As I will show below, I observe evidence for bi-exponential desorption kinetics when I compare the raw experimental data to the first-order kinetic model (equation 7.9). In order to take these deviations into account, I modify equation 7.9 and introduce two desorption rates with rate constants $k_{d}^{f a s t}\left(T_{S}\right)$ and $k_{d}^{\text {slow }}\left(T_{S}\right)$ and amplitudes $B_{T D}^{\text {fast }}\left(T_{S}\right)$ and $B_{T D}^{\text {slow }}\left(T_{S}\right)$.

$$
\Phi_{\text {out }}\left(t, T_{S}\right)=\underbrace{\mathrm{B}_{\mathrm{DS}}\left(T_{S}\right) \Phi_{\mathrm{in}}(t)}_{\text {direct scattering }}+\underbrace{B_{T D}^{\text {fast }}\left(T_{S}\right) k_{d}^{\text {fast }}\left(T_{S}\right) N_{a d}^{(1)}(t)}_{\text {fast desorption }}+\underbrace{B_{T D}^{\text {slow }}\left(T_{S}\right) k_{d}^{\text {slow }}\left(T_{S}\right) N_{a d}^{(2)}(t)}_{\text {slow desorption }}
$$

\subsection{Results}

\section{Speed distributions}

The constant temporal delay, $t_{2}$, between UV excitation (tagging) and REMPI detection is a key parameter for the observation of desorption kinetics. By the choice of a fixed $t_{2}$ delay, the experiment becomes sensitive to only those molecules that leave the surface with a certain final velocity, $s_{f}$. Consequently, velocity spread is suppressed and changes in the temporal profiles of the molecular beam are related to residence time effects. Take note that the method only gives the correct desorption kinetic if the speed distribution of the desorbed molecules is independent of their residence time at the surface. Fig. 7.5 shows time-of-flight profiles between UV excitation and REMPI detection for various experimental conditions. All datasets are normalized to the peak for comparison. The solid lines represent the corresponding fits to the experimental data. The fit function is derived following the procedure described in Appendix B. In this case, the scattered/ desorbed molecules pass two laser beams after they leave the surface. Both UV tagging and REMPI detection are sensitive to the density of molecules in the excitation volume. In order to convert the measured density into a time-dependent flux, I have to multiply the REMPI signal with the speed of the molecules once per laser excitation step. Consequently, I have to apply density-to-flux conversion twice by multiplication of time-dependent density, $S(t)$, with $s^{2}$. This changes equation 9.3 to equation 7.11 . 


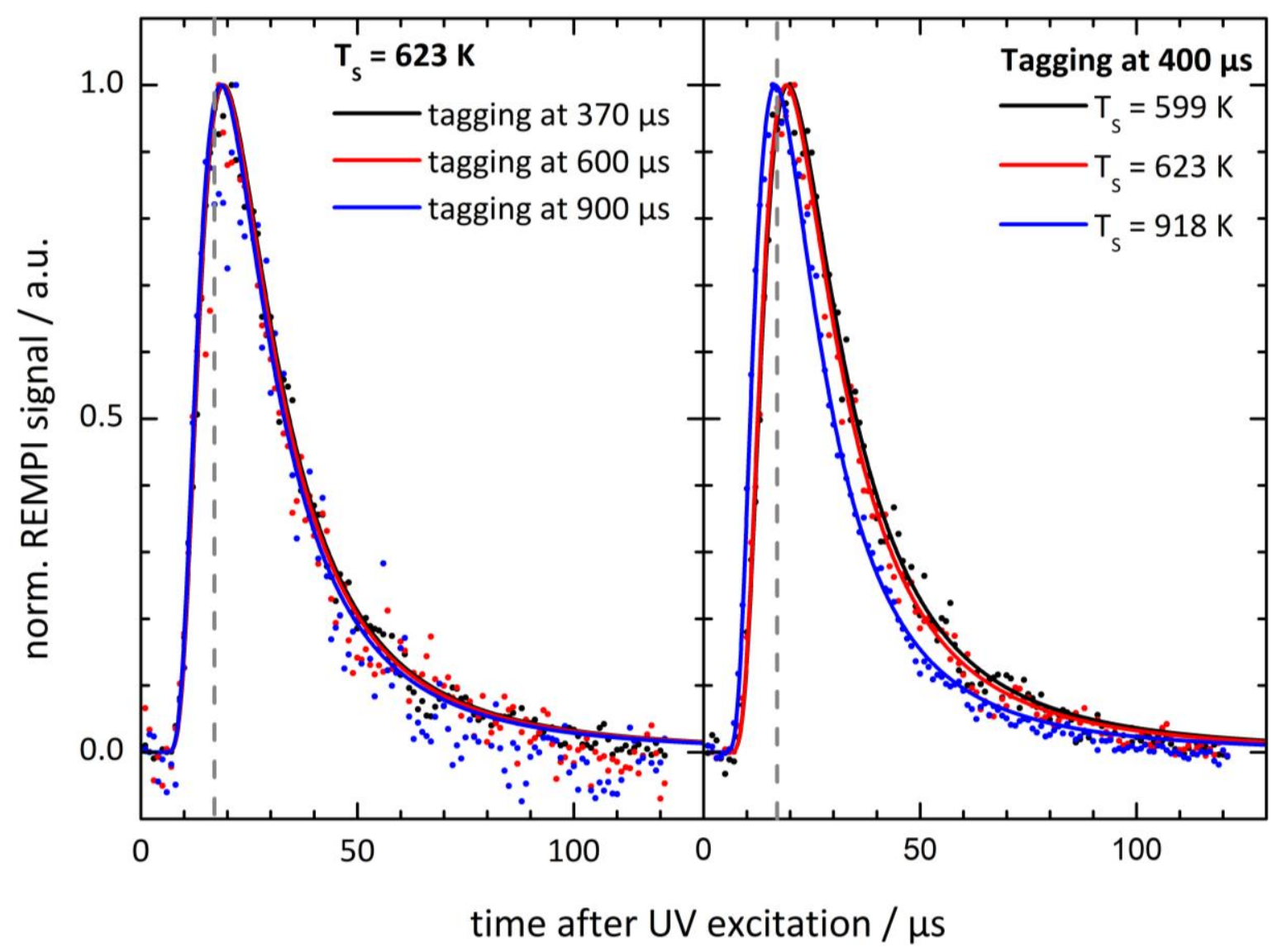

Fig. 7.5: Arrival time distributions between UV excitation and REMPI detection of scattered/ desorbed CO molecules from $\mathrm{Pt}(111)$ at $E_{I}=0.09 \mathrm{eV}$. The solid dots show the experimental data; solid lines represent the corresponding fits. The left panel shows the time-of-flight profiles for $T_{S}=623 \mathrm{~K}$ and tagging the molecules at different delays in the molecular beam. The right panel shows the influence of surface temperature on the time-of-flight profiles. The vertical dashed gray line indicates the delay of $t_{2}=17 \mu$ s used for the desorption kinetic experiments.

$$
\varphi_{s}(s) d s=S(t) \times s^{2} d t=\frac{S(t) \times s^{2}}{\frac{l}{t^{2}}} d s=S(t) \times l d s
$$

For the velocity dependent flux, $P(v)$, of the CO molecules leaving the $\mathrm{Pt}(111)$ surface, I assume a thermal Maxwell-Boltzmann distribution (equation 7.12) with an amplitude $A$ and a translational temperature $T_{\text {trans }}$ as adjustable fit parameters.

$$
\varphi_{S}(s) d s=A \times s^{3} \times e^{-m s^{2} / 2 k_{B} T_{\text {trans }}} d s
$$




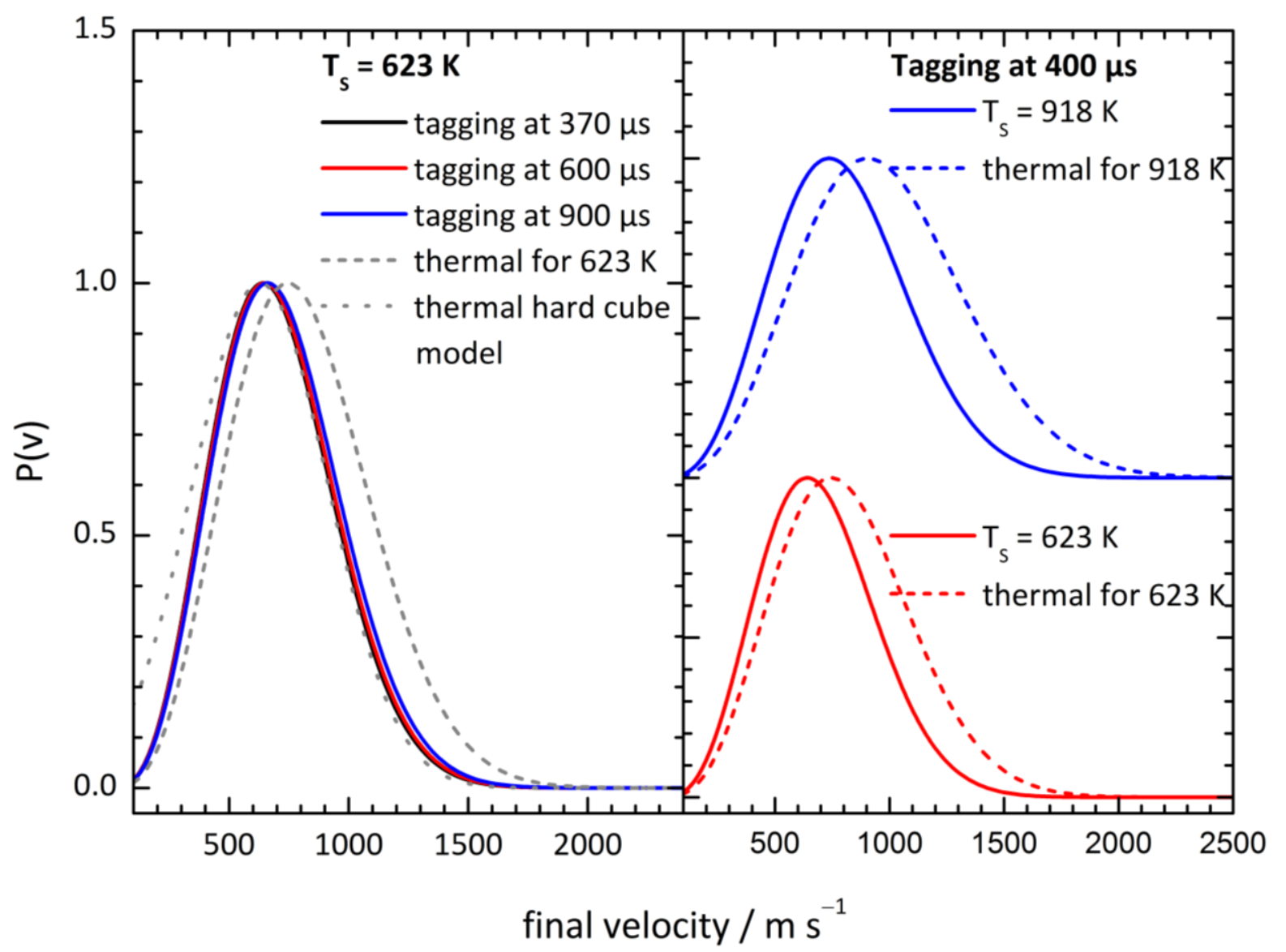

Fig. 7.6: Speed distributions obtained from the fits to the time-of-flight profiles in Fig. 7.5. Left panel: Speed distributions for $T_{S}=623 \mathrm{~K}$ and for tagging at different delay with respect to the molecular beam. Right panel: Speed distributions for tagging at $400 \mu$ s with respect to the molecular beam (see Fig. 7.7) but different surface temperatures. In both panels, the dashed lines show a thermal Maxwell-Boltzmann distribution at the surface temperature. The dotted line in the left panel shows the prediction of the thermal Baule limit for direct scattering (see section 3.3).

The right panel of Fig. 7.5 shows the effect of surface temperature on the speed distributions of CO molecules that pass the UV excitation laser at $t_{1}=400 \mu \mathrm{s}$. The time-of-flight profiles for $T_{S}=599 \mathrm{~K}$ and $623 \mathrm{~K}$ are very similar, in agreement with the small change in surface temperature. The profile at $T_{S}=918 \mathrm{~K}$ is shifted toward earlier arrival times by $\sim 3 \mu \mathrm{s}$ indicating higher final velocities. The corresponding speed distributions for $T_{S}=623 \mathrm{~K}$ and $918 \mathrm{~K}$ are shown in in the right panel Fig. 7.6. The dashed lines indicate the expectation for thermal Maxwell-Boltzmann distributions at the surface temperature. The comparison to the experimental speed distributions shows that $\mathrm{CO}$ molecules recoil significantly slower than expected from the thermal prediction. Accordingly, I find translational temperatures of $T_{\text {trans }}=461 \mathrm{~K}$ and $610 \mathrm{~K}$ that are lower than the surface temperature, $T_{S}=623 \mathrm{~K}$ and $918 \mathrm{~K}$.

Consequently, the measured speed distributions are always colder than the surface temperature. This behavior has been observed earlier for other systems, e.g. $\mathrm{Ar} / 2 \mathrm{H}-\mathrm{W}(100)$ [171], and results from detailed balance arguments, that is, taking into account the $E_{I}$ dependent sticking probability. However, a corresponding analysis suggests that the trapping probability decreases rapidly with increasing $E_{I}$ and predicts almost zero sticking at $E_{I} \geq 0.6 \mathrm{eV}$, which is in clear contrast to the weak $E_{I}$ dependence observed in previously [172] and the significant amount of trapping-desorption observed at $E_{I}=0.65 \mathrm{eV}$ (see section 5). A possible reason for this deviation is that the speed distribution for directly scattered 
molecules at $E_{I}=0.09 \mathrm{eV}\left(s_{i} \sim 780 \mathrm{~m} / \mathrm{s}\right)$ is very similar, and therefore indistinguishable, from that of molecules undergoing trapping followed by desorption. I estimate the final speed distribution using the thermal hard cube model (gray dotted line in left panel of Fig. 7.6) introduced in section 3.3 for $\mathrm{NO} / \mathrm{Au}(111)$ scattering. The prediction for direct scattering overlaps with the thermal MaxwellBoltzmann distribution at $T_{S}=623 \mathrm{~K}$ but peaks at lower final velocities. This overlap can influence the observed speed distributions in the experiment. This hypothesis is supported by the results of Fig. 5.5 for $E_{I}=0.65 \mathrm{eV}$. The data at higher $E_{I}$ enabled the separation of direct scattering and trapping-desorption and demonstrated that the speed distribution of $\mathrm{CO}$ molecules desorbing from $\mathrm{Pt}(111)$ agrees reasonably well with a thermal Maxwell-Boltzmann distributions at the surface temperature. This observation is also consistent with a weak $E_{I}$ dependence of the sticking probability observed previously [172].

However, the key experiment for the observation of reliable desorption kinetic is the verification that the speed distribution of desorbed molecules does not depend on the residence time at the surface. The left panels of Fig. 7.5 and Fig. 7.6 show the dependence of the time-of-flight profile and speed distribution for different times $t_{1}$ in the molecular beam at a surface temperature of $T_{S}=623 \mathrm{~K}$. The time-of-flight and speed distributions at $T_{S}=623 \mathrm{~K}$ are very similar and independent of $t_{1}$ within the scatter of the data. Consequently, the speed distribution of desorbed molecules is independent of residence time to a good approximation.
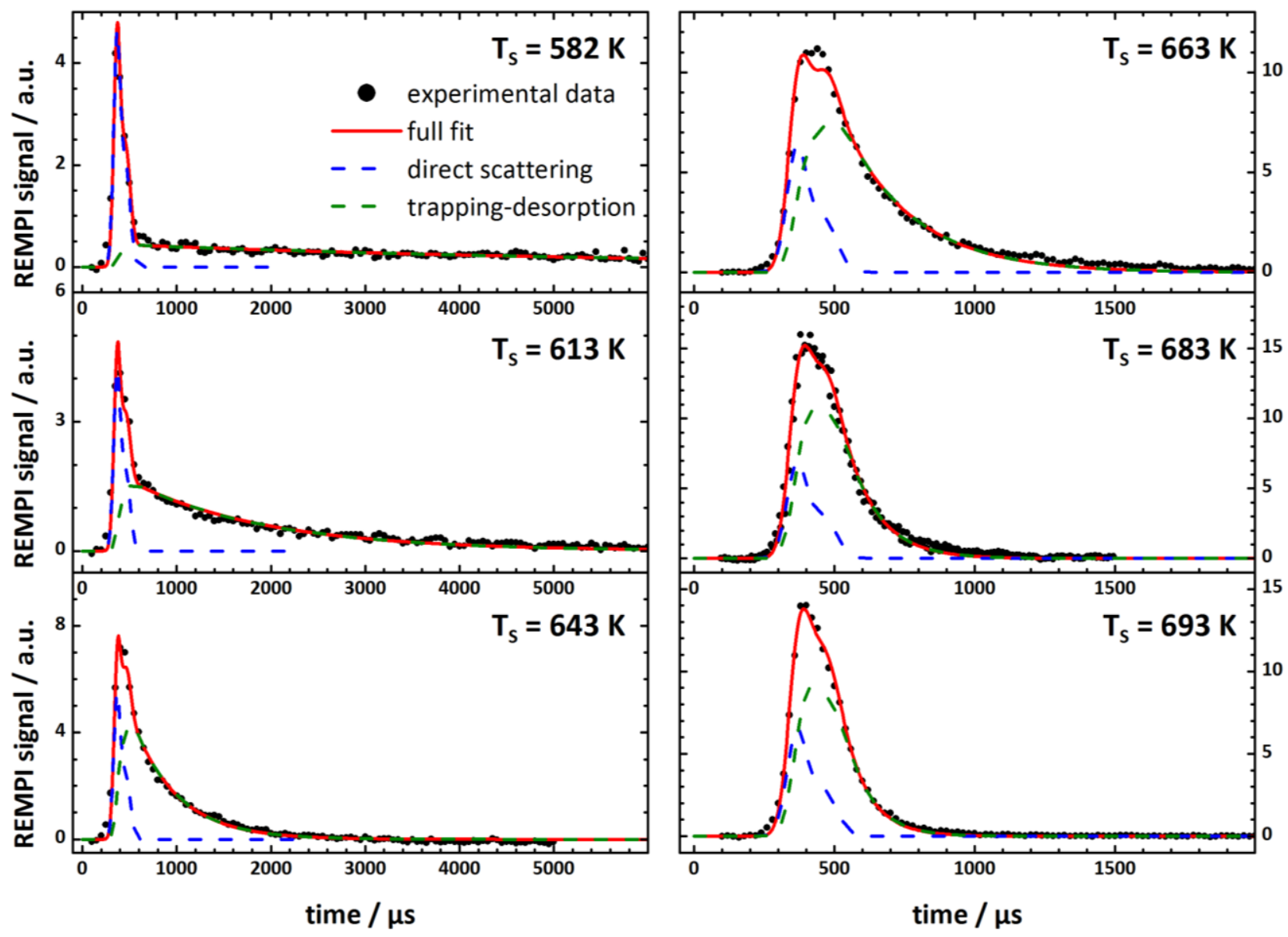

Fig. 7.7: Fit results of the kinetic model presented above for different surface temperatures. The model (red solid line) includes a contribution of direct scattering with zero residence time (blue dashed line) and of trapping desorption (green dashed line). The fitting procedure optimized two amplitude $B_{D S}$ and $B_{T D}$ as well as the rate constant for desorption from the surface. 


\section{Desorption measurements: comparison to first-order kinetic model}

Because the speed distributions for direct scattering and trapping-desorption are very similar in the experiment, the delay $t_{2}$ cannot discriminate between the two mechanisms. For measurements of the $t_{1}$ temporal profile of the CO molecules leaving the surface, I selected a delay $t_{2}=17 \mu$ s (gray dashed line in Fig. 7.5) between UV excitation and REMPI detection, which corresponds to a final speed of $s_{f}=712 \mathrm{~m} \mathrm{~s}^{-1}$. Fig. 7.7 shows the results for surface temperature dependent $t_{1}$ profiles of CO desorption from $\mathrm{Pt}(111)$ in the range from $T_{S}=582 \mathrm{~K}$ to $693 \mathrm{~K}$. The shape of the arrival time distribution exhibits a strong dependence on surface temperature. The profiles extend to longer $t_{1}$ times with decreasing surface temperature while its amplitude decreases at the same time. At the lowest surface temperatures of $T_{S}=582 \mathrm{~K}$ and $613 \mathrm{~K}$, the profiles further show an additional narrow peak for $t_{1}<700 \mu \mathrm{s}$ that does not depend on surface temperature and that is similar to the profile at high $T_{S}$ (Fig. 7.4). I attribute this feature to a direct scattering mechanism.

I use equation 7.9 to fit the experimental data; the results are shown as red solid line in Fig. 7.7. The kinetic model contains a surface temperature dependent rate constant $k_{d}\left(T_{S}\right)$ and two amplitudes, $B_{D S}\left(T_{S}\right)$ and $B_{T D}\left(T_{S}\right)$, to scale the model to the signal size in the experiment. The dependence of the three fit parameters on $T_{S}$ is shown in Fig. 7.8. The first-order rate constant $k_{d}$ increases exponentially with increasing $T_{S}$ as expected from the Arrhenius equation 7.1. The two amplitude factors, $B_{T D}$ and $B_{D S}$, should be independent of $T_{S}$, assuming that the experimental conditions (e.g. the laser intensity) remain constant and that speed distribution, angular distribution and trapping probability do not change with $T_{S}$. The values obtained in the fit fulfill this condition to a very good approximation. Except at the highest $T_{S}$, I find no strong dependence of the two fit parameters on surface temperature outside the measurement error. At $T_{S}=723 \mathrm{~K}$, the change is likely related to uncertainty in the fit because the rate of desorption becomes faster than the molecular beam pulse duration.

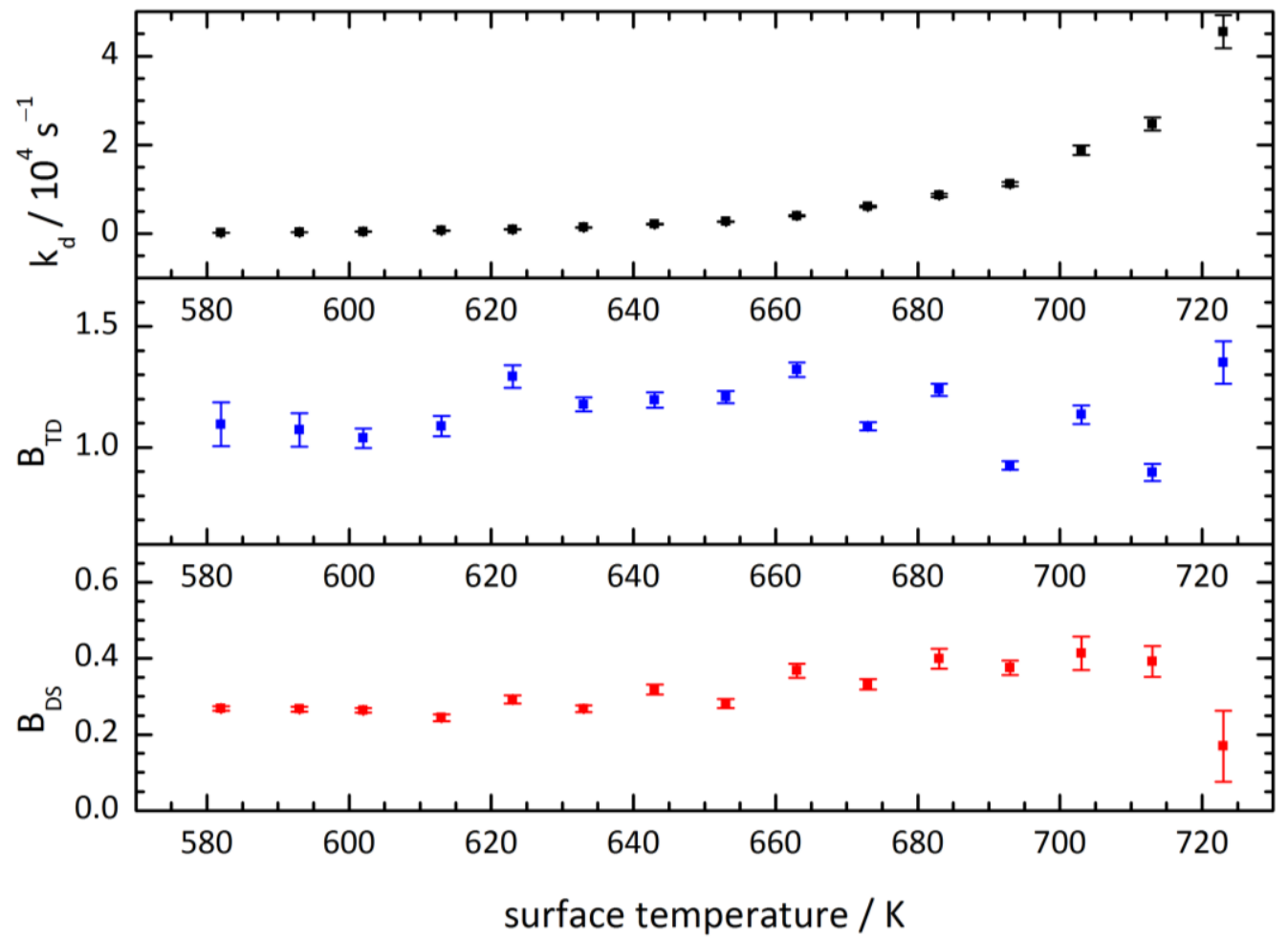

Fig. 7.8: Surface temperature dependence of the fit parameters $k_{d}$ (upper panel), $B_{T D}$ (middle panel) and $B_{D S}$ (lower panel) of the kinetic model for $\mathrm{CO}$ desorption from $\mathrm{Pt}(111)$. The error bars indicate $95 \%$ confidence interval. 
$B_{D S}$ is about four times lower than $B_{T D}$, indicating that the ratio between direct scattering and trappingdesorption is about 1:4 at $\theta_{f} \approx 9^{\circ}$. Furthermore, $B_{D S}$ shows a slight systematic increase with increasing $T_{S}$, which is likely due to the fact that the sharp contribution of direct scattering becomes almost invisible under the 4 times stronger trapping-desorption component; the accurate determination of $B_{D S}$ and $B_{T D}$ becomes impossible. This is also reflected in an increasing scatter in the results for $B_{D S}$ for $T_{S}>700 \mathrm{~K}$. Therefore, the results for these temperatures are not taken into account in the further analysis. Because the incident flux of $\mathrm{CO}$ molecules is modeled with the help of a $t_{1}$ profile at high surface temperature $\left(T_{S}=973 \mathrm{~K}\right)$, the fit results for $B_{T D}$ should be close to 1 , which is the case in the data shown in Fig. 7.8. This property results from the fact that the time integrated flux of desorbing $\mathrm{CO}$ molecules is constant under the assumption that trapping probability, speed distribution and rotational distribution depend only weakly on surface temperature.

I use the fitted $T_{S}$ dependent rate constants, $k_{d}\left(T_{S}\right)$, for determination of the activation energy for desorption, $E_{d}$, and the preexponential factor, $A_{d}$. The logarithmic form of equation 7.1 predicts a linear dependence of $\ln k_{d}$ on $1 / T_{S}$ with a slope $m=-E_{d} / k_{B}$ and an intercept $b=\ln A_{d}$. The result of this analysis is shown in Fig. 7.9. The rate constants in the range from $T_{S}=582 \mathrm{~K}$ to $713 \mathrm{~K}$ show the expected linear behavior on inverse surface temperature and I find no systematic deviation within the error bars, which indicate 95\% confidence interval. The linear fit to the experimental data (weighted by the error at each $k_{d}$ ) yields a activation energy for desorption of $E_{d}=1.321 \pm 0.041 \mathrm{eV}$ and a preexponential factor of $A_{d}=\left(4.7\left\{\begin{array}{l}+4.9 \\ -2.4\end{array}\right) \times 10^{13} \mathrm{~s}^{-1}\right.$. The errors reflect $95 \%$ confidence interval.

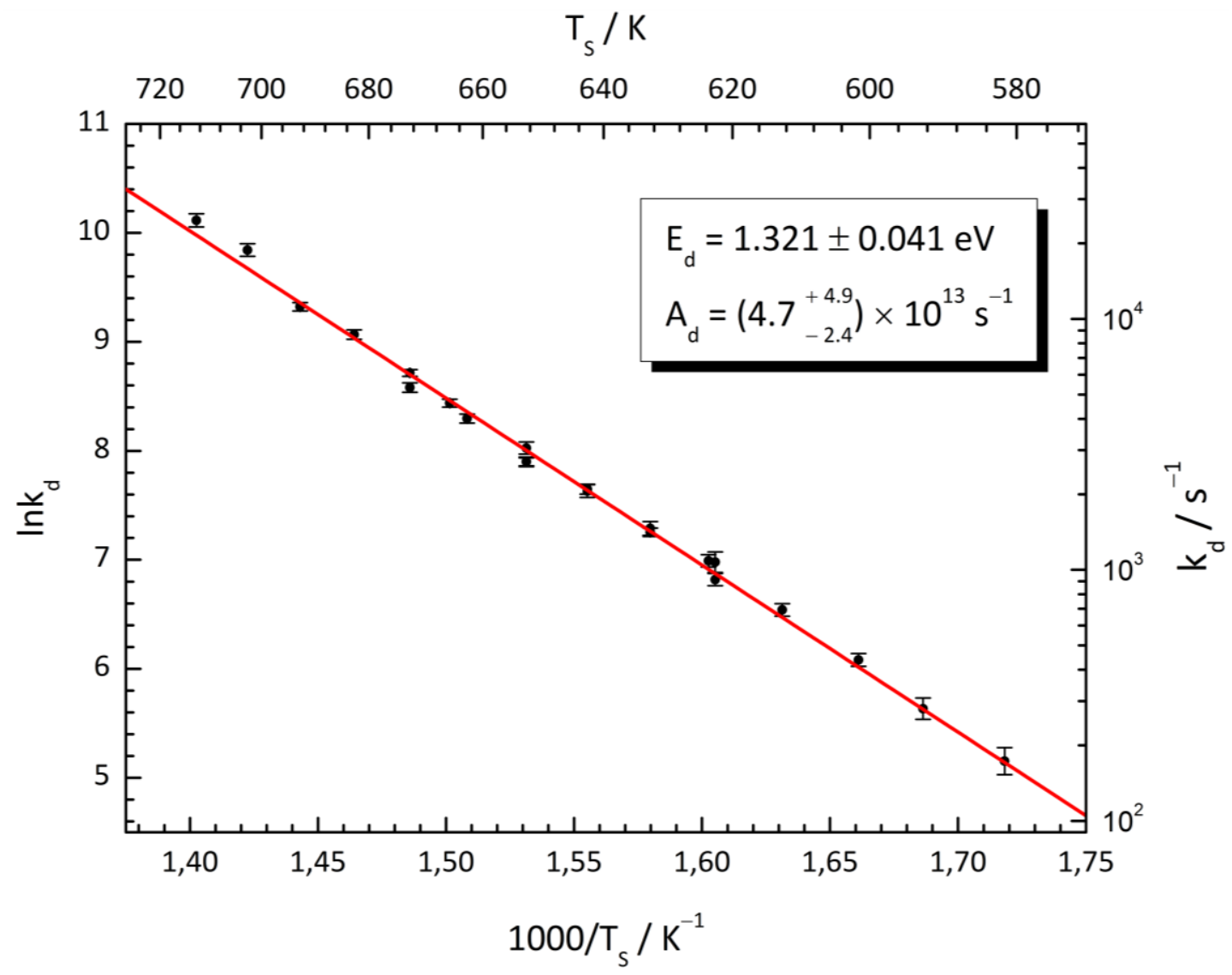

Fig. 7.9: Arrhenius plot of the logarithm of the rate constant $k_{d}$ vs. inverse surface temperature. The solid dots represent the rate constants obtained from the fit of the kinetic model to the experimental data. The error bars indicate a $95 \%$ confidence interval. 

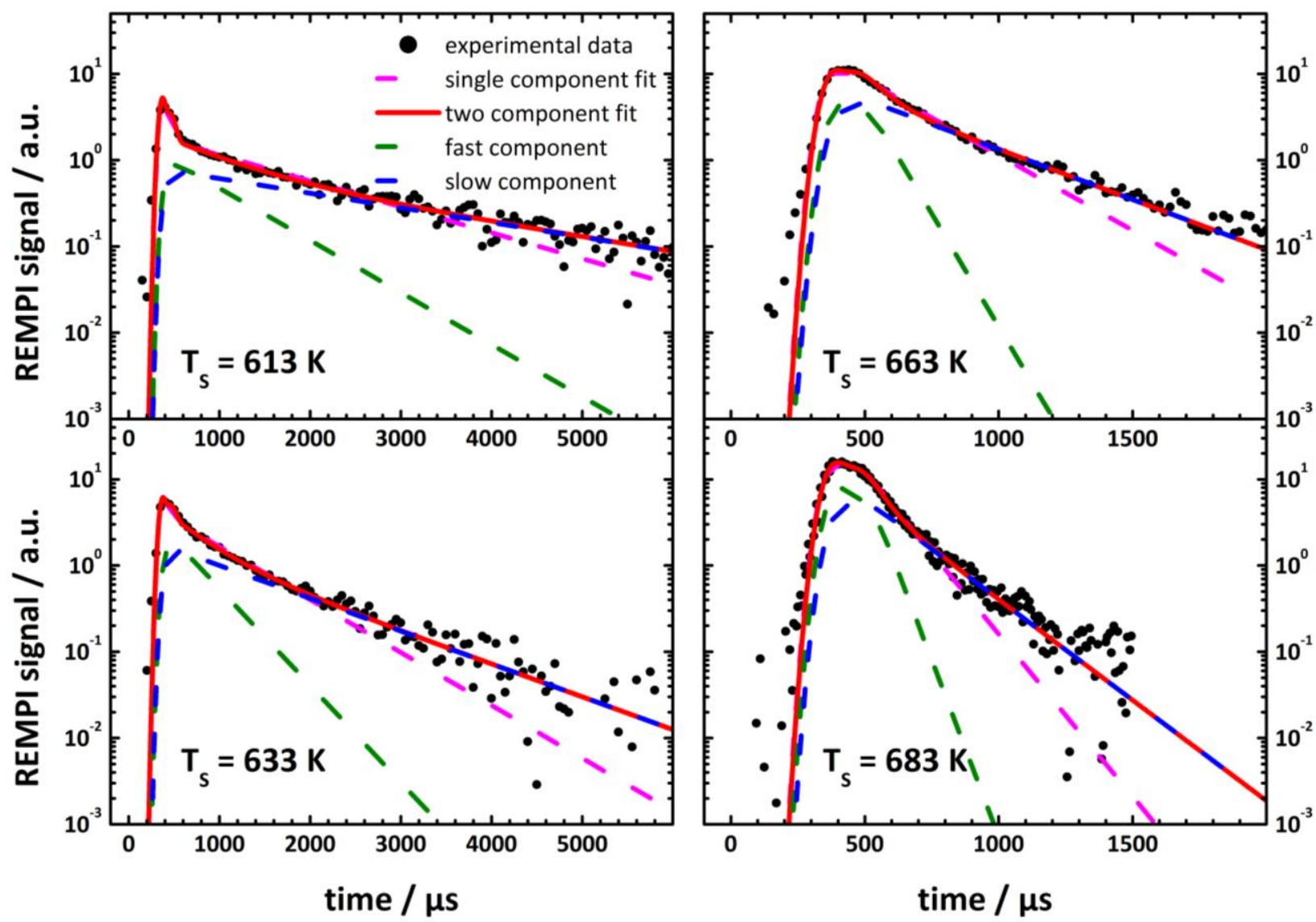

Fig. 7.10: Comparison of the surface temperature dependent velocity selected time-of-flight data (black dots) to the fit a firstorder kinetic rate model for desorption (magenta dashed curves) with the measured intensities on a logarithmic scale. The comparison of experimental data and kinetic model demonstrates that the model shows systematic deviations, indicating a biexponential decay of the desorption signal. The solid red lines show the results for a bimodal fit to the experimental data with a fast (green) and slow (blue) component contributing to the desorption signal.

\section{Deviations from first-order desorption kinetics}

So far, my analysis was based on the assumption that the desorption of $\mathrm{CO}$ from $\mathrm{Pt}(111)$ can be described by a simple first-order kinetic rate model as observed in previous studies [143, 145]. However, a more detailed comparison of the first-order kinetic rate model to the experimental velocity selected residence time data reveals small but systematic deviations. Fig. 7.10 shows the experimental data at four different surface temperatures with the measured intensity on a logarithmic scale. This comparison shows that the first-order kinetic rate model (magenta dashed line) clearly deviates from the experimental data. The data clearly exhibits a higher intensity at large $t_{1}$ than expected from the firstorder kinetic assumption. This observation is clear evidence for a bi-exponential decay of desorption signal, thus for the decay of the CO density at the surface. This is in contrast to observations of previous studies that did not directly measure the real-time kinetics; these observations demonstrate the power of the velocity selected residence time method.

In order to take the biexponential decay of the desorption flux into account, I apply equation 7.10 to fit the experimental data. Note that the model now includes two different kinds of adsorbates $N_{a d}^{(1)}(t)$ and $N_{a d}^{(2)}(t)$, which are depopulated according to the rate constants $k_{d}^{f a s t}\left(T_{S}\right)$ and $k_{d}^{\text {slow }}\left(T_{S}\right)$. The amplitude factors $B_{T D}^{\text {fast }}\left(T_{S}\right)$ and $B_{T D}^{\text {slow }}\left(T_{S}\right)$ reflect the relative intensities of the two components in the model. I 
used equation 7.10 to fit those $t_{1}$ scans again for which I clearly observe a biexponential behavior. Because the fit to this new model is much more sensitive than before, I tried to reduce the number of variable fit parameters. The results Fig. 7.8 for the $T_{S}$ dependence of the fit parameters showed that $B_{D S}$, the amplitude for direct scattering, is approximately independent of surface temperature. Therefore, I hold $B_{D S}\left(T_{S}\right)$ constant at the average value obtained in the range of $T_{S}=582-633 \mathrm{~K}$ in the first-order kinetic model fit. The new rate constants are shown on an Arrhenius plot in Fig. 7.11. The comparison shows that the rate constants, $k_{d 1}^{\text {fast }}$, for the fast component (blue) are always higher than the first-order rate constants, $k_{d}$, (black), whereas the values for $k_{d}^{\text {slow }}$ (red) are always lower. Furthermore, the larger error bars on $k_{d}^{\text {fast }}$ and $k_{d}^{\text {slow }}$ indicate a higher statistical uncertainty in the fit. However, the correct description of the desorption kinetics reduces the systematic error on the desorption rate that results from the wrong kinetic model used in the simple analysis and also in previous studies $[145,173,174]$. The ratio $B_{T D}^{\text {fast }}\left(T_{S}\right): B_{T D}^{\text {slow }}\left(T_{S}\right)$ of fast and slow desorption component is close to unity over the whole range of surface temperatures.

The activation energy for desorption in the fast desorption channel decreases by about $45 \mathrm{meV}$ with respect to the single exponential model while the prefactor decreases by $25 \%$. The changes are more dramatic for the slow component for which I obtain remarkable low values for $E_{d}$ and $A_{d}$ of only $1.05 \mathrm{eV}$ and $1.9 \times 10^{11} \mathrm{~s}^{-1}$. Interestingly, I find that the values for $k_{d}\left(T_{S}\right)$ of the single exponential fit approach $k_{d}^{\text {slow }}\left(T_{S}\right)$ at low surface temperatures and $k_{d}^{\text {fast }}\left(T_{S}\right)$ at high surface temperatures.

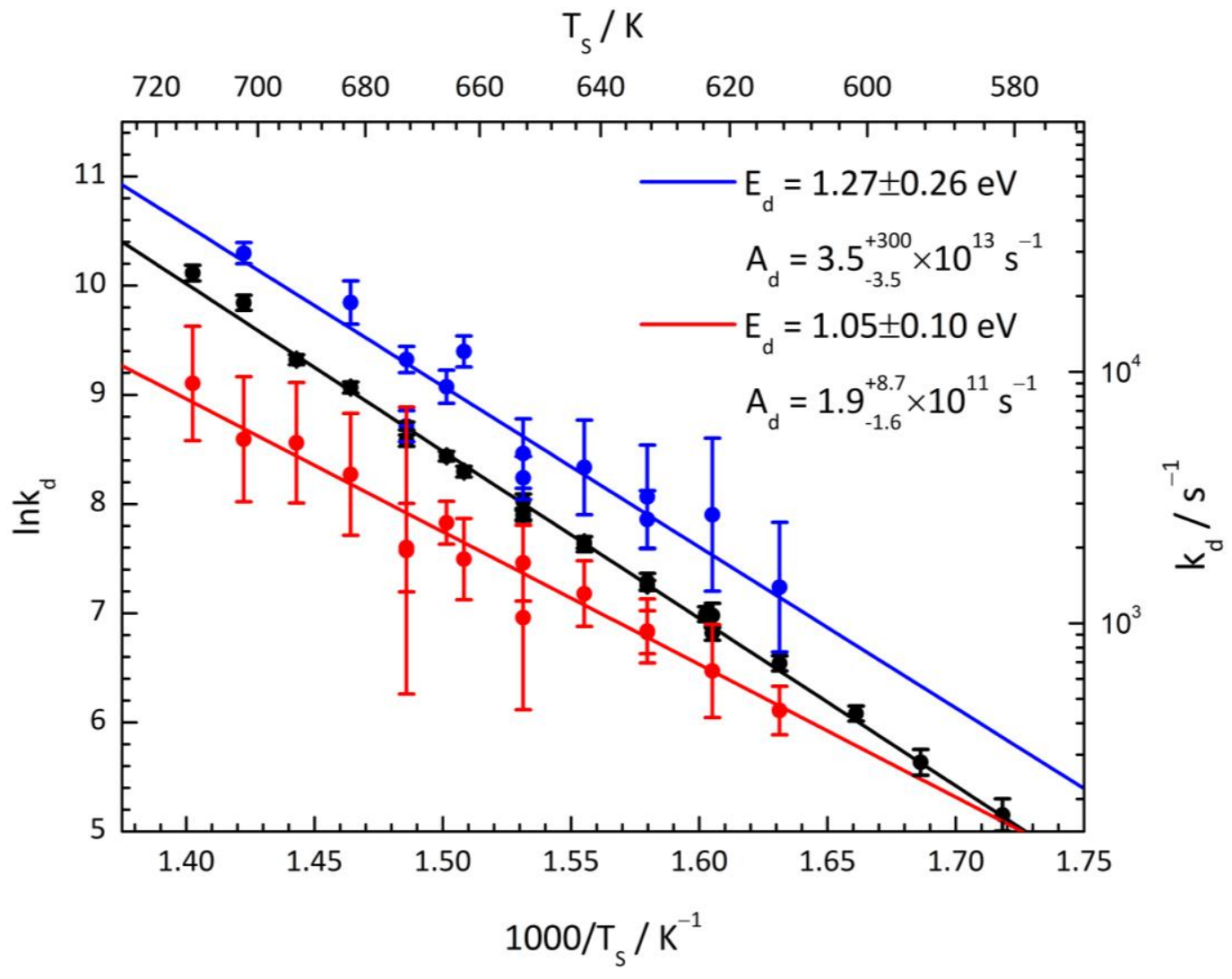

Fig. 7.11: Comparison of the surface temperature dependent rate constants obtained from a bimodal kinetic model (red, blue) to the rate constants obtained from a simple first-order kinetic rate model (black). The lines show the Arrhenius fits to the experimental data. Error bars indicate a $90 \%$ confidence interval. Note that the errors on $k_{d}^{\text {fast }}\left(T_{S}\right)$ (blue) and $k_{d}^{\text {slow }}\left(T_{S}\right)$ are much large than those of the single exponential fit. This is a result of the coupling between the two components in the fit. The new model increases the statistical uncertainty but eliminates the systematic error of the previous model. 


\subsection{Discussion}

Prior to the discussion of the desorption kinetics found in the experiment, I briefly discuss the observed ratio between molecules undergoing direct scattering and those undergoing trapping followed by desorbed is given by the ratio $B_{D S}: B_{T D} \approx 0.25$ in the fit of the kinetic model. This ratio is in good agreement with the sticking probability of $S \approx 0.81$ for CO/Pt(111) at $E_{I}=0.09 \mathrm{eV}$ and $T_{S}=350 \mathrm{~K}$ measured by Harris and Luntz [172]. Lin and Somorjai [145] and Campbell et al. [143] observed constant initial sticking probabilities of $S=0.74$ and $S=0.84 \pm 0.05$ over a range of $T_{S}$ similar to that used here. The observed $B_{D S}: B_{T D}$ ratio of this work is consistent with these results.

\section{First-order kinetic model and comparison to previous work}

The results of the first-order kinetic analysis demonstrate that the velocity selected residence time measurements are applicable for determination of desorption kinetics on a microsecond to millisecond time scale. Moreover, the small errors on the fitted values for $k_{d}\left(T_{S}\right)$, as well as for activation energy $E_{d}$ and prefactor $A_{d}$, show that it is possible to determine kinetic parameters with high accuracy. However, a more detailed comparison of the first-order kinetic model to the experimental data (Fig. 7.10) already revealed deviations from first-order kinetic. This systematic deviation causes a systematic error in $k_{d}\left(T_{S}\right)$. I will discuss this topic in detail below. Nevertheless, the first-order kinetic was used in previous studies and therefore provides the possibility to compare my velocity selected residence time experiments to the results obtained by other methods. The spread in reported activation energies for desorption and prefactors is rather large [138, 139, 143, 144, 146-152, 174] and studies with independent determination of $E_{d}$ and $A_{d}$ are limited. However, both parameters are correlated and should be determined separately.

Four experimental studies are especially suitable for comparison to my results. Winicur et al. used lowenergy molecular beam scattering of $\mathrm{CO}$ from $\mathrm{Pt}(111)$ at $T_{S}=419 \ldots 505 \mathrm{~K}$ and found values of $E_{d}=1.349 \mathrm{eV}$ and $A_{d}=2.7 \times 10^{13} \mathrm{~s}^{-1}$ [151]. The authors used specular scattering of a modulated helium beam to probe to the $\mathrm{CO}$ coverage on the $\mathrm{Pt}(111)$ surface caused by an unmodulated $\mathrm{CO}$ dose beam. The desorption kinetics were obtained by measuring the time-dependent helium signal after the $\mathrm{CO}$ beam was blocked. The time resolution of the experiment was in the order of $10^{-3}-10^{-2} \mathrm{~s}$. The authors found first-order desorption kinetics in agreement with the kinetic model used here. The prediction of the firstorder kinetic model using the parameters of Winicur et al. to calculate $k_{d}\left(T_{S}\right)$ is shown as green lines in left panels of Fig. 7.12. The comparison to the raw experimental data shows a strong disagreement with between the model and the data. The model predicts a slower decay of the CO signal than observed in the experiment indicating that either $A_{d}$ is too small or $E_{d}$ is too high. A comparison of the reported values to my results reveals that both is the case. The prefactor is at the lower limit of the uncertainty, while the desorption energy is at the upper limit of the error bars. The results of Winicur et al. do not agree with my observations.

A second comparable study was performed by Lin and Somorjai [145] who used modulated molecular beam scattering and found Arrhenius parameters of $E_{d}=1.297 \mathrm{eV}$ and $A_{d}=2.9 \times 10^{13} \mathrm{~s}^{-1}$. The authors measured the modulated $(10 \mathrm{~Hz}, 100 \mathrm{~Hz}) \mathrm{CO}$ background pressure in the chamber for different surface temperatures ranging from $350-1100 \mathrm{~K}$ and referenced it to a measurement at $T_{S}=1250 \mathrm{~K}$ with negligible residence time. They determined the desorption kinetics from the phase lag of the pressure rise/ decay in the vacuum chamber. The corresponding prediction of the kinetic model is shown as blue lines in the left panels of Fig. 7.12. The agreement with the experimental data and the fitted model (red 
line) is quite striking; the results match almost perfectly with the measured rate constants (right panel of Fig. 7.12). Of course this method cannot detect the bi-exponential behavior seen in this work.

Veheij and co-workers [174] derived kinetic parameters of $E_{d}=1.39 \mathrm{eV}$ and $A_{d}=4.3 \times 10^{14} \mathrm{~s}^{-1}$ using a pulse shape analysis in a modulated molecular beam scattering experiment. The comparison to my experimental data is shown as orange lines in Fig. 7.12 and shows that the predicted temperature dependent rate constants are systematically too high and do not agree with my observations.

Finally, Campbell et al. [143] used modulated molecular beam scattering, analogous to that of Lin and Somorjai, but used a different kinetic model in the analysis of the experimental results. They assumed a very high mobility, fast surface diffusion of the adsorbed CO molecules, leading to only desorption from step sites or defects. The authors derived an activation energy for desorption of $1.51 \mathrm{eV}$ and a high preexponential factor of $1.25 \times 10^{15} \mathrm{~s}^{-1}$. The prediction of the first-order kinetic rate model for these parameters is shown as red lines in Fig. 7.12. Although Campbell et al. found a much higher value for $E_{d}$, exceeding my result by $0.19 \mathrm{eV}$, the high prefactor compensates for this and I observe a good agreement with the velocity selected residence time data. However, especially the comparison of predicted and observed rate constants for desorption (right panel of Fig. 7.12) shows that the higher $E_{d}$ value leads to an underestimation of $k_{d}$ at low surface temperatures.
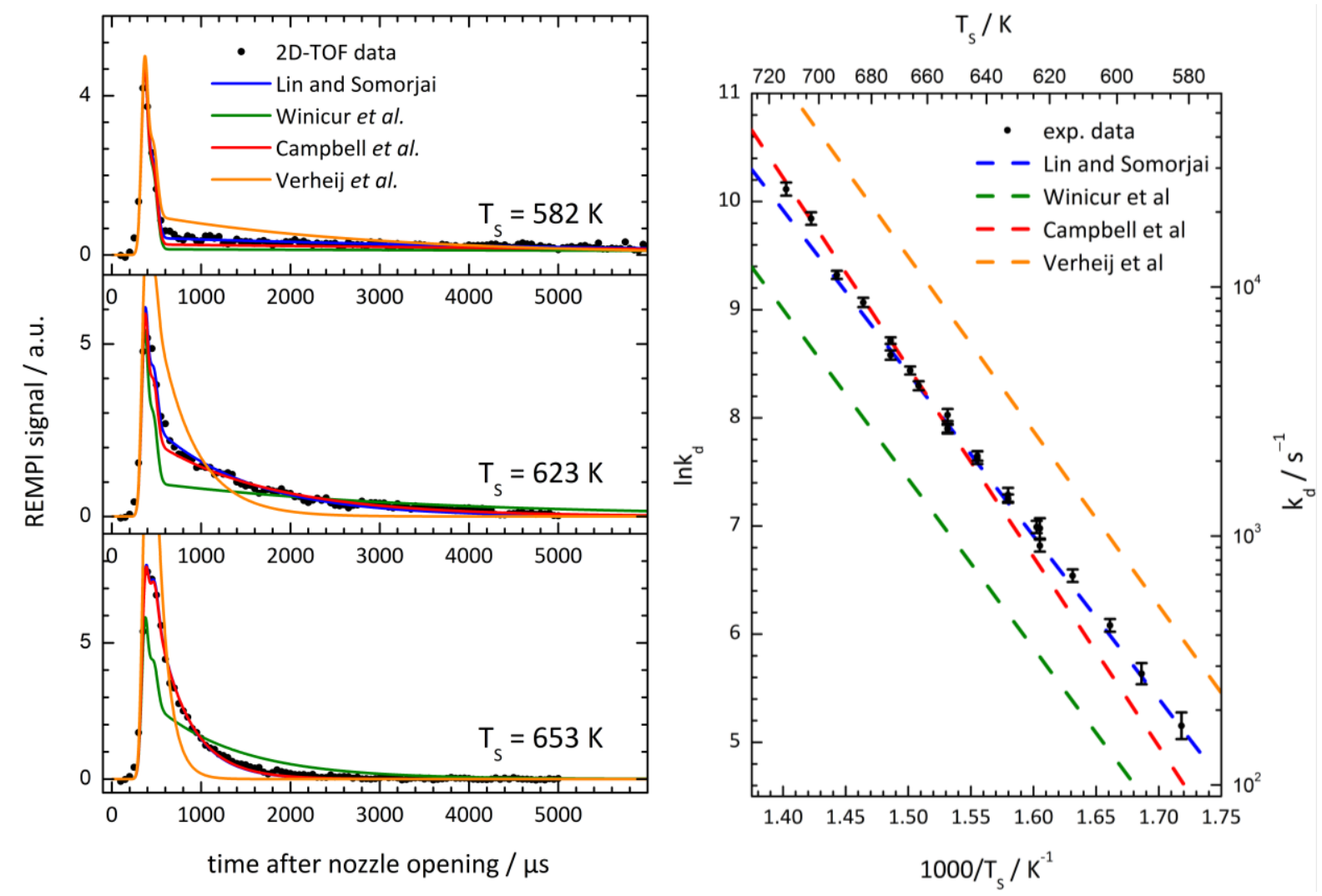

Fig. 7.12: Left panels: Comparison of my raw experimental data (black dots) to the results of previous works using desorption energy and preexponential factor as in input for the kinetic model at three different surface temperatures $T_{S}=582,623$ and $653 \mathrm{~K}$. The colored lines show the predictions of the kinetic model using the results of Lin and Somorjai [145] (blue), Winicur et al. [151] (green), Campbell et al. [143] (red) or Verheij et al. [174] (orange). The amplitude factors $B_{D S}$ and $B_{T D}$ are taken from the fit of the kinetic model to the experimental data. Note that the blue and red curve overlay at $T_{S}=653 \mathrm{~K}$. Right panel: Arrhenius plot of the experimental rate constants to the results of the same studies using the identical color coding. 
The comparison of my experimental data to these four experimental studies demonstrates that the measured real-time microsecond desorption kinetics cannot only be used to determine the Arrhenius parameters, $E_{d}$ and $A_{d}$, but also to test the reliability of the results of previous studies. Already small differences in the prefactor and activation energy lead to strong deviations from the measured kinetic behavior. Moreover, the velocity-selected residence time measurements clearly exhibit the importance of higher order kinetics which have not been observed in previous molecular beam studies.

\section{Evidence for higher order kinetics: the role of steps and surface diffusion}

The observation of two components in the kinetics of $\mathrm{CO}$ desorption from $\mathrm{Pt}(111)$ raises an important question: Which processes do the two rate constants $k_{d}^{\text {fast }}$ and $k_{d}^{\text {slow }}$ describe? First, one might think of desorption from different surface sites (atop, bridge, hollow). However, several studies showed that CO exclusively binds to atop sites on $\mathrm{Pt}(111)$ at low coverages (<0.2 ML) [152, 167-169]. Another possible explanation is the influence of steps or defects on the desorption dynamics. Serri et al. developed a kinetic model for a similar system, the desorption of NO from Pt(111) $[175,176]$. I will refer to it as the STC model. The STC model takes into account the possible role of step sites in the adsorption-desorption dynamics. In general, the Pt(111) crystal is not flat over the whole area illuminated by the molecular beam ( $2 \mathrm{~mm}$ diameter). The crystal has, dependent on quality and preparation, a certain percentage of steps that separate the atomically flat (111) terraces. The AFM analysis (Fig. 7.3) of the Pt crystal used in this work showed a step density $0.5 \%$ over a $5 \mu \mathrm{m} \times 5 \mu \mathrm{m}$ area. Although there might be areas with a higher density of steps, I expect the dose of $\mathrm{CO}$ molecules per pulse to be higher than the number of steps as described above.

The binding of an adsorbate to step sites is often significantly stronger than to the flat terrace adsorption sites. Furthermore, the adsorbed molecules can diffuse at the metal surface, a process that was suggested to be very fast $\left(k_{H} \sim 10^{11} \ldots 10^{13} \mathrm{~s}^{-1}\right)$ for NO on $\mathrm{Pt}(111)$ compared to the desorption rate from the terrace sites $\left(k_{T} \sim 10^{5} \ldots 10^{7} \mathrm{~s}^{-1}\right)$. Here, $k_{H}$ is the site-to-site hopping rate on the terraces and $k_{T}$ is the desorption rate from the terrace sites. Based on the high mobility of the adsorbate on the terraces, Serri and co-workers suggested a mechanism in which the incident NO molecules are adsorbed at the terraces (low step density) and diffuse from the terrace to the step sites in a few picoseconds. Desorption then occurs upon diffusion from the steps to the terraces (step-to-terraces hopping rate $k_{S} \sim 10^{5} \ldots 10^{7} \mathrm{~s}^{-1}$ ) from which they can finally escape into the gas phase. The latter mechanism is based on detailed balance arguments. Because the step density is rather low $(\sim 0.5-1 \%)$ the majority of incident molecules will be adsorbed at the terraces. From detailed balance it follows that desorption also has to occur primarily from the terrace sites. As a consequence, one always observes a single exponential decay in desorption experiments at very low coverage (steps not saturated); the decay results from the kinetics of step-toterrace diffusion and desorption from the terraces.

All these arguments hold if the diffusion on the terraces is much faster than the desorption process and if the steps are not saturated and therefore blocked. I set-up the kinetic model proposed by Serri et al. and tried to apply it to the CO/Pt(111) model [175]. The time dependent change of the population of an adsorption site $\eta_{n}$ is given by the following nonlinear system of coupled differential equations: 


$$
\begin{gathered}
\dot{\eta}_{0}(t)=-2 k_{S} \eta_{0}(t)+2 k_{H}\left(1-\gamma \eta_{0}(t)\right) \eta_{1}(t) \\
\dot{\eta}_{1}(t)=k_{S} \eta_{0}(t)-\left[k_{T}+k_{H}\left(2-\gamma \eta_{0}(t)\right)\right] \eta_{1}(t)+k_{H} \eta_{2}(t) \\
\vdots \\
\dot{\eta}_{m}(t)=-\left(k_{T}+2 k_{H}\right) \eta_{m}(t)+k_{H}\left(\eta_{m-1}(t)+\eta_{m+1}(t)\right)
\end{gathered}
$$

where $\eta_{0}(t)$ corresponds to the step site and $\eta_{1 \ldots m}(t)$ describe the population at the $1^{\text {st }}-m^{\text {th }}$ terrace site. $k_{H}$ is rate constant for site-to-site hopping on the terraces, $k_{S}$ is the rate constant for step-to-terrace hopping and $k_{T}$ is the rate constant for desorption from the terrace sites. The parameter $\gamma$ is an occupancy factor that reflects the saturation population at the steps. The differential equation system can be solved numerically without further assumptions or simplifications [175].

Nevertheless, the model requires the rate constants $k_{H}, k_{T}$ and $k_{S}$ as input parameters. Especially the diffusion rate at the terraces can be an important parameter, because it influences the probability of molecules desorbing from the terrace sites prior to binding to the steps. The hopping rate for CO between $\mathrm{Pt}(111)$ terrace adsorption sites has been measured by Reutt-Robey et al. $[177,178]$ in the range of $T_{S}=95-195 \mathrm{~K}$. The authors derived Arrhenius parameters and obtained a hopping rate constant of $k_{H}\left(T_{S}\right)=8 \times 10^{9} \mathrm{~s}^{-1} e^{-0.19 \mathrm{eV} / k_{B} T_{S}}$, which yields values of $k_{H} \sim 2 \times 10^{8} \mathrm{~s}^{-1}$ in the temperature range from $T_{S}=580-720 \mathrm{~K}$. Next, I postulate that the fast component in Fig. 7.11 corresponds to direct desorption from the terraces. Consequently, I can use the Arrhenius parameters of $E_{d}=1.27 \mathrm{eV}$ and $A_{d}=3.5 \times 10^{13} \mathrm{~s}^{-1}$ for $k_{T}\left(T_{S}\right)$, observed in the Arrhenius analysis of $k_{d}^{f a s t}\left(T_{S}\right)$ in Fig. 7.11. This is an assumption so far and has to be verified by comparison of the results of the model to the experimental observations. Finally, I have to make assumptions for the unknown rate for step-to-terrace diffusion, $k_{S}$. According to the work of Serri et al., the activation energy for this process should be similar to the difference in binding energies of the adsorbate to steps and to terraces plus the activation barrier for $k_{H}\left(T_{S}\right)$. I estimate the difference in activation energy between terrace and step sites from the results of Lin and Somorjai who compared CO desorption from Pt(111) to desorption from a stepped Pt(557) with 6 atoms wide terraces ( $16 \%$ step density) [145]. The authors found activation energies of $E_{d}=1.296 \mathrm{eV}$ and $1.457 \mathrm{eV}$, respectively. I further assume that the prefactor decreases by about one order of magnitude as suggested by Serri et al. for NO/Pt(111). These approximations give $k_{S}\left(T_{S}\right)=5 \times$ $10^{8} S^{-1} e^{-0.31 e V / k_{B} T_{S}}$. Because the STC model includes some rough assumptions, the results of the model should be taken with some care and have to be compared to the experimental results.

Fig. 7.13 shows the desorption fluxes resulting from the STC model for four different surface temperatures assuming $0.5 \%$ (left) and $1 \%$ (right) step density. The top and bottom panels show the results for low (no saturation of steps) and high surface coverage (steps saturated). At low coverage, I find a very fast decay $(<5 \mu \mathrm{s}$ ) of the outgoing flux at early times followed by a slow single exponential decay. The fast decay is related to the diffusion of molecules from the terrace sites to the steps. This view is supported by a slower decay at $0.5 \%$ step density compared to $1 \%$ step sites. A lower step density corresponds to broader terraces at the surface and the adsorbed molecules have to perform more siteto-site hopping processes to reach a step site. The slow component reflects step-to-terrace diffusion followed by desorption from the terrace. 

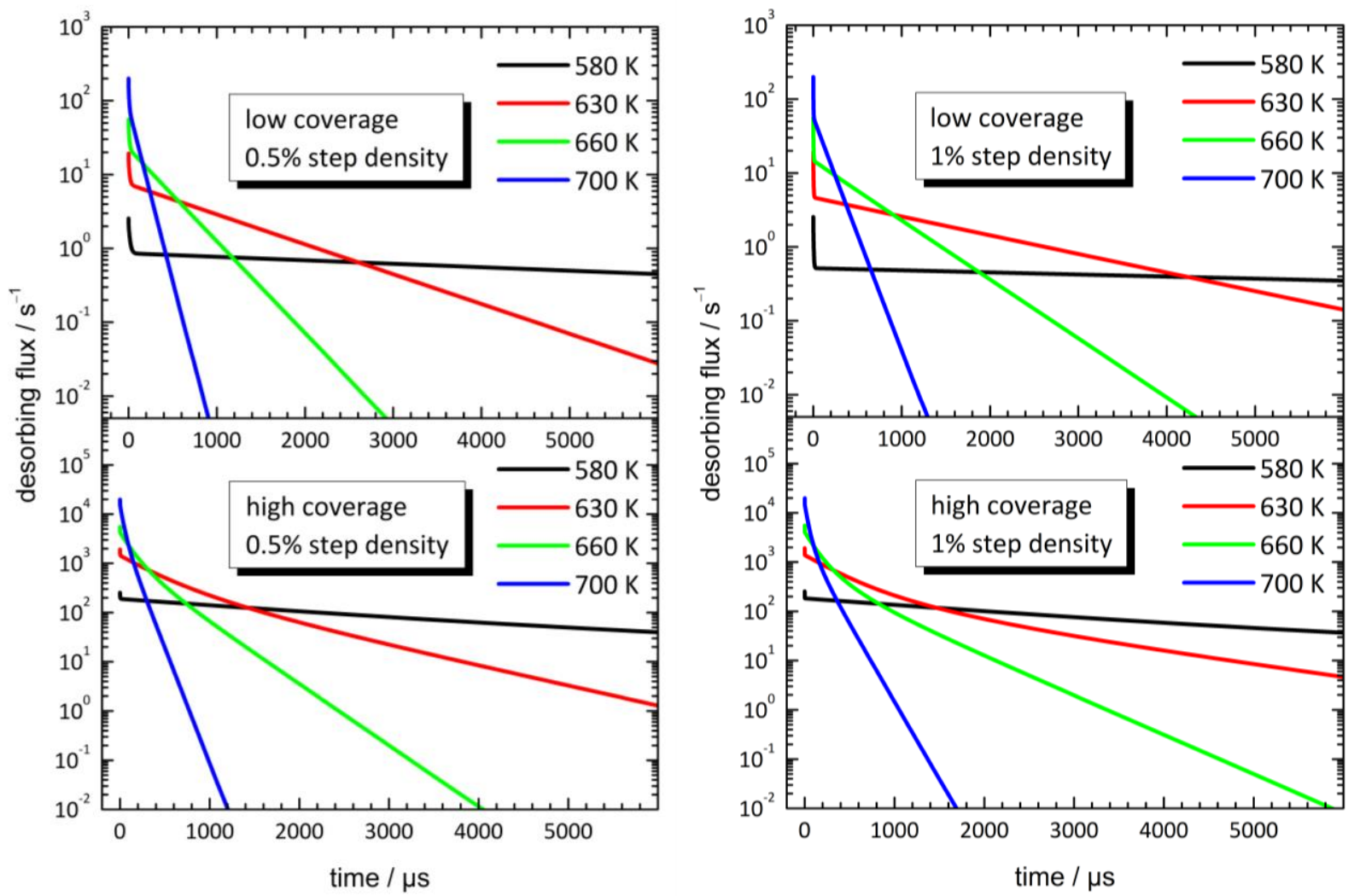

Fig. 7.13: Time dependent desorbing fluxes of $\mathrm{CO}$ adsorbed on $\mathrm{Pt}(111)$ assuming $0.5 \%$ (left panels) and $1 \%$ (right panels) step density and four different surface temperatures. The top and bottom panels show the behavior for very low and high surface coverage. The latter is sufficient for saturation of step sites.

The situation is very different in the high coverage regime corresponding to a surface coverage higher than the density of steps. Consequently, there is a significant population left at the terrace sites after the steps are saturated. This population can directly desorb from the terraces and causes the biexponential behavior in the bottom panels of Fig. 7.13 where the fast component corresponds to direct desorption from terraces sites. This hypothesis requires some further support. I extracted the two rate constants from the model by fitting the simulations with a biexponential decay. The resulting rate constants (dashed lines) are shown as a function of surface temperature in Fig. 7.14 and compared to the rate constants obtained in the experiment (dots). I observe a good agreement between simulation and experiment for the fast component (blue). Remember that I used the Arrhenius law for $k_{d}^{\text {fast }}\left(T_{S}\right)$ to calculate the rate constants for terrace desorption, $k_{T}\left(T_{S}\right)$, in the model. Therefore, the observed agreement verifies the view that the fast component in the biexponential decay results from direct terrace desorption.

The slow component in the STC model is identical to the rate constant found at low coverage in Fig. 7.13; it represents the process of step-to-terrace diffusion followed by desorption from the terrace. In addition, the simulation is in reasonable agreement with the experimental values for $k_{d}^{\text {slow }}\left(T_{S}\right)$. Therefore, I attribute the slow component in the velocity selected residence time measurements to surface diffusion followed by terrace desorption. 


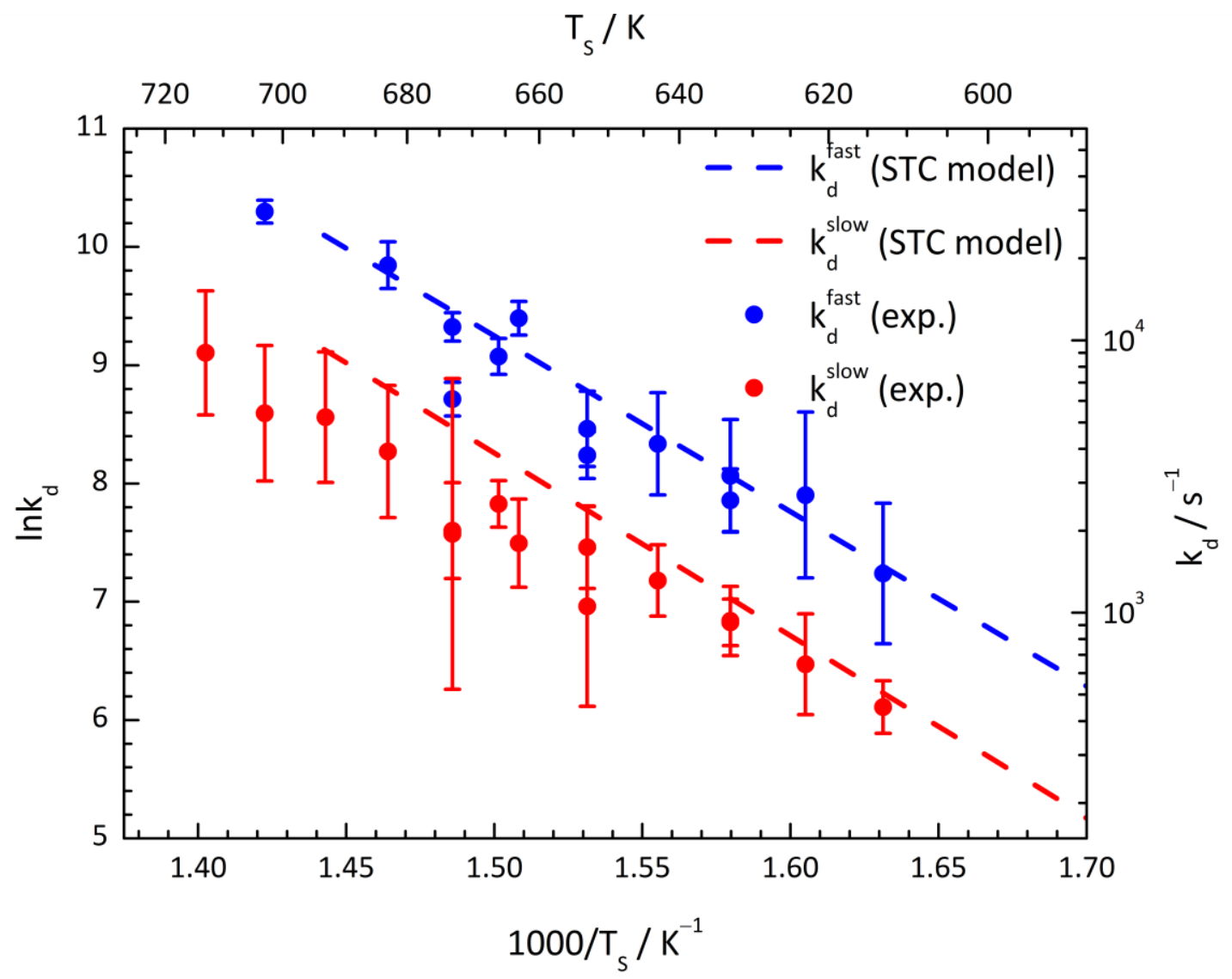

Fig. 7.14: Arrhenius plot showing a comparison of experimental rates constants $k_{d}^{\text {fast }}$ (blue dots) and $k_{d}^{\text {slow }}$ (red dots) to the predictions of the STC model (dashed lines) at high surface coverage (saturation of steps). The STC rate constants are obtained from a biexponential fit to the decay of the high coverage simulation for $0.5 \%$ step density (left panel of Fig. 7.13).

The analysis of desorption kinetics using the STC model illustrates that a biexponential decay of the desorption rate should only be observable if the dose to the surface is high enough to saturate the step sites. This is in agreement with the estimated step density of $0.5-1 \%$ from AFM measurements and the calculated surface dose of $\sim 0.03 \mathrm{ML}$ per molecular beam pulse. Moreover, the STC simulation clearly shows that the fast component, if observed, directly gives the rate constant for terrace desorption. From this important observation it follows that an analysis of these temperature dependent rates will yield the binding energy of $\mathrm{CO}$ to the terrace sites.

\section{Derivation of binding energies}

A necessary step towards comparison to first-principles theory is the derivation of the binding energy, $E_{0}$, from the measured rate constants for desorption. Transition-state theory (TST) represents suitable way to settle this issue because it enables the calculation of thermal rate constants from statistical mechanics. The description of desorption processes in TST has been worked out previously by Tully [179]. Following his analysis, the thermal desorption rate constant is given by the surface temperature dependent sticking probability, $S\left(T_{S}\right)$, multiplied with the standard TST rate expression $R_{T S T}\left(T_{S}\right)$ [179].

$$
k_{d}\left(T_{S}\right)=S\left(T_{S}\right) \times R_{T S T}\left(T_{S}\right)=S\left(T_{S}\right) \times\left(\frac{k_{B} T_{S}}{h}\right) \times \frac{Q^{\ddagger}\left(T_{S}\right)}{Q_{a d}\left(T_{S}\right)} \times e^{-E_{0} / k_{B} T_{S}}
$$


where $E_{0}$ denotes the binding energy and $Q^{\ddagger}$ and $Q_{a d}$ are the partition functions of the transition state and the adsorbate. In absence of an adsorption barrier, as suggested by the $E_{I}$ dependence of the initial sticking probability for CO/Pt(111) [172], $Q^{\ddagger}$ is equal to the partition function of the gas phase molecule.

$$
\begin{gathered}
Q^{\ddagger}\left(T_{S}\right)=Q_{\text {trans }}^{2 D} \times Q_{\text {rot }} \times Q_{v i b} \\
Q^{\ddagger}\left(T_{S}\right)=\frac{2 \pi m k_{B} T_{S}}{h^{2}} \times \frac{8 \pi^{2} I_{C O} k_{B} T_{S}}{h^{2}} \times \frac{1}{1-e^{-\frac{h v_{C O}}{k_{B} T_{S}}}}
\end{gathered}
$$

Note that equation 7.17 only contains a two-dimensional translational partition function because the motion normal to the surface corresponds to the reaction coordinate and is given by the frequency factor $k_{B} T_{S} / h$. The partition function of the adsorbate can be described as an ensemble of independent harmonic oscillators (equation 7.18).

$$
Q_{a d}^{o s c}\left(T_{S}\right)=N_{\text {sites }} \times \prod_{i=1}^{4}\left(\frac{1}{1-e^{-\frac{h v_{i}}{k_{B} T_{S}}}}\right)^{g_{i}}
$$

where $N_{\text {sites }} \approx 1.6 \times 10^{15} \mathrm{~cm}^{-2}$ is the density of available adsorption sites at the surface, the frequencies $v_{1} \ldots v_{4}$ are the vibrational modes of the adorbate and $g_{1} \ldots g_{4}$ are the degeneracies of the respective vibrational modes. The vibrational frequencies for $\mathrm{CO} / \mathrm{Pt}(111)$ have been measured previously by IR reflectance spectroscopy [149] and He diffraction [180]. The authors found vibrational frequencies of $v_{1}=2100 \mathrm{~cm}^{-1}$ (C-O stretch, $g_{1}=1$ ), $v_{2}=480 \mathrm{~cm}^{-1}$ (Pt-CO stretch, $g_{2}=1$ ), $v_{3}=411 \mathrm{~cm}^{-1}$ (hindered rotation, $g_{3}=2$ ) and $v_{4}=48.5 \mathrm{~cm}^{-1}$ (hindered translation, $g_{4}=2$ ).

Alternatively, the motion of the adsorbate on the surface (surface diffusion) can also be described as two-dimensional gas for which the partition functions of hindered rotation and translation are replaced by a 2D translational partition function:

$$
Q_{a d}^{2 D}=Q_{\text {trans }}^{2 D} \times \frac{1}{1-e^{-\frac{h v_{1}}{k_{B} T_{S}}}} \times \frac{1}{1-e^{-\frac{h v_{2}}{k_{B} T_{S}}}}
$$

Depending on the assumption for $Q_{a d}$, I obtain equations 7.20 and 7.21 for the desorption rate constant predicted by TST:

$$
\begin{aligned}
& k_{T S T}^{o S C}\left(T_{S}\right)=S\left(T_{S}\right) \times \frac{k_{B} T_{S}}{h} \times \frac{Q^{\ddagger}\left(T_{S}\right)}{Q_{a d}^{o S C}\left(T_{S}\right)} \times e^{-E_{0} / k_{B} T_{S}} \\
& k_{T S T}^{2 D}\left(T_{S}\right)=S\left(T_{S}\right) \times \frac{k_{B} T_{S}}{h} \times \frac{Q^{\ddagger}\left(T_{S}\right)}{Q_{a d}^{2 D}\left(T_{S}\right)} \times e^{-E_{0} / k_{B} T_{S}}
\end{aligned}
$$




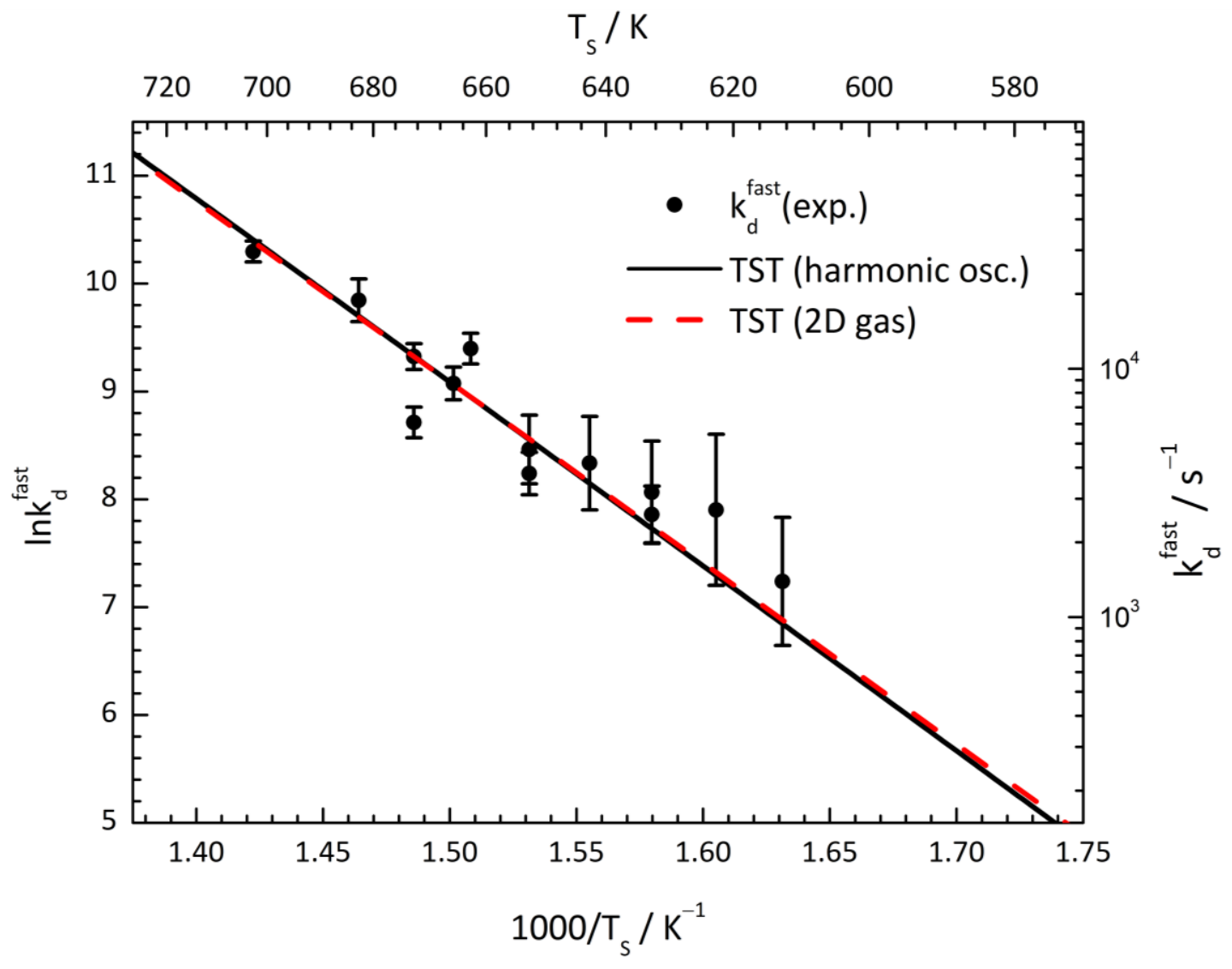

Fig. 7.15: Arrhenius plot of fast desorption rate constants $k_{d}^{f a s t}\left(T_{S}\right)$ (solid symbols). The lines show the fit of transition-state theory to the experimental rate constants with the binding energy, $E_{0}$, as the only adjustable fit parameter. The black solid and red dashed line show the results for the adsorbate treated as an ensemble of harmonic oscillators or as an two dimensional gas.

I assume the sticking probability to be independent of surface temperature which is in agreement with the similar sticking probabilities derived at $T_{S}=350 \mathrm{~K}[172]$ and at higher temperatures $[143,150]$. I chose $S=0.73$ as obtained in the most recent study of Fischer-Wolfarth et al. [181] while other studies reported sticking probabilities ranging from $S=0.7-0.85[143,145]$. However, I find that the final binding energy, $E_{0}$, is only weakly dependent on the assumed sticking probability. Fig. 7.15 shows the results for the fit of the TST expressions to the experimentally determined rate constants. The derived binding energies are given for both models (equations 7.20 and 7.21) in Table 7.1.

Table 7.1: Overview of binding energies $E_{0}$ for $\mathrm{CO}$ to $\mathrm{Pt}(111)$ derived from the fit of the TST expression to the experimental rate constants of the fast component. The values are given for the adsorbate treated as an ensemble of harmonic oscillators as well as treated as a $2 \mathrm{D}$ gas. Energies are given in $\mathrm{eV}, \mathrm{kJ} \mathrm{mol}^{-1}$ and $\mathrm{kcal} \mathrm{mol}^{-1}$.

\begin{tabular}{c|c}
\multicolumn{2}{c}{ Binding energy for CO to Pt(111) terraces } \\
TST with harmonic oscillators & TST with 2D gas \\
\hline $1.51 \pm 0.01 \mathrm{eV}$ & $1.43 \pm 0.01 \mathrm{eV}$ \\
$146 \pm 1 \mathrm{~kJ} \mathrm{~mol}^{-1}$ & $138 \pm 1 \mathrm{~kJ} \mathrm{~mol}^{-1}$ \\
$34.8 \pm 0.3 \mathrm{kcal} \mathrm{mol}^{-1}$ & $33.1 \pm 0.3 \mathrm{kcal} \mathrm{mol}^{-1}$
\end{tabular}




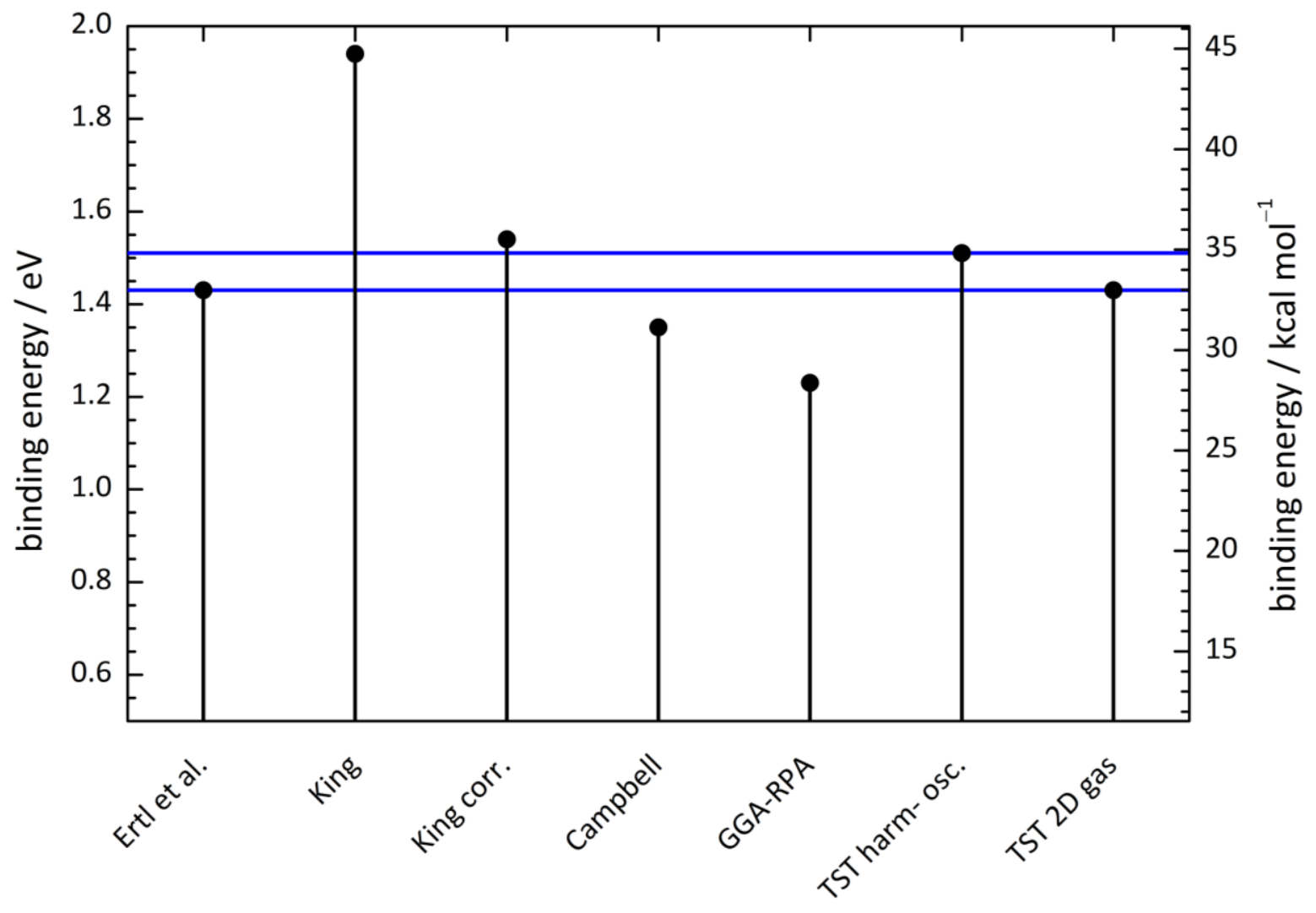

Fig. 7.16: Comparison of the derived binding energy of my work to results of previous studies. The TST analysis of the fast component suggests that binding energy of $\mathrm{CO}$ to $\mathrm{Pt}(111)$ lies in between $1.43 \mathrm{eV}$ and $1.51 \mathrm{eV}$ (blue lines). The results agree well with the measured isosteric heat of adsorption of Ertl et al. [138] and the adsorption energy measured in the group of C. T. Campbell [181]. The same measurement done in the group of D. A. King [182] is higher than the other results which is related to the wrong reflectivity of the crystal in this measurement. The value corrected by Campbell is in better agreement. The results are also compared to the results of theoretical GGA-RPA calculations [165], the value has been corrected for the difference in zero point energy $(\triangle Z P E \approx 0.08 \mathrm{eV})$.

I only apply the fit of the TST expressions for the fast component because it reflect direct desorption from terrace site. The first-order kinetic model does not reflect the correct desorption kinetic while the slow component corresponds to multistep mechanism including surface diffusion and binding at step sites. The fits of the TST expressions for $k_{d}^{f a s t}\left(T_{S}\right)$ give binding energies of $1.51 \mathrm{eV}$ and $1.43 \mathrm{eV}$. The slow component does not correspond to a single desorption process but includes surface diffusion; the firstorder kinetic model (single exponential fit) does not describe the correct desorption kinetics. In Fig. 4.15, these values are compared to previous heat of adsorption measurements that were performed for $\mathrm{CO} / \mathrm{Pt}(111)$ system. My results agree very well with the isosteric heat of adsorption of Ertl et al. [138], which was obtained by measuring the pressure dependent surface coverage at different temperatures (adsorption isotherms). A more direct measure of the adsorption heat is single crystal microcalorimetry. There are two different measurements for the $\mathrm{CO} / \mathrm{Pt}(111)$ system available in the literature. The group of D. A. King obtained a heat of adsorption of $1.93 \mathrm{eV}$ [182], which exceeds my binding energies by 0.4$0.5 \mathrm{eV}$. This result also disagrees with a recent study of Fischer-Wolfarth et al. in the group of C. T. Campbell [181]. The authors obtained a heat of adsorption of $1.35 \mathrm{eV}$, in much better agreement with the binding energies of this and Ertl's work. Fischer-Wolfarth and co-workers suggested that the difference between the two studies, which used the same experimental method, is due to errors in calibration of the calorimeter used in the King work. Calibration is accomplished by photothermal measurements - employing the correct reflectivity is crucial to its success. A correction using the 
reflectivity measured by Fischer-Wolfarth et al. changes the results to $1.54 \mathrm{eV}$ [181], in better agreement with my results and closer to the value of Campbell.

A comparison of the experimental results to first-principles theory is less straight forward. The binding energies calculated from DFT show a remarkably large spread and the reported values for atop binding of $\mathrm{CO}$ on Pt(111) range from 1-2.2 eV, dependent on the choice of XC functional [165]. Furthermore, the DFT calculations generally predict the wrong adsorption site, favoring higher CO coordination at hollow and bridge sites $[142,160,162,163,166]$, whereas experiments clearly show the preferred binding to atop sites $[152,167-169]$. This failure is frequently referred to as the CO/Pt(111) puzzle [142] and related to an underestimation of the CO LUMO energy in DFT. Recent work demonstrated that the correct site order can be restored using DFT in the generalized gradient approximation (GGA) in combination with the random phase approximation (RPA) [165]. However, these GGA-RPA calculations are computationally expensive and currently not possible for periodic systems; the high costs limit its application to the calculation of finite clusters. The reported energy [165] for atop binding of $\mathrm{CO}$ on $\mathrm{Pt}(111)$ is also shown in Fig. 7.16. Note that the binding energy derived from the TST corresponds to the difference in zero point energies between adsorbate and gas phase molecule $(\sim 0.08 \mathrm{eV})$. This difference has been subtracted from the theoretical value to make it comparable to the experimental results. The comparison shows that the GGA-RPA calculation underestimates the binding energies of this work by $0.2-0.28 \mathrm{eV}$.

\subsection{Conclusions}

In summary, I performed velocity selected residence time measurements for CO desorption from $\mathrm{Pt}(111)$ over a wide range of temperatures. The time profiles reveal a biexponential decay of the desorption signal. A comparison to the previously developed adsorption-desorption model of Serri et al. indicates that the fast component of the desorption process is related to direct desorption from terrace sites, whereas the slow component involves adsorption at step-sites, step-to-terrace diffusion and desorption from the terrace sites. I used transition-state theory to derive the binding energy, $E_{0}$, of $\mathrm{CO}$ to $\mathrm{Pt}(111)$ and found a values of $E_{0}=1.43-1.51 \mathrm{eV}$ using different assumptions for the TST rate constant; the obtained binding energies are in good agreement with adsorption heats derived from other methods. Based on the high mobility of the CO molecules at the surface in the STC model, it seems likely that the description of the adsorbate as a $2 \mathrm{D}$ gas is a better description of the system. A comparison to state-ofthe-art DFT calculations at the GGA-RPA level shows that DFT underestimates the binding energy by 0.2$0.28 \mathrm{eV}$.

In general, my results show that the velocity selected residence time method is a promising tool for accurate measurements of desorption kinetics of molecules from surfaces and, thus, for determination of binding energies with high precision. In its current implementation, it is limited to molecules with metastable intermediate states, which can be used to suppress velocity spread in a double resonance detection scheme. For small molecules with long-lived vibrations, IR-UV double resonance is a universal scheme as long as the excited vibrational state can be detected efficiently. However, problems occur for larger polyatomic molecules, e.g. benzene, for which vibrationally excited states are often depopulated due to (ultra-)fast internal vibrational energy redistribution (IVR), or which do not provide an appropriate detection scheme. Vacuum ultraviolet (VUV) ionization in combination with velocity map imaging (VMI) is a promising approach to make this method universally applicable. VUV radiation can, in principle, ionize any molecule and a VMI set-up can be used to limit the detection to a narrow range of final velocities. Moreover, the scheme would not include a major drawback of the double resonance detection scheme used in this work: the use of a double resonance limits the detection to those 
molecules that are scattered into very narrow range of final angles. The main fraction of molecules is therefore lost in the experiment, in contrast to a VUV-VMI scheme.

Furthermore, the determination of $E_{d}, A_{d}$ and $E_{0}$ would become even more accurate for a larger range of surface temperatures. In the experiment, the upper limit for $T_{S}$ is determined by the temporal width of the molecular beam. For higher surface temperatures, the temporal resolution has to be improved by the use of a shorter molecular beam pulse while the number of molecules per pulse has to stay similar in order to maintain a sufficient dose of molecules to ensure step saturation. Alternatively, a second molecular beam could be used to saturate the step sites. The lower limit of $T_{S}$ is, on one hand, determined by the signal intensity (time spread reduces the measured signal) and, on the other hand, by the repetition rate of the experiment (all molecules must be desorbed prior to the next dose/ pulse). Consequently, lower temperatures require a detection scheme with sufficient sensitivity and a variable time delay between the molecular beam pulses. 


\section{Outlook}

The results of my work show that even in systems like NO/Au(111), which were thought to be very well understood, some open questions are remaining. It is well established that the vibrational excitation and relaxation of NO in scattering from $\mathrm{Au}(111)$ occurs upon nonadiabatic V-EHP coupling. My observations further support this view. However, the results of section 3 showed that the theoretical modeling of the system is still far away from being correct. The most promising IESH model, which was found to reproduce relaxation of highly vibrationally excited $\mathrm{NO}(v=15)$ as well as excitation from $v_{i}=0$ to $v_{f}=1,2[25,65,73,74]$, revealed surprisingly large weaknesses in reproducing simple adiabatic effects like translational inelasticity or translation-to-rotation coupling. Facing such dramatic problems of the model in its current implementation, it is pointless to use the IESH model for further interpretation of effects like $\mathrm{T} \leftrightarrow \mathrm{V}$ coupling or $E_{I}$ and $v_{f}$ dependent translation-to-rotation coupling. Further work is needed in order to improve the theory, most likely the adiabatic potential energy surface, which can then be tested by comparison to the detailed experimental observations of my work. It is possible that a simple recalculation of the PES from DFT using a higher density grid of DFT points is not sufficient to settle this issue. Maybe more advanced methods, e.g. embedded correlated wave function theory [119, $130]$, have to be applied to obtain the correct topology of the PES.

Furthermore, CO scattering from $\mathrm{Au}(111)$ slowly establishes as a second benchmark system for comparison between theory and experiment. My results demonstrated that the nonadiabatic coupling in $\mathrm{CO} / \mathrm{Au}(111)$ is about three times weaker than in case of NO/Au(111). Moreover, the observations provide some indications that the lifetime of the $\mathrm{CO}$ vibration on $\mathrm{Au}(111)$ might be longer than expected from previous experiments on $\mathrm{CO} / \mathrm{Cu}(100)$ or $\mathrm{CO} / \mathrm{Pt}(111)$. The basic difference between the systems is that the CO-Au interaction is probably very weak and the $\mathrm{CO}$ is only trapped in the shallow physisorption well, thus it might not form a 'real' chemical bond. In contrast, $\mathrm{CO}$ is strongly bound at $\mathrm{Cu}$ and Pt surfaces (chemisorption). This hypothesis requires the direct measurement of the vibrational lifetime for CO adsorbed at $\mathrm{Au}(111)$ using, for example, common methods like sum-frequency generation. Eliminating this unknown variable in the experiment, it will be possible to estimate the residence time at the surface from the ratio between the direct scattering and trapping desorption peaks in 1D-TOF spectra of vibrationally excited molecules, e.g. in $\mathrm{CO}(v=2 \rightarrow 1)$ scattering.

The experiment on vibrational excitation of $\mathrm{N}_{2}$ scattering from $\mathrm{Pt}(111)$ showed that nonadiabatic coupling can occur even for molecules with very low electron affinity. The absence of vibrational excitation in scattering from $\mathrm{Au}(111)$ indicates that the surface itself plays an important role regarding the strength of nonadiabaticity. This is an issue that has not been investigated in detail, yet. The inert $\mathrm{N}_{2}$ molecule might thereby be an ideal molecule to test different surfaces with varying density of states around the Fermi-level; this would allow the investigation of the laws that scale the nonadiabatic interaction strength between gas phase molecules and surfaces.

Finally, I introduced a new method for the measurement of real-time desorption kinetics on a microsecond time-scale using a velocity selected time-of-flight technique. The application to the wellstudied CO/Pt(111) system showed its power in the sense that it is possible to determine prefactors and activation energies with high accuracy and that the experimental data itself can be compared to other studies and used to judge their reliability. Furthermore, the results showed that the microsecond time resolution in combination with an intense pulsed molecular beam is able to provide deep insights into the adsorption-desorption dynamics. The comparison to a previously introduced kinetic model demonstrates the role of steps on the observed kinetics. Future experiments can investigate the effect of 
different step-densities onto the desorption rates. Furthermore, the method itself can be applied to other systems, e.g. desorption of $\mathrm{CO}$ from Ni or Cu surfaces.

In addition, it is the first time that this velocity selected residence time approach is used and its implementation can be improved significantly. Most importantly, it has the potential to become a universal tool for the determination of rate processes involving molecules to surfaces. This requires on one hand an ionization scheme (e.g. VUV ionization, strong-field ionization), that is applicable to any molecule, and on the other the implementation of velocity map imaging as an universal tool for velocity filtering. 


\section{Appendices}

\subsection{Appendix A: Gain curve of the micro-channel plate detector}

The micro-channel plate (MCP) detector is used for amplification of the ion signal created in the REMPI process. The gain factor depends on the voltage $V_{M C P}$ which is applied across the two MCPs [183]. The amplified signal first grows exponentially with increasing $V_{M C P}$ and reaches saturation if the ion current or amplification becomes too high. Both effects can be seen in Fig. 9.1. The data points were measured for NO scattered off $\mathrm{Au}(111)$ by monitoring the ion signal (averaged over 100 laser pulses) as a function of $V_{M C P}$. For $V_{M C P}<1800 \mathrm{~V}$ the signal grows exponentially but approaches saturation for higher voltages. The gain curve of Fig. 9.1 is used to correct the REMPI intensities for all absolute measurements of this work.
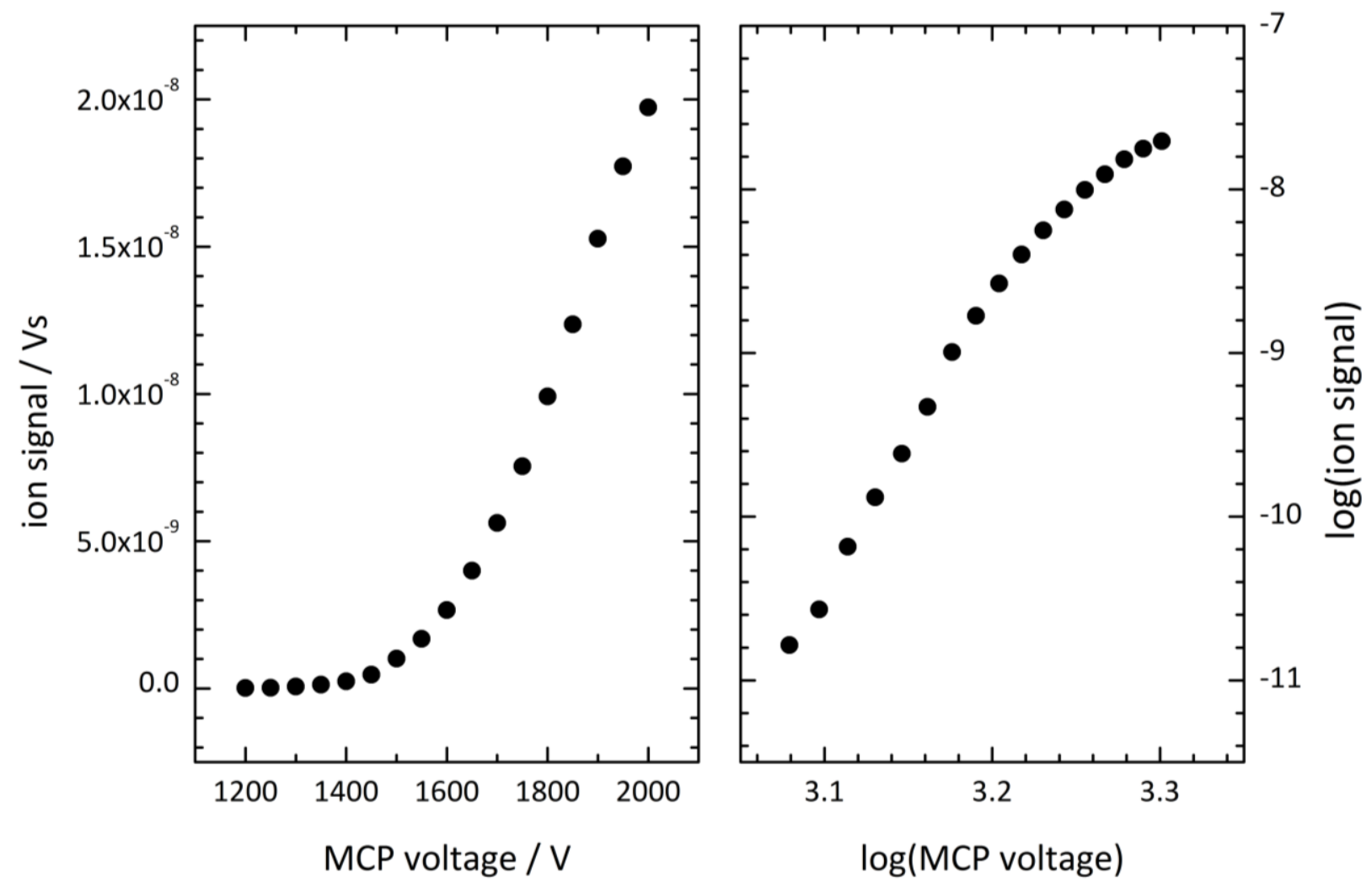

Fig. 9.1: Dependence of the measured ion signal on the micro-channel plate detector as function of detector gain voltage on a linear scale (left panel) and a double logarithmic scale (right panel). 


\subsection{Appendix B: Analysis of state-to-state time-of-flight spectra}

The general procedure for conversion of time-of-flight spectra obtained from laser based detection into speed and translational energy distributions has been described earlier in detail [32]. In the experiment, I measure a time-dependent density $S(t)$ of molecules passing the REMPI detection laser beam. This is a general feature of laser based detection schemes since the observed signal is proportional to the density of molecules in the detection volume. $S(t)$ is converted into a time dependent flux, $\varphi_{t}(t)$, by multiplication with the speed $s$ of the molecules (density-to-flux conversion):

$$
\varphi_{t}(t)=s \cdot S(t)
$$

$\varphi_{t}(t)$ is related to the velocity dependent flux $\varphi_{s}(s)$ by:

$$
\varphi_{s}(s) d s=\varphi(t) d t=S(t) s d t
$$

Furthermore, I have to apply the appropriate Jacobian for conversion from time space into velocity space:

$$
\begin{gathered}
\left|\frac{d s}{d t}\right|=\left|\frac{d(l / t)}{d t}\right|=\frac{l}{t^{2}} \\
\rightarrow \varphi_{s}(s) d s=\frac{S(t) \cdot s}{\frac{l}{t^{2}}} d s=S(t) \cdot t d s
\end{gathered}
$$

The speed dependent flux $\varphi_{S}(s)$ can be converted into energy space using the corresponding Jacobian:

$$
\begin{gathered}
\frac{d E}{d s}=\frac{d\left(1 / 2 \cdot m \cdot s^{2}\right)}{d s}=m \cdot s \\
\rightarrow P(E) d E=\varphi_{s}(s) d s=\frac{\varphi_{s}(s)}{m s} d E=\frac{S(t) \cdot t}{m s}
\end{gathered}
$$

The speed distribution of the incident molecular beam can be well described by the following function:

$$
\varphi\left(s, s_{0}, \alpha_{0}\right)=A \cdot s^{2} \cdot e^{-\left(s-s_{0}\right)^{2} / \alpha_{0}^{2}}
$$

where $A$ is an amplitude factor, $s_{0}$ is the center velocity of distribution and $\alpha_{0}$ is a width parameter which is related to the translational temperature $\alpha_{0}=\sqrt{2 k_{B} T_{\text {trans }} / m}$ of the beam. The speed distribution of the scattered molecules is described as a flowing Maxwell Boltzmann distribution of the form:

$$
\varphi_{f}\left(s, s_{i}, \alpha, \gamma\right)=A \cdot s^{2} \cdot e^{-\left(s-\gamma s_{i}\right)^{2} / \alpha^{2}}
$$

which is coupled to the incident beam velocity, $s_{i}$, by a parameter $\gamma$ that gives the fraction of $s_{i}$ that is maintained in the recoiling molecule after scattering. Both functions can be converted to energy space 
and time-dependent density using the equations above. The raw data is fitted using $S(t)$ and the obtained parameters are used to convert the fit into velocity and energy dependent flux.

In general, it is useful to condense the experimental data for comparison of different effects on the final translational energy distribution. Common values are the most probable energy, $E^{M P}$, and the mean kinetic energy, $\langle E\rangle=\int_{0}^{\infty} E \cdot P(E) d E$.

\subsection{Appendix C: Forward convolution for fitting of NO time-of-flight distributions}

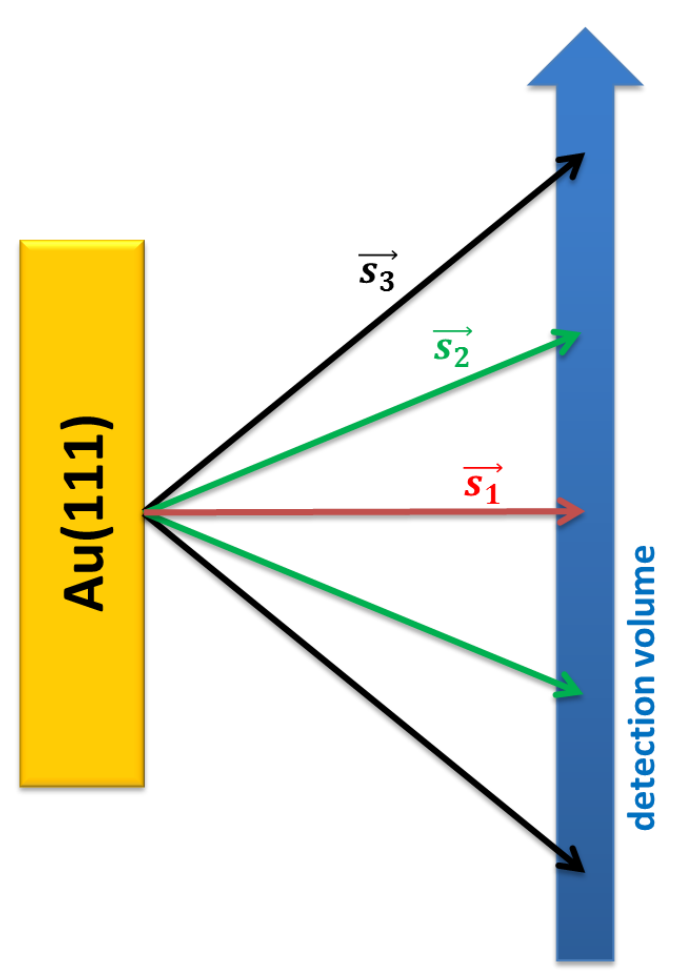

Fig. 9.2: Effect of the angular distribution along the REMPI beam on the detected time-dependent density.
A weakness of the state-to-state time-of-flight experiments (section 2.4) is that only the velocity component perpendicular to the laser beam is resolved. In cases where REMPI detection schemes are used that require absorption of three or more photons (e.g. (2+1) REMPI on CO), the REMPI laser is focused and thus the region of efficient ionization will be very well located at the focal point of the laser beam. However, for detection of NO molecules I used $A \leftarrow X(1+1)$ REMPI. Although this scheme allows for very sensitive detection even with unfocused lasers, it will lead to the effect that basically all molecules along the laser beam will be ionized and detected with similar efficiency. The effect on the detected time-dependent density is illustrated in Fig. 9.2. At the same delay between tagging and detection, the REMPI laser will ionize molecules with different total velocities $\overrightarrow{s_{1}}<\overrightarrow{s_{2}}<\overrightarrow{s_{3}}$ which have the same velocity components $s_{\perp}=s_{1}$ perpendicular to the REMPI beam but different components $s_{\|}$parallel to the detection laser beam. The correction for this effect requires the knowledge of the angular distributions of the scattered molecules. Furthermore, the incident molecular beam is not characterized by only one velocity but by a distribution $\varphi_{i}\left(s_{i}, s_{i 0}, \alpha_{0}\right)$.

I used forward convolution to fit the experimentally observed arrival time distributions $S(t)$. The speed distribution of the incident molecular beam can be taken into account by convolution of $\varphi_{f}\left(s, s_{i}, \alpha, \gamma\right)$ with $\varphi_{i}\left(s_{i}, s_{i 0}, \alpha_{0}\right)$ :

$$
\varphi_{f}(s, \alpha, \gamma)=\int_{0}^{\infty} \varphi_{f}\left(s, s_{i}, \alpha, \gamma\right) \cdot \varphi_{i}\left(s_{i}, s_{i 0}, \alpha_{0}\right) d s_{i}
$$

If one assumes that all molecules along the laser beam are ionized and detected with equal efficiency, the measured time-dependent density $S(t)$ of the molecules is given by: 


$$
S(t)=\int \rho\left(x, d_{y} d_{z}, t\right) d x
$$

with

$$
\rho\left(x, d_{y} d_{z}, t\right)=\left[\frac{\varphi_{f}(r / t, \alpha, \gamma)}{r^{2} t}\right] \cdot \cos ^{m} \theta
$$

where $x$ is the coordinate along the laser propagation direction, $d_{y}$ and $d_{z}$ are the $y, z$ components of the total flight distance $l . \rho\left(x, d_{y} d_{z}, t\right)$ describes the time-dependent density of molecules in Cartesian coordinates. The angular distributions are taken from ref. [1] and are fitted to a $\cos ^{m} \theta$ function, where $m$ is a parameter related to the width of the distribution. The resulting function for $S(t)$ is fitted to the experimental data and the fit parameters $\alpha$ and $\gamma$ are used to derive the corresponding speed and translational energy distributions. 


\subsection{Appendix D: Spectral simulation for $\mathrm{CO}$ and $\mathrm{N}_{2}$ spectra}

The measured $\mathrm{CO}$ and $\mathrm{N}_{2}$ REMPI spectra were fitted to a simulation using the following expression:

$$
I(\lambda)=\sum_{v} A_{v} \sum_{J} N_{J} \cdot e^{-\frac{\left(\lambda-\lambda_{v, J}-\lambda_{o f f}\right)^{2}}{\sigma^{2}}}
$$

The values of $\lambda_{v, J}$ were calculated from the spectroscopic constants given in Table 9.1 and Table 9.2 by:

$$
\begin{aligned}
\Delta E_{v, J}=\frac{c}{\lambda_{v, J}}= & T_{e}^{\prime}-T_{e}^{\prime \prime}+G^{\prime}\left(v^{\prime}\right)-G^{\prime \prime}\left(v^{\prime \prime}\right)+\left(B_{v}^{\prime} J^{\prime}\left(J^{\prime}+1\right)-B_{v}^{\prime \prime} J^{\prime \prime}\left(J^{\prime \prime}+1\right)\right) \\
& -\left(D_{v}^{\prime} J^{\prime 2}\left(J^{\prime}+1\right)^{2}-D_{v}^{\prime \prime} J^{\prime \prime 2}\left(J^{\prime \prime}+1\right)^{2}\right)
\end{aligned}
$$

with $E_{r o t}=2 B_{v} J(J+1)-J^{2}(J+1)^{2} D_{v}$ and $B_{v}=B_{e}-1 / 2 \alpha(v+1 / 2)$.

In case of the (2+1) REMPI schemes for $\mathrm{CO}$ and $\mathrm{N}_{2}$ using a ${ }^{1} \Sigma \leftarrow{ }^{1} \Sigma$ transition, only $\mathrm{O}, \mathrm{Q}$ and $\mathrm{S}$ transitions with $\Delta J=-2,0,+2$ are allowed. For the dominant $\mathrm{Q}$ branch ( 200 times more intense for linear polarized light) with $\Delta J=0$, the expression simplifies to:

$$
\Delta E_{v, J}=\frac{c}{\lambda_{v, J}}=T_{e}^{\prime}-T_{e}^{\prime \prime}+G^{\prime}\left(v^{\prime}\right)-G^{\prime \prime}\left(v^{\prime \prime}\right)+\left(B_{v}^{\prime}-B_{v}^{\prime \prime}\right) J(J+1)-\left(D_{v}^{\prime}-D_{v}^{\prime \prime}\right) J^{2}(J+1)^{2}
$$

with vibrational term energies $G^{\prime}\left(v^{\prime}\right)=\omega_{e}^{\prime}\left(v^{\prime}+1 / 2\right)-\omega_{e} x_{e}^{\prime}\left(v^{\prime}+1 / 2\right)^{2}$ and $G^{\prime \prime}\left(v^{\prime \prime}\right)=\omega_{e}^{\prime \prime}\left(v^{\prime \prime}+\right.$ $1 / 2)-\omega_{e} x_{e}^{\prime \prime}\left(v^{\prime \prime}+1 / 2\right)^{2}$ for the upper and lower states, respectively.

$A_{v}$ is a variable constant to fit the amplitude of a vibrational band in the REMPI spectrum. The linewidth in the spectrum is reproduced by adjusting $\sigma$. The offset in laser wavelength is corrected by shifting the simulation by $\lambda_{\text {off }}$ (typically $\sim 0.055 \mathrm{~nm}$ ). $N_{J}$ is the relative population of the rotational state $J$. The precise expressions for $N_{J}$ are described below in the corresponding sections for CO and $N_{2}$. In the easiest case, the rotational distribution can be described by a simple Boltzmann distribution:

$$
N_{J}=(2 J+1) \cdot e^{-\frac{E_{r o t}(J)}{k_{B} T_{r o t}}}
$$




\section{$(2+1)$ REMPI spectra of CO}

In case of $\mathrm{CO}$ scattering from $\mathrm{Au}(111)$ and $\mathrm{Pt}(111)$, the rotational population cannot be described by a simple thermal distribution since both systems show rotational rainbow scattering. A possible way to model the rotational distribution is the combination of a thermal distribution with a Gaussian function that is centered at high rotational states.

$$
N_{J}=(2 J+1) \cdot e^{-\frac{E_{r o t}(J)}{k_{B} T_{r o t}}}+a \cdot e^{-\frac{\left(J-J_{c}\right)^{2}}{b^{2}}}
$$

This formula is used for the description of rotational distributions for $\operatorname{CO}(v=0 \rightarrow 0)$ scattering. Vibrationally inelastic $0 \rightarrow 1$ scattering can be described by a simple thermal rotational distribution (equation 9.13). The spectroscopic constant for the simulation are given in Table 9.1.

Table 9.1: Spectroscopic constants used for fitting of the $\operatorname{COX}{ }^{1} \Sigma^{+} \leftarrow B{ }^{1} \Sigma^{+}$transition.

\begin{tabular}{ccc}
\hline Constant & Value & Reference \\
\hline & \multicolumn{1}{c|}{$X^{1} \Sigma^{+}$} & \\
\hline$T_{e} / \mathrm{cm}^{-1}$ & 0 & - \\
\hline$\omega_{e}^{\prime \prime} / \mathrm{cm}^{-1}$ & 2169.8135802 & {$[184]$} \\
\hline$\omega_{e} x_{e}^{\prime \prime} / \mathrm{cm}^{-1}$ & 13.2883076 & {$[184]$} \\
\hline$B_{e}^{\prime \prime} / \mathrm{cm}^{-1}$ & 1.9312808724 & {$[184]$} \\
\hline$D_{v}^{\prime \prime} / \mathrm{cm}^{-1}$ & $6.12087 \times 10^{-6}(v=0)$ & {$[184]$} \\
\hline$\alpha_{e}^{\prime \prime} / \mathrm{cm}^{-1}$ & $6.12008 \times 10^{-6}(v=1)$ & {$[184]$} \\
\hline & 0.0175044121 & {$[185]$} \\
\hline$T_{e} / \mathrm{cm}^{-1}$ & $B{ }^{1} \Sigma^{+}$ & {$[185]$} \\
\hline$\omega_{e}^{\prime} / \mathrm{cm}^{-1}$ & 86945.21 & {$[185]$} \\
\hline$\omega_{e} x_{e}^{\prime} / \mathrm{cm}^{-1}$ & 2112.7 & {$[185]$} \\
\hline$B_{e}^{\prime} / \mathrm{cm}^{-1}$ & 15.22 & {$[185]$} \\
\hline$D_{v}^{\prime} / \mathrm{cm}^{-1}$ & 1.9612 & {$[185]$}
\end{tabular}




\section{(2+1) REMPI spectra of $\mathrm{N}_{2}$}

The rotational population of $\mathrm{N}_{2}$ scattering from $\mathrm{Pt}(111)$ can be well described by a simple thermal distribution with a single rotational temperature $T_{\text {rot }}$. Due to the nuclear spin of $I=1$ of the nitrogen atom, $N_{J}$ has to include nuclear spin statistics. This leads to the effect that odd $J$ states have only half the intensity compared to even $J$ states. Therefore $N_{J}$ can be written as:

$$
N_{J}=\left(1.5+0.5 \cdot(-1)^{J}\right) \cdot(2 J+1) \cdot e^{-\frac{E_{r o t}(J)}{k_{B} T_{r o t}}}
$$

The final simulation is fitted to the experimental spectrum by optimization of the parameters $A_{v}, T_{\text {rot }}$, $\lambda_{o f f}$ and $\sigma$. The spectroscopic constants for the simulation of the (2+1) REMPI spectra are given in Table 9.2.

Table 9.2: Spectroscopic constants for the $X^{1} \Sigma_{g}^{+}$and the $a^{\prime \prime}{ }^{1} \Sigma_{g}^{+}$state of $\mathrm{N}_{2}$.

\begin{tabular}{|c|c|c|}
\hline Constant & Value & Reference \\
\hline \multicolumn{3}{|c|}{$X^{1} \Sigma_{g}^{+}$} \\
\hline$T_{e} / \mathrm{cm}^{-1}$ & 0 & - \\
\hline$\omega_{e}^{\prime \prime} / \mathrm{cm}^{-1}$ & 2358.57 & [186] \\
\hline$\omega_{e} x_{e}^{\prime \prime} / \mathrm{cm}^{-1}$ & 14.324 & [186] \\
\hline$B_{e}^{\prime \prime} / \mathrm{cm}^{-1}$ & 1.99824 & [186] \\
\hline$D_{v}^{\prime \prime} / \mathrm{cm}^{-1}$ & $5.76 \times 10^{-6}$ & [186] \\
\hline$\alpha_{e}^{\prime \prime} / \mathrm{cm}^{-1}$ & 0.017318 & [186] \\
\hline \multicolumn{3}{|c|}{$a^{\prime \prime 1} \Sigma_{g}^{+}$} \\
\hline$T_{e} / \mathrm{cm}^{-1}$ & 98938 & [106] \\
\hline$\omega_{e}^{\prime} / \mathrm{cm}^{-1}$ & 2167 & [106] \\
\hline$\omega_{e} x_{e}^{\prime} / \mathrm{cm}^{-1}$ & 14.451 & Calculated from $v_{00}, v_{11}$ and $v_{22}$ of [106] \\
\hline$B_{e}^{\prime} / \mathrm{cm}^{-1}$ & 1.938 & {$[106]$} \\
\hline$D_{v}^{\prime} / \mathrm{cm}^{-1}$ & $6.2 \times 10^{-6}$ & {$[106]$} \\
\hline$\alpha_{e}^{\prime} / \mathrm{cm}^{-1}$ & 0.08934 & Calculated from values for $B_{v}^{\prime}$ of [106] \\
\hline
\end{tabular}




\section{References}

1. Cooper, R., et al., On the determination of absolute vibrational excitation probabilities in molecule-surface scattering: Case study of NO on Au(111). Journal of Chemical Physics, 2012. 137(6): p. 064705-12.

2. Golibrzuch, K., et al., Experimental and Theoretical Study of Multi-Quantum Vibrational Excitation: $N O(v=0 \rightarrow 1,2,3)$ in Collisions with $A u(111)$. Journal of Physical Chemistry A, 2013. 117(32): p. 7091-7101.

3. Huang, Y., et al., Vibrational Promotion of Electron Transfer. Science, 2000. 290(5489): p. 111114.

4. Kay, B.D., T.D. Raymond, and M.E. Coltrin, Observation of Direct Multiquantum Vibrational Excitation in Gas-Surface Scattering: NH_\{3\} on Au(111). Physical Review Letters, 1987. 59(24): p. 2792-2794.

5. Senn, P., The computation of RKR potential energy curves of diatomic molecules using mathematica. Computers \& Chemistry, 1995. 19(4): p. 437-439.

6. Ertl, G., Elementary Steps in Heterogeneous Catalysis. Angewandte Chemie International Edition in English, 1990. 29(11): p. 1219-1227.

7. Rahinov, I., et al., Quantifying the breakdown of the Born-Oppenheimer approximation in surface chemistry. Physical Chemistry Chemical Physics, 2011. 13(28): p. 12680-12692.

8. Rettner, C.T., et al., OBSERVATION OF DIRECT VIBRATIONAL-EXCITATION IN GAS-SURFACE COLLISIONS - NO ON AG(111). Physical Review Letters, 1985. 55(18): p. 1904-1907.

9. Kimman, J., et al., Correlation Between Kinetic-Energy Transfer to Rotation and to Phonons in Gas-Surface Collisions of NO with Ag(111). Physical Review Letters, 1986. 57(16): p. 2053-2056.

10. Rettner, C.T., J. Kimman, and D.J. Auerbach, Inelastic scattering of NO from Ag(111): Internal state, angle, and velocity resolved measurements. The Journal of Chemical Physics, 1991. 94(1): p. 734-750.

11. Siders, J.L.W. and G.O. Sitz, Observation and characterization of direct inelastic and trappingdesorption channels in the scattering of $\mathrm{N} 2$ from Cu(110). The Journal of Chemical Physics, 1994. 101(7): p. 6264-6270.

12. Sitz, G.O., Gas surface interactions studied with state-prepared molecules. Reports on Progress in Physics, 2002. 65(8): p. 1165.

13. Sitz, G.O., A.C. Kummel, and R.N. Zare, Direct inelastic scattering of N2 from Ag(111). I. Rotational populations and alignment. The Journal of Chemical Physics, 1988. 89(4): p. 25582571.

14. Beck, R.D., et al., Vibrational Mode-Specific Reaction of Methane on a Nickel Surface. Science, 2003. 302(5642): p. 98-100.

15. Bisson, R., et al., State-Resolved Reactivity of $\mathrm{CH} 4(2 \mathrm{v} 3)$ on Pt(111) and Ni(111): Effects of Barrier Height and Transition State Locationt. The Journal of Physical Chemistry A, 2007. 111(49): p. 12679-12683.

16. Maroni, P., et al., State-Resolved Gas-Surface Reactivity of Methane in the Symmetric C-H Stretch Vibration on Ni(100). Physical Review Letters, 2005. 94(24): p. 246104.

17. Yoder, B.L., R. Bisson, and R.D. Beck, Steric Effects in the Chemisorption of Vibrationally Excited Methane on Ni(100). Science, 2010. 329(5991): p. 553-556.

18. Nave, S. and B. Jackson, Methane Dissociation on Ni(111): The Role of Lattice Reconstruction. Physical Review Letters, 2007. 98(17): p. 173003.

19. Tiwari, A.K., S. Nave, and B. Jackson, Methane Dissociation on Ni(111): A New Understanding of the Lattice Effect. Physical Review Letters, 2009. 103(25): p. 253201. 
20. Juurlink, L.B.F., D.R. Killelea, and A.L. Utz, State-resolved probes of methane dissociation dynamics. Progress in Surface Science, 2009. 84(3-4): p. 69-134.

21. Juurlink, L.B.F., et al., Eigenstate-Resolved Studies of Gas-Surface Reactivity: $\left.C_{-} H_{4}\right\}$ ( v_\{3\}) Dissociation on Ni(100). Physical Review Letters, 1999. 83(4): p. 868-871.

22. Juurlink, L.B.F., et al., Comparative Study of $\mathrm{C}-\mathrm{H}$ Stretch and Bend Vibrations in Methane Activation on Ni(100) and Ni(111). Physical Review Letters, 2005. 94(20): p. 208303.

23. Killelea, D.R., et al., Surface Temperature Dependence of Methane Activation on Ni(111). The Journal of Physical Chemistry C, 2009. 113(48): p. 20618-20622.

24. Smith, R.R., et al., Preference for Vibrational over Translational Energy in a Gas-Surface Reaction. Science, 2004. 304(5673): p. 992-995.

25. Cooper, R., et al., Multiquantum Vibrational Excitation of NO Scattered from Au(111): Quantitative Comparison of Benchmark Data to Ab Initio Theories of Nonadiabatic MoleculeSurface Interactions. Angewandte Chemie, 2012. 124(20): p. 5038-5042.

26. Cooper, R., et al., On the determination of absolute vibrational excitation probabilities in molecule-surface scattering: Case study of NO on Au(111). The Journal of Chemical Physics, 2012. 137(6): p. 064705.

27. Cooper, R., et al., Vibrational overtone excitation in electron mediated energy transfer at metal surfaces. Chemical Science, 2010. 1(1): p. 55-61.

28. Gratz, F., et al., Vibrational enhancement of electron emission in $\mathrm{CO}$ (a3Pi) quenching at a clean metal surface. Phys Chem Chem Phys, 2013. 15(36): p. 14951-5.

29. LaRue, J., et al., Vibrationally promoted electron emission at a metal surface: electron kinetic energy distributions. Physical Chemistry Chemical Physics, 2011. 13(1): p. 97-99.

30. Matsiev, D., et al., On the temperature dependence of electronically non-adiabatic vibrational energy transfer in molecule-surface collisions. Physical Chemistry Chemical Physics, 2011. 13(18): p. 8153-8162.

31. Nahler, N.H., et al., Inverse Velocity Dependence of Vibrationally Promoted Electron Emission from a Metal Surface. Science, 2008. 321(5893): p. 1191-1194.

32. Rahinov, I., et al., Efficient vibrational and translational excitations of a solid metal surface: Stateto-state time-of-flight measurements of $\mathrm{HCl}(v=2, J=1)$ scattering from $\mathrm{Au}(111)$. Journal of Chemical Physics, 2008. 129(21).

33. Ran, Q., et al., Observation of a change of vibrational excitation mechanism with surface temperature: $\mathrm{HCl}$ collisions with $\mathrm{Au}(111)$. Physical Review Letters, 2007. 98(23).

34. White, J.D., et al., Conversion of large-amplitude vibration to electron excitation at a metal surface. Nature, 2005. 433(7025): p. 503-505.

35. Bartels, N., et al., Observation of orientation-dependent electron transfer in molecule-surface collisions. Proceedings of the National Academy of Sciences, 2013.

36. Groot, I.M.N., et al., Dynamics of hydrogen dissociation on stepped platinum. The Journal of Chemical Physics, 2008. 129(22): p. -.

37. van der Niet, M.J.T.C., et al., Hydrophobic interactions between water and pre-adsorbed D on the stepped Pt(533) surface. Physical Chemistry Chemical Physics, 2008. 10(47): p. 7169-7179.

38. van der Niet, M.J.T.C., et al., The influence of step geometry on the desorption characteristics of O2, D2, and H2O from stepped Pt surfaces. The Journal of Chemical Physics, 2010. 132(17): p. -.

39. Muhlhausen, C.W., L.R. Williams, and J.C. Tully, Dynamics of gas-surface interactions: Scattering and desorption of NO from Ag(111) and Pt(111). The Journal of Chemical Physics, 1985. 83(5): p. 2594-2606.

40. Born, M. and R. Oppenheimer, Zur Quantentheorie der Molekeln. Annalen der Physik, 1927. 389(20): p. 457-484.

41. Bartels, C., et al., Energy transfer at metal surfaces: the need to go beyond the electronic friction picture. Chemical Science, 2011. 2(9): p. 1647-1655. 
42. Wodtke *, A.M., J.C. Tully, and D.J. Auerbach, Electronically non-adiabatic interactions of molecules at metal surfaces: Can we trust the Born-Oppenheimer approximation for surface chemistry? International Reviews in Physical Chemistry, 2004. 23(4): p. 513-539.

43. Newns, D.M., Electron-hole pair mechanism for excitation of intramolecular vibrations in molecule-surface scattering. Surface Science, 1986. 171(3): p. 600-614.

44. Rettner, C.T., et al., DIRECT VIBRATIONAL-EXCITATION IN GAS SURFACE COLLISIONS OF NO WITH $A G(111)$. Surface Science, 1987. 192(1): p. 107-130.

45. Silva, M., et al., THE DYNAMICS OF "STRETCHED MOLECULES": Experimental Studies of Highly Vibrationally Excited Molecules With Stimulated Emission Pumping. Annual Review of Physical Chemistry, 2001. 52(1): p. 811-852.

46. Rettner, C.T., The search for direct vibrational excitation in gas-surface collisions of CO with Au(111). The Journal of Chemical Physics, 1993. 99(7): p. 5481-5489.

47. Persson, B.N.J. and M. Persson, Vibrational Lifetime for Co Adsorbed on Cu(100). Solid State Communications, 1980. 36(2): p. 175-179.

48. Beckerle, J.D., et al., Ultrafast infrared response of adsorbates on metal surfaces: Vibrational lifetime of CO/Pt(111). Physical Review Letters, 1990. 64(17): p. 2090-2093.

49. Krishna, V. and J.C. Tully, Vibrational lifetimes of molecular adsorbates on metal surfaces. The Journal of Chemical Physics, 2006. 125(5): p. -.

50. Morin, M., N.J. Levinos, and A.L. Harris, Vibrational energy transfer of CO/Cu(100): Nonadiabatic vibration/electron coupling. The Journal of Chemical Physics, 1992. 96(5): p. 3950-3956.

51. Head-Gordon, M. and J.C. Tully, Vibrational relaxation on metal surfaces: Molecular-orbital theory and application to $\mathrm{CO} / \mathrm{Cu}(100)$. The Journal of Chemical Physics, 1992. 96(5): p. 39393949.

52. Tully, J.C., M. Gomez, and M. Head-Gordon, Electronic and phonon mechanisms of vibrational relaxation: $\mathrm{CO}$ on Cu(100). Journal of Vacuum Science \&amp; Technology A, 1993. 11(4): p. 19141920.

53. Chang, H.-C. and G.E. Ewing, Infrared fluorescence from a monolayer of $\mathrm{CO}$ on $\mathrm{NaCl}(100)$. Physical Review Letters, 1990. 65(17): p. 2125-2128.

54. Head-Gordon, M. and J.C. Tully, VIBRATIONAL-RELAXATION ON METAL-SURFACES - MOLECULARORBITAL THEORY AND APPLICATION TO CO/CU(100). Journal of Chemical Physics, 1992. 96(5): p. 3939-3949.

55. Persson, B.N.J. and R. Ryberg, Collective Vibrational-Modes in Isotopic Mixtures of Co Adsorbed on $\mathrm{Cu}(100)$. Solid State Communications, 1980. 36(7): p. 613-617.

56. Tully, J.C., M. Gomez, and M. Headgordon, ELECTRONIC AND PHONON MECHANISMS OF VIBRATIONAL-RELAXATION - CO ON CU(100). Journal of Vacuum Science \& Technology a-Vacuum Surfaces and Films, 1993. 11(4): p. 1914-1920.

57. Rettner, C.T., H.A. Michelsen, and D.J. Auerbach, Determination of quantum-state-specific gassurface energy transfer and adsorption probabilities as a function of kinetic energy. Chemical Physics, 1993. 175(1): p. 157-169.

58. Kroes, G.-J., et al., Apparent failure of the Born-Oppenheimer static surface model for vibrational excitation of molecular hydrogen on copper. Proceedings of the National Academy of Sciences, 2010. 107(49): p. 20881-20886.

59. Díaz, C., et al., Chemically Accurate Simulation of a Prototypical Surface Reaction: H2 Dissociation on $\mathrm{Cu}(111)$. Science, 2009. 326(5954): p. 832-834.

60. Rettner, C.T., D.J. Auerbach, and H.A. Michelsen, Observation of direct vibrational excitation in collisions of $H_{-}\{2\}$ and $D_{-}\{2\}$ with a $\mathrm{Cu}(111)$ surface. Physical Review Letters, 1992. 68(16): $p$. 2547-2550.

61. Darling, G.R. and S. Holloway, Translation-to-vibrational excitation in the dissociative adsorption of D2. The Journal of Chemical Physics, 1992. 97(1): p. 734-736. 
62. Hohenberg, P. and W. Kohn, Inhomogeneous Electron Gas. Physical Review, 1964. 136(3B): p. B864-B871.

63. Kohn, W. and L.J. Sham, Self-Consistent Equations Including Exchange and Correlation Effects. Physical Review, 1965. 140(4A): p. A1133-A1138.

64. Monturet, S. and P. Saalfrank, Role of electronic friction during the scattering of vibrationally excited nitric oxide molecules from Au(111). Physical Review B, 2010. 82(7): p. 075404.

65. Shenvi, N., S. Roy, and J.C. Tully, Dynamical Steering and Electronic Excitation in NO Scattering from a Gold Surface. Science, 2009. 326(5954): p. 829-832.

66. Osterlund, L., I. Zoric, and B. Kasemo, Dissociative sticking of O-2 on Al(111). Physical Review B, 1997. 55(23): p. 15452-15455.

67. Behler, J., et al., Dissociation of $0-2$ at Al(111): The role of spin selection rules. Physical Review Letters, 2005. 94(3).

68. Libisch, F., et al., Origin of the Energy Barrier to Chemical Reactions of O-2 on Al(111): Evidence for Charge Transfer, Not Spin Selection. Physical Review Letters, 2012. 109(19).

69. Schaich, W.L., Brownian motion model of surface chemical reactions. Derivation in the large mass limit. The Journal of Chemical Physics, 1974. 60(3): p. 1087-1093.

70. Schaich, W.L., Model calculation of brownian motion parameters at a metal surface. Surface Science, 1975. 49(1): p. 221-236.

71. d'Agliano, E.G., et al., Brownian motion model of the interactions between chemical species and metallic electrons: Bootstrap derivation and parameter evaluation. Physical Review B, 1975. 11(6): p. 2122-2143.

72. Tully, J.C., Molecular dynamics with electronic transitions. The Journal of Chemical Physics, 1990. 93(2): p. 1061-1071.

73. Roy, S., N.A. Shenvi, and J.C. Tully, Model Hamiltonian for the interaction of NO with the Au(111) surface. The Journal of Chemical Physics, 2009. 130(17): p. - .

74. Shenvi, N., S. Roy, and J.C. Tully, Nonadiabatic dynamics at metal surfaces: Independent-electron surface hopping. The Journal of Chemical Physics, 2009. 130(17): p. -.

75. Lykke, K.R. and B.D. Kay, Rotationally inelastic gas--surface scattering: $\mathrm{HCl}$ from $\mathrm{Au}(111)$. The Journal of Chemical Physics, 1990. 92(4): p. 2614-2623.

76. Geuzebroek, F.H., et al., Rotational excitation of oriented molecules as a probe of moleculesurface interaction. The Journal of Physical Chemistry, 1991. 95(21): p. 8409-8421.

77. Wiskerke, A.E., et al., Survival mechanism for rotational rainbows in highly attractive moleculesurface systems: NO scattering from Pt(111). Chemical Physics Letters, 1993. 216(1-2): p. 93-99.

78. Cowin, J.P., et al., HD scattering from Pt(111): Rotational excitation probabilities. The Journal of Chemical Physics, 1983. 79(7): p. 3537-3549.

79. Lykke, K.R. and B.D. Kay, Rotational rainbows in the inelastic scattering of $N 2$ from Au(111). Journal of Physics: Condensed Matter, 1991. 3(S): p. S65.

80. Jacobs, D.C. and R.N. Zare, Simplified trajectory method for modeling gas-surface scattering: The NO/Pt(111) system. The Journal of Chemical Physics, 1989. 91(5): p. 3196-3207.

81. Jacobs, D.C., et al., Rotational population and alignment distributions for inelastic scattering and trapping/desorption of NO on Pt(111). The Journal of Chemical Physics, 1989. 91(5): p. 31823195.

82. Ran, Q., et al., An advanced molecule-surface scattering instrument for study of vibrational energy transfer in gas-solid collisions. Review of Scientific Instruments, 2007. 78(10).

83. Moore, J.H., C.C. Davis, and M.A. Coplan, Building scientific apparatus: a practical guide to design and construction. 1989: Addison-Wesley, Advanced Book Program.

84. Scoles, G., Atomic and Molecular Bean Methods: 1. 1988: Oxford University Press, Incorporated.

85. Zaera, F. and G.A. Somorjai, Hydrogenation of ethylene over platinum (111) single-crystal surfaces. Journal of the American Chemical Society, 1984. 106(8): p. 2288-2293. 
86. Aberdam, D., et al., Structural changes of a Pt(111) electrode induced by electrosorption of oxygen in acidic solutions: A coupled voltammetry, LEED and AES study. Surface Science, 1986. 171(2): p. 303-330.

87. Golibrzuch, K., et al., State-to-State Time-of-Flight Measurements of NO Scattering from Au(111): Direct Observation of Translation-to-Vibration Coupling in Electronically Nonadiabatic Energy Transfer. The Journal of Physical Chemistry A, 2013.

88. Huang, Y., et al., Observation of Vibrational Excitation and Deexcitation for NO ( $v=2)$ Scattering from Au(111): Evidence for Electron-Hole-Pair Mediated Energy Transfer. Physical Review Letters, 2000. 84(13): p. 2985-2988.

89. Ory, H.A., A.P. Gittleman, and J.P. Maddox, FRANCK-CONDON FACTORS FOR NO BETA + GAMMA BAND SYSTEMS. Astrophysical Journal, 1964. 139(1): p. 346-\&.

90. Herzberg, G., Spectra of diatomic molecules. 1950: Van Nostrand.

91. Amiot, C., R. Bacis, and G. Guelachvili, Infrared study of the $X 2 \Pi v=0,1,2$ levels of $14 N 160$. Preliminary results on the $v=0,1$ levels of 14N170, 14N180, and 15N160. Canadian Journal of Physics, 1978. 56(2): p. 251-265.

92. Kittrell, C., et al., Two-photon excitation of the $D 1 \Delta-X 1 \Sigma+$ transition in carbon monoxide. The Journal of Chemical Physics, 1983. 78(6): p. 3623-3624.

93. Garetz, B.A., C. Kittrell, and A.C. Le Floch, Analysis of the two-photon $D 1 \Delta-X 1 \Sigma+$ transition in CO: Perturbations in the (10-0) band. The Journal of Chemical Physics, 1991. 94(2): p. 843-853.

94. Bray, R.G. and R.M. Hochstrasser, Two-photon absorption by rotating diatomic molecules. Molecular Physics, 1976. 31(4): p. 1199-1211.

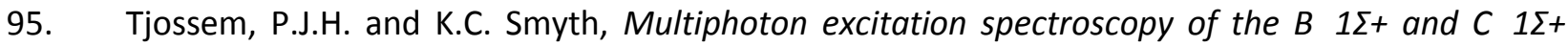
Rydberg states of CO. The Journal of Chemical Physics, 1989. 91(4): p. 2041-2048.

96. Baker, J., et al., A 2+1 REMPI study of the $E-X$ transition in CO. Indirect predissociations in the $E$ $1 \Pi$ state. Chemical Physics, 1993. 178(1-3): p. 569-579.

97. Imhof, R.E., F.H. Read, and S.T. Beckett, Determination of the transition moment of the $B 1 \Sigma+-X$ $1 \Sigma+$ transition in CO. Journal of Physics B: Atomic and Molecular Physics, 1972. 5(4): p. 896.

98. Borst, W.L. and E.C. Zipf, Lifetimes of Metastable CO and <span class="aps-inlineformula" $><$ math $><$ mrow $><m s u b><m$ row $><m i$

mathvariant="normal" $>N</$ mi $></$ mrow $><$ mrow $><m n>2</ m n></$ mrow $></$ msub $></$ mrow $></$ mat $h></$ span $>$ Molecules. Physical Review A, 1971. 3(3): p. 979-989.

99. Lawrence, G.M., Quenching and radiation rates of CO (a 3П). Chemical Physics Letters, 1971. 9(6): p. 575-577.

100. Slanger, T.G. and G. Black, $\mathrm{CO}(a$ 3ח), Its Production, Detection, Deactivation, and Radiative Lifetime. The Journal of Chemical Physics, 1971. 55(5): p. 2164-2173.

101. Johnson, C.E. and R.S. Van Dyck, Lifetime of the a3/l Metastable State of Carbon Monoxide. The Journal of Chemical Physics, 1972. 56(4): p. 1506-1510.

102. Field, R.W., et al., Fine structure and perturbation analysis of the a3ח state of CO. Journal of Molecular Spectroscopy, 1972. 44(2): p. 347-382.

103. Gilijamse, J.J., et al., The radiative lifetime of metastable $C O(a \Pi 3, v=0)$. The Journal of Chemical Physics, 2007. 127(22): p. -.

104. Sitz, G.O., A.C. Kummel, and R.N. Zare, Population and alignment of N2 scattered from Ag(111). Journal of Vacuum Science \&amp; Technology A, 1987. 5(4): p. 513-517.

105. Carleton, K.L., K.H. Welge, and S.R. Leone, Detection of nitrogen rotational distributions by resonant $2+2$ multiphoton ionization through the a1 $\mathrm{mg}$ state. Chemical Physics Letters, 1985. 115(6): p. 492-495.

106. Hanisco, T.F. and A.C. Kummel, Resonantly enhanced multiphoton ionization of nitrogen $a^{\prime \prime} 1 . S I G M A \cdot g+\left(v^{\prime}=v^{\prime \prime}\right) \cdot$ rarw. X1.SIGMA.g+ $\left(v^{\prime \prime}=0-2\right)$. 1. Determination of rotational populations and virtual state character. The Journal of Physical Chemistry, 1991. 95(22): p. 8565-8574. 
107. Cooper, R., et al., Efficient translational excitation of a solid metal surface: State-to-state translational energy distributions of vibrational ground state $\mathrm{HCl}$ scattering from $\mathrm{Au}(111)$. Journal of Vacuum Science \&amp; Technology A, 2009. 27(4): p. 907-912.

108. Hippler, M. and J. Pfab, Detection and probing of nitric oxide (NO) by two-colour laser photoionisation (REMPI) spectroscopy on the $A \leftarrow X$ transition. Chemical Physics Letters, 1995. 243(5-6): p. 500-505.

109. Zacharias, H., R. Schmiedl, and K.H. Welge, State selective step-wise photoionization of NO with mass spectroscopic ion detection. Applied physics, 1980. 21(2): p. 127-133.

110. Cervenan, M.R. and N.R. Isenor, Multiphoton ionization yield curves for gaussian laser beams. Optics Communications, 1975. 13(2): p. 175-178.

111. Luque, J. and D.R. Croslay, LIFBASE: Database and Spectral Simulation Program (Version 1.5). SRI International Report MP, 1999: p. 99-009.

112. Wodtke, A.M., H. Yuhui, and D.J. Auerbach, Insensitivity of trapping at surfaces to molecular vibration. Chemical Physics Letters, 2005. 413(4-6): p. 326-330.

113. Newns, D.M., Fermi-Thomas Response of a Metal Surface to an External Point Charge. The Journal of Chemical Physics, 1969. 50(10): p. 4572-4575.

114. Anderson, P.W., Localized Magnetic States in Metals. Physical Review, 1961. 124(1): p. 41-53.

115. Wodtke, A.M., Y. Huang, and D.J. Auerbach, Interaction of NO(v=12) with LiF(001): Evidence for anomalously large vibrational relaxation rates. The Journal of Chemical Physics, 2003. 118(17): p. 8033-8041.

116. Zacharias, H., et al., Ionization probabilities of $A(2)$ Sigma $(+)\left(v^{\prime}=0,1,2\right)$ and $B-2$ Pi $\left(v^{\prime}=0,2\right)$ states of NO. Journal of Chemical Physics, 1996. 105(1): p. 111-117.

117. Wodtke, A.M., H. Yuhui, and D.J. Auerbach, Observation of orientational influences on vibrational energy transfer at metal surfaces: Rotational cooling associated with vibrational relaxation at a metal surface. Chemical Physics Letters, 2005. 414(1-3): p. 138-142.

118. Golibrzuch, K., et al., The importance of accurate adiabatic interaction potentials for the correct description of electronically nonadiabatic vibrational energy transfer: A combined experimental and theoretical study of $N O(v=3)$ collisions with a $\mathrm{Au}(111)$ surface. Journal of Chemical Physics, 2014. 140(4).

119. Huang, C., M. Pavone, and E.A. Carter, Quantum mechanical embedding theory based on a unique embedding potential. The Journal of Chemical Physics, 2011. 134(15): p. -.

120. Golibrzuch, K., et al., State-to-State Time-of-Flight Measurements of NO Scattering from Au(111): Direct Observation of Translation-to-Vibration Coupling in Electronically Nonadiabatic Energy Transfer. Journal of Physical Chemistry A, 2013. 117(36): p. 8750-8760.

121. Golibrzuch, K., et al., Incidence energy dependent state-to-state time-of-flight measurements of $\mathrm{NO}(v=3)$ collisions with $\mathrm{Au}(111)$ : the fate of incidence vibrational and translational energy. Physical Chemistry Chemical Physics, 2014. 16(16): p. 7602-7610.

122. Baule, B., Theoretische Behandlung der Erscheinungen in verdünnten Gasen. Annalen der Physik, 1914. 349(9): p. 145-176.

123. Logan, R.M. and R.E. Stickney, Simple Classical Model for the Scattering of Gas Atoms from a Solid Surface. The Journal of Chemical Physics, 1966. 44(1): p. 195-201.

124. Beckerle, J.D., et al., Subpicosecond transient infrared spectroscopy of adsorbates. Vibrational dynamics of CO/Pt(111). The Journal of Chemical Physics, 1991. 95(7): p. 5403-5418.

125. Janke Svenja, M., et al., Toward Detection of Electron-Hole Pair Excitation in H-atom Collisions with Au(111): Adiabatic Molecular Dynamics with a Semi-Empirical Full-Dimensional Potential Energy Surface, in Zeitschrift für Physikalische Chemie. 2013. p. 1467.

126. Martin-Gondre, L., et al., Competition between Electron and Phonon Excitations in the Scattering of Nitrogen Atoms and Molecules off Tungsten and Silver Metal Surfaces. Physical Review Letters, 2012. 108(9). 
127. Roth, C., J. Häger, and H. Walther, Scattering of NO molecules from a diamond (110) surface. The Journal of Chemical Physics, 1992. 97(9): p. 6880-6889.

128. Bartels, N., et al., Dynamical steering in an electron transfer surface reaction: Oriented NO( $v=3$, $0.08<E-i<0.89 \mathrm{eV}$ ) relaxation in collisions with a Au(111) surface. Journal of Chemical Physics, 2014. 140(5).

129. Bartels, N., et al., Observation of orientation-dependent electron transfer in molecule-surface collisions. Proceedings of the National Academy of Sciences of the United States of America, 2013. 110(44): p. 17738-17743.

130. Huang, P. and E.A. Carter, Self-consistent embedding theory for locally correlated configuration interaction wave functions in condensed matter. The Journal of Chemical Physics, 2006. 125(8): p. -.

131. Morrill, J.S., et al., The (X (2)Pi(g)) ns sigma(g) (1,3)Pi(g) Rydberg states of O-2: Spectra, structures, and interactions. Journal of Chemical Physics, 1999. 111(1): p. 173-185.

132. Schafer, T., et al., Observation of direct vibrational excitation in gas-surface collisions of $\mathrm{CO}$ with Au(111): a new model system for surface dynamics. Physical Chemistry Chemical Physics, 2013. 15(6): p. 1863-1867.

133. Peterson, A.A., et al., Finite-Size Effects in $O$ and CO Adsorption for the Late Transition Metals. Topics in Catalysis, 2012. 55(19-20): p. 1276-1282.

134. Bartels, N., et al., Production of a beam of highly vibrationally excited CO using perturbations. The Journal of Chemical Physics, 2012. 136(21): p. - .

135. Wurm, S., P. Feulner, and D. Menzel, Resonance-enhanced multiphoton ionization spectroscopy of X(1)Sigma(+) and a (3)Pi carbon monoxide using electron stimulated desorption as a source for rovibronically excited species. Journal of Chemical Physics, $1996.105(16):$ p. 6673-6687.

136. Harris, J. and B. Kasemo, ON PRECURSOR MECHANISMS FOR SURFACE-REACTIONS. Surface Science, 1981. 105(2-3): p. L281-L287.

137. Christmann, K., G. Ertl, and T. Pignet, Adsorption of hydrogen on a Pt(111) surface. Surface Science, 1976. 54(2): p. 365-392.

138. Ertl, G., M. Neumann, and K.M. Streit, Chemisorption of CO on the Pt(111) surface. Surface Science, 1977. 64(2): p. 393-410.

139. Hopster, H. and H. Ibach, Adsorption of CO on Pt(111) and Pt 6(111) $\times$ (111) studied by high resolution electron energy loss spectroscopy and thermal desorption spectroscopy. Surface Science, 1978. 77(1): p. 109-117.

140. Campbell, C.T., et al., A molecular beam study of the catalytic oxidation of CO on a Pt(111) surface. The Journal of Chemical Physics, 1980. 73(11): p. 5862-5873.

141. Heyden, B.E. and A.M. Bradshaw, The adsorption of CO on Pt(111) studied by infrared reflection-Absortion spectroscopy. Surface Science, 1983. 125(3): p. 787-802.

142. Feibelman, P.J., et al., The CO/Pt(111) puzzle. Journal of Physical Chemistry B, 2001. 105(18): p. 4018-4025.

143. Campbell, C.T., et al., A molecular beam investigation of the interactions of CO with a Pt(111) surface. Surface Science, 1981. 107(1): p. 207-219.

144. Collins, D.M. and W.E. Spicer, The adsorption of $\mathrm{CO}, \mathrm{O} 2$, and $\mathrm{H} 2$ on Pt: I. Thermal desorption spectroscopy studies. Surface Science, 1977. 69(1): p. 85-113.

145. Lin, T.H. and G.A. Somorjai, Modulated molecular beam scattering of CO and NO from Pt(111) and the stepped Pt(557) crystal surfaces. Surface Science, 1981. 107(2-3): p. 573-585.

146. McCabe, R.W. and L.D. Schmidt, binding states of $\mathrm{CO}$ and $\mathrm{H} 2$ on clean and oxidized (111)Pt. Surface Science, 1977. 65(1): p. 189-209.

147. Norton, P.R., J.W. Goodale, and E.B. Selkirk, Adsorption of co on Pt(111) studied by photoemission, thermal desorption spectroscopy and high resolution dynamic measurements of work function. Surface Science, 1979. 83(1): p. 189-227. 
148. HORN, K. and J. PRITCHARD, INFRARED SPECTRA OF ORDERED AND DISORDERED OVERLAYERS ON METALS : CARBON MONOXIDE ON A PLATINUM(111) SINGLE CRYSTAL SURFACE. J. PhyS. Colloques, 1977. 38(C4): p. C4-164-C4-171.

149. Hayden, B.E. and A.M. Bradshaw, The Adsorption of Co on Pt(111) Studied by Infrared ReflectionAbsorption Spectroscopy. Surface Science, 1983. 125(3): p. 787-802.

150. Lin, T.H. and G.A. Somorjai, Modulated Molecular-Beam Scattering of Co and No from Pt(111) and the Stepped Pt(557) Crystal-Surfaces. Surface Science, 1981. 107(2-3): p. 573-585.

151. Winicur, D.H., et al., Co Desorption and Adsorption on Pt(111). Surface Science, 1981. 109(1): p. 263-275.

152. Steininger, H., S. Lehwald, and H. Ibach, On the Adsorption of Co on Pt(111). Surface Science, 1982. 123(2-3): p. 264-282.

153. Polanyi, J.C. and W.H. Wong, Location of Energy Barriers. I. Effect on the Dynamics of Reactions $A+B C$. The Journal of Chemical Physics, 1969. 51(4): p. 1439-1450.

154. Compton, R.N., et al., Threshold Electron Impact Excitation of Atoms and Molecules: Detection of Triplet and Temporary Negative Ion States. The Journal of Chemical Physics, 1968. 48(2): p. 901909.

155. Ehrhardt, H., et al., Resonance Scattering of Slow Electrons from $\mathrm{H} 2$ and Co Angular Distributions. Physical Review, 1968. 173(1): p. 222-\&.

156. Janda, K.C., et al., Direct-inelastic and trapping-desorption scattering of $\mathrm{N} 2$ and $\mathrm{CH} 4$ from Pt(111). Surface Science, 1983. 130(2): p. 395-409.

157. Lykke, K.R. and B.D. Kay, Two-photon spectroscopy of N2: Multiphoton ionization, laser-induced fluorescence, and direct absorption via the $a^{\prime} 1 \Sigma+g$ state. The Journal of Chemical Physics, 1991. 95(4): p. 2252-2258.

158. Rettner, C.T. and N.R. Ashfold, Dynamics of gas-surface interactions. 1991: Royal Society of Chemistry.

159. Redhead, P.A., Thermal desorption of gases. Vacuum, 1962. 12(4): p. 203-211.

160. Gil, A., et al., Site preference of CO chemisorbed on Pt(111) from density functional calculations. Surface Science, 2003. 530(1-2): p. 71-86.

161. Hu, Q.M., K. Reuter, and M. Scheffler, Towards an exact treatment of exchange and correlation in materials: Application to the "CO adsorption puzzle" and other systems. Physical Review Letters, 2007. 98(17).

162. Hammer, B., Y. Morikawa, and J.K. Norskov, CO chemisorption at metal surfaces and overlayers. Physical Review Letters, 1996. 76(12): p. 2141-2144.

163. Sun, J.W., et al., Improved lattice constants, surface energies, and CO desorption energies from a semilocal density functional. Physical Review B, 2011. 83(12).

164. Ren, X.G., P. Rinke, and M. Scheffler, Exploring the random phase approximation: Application to CO adsorbed on $\mathrm{Cu}(111)$. Physical Review B, 2009. 80(4).

165. Schimka, L., et al., Accurate surface and adsorption energies from many-body perturbation theory. Nature Materials, 2010. 9(9): p. 741-744.

166. Feibelman, P.J., et al., The CO/Pt(111) Puzzlet. The Journal of Physical Chemistry B, 2000. 105(18): p. 4018-4025.

167. Blackman, G.S., et al., Mix of Molecular Adsorption Sites Detected for Disordered Co on Pt(111) by Diffuse Low-Energy Electron-Diffraction. Physical Review Letters, 1988. 61(20): p. 2352-2355.

168. Ogletree, D.F., M.A. Vanhove, and G.A. Somorjai, Leed Intensity Analysis of the Structures of Clean Pt(111) and of Co Adsorbed on Pt(111) in the C(4x2) Arrangement. Surface Science, 1986. 173(2-3): p. 351-365.

169. Hayden, B.E., et al., An Infrared Study of the Adsorption of Co on a Stepped Platinum Surface. Surface Science, 1985. 149(2-3): p. 394-406. 
170. Hermann, K., Appendix E: Parameter Tables of Crystals, in Crystallography and Surface Structure. 2011, Wiley-VCH Verlag GmbH \& Co. KGaA. p. 265-266.

171. Rettner, C.T., E.K. Schweizer, and C.B. Mullins, Desorption and Trapping of Argon at a $2 h-W(100)$ Surface and a Test of the Applicability of Detailed Balance to a Nonequilibrium System. Journal of Chemical Physics, 1989. 90(7): p. 3800-3813.

172. Harris, J. and A.C. Luntz, Sticking and Scattering in the Molecular Chemisorption Regime - Co on Pt(111). Journal of Chemical Physics, 1989. 91(10): p. 6421-6428.

173. Campbell, C.T., et al., A Molecular-Beam Investigation of the Interactions of Co with a Pt(111)Surface. Surface Science, 1981. 107(1): p. 207-219.

174. Verheij, L.K., et al., A molecular beam study of the interaction of CO molecules with a Pt(111) surface using pulse shape analysis. Surface Science, 1987. 182(3): p. 390-410.

175. Serri, J.A., J.C. Tully, and M.J. Cardillo, The Influence of Steps on the Desorption-Kinetics of No from Pt(111). Journal of Chemical Physics, 1983. 79(3): p. 1530-1540.

176. Serri, J.A., M.J. Cardillo, and G.E. Becker, A Molecular-Beam Study of the No Interaction with Pt(111). Journal of Chemical Physics, 1982. 77(4): p. 2175-2189.

177. Reuttrobey, J.E., et al., Microscopic Co Diffusion on a Pt(111) Surface by Time-Resolved InfraredSpectroscopy. Physical Review Letters, 1988. 61(24): p. 2778-2781.

178. Reuttrobey, J.E., et al., Co Diffusion on Pt(111) with Time-Resolved Infrared-Pulsed MolecularBeam Methods - Critical Tests and Analysis. Journal of Chemical Physics, 1990. 93(12): p. 91139129.

179. Tully, J.C., The dynamics of adsorption and desorption. Surface Science, 1994. 299-300(0): p. 667-677.

180. Lahee, A.M., J.P. Toennies, and C. Woll, Low-Energy Adsorbate Vibrational-Modes Observed with Inelastic Helium Atom Scattering - Co on Pt(111). Surface Science, 1986. 177(2): p. 371-388.

181. Fischer-Wolfarth, J.-H., et al., An improved single crystal adsorption calorimeter for determining gas adsorption and reaction energies on complex model catalysts. Review of Scientific Instruments, 2011. 82(2): p. -.

182. Yeo, Y.Y., L. Vattuone, and D.A. King, Calorimetric heats for $\mathrm{CO}$ and oxygen adsorption and for the catalytic CO oxidation reaction on Pt\{111\}. The Journal of Chemical Physics, 1997. 106(1): p. 392401.

183. Ladislas Wiza, J., Microchannel plate detectors. Nuclear Instruments and Methods, 1979. 162(1): p. 587-601.

184. Mantz, A.W., et al., Ground state molecular constants of 12C160. Journal of Molecular Spectroscopy, 1975. 57(1): p. 155-159.

185. Tilford, S.G. and J.T. Vanderslice, High resolution vacuum ultraviolet absorption spectra of the

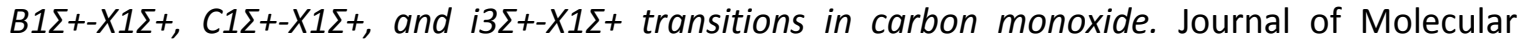
Spectroscopy, 1968. 26(4): p. 419-431.

186. Lofthus, A. and P.H. Krupenie, The spectrum of molecular nitrogen. Journal of Physical and Chemical Reference Data, 1977. 6(1): p. 113-307. 


\section{Curriculum Vitae}

\section{Personal details:}

Diplom-Chemiker Kai Golibrzuch

Gerhart-Hauptmann-Straße 8

37154 Northeim

Birthday:

Nationality:

Family status:

Education:

1992-1996

1996-1998

1998-2005

03/2006- $12 / 2010$

03/2010-09/2010

01/2011-09/2014

Military training:

01.07.2005-31.03.2006

Further education:

10/2011

$10 / 2011-09 / 2013$
04.02.1986 in Northeim

German

married

Elementary school Höckelheim

Thomas-Mann-school Northeim

Gymnasium Corvinianum Northeim, A level with grade 1.9

Undergraduate studies in Chemistry at Georg-August-University Göttingen

Diploma degree (1.0)

Diploma thesis in the group of Prof. Jürgen Troe/ Prof. Thomas Lenzer on „Untersuchungen zur Photochemie von $\beta$-Carotin-Derivaten“

Awarded with the book price of the Bunsen-Society

Graduate studies at Georg-August-University Göttingen in the group of Prof. Alec M. Wodtke on "Quantum-state resolved scattering of molecules from surfaces"

Panzerpionierbataillon 1 in Holzminden

Training at Sirah Laser- und Plasmatechnik on the Matisse single-mode cw ring laser

Distance studies on lasertechnology at the Friedrich-Schiller University Jena, instructed by Doz. Dr. G. Staupendahl 